\title{
Natural Gas Annual 1993 Supplement: Company Profiles
}

\author{
February 1995
}

\author{
Energy Information Administration \\ Office of Oil and Gas \\ U.S. Department of Energy \\ Washington, DC 20585
}

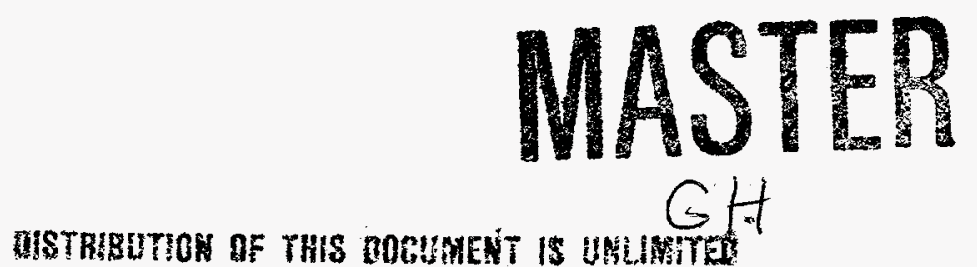




\section{Contacts}

The Natural Gas Annual Supplement is prepared by the Energy Information Administration, Office of Oil and Gas, Reserves and Natural Gas Division.

General information for this document may be obtained from Kendrick E. Brown, Jr. (202/586-6077), Chief of the Data Operations Branch. Questions and comments concerning the contents of the Natural Gas Annual Supplement may be directed to Ann M. Ducca (202/586-
6137) and Margo Natof (202/586-6303). Inquiries about specific companies and data presented in the tables may be referred to Ellis Maupin (202/586-6178), Sylvia Norris (202/586-6106) and Dolly Tolson (202/586-6664).

The data reported on the Form EIA-176, "Annual Report of Natural and Supplemental Gas Supply and Disposition," are available on diskette. Contact Sheila Darnell (202/586-6165) for information about diskettes. 


\section{DISCLAIMER}

This report was prepared as an account of work sponsored by an agency of the United States Government. Neither the United States Government nor any agency thereof, nor any of their employees, make any warranty, express or implied, or assumes any legal liability or responsibility for the accuracy, completeness, or usefulness of any information, apparatus, product, or process disclosed, or represents that its use would not infringe privately owned rights. Reference herein to any specific commercial product, process, or service by trade name, trademark, manufacturer, or otherwise does not necessarily constitute or imply its endorsement, recommendation, or favoring by the United States Government or any agency thereof. The views and opinions of authors expressed herein do not necessarily state or reflect those of the United States Government or any agency thereof. 


\section{DISCLAIMER}

Portions of this document may be illegible in electronic image products. Images are produced from the best available original document. 


\section{Preface}

The Natural Gas Annual provides information on the supply and disposition of natural gas to a wide audience including industry, consumers, Federal and State agencies, and educational institutions. This report, the Natural Gas Annual 1993 Supplement: Company Profiles, presents a detailed profile of selected companies.

The data in the Natural Gas Annual publications are taken from surveys conducted by the Energy Information Administration (EIA), U.S. Department of Energy (DOE), to fulfill its responsibilities for gathering and reporting energy data. All of the information presented in this volume is taken from the mandatory Form EIA-176, "Annual Report of Natural and Supplemental Gas Supply and Disposition." Data collected on the Form EIA-176 are not proprietary.

All volumes of natural gas in this publication are reported at 14.73 pounds per square inch absolute and 60 degrees Fahrenheit, except where noted. A glossary of terms is provided to assist users in understanding the data presented. A description of the data collection surveys appears in Appendix A of the Natural Gas Annual 1993. 



\section{Contents}

Overview $\ldots \ldots \ldots \ldots \ldots \ldots \ldots \ldots \ldots \ldots \ldots \ldots \ldots \ldots \ldots \ldots$

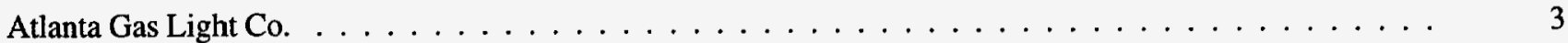

Baltimore Gas and Electric $C_{0} \ldots \ldots \ldots \ldots \ldots \ldots \ldots \ldots \ldots \ldots \ldots$

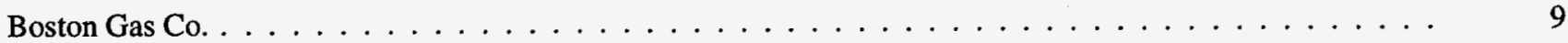

Brooklyn Union Gas Co. . . . . . . . . . . . . . . . . . . . . . 11

Coastal Corp . . . . . . . . . . . . . . . . . . . . . . . . . . 13

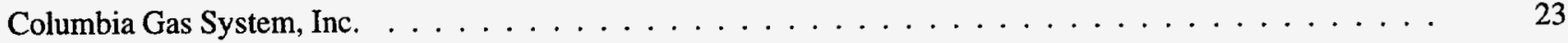

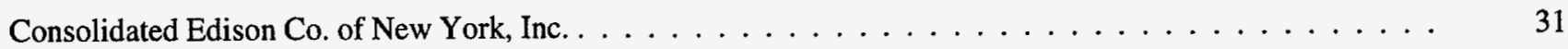

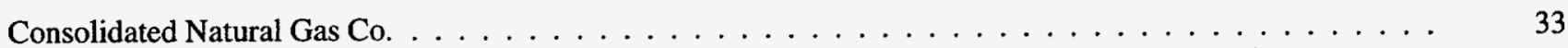

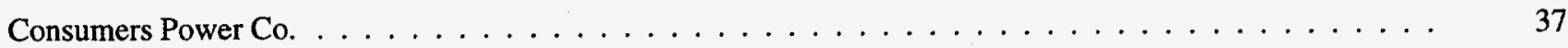

El Paso Natural Gas Co. . . . . . . . . . . . . . . . . . . . . . . . . . . 39

Enron Corp. . . . . . . . . . . . . . . . . . . . . . . 43

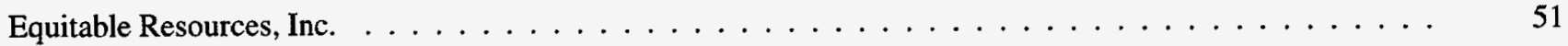

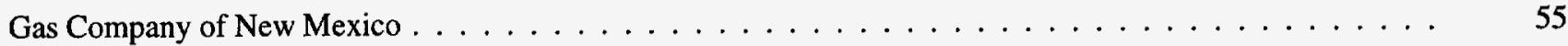

Iroquois Gas Transmission System $\ldots \ldots \ldots \ldots \ldots \ldots \ldots \ldots \ldots \ldots \ldots \ldots$

K N Energy, Inc. . . . . . . . . . . . . . . . . . . . . . . . . . . . . . . . 59

Koch Gateway Pipeline $\mathrm{Co} \ldots \ldots \ldots \ldots \ldots \ldots \ldots \ldots$

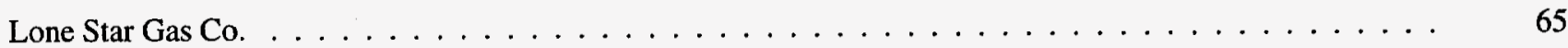

Michigan Consolidated Gas Co. . . . . . . . . . . . . . . . . . . . . . . . . . 69

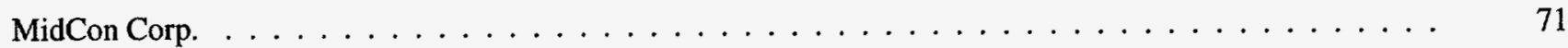

National Fuel Gas Co. . . . . . . . . . . . . . . . . . . . . . . 77

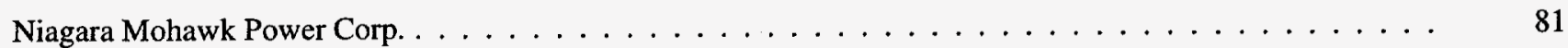

NorAm Energy Corp., Inc. . . . . . . . . . . . . . . . . . . . . . . . . . . . . . 83

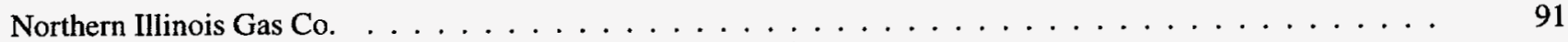

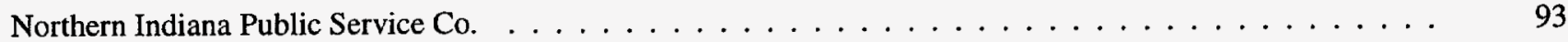

Northwest Natural Gas Co. . . . . . . . . . . . . . . . . . . . . . . . . . 95

ONEOK Inc. . . . . . . . . . . . . . . . . . . . . . . . . . . . . . . 97

Pacific Gas and Electric Co . . . . . . . . . . . . . . . . . . . . . . . . . 101

Panhandle Eastern Corp. . . . . . . . . . . . . . . . . . . . . . . . . 105

Peoples Gas Light and Coke Co. . . . . . . . . . . . . . . . . . . . . . 115

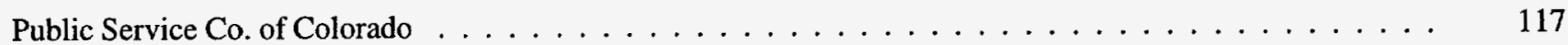

Public Service Electric and Gas Co. . . . . . . . . . . . . . . . . . . . . . . . . 119 
Questar Corp. .

Sabine Pipe line Co.

San Diego Gas and Electric Co.

Southern California Gas Co.

Southern Natural Gas Co.

Southwest Gas Corp

Tenneco, Inc.

Transco Gas Co.

Valero Energy Corp.

Washington Gas Light Co.

Western Resources, Inc.

The Williams Companies, Inc.

Williston Basin Interstate Pipeline Co.

Wisconsin Gas Co.

Appendices

A. Summary of Data Collection and Report Methodology

B. Selected Natural Gas and Related Reports 
1. Atlanta Gas Light Co., Natural Gas Data, 1993

2. Baltimore Gas and Electric Co., Natural Gas Data, $1993 \ldots \ldots \ldots$

3. Boston Gas Co., Natural Gas Data, 1993

4. Brooklyn Union Gas Co., Natural Gas Data, 1993

5. Coastal Corp., Natural Gas Data, 1993

Coastal Corp., Interstate Flows of Natural Gas, $1993 \ldots \ldots \ldots \ldots \ldots$

Columbia Gas System, Inc., Natural Gas Data, 1993.

Columbia Gas System, Inc., Interstate Flows of Natural Gas, 1993

Consolidated Edison Co. of New York, Inc. Natural Gas Data, 1993

10.

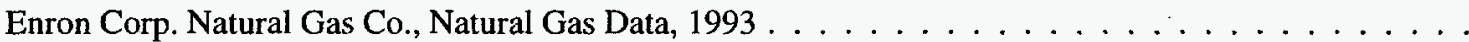

Enron Corp. Natural Gas Co., Interstate Flows of Natural Gas, $1993 \ldots \ldots$. . . . . . . . . . . . . .

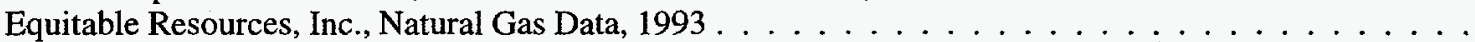

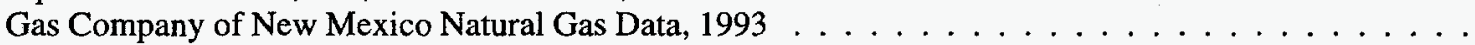

Iroquois Gas Transmission System Natural Gas Data, 1993

K N Energy, Inc., Natural Gas Data, 1993

K N Energy, Inc., Interstate Flows of Natural Gas $1993 \ldots \ldots \ldots$

Koch Gateway Pipeline Co., Natural Gas Data, $1993 \ldots \ldots \ldots \ldots \ldots$

Koch Gateway Pipeline Co., Interstate Flows of Natural Gas, 1993 . . . . . . . . . . . . . . . . . .

Lone Star Gas Co., Natural Gas Data, 1993

Michigan Consolidated Gas Co., Natural Gas Data, $1993 \ldots \ldots \ldots$

MidCon Corp., Natural Gas Data, $1993 \ldots \ldots \ldots \ldots \ldots \ldots \ldots$

MidCon Corp., Interstate Flows of Natural Gas, $1993 \ldots \ldots \ldots$. . . . . . . . . . . . . . . .

National Fuel Gas Co., Natural Gas Data, 1993

National Fuel Gas Co., Interstate Flows of Natural Gas, $1993 \ldots \ldots \ldots \ldots$

Niagara Mohawk Power Corp., Natural Gas Data, $1993 \ldots \ldots$. . . . . . . . . . . . . .

NorAm Energy Corp., Inc., 1993

NorAm Energy Corp., Inc., Interstate Flows of Natural Gas Data, $1993 \ldots \ldots \ldots$

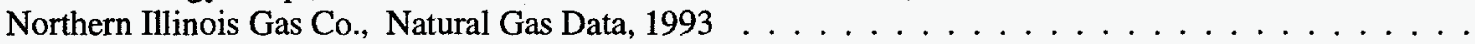

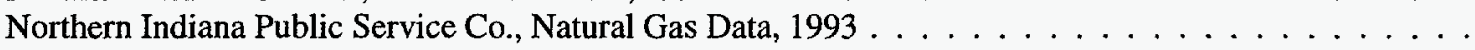

Northwest Natural Gas Co., Natural Gas Data, 1993.

ONEOK, Inc., Natural Gas Data, 1993

Pacific Gas and Electric Co., Natural Gas Data, 1993

38.

Pacific Gas and Electric Co., Interstate Flows of Natural Gas, 1993 . . . . . . . . . . . . . . .

39.

Panhandle Eastern Corp., Natural Gas Data, 1993

40.

Panhandle Eastern Corp., Interstate Flows of Natural Gas, 1993 . . . . . . . . . . . . . . . . .

Peoples Gas Light and Coke Co., Natural Gas Data, 1993

Public Service Co. of Colorado Natural Gas Data, $1993 \ldots \ldots$. . . . . . . . . . . . . . . . .

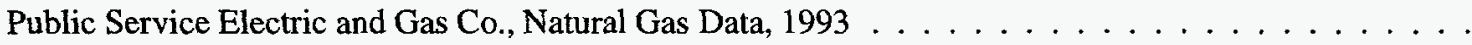


50. Southern Natural Gas Co., Interstate Flows of Natural Gas, 1993 . . . . . . . . . . . . . . . . . . . . . . . . . . . . . . .

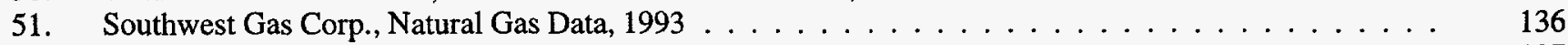

52. Southwest Gas Corp., Interstate Flows of Natural Gas, $1993 \ldots \ldots \ldots$

53. Tenneco, Inc., Natural Gas Data, $1993 \ldots \ldots \ldots$

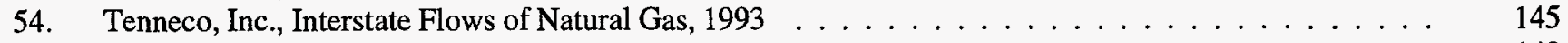

55. Transco Gas Co., Natural Gas Data, $1993 \ldots \ldots \ldots$

56. Transco Gas Co., Interstate Flows of Natural Gas, $1993 \ldots \ldots \ldots$

57. Valero Energy Corp., Natural Gas Data, $1993 \ldots \ldots \ldots$

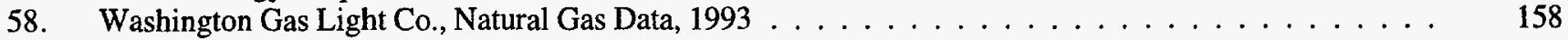

59. Washington Gas Light Co., Interstate Flows of Natural Gas, 1993 . . . . . . . . . . . . . . . . . . 159

60. Western Resources Inc., Natural Gas Data, $1993 \ldots \ldots \ldots$

61. The Williams Companies, Inc., Natural Gas Data, 1993 . . . . . . . . . . . . . . . . . . . . . . . . . 164

62. The Williams Companies, Inc., Interstate Flows of Natural Gas, $1993 \ldots \ldots$

64. Williston Basin Interstate Pipeline Co., Natural Gas Data, 1993 . . . . . . . . . . . . . . . . . . . . . 168

64. Williston Basin Interstate Pipeline Co., Interstate Flows of Natural Gas, 1993 . . . . . . . . . . . . 171

65. Wisconsin Gas Co., Natural Gas Data, $1993 \ldots \ldots \ldots$

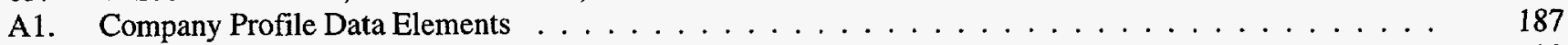

A2. Alphabetical Listing of Affiliate Companies . . . . . . . . . . . . . . . . . . 188 


\section{Illustrations}

1. Atlanta Gas Light Co., 1993

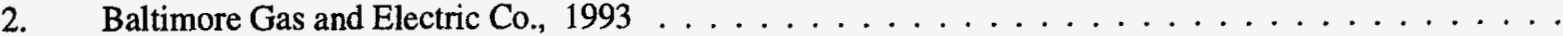

Boston Gas Co., 1993

Brooklyn Union Gas Co., $1993 \ldots \ldots \ldots \ldots \ldots \ldots$

5. Coastal Corp., 1993

Consolidated Edison Co. of New York, Inc., $1993 \ldots \ldots \ldots \ldots \ldots \ldots$ Consolidated Natural Gas Co., 1993

10. El Paso Natural Gas Co., 1993

16. K N Energy, Inc., 1993

17. Koch Gateway Pipeline Co., 1993

18. Lone Star Gas Co., 1993

19. Michigan Consolidated Gas Co., 1993

20. MidCon Corp., 1993

21. National Fuel Gas Co., 1993

22. Niagara Mohawk Power Corp., 1993

23. NorAm Energy Corp., Inc., 1993 


\section{Overview}

A complex grid of natural gas pipelines, extending a quarter of a million miles, crisscrosses the Nation and connects all States except Alaska, Hawaii, and Vermont. This extensive network reaches more than 50 million residential customers and more than 4 million other customers. The demand along this grid is met by the operations of natural gas pipeline companies and local distribution companies.

The companies appearing in this report, the Natural Gas Annual 1993 Supplement: Company Profiles, are major interstate natural gas pipeline companies, large distribution companies, or combination companies with both pipeline and distribution operations. The report contains profiles of 45 corporate families. The profiles describe briefly each company, where it operates, and selected issues that the company faces.

The tables accompanying each profile present data reported on Form EIA-176, "Annual Report of Natural and Supplemental Gas Supply and Disposition." Other sources of information include industry literature and corporate annual reports.

Companies were selected for the profiles in a multistep process using knowledge of the industry and specific information included on Form EIA-176 submissions. First, the 20 interstate pipeline companies (representing distinct corporate families) with the greatest reported volumes of gas transported were selected. The other companies, not in the same corporate family as any of the first group, were selected from the top companies in volumes delivered to end users. Finally, additional companies belonging to the same corporate family as companies in either of the first two groups were included in the profiles.

Form EIA-176 is submitted by all respondents from an identified universe of operators of fields, wells, or natural gas processing plants that distribute gas to end users or transport gas to or across a State border; operators of synthetic natural gas plants; natural gas distributors; natural gas pipeline companies; and companies that operate underground natural gas storage facilities. Each respondent reports a supply and disposition balance for each State in which he operates. For the 1993 survey, 2,039 responses were received from approximately $1,800 \mathrm{com}-$ panies.

Data are reported on a custody basis. All volumes of natural gas physically received onsystem are reported regardless of ownership. Concurrently, all volumes owned by the respondent company but not in its possession are not reported. Thus, double-counting is avoided. Natural gas not owned but in the possession of the respondent is reported as received or transported for the account of others, as appropriate. Exchange agreements are treated similarly.

The purpose of this report is to show the movement of natural gas through the various States served by the 45 large companies profiled. The companies in the report are interstate pipeline companies or local distribution companies (LDC's). Interstate pipeline companies acquire gas supplies from company-owned production, purchases from producers, and receipts for transportation for the account of others.

The Federal Energy Regulatory Commission (FERC) promulgated its Order 636, the restructuring order for the natural gas industry. It provides for the "unbundling" of all services and rates for those services. More specifically, pipeline companies were required by the new rule to separate transmission and storage services from the sale of natural gas. All pipeline companies were required to file a plan to "unbundle" the product and services, and upon FERC approval of the plan, to implement the plan on November 1, 1993.

LDC's deliver natural gas directly to consumers, including individual residences, commercial operations, industrial facilities, and electric utilities. The LDC's that are the leading suppliers of natural gas to consumers are: Atlanta Gas Light Co., serving the Atlanta and Chattanooga areas; Consolidated Edison Co. of New York, Inc., serving New York City; Consumers Power Co., serving the central area of Michigan's Lower Peninsula; Michigan Consolidated Gas Co., serving the metropolitan area of Detroit and northern and western Michigan; Northern Illinois Gas Co., serving the northern third of Illinois, except Chicago; Northern Indiana Public Service Co., serving the northern third of Indiana; Pacific Gas and Electric Co., serving northern and central California; Peoples Gas Light and Coke Co., serving the city of Chicago and nearby areas in Northeastern Illinois; Public Service Electric and Gas Co., serving northern and central New Jersey; and Southern California Gas Co., serving southern California.

Natural gas company profiles are presented with pipeline system and service area maps. Company supply and disposition data are presented for the States of operation as reported on the Form EIA-176. 



\section{Atlanta Gas Light Co.}

Atlanta Gas Light Company, incorporated in 1856, is the oldest corporation in Georgia to operate continuously under its original charter. It is the largest natural gas distribution company in the Southeastern United States and the eighth largest in the United States. While its principal business is natural gas, the company operates in several diverse areas.

During 1993, Atlanta Gas Light served 1,237,000 customers in 228 communities in Georgia, an increase over 1992 of approximately 2,000 commercial and 30,500 residential customers. Through its wholly owned subsidiary, Chattanooga Gas Company, 45,000 customers in Chattanooga and Cleveland, Tennessee were served.
As of September 1993, the Atlanta Gas Light natural gas system consisted of 23,893 miles of pipeline and the equivalent of 6 billion cubic feet of liquified natural gas (LNG), storage capacity in three LNG storage facilities. Chattanooga Gas' system was comprised of 1,216 miles of pipeline and the equivalent of 1 billion cubic feet of LNG storage capacity in its facility.

In 1993, Atlanta Gas Light significantly expanded its efforts to promote the use of natural gas vehicles (NGV) and natural gas cooling equipment. Federal and State programs initiated in 1993 served to bolster the use of NGV's in Georgia. The Department of Energy designated Atlanta as the first city in its "Clean Cities" program,

Figure 1. Atlanta Gas Light Co., 1993

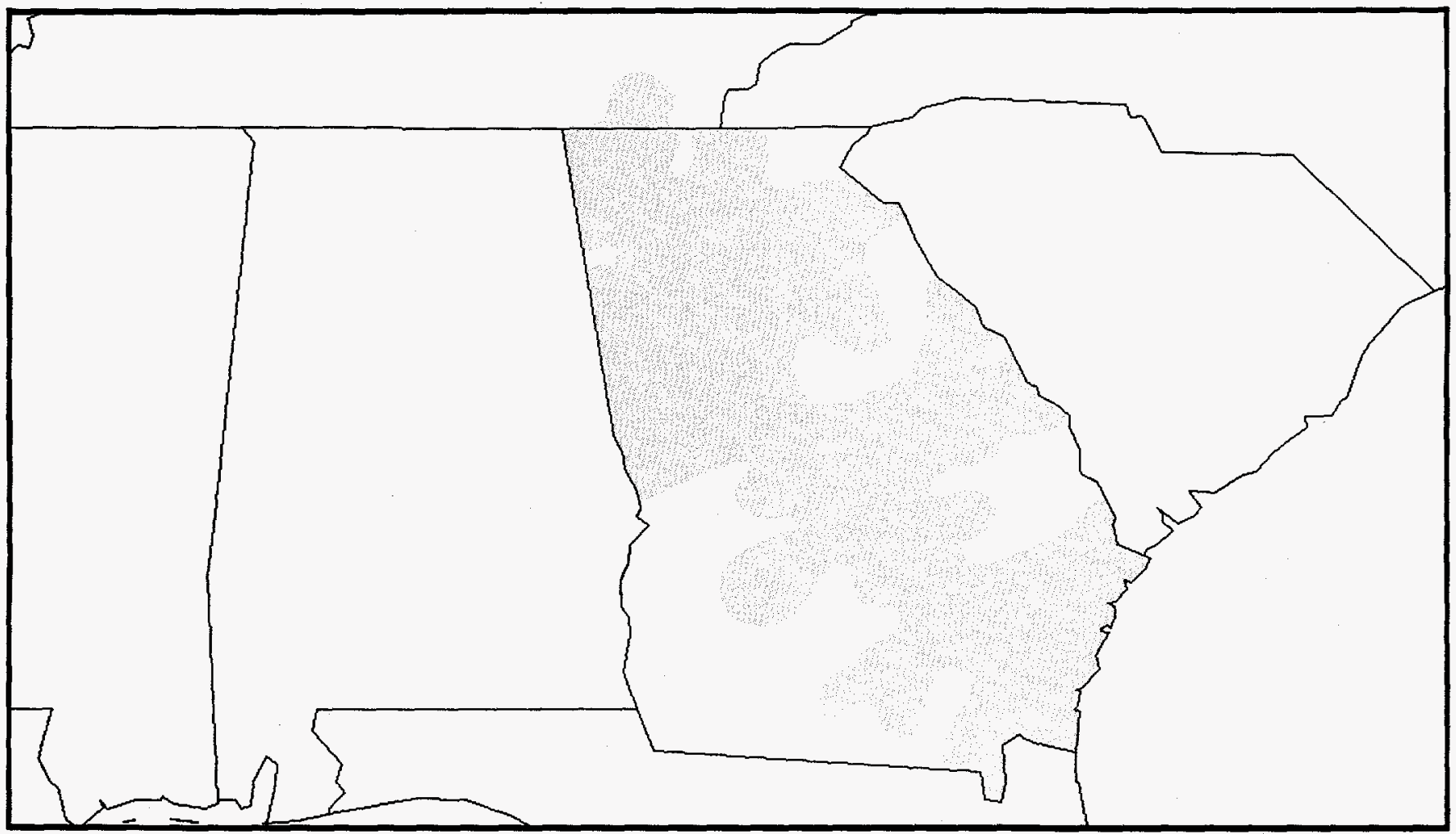

Source: Energy Information Administration (EIA), Form EIA-176, "Annual Report of Natura! and Supplemental Gas Supply and Disposition."

Service Area 
and the State of Georgia awarded 16 1-year grants to convert nearly 300 vehicles to alternative fuels (all chose natural gas). Within Georgia alone, local, State, and Federal government agencies added approximately 400 natural gas-powered cars and trucks to their fleets. During 1993, nearly 1,000 NGV's were on the road in Georgia, roughly doubling that of 1992. Atlanta Gas Light anticipates that Georgia will add another 1,000 NGV's to agency fleets in 1994.

In addition, the NGV Southeast Technology Center, which opened in Atlanta in 1993, gives customers access to the latest NGV technologies and vehicle conversion services. The company also provides natural gas for a number of NGV customers under a program that allows the company to install at no up-front cost an on-site refueling station that accommodates up to 50 fleets. In addition, the company continues to work with fuel outlet owners to open public refueling stations. The two efforts will soon make it possible to drive from one end of the company service area to the other with easy access to compressed natural gas (CNG). At the end of 1993, the Atlanta area had seven NGV public refueling stations in operation.

Atlanta Gas Light currently heads an international consortium developing an adsorbed natural gas (ANG) storage system to replace the CNG system currently used in NGV's. The ANG system will store the gas in a flat, extruded aluminum tank containing a carbon adsorbent. Such tanks can be more easily fitted into various vehicle designs and should be cost-competitive with conventional CNG storage units. DOE has awarded the consortium federal funding for a demonstration project. Based in part on the demonstration results, the consortium will decide within 2 years whether to pursue commercialization of this technology.

Successes in the cooling market in 1993 included: an Atlanta condominium complex at which a 450-ton electric chiller was replaced with three 150-ton engine-driven gas chilling units that are expected to save condominium owners a total of $\$ 60,000$ annually; a new Nestle's distribution center at which 600 tons of gas-cooling equipment was installed that is expected to cut operating costs by about 50 percent; and installation of natural gas desiccant cooling in Cub Foods' grocery stores throughout Georgia. In 1994, Wal-Mart plans to use desiccant cooling in all of its Super Sam's Warehouses in Georgia, and the Medical College of Georgia is installing a large system in one of its facilities.

During 1994, Atlanta Gas Light company expects to enter into several agreements involving large-scale cogeneration projects, and is also optimistic that it will receive some of the electric generation peaking plant business in Georgia. 
Table 1. Atlanta Gas Light Co., Natural Gas Data, 1993

(Thousand Cubic Feet)

\begin{tabular}{r|r|r|r|}
\hline Supply/Disposition & Atlanta Gas Light Co. & Chattanooga Gas Co. & Georgia \\
\cline { 2 - 4 }
\end{tabular}

\section{SUPPLY}

Produced Onsystem

Receipts

Purchases ...

Transpontedfexchange Gas

inters

$175,524,695$

$70,103,559$

$11,308,939$

$4,451,646$

Company-owned Gas

Transported/Exchange Gas

Storage Withdrawals

Received from Storage Operators

$70,103,559$

$4,451,646$

Other Supplies

0
0
$1,551,993$
0

168,732

Total Supplies

$247,348,979$

0
0
529,031
910,703
0

DISPOSmON

Lease and Plant Use

.

Interstate Movements

Company-owned Gas

Transported/Exchange Gas

Exchange Gas Deliveries

Storage Iniections

Deliveries of Company-owned Gas

To Storage Operators

To Other Pipelines

To Resellers ...

To Consumers

Residential

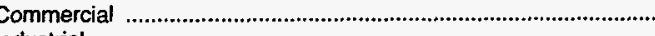

Industrial .......

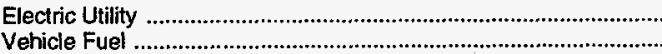

Transported for the Account of Others

To Storage Operators

To Other Pipelines

To Resellers

To Resellers
To Consumers

Residential.

Commerciat

Industrial

Electric Utility

Electric Utility

Pipeline/Compressor Fuel

Other Disposition

Unaccounted For

(1)

$17,200,319$

Total Dispos

$\begin{array}{rr}0 & 0 \\ 0 & 0 \\ 0 & 0 \\ 0 & 0 \\ 0 & 0 \\ 1,521,699 & 809,237 \\ 0 & 124,598 \\ 0 & 0 \\ 0 & 0 \\ & \\ 96,176,275 & 3,452,236 \\ 43,653,576 & 3,125,739 \\ 29,309,876 & 5,053,575 \\ 3,079,567 & 0 \\ 0 & 0 \\ 0 & 0 \\ 0 & 0 \\ 0 & 0 \\ 0 & 0 \\ 8,487,453 & 0 \\ 63,813,195 & 0 \\ 0 & 174,262 \\ 0 & 0 \\ 826,965 & 0,451,646 \\ 0 & 0 \\ 3,480,373 & 0,319\end{array}$

Source: Energy Information Administration (EIA), Form EIA-176, "Annual Report of Natural and Supplemental Gas Suppiy and Disposition." 



\section{Baltimore Gas and Electric Co.}

Baltimore Gas and Electric Company (BG\&E) is a publicly held corporation that provides natural gas and electricity service to the central Maryland region, including the city of Baltimore. BG\&E is the Nation's oldest natural gas utility.

BG\&E's natural gas service area includes 617 square miles and more than one-half million customers. The vast majority of its natural gas is purchased from suppliers, although the company can supplement these supplies with its own liquefied gas and propane facilities. With 10 local power plants, one of which is a nuclear power plant, the company supplies electricity to more than one million customers in a 2,300-square-mile area.

During 1993, many developments impacted BG\&E's natural gas operations. The company increased its natural gas customer base by 6,500. A joint project between BG\&E and Crown Central Petroleum yielded Maryland's first public natural gas vehicle fueling station in Annapolis. BG\&E received the first approval in nearly 30 years to establish a new municipal natural gas franchise, located in the town of Mount Airy. Also, the construction of a 140-megawatt natural gas unit at the Perryman site in Harford County was begun. It is expected to be operational in June 1995.

Figure 2. Baltimore Gas and Electric Co., 1993

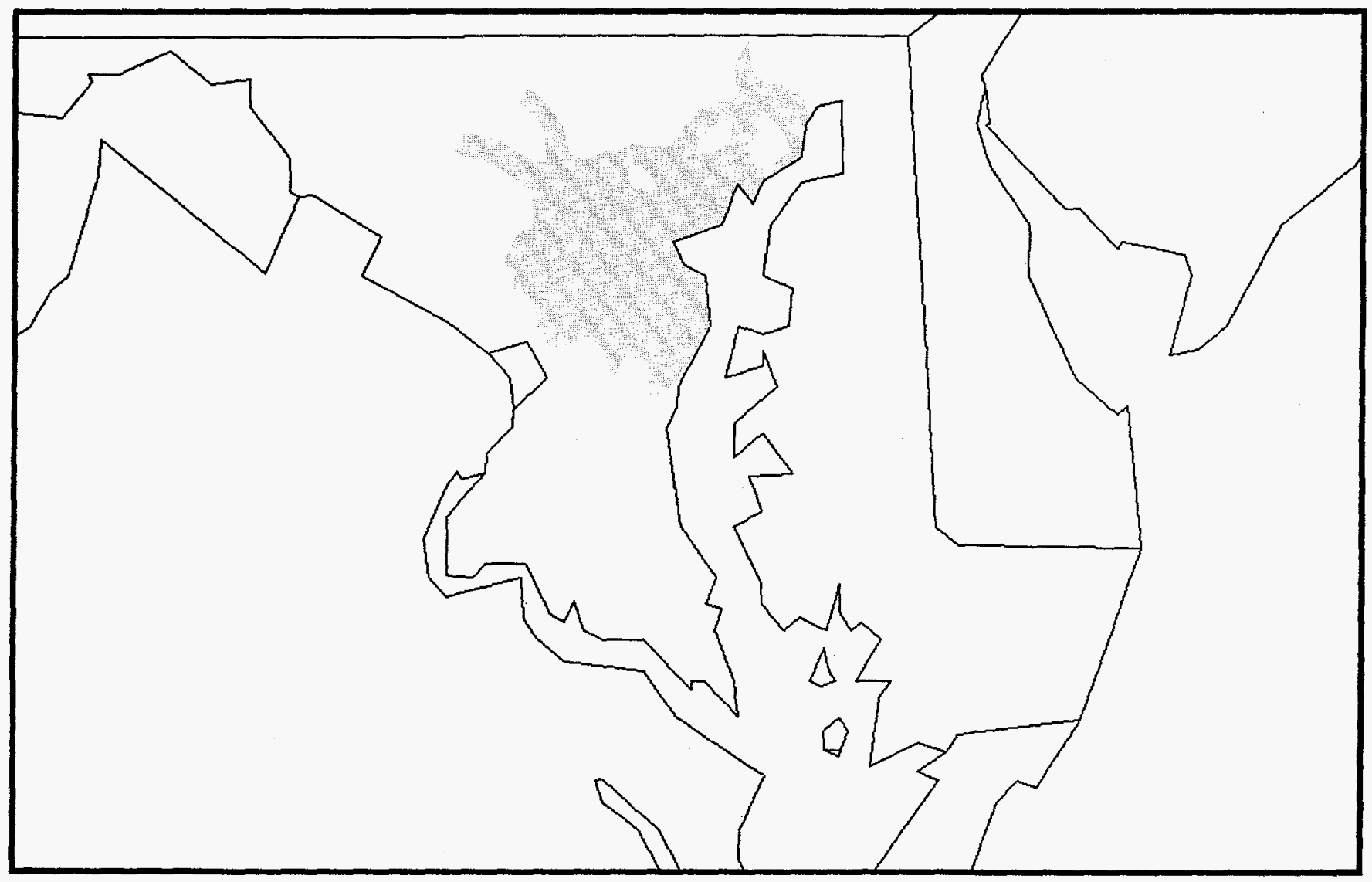

Source: Energy Information Administration (EIA), Form ElA-176, "Annual Report of Natural and Supplemental Gas Supply and Disposition." 
Table 2. Baltimore Gas and Electric Co., Natural Gas Data, 1993 (Thousand Cubic Feet)

\begin{tabular}{|c|c|}
\hline \multirow{2}{*}{ Supply/Disposition } & Baltimore Gas and Electric Co. \\
\hline & Maryland \\
\hline \multicolumn{2}{|l|}{ SUPPLY } \\
\hline \multirow{2}{*}{\multicolumn{2}{|c|}{$\begin{array}{l}\text { Produced Onsystem } \\
\text { Receipts }\end{array}$}} \\
\hline & \\
\hline \multicolumn{2}{|l|}{$\begin{array}{l}\text { Receipts } \\
\text { Purchases }\end{array}$} \\
\hline 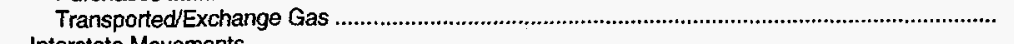 & \\
\hline \multicolumn{2}{|l|}{ Interstate Movements } \\
\hline 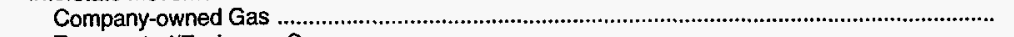 & 0 \\
\hline 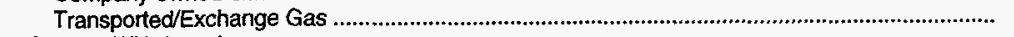 & 0 \\
\hline 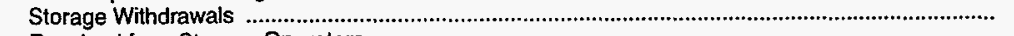 & 610,190 \\
\hline Received from Storage Operators & 0 \\
\hline 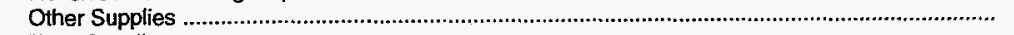 & 249,760 \\
\hline 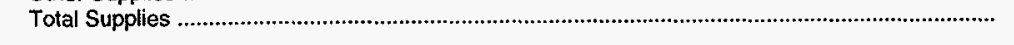 & $112,444,251$ \\
\hline \multicolumn{2}{|l|}{ DISPOSITION } \\
\hline \multicolumn{2}{|l|}{ Lease and Plant Use } \\
\hline 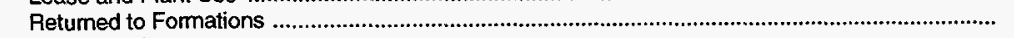 & 0 \\
\hline \multicolumn{2}{|l|}{$\begin{array}{l}\text { Retumed to romations } \\
\text { Interstate Movements }\end{array}$} \\
\hline 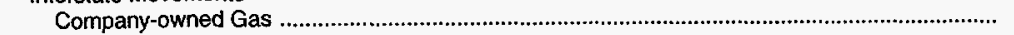 & 0 \\
\hline 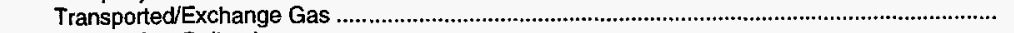 & 0 \\
\hline Exchange Gas Deliveries & 0 \\
\hline Storage Injections & 695,611 \\
\hline \multicolumn{2}{|l|}{ Deliveries of Company-owned Gas } \\
\hline 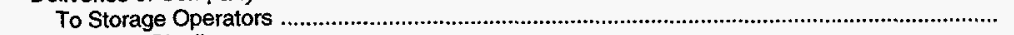 & 0 \\
\hline 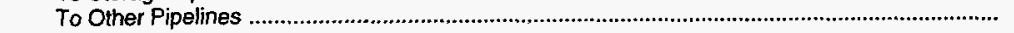 & 0 \\
\hline 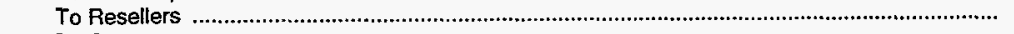 & 0 \\
\hline \multicolumn{2}{|l|}{ To Consumers } \\
\hline 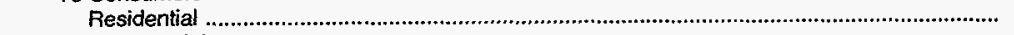 & $38,579,959$ \\
\hline 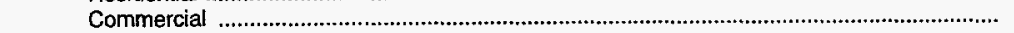 & $21,499,822$ \\
\hline 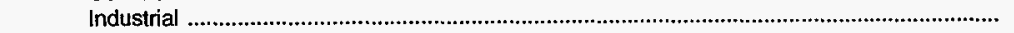 & $6,542,641$ \\
\hline 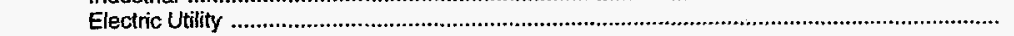 & 0 \\
\hline Vehicle Fuel & 19,272 \\
\hline \multicolumn{2}{|l|}{ Transported for the Account of Others } \\
\hline 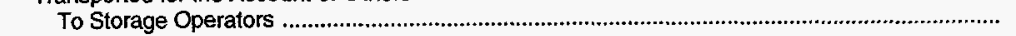 & 0 \\
\hline To Other Pipelines & 0 \\
\hline 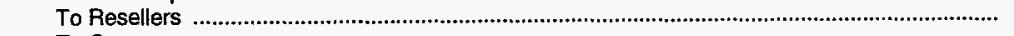 & 0 \\
\hline \multicolumn{2}{|l|}{ To Consumers } \\
\hline 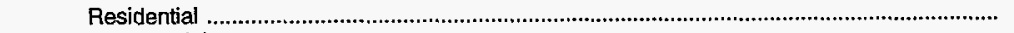 & 0 \\
\hline 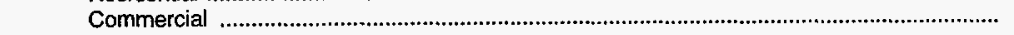 & 652,100 \\
\hline Industrial & $37,636,886$ \\
\hline 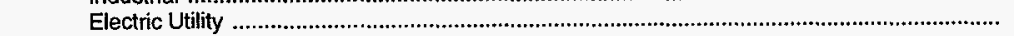 & $3,034,445$ \\
\hline Vehicle Fuel & 0 \\
\hline 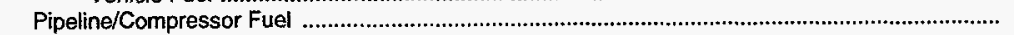 & 21,859 \\
\hline Other Disposition & 0 \\
\hline 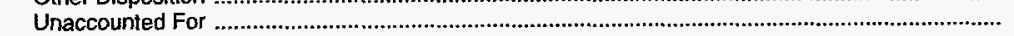 & $3,761,656$ \\
\hline 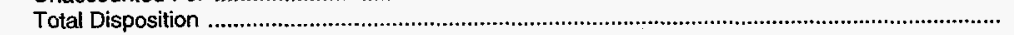 & $112,444,251$ \\
\hline
\end{tabular}

Source: Energy Information Administration (EIA), Form ElA-176, "Annual Report of Natural and Supplemental Gas Supply and Disposition." 


\section{Boston Gas Co.}

Boston Gas Company is the largest gas distributor company in New England. It is a subsidiary of Eastern Enterprises, a diversified holding company. Boston Gas operates in the city of Boston and in over 70 other cities and towns throughout eastern and central Massachusetts. The company serves over 500,000 residential, commercial, industrial, and electric utility users, and delivered over 100 billion cubic feet of natural gas to end users during 1993.

During 1993, Boston Gas Company expanded its service by providing natural gas to power plants at Brandeis University and Wellesley College. It also began provid- ing service to Boston Edison's South Boston power plant which converted to natural gas in order to meet environmental standards.

Boston Gas has only a small share of the market of potential commercial or industrial users in its service area. In the residential sector, almost half of Boston Gas Company's customers do not use gas for home heating. The company, however, expects to increase gas sales in the future as a result of the environmental benefits derived from using natural gas. New markets for products like gas air conditioning and natural gas vehicles are being explored by the company.

Figure 3. Boston Gas Co., 1993

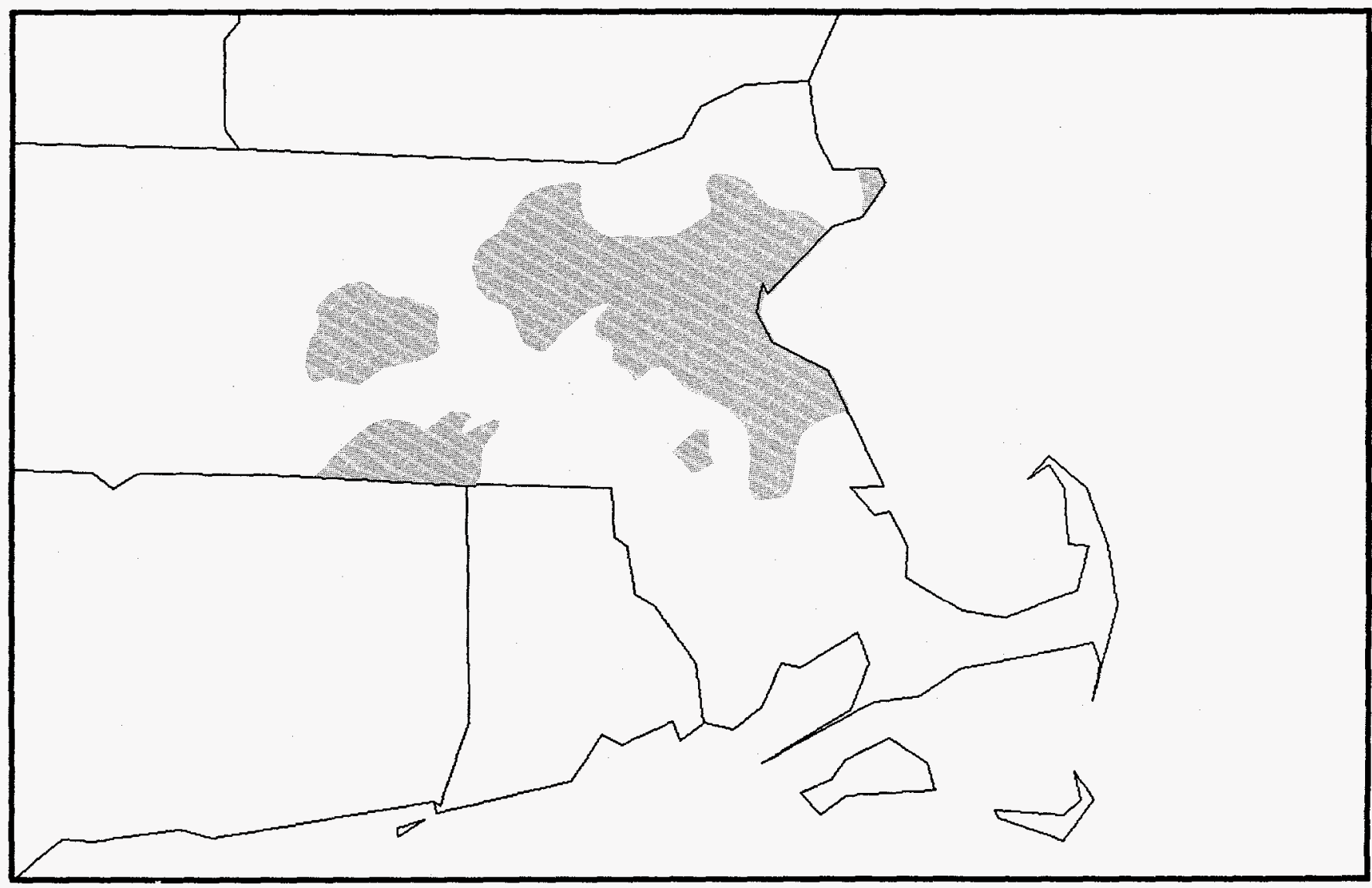

Source: Energy Information Administration (EIA), Form ElA-176, "Annual Report of Natural and Supplemental Gas Supply and Disposition."

\section{Service Areas}


Table 3. Boston Gas Co., Natural Gas Data, 1993 (Thousand Cubic Feet)

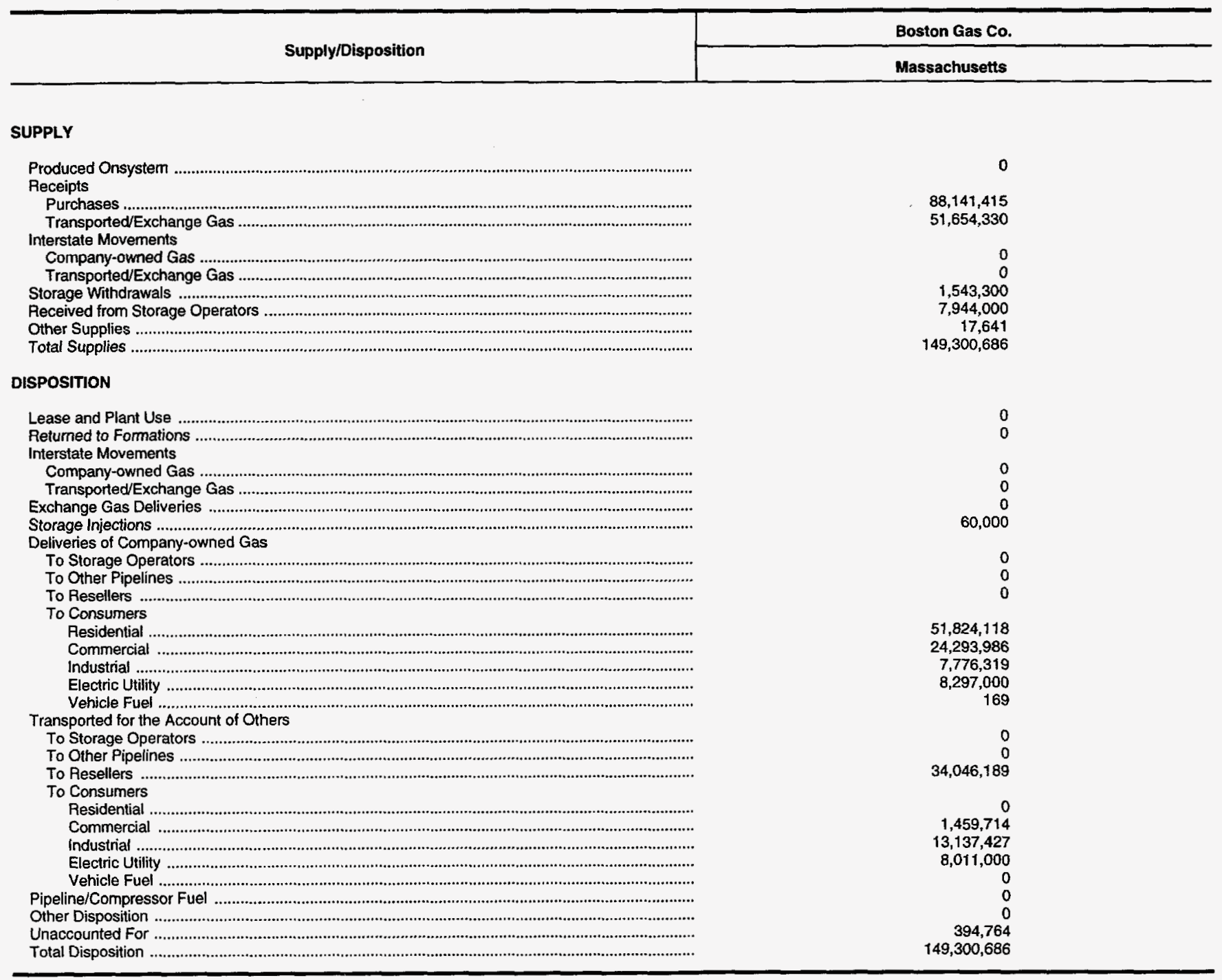

Source: Energy Information Administration (EIA), Form EIA-176, "Annual Report of Natural and Supplemental Gas Supply and Disposition."

The company submitted a proposal to the Massachusetts Department of Public Utilities in 1992 proposing the purchase and consolidation of the 10 gas distribution companies in Massachusetts into one large utility. This plan was not a firm proposal to buy any or all of the other gas distribution companies in the State. If implemented, Boston Gas believes it could save $\$ 100$ million yearly and improve overall service in the State. The savings would come from greater purchasing power, consolidating functions, and eliminating redundant services. The plan con- tinues to be promoted by Boston Gas, but no consolidations have taken place in either 1993 or 1994 . The merits of the plan were discussed in the 1993 sessions of the Massachusetts legislature.

During 1993, Boston Gas weathered a 17-week work stoppage as a result of labor disputes. Milder than usual winter weather and the labor disputes are factors cited by the company for level sales in a period during which the company had projected strong growth. 


\section{Brooklyn Union Gas Co.}

Brooklyn Union Gas Company is a natural gas utility that distributes natural gas to three densely populated boroughs of New York City. It has over 1.1 million customers in Brooklyn, Queens, and Staten Island. Since the implementation of FERC Order 636, Brooklyn Union has been shopping for its own supply of natural gas to ensure the best prices for its customers. To date, the company has agreements for direct purchases of natural gas from eight major suppliers.

Brooklyn Union has been a leader in natural gas technology in the New York area. For instance, it provides service for a 700-ton natural gas refrigeration system and a 175-horsepower air compressor for the Coca-Cola Bottling Company. Two hospitals in Brooklyn have agreed to install small natural gas cogeneration plants. The company is also marketing natural gas heat pumps for use by small commercial businesses as well as residences. It is also promoting a natural gas fuel cell that produces electricity and usable heat by a chemical process that does not create harmful emissions. A 200-kilowatt fuel cell was used commercially for the first time at St. Vincent's Medical Center in New York City.

The company is also very active in the field of cogeneration. It participates in the construction, design, operation, and ownership of natural gas-fired cogeneration plants. A 100-megawatt plant at JFK International Airport was completed in 1994 that delivers power to the entire airport complex. The company has acquired an 11-percent own-

Figure 4. Brooklyn Union Gas Co., 1993

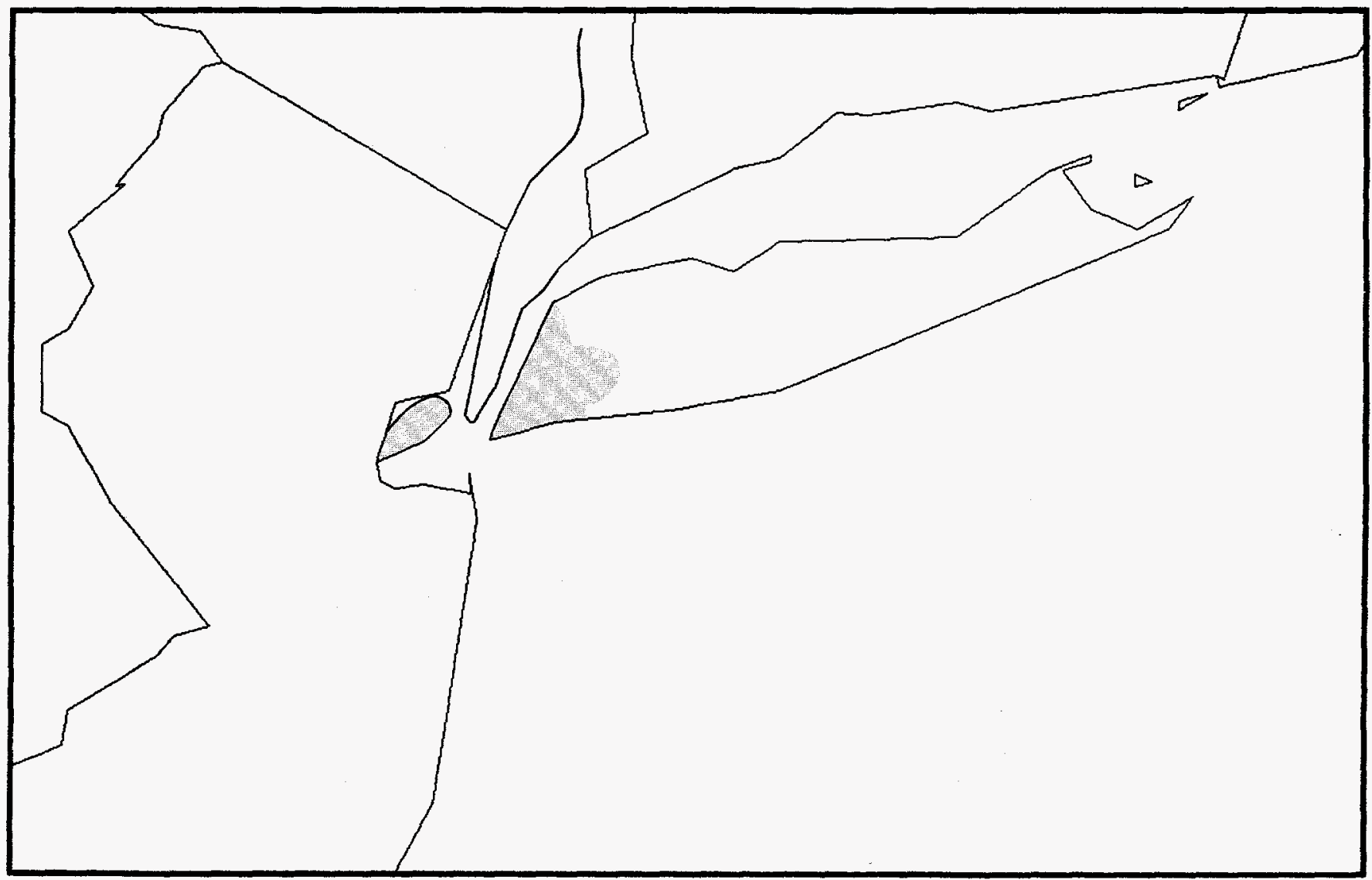

Source: Energy Information Administration (EIA), Form EIA-176, "Annual Report of Natural and Supplemental Gas Supply and Disposition."

Service Areas 
Table 4. Brooklyn Union Gas Co., Natural Gas Data, 1993 (Thousand Cubic Feet)

\begin{tabular}{l|l|r|}
\hline \multirow{2}{*}{ Supply/Disposition } & Brooklyn Union Gas Co. \\
\cline { 2 - 2 } & New York \\
\hline
\end{tabular}

SUPPLY

Produced Onsystem

Receipts

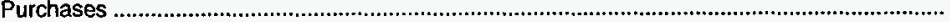

Transported/Exchange Gas

Interstate Movements

Company-owned Gas

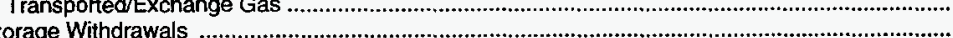

Received from Storage Operators

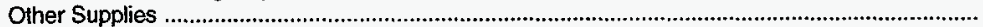

Other Supplies

ISPOSITION
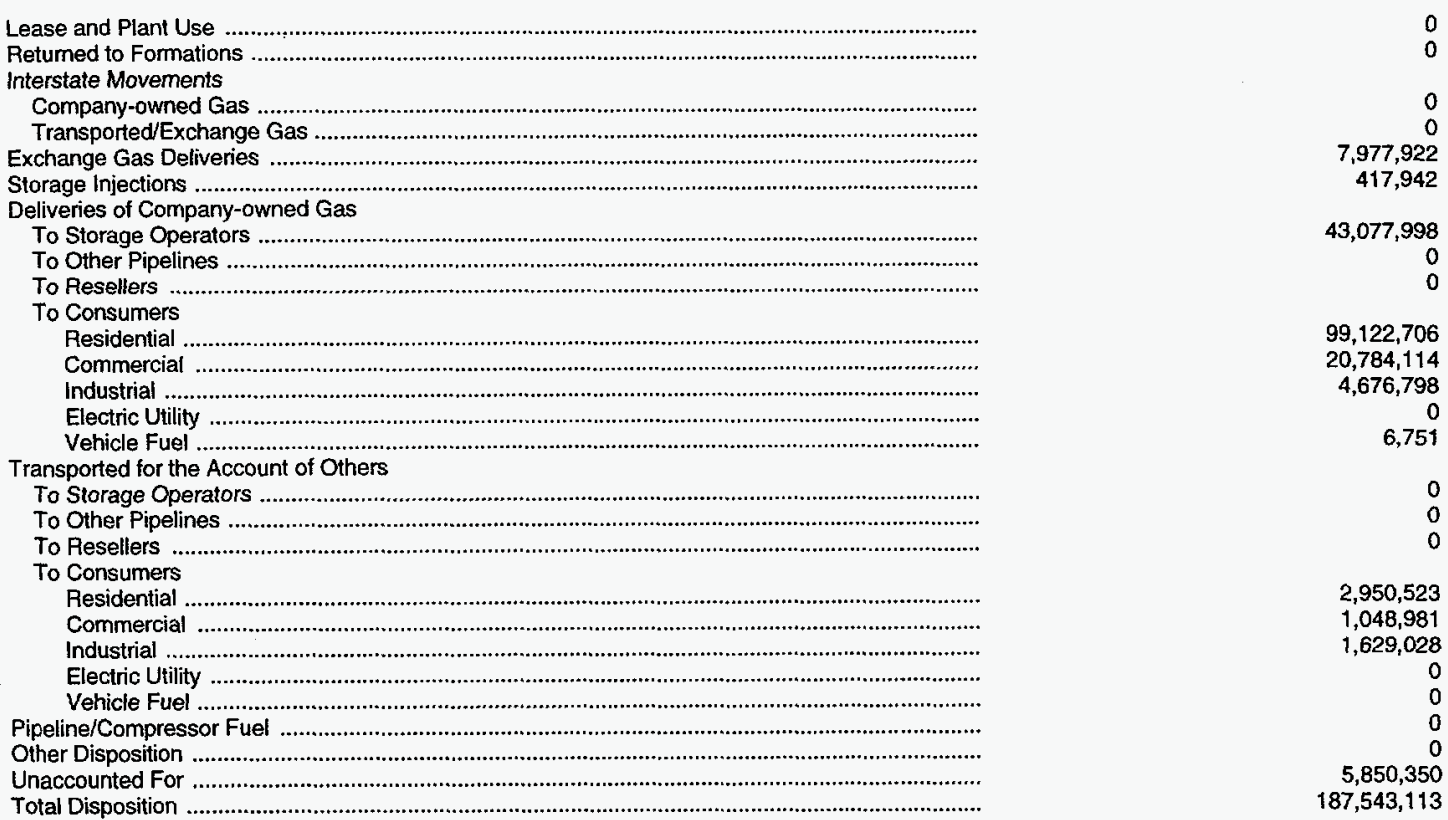

Source: Energy Information Administration (EIA), Form ElA-176, "Annual Report of Natural and Supplemental Gas Supply and Disposition."

ership in a 174-megawatt plant in Lockport, New York. Since 1989 , it has owned 45 percent of a plant that provides heat and power to Grumman Aerospace Corporation. It is a partner in a new 40-megawatt plant that is due to be completed in 1995 at the Stony Brook campus of the University of New York.

Brooklyn Union owns an 11-percent interest in the Iroquois Gas Transmission System. The company has also agreed to participate in the construction and operation of the Mayflower Gas Transmission System, a 200-mile pipeline that will connect Iroquois to markets from eastern New York to Boston, Massachusetts. A new compression station in Wright, New York has been completed, and a second station has been proposed at
Croghan, New York. These two stations could increase capacity for Brooklyn Union by 20 percent. Brooklyn Union is also participating in the development of the 38-mile Liberty Pipeline project, resulting in a system extending from New Jersey, across the lower New York Bay to Queens, New York.

Brooklyn Union also plays an important role in promoting the use of natural gas vehicles (NGV's) in the greater New York City market. The government of the City of New York will have 700 NGV's in operation by the end of 1994. In 1995, the General Services Administration in New York City will add 180 NGV's. Presently, Brooklyn Union's own vehicle fleet includes more than 500 company-owned NGV's, and the company plans to convert its entire fleet to natural gas by 1996. 


\section{Coastal Corp.}

Coastal Corporation is a major energy company based in Houston, Texas, that has diverse interests in the energy industry. Its Natural Gas Group is its most profitable division. Coastal Natural Gas owns or operates natural gas pipelines that comprise an extensive network located primarily in the U.S. mid-continent. This network also reaches into Colorado and Wyoming in the West and accesses the Northeast natural gas pipeline grid. The Coastal network includes ANR Pipeline Company, Colorado Interstate Gas Company, and Wyoming Interstate Company, plus the Empire State Pipeline project which was completed during 1993. Together, Coastal's pipelines now span more than 20,000 miles.
The company continues to expand its pipeline network and is currently participating in the SunShine Project (40-percent ownership) in North Florida, a proposed 715 miles of natural gas pipeline. Partnership proposals in the Northeast include: the Intercoastal Pipeline (20-percent ownership); the Mayflower Pipeline (45-percent ownership); and the Liberty Pipeline (25-percent ownership). The Mayflower, Intercoastal, and Liberty projects, together with Empire (45-percent ownership), plus the company's connections with the Iroquois system, will significantly expand the Coastal presence in the northeastern natural gas market. This northeastern presence is also bolstered by its access to the TransCanada Pipeline.

Figure 5. Coastal Corp., 1993

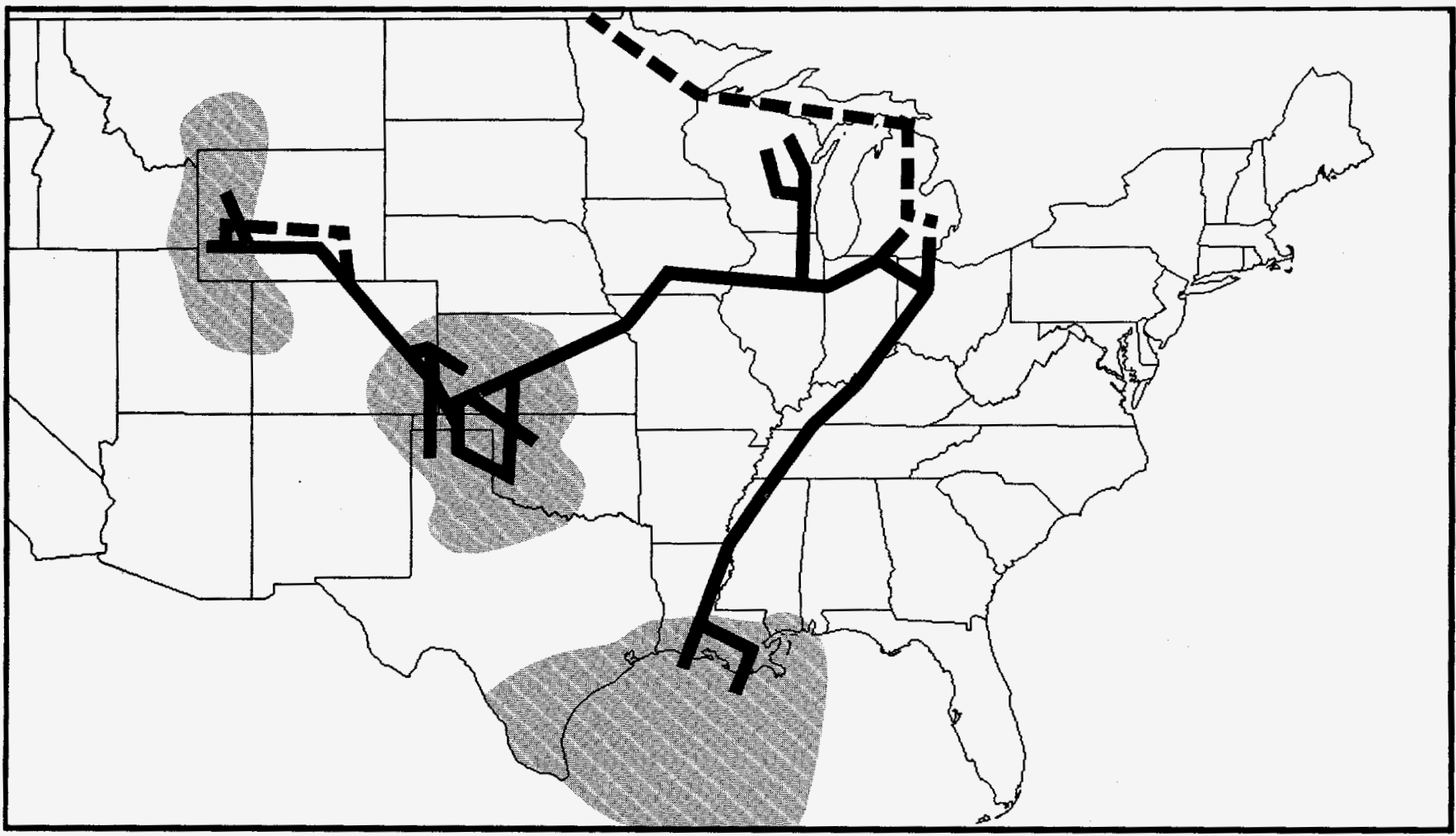

Source: Energy Information Administration (EIA), Form ElA-176, "Annual Report of Natural and Supplemental Gas Supply and Disposition." 
Table 5. Coastal Corp., Natural Gas Data, 1993

(Thousand Cubic Feet)

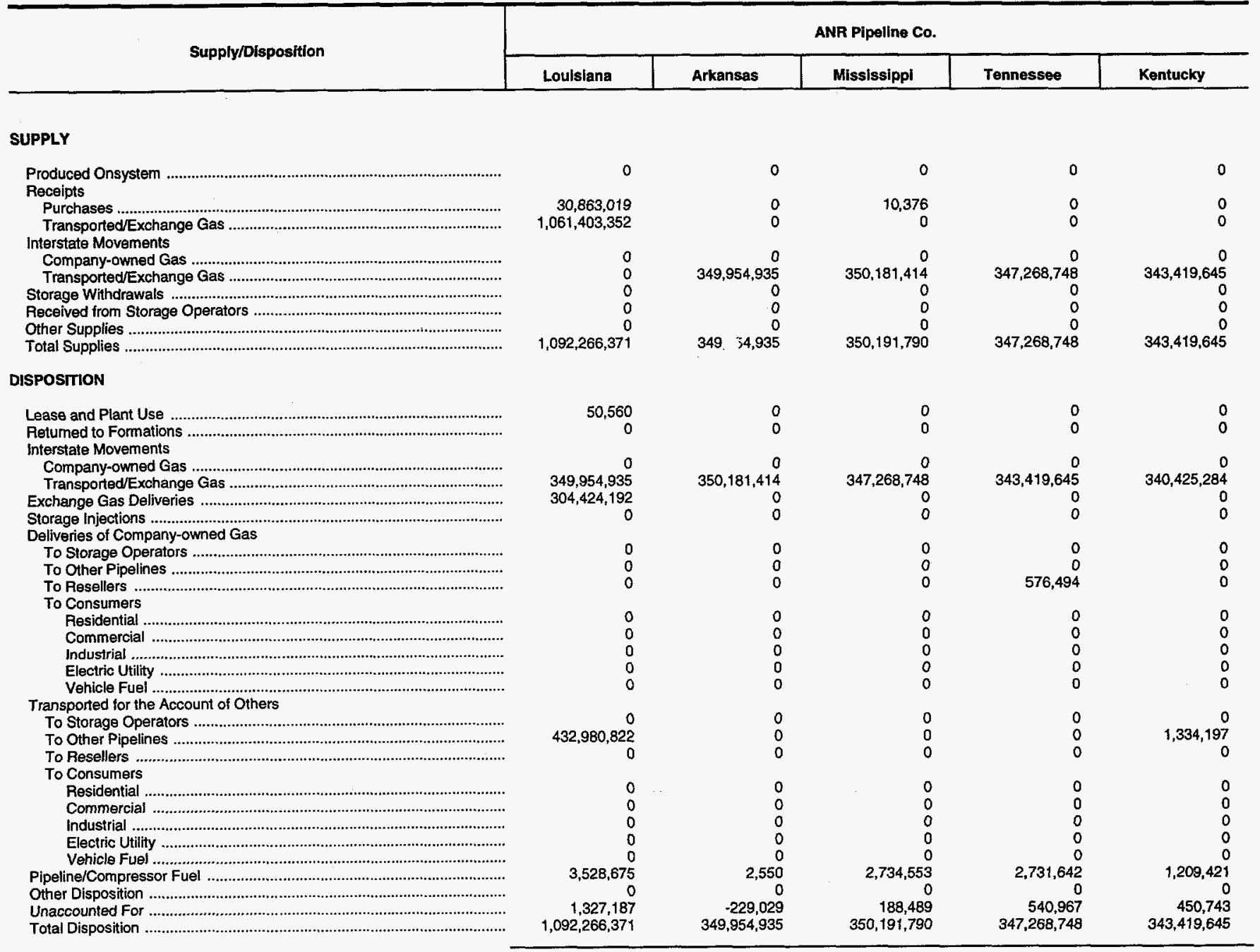

Coastal's other natural gas pipeline subsidiaries are Great Lakes Gas Transmission Company (50-percent ownership); High Island Offshore System (40-percent ownership); U-T Offshore System (33 1/3-percent ownership); and Iroquois Gas Transmission System (9.4-percent ownership).

During 1993, Coastal increased its storage and deliverability capacity in Colorado and Michigan. The company's underground storage facilities now have a working capacity of more than 300 billion cubic feet.
ANR Storage Company is one of Coastal's storage subsidiaries. As the natural gas market continues to develop in its new deregulated environment, new marketing techniques have emerged. One such technique is the creation of market hubs where natural gas trading activities are conducted. Coastal Gas Marketing, the marketing subsidiary for the company, has created natural gas hubs in the Midwest and the Northeast. Currently, it also plans to establish hubs in the West. 
Table 5. Coastal Corp., Natural Gas Data, 1993 (Continued) (Thousand Cubic Feet)

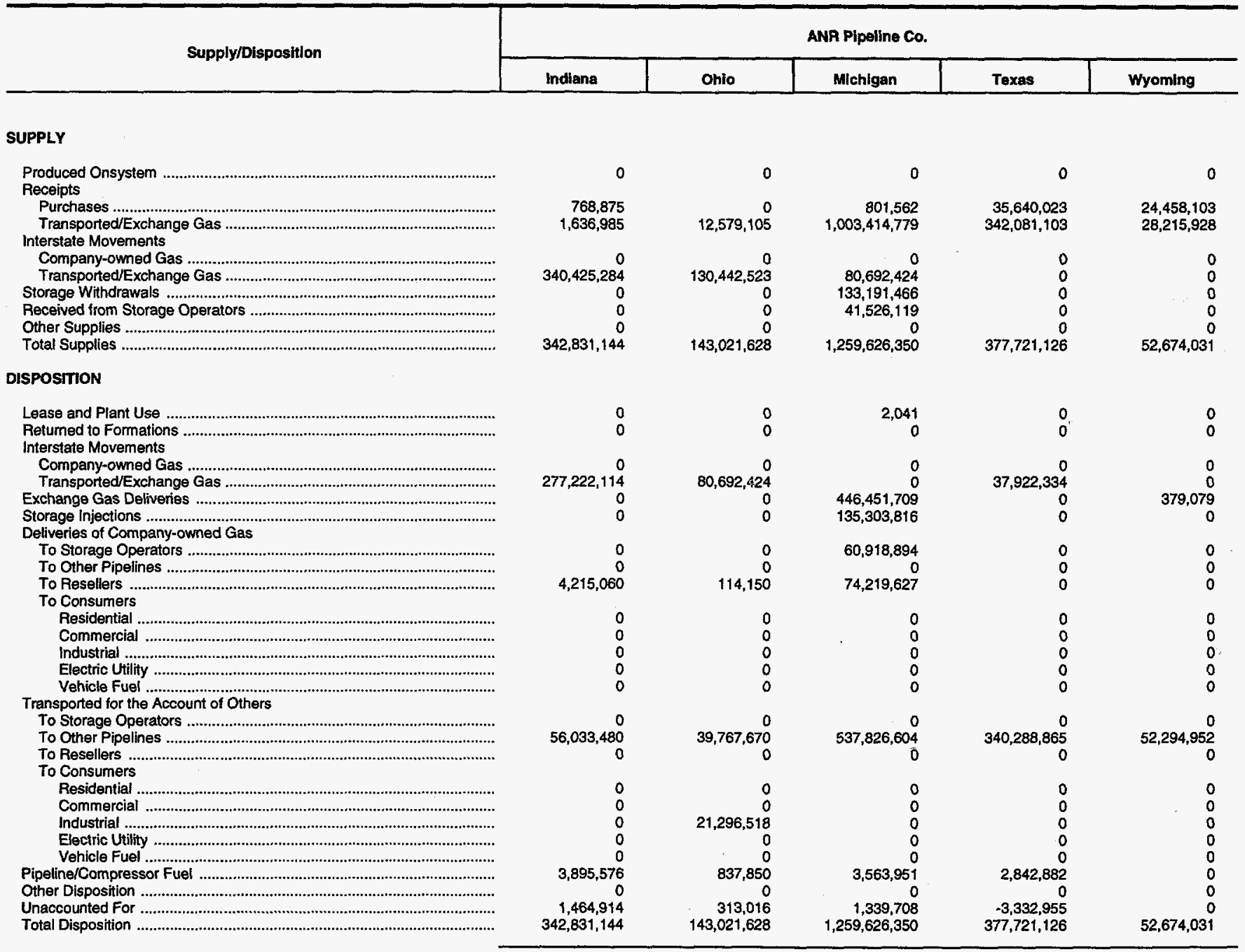


Table 5. Coastal Corp., Natural Gas Data, 1993 (Continued) (Thousand Cubic Feet)

\begin{tabular}{|c|c|c|c|c|c|}
\hline \multirow{2}{*}{ Supply/Disposition } & \multicolumn{5}{|c|}{ ANR Pipeline Co. } \\
\hline & Okiahoma & Kansas & Nebraska & Missouri & lowa \\
\hline \multicolumn{6}{|l|}{ SUPPLY } \\
\hline $\begin{array}{l}\text { Produced Onsystem } \\
\text { Receipts }\end{array}$ & 0 & 0 & 0 & 0 & 0 \\
\hline 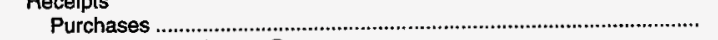 & $30,202,960$ & $1,193,084$ & 0 & 0 & \\
\hline 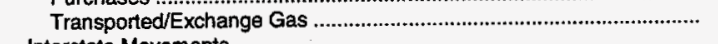 & $218,228,978$ & $135,798,977$ & 0 & 0 & $10,279,912$ \\
\hline \multicolumn{6}{|l|}{ 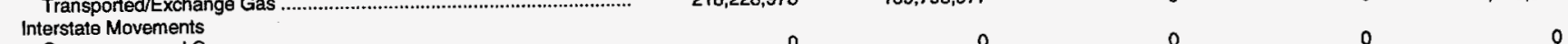 } \\
\hline & 0 & 0 & 0 & 0 & 0 \\
\hline & $37,922,334$ & $197,092,287$ & $191,167,236$ & $191,199,590$ & $188,155,690$ \\
\hline 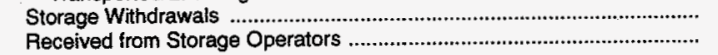 & 0 & 0 & 0 & 0 & 0 \\
\hline & 0 & 0 & 0 & 0 & 0 \\
\hline & 0 & 0 & 0 & 0 & 0 \\
\hline & $286,354,272$ & $334,084,348$ & $191,167,236$ & $191,199,590$ & $198,435,602$ \\
\hline \multicolumn{6}{|l|}{ DISPOSITION } \\
\hline Lease and Plant Use & 574,795 & 0 & 0 & 0 & 0 \\
\hline 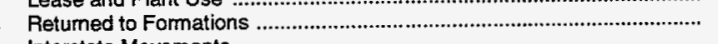 & 0 & 0 & 0 & 0 & 0 \\
\hline \multicolumn{6}{|l|}{ Interstate Movements } \\
\hline & $\begin{array}{r}0 \\
197,092,287\end{array}$ & $\begin{array}{r}0 \\
191,167,236\end{array}$ & $\begin{array}{r}0 \\
191,199,590\end{array}$ & $188,155,690$ & $168,303,806$ \\
\hline $\begin{array}{l}\text { Transported/Exchange Gas } \\
\text { Exchange Gas Deliveries }\end{array}$ & $\begin{array}{r}197,092,287 \\
46,772,163\end{array}$ & $\begin{array}{l}191,167,236 \\
127,845,057\end{array}$ & $\begin{array}{r}191,199,590 \\
0\end{array}$ & 0 & $\begin{array}{r}100,300,000 \\
0\end{array}$ \\
\hline & $\begin{array}{r}40, S / 2,103 \\
0\end{array}$ & $\begin{array}{r}0 \\
121,040,031 \\
0\end{array}$ & 0 & 0 & 0 \\
\hline \multicolumn{6}{|l|}{ Storage Injections } \\
\hline To Storage Operators & 0 & 0 & 0 & 0 & 0 \\
\hline 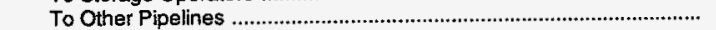 & 0 & 0 & 0 & 0 & 0 \\
\hline 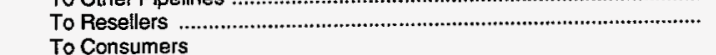 & 0 & 33,011 & 0 & $1,379,246$ & $2,191,918$ \\
\hline 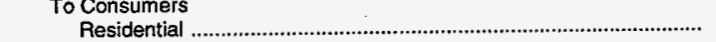 & 0 & 0 & 0 & 0 & 0 \\
\hline $\begin{array}{l}\text { Residential } \\
\text { Commercial }\end{array}$ & 0 & 0 & 0 & 0 & 0 \\
\hline $\begin{array}{l}\text { Commercial } \\
\text { Industrial }\end{array}$ & 0 & 0 & 0 & 0 & 0 \\
\hline 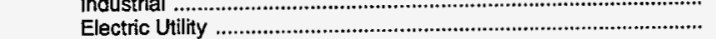 & 0 & 0 & 0 & 0 & 0 \\
\hline 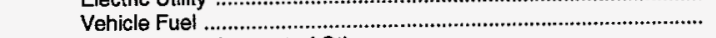 & 0 & 0 & 0 & 0 & 0 \\
\hline \multicolumn{6}{|l|}{ Transported for the Account of Others } \\
\hline 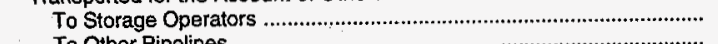 & 0 & 0 & 0 & $\begin{array}{r}0 \\
488,060\end{array}$ & 25.548532 \\
\hline $\begin{array}{l}\text { To Other Pipelines } \\
\text { To Resellers }\end{array}$ & $38,099,156$ & $9,678,980$ & $\begin{array}{l}0 \\
0\end{array}$ & $\begin{array}{r}488,060 \\
0\end{array}$ & $25,548,532$ \\
\hline \multicolumn{6}{|l|}{$\begin{array}{l}\text { To Resellers } \\
\text { To Consumers }\end{array}$} \\
\hline 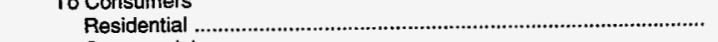 & 0 & 0 & 0 & 0 & 0 \\
\hline 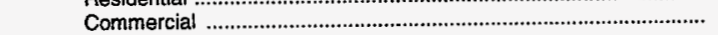 & 0 & 0 & 0 & 0 & 0 \\
\hline 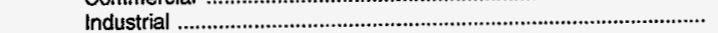 & 0 & 0 & 0 & 0 & 0 \\
\hline 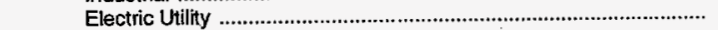 & 0 & 0 & 0 & 0 & 0 \\
\hline 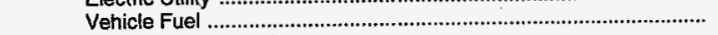 & 0 & 0 & 0 & 0 & 0 \\
\hline 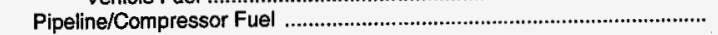 & $2,776,659$ & $3,895,149$ & 364 & 851,059 & $1,740,273$ \\
\hline 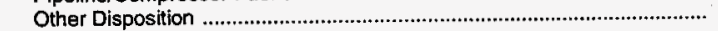 & 0 & 0 & 0 & 0 & 0 \\
\hline 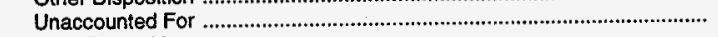 & $1,039,212$ & $1,464,915$ & $-32,718$ & 325,535 & 651,073 \\
\hline 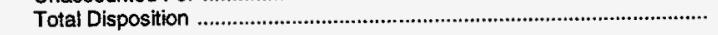 & $286,354,272$ & $334,084,348$ & $191,167,236$ & $191,199,590$ & $198,435,602$ \\
\hline
\end{tabular}


Table 5. Coastal Corp., Natural Gas Data, 1993 (Continued) (Thousand Cubic Feet)

\begin{tabular}{|c|c|c|c|c|c|}
\hline \multirow{2}{*}{ Supply/Disposition } & \multicolumn{2}{|c|}{ ANR Pipeline Co. } & \multirow{2}{*}{$\begin{array}{c}\text { Eaton Rapids Gas } \\
\text { Storage System } \\
\text { Michigan }\end{array}$} & \multirow{2}{*}{$\begin{array}{c}\text { ANR Storage Co. } \\
\text { Michigan }\end{array}$} & \multirow{2}{*}{$\begin{array}{c}\begin{array}{c}\text { ANR Production } \\
\text { Co. }\end{array} \\
\text { Utah }\end{array}$} \\
\hline & Illinois & Wisconsin & & & \\
\hline \multicolumn{6}{|l|}{ SUPPLY } \\
\hline $\begin{array}{l}\text { Produced Onsystem } \\
\text { Receipts }\end{array}$ & 0 & 0 & 0 & 0 & $7,645,755$ \\
\hline 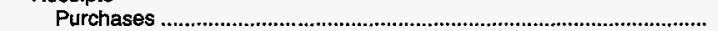 & 138,110 & 86,208 & 0 & 0 & $1,462,011$ \\
\hline 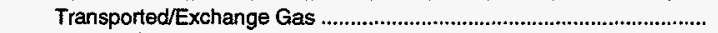 & $21,011,765$ & $92,195,624$ & $11,728,974$ & $37,181,900$ & 0 \\
\hline \multicolumn{6}{|l|}{ Interstate Movements } \\
\hline 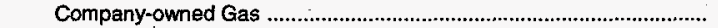 & 0 & 0 & 0 & 0 & 0 \\
\hline 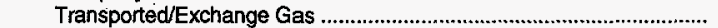 & $315,083,397$ & $180,338,002$ & 0 & 0 & 0 \\
\hline Storage Withdrawals & 0 & 0 & $10,099,188$ & $39,477,000$ & 0 \\
\hline 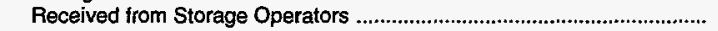 & 0 & 0 & 0 & 0 & 0 \\
\hline 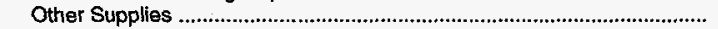 & 0 & 0 & 0 & 0 & 0 \\
\hline 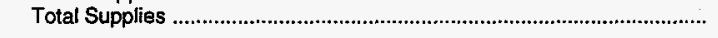 & $336,233,272$ & $272,619,834$ & $21,828,162$ & $76,658,900$ & $9,107,766$ \\
\hline \multicolumn{6}{|l|}{ DISPOSITION } \\
\hline Lease and Plant Use & 0 & 0 & 0 & 0 & $3,189,116$ \\
\hline $\begin{array}{l}\text { Returned to Formations } \\
\text { Interstate Movements }\end{array}$ & 0 & 0 & 0 & 0 & 0 \\
\hline 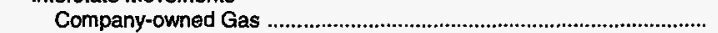 & 0 & 0 & 0 & 0 & 0 \\
\hline 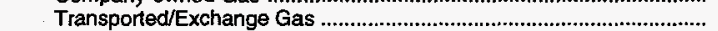 & $180,338,002$ & 0 & 0 & 0 & 0 \\
\hline Exchange Gas Deliveries & 0 & 0 & $10,027,004$ & $39,408,600$ & 0 \\
\hline \multirow{2}{*}{\multicolumn{6}{|c|}{ Deliveries of Company-owned Gas }} \\
\hline & & & & & \\
\hline To Storage Operators & 0 & 0 & 0 & 0 & 0 \\
\hline 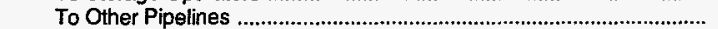 & 0 & 0 & 0 & 0 & $5,827,597$ \\
\hline 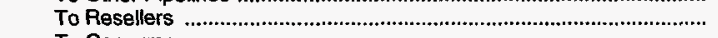 & 743,691 & $144,305,694$ & 0 & 0 & 0 \\
\hline \multicolumn{6}{|l|}{ To Consumers } \\
\hline 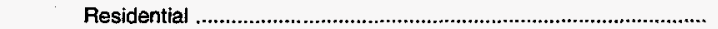 & 0 & 0 & 0 & 0 & 0 \\
\hline 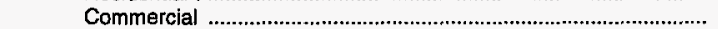 & 0 & 0 & 0 & 0 & 0 \\
\hline 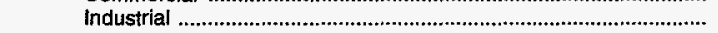 & 0 & 0 & 0 & 0 & 0 \\
\hline 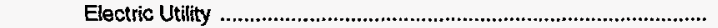 & 0 & 0 & 0 & 0 & 0 \\
\hline 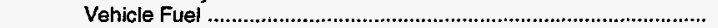 & 0 & 0 & 0 & 0 & 0 \\
\hline \multicolumn{6}{|l|}{ Transported for the Account of Others } \\
\hline To Storage Operators & 0 & 0 & 0 & 0 & 0 \\
\hline 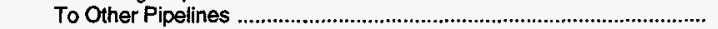 & $152,616,161$ & $127,076,263$ & 0 & 0 & 0 \\
\hline 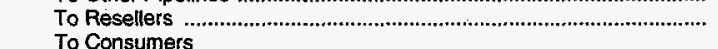 & 0 & 0 & 0 & 0 & 0 \\
\hline 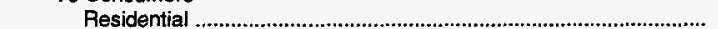 & 0 & 0 & 0 & 0 & 0 \\
\hline 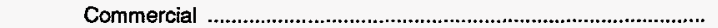 & 0 & 0 & 0 & 0 & 0 \\
\hline 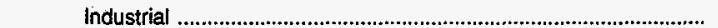 & 0 & 0 & 0 & 0 & 0 \\
\hline 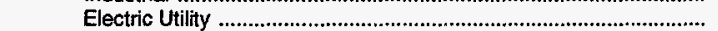 & 0 & 0 & 0 & 0 & 0 \\
\hline Vehicle Fuel & 0 & 0 & 0 & 0 & 0 \\
\hline 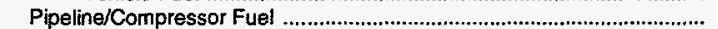 & $1,846,783$ & 899,820 & 251,970 & 542,900 & 0 \\
\hline Other Disposition & & 0 & 0 & 0 & 0 \\
\hline 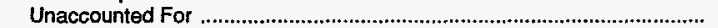 & 688,635 & 338,057 & 0 & 0 & 91,053 \\
\hline 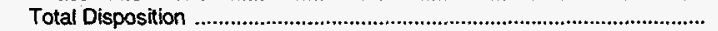 & $336,233,272$ & $272,619,834$ & $21,828,162$ & $76,658,900$ & $9,107,766$ \\
\hline
\end{tabular}


Table 5. Coastal Corp., Natural Gas Data, 1993 (Continued) (Thousand Cubic Feet)

\begin{tabular}{|c|c|c|c|c|c|}
\hline \multirow{2}{*}{ Supply/Disposition } & \multicolumn{5}{|c|}{ Colorado Interstate Gas Co. } \\
\hline & Texas & Kansas & Oklahoma & Colorado & Wyoming \\
\hline \multicolumn{6}{|l|}{ SUPPLY } \\
\hline $\begin{array}{l}\text { Produced Onsystem } \\
\text { Receipts }\end{array}$ & $46,939,131$ & 66,570 & $1,148,862$ & 0 & 0 \\
\hline 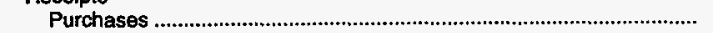 & $3,880,955$ & $11,035,959$ & $8,431,013$ & $1,835,296$ & $13,147,679$ \\
\hline 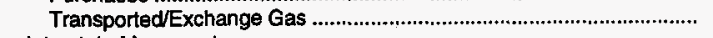 & $15,785,925$ & $47,302,901$ & $28,363,488$ & $124,884,109$ & $182,552,611$ \\
\hline \multicolumn{6}{|l|}{ Interstate Movements } \\
\hline 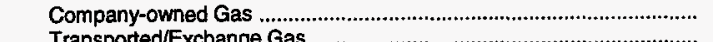 & $\begin{array}{r}0 \\
0\end{array}$ & $0 \begin{array}{r}0 \\
0.196291\end{array}$ & $\begin{array}{r}0 \\
71061691\end{array}$ & $\begin{array}{r}0 \\
243889934\end{array}$ & $\begin{array}{r}0 \\
45104135\end{array}$ \\
\hline & $\begin{array}{r}12,920,970 \\
0\end{array}$ & $\begin{array}{r}9,196,291 \\
10,153,676\end{array}$ & $\begin{array}{r}71,061,491 \\
0\end{array}$ & $\begin{array}{r}243,889,934 \\
41,792,395\end{array}$ & $\begin{array}{r}45,104,135 \\
0\end{array}$ \\
\hline $\begin{array}{l}\text { Storage Withdrawals } \\
\text { Received from Storage Operators }\end{array}$ & $\begin{array}{l}0 \\
0\end{array}$ & $\begin{array}{r}10,153,60 \\
0\end{array}$ & 0 & $\begin{array}{r}41,192,395 \\
0\end{array}$ & 0 \\
\hline Other Supplies & 0 & 0 & 0 & $7,965,890$ & 0 \\
\hline Total Supplies & $79,526,981$ & $77,755,397$ & $109,004,854$ & $420,367,624$ & $240,804,425$ \\
\hline \multicolumn{6}{|l|}{ DISPOSITION } \\
\hline Leas $\theta$ and Plant Use & $2,990,335$ & $1,563,863$ & $1,508,324$ & 0 & $3,145,271$ \\
\hline 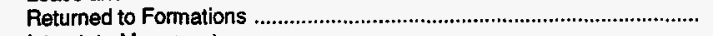 & 0 & 0 & 0 & 0 & 0 \\
\hline \multicolumn{6}{|l|}{ Interstate Movements } \\
\hline 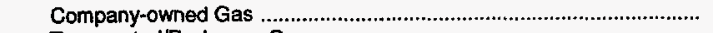 & 0 & 0 & 0 & 0 & 0 \\
\hline 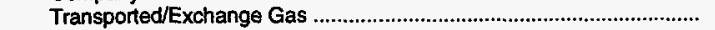 & 0 & 427,622 & $38,295,175$ & $124,934,295$ & $173,411,594$ \\
\hline Exchange Gas Deliveries & 381,444 & 73,912 & 881,045 & 0 & $1,020,739$ \\
\hline 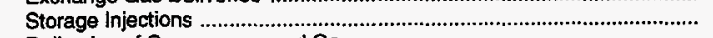 & 0 & $11,364,559$ & 0 & $41,814,523$ & 0 \\
\hline \multicolumn{6}{|l|}{ Deliveries of Company-owned Gas } \\
\hline To Storage Operators & 0 & 0 & 0 & 0 & 0 \\
\hline 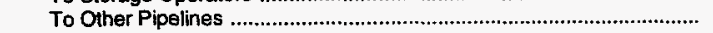 & 0 & 467,211 & 0 & $1,108,909$ & 0 \\
\hline 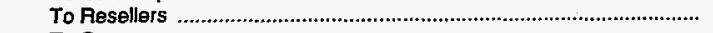 & $34,770,745$ & 15 & 15,889 & $41,407,240$ & $1,223,517$ \\
\hline \multicolumn{6}{|l|}{ To Consumers } \\
\hline 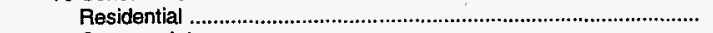 & 0 & 0 & 0 & 0 & 0 \\
\hline 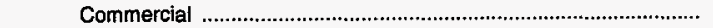 & 0 & 0 & 0 & 0 & 0 \\
\hline Industrial & 576,592 & 0 & 0 & 0 & 1,312 \\
\hline 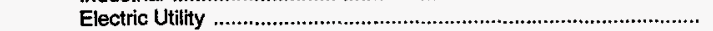 & 0 & 0 & 0 & 56,426 & 0 \\
\hline 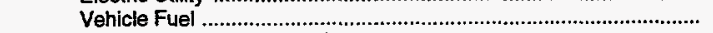 & 0 & 0 & 0 & 0 & 0 \\
\hline \multicolumn{6}{|l|}{ Transported for the Account of Others } \\
\hline 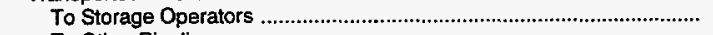 & 0 & 0 & 0 & 0 & 0 \\
\hline 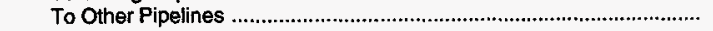 & $28,273,034$ & $60,445,019$ & $67,195,149$ & $75,909,710$ & $59,201,005$ \\
\hline 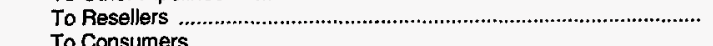 & 0 & 135,173 & 8,251 & $116,483,522$ & $13,500,825$ \\
\hline \multicolumn{6}{|l|}{ To Consumers } \\
\hline 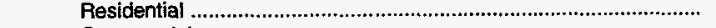 & $\begin{array}{l}0 \\
0\end{array}$ & $\begin{array}{l}0 \\
0\end{array}$ & $\begin{array}{l}0 \\
0\end{array}$ & 0 & $\begin{array}{l}0 \\
0\end{array}$ \\
\hline 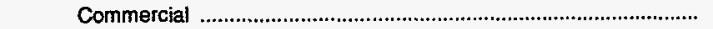 & 0 & 0 & & & 00 \\
\hline 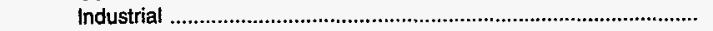 & $8,754,345$ & 0 & 0 & $4,495,219$ & $2,282,376$ \\
\hline 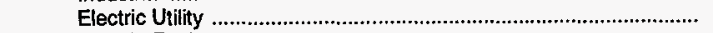 & 0 & 0 & 0 & $2,596,430$ & 0 \\
\hline 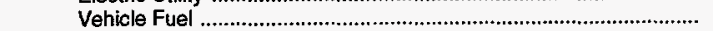 & 0 & 0 & 0 & 0 & 0 \\
\hline 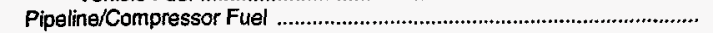 & $3,581,139$ & $3,278,023$ & $1,101,021$ & $2,645,312$ & $1,638,987$ \\
\hline Other Disposition & 0 & 0 & 0 & 0 & 0 \\
\hline 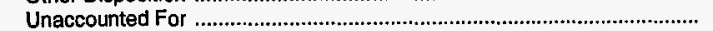 & 199,347 & 0 & 0 & $8,916,038$ & \\
\hline Total Disposition & $79,526,981$ & $77,755,397$ & $109,004,854$ & $420,367,624$ & $255,425,626$ \\
\hline
\end{tabular}


Table 5. Coastal Corp., Natural Gas Data, 1993 (Continued) (Thousand Cubic Feet)

\begin{tabular}{|c|c|c|c|c|c|}
\hline \multirow{2}{*}{ Supply/Disposition } & \multicolumn{2}{|c|}{ Wyoming Interstate Co. } & \multicolumn{3}{|c|}{ Great Lakes Gas Transmission Ltd. } \\
\hline & Wyoming & Colorado & Minnesota & Wisconsin & Michigan \\
\hline \multicolumn{6}{|l|}{ SUPPLY } \\
\hline $\begin{array}{l}\text { Produced Onsystem } \\
\text { Receipts }\end{array}$ & 0 & 0 & 0 & 0 & 0 \\
\hline Purchases & 0 & 0 & 0 & 0 & 0 \\
\hline 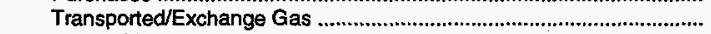 & $68,575,316$ & $9,504,008$ & 0 & 0 & $196,497,824$ \\
\hline \multicolumn{6}{|l|}{ Interstate Movements } \\
\hline 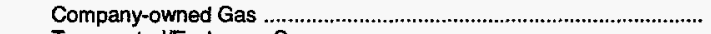 & 0 & 0 & 123,038 & 0 & 0 \\
\hline 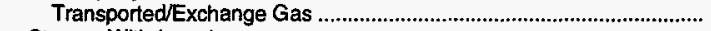 & 0 & $68,654,906$ & $565,129,130$ & $670,311,093$ & $667,067,856$ \\
\hline Storage Withdrawals & 0 & 0 & 0 & 0 & 0 \\
\hline Received from Storage Operators & 0 & 0 & 0 & 0 & 0 \\
\hline 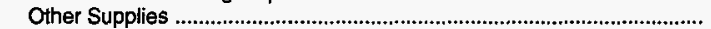 & 0 & 0 & 0 & 0 & 0 \\
\hline 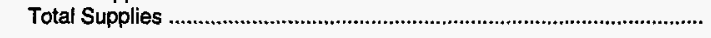 & $68,575,316$ & $78,158,914$ & $565,252,168$ & $670,311,093$ & $863,565,680$ \\
\hline \multicolumn{6}{|l|}{ DISPOSITION } \\
\hline Lease and Plant Use & 0 & 0 & 0 & 0 & 0 \\
\hline Returned to Formations & 0 & 0 & 0 & 0 & 0 \\
\hline \multicolumn{6}{|l|}{ Interstate Movements } \\
\hline Company-owned Gas & 0 & 0 & 0 & 0 & 0 \\
\hline 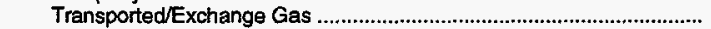 & $68,654,906$ & 0 & $670,311,093$ & $667,067,856$ & $355,334,407$ \\
\hline 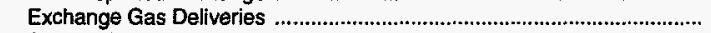 & 0 & 0 & 0 & 0 & $110,827,256$ \\
\hline 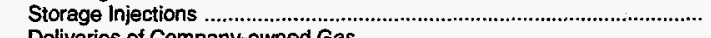 & 0 & 0 & 0 & 0 & 0 \\
\hline \multicolumn{6}{|l|}{ Deliveries of Company-owned Gas } \\
\hline To Storage Operators & 0 & 0 & 0 & 0 & 0 \\
\hline 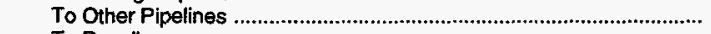 & 0 & 0 & 0 & 0 & 0 \\
\hline To Resellers & 0 & 0 & 0 & 0 & 0 \\
\hline \multicolumn{6}{|l|}{ To Consumers } \\
\hline 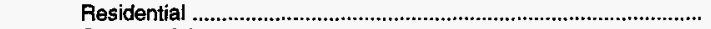 & 0 & 0 & 0 & 0 & 0 \\
\hline 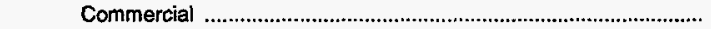 & 0 & 0 & 0 & 0 & 0 \\
\hline Industrial & 0 & 0 & 0 & 0 & 0 \\
\hline 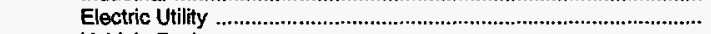 & 0 & 0 & 0 & 0 & 0 \\
\hline Vehicle Fuel & 0 & 0 & 0 & 0 & 0 \\
\hline \multicolumn{6}{|l|}{ Transported for the Account of Others } \\
\hline To Storage Operators & 0 & 0 & 0 & 0 & 0 \\
\hline 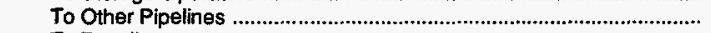 & 0 & $78,108,377$ & $57,814,603$ & $1,011,376$ & $284,501,271$ \\
\hline 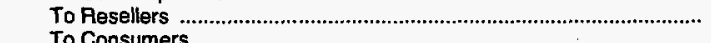 & 0 & 0 & 0 & 0 & 0 \\
\hline \multicolumn{2}{|l|}{ To Consumers } & 0 & & 0 & 0 \\
\hline 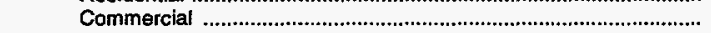 & 0 & 0 & 0 & 0 & 0 \\
\hline 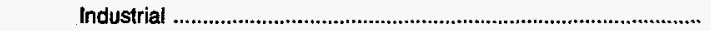 & 0 & 0 & 0 & 0 & 0 \\
\hline 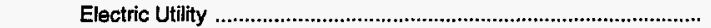 & 0 & 0 & 0 & 0 & 0 \\
\hline 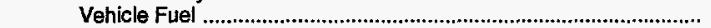 & 0 & 0 & 0 & 0 & 0 \\
\hline Pipeline/Compressor Fuel & 0 & 0 & $12,086,474$ & $2,222,341$ & $13,432,550$ \\
\hline Other Disposition & 0 & 0 & $\quad 73,061$ & 9,520 & 120,105 \\
\hline 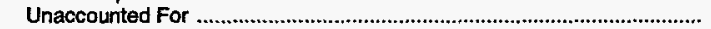 & 0 & 50,537 & $-175,033,063$ & 0 & $99,350,091$ \\
\hline 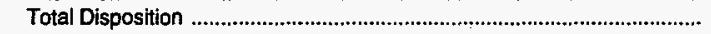 & $68,654,906$ & $78,158,914$ & $565,252,168$ & $670,311,093$ & $863,565,680$ \\
\hline
\end{tabular}


Table 5. Coastal Corp., Natural Gas Data, 1993 (Continued) (Thousand Cubic Feet)

\begin{tabular}{|c|c|c|c|c|}
\hline \multirow{2}{*}{ Supply/Disposition } & \multirow{2}{*}{$\begin{array}{c}\begin{array}{c}\text { Steuben Gas Storage } \\
\text { Co. }\end{array} \\
\text { New York } \\
\end{array}$} & \multicolumn{2}{|c|}{ High Island Offshore System } & \multirow{2}{*}{ U-T Offshore System } \\
\hline & & Texas & Louisiana & \\
\hline \multicolumn{5}{|l|}{ SUPPLY } \\
\hline $\begin{array}{l}\text { Produced Onsystem } \\
\text { Receipts }\end{array}$ & 0 & 0 & 0 & 0 \\
\hline 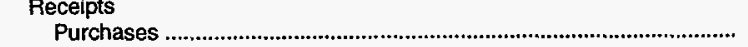 & 0 & 0 & 0 & 0 \\
\hline 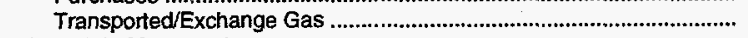 & $5,573,593$ & $301,414,824$ & $14,516,038$ & $135,565,337$ \\
\hline \multicolumn{5}{|l|}{ Interstate Movements } \\
\hline Company-owned Gas & 0 & 0 & 0 & 0 \\
\hline 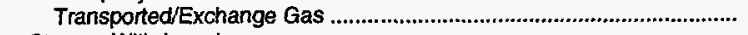 & 0 & 0 & $296,956,480$ & 0 \\
\hline 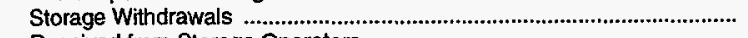 & $4,866,474$ & 0 & 0 & 0 \\
\hline 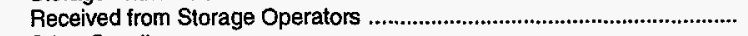 & 0 & 0 & 0 & 0 \\
\hline 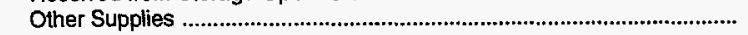 & 0 & 0 & 0 & 0 \\
\hline 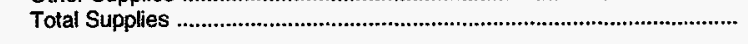 & $10,440,067$ & $301,414,824$ & $311,472,518$ & $135,565,337$ \\
\hline \multicolumn{5}{|l|}{ DISPOSITION } \\
\hline Lease and Plant Use & 0 & 0 & 0 & 0 \\
\hline 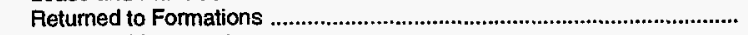 & 0 & 0 & 0 & 0 \\
\hline \multicolumn{5}{|l|}{ Interstate Movements } \\
\hline 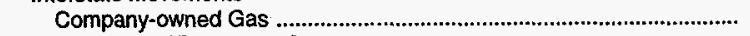 & 0 & 0 & 0 & 0 \\
\hline 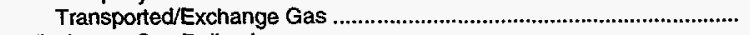 & 0 & $296,956,480$ & 0 & 0 \\
\hline 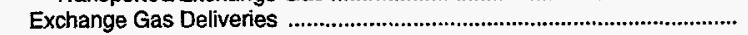 & $4,848,035$ & 0 & 0 & 0 \\
\hline Storage Injections & $5,522,050$ & 0 & 0 & 0 \\
\hline \multicolumn{5}{|l|}{ Deliveries of Company-owned Gas } \\
\hline To Storage Operators & 0 & 0 & 0 & 0 \\
\hline 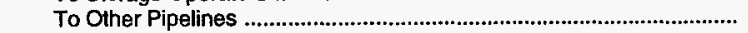 & 0 & 0 & 0 & 0 \\
\hline 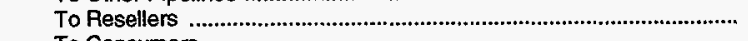 & 0 & 0 & 0 & 0 \\
\hline \multicolumn{5}{|l|}{ To Consumers } \\
\hline 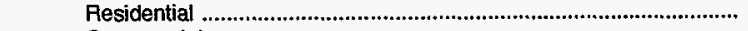 & 0 & 0 & 0 & 0 \\
\hline 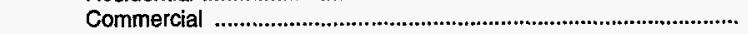 & 0 & 0 & 0 & 0 \\
\hline 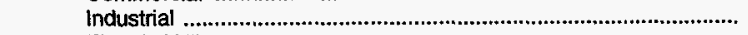 & 0 & 0 & 0 & 0 \\
\hline 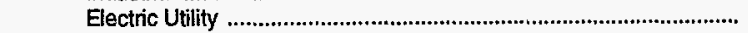 & 0 & 0 & 0 & 0 \\
\hline 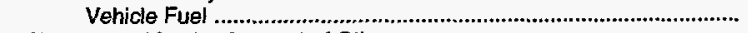 & 0 & 0 & 0 & 0 \\
\hline \multicolumn{5}{|l|}{ Transported for the Account of Others } \\
\hline To Storage Operators & 0 & 0 & 0 & 0 \\
\hline 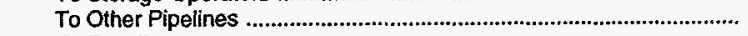 & 0 & 0 & $310,291,386$ & $135,501,458$ \\
\hline 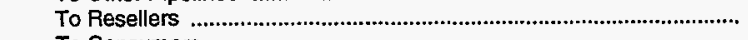 & 0 & 0 & 0 & 0 \\
\hline \multicolumn{5}{|l|}{ To Consumers } \\
\hline 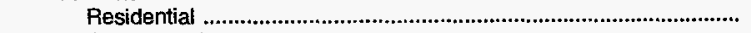 & 0 & 0 & 0 & 0 \\
\hline 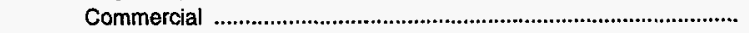 & 0 & 0 & 0 & 0 \\
\hline 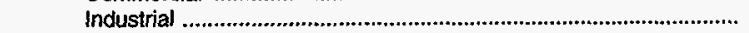 & 0 & 0 & 0 & 0 \\
\hline 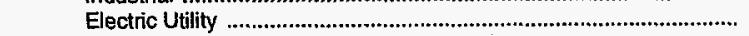 & 0 & 0 & 0 & 0 \\
\hline Vehicle Fuel & 0 & 0 & 0 & 0 \\
\hline 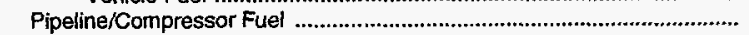 & 69,982 & $1,411,628$ & 373,977 & 3,251 \\
\hline 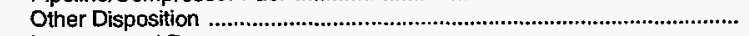 & 0 & 0 & 0 & 0 \\
\hline 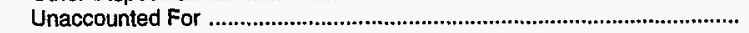 & 0 & $3,046,716$ & 807,155 & 60,628 \\
\hline 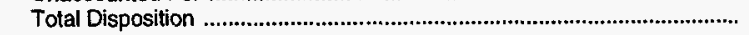 & $10,440,067$ & $301,414,824$ & $311,472,518$ & $135,565,337$ \\
\hline
\end{tabular}

Source: Energy Information Administration (EIA), Form ElA-176, "Annual Report of Natural and Supplemental Gas Supply and Disposition." 
Table 6. Coastal Corp., Interstate Flows of Natural Gas, 1993 (Thousand Cubic Feet)

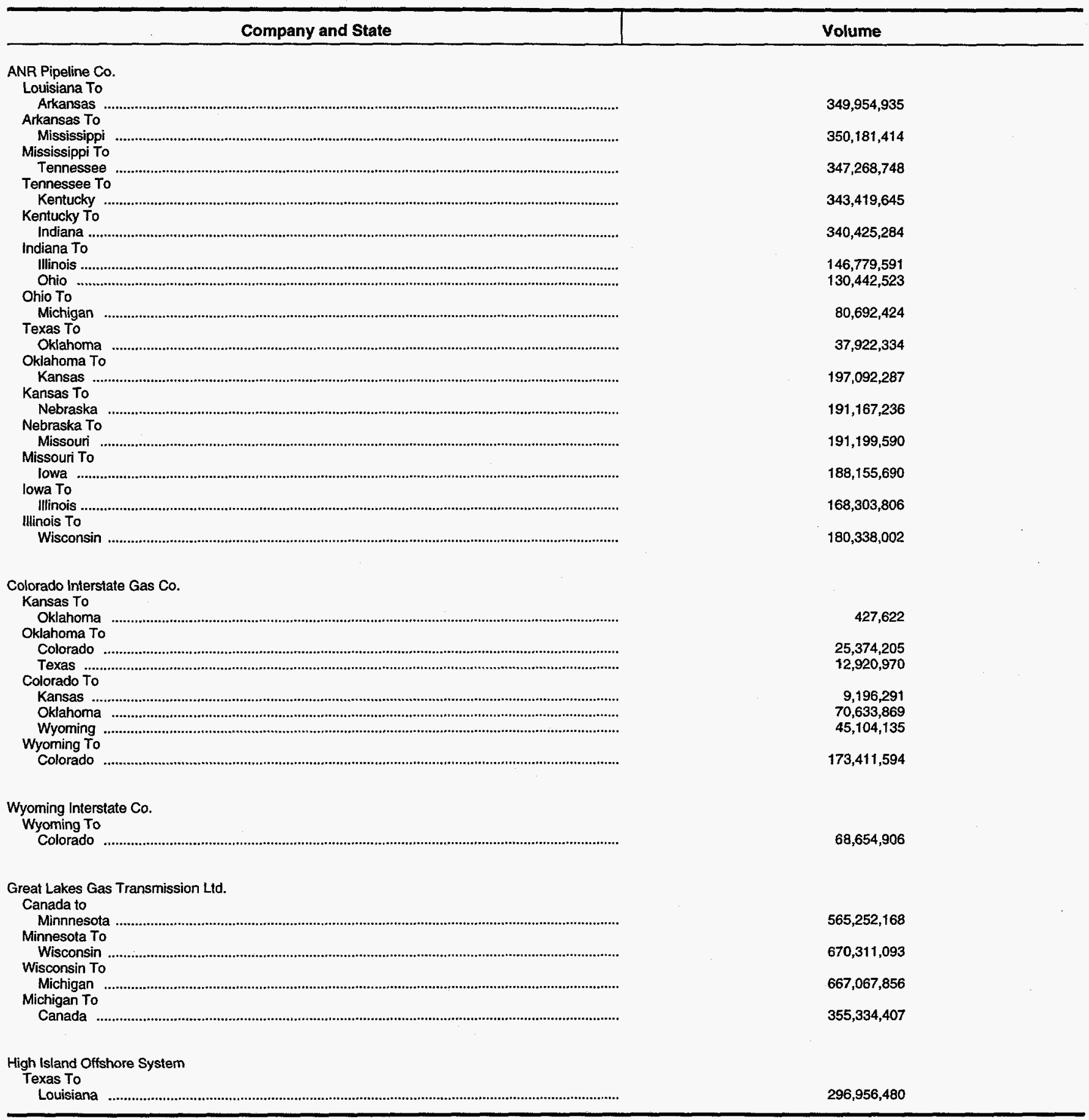

Source: Energy Information Administration (EIA), Form EIA-176, "Annual Report of Natural and Supplemental Gas Supply and Disposition." 



\section{Columbia Gas System, Inc.}

Columbia Gas System, one of the Nation's largest natural gas systems, consists of a holding company, a service company, and 17 diverse subsidiaries. These subsidiaries are involved in the exploration, production, purchase, marketing, storage, transportation, and distribution of natural gas. Columbia's transmission subsidiaries, Columbia Gas Transmission and Columbia Gulf Transmission, operate a 23,000-mile pipeline network that extends from offshore in the Gulf of Mexico to Lake Erie, New York State, and to the eastern seaboard.

Columbia Gas System, Inc., and Columbia Gas Transmission Corporation, its principal pipeline subsidiary, have been operating under the protection of Chapter 11 of the
Federal Bankruptcy Code since July 1991. Both companies hope to emerge from this status in the near future.

Columbia Gas Transmission operates one of the largest natural gas storage systems in the Nation with a storage capacity totaling 775 billion cubic feet. To improve injection and withdrawal rates, Columbia drilled a horizontal well in one of its storage fields. Early test results are promising. Columbia Gas Transmission also maintains competitively priced natural gas transportation and storage services for local distribution companies and industrial and commercial customers who contract directly with producers or marketers for their gas supplies.

Figure 6. Columbia Gas System, Inc., 1993

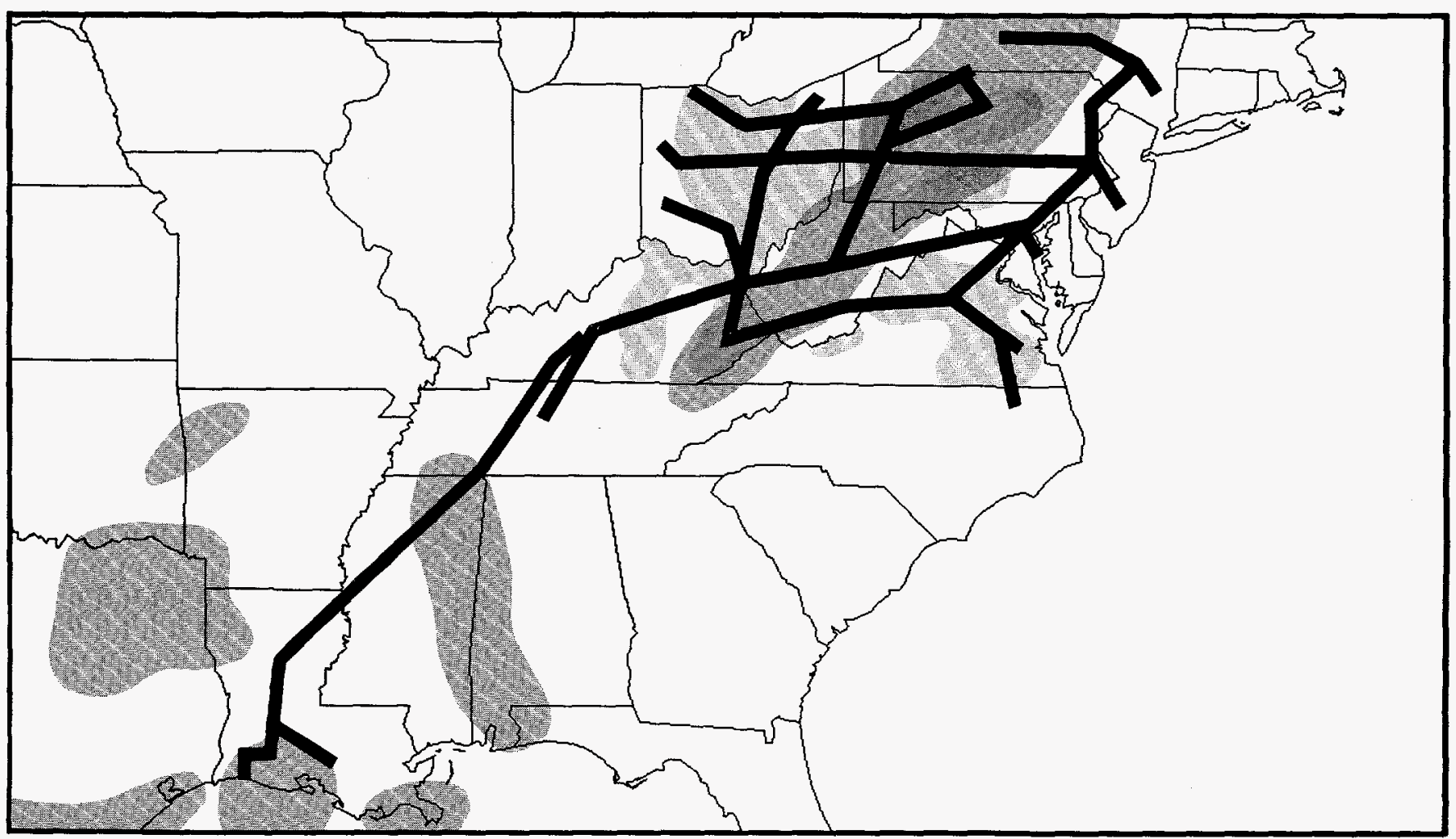

Source: Energy Information Administration (EIA), Form EIA-176, "Annual Report of Natural and Supplemental Gas Supply and Disposition." 
Table 7. Columbia Gas System, Inc., Natural Gas Data, 1993

(Thousand Cubic Feet)

\begin{tabular}{|c|c|c|c|c|c|}
\hline \multirow{2}{*}{ Supply/Disposition } & \multicolumn{5}{|c|}{ Columbia Gas Transmission Corp. } \\
\hline & Tennessee & Kentucky & West Virginia & Virginia & Maryland \\
\hline \multicolumn{6}{|l|}{ SUPPLY } \\
\hline $\begin{array}{l}\text { Produced Onsystem } \\
\text { Receipts }\end{array}$ & 0 & 0 & 0 & 0 & 0 \\
\hline 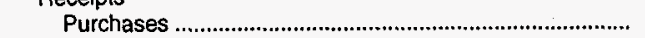 & 0 & $139,576,780$ & $3,946,853$ & $1,668,516$ & 2,407 \\
\hline 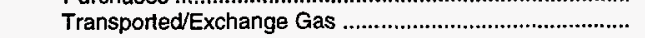 & 0 & $502,361,312$ & $236,891,987$ & 11,470 & 29,703 \\
\hline \multicolumn{6}{|l|}{ Interstate Movements } \\
\hline 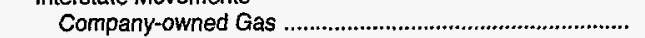 & 0 & 0 & 0 & 0 & 0 \\
\hline 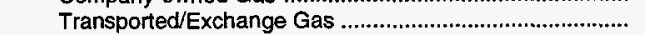 & 717,156 & 0 & $587,024,840$ & $315,644,187$ & $158,662,678$ \\
\hline Storage Withdrawals & 0 & $3,217,357$ & $69,708,952$ & 0 & $4,240,560$ \\
\hline Received from Storage Operators & 49,505 & 0 & 0 & $13,328,729$ & 0 \\
\hline Other Supplies & 0 & 0 & 0 & 0 & 0 \\
\hline 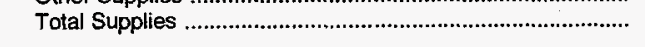 & 766,661 & $645,155,449$ & $897,572,632$ & $330,652,902$ & $162,935,348$ \\
\hline \multicolumn{6}{|l|}{ DISPOSITION } \\
\hline Lease and Plant Use & 0 & 0 & $3,336,261$ & 0 & 0 \\
\hline Returned to Formations & 0 & 0 & 0 & 0 & 0 \\
\hline \multicolumn{6}{|l|}{ Interstate Movements } \\
\hline Company-owned Gas & 0 & $330,295,578$ & 0 & 0 & 0 \\
\hline 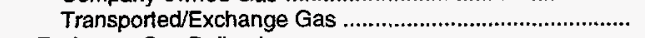 & 0 & $268,798,979$ & $742,160,793$ & $167,396,564$ & $34,440,700$ \\
\hline 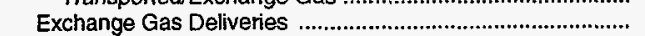 & 0 & 0 & 0 & 0 & 0 \\
\hline Storage Injections & 0 & 861,628 & $80,022,851$ & 0 & 46,248 \\
\hline \multicolumn{6}{|l|}{ Deliveries of Company-owned Gas } \\
\hline To Storage Operators & 0 & 0 & 0 & $1,606,706$ & 0 \\
\hline To Other Pipelines & 0 & 0 & $5,161,147$ & 0 & 0 \\
\hline 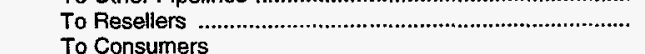 & 718,117 & $7,566,606$ & 0 & $20,626,391$ & $22,372,199$ \\
\hline 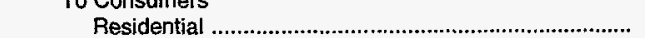 & 0 & 0 & 0 & 0 & 0 \\
\hline Commercial & 0 & 0 & 0 & 0 & 0 \\
\hline Industrial & 0 & 0 & 0 & 0 & 0 \\
\hline Electric Utility & 0 & 0 & 0 & 0 & 0 \\
\hline Vehicle Fuel & 0 & 0 & 0 & 0 & 0 \\
\hline \multicolumn{6}{|l|}{ Transported for the Account of Others } \\
\hline 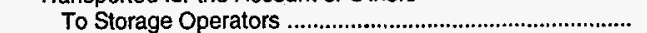 & 0 & 0 & 0 & 0 & 0 \\
\hline To Other Pipelines & 0 & 0 & 0 & 0 & 0 \\
\hline 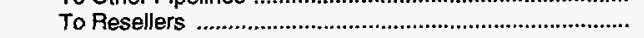 & 48,544 & $38,992,200$ & $59,582,324$ & $127,700,511$ & $106,076,201$ \\
\hline \multicolumn{6}{|l|}{ To Consumers } \\
\hline 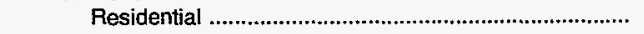 & 0 & 0 & 0 & 0 & 0 \\
\hline 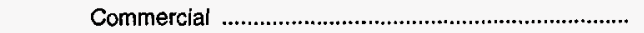 & 0 & 0 & 0 & 0 & 0 \\
\hline 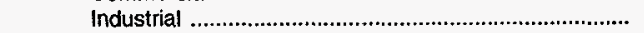 & 0 & 0 & 0 & $13,932,683$ & 0 \\
\hline Electric Utility & 0 & 0 & 0 & 0 & 0 \\
\hline Vehicle Fuel & 0 & 0 & 0 & 0 & 0 \\
\hline 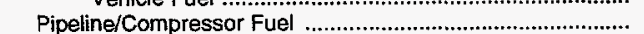 & 0 & 0 & $7,133,399$ & 0 & 0 \\
\hline Other Disposition & 0 & 0 & 0 & 0 & 0 \\
\hline Unaccounted For & 0 & $-1,359,542$ & 175,857 & $-609,953$ & 0 \\
\hline Total Disposition & 766,661 & $645,155,449$ & $897,572,632$ & $330,652,902$ & $162,935,348$ \\
\hline
\end{tabular}

Columbia Gas distribution companies are participating in gas industry efforts to promote natural gas vehicles as alternatives to regular-fueled fleet and mass transit vehicles. In March 1993, Columbia Gas of Ohio opened the Nation's largest publicly available natural gas vehicle fueling station in Columbus, Ohio. Columbia currently operates five other publicly available fueling stations. Plans are in place to develop up to one hundred additional fueling stations in the next 5 years.

Columbia LNG Corporation owns the largest liquefied natural gas receiving and regasification terminal in North America at Cove Point, Maryland. Although this facility has been idle since 1980, the Federal Energy Regulatory Commission (FERC) gave their approval for Cove Point LNG to be recommissioned to provide peaking services scheduled to begin in 1995. Cove Point LNG is in the process of obtaining the terminal from Columbia Gas Transmission and Columbia LNG, along with an 87-mile pipeline that connects the facility to an interconnect with Columbia Gas Transmission and CNG Transmission in Virginia. A 15 million cubic feet per day liquefaction plant will be constructed at the Cove Point site so deliveries during the summer can be liquefied and stored in existing LNG tanks for regasification and delivery during the winter. 
Table 7. Columbia Gas System, Inc., Natural Gas Data, 1993 (Continued) (Thousand Cubic Feet)

\begin{tabular}{|c|c|c|c|c|c|}
\hline \multirow{2}{*}{ Supply/Disposition } & \multicolumn{5}{|c|}{ Columbia Gas Transmission Corp. } \\
\hline & Delaware & North Carolina & Ohio & Pennsylvania & New York \\
\hline \multicolumn{6}{|l|}{ SUPPLY } \\
\hline $\begin{array}{l}\text { Produced Onsystem } \\
\text { Receipts }\end{array}$ & 0 & 0 & 0 & 0 & 0 \\
\hline 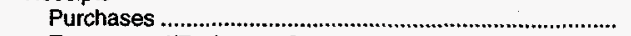 & 0 & 0 & $13,371,867$ & $23,000,654$ & 0 \\
\hline 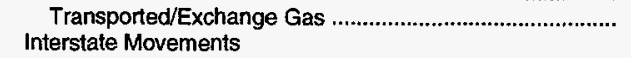 & 0 & 0 & $95,549,140$ & $12,557,862$ & $3,543,246$ \\
\hline Company-owned Gas & 0 & 0 & 0 & 0 & 0 \\
\hline 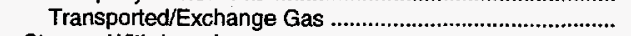 & $6,156,298$ & $8,733,886$ & $261,020,236$ & $219,884,717$ & $34,620,799$ \\
\hline 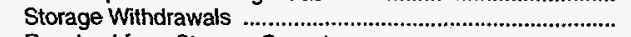 & 0 & 0 & $124,742,449$ & $17,735,859$ & $6,005,829$ \\
\hline Received from Storage Operators & 0 & 739,963 & 0 & $1,196,389$ & 0 \\
\hline Other Supplies & 0 & 0 & 0 & 0 & 0 \\
\hline 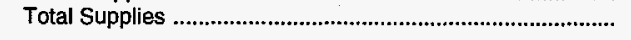 & $6,156,298$ & $9,473,849$ & $494,683,692$ & $274,375,481$ & $44,169,874$ \\
\hline \multicolumn{6}{|l|}{ DISPOSITION } \\
\hline Lease and Plant Use & 0 & 0 & 98,854 & 13,896 & 0 \\
\hline $\begin{array}{l}\text { Returned to Formations } \\
\text { Interstate Movements }\end{array}$ & 0 & 0 & 0 & 0 & 0 \\
\hline Company-owned Gas & 0 & 0 & 0 & 0 & 0 \\
\hline 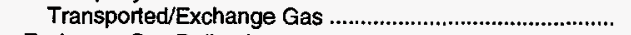 & 0 & 0 & 0 & $85,638,095$ & 0 \\
\hline 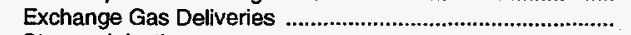 & 0 & 0 & 0 & 0 & 0 \\
\hline Storage Injections & 0 & 0 & $112,018,037$ & $10,988,117$ & $4,224,306$ \\
\hline \multicolumn{6}{|l|}{ Deliveries of Company-owned Gas } \\
\hline To Storage Operators & 0 & 452 & 0 & $1,724,146$ & 0 \\
\hline 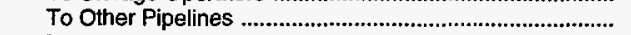 & 0 & 0 & 0 & 0 & 0 \\
\hline 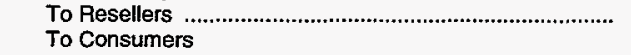 & $1,165,378$ & $4,437,273$ & $73,805,042$ & $18,367,375$ & $7,045,083$ \\
\hline 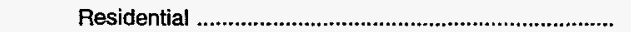 & 0 & 0 & 0 & 0 & 0 \\
\hline 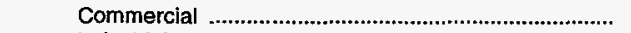 & 0 & 0 & 0 & 0 & 0 \\
\hline 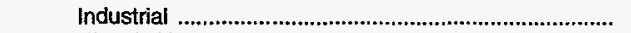 & 0 & 0 & 0 & 0 & 0 \\
\hline Electric Utility & 0 & 0 & 0 & 0 & 0 \\
\hline 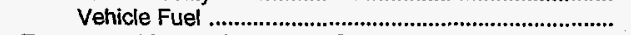 & 0 & 0 & 0 & 0 & 0 \\
\hline \multicolumn{6}{|l|}{ Transported for the Account of Others } \\
\hline 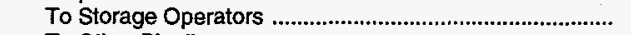 & 0 & 0 & 0 & 0 & 0 \\
\hline 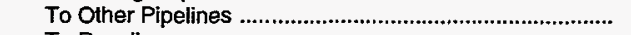 & 0 & 0 & 0 & 0 & 0 \\
\hline 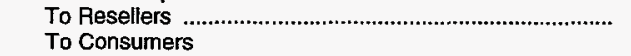 & $4,990,920$ & $5,036,124$ & $308,769,824$ & $155,712,607$ & $33,234,133$ \\
\hline 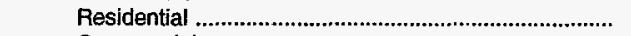 & 0 & 0 & 0 & 0 & 0 \\
\hline 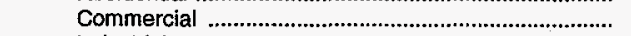 & 0 & 0 & 0 & 0 & 0 \\
\hline Industrial & 0 & 0 & 0 & 0 & 0 \\
\hline 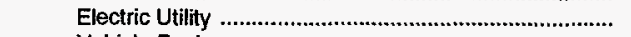 & 0 & 0 & 0 & 0 & 0 \\
\hline Vehicle Fuel & 0 & 0 & 0 & 0 & 0 \\
\hline 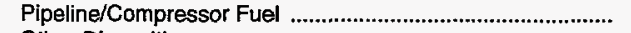 & 0 & 0 & 0 & $1,931,245$ & 0 \\
\hline 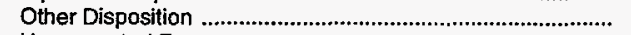 & 0 & 0 & 0 & 0 & 0 \\
\hline 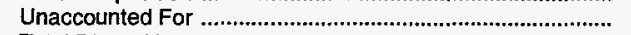 & 0 & 0 & $-8,065$ & 0 & $-333,648$ \\
\hline Total Disposition & $6,156,298$ & $9,473,849$ & $494,683,692$ & $274,375,481$ & $44,169,874$ \\
\hline
\end{tabular}

Five distribution subsidiaries provide natural gas service to more than 1.9 million residential, commercial, industrial, and electric generation customers in Kentucky, Maryland, Ohio, Pennsylvania, and Virginia. Not only are these subsidiaries developing and managing natural gas supplies for high priority customers, they are also transporting natural gas for industrial and commercial customers who purchase natural gas from other sources. In addition, through the subsidiary TriStar Ventures Corporation, three cogeneration facilities are now operating and several more are under development. 
Table 7. Columbia Gas System, Inc., Natural Gas Data, 1993 (Continued) (Thousand Cubic Feet)

\begin{tabular}{|c|c|c|c|c|c|}
\hline \multirow{2}{*}{ Supply/Disposition } & \multirow{2}{*}{$\begin{array}{c}\begin{array}{c}\text { Columbia Gas } \\
\text { Transmission } \\
\text { Corp. }\end{array} \\
\text { New Jersey } \\
\end{array}$} & \multicolumn{4}{|c|}{ Columbia Gulf Transmission Corp. } \\
\hline & & Louisiana & Mississippi & Tennessee & Kentucky \\
\hline \multicolumn{6}{|l|}{ SUPPLY } \\
\hline $\begin{array}{l}\text { Produced Onsystem } \\
\text { Receipts }\end{array}$ & 0 & 0 & 0 & 0 & 0 \\
\hline 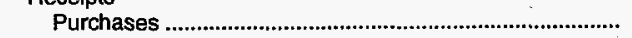 & 0 & 0 & 0 & 0 & 0 \\
\hline 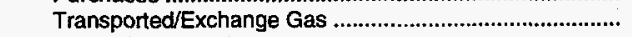 & 0 & $823,588,669$ & 0 & 0 & $2,102,473$ \\
\hline \multicolumn{6}{|l|}{ Interstate Movements } \\
\hline 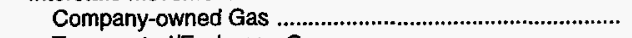 & 0 & 0 & 0 & 0 & 0 \\
\hline 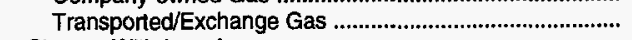 & $36,265,912$ & 0 & $579,779,316$ & $572,857,815$ & $561,225,089$ \\
\hline Storage Withdrawals & 0 & 0 & 0 & 0 & 0 \\
\hline 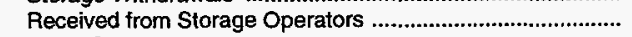 & 625,555 & 0 & 0 & 0 & 0 \\
\hline 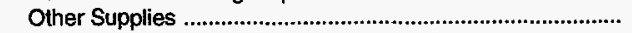 & 0 & 0 & 0 & 0 & 0 \\
\hline 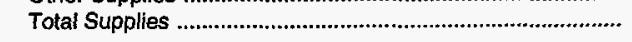 & $36,891,467$ & $823,588,669$ & $579,779,316$ & $572,857,815$ & $563,327,562$ \\
\hline \multicolumn{6}{|l|}{ DISPOSITION } \\
\hline 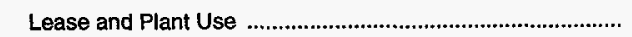 & 0 & $7,198,691$ & 0 & 0 & 0 \\
\hline 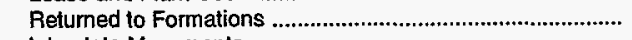 & 0 & 0 & 0 & 0 & 0 \\
\hline Interstate Movements & & & & & \\
\hline 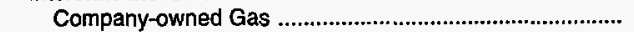 & 0 & 0 & 0 & 0 & 0 \\
\hline 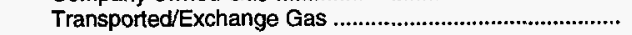 & 0 & $579,779,316$ & $572,857,815$ & $561,225,089$ & 0 \\
\hline 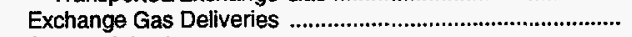 & 0 & $227,425,194$ & 7,134 & $7,245,608$ & $560,487,756$ \\
\hline 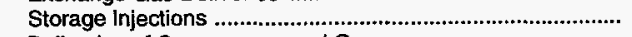 & 0 & 0 & 0 & 0 & 0 \\
\hline \multicolumn{6}{|l|}{ Deliveries of Company-owned Gas } \\
\hline 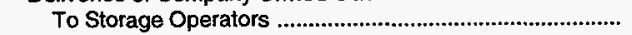 & 72,095 & 0 & 0 & 0 & 0 \\
\hline 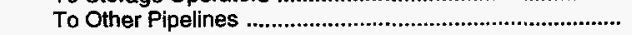 & 0 & 0 & 0 & 0 & 0 \\
\hline 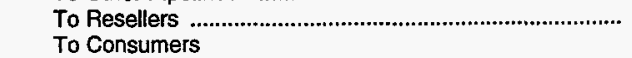 & $3,349,932$ & 0 & 0 & 0 & 0 \\
\hline 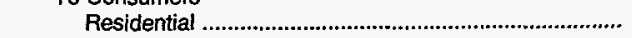 & 0 & 0 & 0 & 0 & 0 \\
\hline 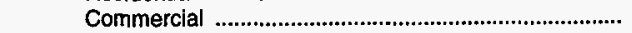 & 0 & 0 & 0 & 0 & 0 \\
\hline Industrial & 0 & 29,048 & 0 & 0 & 0 \\
\hline Electric Utility & 0 & 0 & 0 & 0 & 0 \\
\hline 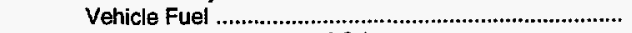 & 0 & 0 & 0 & 0 & 0 \\
\hline \multicolumn{6}{|l|}{ Transported for the Account of Others } \\
\hline 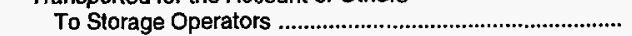 & 0 & 0 & 0 & 0 & 0 \\
\hline To Other Pipelines & 0 & 0 & 0 & 0 & 0 \\
\hline 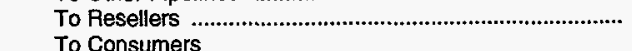 & $33,469,440$ & 0 & 0 & 0 & 0 \\
\hline 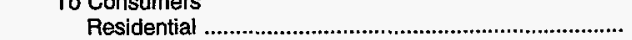 & 0 & 0 & 0 & 0 & 0 \\
\hline 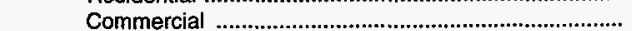 & 0 & 0 & 0 & 0 & 0 \\
\hline Industrial & 0 & 0 & 0 & 0 & 0 \\
\hline 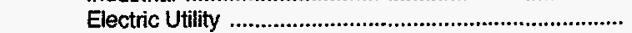 & 0 & 0 & 0 & 0 & 0 \\
\hline Vehicle Fuel & 0 & 0 & 0 & 0 & 0 \\
\hline 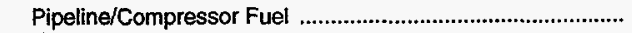 & 0 & $7,207,800$ & $5,442,889$ & $3,453,475$ & $2,263,443$ \\
\hline 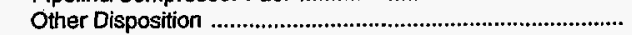 & 0 & 0 & 0 & 0 & 0 \\
\hline Unaccounted For & 0 & $1,948,620$ & $1,471,478$ & 933,643 & 576,363 \\
\hline Total Disposition & $36,891,467$ & $823,588,669$ & $579,779,316$ & $572,857,815$ & $563,327,562$ \\
\hline
\end{tabular}


Table 7. Columbia Gas System, Inc., Natural Gas Data, 1993 (Continued) (Thousand Cubic Feet)

\begin{tabular}{|c|c|c|c|c|c|}
\hline \multirow{2}{*}{ Supply/Disposition } & \multicolumn{2}{|c|}{ Columbia Gulf Transmission Corp. } & \multicolumn{3}{|c|}{ Columbia Gas Distribution Companies } \\
\hline & Wyoming & Texas & Kentucky & Ohio & Pennsylvania \\
\hline \multicolumn{6}{|l|}{ SUPPLY } \\
\hline $\begin{array}{l}\text { Produced Onsystem } \\
\text { Receipts }\end{array}$ & 0 & 0 & 0 & 0 & 0 \\
\hline 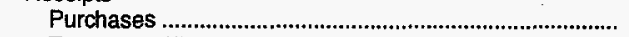 & 0 & 0 & $11,878,627$ & $113,159,769$ & $36,984,538$ \\
\hline 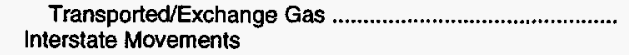 & $31,452,723$ & 570,330 & $34,000,983$ & $281,648,855$ & $95,821,237$ \\
\hline Company-owned Gas & 0 & 0 & 0 & 0 & 0 \\
\hline 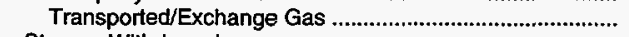 & 0 & 0 & 0 & 0 & 0 \\
\hline 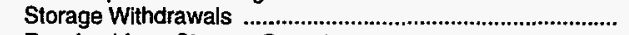 & 0 & 0 & 0 & 0 & 96,460 \\
\hline 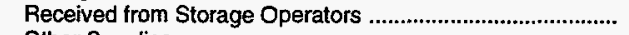 & 0 & 0 & 0 & 0 & 0 \\
\hline 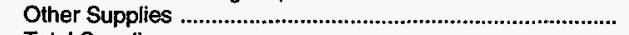 & 0 & 0 & 14,386 & 0 & 0 \\
\hline 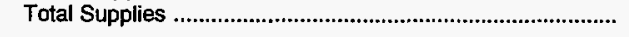 & $31,452,723$ & 570,330 & $45,893,996$ & $394,808,624$ & $132,902,235$ \\
\hline \multicolumn{6}{|l|}{ DISPOSITION } \\
\hline Lease and Plant Use & 0 & 0 & 0 & 0 & 0 \\
\hline $\begin{array}{l}\text { Returned to Formations } \\
\text { Interstate Movements }\end{array}$ & 0 & 0 & 0 & 0 & 0 \\
\hline Company-owned Gas & 0 & 0 & 0 & 0 & 0 \\
\hline 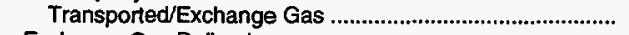 & 0 & 0 & 0 & 0 & 0 \\
\hline 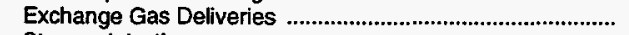 & $31,324,887$ & 570,311 & 691,382 & $7,027,645$ & $2,902,948$ \\
\hline 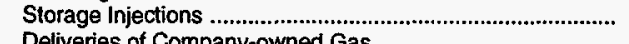 & 0 & 0 & 0 & 0 & 89,612 \\
\hline \multicolumn{6}{|l|}{ Deliveries of Company-owned Gas } \\
\hline To Storage Operators & 0 & 0 & $8,862,325$ & $79,376,168$ & $27,863,451$ \\
\hline 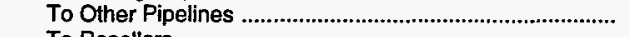 & 0 & 0 & 0 & 0 & 0 \\
\hline $\begin{array}{l}\text { To Resellers ................................................................... } \\
\text { To Consumers }\end{array}$ & 0 & 0 & 127,100 & 0 & 0 \\
\hline 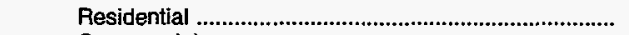 & 0 & 0 & $11,863,059$ & $129,796,954$ & $38,771,036$ \\
\hline 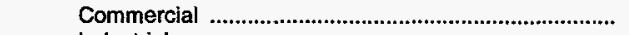 & 0 & 0 & $6,231,164$ & $50,009,134$ & $17,032,554$ \\
\hline 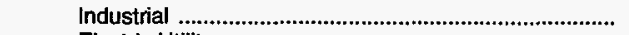 & 0 & 0 & 322,954 & $5,687,123$ & $5,230,970$ \\
\hline 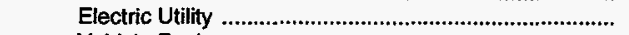 & 0 & 0 & 21,617 & 10,557 & 26,457 \\
\hline Vehicle Fuel & 0 & 0 & 34 & 2,023 & 1,646 \\
\hline \multicolumn{6}{|l|}{ Transported for the Account of Others } \\
\hline 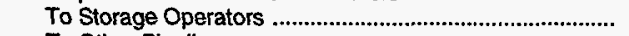 & 0 & 0 & 0 & 0 & 0 \\
\hline 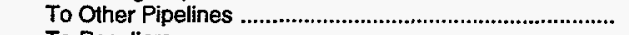 & 0 & 0 & 0 & 0 & 0 \\
\hline 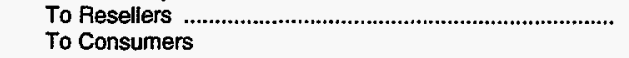 & 0 & 0 & 0 & 0 & 0 \\
\hline 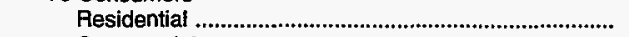 & 0 & 0 & 0 & 0 & 0 \\
\hline 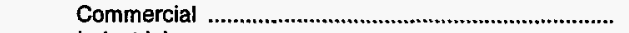 & 0 & 0 & $1,478,289$ & $13,882,442$ & $4,714,306$ \\
\hline 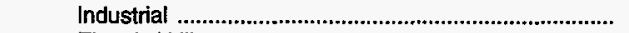 & 0 & 0 & $15,789,102$ & $104,683,994$ & $33,942,353$ \\
\hline 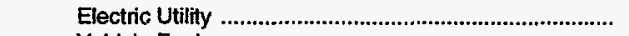 & 0 & 0 & 0 & 36,841 & 152,364 \\
\hline Vehicle Fuel & 0 & 0 & 0 & 0 & 0 \\
\hline 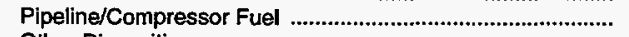 & 103,295 & 27,649 & 19,069 & 185,636 & 60,091 \\
\hline Other Disposition & 0 & 0 & 4,923 & 16,969 & 10,040 \\
\hline 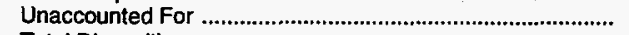 & 24,541 & $-27,630$ & 482,978 & $4,093,138$ & $2,104,407$ \\
\hline 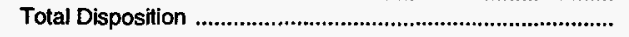 & $31,452,723$ & 570,330 & $45,893,996$ & $394,808,624$ & $132,902,235$ \\
\hline
\end{tabular}


Table 7. Columbia Gas System, Inc., Natural Gas Data, 1993 (Continued) (Thousand Cubic Feet)

\begin{tabular}{|c|c|c|c|c|}
\hline \multirow{2}{*}{ Supply/Disposition } & \multirow{2}{*}{$\begin{array}{c}\text { Columbia Gas Distribution } \\
\text { Companies } \\
\text { Maryland }\end{array}$} & \multirow{2}{*}{$\begin{array}{c}\text { Commonwealth Gas } \\
\text { Service, Inc. }\end{array}$} & \multicolumn{2}{|c|}{ Columbia LNG Corp. } \\
\hline & & & Maryland & Virginia \\
\hline \multicolumn{5}{|l|}{ SUPPLY } \\
\hline $\begin{array}{l}\text { Produced Onsystem } \\
\text { Receipts }\end{array}$ & 0 & 0 & 0 & 0 \\
\hline 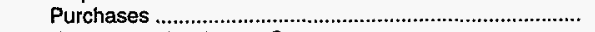 & $2,392,521$ & $16,273,779$ & 0 & 0 \\
\hline 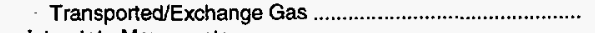 & $6,150,548$ & $55,578,042$ & 0 & $4,972,827$ \\
\hline \multicolumn{5}{|l|}{ Interstate Movements } \\
\hline 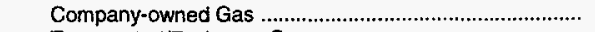 & 0 & 0 & 0 & 0 \\
\hline 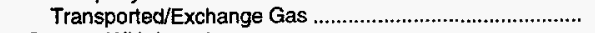 & 0 & 0 & $4,972,827$ & 0 \\
\hline 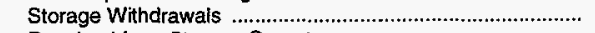 & 0 & 0 & 0 & 0 \\
\hline 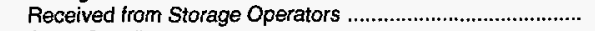 & 0 & 0 & 0 & 0 \\
\hline Other Supplies & 0 & 199,883 & 0 & $\frac{0}{20}$ \\
\hline 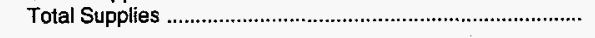 & $8,543,069$ & $72,051,704$ & $4,972,827$ & $4,972,827$ \\
\hline \multicolumn{5}{|l|}{ DISPOSTION } \\
\hline 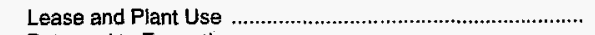 & 0 & 0 & 0 & 0 \\
\hline 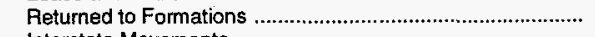 & 0 & 0 & 0 & 0 \\
\hline \multicolumn{5}{|l|}{ Interstate Movements } \\
\hline 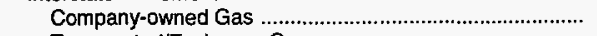 & 0 & 0 & 0 & 0 \\
\hline 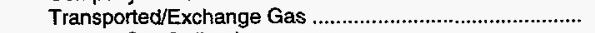 & 0 & 0 & 0 & $4,972,827$ \\
\hline 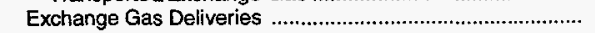 & 178,415 & 545,800 & 0 & 0 \\
\hline 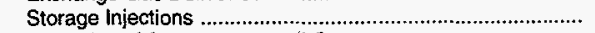 & 0 & 0 & 0 & 0 \\
\hline \multicolumn{5}{|l|}{ Deliveries of Company-owned Gas } \\
\hline 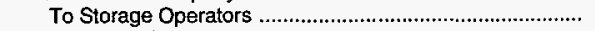 & $1,673,623$ & $5,367,441$ & 0 & 0 \\
\hline 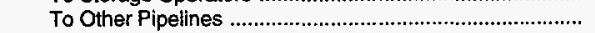 & 0 & 0 & 0 & 0 \\
\hline 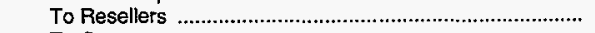 & 0 & 0 & 0 & 0 \\
\hline \multicolumn{5}{|l|}{ To Consumers } \\
\hline 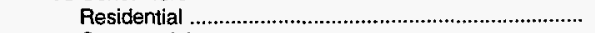 & $2,834,509$ & $11,444,975$ & 0 & 0 \\
\hline 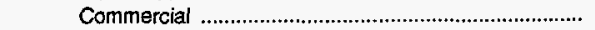 & $1,629,442$ & $8,506,351$ & 0 & 0 \\
\hline 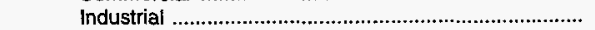 & 192,062 & $2,538,325$ & 0 & 0 \\
\hline Electric Utility & 0 & 8,451 & 0 & 0 \\
\hline 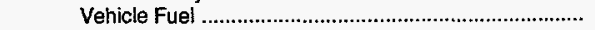 & 0 & 1,460 & 0 & 0 \\
\hline \multicolumn{5}{|l|}{ Transported for the Account of Others } \\
\hline 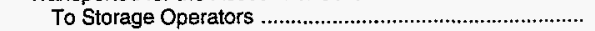 & 0 & 0 & 0 & 0 \\
\hline 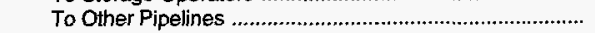 & 0 & 0 & 0 & 0 \\
\hline 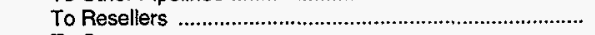 & 0 & 0 & $4,972,827$ & 0 \\
\hline \multicolumn{5}{|l|}{ To Consumers } \\
\hline 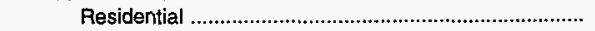 & 0 & 0 & 0 & 0 \\
\hline 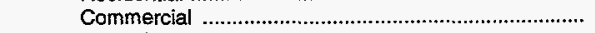 & 567,094 & $1,668,787$ & 0 & 0 \\
\hline 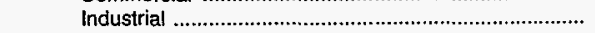 & $1,205,312$ & $20,586,065$ & 0 & 0 \\
\hline 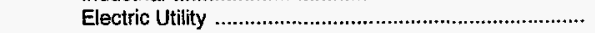 & 0 & $18,767,659$ & 0 & 0 \\
\hline Vehicle Fuel & 0 & 0 & 0 & 0 \\
\hline 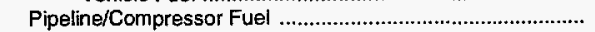 & 5,228 & 16,756 & 0 & 0 \\
\hline 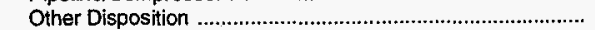 & 779 & 4,338 & 0 & 0 \\
\hline Unaccounted For & 256,605 & $2,595,296$ & 0 & 0 \\
\hline 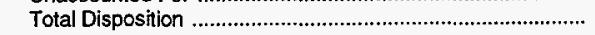 & $8,543,069$ & $72,051,704$ & $4,972,827$ & $4,972,827$ \\
\hline
\end{tabular}

Source: Energy Information Administration (EIA), Form EIA-176, "Annual Report of Natural and Supplemental Gas Supply and Disposition." 
Table 8. Columbia Gas System, Inc., Interstate Flows of Natural Gas, 1993

(Thousand Cubic Feet)

\begin{tabular}{|c|c|}
\hline Company and State & Volume \\
\hline \multicolumn{2}{|l|}{$\begin{array}{l}\text { Columbia Gas Transmission Corp. } \\
\text { Kentucky To }\end{array}$} \\
\hline Ohio & $11,352,561$ \\
\hline 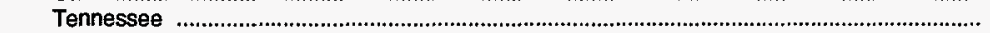 & 717,156 \\
\hline West Virginia & $587,024,840$ \\
\hline \multicolumn{2}{|l|}{ West Virginia To } \\
\hline Ohio & $241,072,589$ \\
\hline Pennsylvania & $185,444,017$ \\
\hline 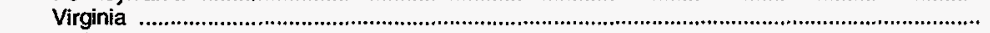 & $315,644,187$ \\
\hline \multicolumn{2}{|l|}{ Virginia To } \\
\hline Maryland & $158,662,678$ \\
\hline North Caroline & $8,733,886$ \\
\hline \multicolumn{2}{|l|}{ Maryland To } \\
\hline \multicolumn{2}{|l|}{$\begin{array}{l}\text { Pennsylvania } \\
\text { Pennsylvania To }\end{array}$} \\
\hline Delaware & $6,156,298$ \\
\hline New Jersey & $36,265,912$ \\
\hline New York & $34,620,799$ \\
\hline Ohio & $\mathbf{8 , 5 9 5 , 0 8 6}$ \\
\hline \multicolumn{2}{|l|}{$\begin{array}{l}\text { Columbia Gulf Transmission Corp. } \\
\text { Louisiana To }\end{array}$} \\
\hline Mississippi & $579,779,316$ \\
\hline \multicolumn{2}{|l|}{ Mississippi To } \\
\hline Tennessee & $572,857,815$ \\
\hline \multicolumn{2}{|l|}{ Tennessee To } \\
\hline 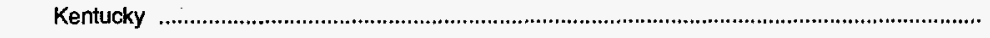 & $561,225,089$ \\
\hline \multicolumn{2}{|l|}{ Columbia LNG Corp. } \\
\hline Virginia To & $4,972,827$ \\
\hline
\end{tabular}

Source: Energy Information Administration (E|A), Form EIA-176, "Annual Report of Natural and Supplemental Gas Supply and Disposition." 



\section{Consolidated Edison Co., of New York, Inc.}

Consolidated Edison Company of New York (Con Edison) is a publicly held utility that provides electric, gas, and steam supply system services throughout most of New York City. Con Edison supplies natural gas to Manhattan, the Bronx, and parts of Queens, and to Westchester County. The company is principally engaged in the generation, transmission, and distribution of electricity, providing electric service to nearly all of New York City and most of Westchester County, an area with a combined population of more than 8 million. The New York Power Authority uses Con Edison's facilities to provide electric service to most governmental customers within Con Edison's service area. The company also supplies steam to parts of Manhattan. Con Edison's natural gas system consists of approximately 4,200 miles of distribution pipeline, 438 miles of plastic mains, and 2,495 miles of service line.

Con Edison owns a controlling interest in Honeoye Storage Corporation of Canandaigua, New York. Formed in 1973 , Honeoye is principally engaged in the storage and transmission of natural gas. Con Edison also has a natural gas liquefaction facility and a liquefied gas storage tank in Queens, New York.

Con Edison actively encourages owners of vehicle fleets to switch to compressed natural gas as a fuel. The company itself has more than 300 vehicles fueled by natural

Figure 7. Consolidated Edison Co., of New York, Inc., 1993

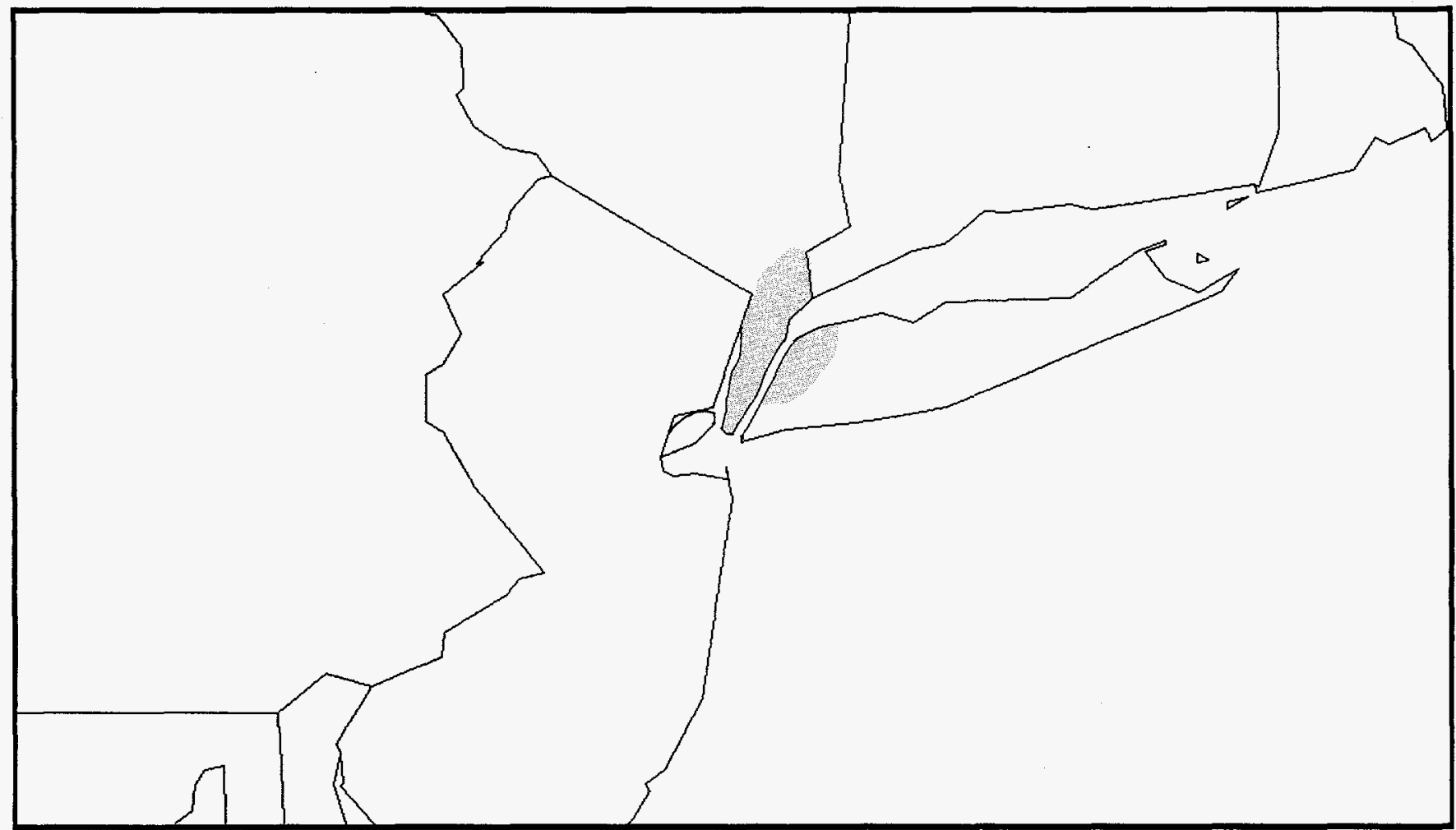

Source: Energy Information Administration (EIA), Form EIA-176, "Annual Report of Natural and Supplemental Gas Supply and Disposition."

Service Area 


\begin{tabular}{|c|c|}
\hline \multirow{2}{*}{ Supply/Disposition } & Consolidated Edison Co. of New York, Inc. \\
\hline & New York \\
\hline \multicolumn{2}{|l|}{ SUPPLY } \\
\hline \multicolumn{2}{|l|}{ 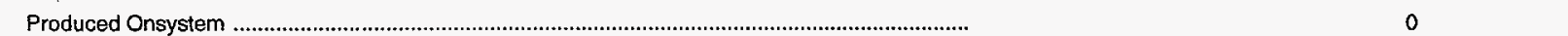 } \\
\hline \multicolumn{2}{|l|}{ Receipts } \\
\hline 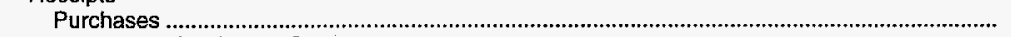 & $26,940,071$ \\
\hline 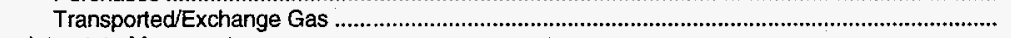 & $201,011,410$ \\
\hline \multicolumn{2}{|l|}{ Interstate Movements } \\
\hline Company-owned Gas & 0 \\
\hline 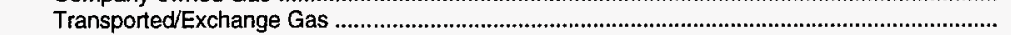 & 0 \\
\hline Storage Withdrawals & 146,426 \\
\hline Received from Storage Operators & $21,162,164$ \\
\hline Other Supplies & 0 \\
\hline 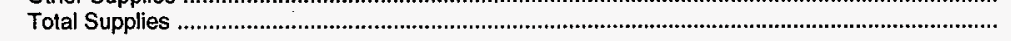 & $249,260,071$ \\
\hline \multicolumn{2}{|l|}{ DISPOSITION } \\
\hline Lease and Plant Use & 0 \\
\hline 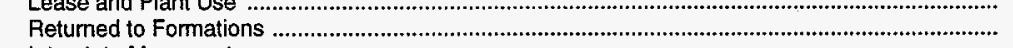 & 0 \\
\hline \multicolumn{2}{|l|}{ Interstate Movements } \\
\hline 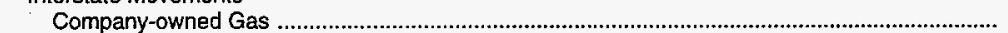 & 0 \\
\hline 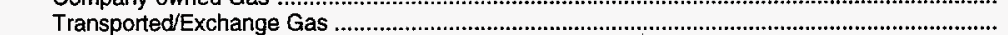 & 0 \\
\hline Exchange Gas Deliveries & 0 \\
\hline Storage Injections & 234,962 \\
\hline \multicolumn{2}{|l|}{ Deliveries of Company-owned Gas } \\
\hline \multirow{4}{*}{ 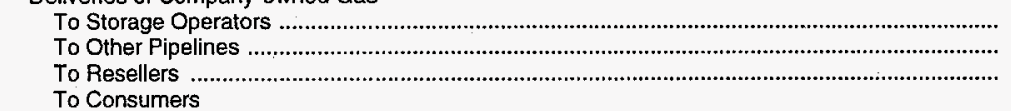 } & $20,848,048$ \\
\hline & 0 \\
\hline & 0 \\
\hline & \\
\hline 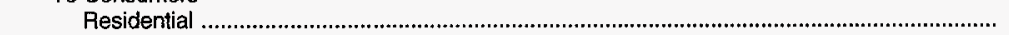 & $51,700,875$ \\
\hline \multirow{2}{*}{ Commercial } & $57,696,779$ \\
\hline & $5,783,585$ \\
\hline 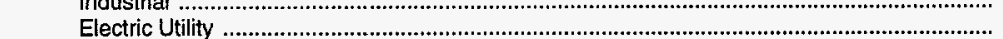 & $89,050,074$ \\
\hline Vehicle Fuel & 2,446 \\
\hline \multicolumn{2}{|l|}{ Transported for the Account of Others } \\
\hline \multirow{4}{*}{ 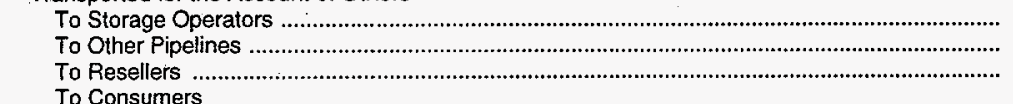 } & 0 \\
\hline & 0 \\
\hline & 0 \\
\hline & \\
\hline To Consumers & 0 \\
\hline Commercial & 0 \\
\hline Industrial & $4,774,405$ \\
\hline Electric Utility & $15,455,068$ \\
\hline Vehicle Fuel & 0 \\
\hline Pipeline/Compressor Fuel & 12 \\
\hline 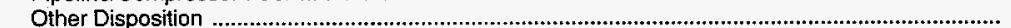 & 0 \\
\hline 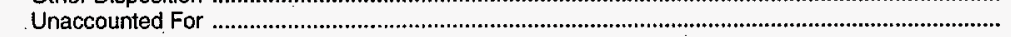 & $3,713,817$ \\
\hline Total Disposition & $249,260,071$ \\
\hline
\end{tabular}

Source: Energy Information Administration (EIA), Form EIA-176, "Annual Report of Natural and Supplemental Gas Supply and Disposition."

gas and plans to increase this number to 1,000 in the next few years. It operates six fueling stations and plans to add three more. Con Edison is working with the City of New York to open a fueling station in Manhattan to be used by the natural gas vehicle fleet operated by New York City agencies.

Continuing its efforts to promote the use of natural gas heating installations, Con Edison saw the conversion to natural gas heat of nearly 400 buildings with five or more residential units and over 2,000 one- to- four- family homes during 1993. For new construction, more than 160 multiple-dwelling units and about 1,700 new single-family homes chose gas heat.

Con Edison is making extensive use of trenchless technologies to replace or reline cast iron and steel natural gas mains with plastic pipe. These are "no-dig" methods that do not require traditional trenching to maintain and replace existing gas mains, thus reducing excavation, restoration, and labor costs. 


\section{Consolidated Natural Gas Co.}

Consolidated Natural Gas Company (CNG), based in Pittsburgh, Pennsylvania and New York City, consists of 15 subsidiaries. A primary mission of CNG is to produce and purchase natural gas throughout the Nation. It sells and transports gas to markets in the Midwest, Mid-Atlantic, and Northeast, serving both utilities and residential consumers. Other operations include the sale of gas byproducts and petroleum production. CNG also operates the largest underground storage system for natural gas in the United States.

In 1993, CNG Transmission tremendously expanded its pipeline capabilities and storage facilities when it completed the construction of its Lebanon-to-Leidy pipeline project. This project enhances the Company's ability to move gas from a supply hub at Lebanon, Ohio, to the large Leidy storage field in north central Pennsylvania. The Lebanon-to-Leidy pipeline increases CNG's East Coast delivery capacity by 380 million cubic feet per day.

In late 1993, CNG, along with Texaco, the owner of Sabine Pipeline, began exploring the possibilities of creating a "super-hub," that would make it easier to transport gas between various points and in different directions. The hub began operating in July 1994 throughout the entire CNG pipeline system serving New York, Ohio, Pennsylvania, and West Virginia.

Figure 8. Consolidated Natural Gas Co., 1993

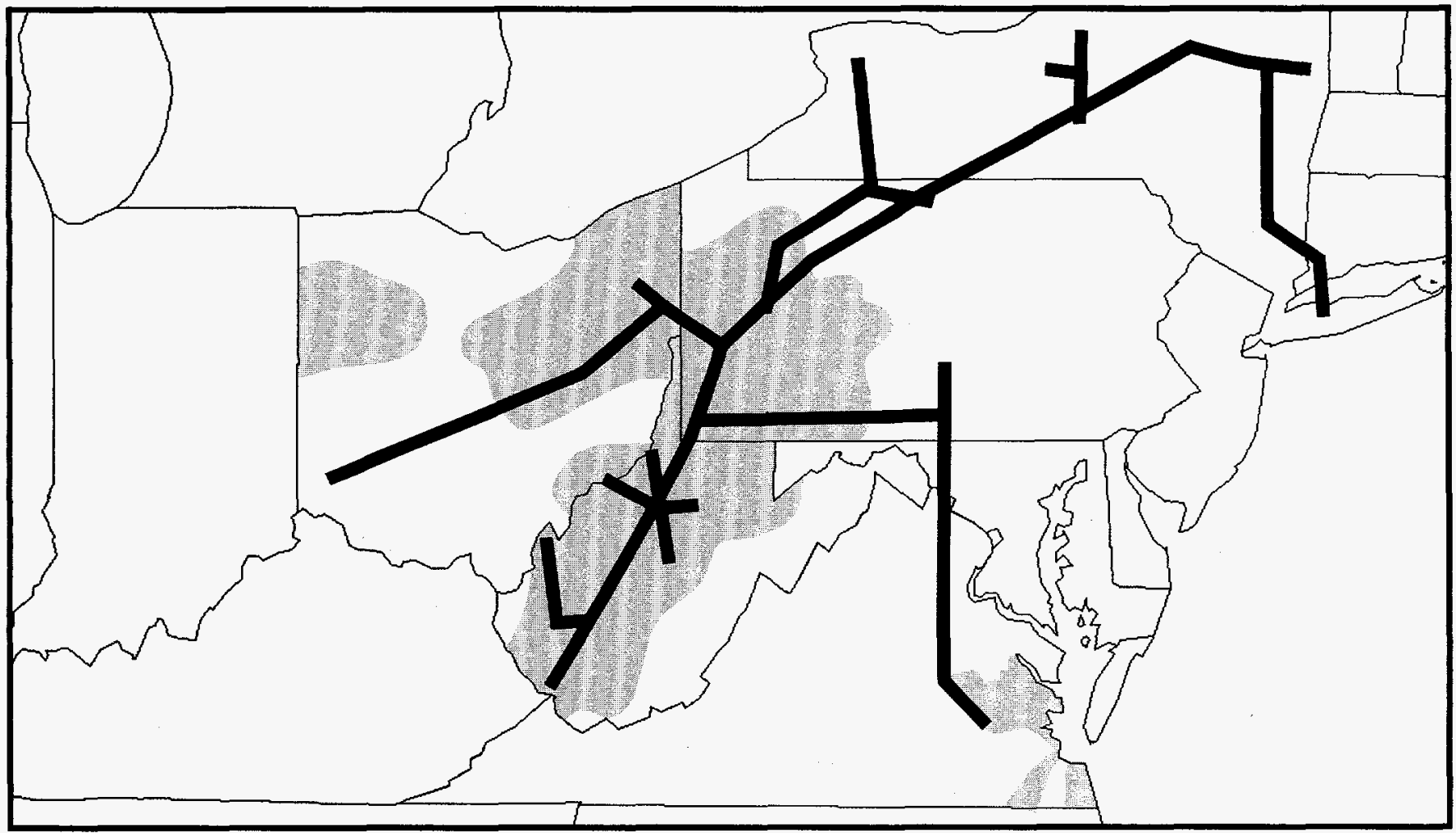

Source: Energy Information Administration (EIA), Form EIA-176, "Annual Report of Natural and Supplemental Gas Supply and Disposition." 
Table 10. Consolidated Natural Gas Co., Natural Gas Data, 1993

(Thousand Cubic Feet)

\begin{tabular}{|c|c|c|c|c|c|}
\hline \multirow{2}{*}{ Supply/Disposition } & \multicolumn{5}{|c|}{ CNG Transmission Corp. } \\
\hline & Louisiana & Pennsylvania & Maryland & Virginia & West Virginia \\
\hline \multicolumn{6}{|l|}{ SUPPLY } \\
\hline $\begin{array}{l}\text { Produced Onsystem } \\
\text { Receipts }\end{array}$ & 0 & $2,516,506$ & 0 & 0 & $10,387,078$ \\
\hline 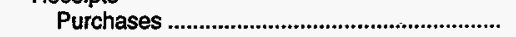 & 0 & $2,744,639$ & 0 & 0 & 197,234 \\
\hline 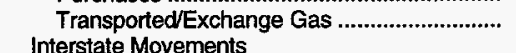 & $25,190,125$ & $503,820,816$ & $6,321,674$ & $21,622,581$ & $198,854,504$ \\
\hline interstate Movewents & 0 & 0 & 0 & 0 & 0 \\
\hline 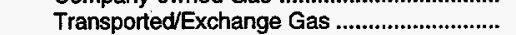 & 0 & $140,295,138$ & $36,138,729$ & $19,645,512$ & 0 \\
\hline Storage Withdrawals & 0 & $275,122,000$ & 0 & 0 & $60,769,000$ \\
\hline 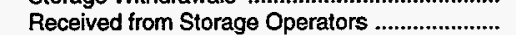 & 0 & 0 & 0 & 0 & 0 \\
\hline 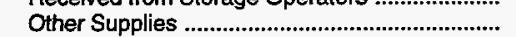 & 0 & 0 & 0 & 0 & 0 \\
\hline Total Supplies & $25,190,125$ & $924,499,099$ & $42,460,403$ & $41,268,093$ & $270,207,816$ \\
\hline \multicolumn{6}{|l|}{ DISPOSITION } \\
\hline Lease and Plant Use & 0 & 0 & 0 & 0 & $9,299,403$ \\
\hline 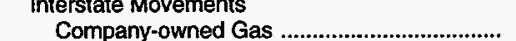 & 0 & 0 & 0 & 0 & 0 \\
\hline Transported/Exchange Gas & 0 & $145,078,413$ & $19,645,512$ & 0 & $145,624,016$ \\
\hline 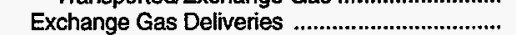 & 0 & 467,421 & 0 & 0 & $2,132,307$ \\
\hline Storage Injections & 0 & $276,264,000$ & 0 & 0 & $56,054,000$ \\
\hline \multicolumn{6}{|l|}{ Deliveries of Company-owned Gas } \\
\hline 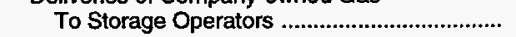 & 0 & 0 & 0 & 0 & 0 \\
\hline 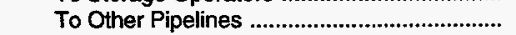 & 0 & 0 & 0 & 0 & 0 \\
\hline & 0 & $31,766,525$ & $8,643,471$ & $7,606,091$ & $11,588,516$ \\
\hline & 0 & 0 & 0 & 0 & 0 \\
\hline 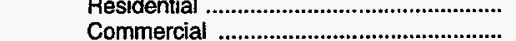 & 0 & 0 & 0 & $\begin{array}{l}0 \\
0\end{array}$ & $\begin{array}{l}0 \\
0\end{array}$ \\
\hline 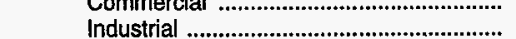 & 0 & 0 & 0 & 0 & 0 \\
\hline 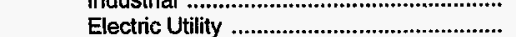 & 0 & 0 & 0 & 0 & 0 \\
\hline Vehicle Fuel & 0 & 0 & 0 & 0 & 0 \\
\hline \multicolumn{6}{|l|}{ Transported for the Account of Others } \\
\hline 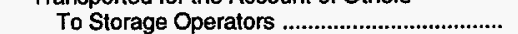 & 0 & 0 & 0 & 0 & 0 \\
\hline 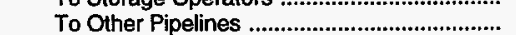 & $22,283,824$ & $442,450,596$ & 0 & 239,040 & $13,001,692$ \\
\hline 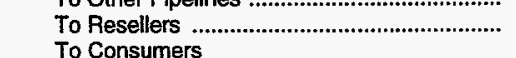 & 0 & $35,944,134$ & $14,153,807$ & $32,966,350$ & $16,019,138$ \\
\hline 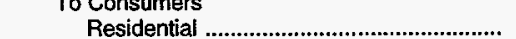 & 0 & 0 & 0 & 0 & 0 \\
\hline 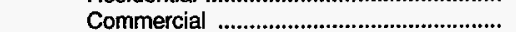 & 0 & 0 & 0 & 0 & 0 \\
\hline 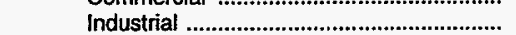 & 0 & 0 & 0 & 0 & 42,804 \\
\hline 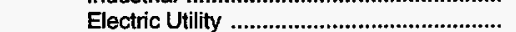 & 0 & 0 & 0 & 0 & 0 \\
\hline Vehicle Fuel & 0 & 0 & 0 & 0 & 0 \\
\hline Pipeline/Compressor Fuel & $2,906,301$ & $7,965,785$ & 6,581 & 456,612 & $13,766,876$ \\
\hline Other Disposition & 0 & 265,972 & 11,032 & 0 & $2,679,064$ \\
\hline Unaccounted For & 0 & $-15,703,747$ & 0 & 0 & 0 \\
\hline Total Disposition & $25,190,125$ & $924,499,099$ & $42,460,403$ & $41,268,093$ & $270,207,816$ \\
\hline
\end{tabular}

In 1993, CNG promoted the clean-burning and energy-efficient features of natural gas. CNG provides natural gas service to the Doswell Power Plant, a 650-megawatt facility in Doswell, Virginia, which is one of the largest independent power plants in the United States. Natural gas is also being used at coal-fired power plants. In addition, in several CNG service areas, some hospitals and public facilities are now using natural-gas-powered air-conditioning units.

Natural gas vehicles (NGV) are becoming more cost effective. In early 1994, CNG's Hope Gas Inc., a local utility in Clarksburg, West Virginia, completed a 3-year program to determine how many NGV's in West Virginia would be in range of a fueling station. Twenty public NGV fueling stations now operate in West Virginia, 10 of which were set up and operated by Hope Gas. Natural gas is also being used to power trolleys in Virginia Beach, Virginia, and Cleveland, Ohio.

In 1994, CNG promoted the York Triathlon natural-gaspowered home heating and cooling system. With the use of a variable-speed natural gas engine, this system provides heat in cold weather and drives a cooling unit in warm weather. 
Table 10. Consolidated Natural Gas Co., Natural Gas Data, 1993 (Continued) (Thousand Cubic Feet)

\begin{tabular}{|c|c|c|c|c|}
\hline \multirow{2}{*}{ Supply/Disposition } & \multicolumn{2}{|c|}{ CNG Transmission Corp. } & \multirow{2}{*}{$\frac{\text { East Ohio Gas Co. }}{\text { Ohio }}$} & \multirow{2}{*}{$\frac{\text { Peoples Natural Gas Co. }}{\text { Pennsylvania }}$} \\
\hline & Ohio & New York & & \\
\hline \multicolumn{5}{|l|}{ SUPPLY } \\
\hline $\begin{array}{l}\text { Produced Onsystem } \\
\text { Receipts }\end{array}$ & 0 & 103,843 & $1,009,000$ & $3,835,553$ \\
\hline 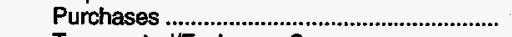 & 0 & 189,837 & $218,173,399$ & $22,147,260$ \\
\hline $\begin{array}{l}\text { Transported/Exchange Gas ............................... } \\
\text { Interstate Movements }\end{array}$ & $121,689,463$ & $152,411,171$ & $84,802,485$ & $63,943,562$ \\
\hline 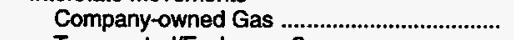 & 0 & 0 & 0 & 0 \\
\hline 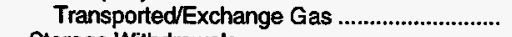 & $46,768,145$ & $108,939,684$ & 0 & 0 \\
\hline 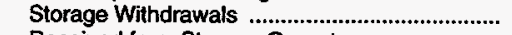 & 0 & $16,858,000$ & $62,741,464$ & $17,134,954$ \\
\hline Received from Storage Operators ........................... & 0 & 0 & $16,543,578$ & 0 \\
\hline 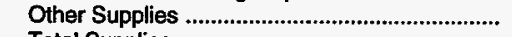 & 0 & 0 & 0 & 0 \\
\hline 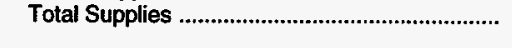 & $168,457,608$ & $278,502,535$ & $383,269,926$ & $107,061,329$ \\
\hline \multicolumn{5}{|l|}{ DISPOSITION } \\
\hline Lease and Plant Use & 0 & 0 & 8,256 & 644,932 \\
\hline 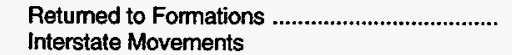 & 0 & 0 & 0 & 0 \\
\hline 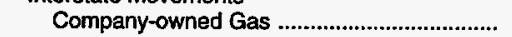 & 0 & 0 & 0 & \\
\hline 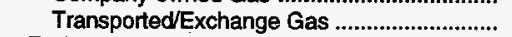 & $41,439,267$ & 0 & 0 & $\begin{array}{l}0 \\
0\end{array}$ \\
\hline 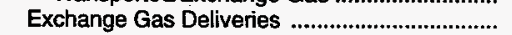 & 0 & 0 & 57,653 & \\
\hline 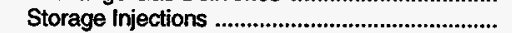 & 0 & $15,756,000$ & $62,486,329$ & $\begin{array}{r}946,765 \\
22,510,680\end{array}$ \\
\hline \multicolumn{5}{|l|}{ Deliveries of Company-owned Gas } \\
\hline To Storage Operators & 0 & 0 & $29,672,925$ & 0 \\
\hline 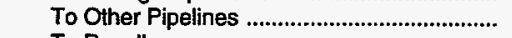 & 0 & 0 & 0 & 0 \\
\hline 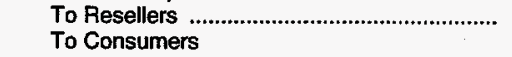 & $16,783,184$ & $36,612,117$ & 0 & 1,443 \\
\hline 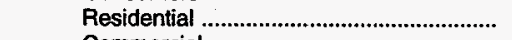 & 0 & 0 & $140,511,831$ & $41,774,726$ \\
\hline 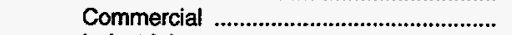 & 0 & 0 & $47,221,650$ & $7,258,835$ \\
\hline 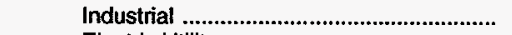 & 0 & 0 & $9,324,417$ & 728,942 \\
\hline 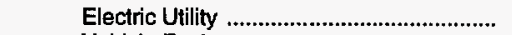 & 0 & 0 & 221,257 & 8,523 \\
\hline 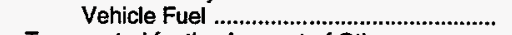 & 0 & 0 & 42,162 & 168 \\
\hline \multicolumn{5}{|l|}{ Transported for the Account of Others } \\
\hline 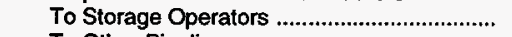 & 0 & 0 & 0 & 0 \\
\hline 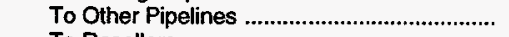 & $18,440,100$ & 0 & $6,167,823$ & 0 \\
\hline $\begin{array}{l}\text { To Resellers } \\
\text { To Consumers }\end{array}$ & $90,352,766$ & $225,346,776$ & 0 & 0 \\
\hline 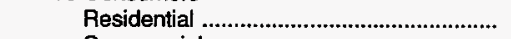 & 0 & 0 & 0 & $1,393,618$ \\
\hline 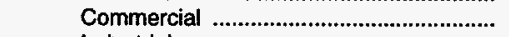 & 0 & 0 & $7,282,160$ & $8,237,776$ \\
\hline 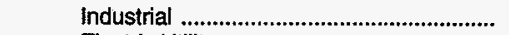 & 0 & 0 & $71,129,868$ & $19,695,544$ \\
\hline 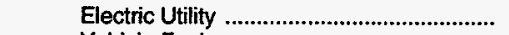 & 0 & 0 & 133,004 & 484,274 \\
\hline 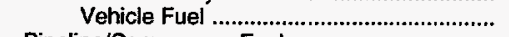 & 0 & 0 & 0 & 0 \\
\hline Pipeline/Compressor Fuel ................................... & $1,442,291$ & 787,476 & $2,416,465$ & 767,307 \\
\hline 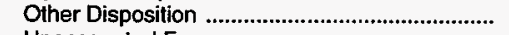 & 0 & 166 & 0 & 0 \\
\hline 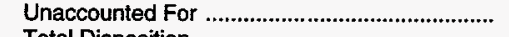 & $\begin{array}{r}0 \\
0\end{array}$ & 0 & $6,594,126$ & $2,607,796$ \\
\hline 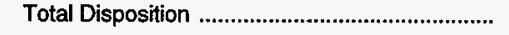 & $168,457,608$ & $278,502,535$ & $383,269,926$ & $107,061,329$ \\
\hline
\end{tabular}


Table 10. Consolidated Natural Gas Co., Natural Gas Data, 1993 (Continued) (Thousand Cubic Feet)

\begin{tabular}{|c|c|c|c|c|}
\hline \multirow{2}{*}{ Supply/Disposition } & Virginia Natural Gas, Inc. & Hope Gas, Inc. & West Ohio Gas Co. & River Gas Co. \\
\hline & Virginia & West Virginia & Ohio & Ohio \\
\hline \multicolumn{5}{|l|}{ SUPPLY } \\
\hline \multicolumn{2}{|l|}{ Receipts } & 994,456 & 0 & 4,225 \\
\hline Purchases & $\begin{array}{l}25,543,405 \\
29,963,412\end{array}$ & $\begin{array}{l}15,704,781 \\
15,435,610\end{array}$ & $\begin{array}{r}11,211,847 \\
6,297,438\end{array}$ & $\begin{array}{l}2,692,633 \\
3,712,649\end{array}$ \\
\hline \multicolumn{5}{|l|}{ Interstate Movements } \\
\hline 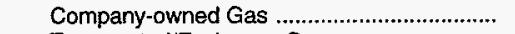 & 0 & 0 & 0 & 0 \\
\hline 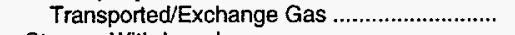 & 0 & 0 & 0 & 0 \\
\hline 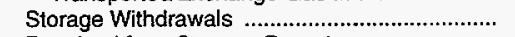 & 0 & 0 & 0 & 0 \\
\hline Received from Storage Operators .......................... & 0 & $3,172,976$ & $2,132,988$ & 904,432 \\
\hline 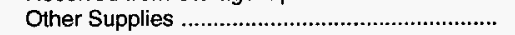 & 38,469 & 0 & 0 & 0 \\
\hline 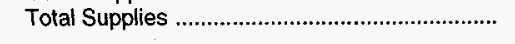 & $55,545,286$ & $35,307,823$ & $19,642,273$ & $7,313,939$ \\
\hline \multicolumn{5}{|l|}{ DISPOSITION } \\
\hline 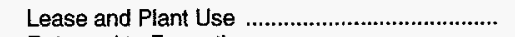 & 0 & 0 & 0 & 33 \\
\hline 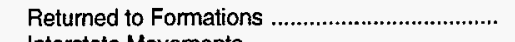 & 0 & 0 & 0 & 0 \\
\hline \multicolumn{5}{|l|}{ Interstate Movements } \\
\hline 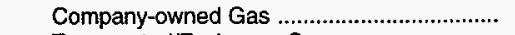 & 0 & 0 & 0 & 0 \\
\hline 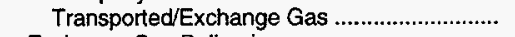 & 0 & 0 & 0 & 0 \\
\hline Exchange Gas Deliveries ................................. & 0 & 0 & 0 & 0 \\
\hline 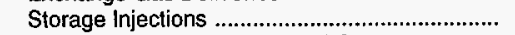 & 0 & 0 & 0 & 0 \\
\hline \multicolumn{5}{|l|}{ Deliveries of Company-owned Gas } \\
\hline 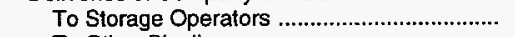 & 0 & $4,895,972$ & 0 & $1,028,338$ \\
\hline 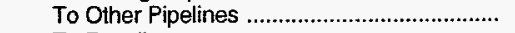 & 0 & 0 & 0 & 0 \\
\hline 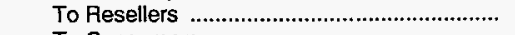 & 0 & 297,711 & 0 & 0 \\
\hline \multicolumn{5}{|l|}{ To Consumers } \\
\hline 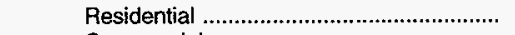 & $12,119,045$ & $10,554,372$ & $5,898,971$ & $1,695,529$ \\
\hline 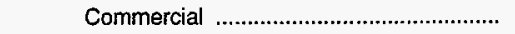 & $10,620,252$ & $3,415,423$ & $3,165,770$ & 652,194 \\
\hline Industrial ................................................... & $2,010,938$ & 129,711 & 268,483 & 109,928 \\
\hline Electric Utility ............................................ & 17,530 & 0 & 0 & 0 \\
\hline 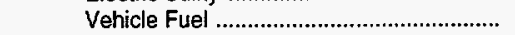 & 0 & 0 & 0 & 0 \\
\hline \multicolumn{5}{|l|}{ Transported for the Account of Others } \\
\hline 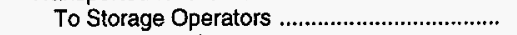 & 0 & 0 & $3,463,029$ & 0 \\
\hline 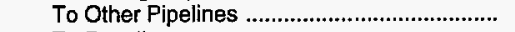 & 0 & 0 & 0 & $1,647,400$ \\
\hline 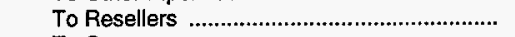 & 0 & 0 & 0 & 0 \\
\hline \multicolumn{5}{|l|}{ To Consumers } \\
\hline 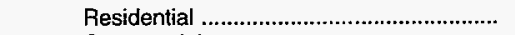 & 0 & 0 & 0 & 0 \\
\hline 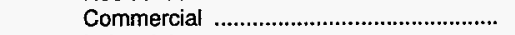 & $2,638,238$ & 805,512 & 532,626 & 95,039 \\
\hline 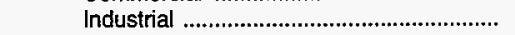 & $18,033,901$ & $13,720,157$ & $5,764,812$ & $1,970,210$ \\
\hline 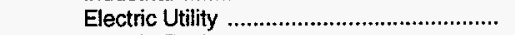 & $7,054,943$ & 0 & 0 & 0 \\
\hline 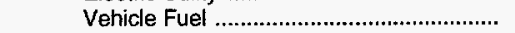 & 0 & 0 & 0 & 0 \\
\hline Pipeline/Compressor Fuel ....................................... & 19,037 & 13,212 & 0 & 12,851 \\
\hline Other Disposition ................................................ & 0 & 232,051 & 68,555 & 0 \\
\hline 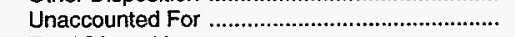 & $3,031,402$ & $1,243,702$ & 480,027 & 102,417 \\
\hline Total Disposition .................................................... & $55,545,286$ & $35,307,823$ & $19,642,273$ & $7,313,939$ \\
\hline
\end{tabular}

Source: Energy Information Administration (EIA), Form EIA-176, "Annual Feport of Natural and Supplemental Gas Supply and Disposition."

Table 11. Consolidated Natural Gas Co., Interstate Flows of Natural Gas, 1993 (Thousand Cubic Feet)

\begin{tabular}{|c|c|}
\hline Company and State & Volume \\
\hline $\begin{array}{l}\text { CNG Transmission Corp. } \\
\text { Pennsylvania To } \\
\text { Maryland } \\
\text { New York } \\
\text { Maryland To } \\
\text { Virginia } \\
\text { West Virginia To } \\
\text { Ohio } \\
\text { Pennsylvania } \\
\text { Pennsylvania }\end{array}$ & $\begin{array}{r}36,138,729 \\
108,939,684 \\
19,645,512 \\
46,768,145 \\
98,855,871 \\
41,439,267\end{array}$ \\
\hline
\end{tabular}

Source: Energy Information Administration (EIA), Form EIA-176, "Annual Report of Natural and Supplemental Gas Supply and Disposition." 


\section{Consumers Power Co.}

Consumers Power Company is the principal subsidiary of CMS Energy Corporation, Michigan's largest natural gas and electric utility, and the Nation's fourth largest natural gas and electric utility. CMS Energy Corporation was formed in 1987 as a holding company for, and direct owner of, Consumers Power Company and the then newly formed CMS Enterprise Company. In addition, CMS Enterprise has five other direct subsidiaries.

Consumers Power serves approximately 1.5 million residences in 215 Michigan cities and villages. Its 22 thousand miles of pipeline make it the Nation's sixth largest natural gas distribution utility company. Consumers Power manages the purchasing, production, transportation, storage, and distribution of natural gas in 40 of the
68 counties in Michigan's Lower Peninsula. Consumers Power's natural gas storage capacities--one of the Nation's largest--and its 14 storage fields together account for more than 60 percent of the natural gas delivered in the State. Consumers' storage fields are located in Allegheny, St. Clair, Macomb, Oakland, Washtenaw, and Wayne Counties.

Consumers Power has participated in the construction of a 25-mile gas pipeline facility in the northwestern portion of Michigan's Lower Peninsula. This pipeline extends the parent company's existing Antrim gas-gathering system from Otsego County's Chester Township to an interconnection with Consumers Power gas facilities at GooseCreek. When completed, the pipeline will transport

Figure 9. Consumers Power Co., 1993

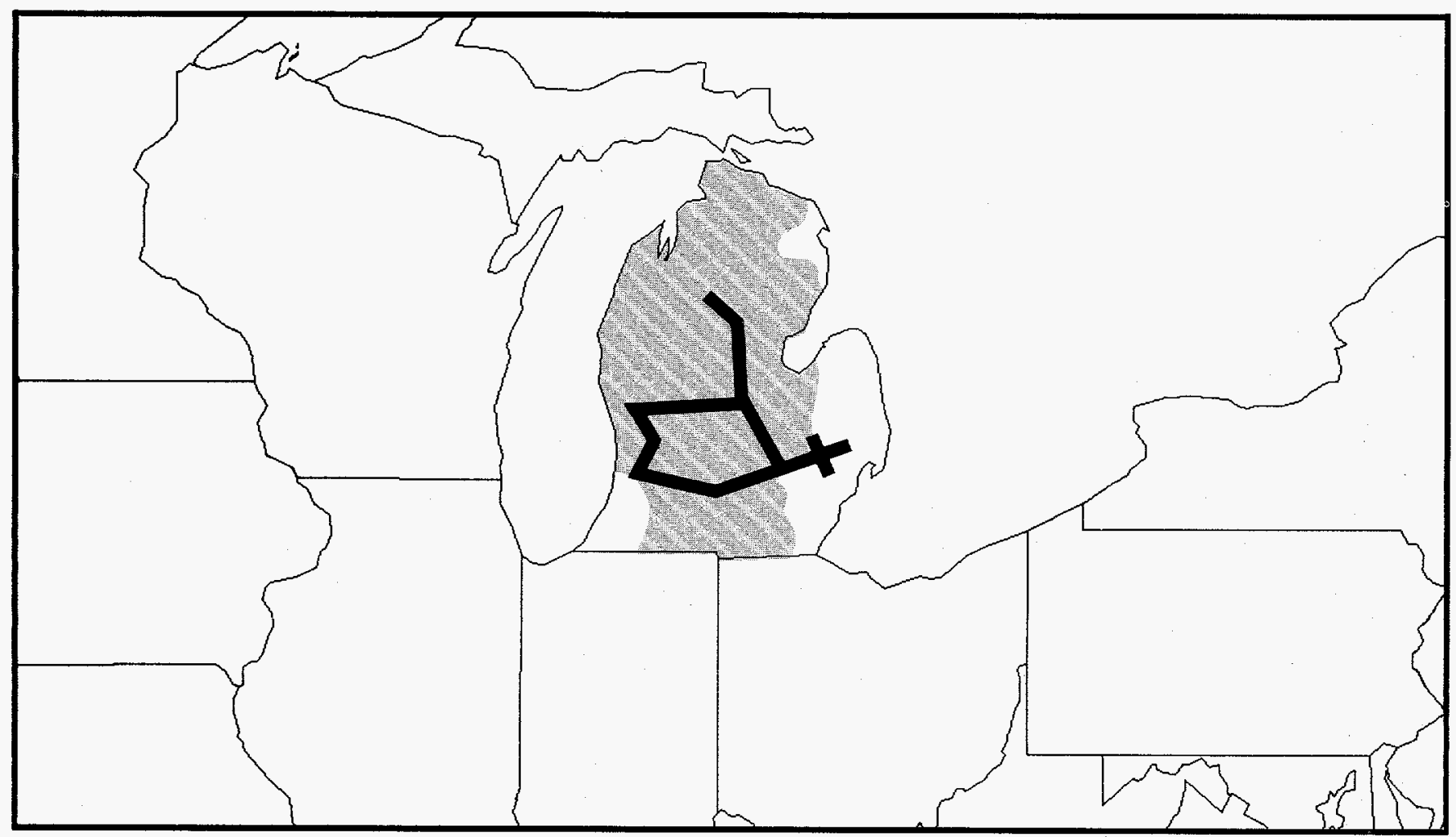

Source: Energy Information Administration (EIA), Form ElA-176, "Annual Report of Natural and Supplemental Gas Supply and Disposition." 
Table 12. Consumers Power Co., Natural Gas Data, 1993 (Thousand Cubic Feet)

\begin{tabular}{|c|c|c|}
\hline \multirow{2}{*}{ Supply/Disposition } & \multirow{2}{*}{$\frac{\text { Consumers Power Co. }}{\text { Michigan }}$} & \multirow{2}{*}{$\frac{\text { Michigan Gas Storage Co. }}{\text { Michigan }}$} \\
\hline & & \\
\hline \multicolumn{3}{|l|}{ SUPPLY } \\
\hline \multicolumn{3}{|l|}{ Receipts } \\
\hline 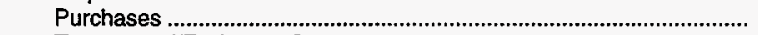 & $38,004,792$ & 0 \\
\hline 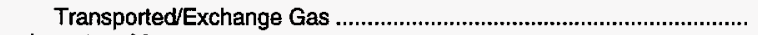 & $198,352,598$ & $320,953,318$ \\
\hline \multicolumn{3}{|l|}{ Interstate Movements } \\
\hline Company-owned Gas & $96,623,364$ & 0 \\
\hline 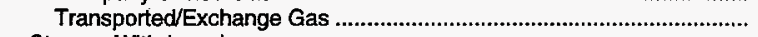 & $77,807,741$ & 0 \\
\hline Storage Withdrawals & $82,816,807$ & $26,424,594$ \\
\hline 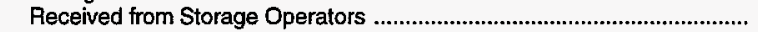 & $26,430,405$ & 0 \\
\hline Other Supplies & 0 & 0 \\
\hline 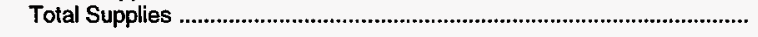 & $520,038,670$ & $347,377,912$ \\
\hline \multicolumn{3}{|l|}{ DISPOSITION } \\
\hline Lease and Plant Use & 1,571 & 0 \\
\hline Returned to Formations & 0 & 0 \\
\hline \multicolumn{3}{|l|}{ Interstate Movements } \\
\hline Company-owned Gas & 0 & 0 \\
\hline 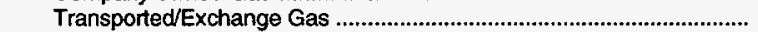 & 431,169 & 0 \\
\hline 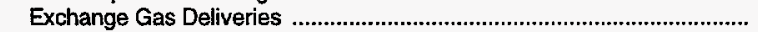 & $1,082,649$ & 0 \\
\hline Storage Injections & $88,909,437$ & $26,430,405$ \\
\hline \multicolumn{3}{|l|}{ Deliveries of Company-owned Gas } \\
\hline 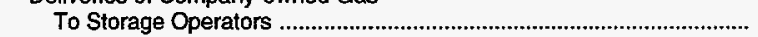 & $26,275,404$ & 0 \\
\hline 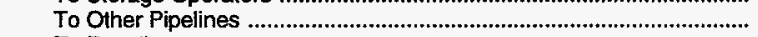 & 0 & 0 \\
\hline 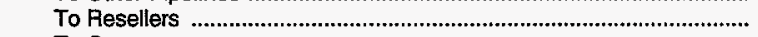 & 0 & 576,643 \\
\hline \multicolumn{3}{|l|}{ To Consumers } \\
\hline Residential & $173,913,246$ & 0 \\
\hline 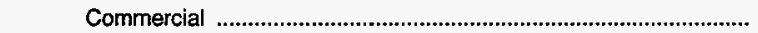 & $55,577,604$ & 0 \\
\hline Industrial & $13,843,380$ & 0 \\
\hline 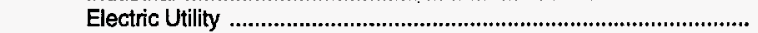 & 229,557 & 0 \\
\hline Vehicle Fuel & 0 & 0 \\
\hline \multicolumn{3}{|l|}{ Transported for the Account of Others } \\
\hline To Storage Operators & 0 & 0 \\
\hline 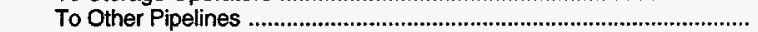 & $4,449,874$ & $319,239,092$ \\
\hline 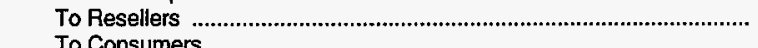 & $13,820,307$ & 0 \\
\hline \multicolumn{3}{|l|}{ To Consumers } \\
\hline 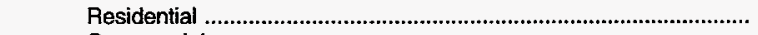 & $2,304,037$ & 0 \\
\hline Commercial & $16,460,090$ & 0 \\
\hline 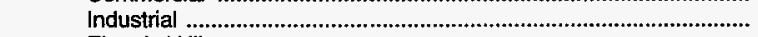 & $124,108,777$ & 0 \\
\hline Electric Utility & $1,447,709$ & 0 \\
\hline Vehicle Fuel & 227 & 0 \\
\hline Pipeline/Compressor Fuel & $1,911,081$ & $1,025,134$ \\
\hline Other Disposition & 0 & 0 \\
\hline Unaccounted For & $-4,727,449$ & 106,638 \\
\hline Total Disposition & $520,038,670$ & $347,377,912$ \\
\hline
\end{tabular}

Source: Energy Information Administration (EIA), Form EIA-176, "Annual Report of Natural and Supplemental Gas Supply and Disposition."

natural gas to a proposed carbon dioxide removal plant before the natural gas is delivered to the Consumers Power transmission system. This carbon dioxide removal facility will have a capacity of approximately 280 million cubic feet per day to meet the growing needs of Antrim gas producers.

Another subsidiary of CMS Energy, NOMECO Oil and Gas Company, is among the 50 largest independent oil and gas producers in the United States. During 1993, NOMECO had an ownership interest in more than 780 producing Antrim wells, with 136 billion cubic feet of proven gas reserves. An additional 100 wells were completed and awaiting pipeline connection, while another 80,000 acres were under exploration. 


\section{El Paso Natural Gas Co.}

El Paso Natural Gas Company, incorporated in 1928, owns and operates a 17,000-mile field and mainline natural gas transmission system. This system supplies natural gas from the San Juan Basin located in New Mexico, the Anadarko Basin located in Oklahoma, and the Permian Basin located in Texas (three of the most abundant areas of natural gas supply in the Nation) to markets in Arizona, California, Nevada, New Mexico, Texas, and Mexico. Mojave Pipeline Company and El Paso Gas Marketing Company are wholly owned subsidiaries of El Paso Natural Gas Company.

El Paso is involved in a number of major projects and the areas affected are described below:
The North/South Transfer Project includes construction of a 30-inch-diameter line to run parallel to, and around, the Havasu crossover line. Changes to the Dutch Flat compressor station will make it capable of transferring over 400 million cubic feet per day more than its present capability between the northern and southern legs of its system. With approval by the Federal Energy Regulatory Commission, the project is expected to be operational by the third quarter of 1995.

The San Juan Triangle Expansion Project includes a plan to increase, by approximately 300 million cubic feet per day, the design transportation capacity of the present system. The project will include construction of approx-

Figure 10. El Paso Natural Gas Co., 1993

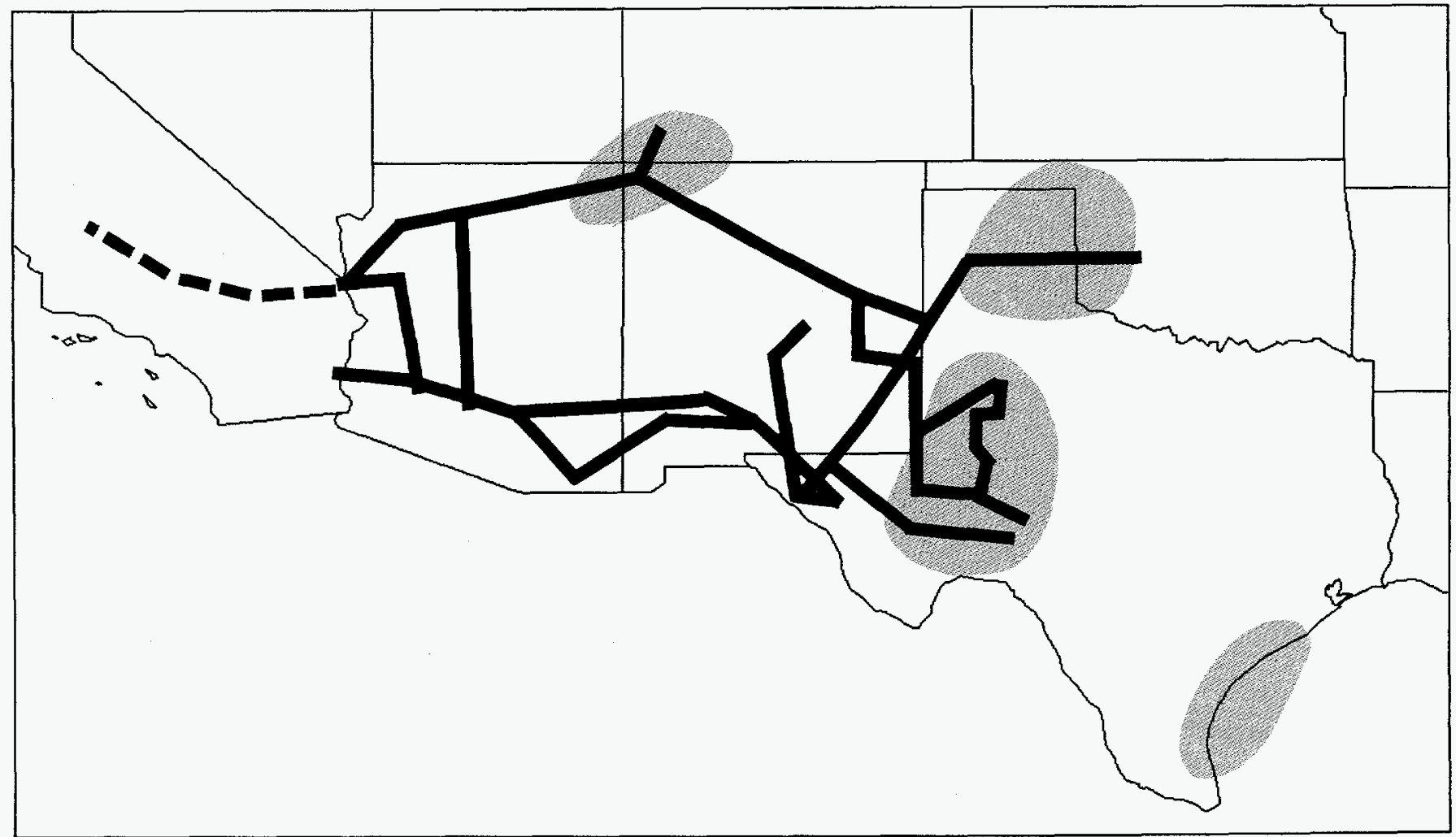

Source: Energy Information Administration (EIA), Form EIA-176, "Annual Report of Natural and Supplemental Gas Supply and Disposition."

$$
\text { Supply Areas El Paso Natural Gas Co. }
$$


Table 13. El Paso Natural Gas Co., Natural Gas Data, 1993 (Thousand Cubic Feet)

\begin{tabular}{|c|c|c|c|c|}
\hline \multirow{2}{*}{ Supply/Disposition } & \multicolumn{4}{|c|}{ El Paso Natural Gas Co. } \\
\hline & New Mexico & Texas & Oklahoma & Arizona \\
\hline \multicolumn{5}{|l|}{ SUPPLY } \\
\hline $\begin{array}{l}\text { Produced Onsystem } \\
\text { Receipts }\end{array}$ & 0 & 0 & 0 & 0 \\
\hline Purchases .... & $\begin{array}{r}24,411,493 \\
813,627,147\end{array}$ & $\begin{array}{r}13,651,540 \\
317,616,615\end{array}$ & $\begin{array}{r}805,560 \\
40,728,022\end{array}$ & $\begin{array}{r}0 \\
298,131\end{array}$ \\
\hline 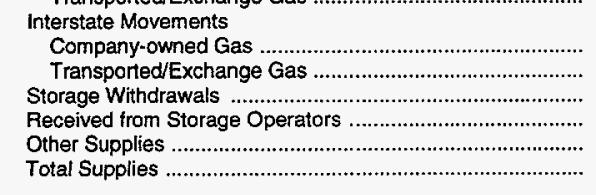 & $\begin{array}{r}0 \\
243,508,159 \\
20,160,040 \\
0 \\
0 \\
1,101,706,839\end{array}$ & $\begin{array}{r}0 \\
11,084,684 \\
0 \\
0 \\
0 \\
342,352,839\end{array}$ & $\begin{array}{r}0 \\
0 \\
0 \\
0 \\
0 \\
41,533,582\end{array}$ & $\begin{array}{r}0 \\
990,534,729 \\
0 \\
0 \\
0 \\
990,832,860\end{array}$ \\
\hline \multicolumn{5}{|l|}{ DISPOSITION } \\
\hline $\begin{array}{l}\text { Lease and Plant Use } \\
\text { Returned to Formations } \\
\text { Interstate Movements }\end{array}$ & $\begin{array}{r}12,519,182 \\
0\end{array}$ & $\begin{array}{l}0 \\
0\end{array}$ & $\begin{array}{l}0 \\
0\end{array}$ & $\begin{array}{l}0 \\
0\end{array}$ \\
\hline 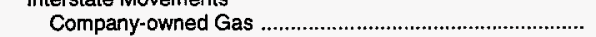 & 0 & 0 & 0 & 0 \\
\hline 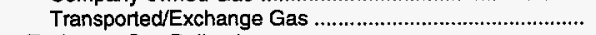 & $990,534,729$ & $45,207,631$ & $11,084,684$ & $708,674,921$ \\
\hline 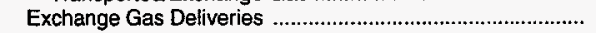 & 14,792 & 0 & $1,351,585$ & 0 \\
\hline 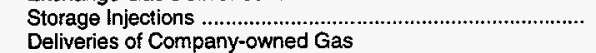 & $11,444,574$ & 0 & 0 & 0 \\
\hline To Storage Operators & 0 & 0 & 0 & 0 \\
\hline To Other Pipelines & 0 & 0 & 0 & 0 \\
\hline 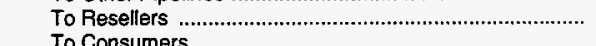 & 0 & 0 & 0 & 0 \\
\hline 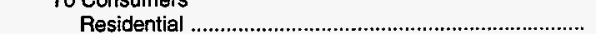 & 0 & 0 & 0 & 0 \\
\hline 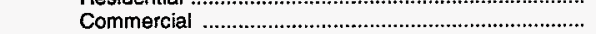 & 0 & 0 & 0 & 0 \\
\hline Industrial & 0 & 0 & 0 & 0 \\
\hline 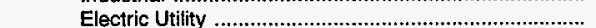 & 0 & 0 & 0 & 0 \\
\hline Vehicle Fuel & 0 & 0 & 0 & 0 \\
\hline Transported for the Account of Others & & & & \\
\hline To Storage Operators & 0 & 0 & 0 & 0 \\
\hline To Other Pipelines & 0 & $249,917,395$ & $28,553,767$ & 0 \\
\hline 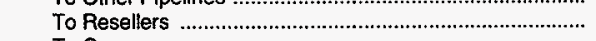 & $19,915,471$ & $31,352,158$ & 1,803 & $63,620,745$ \\
\hline To Consumers & & & & \\
\hline $\begin{array}{l}\text { Residential } \\
\text { Commercial }\end{array}$ & $\begin{array}{l}0 \\
0\end{array}$ & $\begin{array}{l}0 \\
0\end{array}$ & 0 & 0 \\
\hline $\begin{array}{l}\text { Commercial } \\
\text { Industrial }\end{array}$ & & 3.326 .432 & 0 & \\
\hline 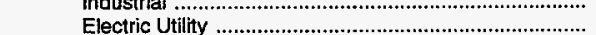 & $\begin{array}{r}4,740,296 \\
10,741,041\end{array}$ & $\begin{array}{l}3,326,432 \\
1,373,679\end{array}$ & $\begin{array}{l}0 \\
0\end{array}$ & $\begin{array}{r}9,693,358 \\
27,005,994\end{array}$ \\
\hline Vehicle Fuel & $\begin{array}{r}10,741,041 \\
0\end{array}$ & $\begin{array}{r}1,373,679 \\
0\end{array}$ & 0 & $\begin{array}{r}26,005,994 \\
0\end{array}$ \\
\hline Pipeline/Compressor Fuel & $51,796,754$ & $11,175,544$ & 541,743 & $15,397,512$ \\
\hline Other Disposition & 0 & 0 & 0 & 0 \\
\hline Unaccounted For & 0 & 0 & 0 & $166,440,330$ \\
\hline Total Disposition & $1,101,706,839$ & $342,352,839$ & $41,533,582$ & $990,832,860$ \\
\hline
\end{tabular}

imately 30 miles of a 34 -inch pipeline loop as well as a replacement compressor. During the past few years, production from Fruitland coal seams wells in the San Juan Basin has increased over 116, percent and the number of wells has increased approximately 60 percent. This expansion project will help accommodate the increase in production.

Meridian Oil and El Paso have planned an agreement wherein Meridian will dedicate 400 million cubic feet per day of the San Juan Basin's gas reserves for gathering and processing by $\mathrm{El}$ Paso in facilities located in northwestern
New Mexico. These quantities will then be transported by El Paso to markets on the eastern end of its system. Construction of a new gas liquids extraction plant at the existing Chaco Plant in New Mexico is also being planned. Approval on this project is still pending. If approved, operation of the plant will begin in 1996.

Mojave Pipeline Company began operating on March 1, 1992. Mojave's system, which is exclusively concerned with natural gas transportation, owns and operates approximately 400 miles of pipeline with a natural gas delivery capacity of 400 million cubic feet per day. In 1993, 
Table 13. El Paso Natural Gas Co., Natural Gas Data, 1993 (Continued) (Thousand Cubic Feet)

\begin{tabular}{|c|c|c|c|}
\hline \multirow{2}{*}{ Supply/Disposition } & \multirow{2}{*}{$\frac{\text { El Paso Natural Gas Co. }}{\text { Colorado }}$} & \multicolumn{2}{|c|}{ Mojave Pipeline Co. } \\
\hline & & Califormia & Arizona \\
\hline \multicolumn{4}{|l|}{ SUPPLY } \\
\hline \multicolumn{3}{|l|}{ Receipts } & 0 \\
\hline 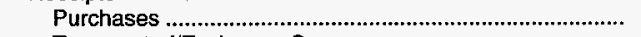 & $8,973,861$ & 0 & 0 \\
\hline 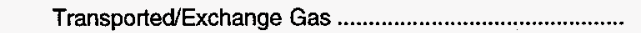 & $190,494,773$ & 0 & $85,615,554$ \\
\hline \multicolumn{4}{|l|}{ Interstate Movements } \\
\hline 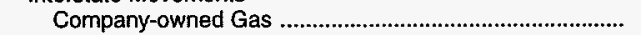 & 0 & 0 & 0 \\
\hline 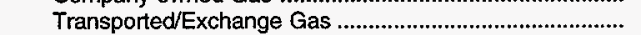 & 0 & $85,027,281$ & 0 \\
\hline Storage Withdrawals & 0 & 0 & 0 \\
\hline 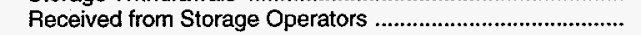 & 0 & 0 & 0 \\
\hline Other Supplies & 0 & 0 & 0 \\
\hline 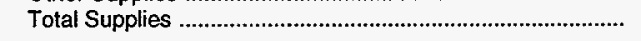 & $199,468,634$ & $85,027,281$ & $85,615,554$ \\
\hline \multicolumn{4}{|l|}{ DISPOSITION } \\
\hline Lease and Plant Use & 0 & 0 & 0 \\
\hline 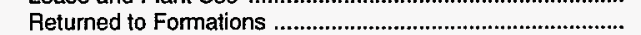 & 0 & 0 & 0 \\
\hline \multicolumn{4}{|l|}{ Interstate Movements } \\
\hline 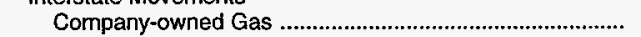 & 0 & 0 & 0 \\
\hline 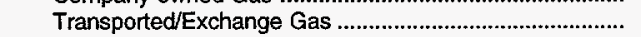 & $198,300,528$ & 0 & $85,027,281$ \\
\hline 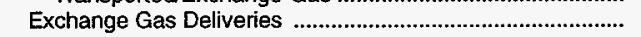 & 0 & 0 & 0 \\
\hline Storage Injections & 0 & 0 & 0 \\
\hline \multicolumn{4}{|l|}{ Deliveries of Company-owned Gas } \\
\hline To Storage Operators & 0 & 0 & 0 \\
\hline To Other Pipelines & 0 & 0 & 0 \\
\hline To Resellers & 0 & 0 & 0 \\
\hline \multicolumn{4}{|l|}{ To Consumers } \\
\hline 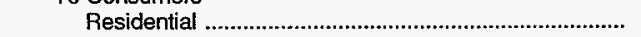 & 0 & 0 & 0 \\
\hline 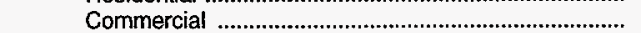 & 0 & 0 & 0 \\
\hline 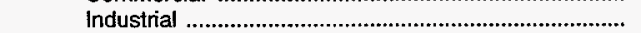 & 0 & 0 & 0 \\
\hline Electric Utility & 0 & 0 & 0 \\
\hline Vehicle Fuel & 0 & 0 & 0 \\
\hline \multicolumn{4}{|l|}{ Transported for the Account of Others } \\
\hline 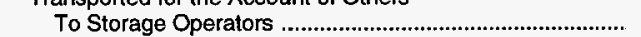 & 0 & 0 & 0 \\
\hline To Other Pipelines & 212,118 & $6,194,718$ & 0 \\
\hline To Resellers & 82,588 & 0 & 0 \\
\hline \multicolumn{4}{|l|}{ To Consumers } \\
\hline 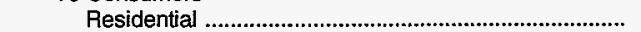 & 0 & 0 & 0 \\
\hline 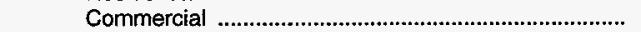 & 0 & 0 & 0 \\
\hline 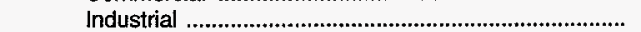 & 0 & $78,832,563$ & 0 \\
\hline 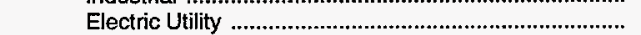 & 0 & 0 & 0 \\
\hline Vehicle Fuel & 0 & 0 & 0 \\
\hline Pipeline/Compressor Fuel & 873,400 & 0 & 588,273 \\
\hline Other Disposition & 0 & 149,468 & 16,706 \\
\hline Unaccounted For & 0 & $-149,468$ & $-16,706$ \\
\hline 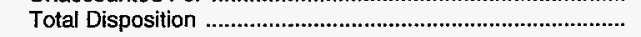 & $199,468,634$ & $85,027,281$ & $85,615,554$ \\
\hline
\end{tabular}

Source: Energy Information Administration (EIA), Form EIA-176, "Annual Report of Natural and Supplemental Gas Supply and Disposition."

Mojave filed an application to build and operate a 475 million cubic feet per day expansion of its present pipeline system to extend into the San Francisco and Sacramento areas. Among interested parties there has been considerable opposition to and support for this proposal. The Federal Energy Regulatory Commission has ruled that this expansion proposal is under its jurisdiction and not that of California. If approved, 557 miles of pipeline will be built, along with appropriate facilities to deliver gas through 37 delivery points. Customers are expected to be large end users and municipalities in these service areas. 
Table 14. EI Paso Natural Gas Co., Interstate Flows of Natural Gas, 1993 (Thousand Cubic Feet)

\begin{tabular}{|c|c|}
\hline Company and State & Volume \\
\hline \multicolumn{2}{|l|}{$\begin{array}{l}\text { El Paso Natural Gas Co. } \\
\text { Oklahoma To }\end{array}$} \\
\hline Texas & $11,084,684$ \\
\hline New Mexico & $45,207,631$ \\
\hline New Mexico To & \\
\hline $\begin{array}{l}\text { Arizona } \\
\text { Arizona To }\end{array}$ & $990,534,729$ \\
\hline California & $669,528,417$ \\
\hline Nevada & $35,893,045$ \\
\hline Mexico & $3,253,459$ \\
\hline Colorado To & \\
\hline 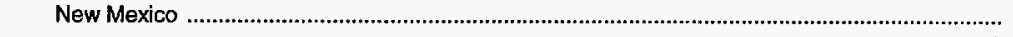 & $198,300,528$ \\
\hline $\begin{array}{l}\text { Mojave Pipeline Co. } \\
\text { Arizona To }\end{array}$ & \\
\hline California & $85,027,281$ \\
\hline
\end{tabular}

Source: Energy Information Administration (E|A), Form ElA-176, "Annual Report of Natural and Supplemental Gas Supply and Disposition." 


\section{Enron Corp.}

Enron Corporation, based in Houston, Texas, has natural gas interests located throughout the United States as well as in other countries. Enron has four major divisions: Enron Operations Corp., handles the operation of physical assets; Enron Gas Services performs North American marketing, financial, and development functions; Enron International performs international marketing and development functions; and Enron Oil and Gas Co., conducts exploration and production activities.

Enron Operations builds, manages, and operates Enron's corporate assets, both domestic and international, except those belonging to Enron Oil and Gas. In the United States, it operates 40,000 miles of natural gas pipeline. This system includes four interstate natural gas pipelines:
Northern Border Pipeline, Northern Natural Gas, Transwestern Pipeline Company, and Florida Gas Transmission. One intrastate pipeline in Texas, Houston Pipe Line Co., is also part of the Enron network.

Northern Natural Gas is the largest pipeline in the Enron system, spanning more than 23,500 miles and serving the upper Midwestern United States. The Transwestern Pipeline extends from supply areas in New Mexico, Oklahoma, and Texas to California. This system covers more than 4,500 miles with a capacity exceeding 1.1 billion cubic feet per day. In the new, deregulated environment for the natural gas industry, Transwestern remains an active participant in the highly competitive California market.

Figure 11. Enron Corp., 1993

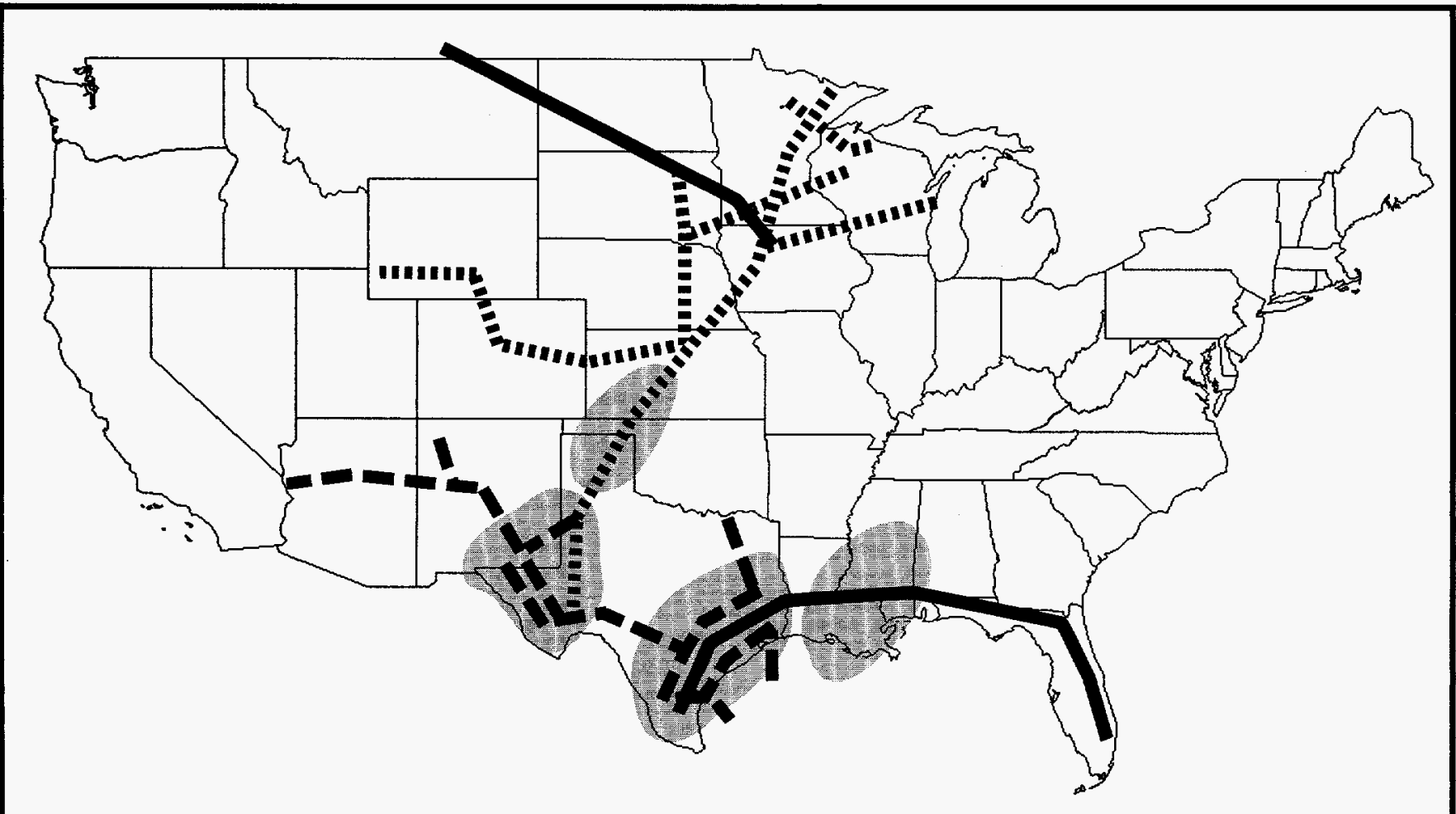

Source: Energy Information Administration (EIA), Form EIA-176, "Annual Report of Natural and Supplemental Gas Supply and Disposition."

Supply Areas

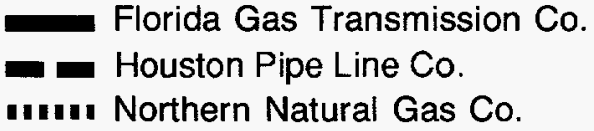

Northern Border Pipeline Co. Transwestern Pipeline Co. 
Table 15. Enron Corp., Natural Gas Data, 1993

(Thousand Cubic Feet)

\begin{tabular}{|c|c|c|c|c|c|c|}
\hline \multirow{2}{*}{ Supply/Disposition } & \multicolumn{5}{|c|}{ Florida Gas Transmission Co. } & \multirow{2}{*}{$\begin{array}{c}\begin{array}{c}\text { Houston Pipe } \\
\text { Line Inc. }\end{array} \\
\text { Texas }\end{array}$} \\
\hline & Texas & Louisiana & Mississippi & Alabama & Florida & \\
\hline \multicolumn{7}{|l|}{ SUPPLY } \\
\hline \multicolumn{6}{|l|}{ Receipts } & 0 \\
\hline Purchases & $4,836,814$ & $10,379,698$ & 390,770 & 51,780 & 23,426 & $50,636,153$ \\
\hline 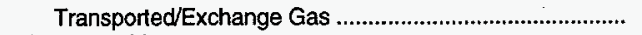 & $77,514,831$ & $233,308,000$ & $18,723,999$ & $7,003,691$ & $2,243,190$ & $678,301,055$ \\
\hline \multicolumn{7}{|l|}{ Interstale Movements } \\
\hline 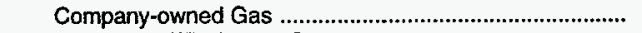 & 0 & 0 & 0 & 0 & 0 & 0 \\
\hline 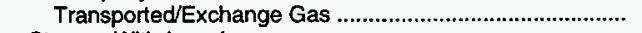 & 0 & $74,119,338$ & $261,405,956$ & $279,423,588$ & $285,926,289$ & 0 \\
\hline Storage Withdrawals & 0 & 0 & 0 & 0 & 0 & $30,151,124$ \\
\hline 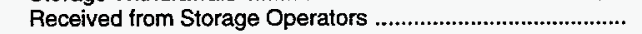 & 0 & 0 & 0 & 0 & 0 & 0 \\
\hline 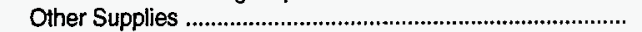 & 0 & 0 & 0 & 0 & 0 & 0 \\
\hline 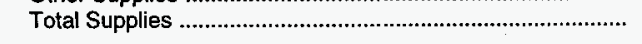 & $82,351,645$ & $317,807,036$ & $280,520,725$ & $286,479,059$ & $288,192,905$ & $759,088,332$ \\
\hline \multicolumn{7}{|l|}{ DISPOSITION } \\
\hline Lease and Plant Use & 0 & 0 & 0 & 0 & 72,127 & $4,118,157$ \\
\hline Returned to Formations & 0 & 0 & 0 & 0 & 0 & 0 \\
\hline Interstate Movements & & & & & & \\
\hline & $\begin{array}{r}0 \\
74119338\end{array}$ & $\begin{array}{r}0 \\
261,405,956\end{array}$ & $\begin{array}{r}0 \\
279,423,588\end{array}$ & $\begin{array}{r}0 \\
285,926,289\end{array}$ & 0 & $\begin{array}{l}0 \\
0\end{array}$ \\
\hline $\begin{array}{l}\text { Transported/Exchange Gas } \\
\text { Exchange Gas Deliveries }\end{array}$ & $\begin{array}{r}74,119,338 \\
0\end{array}$ & $\begin{array}{r}261,405,956 \\
0\end{array}$ & $\begin{array}{r}279,423,588 \\
0\end{array}$ & & $\begin{array}{l}0 \\
0\end{array}$ & $\begin{array}{r}0 \\
9,409,057\end{array}$ \\
\hline & $\begin{array}{l}0 \\
0\end{array}$ & 0 & 0 & $\begin{array}{l}0 \\
0\end{array}$ & $\begin{array}{l}0 \\
0\end{array}$ & $\begin{array}{r}9,409,057 \\
44,572,308\end{array}$ \\
\hline \multicolumn{7}{|l|}{ Deliveries of Company-owned Gas } \\
\hline To Storage Operators & 0 & 0 & 0 & 0 & 0 & 0 \\
\hline 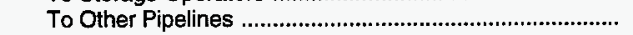 & 0 & 0 & 0 & 0 & 0 & 0 \\
\hline 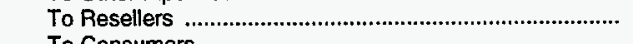 & 60,542 & 0 & 0 & 0 & $9,743,420$ & $28,080,970$ \\
\hline \\
\hline 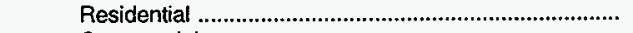 & 0 & 0 & 0 & 0 & 0 & $\begin{array}{r}0 \\
19.720\end{array}$ \\
\hline 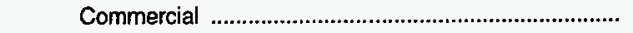 & 0 & 0 & 0 & 0 & 0 & $\begin{array}{r}19,720 \\
186.830\end{array}$ \\
\hline 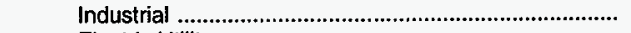 & 5,093 & 9,147 & 0 & 0 & $3,313,201$ & $\begin{array}{r}186,830 \\
0\end{array}$ \\
\hline 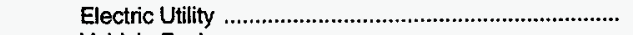 & 0 & 0 & 0 & 0 & $1,446,134$ & $\begin{array}{l}0 \\
0\end{array}$ \\
\hline \multirow{2}{*}{\multicolumn{6}{|c|}{ Transported for the Account of Others }} & \\
\hline & 0 & 0 & 0 & & 0 & 0 \\
\hline $\begin{array}{l}\text { To Storage Operators } \\
\text { To Other Pipelines }\end{array}$ & $7,286,006$ & $54,431,123$ & 678,183 & 30,949 & 0 & $21,595,550$ \\
\hline $\begin{array}{l}\text { To Other Pipelines } \\
\text { To Resellers }\end{array}$ & 0 & 0 & 0 & 0 & $86,468,230$ & $429,204,418$ \\
\hline \multicolumn{7}{|l|}{ To Consumers } \\
\hline 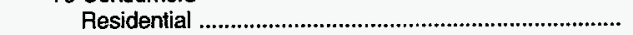 & 0 & 0 & 0 & 0 & 0 & 0 \\
\hline 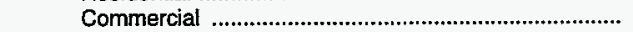 & 0 & 0 & 0 & 0 & 0 & 0 \\
\hline Industrial & 0 & 0 & 0 & 0 & $7,291,799$ & $170,613,463$ \\
\hline 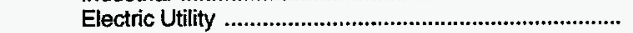 & 0 & 0 & 0 & 0 & $173,293,264$ & $56,887,624$ \\
\hline Vehicle Fuel & 0 & 0 & 0 & 0 & 0 & 0 \\
\hline 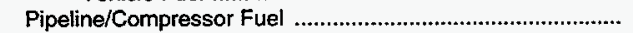 & 780,466 & $1,664,307$ & 395,696 & 513,236 & $4,313,385$ & 211,193 \\
\hline Other Disposition & 0 & 0 & 0 & 0 & $2,248,588$ & \\
\hline 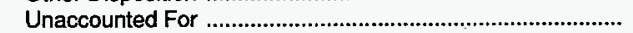 & 100,200 & 296,503 & 23,258 & 8,585 & 2,757 & $-5,810,958$ \\
\hline 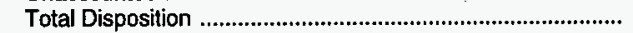 & $82,351,645$ & $317,807,036$ & $280,520,725$ & $286,479,059$ & $288,192,905$ & $759,088,332$ \\
\hline
\end{tabular}

Enron provides operations management for the Northern Border Pipeline that reaches from a point in Montana on the U.S./Canadian border to markets in Iowa. The Northern Border Pipeline is 70 percent owned by Northern Border Partners, L.P., and Enron has a 13-percent interest in Northern Border Partners.

Florida Gas Transmission, a subsidiary of Citrus Corp., is increasing its existing capacity by more than 1.4 billion cubic feet per day. The 4,400-mile pipeline extends from Texas, through Louisiana, Mississippi, and Alabama to Florida. Citrus is jointly owned by Enron and Sonat Inc., and Enron operates Florida Gas Transmission.
Enron Gas Services, in partnership with Western Resources, Inc., reached an agreement to develop the Kansas Mid-Continent Market Center, which will offer transportation, wheeling, parking, balancing, and gas storage services. Companies that will use the center through interconnections are ANR Pipeline Co., Colorado Interstate Gas Co., K N Energy, MESA Operating Co., Natural Gas Pipeline Co., of America, Northern Natural Gas Co., Panhandle Eastern Pipe Line Co., and Williams Natural Gas Co.

In accordance with the deregulation of the natural gas industry under FERC Order 636, the center will provide 
Table 15. Enron Corp., Natural Gas Data, 1993 (Continued) (Thousand Cubic Feet)

\begin{tabular}{|c|c|c|c|c|c|c|}
\hline \multirow{2}{*}{ Supply/Disposition } & \multirow{2}{*}{\begin{tabular}{|c|}
$\begin{array}{c}\text { Enron Industrial } \\
\text { Natural Gas Co. }\end{array}$ \\
Texas
\end{tabular}} & \multicolumn{5}{|c|}{ Northern Natural Gas Co. } \\
\hline & & Louisiana & New Mexico & Texas & Oklahoma & Wyoming \\
\hline \multicolumn{7}{|l|}{ SUPPLY } \\
\hline $\begin{array}{l}\text { Produced Onsystem } \\
\text { Receipts }\end{array}$ & 0 & 0 & 0 & 0 & 0 & 0 \\
\hline 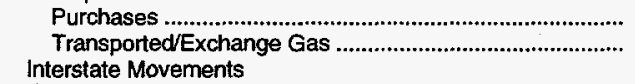 & $\begin{array}{r}0 \\
221,067,728\end{array}$ & $\begin{array}{r}0 \\
23,644,611\end{array}$ & $\begin{array}{r}2,929,670 \\
35,734,872\end{array}$ & $\begin{array}{r}5,715,458 \\
538,436,351\end{array}$ & $\begin{array}{r}3,680,804 \\
118,335,428\end{array}$ & $\begin{array}{r}3,767 \\
528,446\end{array}$ \\
\hline 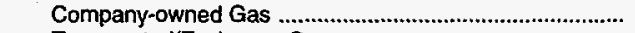 & 0 & 0 & 0 & 0 & 0 & 0 \\
\hline 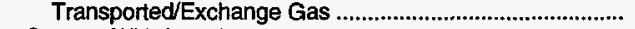 & 0 & 0 & 0 & $49,057,779$ & $97,216,841$ & 0 \\
\hline Storage Withdrawals & 0 & 0 & 0 & 0 & 0 & 0 \\
\hline 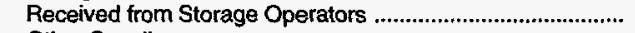 & $6,612,605$ & 0 & 0 & 0 & 0 & 0 \\
\hline Other Supplies & 0 & 0 & 0 & 0 & 0 & 0 \\
\hline 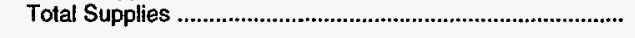 & $227,680,333$ & $23,644,611$ & $38,664,542$ & $593,209,588$ & $219,233,073$ & 532,213 \\
\hline \multicolumn{7}{|l|}{ DISPOSITION } \\
\hline 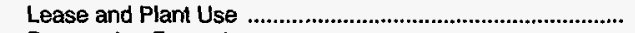 & 0 & 0 & 0 & 0 & 0 & 0 \\
\hline $\begin{array}{l}\text { Retumed to Formations } \\
\text { interstate Movements }\end{array}$ & 0 & 0 & 0 & 0 & 0 & 0 \\
\hline 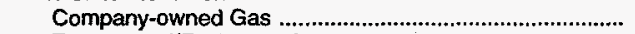 & 0 & 0 & 0 & 0 & 0 & 0 \\
\hline 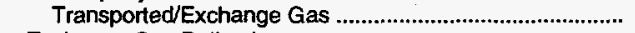 & 0 & 0 & $20,296,066$ & $97,216,841$ & $196,473,028$ & 0 \\
\hline Exchange Gas Deliveries & 0 & 0 & 0 & 0 & 0 & 0 \\
\hline 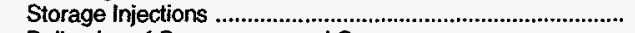 & 0 & 0 & 0 & 0 & 0 & 0 \\
\hline \multicolumn{7}{|l|}{ Deliveries of Company-owned Gas } \\
\hline 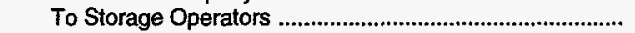 & 0 & 0 & 0 & 0 & 0 & 0 \\
\hline 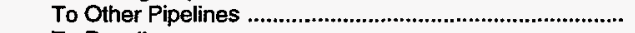 & $24,497,362$ & 0 & 0 & 0 & 0 & 0 \\
\hline 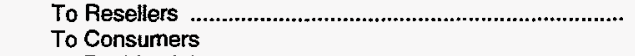 & $29,093,770$ & 0 & 0 & 0 & 0 & 0 \\
\hline Residential & 0 & 0 & 0 & 0 & 0 & 0 \\
\hline 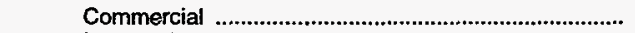 & 469,312 & 0 & 0 & 0 & 0 & 0 \\
\hline Industrial & $128,966,295$ & 0 & 0 & 0 & 0 & 0 \\
\hline Electric Utility & $44,653,904$ & 0 & 0 & 0 & 0 & 0 \\
\hline Vehicle Fuel & 0 & 0 & 0 & 0 & 0 & 0 \\
\hline \multicolumn{7}{|l|}{ Transported for the Account of Others } \\
\hline To Storage Operators & 0 & 0 & 0 & 0 & 0 & 0 \\
\hline 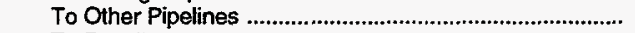 & 0 & $23,537,294$ & $14,800,561$ & $488,405,865$ & $17,967,776$ & 531,391 \\
\hline 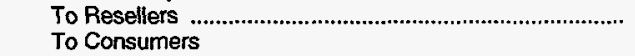 & 0 & 0 & 0 & 0 & 0 & 0 \\
\hline 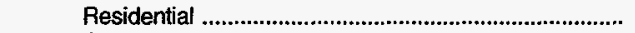 & 0 & 0 & 0 & 0 & 0 & 0 \\
\hline 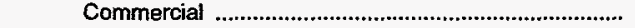 & 0 & 0 & 0 & 0 & 0 & 0 \\
\hline Industrial & 0 & 0 & 0 & 0 & 0 & 0 \\
\hline 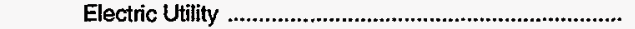 & 0 & 0 & 0 & 0 & 0 & 0 \\
\hline Vehicle Fuel & 0 & 0 & 0 & 0 & 0 & 0 \\
\hline 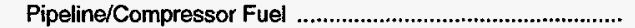 & 0 & 0 & $3,407,894$ & $4,916,763$ & $3,814,536$ & 0 \\
\hline Other Disposition & 0 & 0 & 0 & 0 & 0 & 0 \\
\hline 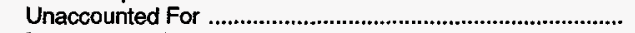 & -310 & 107,317 & 160,021 & $2,670,119$ & 977,733 & 822 \\
\hline Total Disposition & $227,680,333$ & $23,644,611$ & $38,664,542$ & $593,209,588$ & $219,233,073$ & 532,213 \\
\hline
\end{tabular}

unbundled services. It should also help to balance capacity constraints in the area caused by increasing supplies and also enhance the natural gas industry's ability to keep prices competitive.

Enron has made a number of commitments involving the development of electric power generation projects. The company has power facilities in Clear Lake, Texas; Texas
City, Texas; Richmond, Virginia; and Milford, Massachusetts. Enron also holds a natural gas supply contract, the Sithe Energies contract, to provide 1.5 trillion cubic feet of gas over 20 years to an independent power project in upstate New York. This is one of the largest gas supply contracts for a North American independent power producer. 
Table 15. Enron Corp., Natural Gas Data, 1993 (Continued)

(Thousand Cubic Feet)

Northern Natural Gas Co.

Supply/Disposition

\begin{tabular}{|l|l|l|l}
\hline Colorado & Kansas & Nebraska & lowa \\
\hline
\end{tabular}

Montana

South Dakota

SUPPLY

Produced Onsystem

Receipts

Purchases...

.

Transported/Exchange Gas

Interstate Movements

Company-owned Gas

Transported/Exchange Gas........

Storage Withdrawals

Received from Storage Operators

Other Supplies

Total Supplies

\section{DISPOSITION}

Lease and Plant Use

Returned to Formation

Interstate Movements

Company-owned Gas

Transported/Exchange Gas

Exchange Gas Deliveries

Storage Injections .

Deliveries of Company-owned Gas

To Storage Operators

To Other Pipelines

To Resellers

To Consumers

Residential

Commercial

Industrial ......

Electric Utility

Vehicle Fuel

Transported for the Account of Others

To Storage Operators

To Other Pipelines

To Resellers

To Consumers

Residential

Commercial

Industrial ...

Electric Utility

Vehicle Fuel

Pipeline/Compressor Fuel

Other Disposition

Unaccounted For

Total Disposition
0

786,757

$3,118,584$

$4,385,456$

$191,003,334$

0
$167,711,315$

$38,006,557$

0

$401,106,662$

$3,905,341$

401,106,66

29,604

0
$297,327,844$

0

$36,980,577$

$247,503,578$

0
0

0
$24,557,066$
0
$297,327,844$
0
0
0

$321,884,910$

0
$236,716,352$
$28,186,127$

$\begin{array}{rr}0 & 0 \\ 0 & 0 \\ 0 & 0 \\ 247,503,578 & 359,329,202 \\ 0 & 0 \\ 0 & 31,792,565\end{array}$

0
0
0

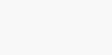

0
0
0

$3,804,09$

$3,804,096$
0

0
$53,747,540$

$71,299,731$

$227,027,198$

0
0
0
0
0
$1,622,844$
0
$1,458,757$
$321,884,910$

$\begin{array}{rr}11,420,385 & 1,622,844 \\ 0 & 0 \\ 1,600,712 & 1,458,757 \\ 401,106,662 & 321,884,910\end{array}$

$81,458,264$
$276,734,590$

0
$623,095,333$

$31,792,565$

0
$18,001,158$

$\begin{array}{rr}0 & 0 \\ 17,573,533 & 0 \\ 849,651 & 8,329,838 \\ 0 & 0 \\ 0 & 13,213,140 \\ 0 & 0 \\ 0 & 0 \\ 0 & 0\end{array}$

$21,542,978$

$\begin{array}{rr}0 & 0 \\ 0 & 0 \\ 0 & 0 \\ 105,661 & 675,560 \\ 0 & 0 \\ 0 & 0\end{array}$

0

0
5,560
0

$$
0
$$

0
0
0

0

0
0
0

0
0

0
$20,769,640$
0

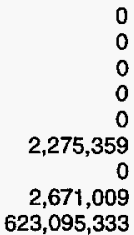


Table 15. Enron Corp., Natural Gas Data, 1993 (Continued) (Thousand Cubic Feet)

\begin{tabular}{|c|c|c|c|c|c|c|}
\hline \multirow{2}{*}{ Supply/Disposition } & \multicolumn{4}{|c|}{ Northern Natural Gas Co. } & \multicolumn{2}{|c|}{ Northern Border Pipeline Co. } \\
\hline & Minnesota & Wisconsin & Michigan & Illinois & Montana & North Dakota \\
\hline \multicolumn{7}{|l|}{ SUPPLY } \\
\hline $\begin{array}{l}\text { Produced Onsystem } \\
\text { Receipts }\end{array}$ & 0 & 0 & 0 & 0 & 0 & 0 \\
\hline Purchases & $48,457,547$ & 0 & 0 & 0 & 0 & 0 \\
\hline 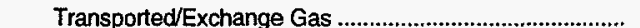 & $79,937,478$ & 0 & 0 & 0 & 0 & $85,941,597$ \\
\hline \multicolumn{7}{|l|}{ Interstate Movements } \\
\hline Company-owned Gas & 0 & 0 & 0 & 0 & 0 & 0 \\
\hline 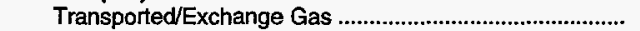 & $195,765,379$ & $124,292,527$ & $19,748,563$ & $161,942,330$ & $490,065,897$ & $491,493,537$ \\
\hline 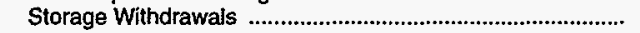 & $5,148,033$ & 0 & 0 & 0 & 0 & 0 \\
\hline Received from Storage Operators & 0 & 0 & 0 & 0 & 0 & 0 \\
\hline Other Supplies & 0 & 0 & 0 & 0 & 0 & 0 \\
\hline 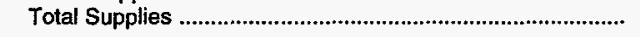 & $329,308,437$ & $124,292,527$ & $19,748,563$ & $161,942,330$ & $490,065,897$ & $577,435,134$ \\
\hline \multicolumn{7}{|l|}{ DISPOSITION } \\
\hline Lease and Plant Use & 0 & 0 & 0 & 0 & 0 & 0 \\
\hline Returned to Formations & 0 & 0 & 0 & 0 & 0 & 0 \\
\hline \multicolumn{7}{|l|}{ Interstate Movements } \\
\hline Company-owned Gas & 0 & 0 & 0 & 0 & 0 & 0 \\
\hline 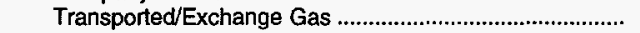 & $42,910,690$ & $19,748,563$ & 0 & $81,510,698$ & $491,493,537$. & $571,847,785$ \\
\hline 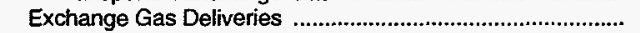 & 0 & 0 & 0 & 0 & 0 & 0 \\
\hline Storage Injections & $5,321,191$ & 0 & 0 & 0 & 0 & 0 \\
\hline \multicolumn{7}{|l|}{ Deliveries of Company-owned Gas } \\
\hline 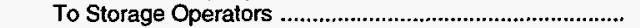 & 0 & 0 & 0 & 0 & 0 & 0 \\
\hline 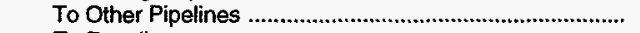 & 0 & 0 & 0 & 0 & 0 & 0 \\
\hline $\begin{array}{l}\text { To Resellers } \\
\text { To Consumers }\end{array}$ & 0 & 0 & 0 & 0 & 0 & 0 \\
\hline 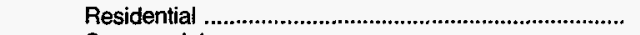 & 0 & 0 & 0 & 0 & 0 & 0 \\
\hline 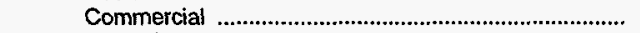 & 0 & 0 & 0 & 0 & 0 & 0 \\
\hline Industrial & 0 & 0 & 0 & 0 & 0 & 0 \\
\hline 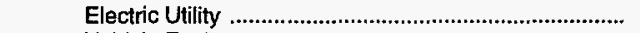 & 0 & 0 & 0 & 0 & 0 & 0 \\
\hline Vehicle Fuel & 0 & 0 & 0 & 0 & 0 & 0 \\
\hline \multicolumn{7}{|l|}{ Transported for the Account of Others } \\
\hline 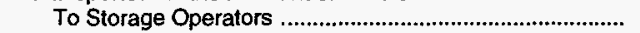 & 0 & 0 & 0 & 0 & 0 & 0 \\
\hline To Other Pipelines & $274,963,765$ & $103,781,587$ & $19,658,929$ & $79,471,773$ & 0 & $1,199,948$ \\
\hline $\begin{array}{l}\text { To Resellers } \\
\text { To Consumers }\end{array}$ & 0 & 0 & 0 & 0 & 0 & 0 \\
\hline 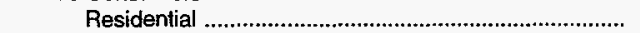 & 0 & 0 & 0 & 0 & 0 & 0 \\
\hline 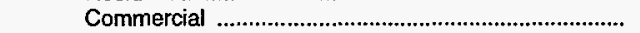 & 0 & 0 & 0 & 0 & 0 & 0 \\
\hline 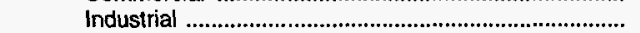 & $6,943,189$ & 0 & 0 & 0 & 0 & 0 \\
\hline 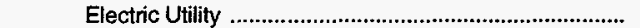 & 0 & 0 & 0 & 0 & 0 & 0 \\
\hline Vehicle Fuel & 0 & 0 & 0 & 0 & 0 & $\mathbf{0}$ \\
\hline Pipeline/Compressor Fuel & 649,435 & 0 & 0 & 225,867 & 581,720 & $3,867,260$ \\
\hline 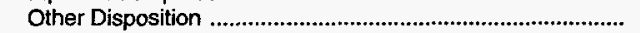 & 0 & 0 & 0 & 0 & 105,972 & 162,616 \\
\hline 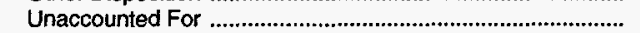 & $-1,479,833$ & 762,377 & 89,634 & 733,992 & $-2,115,332$ & 357,525 \\
\hline 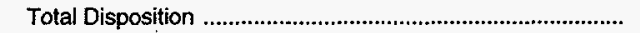 & $329,308,437$ & $124,292,527$ & $19,748,563$ & $161,942,330$ & $490,065,897$ & $577,435,134$ \\
\hline
\end{tabular}


Table 15. Enron Corp., Natural Gas Data, 1993 (Continued) (Thousand Cubic Feet)

\begin{tabular}{|c|c|c|c|c|c|}
\hline \multirow{2}{*}{ Supply/Disposition } & \multicolumn{3}{|c|}{ Northern Border Pipeline Co. } & \multicolumn{2}{|c|}{ Transwestern Pipeline Co. } \\
\hline & South Dakota & Minnesota & lowa & Oklahoma & Texas \\
\hline \multicolumn{6}{|l|}{ SUPPLY } \\
\hline $\begin{array}{l}\text { Produced Onsystem } \\
\text { Receipts }\end{array}$ & 0 & 0 & 0 & 0 & 0 \\
\hline 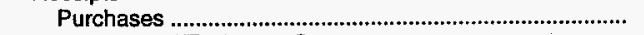 & 0 & 0 & 0 & 421,479 & $1,133,538$ \\
\hline 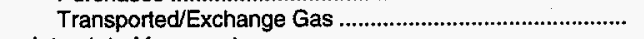 & 0 & 0 & 0 & $7,112,339$ & $68,971,006$ \\
\hline \multicolumn{6}{|l|}{ Interstate Movements } \\
\hline 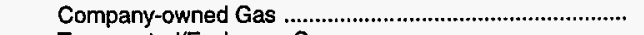 & $\begin{array}{r}0 \\
0\end{array}$ & 0 & 0 & 0 & 0 \\
\hline 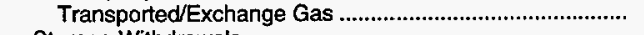 & $571,847,785$ & $560,516,332$ & $511,422,290$ & $5,456,945$ & $14,448,171$ \\
\hline 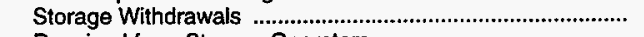 & 0 & 0 & 0 & 0 & 0 \\
\hline 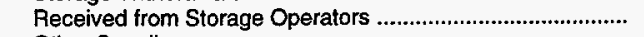 & 0 & 0 & 0 & 0 & 0 \\
\hline 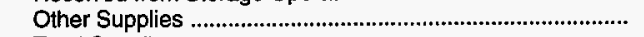 & 0 & 0 & 0 & 0 & 0 \\
\hline 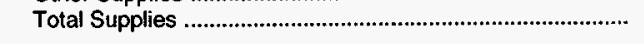 & $571,847,785$ & $560,516,332$ & $511,422,290$ & $12,990,763$ & $84,552,715$ \\
\hline \multicolumn{6}{|l|}{ DISPOSITION } \\
\hline 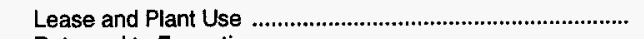 & 0 & 0 & 0 & 0 & 5,889 \\
\hline 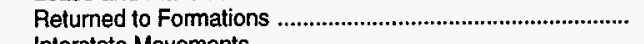 & 0 & 0 & 0 & 0 & 0 \\
\hline \multicolumn{6}{|l|}{ Interstate Movements } \\
\hline 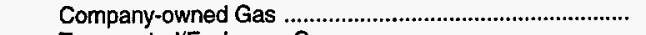 & 0 & 0 & 0 & 0 & 0 \\
\hline 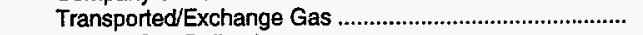 & $560,516,332$ & $511,422,290$ & 0 & 0 & $5,456,945$ \\
\hline 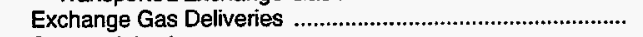 & 0 & 0 & 0 & 0 & 0 \\
\hline Storage Injections & 0 & 0 & 0 & 0 & 0 \\
\hline \multicolumn{6}{|l|}{ Deliveries of Company-owned Gas } \\
\hline 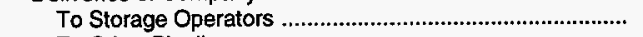 & 0 & 0 & 0 & 0 & 0 \\
\hline 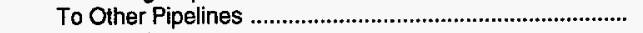 & 0 & 0 & 0 & 0 & 0 \\
\hline 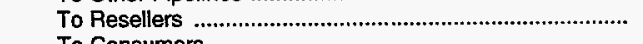 & 0 & 0 & 0 & 237,461 & $2,948,876$ \\
\hline To Consumers & & & & & \\
\hline 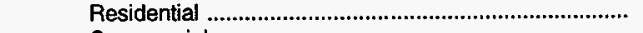 & 0 & 0 & 0 & 0 & $\begin{array}{r}0 \\
536\end{array}$ \\
\hline 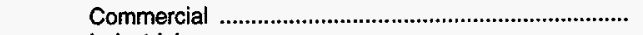 & 0 & 0 & 0 & 0 & 44,536 \\
\hline 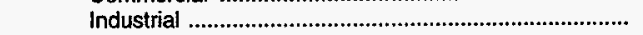 & 0 & 0 & 0 & 0 & 0 \\
\hline 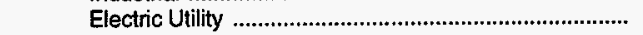 & 0 & 0 & 0 & 0 & 0 \\
\hline Vehicle Fuel & 0 & 0 & 0 & 0 & 0 \\
\hline \multicolumn{6}{|l|}{ Transported for the Account of Others } \\
\hline 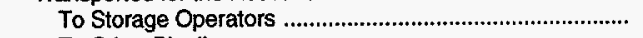 & 0 & 0 & 0 & 0 & 0 \\
\hline 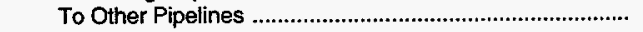 & $8,513,348$ & $47,619,840$ & $511,053,199$ & $12,529,992$ & 0 \\
\hline 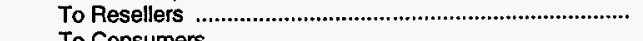 & 0 & 0 & 0 & 0 & $72,672,529$ \\
\hline \multicolumn{6}{|l|}{ To Consumers } \\
\hline 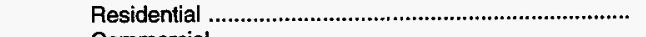 & 0 & 0 & 0 & 0 & 0 \\
\hline 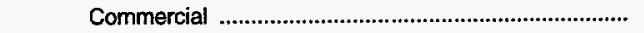 & 0 & 0 & 0 & 0 & 0 \\
\hline 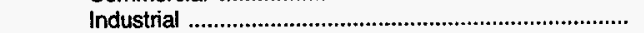 & 0 & 0 & 0 & 0 & 0 \\
\hline 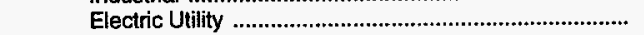 & 0 & 0 & 0 & 0 & 0 \\
\hline 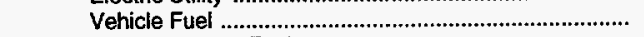 & 0 & 0 & 0 & 0 & 0 \\
\hline 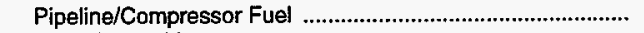 & $2,476,604$ & $1,230,091$ & 204 & 223,310 & $3,423,940$ \\
\hline 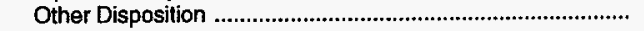 & 106,766 & 76,318 & 115,328 & 0 & 0 \\
\hline Unaccounted For & 234,735 & 167,793 & 253,559 & 0 & \\
\hline 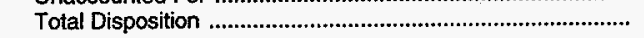 & $571,847,785$ & $560,516,332$ & $511,422,290$ & $12,990,763$ & $84,552,715$ \\
\hline
\end{tabular}


Table 15. Enron Corp., Natural Gas Data, 1993 (Continued) (Thousand Cubic Feet)

\begin{tabular}{|c|c|c|c|c|c|}
\hline \multirow{2}{*}{ Supply/Disposition } & \multicolumn{2}{|c|}{ Transwestern Pipeline Co. } & \multicolumn{2}{|c|}{ Black Marlin Pipeline Co. } & \multirow{2}{*}{$\begin{array}{c}\text { Intratex Gas Co. } \\
\text { Texas }\end{array}$} \\
\hline & New Mexico & Arizona & Texas & Oklahoma & \\
\hline \multicolumn{6}{|l|}{ SUPPLY } \\
\hline $\begin{array}{l}\text { Produced Onsystem } \\
\text { Receipts }\end{array}$ & 0 & 0 & 0 & 0 & 0 \\
\hline 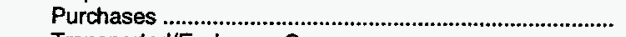 & $2,553,593$ & 0 & 0 & 0 & 527,749 \\
\hline & $319,010,322$ & 114,286 & $63,191,689$ & $13,200,173$ & $276,792,091$ \\
\hline 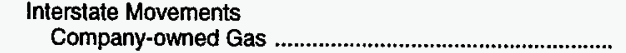 & & & & & \\
\hline Company-owned Gas & 0 & 0 & 0 & 0 & 0 \\
\hline 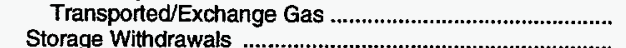 & 0 & $280,114,424$ & $13,200,173$ & 0 & 0 \\
\hline & 0 & 0 & 0 & 0 & 0 \\
\hline Received from Storage Operators & 0 & 0 & 0 & 0 & 0 \\
\hline 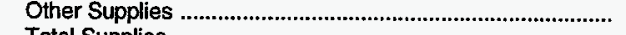 & 0 & 0 & 0 & 0 & 0 \\
\hline 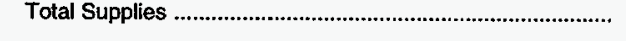 & $321,563,915$ & $280,228,710$ & $76,391,862$ & $13,200,173$ & $277,319,840$ \\
\hline \multicolumn{6}{|l|}{ DISPOSITION } \\
\hline Lease and Plant Use & 1,106 & 0 & 0 & 0 & $1,108,301$ \\
\hline $\begin{array}{l}\text { Returned to Formations } \\
\text { Interstate Movements }\end{array}$ & 0 & 0 & 0 & 0 & 0 \\
\hline 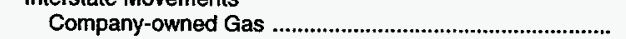 & & & & & \\
\hline 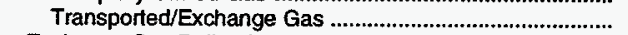 & $\begin{array}{r}0 \\
294,562,595\end{array}$ & $\begin{array}{r}0 \\
07894617\end{array}$ & 0 & 0 & 0 \\
\hline 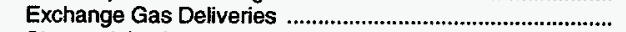 & $\begin{array}{r}294,562,595 \\
0\end{array}$ & $278,844,617$ & 0 & $13,200,173$ & 0 \\
\hline Storage Injections & $\begin{array}{l}0 \\
0\end{array}$ & $\begin{array}{l}0 \\
0\end{array}$ & 0 & $\begin{array}{r}0 \\
0\end{array}$ & 0 \\
\hline \multicolumn{6}{|l|}{ Deliveries of Company-owned Gas } \\
\hline 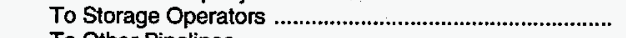 & 0 & 0 & 0 & 0 & 0 \\
\hline 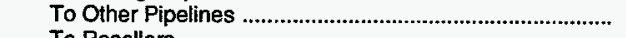 & 0 & 0 & 0 & 0 & 0 \\
\hline 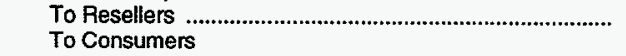 & $3,888,862$ & 0 & 0 & 0 & 90,518 \\
\hline 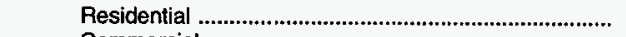 & 0 & 0 & 0 & 0 & 0 \\
\hline 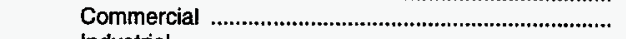 & 18,838 & 30,433 & 0 & 0 & 0 \\
\hline 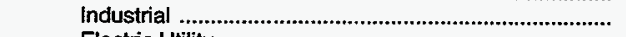 & 0 & 0 & 0 & 0 & 0 \\
\hline Electric Utility & 0 & 0 & 0 & 0 & 0 \\
\hline Vehicle Fuel & 0 & 0 & 0 & 0 & 0 \\
\hline \multicolumn{6}{|l|}{ Transported for the Account of Others } \\
\hline To Storage Operators & 0 & 0 & 0 & 0 & 0 \\
\hline 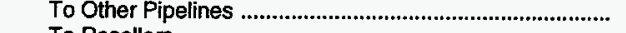 & 0 & 0 & $76,391,862$ & 0 & $254,327,070$ \\
\hline $\begin{array}{l}\text { To Resellers ................................................................. } \\
\text { To Consumers }\end{array}$ & $18,573,737$ & 0 & 0 & 0 & 0 \\
\hline 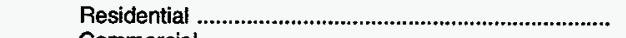 & 0 & 0 & 0 & 0 & 0 \\
\hline 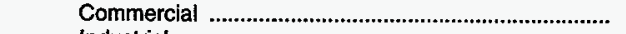 & 0 & 0 & 0 & 0 & 0 \\
\hline 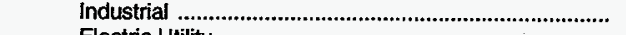 & 0 & 0 & 0 & 0 & $1,384,574$ \\
\hline 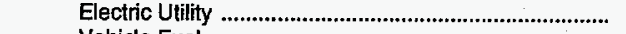 & 0 & 0 & 0 & 0 & $18,140,977$ \\
\hline Vehide Fuel & 0 & 0 & 0 & 0 & 0 \\
\hline 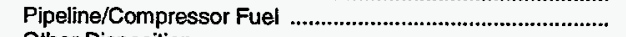 & $4,518,777$ & $1,353,660$ & 0 & 0 & $2,163,343$ \\
\hline 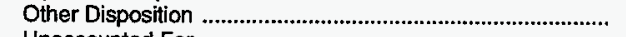 & 0 & 0 & 0 & 0 & 0 \\
\hline Unaccounted For & 0 & 0 & 0 & 0 & 105,057 \\
\hline 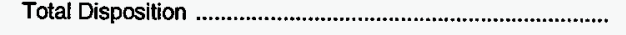 & $321,563,915$ & $280,228,710$ & $76,391,862$ & $13,200,173$ & $277,319,840$ \\
\hline
\end{tabular}

Source: Energy Information Administration (EIA), Form ElA-176, "Annual Report of Natural and Supplemental Gas Supply and Disposition." 
Table 16. Enron Corp., Interstate Flows of Natural Gas, 1993

(Thousand Cubic Feet)

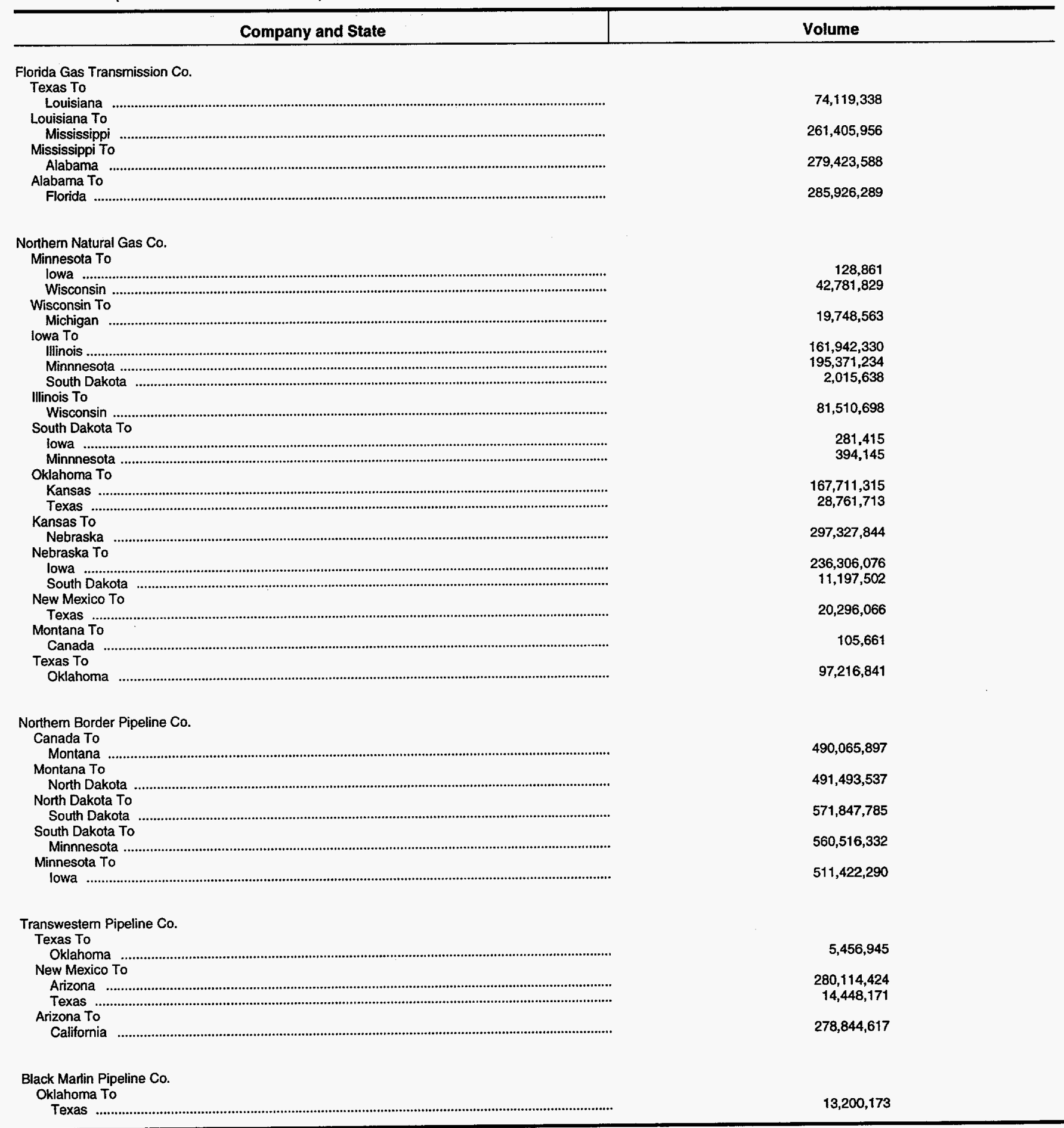

Source: Energy Information Administration (EIA), Form EIA-176, "Annual Report of Natural and Supplemental Gas Supply and Disposition." 


\section{Equitable Resources, Inc.}

Equitable Resources, Inc., is an integrated energy company that conducts its business through two entities, Energy Resources and Utility Services. The Energy Resources section is involved in the exploration, development, and production of natural gas and oil. The function of Utility Services is to purchase, gather, transport, and distribute natural gas.

Its pipeline operations in Pennsylvania and West Virginia are conducted by Equitrans, Inc., and in Kentucky by Kentucky West Virginia Gas Company. The Equitable Gas Company provides retail natural gas sales and transportation services to more than 265,000 customers in southwestern Pennsylvania, northern West Virginia and eastern Kentucky.
In 1993, Equitable acquired the Louisiana Intrastate Gas Company (LIG), the largest intrastate gas pipeline in the State. This 1,900-mile intrastate transmission system links the Gulf of Mexico supply to every market in the United States through more than one hundred interconnects with other pipelines. In addition, four large natural gas processing plants are directly connected to LIG's system. The company expects to open its new Jefferson Island underground gas storage facility near Henry, Louisiana, by October 1995. Equitable plans to establish a hub in Louisiana using LIG facilities, the new salt dome storage system, and a proposed storage interconnection to the Henry Hub.

Figure 12. Equitable Resources, Inc., 1993

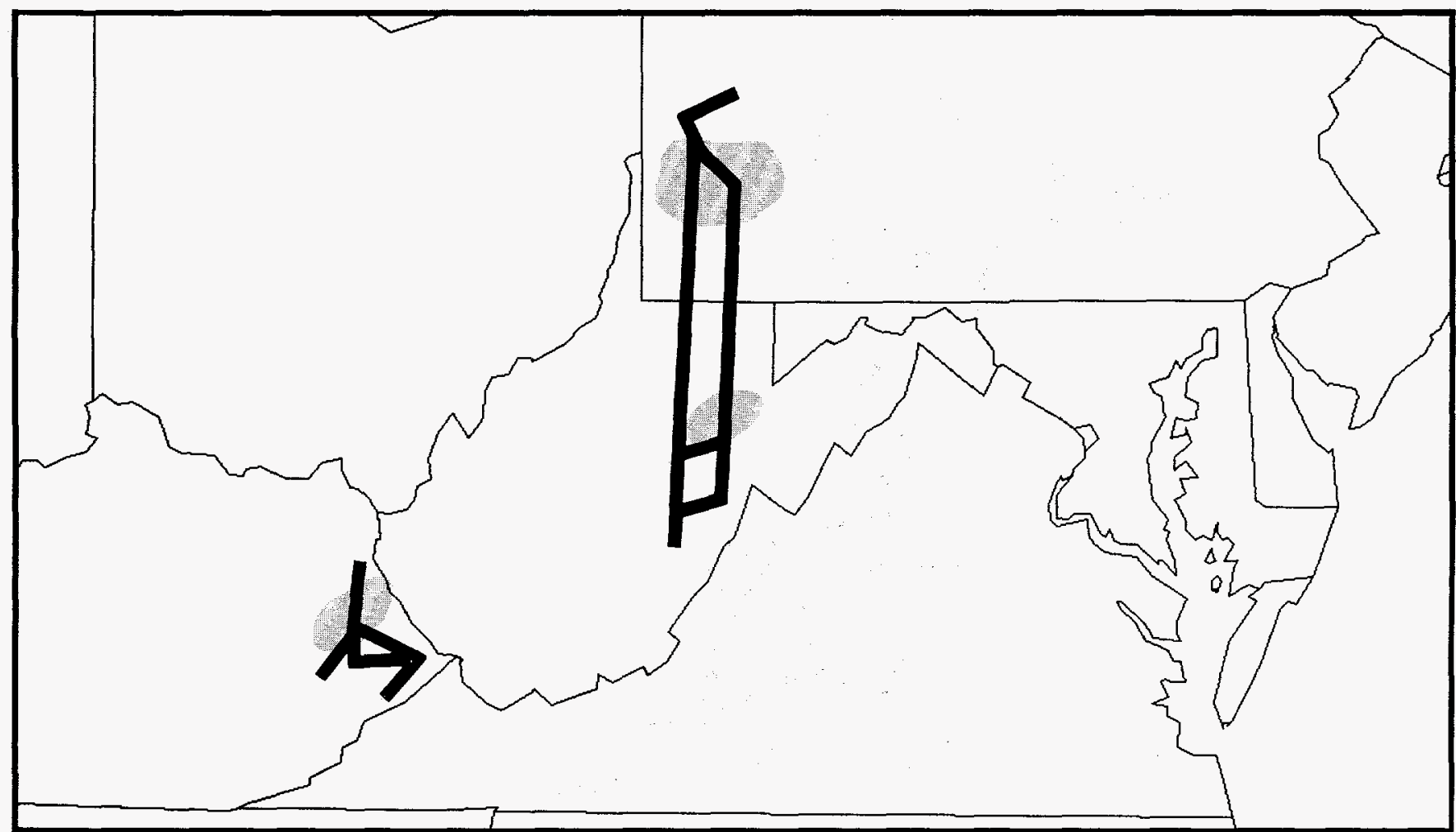

Source: Energy Information Administration (EIA), Form ElA-176, "Annual Report of Natural and Supplemental Gas Supply and Disposition." 
Equitable also plans to develop three new storage fields in association with various partners. One project, in Pennsylvania, is scheduled to begin operations in 1995 . The other two projects, in New York State, are projected to open in 1996.

Equitable continues to explore opportunities in such expanding markets as fuel cells, natural gas cooling, and natural gas vehicles (NGV). Equitable was one of the first utilities to install a fuel cell. The fuel cell converts natural gas fuel to electricity and to heat by a chemical process, not by combustion, and thus does not produce emissions.

Equitable has installed 35 natural gas cooling units through 1993 and projects a doubling of installed tonnage by the year 2000 . Equitable also projects serving an estimated 20,000 NGV's in the Pittsburgh area by the year 2000. Two more unusual NGV applications currently served by Equitable are a Zamboni ice resurfacing machine and a forklift truck fleet.

Figure 13. Louisiana Interastate Gas Company, 1993

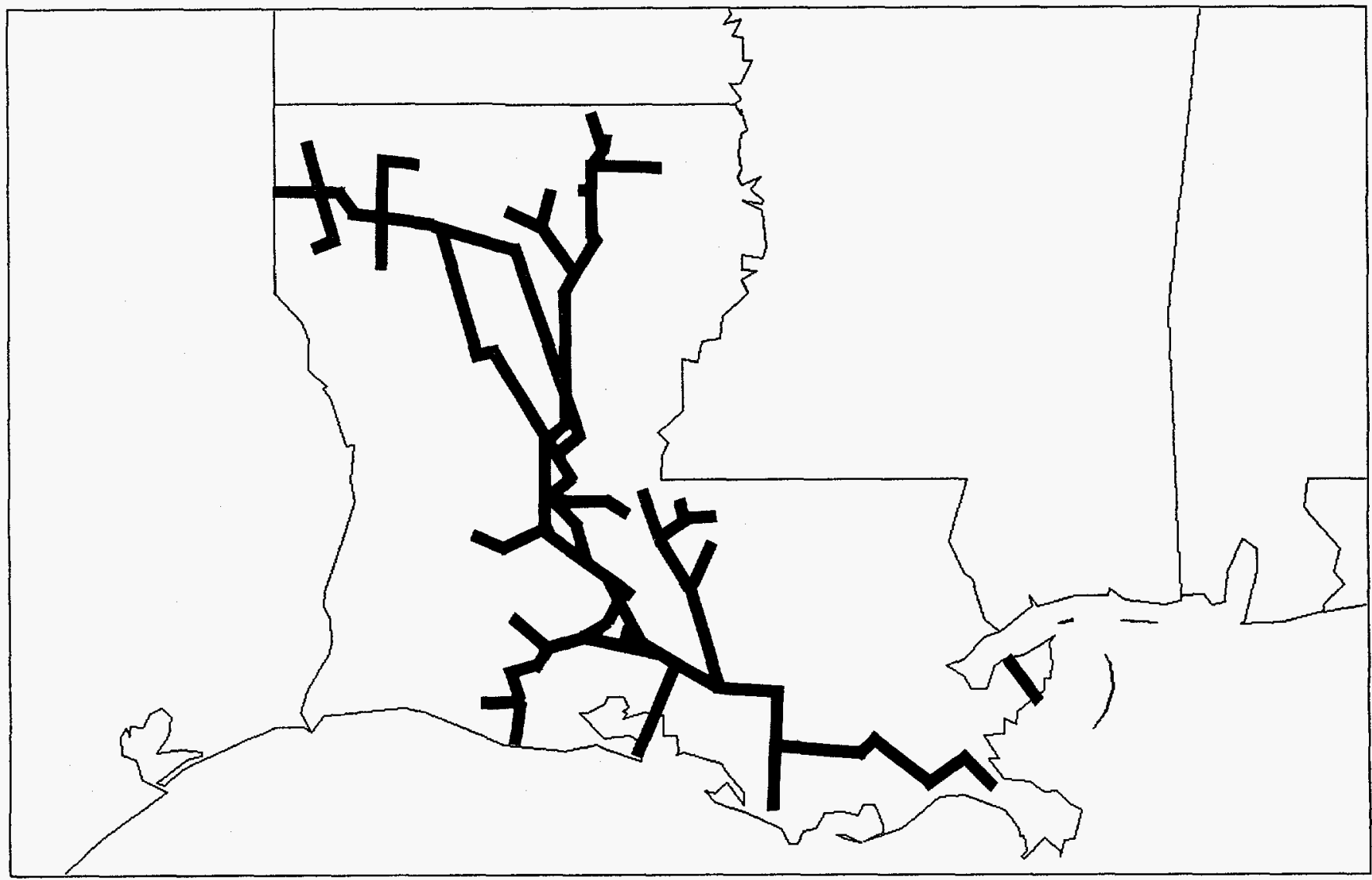


Table 17. Equitable Resources, Inc., Natural Gas Data, 1993 (Thousand Cubic Feet)

\begin{tabular}{|c|c|c|c|c|}
\hline \multirow{2}{*}{ Supply/Disposition } & \multicolumn{2}{|c|}{ Equitrans, inc. } & \multicolumn{2}{|c|}{ Equitable Gas Co. } \\
\hline & West Virginia & Pennsylvania & Kentucky & West Virginia \\
\hline \multicolumn{5}{|l|}{ SUPPLY } \\
\hline \multicolumn{4}{|l|}{ Receipts } & \\
\hline 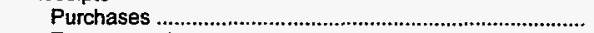 & $1,466,897$ & $27,629,678$ & 511,382 & $1,558,094$ \\
\hline 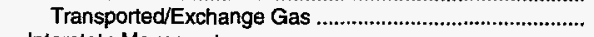 & $16,821,144$ & $36,965,474$ & 0 & 573,117 \\
\hline \multicolumn{5}{|l|}{ Interstate Movements } \\
\hline Company-owned Gas & 0 & 0 & 0 & 0 \\
\hline 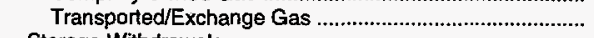 & $6,046,263$ & $18,833,240$ & 0 & 0 \\
\hline 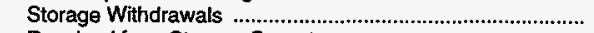 & $15,792,180$ & $11,038,123$ & 0 & 0 \\
\hline 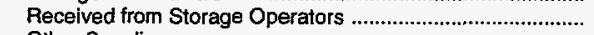 & 0 & 0 & 0 & 487,840 \\
\hline 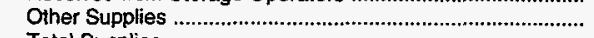 & 0 & 0 & 0 & 401,040 \\
\hline 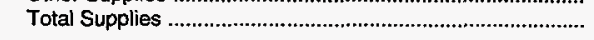 & $41,013,091$ & $94,842,260$ & 511,382 & $2,619,051$ \\
\hline \multicolumn{5}{|l|}{ DISPOSITION } \\
\hline 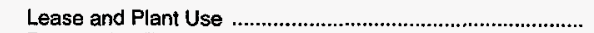 & $1,730,973$ & 383,259 & 0 & 0 \\
\hline 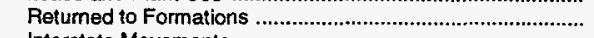 & 0 & 0 & 0 & 0 \\
\hline \multicolumn{5}{|l|}{ Interstate Movements } \\
\hline 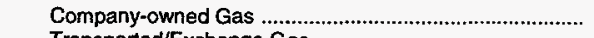 & 0 & 0 & 0 & 0 \\
\hline 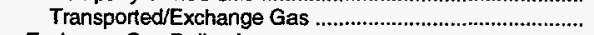 & $18,833,240$ & $6,046,263$ & 0 & 0 \\
\hline 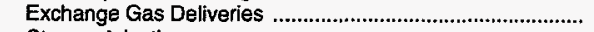 & 253,706 & 753,773 & 0 & 0 \\
\hline \multirow{2}{*}{\multicolumn{5}{|c|}{ Deliveries of Company-owned Gas }} \\
\hline & & & & \\
\hline To Storage Operators & 0 & 0 & 0 & 570,112 \\
\hline To Other Pipelines & 0 & 0 & 0 & 0 \\
\hline \multicolumn{5}{|l|}{ To Consumers } \\
\hline Residential & 0 & 0 & 501,547 & 990,184 \\
\hline 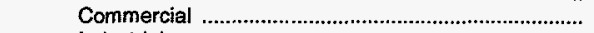 & 0 & 0 & 0 & 248,463 \\
\hline Industrial & 0 & 0 & 10,333 & 5,660 \\
\hline 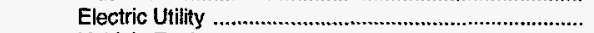 & 0 & 0 & 0 & 0 \\
\hline 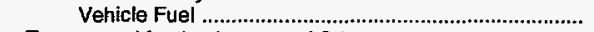 & 0 & 0 & 0 & 0 \\
\hline \multicolumn{5}{|l|}{ Transported for the Account of Others } \\
\hline To Storage Operators & 0 & 0 & 0 & 0 \\
\hline 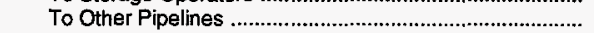 & 366,838 & $32,325,108$ & 0 & 0 \\
\hline \multicolumn{2}{|l|}{ To Consumers } & $19,017,932$ & 0 & 0 \\
\hline 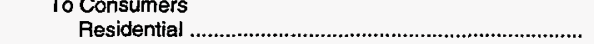 & 0 & 0 & 0 & . \\
\hline 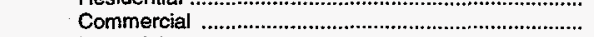 & 0 & 0 & 0 & 59,685 \\
\hline 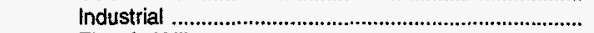 & 0 & 0 & 0 & 245,060 \\
\hline 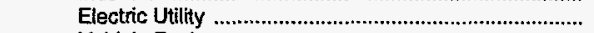 & 0 & 0 & 0 & 278,614 \\
\hline Vehicle Fuel & 0 & 0 & 0 & 0 \\
\hline 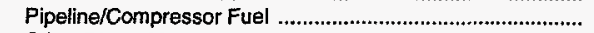 & 146,388 & 57,648 & 0 & 474 \\
\hline 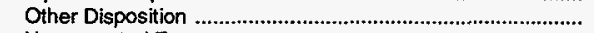 & 0 & 0 & 0 & 0 \\
\hline 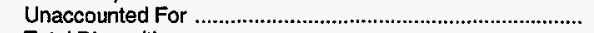 & $-154,530$ & $3,058,509$ & -498 & 220,799 \\
\hline Total Disposition & $41,013,091$ & $94,842,260$ & 511,382 & $2,619,051$ \\
\hline
\end{tabular}


Table 17. Equitable Resources, Inc., Natural Gas Data, 1993 (Continued) (Thousand Cubic Feet)

\begin{tabular}{|c|c|c|c|}
\hline \multirow{2}{*}{ Supply/Disposition } & Equitable Gas Co. & Kentucky West Virginia Gas Co. & Louisiana Intrastate Gas Co. \\
\hline & Pennsylvania & Kentucky & Louisiana \\
\hline \multicolumn{4}{|l|}{ SUPPLY } \\
\hline $\begin{array}{l}\text { Produced Onsystem } \\
\text { Receipts }\end{array}$ & 144,031 & 0 & 0 \\
\hline 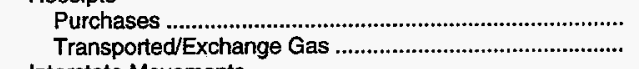 & $\begin{array}{r}44,286,661 \\
9,591,962\end{array}$ & $\begin{array}{r}5,710,662 \\
26,500,913\end{array}$ & $\begin{array}{r}12,439,331 \\
176,682,231\end{array}$ \\
\hline 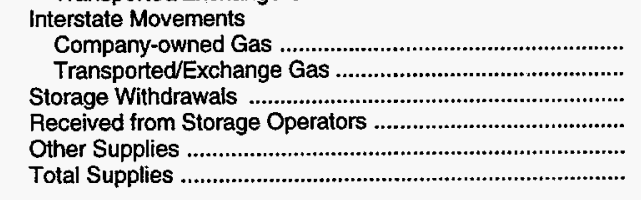 & $\begin{array}{r}0 \\
0 \\
0 \\
3,819,530 \\
0 \\
57,842,184\end{array}$ & $\begin{array}{r}0 \\
0 \\
0 \\
0 \\
0 \\
32,211,575\end{array}$ & $\begin{array}{r}0 \\
0 \\
0 \\
0 \\
0 \\
189,121,562\end{array}$ \\
\hline \multicolumn{4}{|l|}{ DISPOSITION } \\
\hline 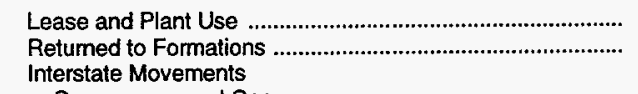 & $\begin{array}{l}0 \\
0\end{array}$ & $\begin{array}{r}1,914,371 \\
0\end{array}$ & $\begin{array}{r}6,708,000 \\
0\end{array}$ \\
\hline 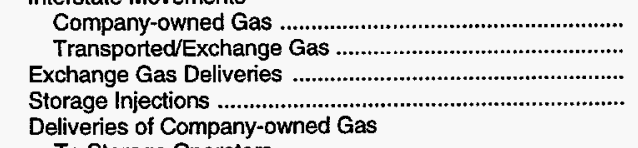 & $\begin{array}{l}0 \\
0 \\
0 \\
0\end{array}$ & $\begin{array}{r}0 \\
0 \\
10,838 \\
0\end{array}$ & $\begin{array}{l}0 \\
0 \\
0 \\
0\end{array}$ \\
\hline 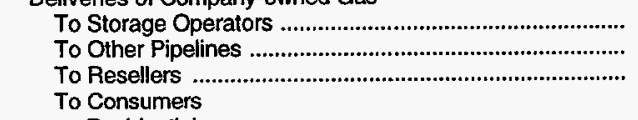 & $\begin{array}{r}7,640,929 \\
0 \\
0\end{array}$ & $\begin{array}{r}0 \\
0 \\
2,763,688\end{array}$ & $\begin{array}{l}0 \\
0 \\
0\end{array}$ \\
\hline 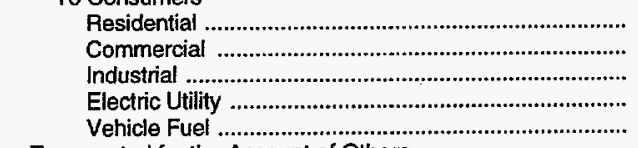 & $\begin{array}{r}28,488,194 \\
7,986,221 \\
1,751,142 \\
0 \\
0\end{array}$ & $\begin{array}{r}0 \\
0 \\
293,966 \\
0 \\
0\end{array}$ & $\begin{array}{r}0 \\
0 \\
7,036,415 \\
4,164,300 \\
0\end{array}$ \\
\hline $\begin{array}{l}\text { Transported for the Account of Others } \\
\text { To Storage Operators } \\
\text { To Other Pipelines } \\
\text { To Resellers } \\
\text { To Consumers }\end{array}$ & $\begin{array}{r}0 \\
0 \\
946,382\end{array}$ & $\begin{array}{r}0 \\
25,290,451 \\
0\end{array}$ & $\begin{array}{r}0 \\
16,921,025 \\
0\end{array}$ \\
\hline 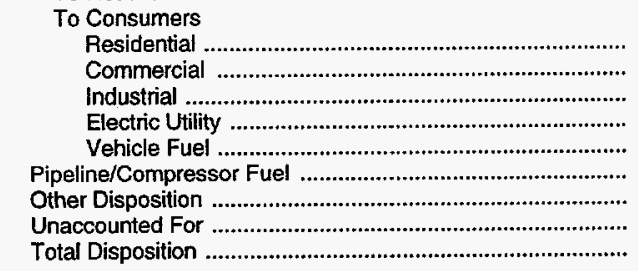 & $\begin{array}{r}3,497 \\
6,078,411 \\
2,828,220 \\
0 \\
0 \\
84,589 \\
0 \\
2,034,599 \\
57,842,184\end{array}$ & $\begin{array}{r}0 \\
0 \\
0 \\
0 \\
0 \\
437,656 \\
1,022,229 \\
478,376 \\
32,211,575\end{array}$ & $\begin{array}{r}0 \\
0 \\
130,882,158 \\
23,641,379 \\
0 \\
0 \\
0 \\
-231,715 \\
189,121,562\end{array}$ \\
\hline
\end{tabular}

Source: Energy Information Administration (EIA), Form EIA-176, "Annual Report of Natural and Supplemental Gas Supply and Disposition." 


\section{Gas Company of New Mexico}

Public Service Company of New Mexico (PNM) is a publicly owned combination gas and electric utility serving numerous communities in the State of New Mexico. Natural gas distribution operations are conducted by PNM's subsidiary, Gas Company of New Mexico. Other subsidiaries, Sunterra Gas Gathering Company and Sunterra Gas Processing Company provide gathering and processing services, respectively. The company's processing facilities in northwestern New Mexico have the capacity to process 300 thousand cubic feet of gas per day. PNM also owns facilities in Santa Fe, New Mexico for the storage, transmission, distribution, and sale of water. In 1993, PNM derived 67.5 percent of its utility operations revenues from electric operations, 31.0 percent from natural gas operations, and 1.5 percent from water operations.
GasCo distributes natural gas to approximately 371,000 customers in Albuquerque, Santa Fe, and other communities, with Albuquerque accounting for slightly more than half of the company's total natural gas customers. Sales and transportation services accounted for 66 percent and 34 percent, respectively, of GasCo's total gas deliveries to end users. GasCo provides most of the State's natural gas requirements, accounting for more than 80 percent of residential and commercial natural gas consumption, and more than 60 percent of the natural gas consumed by industrial customers.

At the end of 1993, GasCo owned 9,471 miles of distribution lines, 552 miles of gathering pipe, and 1,355 miles of transmission lines. It held leases from the Department of Energy on 128 miles of pipe for transportation of natural

Figure 14. Gas Company of New Mexico, 1993

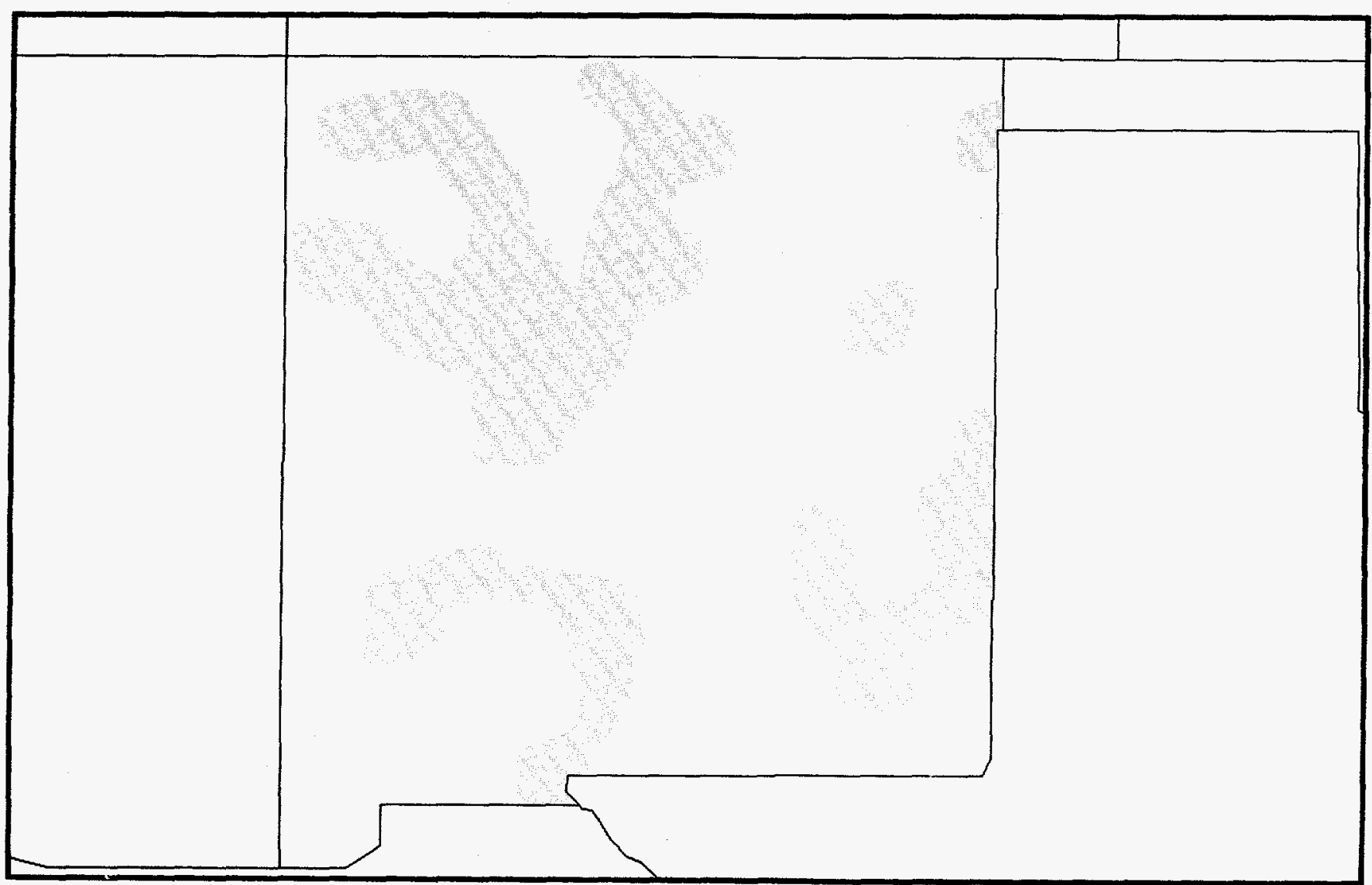

Source: Energy Information Administration (EIA), Form ElA-176, "Annual Report of Natural and Supplemental Gas Supply and Disposition." 


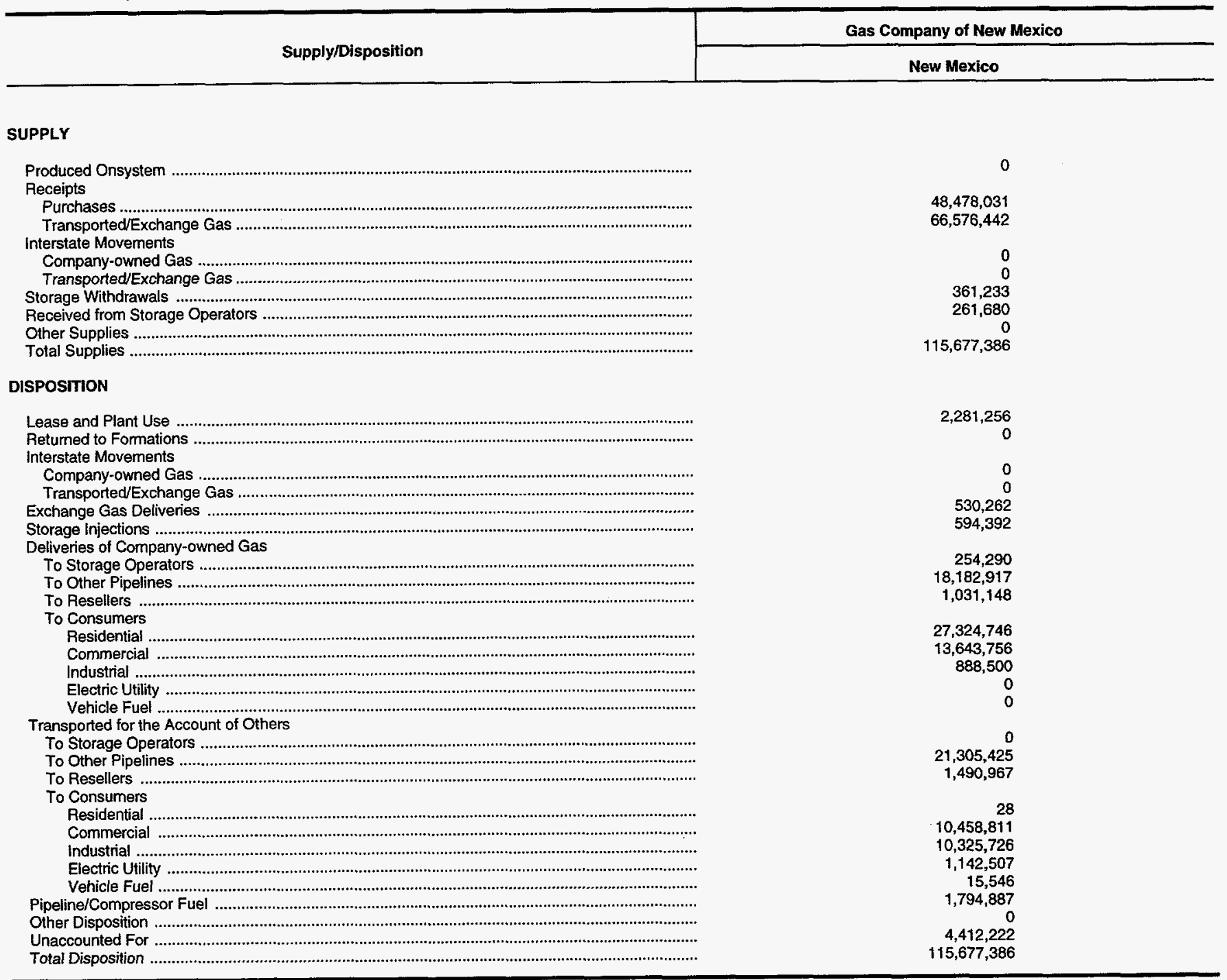

Source: Energy Information Administration (EIA), Form EIA-176, "Annual Report of Natural and Supplemental Gas Supply and Disposition."

gas to Los Alamos and other northern New Mexico communities.

GasCo uses natural gas- powered vehicles as a part of the company's fleet of service vehicles and successfully assisted in numerous vehicle conversion programs at both city and State levels. For instance, the public transportation division of the City of Santa Fe has converted its entire fleet to natural gas-powered vehicles. The company and the State won a President's Environment and Conservation Challenge Award for working together on a program to promote the use of natural gas vehicles. 


\section{Iroquois Gas Transmission System}

Iroquois Gas Transmission System is a transportationonly pipeline company providing gas service from Canada to the Northeastern United States. Owned by a partnership of 12 U.S. and Canadian energy companies, Iroquois began operations in December 1991. At that time, it had 192 miles of pipeline extending from the U.S.-Canadian border at Waddington, New York to Wright, New York. The entire 375-mile Iroquois system, completed in January 1992, transports gas to customers in Connecticut, Massachusetts, New Hampshire, New Jersey, New York, and Rhode Island.

The company provides direct deliveries to shippers as well as upstream transportation service for shippers on the Tennessee and Algonquin systems. As it extends from the U.S.-Canadian border across the States of New York and Connecticut to South Commack and Long Island, Iroquois interconnects with Tennessee Gas Pipeline Co. at Wright, New York and Shelton, Connecticut; with Consolidated Natural Gas Transmission at Canajoharie, New York; and with Algonquin Gas Transmission Co. at Brookfield, Connecticut. Of the gas delivered in 1993, 80 percent is delivered to local distribution companies, and the remaining 20 percent is delivered directly to power generators. New York and New Jersey use 49 percent of the gas, while the balance goes to consumers in Connecticut, Massachusetts, New Hampshire, and Rhode Island. In 1993, Iroquois had a total disposition of 419 billion cubic feet of natural gas compared to 333 billion cubic feet in 1992, an increase of 26 percent.

Figure 15. Iroquois Gas Transmission System, 1993

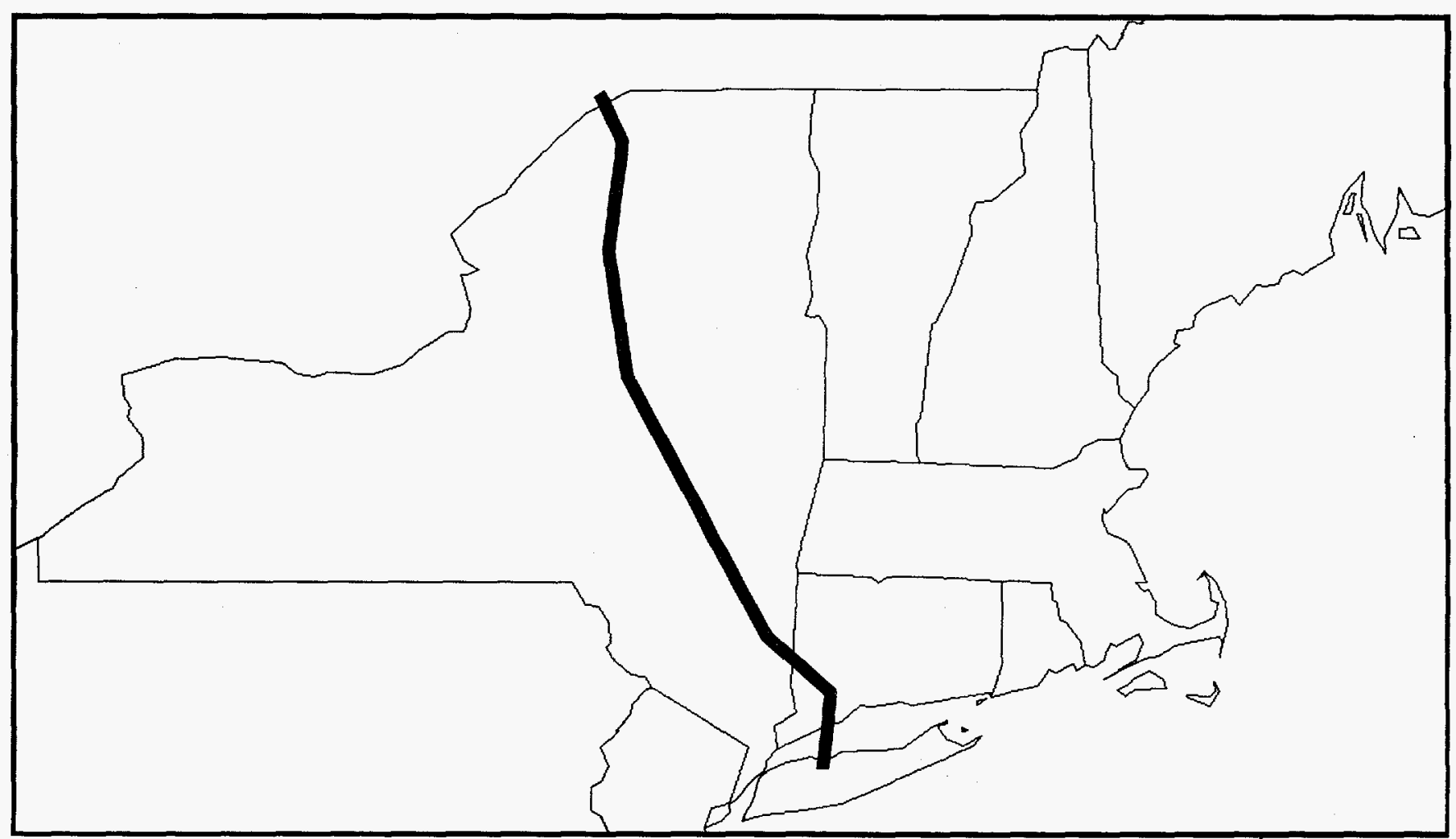

Source: Energy Information Administration (EIA), Form EIA-176, "Annual Report of Natural and Supplemental Gas Supply and Disposition." 
Table 19. Iroquois Gas Transmission System Natural Gas Data, 1993

(Thousand Cubic Feet)

\begin{tabular}{l|l|l}
\hline \multirow{2}{*}{ Supply/Disposition } & \multicolumn{2}{|c}{ Iroquois Gas Transmission System } \\
\cline { 2 - 3 } & New York & Connecticut \\
\hline
\end{tabular}

SUPPLY

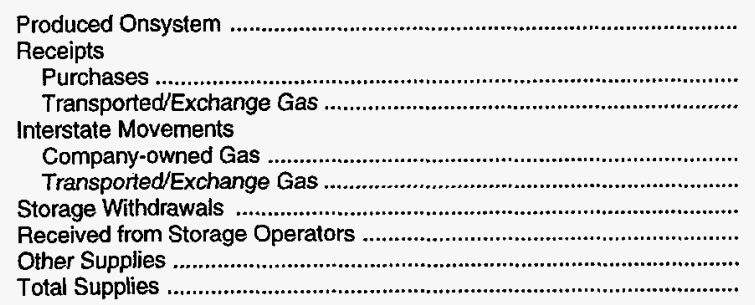

\section{DISPOSITION}

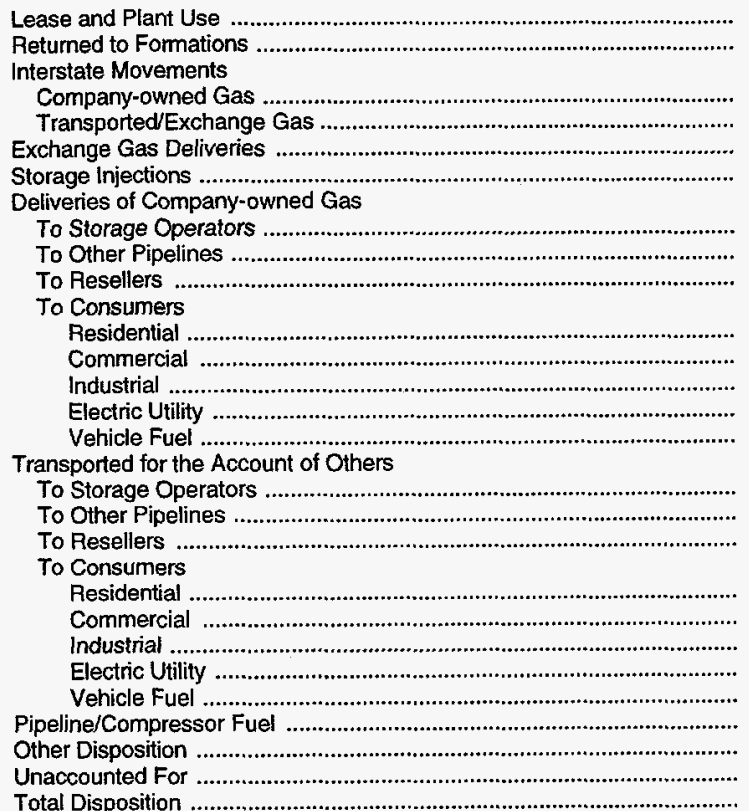

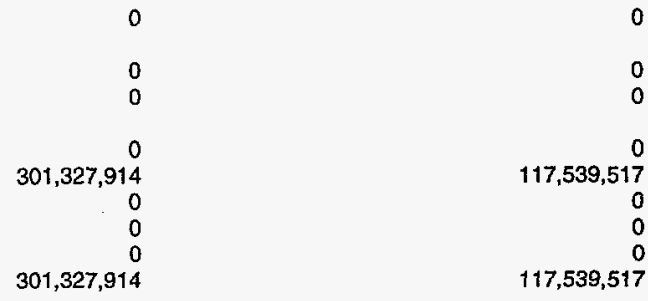

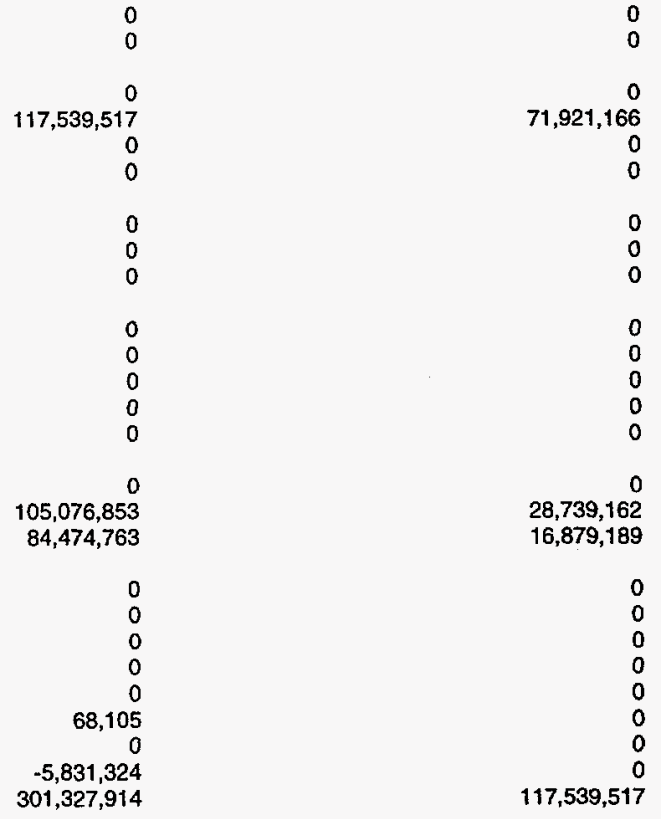

Source: Energy Information Administration (EIA), Form ElA-176, "Annual Report of Natural and Supplemental Gas Supply and Disposition."

During the past year, Iroquois brought several new facilities on-line. The most important was the compressor station located in Wright, New York. The two solar-powered compressor units at this station are among the first to be used in the Northeast and produce fewer of the polluting nitrogen oxides than the more conventional compressor stations. The station is operated at 15 decibels below the FERC noise guidelines by utilizing state-of-the-art noise abatement techniques and materials. Two meter stations were also placed into operation. One--at Northport, Long Island--will provide natural gas to the first of four generating units. The second unit is to be converted in 1994. Each unit consumes approximately 95 million cubic feet per day. The second meter station was placed in operation at Edwards, New York to provide natural gas to a cogeneration plant. Future plans call for construction of a lateral and meter station in Milford, Connecticut, and construction of a compressor station along with a new meter station at Crogham, New York, which will establish new delivery points. A total of 16 meter stations and 2 compression stations comprise the facilities along the Iroquois Gas Transmission System.

While it does not deliver gas directly to end users who can use it as a vehicle fuel, Iroquois supports the development of the natural gas vehicle market. A natural gas vehicle demonstration performed at the State Capitol in Albany by Iroquois, its LDC customers, and other organizations resulted in State legislation that mandates that at least 10 percent of all cars and light trucks purchased by the State in 1993 and 1994 be powered by natural gas or electricity. 


\section{K N Energy, Inc.}

$\mathrm{K} N$ Energy, Inc. ( $\mathrm{K} \mathrm{N}$ ) is a natural gas services company that provides gas reserves development, gas gathering, processing, marketing, storage, transportation, and gas distribution services. The company operates in seven States and serves its customers through three pipeline systems, covering more than 18,000 miles. Its principal operations supply natural gas to consumers in Colorado, Kansas, Nebraska, and Wyoming. K N's interstate pipeline has access to the natural gas supply areas of the Mid-Continent and the Rocky Mountains.

$\mathrm{K} \mathrm{N}$ operates its interstate transmission pipeline and storage facilities through $\mathrm{K} \mathbf{N}$ Interstate Gas Transmission Co., a separate business unit subsidiary. Although K N's local distribution operation is also operated as a separate business unit, it remains part of $\mathrm{K} N$ Energy, Inc. In addition, the company supplies natural gas to consumers through two intrastate divisions, Rocky Mountain Natural Gas in Colorado and Northern Gas of Wyoming.

K N Gas Gathering, Inc., a wholly owned subsidiary, operates K N's gathering and principal processing facilities. In another nonregulated business activity, $\mathrm{K} N$ develops natural gas reserves through two wholly owned subsidiaries, K N Production Company and GASCO, Inc. K N Gas Marketing, Inc., markets natural gas and related services.

Several additions to the $\mathrm{K} N$ system were completed during the past 2 years. Northern Gas of Wyoming pur-

Figure 16. K N Energy, Inc., 1993

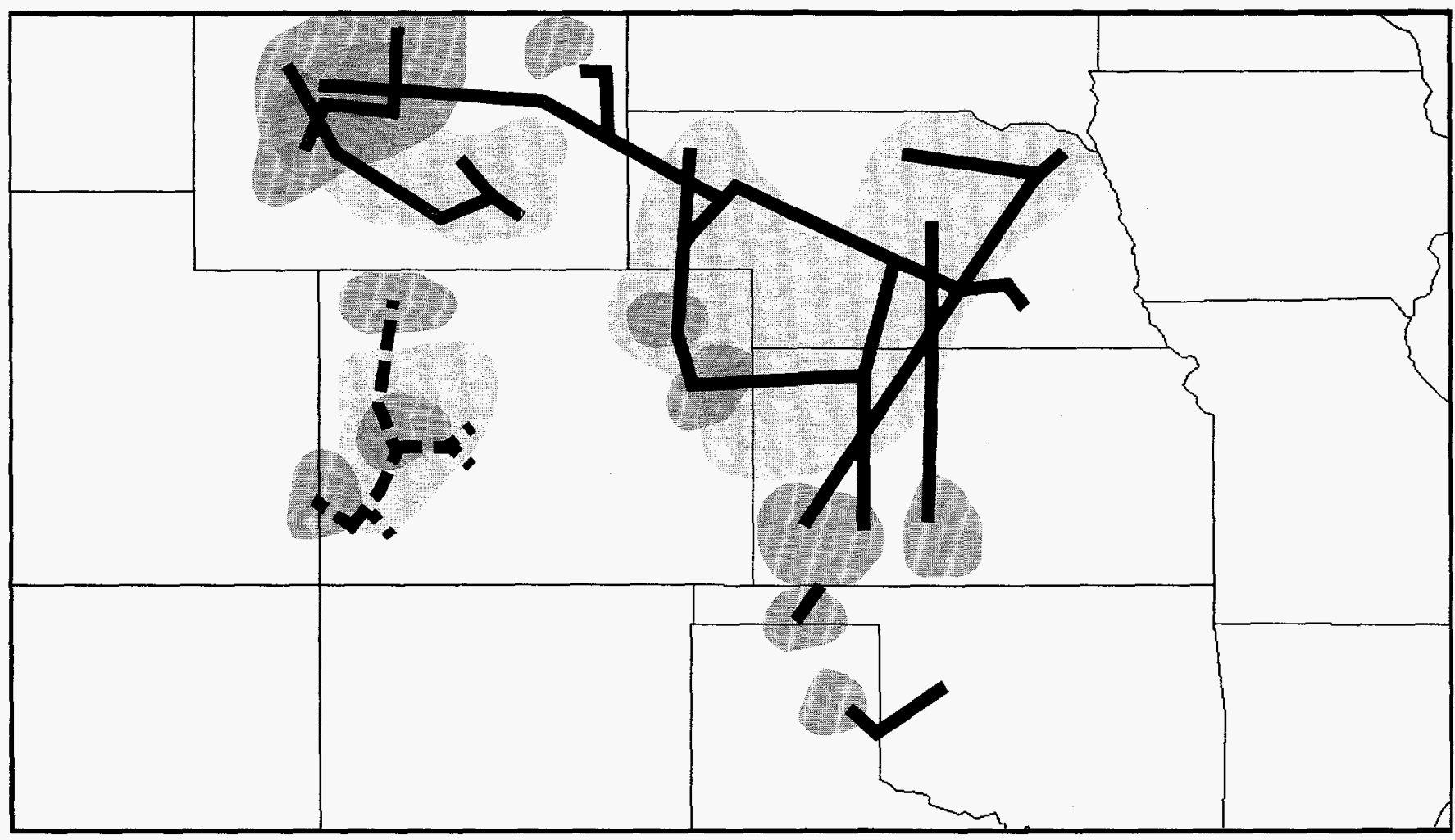

Source: Energy Information Administration (EIA), Form EIA-176, "Annual Report of Natural and Supplemental Gas Supply and Disposition."

Supply Areas

Service Areas
K N Energy, Inc.

- Colorado Intrastate System
Wyoming Intrastate System 
Table 20. K N Energy, Inc., Natural Gas Data, 1993

(Thousand Cubic Feet)

\begin{tabular}{l|l|l|l|l|c|c}
\hline \multirow{2}{*}{ Supply/Disposition } & \multicolumn{9}{|c}{ KN Energy, Inc. } \\
\cline { 2 - 8 } & Texas & Oklahoma & Kansas & Nebraska & Colorado & Wyoming \\
\hline
\end{tabular}

SUPPLY

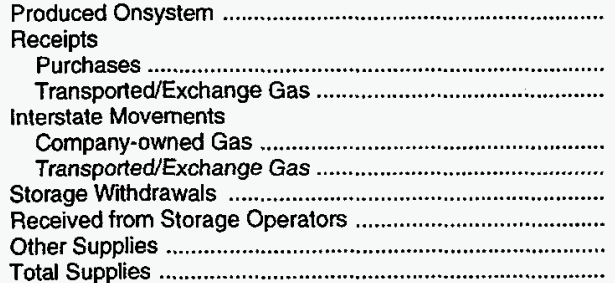

0
620
$16,459,675$
0
$10,107,348$
0
0
0
$26,567,643$

DISPOSITION

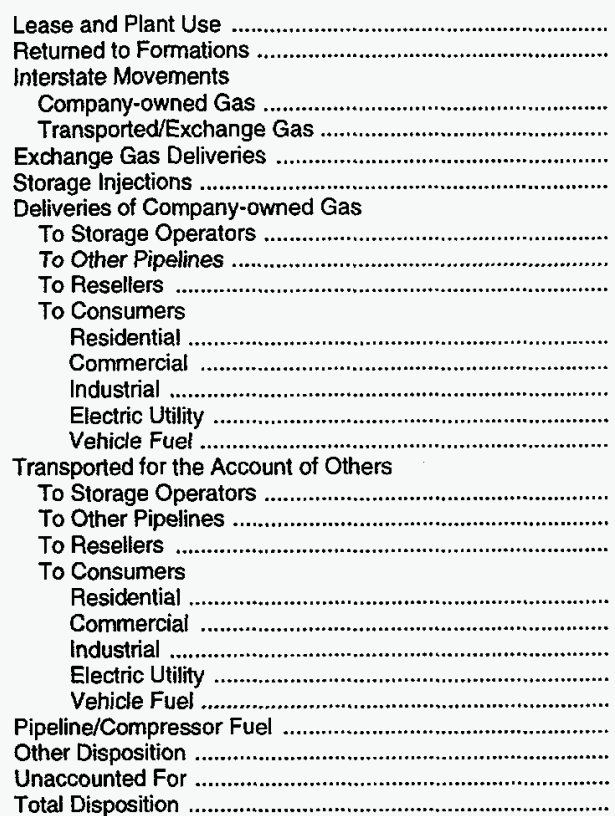
724,625
$28,164,030$

$\begin{array}{rr}0 & 1,720,396 \\ 0 & 0 \\ 0 & 0 \\ 0 & 10,107,348 \\ 0 & 548,822 \\ 0 & 0 \\ & \\ 0 & 0 \\ 0 & 0 \\ 0 & 0 \\ & \\ 185 & 1,836 \\ 1,331 & 32,859 \\ 0 & 0 \\ 0 & 0 \\ 0 & 0 \\ 0 & 0 \\ 0 & 0 \\ 0 & 0 \\ 0 & 0 \\ 0 & 0 \\ 0 & 0 \\ 0 & 0 \\ 0 & 1,258 \\ 28,407,940 & 0 \\ 0 & 121,433 \\ -122,491 & 0 \\ 26,567,643 & 0,888,655\end{array}$
$28,164,03$ 0
0
$28,888,655$
0

$35,053,074$
$16,303,473$
0
0
$4,530,156$
222,698
0
0
$56,109,401$

0
356,974
$1,668,780$
0
$47,004,145$
$11,718,180$
0
781,109
$61,529,188$

0 $56,109,401$ $61,529,188$ 408,355

$4,435,617$ $43,016,938$

0
$14,347,984$
42,775
0
0
$29,522,859$

0
0
0
0
0
$47,452,555$

$6,724,925$

$19,111,975$

2,601,624

222,519

0
0
$2,768,488$

$2,904,375$

$5,602,436$

45,288

61,256

$16,471,397$

0
55,660

718,750

0
0

$2,049,290$

$-3,228,582$

$56,109,401$

$\begin{array}{rrr}0 & 325,117 & 625,862 \\ 0 & 0 & 0 \\ 14,492,758 & 6,567,416 & 25,710,136 \\ 0 & 0 & 0 \\ 0 & 0 & 0 \\ 14,485,380 & 22,479 & 0 \\ 0 & 0 & 0 \\ 0 & 0 & 0 \\ 6,374,916 & 5,672,745 & 2,392,276 \\ 8,731,286 & 610,139 & 932,616 \\ 8,289,337 & 435,380 & 688,804 \\ 84,522 & 27,883 & 0 \\ 16,742 & 0 & 0 \\ 0 & 0 & 0\end{array}$

$\begin{array}{rrr}0 & 0 & 0 \\ 0 & 14,027,082 & 18,524,049 \\ 3,056,601 & 0 & 0\end{array}$

0
$2,756,427$
$2,044,684$
474,622
0
664,371
0
57,542
$61,529,188$

0
0
46,445
0
0
364,108
0
$-1,831,741$
$47,452,555$

chased a natural gas distribution system serving almost 7,000 customers in northeast Wyoming. K N Gas Gathering, Inc., acquired a nonregulated natural gas gathering and processing facility located near Douglas, Wyoming. GASCO, Inc., bought oil and gas producing property in the Piceance Basin, located in western Colorado. $\mathrm{K} \mathrm{N}$ also acquired two natural gas gathering systems, located in the major Rocky Mountain production areas. The first was the 1,250-mile Wattenberg system in the Denver-Julesburg Basin in Colorado. The second comprises 110 miles of natural gas pipeline and related field facilities in the Wind River Basin of Wyoming.

Regulatory work for the TransColorado transmission pipeline project in western Colorado--a joint venture among K N subsidiaries, Questar Corporation, and Public
Service Company of Colorado--is almost completed. The pipeline is expected to have a daily capacity of 300 million cubic feet of gas and will access various Rocky Mountain region reserve basins. Construction is expected to begin in 1995 .

Distribution of K N's gas sales in 1993 continue to demonstrate the company's diversity. The company delivered 94 billion cubic feet to other pipelines, 39 billion cubic feet to resellers, 21 billion cubic feet to residential customers, 24 billion cubic feet to commercial customers, 18 billion cubic feet to industrial customers, and small amounts to electric utility customers. K N's major storage fields located at Huntsman and Big Springs, Nebraska, enable it to meet heavy winter demand without disruption. 
Table 20. K N Energy, Inc., Natural Gas Data, 1993 (Continued) (Thousand Cubic Feet)

\begin{tabular}{l|c|c|c|c|c|c|c|c|}
\hline Supply/Disposition & K N Energy, Inc. & $\begin{array}{c}\text { Northern Gas } \\
\text { Co. }\end{array}$ & $\begin{array}{c}\text { Northern Gas of } \\
\text { Wyoming }\end{array}$ & $\begin{array}{l}\text { Rocky Mountain } \\
\text { Natural Gas Div. }\end{array}$ & $\begin{array}{c}\text { Rocky Mountain } \\
\text { Natural Gas Co. } \\
\text { Gathering Co. }\end{array}$ \\
\cline { 2 - 5 } & Montana & Wyoming & Wyoming & Colorado & Colorado & Colorado \\
\hline
\end{tabular}

SUPPLY

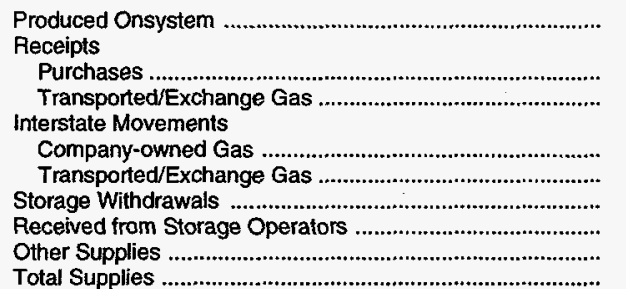

Total Supplies

\section{DISPOSITION}

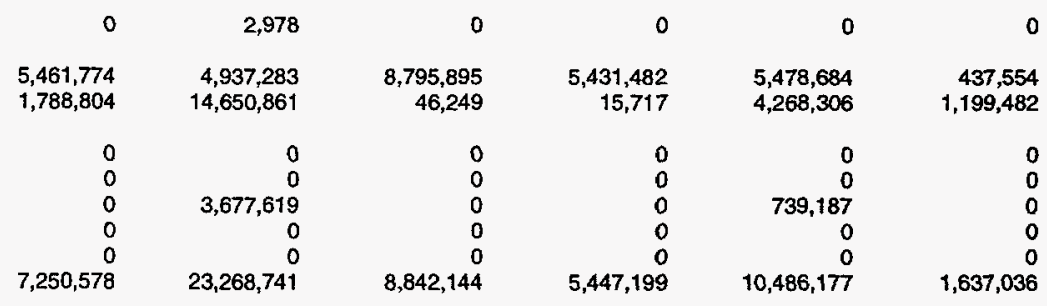

\footnotetext{
Lease and Plant Use

Returned to Formations

Interstate Movements

Company-owned Gas

Transported/Exchange Gas

Exchange Gas Deliveries

Storage Injections .....

Deliveries of Company-owned Gas

To Storage Operators

To Resellers

To Consumers

Residential

Commercial

Industrial ...

Electric Utility

Transported for the Account of Others

To Storage Operators

To Other Pipelines

To Resellers .

To Consumers

Residential

Industrial.

Electric Utility

Vehicle Fuel

Pipeline/Compressor Fuel

Other Disposition

Unaccounted For

Total Disposition
}

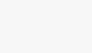

0

554

0
0
0
0

$1,637,036$

\begin{tabular}{|c|c|c|}
\hline $\begin{array}{l}0 \\
0\end{array}$ & $\begin{array}{l}0 \\
0\end{array}$ & $\begin{array}{l}0 \\
0\end{array}$ \\
\hline 0 & 0 & 0 \\
\hline 0 & 0 & 0 \\
\hline 0 & 0 & 0 \\
\hline 0 & 707,779 & 0 \\
\hline 0 & 0 & 0 \\
\hline 0 & 0 & 0 \\
\hline 0 & $4,974,260$ & 414,421 \\
\hline $2,948,596$ & 0 & 0 \\
\hline $2,277,310$ & 0 & 0 \\
\hline 22,313 & 0 & 0 \\
\hline 0 & 0 & 0 \\
\hline 0 & 0 & 0 \\
\hline 0 & 0 & 0 \\
\hline 0 & 0 & 0 \\
\hline 0 & $4,384,190$ & $1,200,154$ \\
\hline 0 & 0 & 0 \\
\hline 0 & 0 & 0 \\
\hline 13,326 & 0 & 0 \\
\hline 0 & 0 & 0 \\
\hline 0 & 0 & 0 \\
\hline 20,858 & 343,940 & 0 \\
\hline 0 & 0 & 0 \\
\hline 164,796 & 76,008 & 22,461 \\
\hline $5,447,199$ & $10,486,177$ & $1,637,036$ \\
\hline
\end{tabular}

Source: Energy Information Administration (EIA), Form EIA-176, "Annual Report of Natural and Supplemental Gas Supply and Disposition." 
Table 20. K N Energy, Inc., Natural Gas Data, 1993 (Continued) (Thousand Cubic Feet)

\begin{tabular}{|c|c|c|c|c|}
\hline \multirow{2}{*}{ Supply/Disposition } & Northern Gas of Wyoming & $\begin{array}{c}\text { Rocky Mountain Natural } \\
\text { Gas Div. }\end{array}$ & $\begin{array}{c}\text { Rocky Mountain Natural } \\
\text { Gas Co. }\end{array}$ & RMNG Gathering Co. \\
\hline & Wyoming & Colorado & Colorado & Colorado \\
\hline
\end{tabular}

SUPPLY

Produced Onsystem

Receipts

Purchases $\quad 8,795,895$

Transported/Exchange Gas .......................... $\quad 46,249$

Interstate Movements

Company-owned Gas

Transported/Exchange Gas .....................

Storage Withdrawals ..........................................

Received from Storage Operators .....................

Other Supplies .

Total Supplies

DISPOSITION

Lease and Plant Use

Retumed to Formations

Interstate Movements

Gompany-owned Gas .

Transported/Exchange Gas

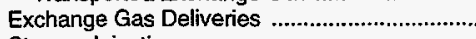

Storage Injections ...................................

To Storage Operators

.

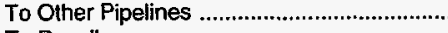

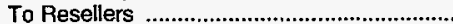

To Consumers

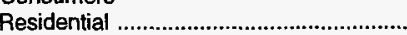

Commercial

Industrial ...........

Electric Utility .

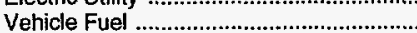

Transported for the Account of Others

To Storage Operators

To Other Pipelines

To

To Consumers

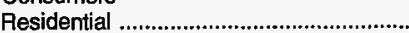

Commercial

industrial ........

Electric Utility

Vehicle Fuel

Other Disposition

Unaccounted For ..........

Total Disposition
$8,842,144$

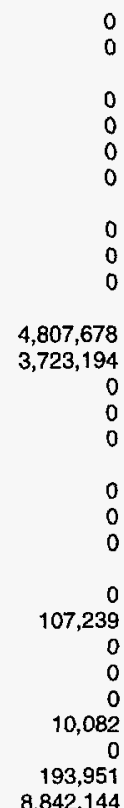

$5,431,482$

15,717

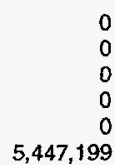

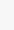

西

西

$, 447,199$

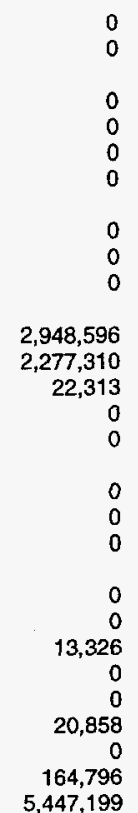

$\begin{array}{rr}0 & 0 \\ 5,478,684 & 437,554 \\ 4,268,306 & 1,199,482 \\ & \\ 0 & 0 \\ 0 & 0 \\ 739,187 & 0 \\ 0 & 0 \\ 0 & 0 \\ 10,486,177 & 1,637,036\end{array}$

$\begin{array}{rr}0 & 0 \\ 0 & 0 \\ 0 & 0 \\ 0 & 0 \\ 0 & 0 \\ 707,779 & 0 \\ 0 & \\ 0 & 0 \\ 4,974,260 & 0\end{array}$

0
0
0

4,421

Source: Energy Information Administration (EIA), Form EIA-176, "Annual Report of Natural and Supplemental Gas Supply and Disposition."

Table 21. K N Energy, Inc., Interstate Flows of Natural Gas, 1993 (Thousand Cubic Feet)

\begin{tabular}{|c|c|}
\hline Company and State & Volume \\
\hline \multicolumn{2}{|l|}{ KN Energy, Inc. } \\
\hline Texas ... & $10,107,348$ \\
\hline \multicolumn{2}{|l|}{ Kansas To } \\
\hline . & $19,111,975$ \\
\hline \multicolumn{2}{|l|}{ Nebraska To } \\
\hline 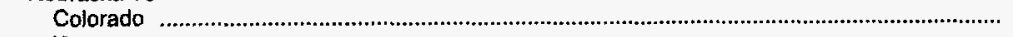 & $14,347,984$ \\
\hline 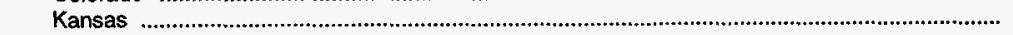 & 144,774 \\
\hline \multicolumn{2}{|l|}{ Colorado To } \\
\hline 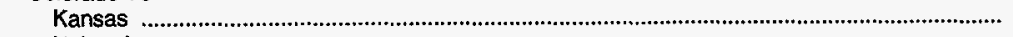 & $4,385,382$ \\
\hline Nebraska & $2,182,034$ \\
\hline \multicolumn{2}{|l|}{ Wyoming To } \\
\hline 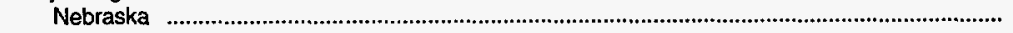 & $25,710,136$ \\
\hline
\end{tabular}

Source: Energy Information Administration (EIA), Form ElA-176, "Annual Report of Natural and Supplemental Gas Supply and Disposition." 


\section{Koch Gateway Pipeline Co.}

Koch Gateway Pipeline Company is a privately held corporation that was acquired by Koch Industries, Inc., in late 1992 when it was known as United Gas Pipeline Company. The Koch natural gas pipeline system is located in the Southcentral United States in Alabama, Louisiana, Mississippi, and Texas. With the acquisition of United's assets, the company now owns the following subsidiaries: Gateway Pipeline Company, Trans-Anadarko Pipeline Company; United Offshore Company, Cypress Pipeline Company, Unisouth Pipeline Company, Laser Marketing Company, Gulf South Pipeline Company, Texas Gulf South Pipeline Company, Mississippi Gulf South Pipeline Company, Florida Gulf South Pipeline Company, and Alabama Gulf South Pipeline Company.
In an historic decision in March 1994, the Federal Energy Regulatory Commission (FERC) approved market-based rates for Koch Gateway storage services. FERC stated that Koch's filing provided sufficient data to show that Koch could not exercise "market power" over its storage services. To expand its pipeline system, Koch has expressed an interest in building an interconnect from Tennessee Gas Pipeline's Mobile Bay gathering facilities to Tennessee's 500 line in Hancock County, Mississippi. It is expected that Koch will ship as much as 300 million cubic feet per day to the Tennessee 500 line through this interconnect once it is completed. Completion of the project is expected in time to provide gas to the area for the 1995-96 winter heating season.

Figure 17. Koch Gateway Pipeline Co., 1993

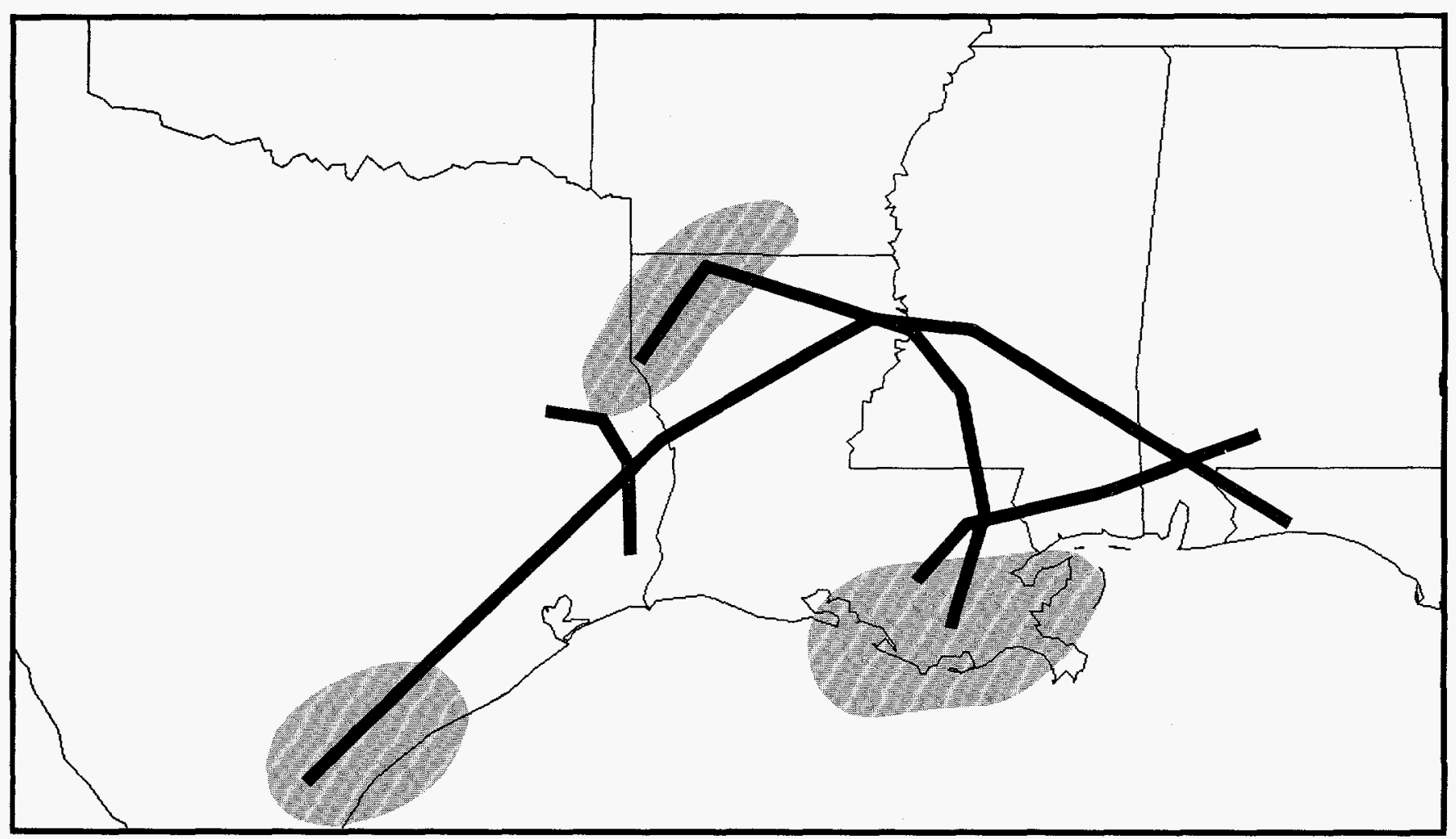

Source: Energy Information Administration (E|A), Form EIA-176, "Annual Report of Natural and Supplemental Gas Supply and Disposition." 
Table 22. Koch Gateway Pipeline Co., Natural Gas Data, 1993

(Thousand Cubic Feet)

\begin{tabular}{|c|c|c|c|c|c|}
\hline \multirow{2}{*}{ Supply/Disposition } & \multicolumn{5}{|c|}{ Koch Gateway Pipeline Co. } \\
\hline & Texas & Louisiana & Mississippi & Alabama & Florida \\
\hline \multicolumn{6}{|l|}{ SUPPLY } \\
\hline $\begin{array}{l}\text { Produced Onsystem } \\
\text { Receipts }\end{array}$ & 0 & 0 & 0 & 0 & 0 \\
\hline 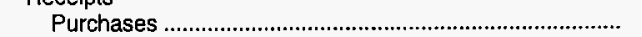 & $32,482,483$ & $24,577,231$ & $4,256,662$ & $5,231,543$ & 0 \\
\hline 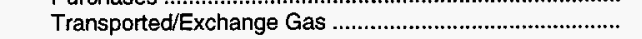 & $304,012,834$ & $401,327,071$ & $56,245,892$ & $67,700,141$ & 0 \\
\hline \multicolumn{6}{|l|}{ Interstate Movements } \\
\hline 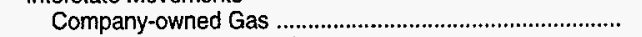 & 0 & 0 & 0 & 0 & 0 \\
\hline 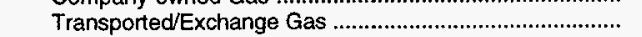 & 0 & $182,461,440$ & $113,372,338$ & 0 & $37,887,825$ \\
\hline Storage Withdrawals & 0 & $52,015,362$ & $7,148,344$ & 0 & 0 \\
\hline 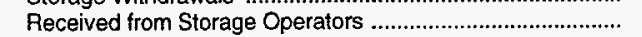 & 0 & 0 & $1,094,467$ & 0 & 0 \\
\hline 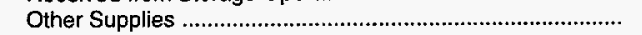 & 0 & 0 & 0 & 0 & 0 \\
\hline Total Supplies & $336,495,317$ & $660,381,104$ & $182,117,703$ & $72,931,684$ & $37,887,825$ \\
\hline \multicolumn{6}{|l|}{ DISPOSITION } \\
\hline Lease and Plant Use & $3,959,782$ & $2,397,964$ & 0 & 0 & 0 \\
\hline 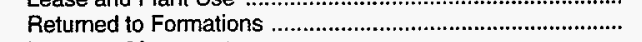 & 0 & 0 & 0 & 0 & 0 \\
\hline \multicolumn{6}{|l|}{ interstate Movements } \\
\hline 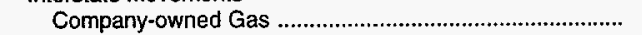 & 0 & 0 & 0 & 0 & 0 \\
\hline 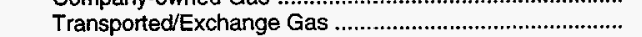 & $182,461,440$ & $99,582,089$ & 0 & $51,678,074$ & 0 \\
\hline Exchange Gas Deliveries & $103,533,539$ & $118,020,346$ & $4,133,381$ & $1,096,186$ & 0 \\
\hline Storage Injections & 0 & $61,455,491$ & $6,086,782$ & 0 & 0 \\
\hline \multicolumn{6}{|l|}{ Deliveries of Company-owned Gas } \\
\hline To Storage Operators & 0 & 0 & 0 & 0 & 0 \\
\hline To Other Pipelines & 0 & 0 & 0 & 0 & 0 \\
\hline 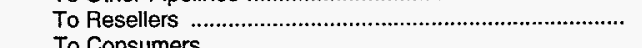 & $4,297,838$ & $12,196,087$ & $9,618,251$ & $1,400,055$ & $1,461,157$ \\
\hline \multicolumn{4}{|l|}{ To Consumers } & & \\
\hline $\begin{array}{l}\text { Residential } \\
\text { Commercial }\end{array}$ & 0 & 0 & 0 & 0 & 0 \\
\hline $\begin{array}{l}\text { Commercial } \\
\text { Industrial }\end{array}$ & 3,820 & 185,869 & 40,472 & 0 & 0 \\
\hline $\begin{array}{l}\text { Industrial } \\
\text { Electric Utility }\end{array}$ & 0 & 0 & $12,193,561$ & 0 & 0 \\
\hline Vehicle Fuel & 0 & 0 & 0 & 0 & 0 \\
\hline \multicolumn{6}{|l|}{ Transported for the Account of Others } \\
\hline To Storage Operators & 0 & 0 & 0 & 0 & 0 \\
\hline To Other Pipelines & $24,630,376$ & $177,251,540$ & $92,410,405$ & 324,395 & 0 \\
\hline To Resellers & $6,231,965$ & $31,980,618$ & $24,506,890$ & $10,741,261$ & $6,155,886$ \\
\hline \multicolumn{6}{|l|}{ To Consumers } \\
\hline 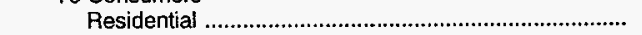 & 0 & 0 & 0 & 0 & 0 \\
\hline 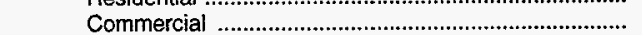 & 190,798 & 472,399 & 590,259 & 0 & 911,465 \\
\hline Industrial & $3,997,814$ & $104,933,072$ & $23,013,614$ & $7,581,108$ & $28,130,580$ \\
\hline 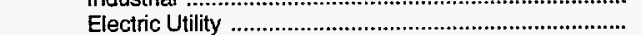 & $3,703,441$ & $46,405,905$ & $8,140,112$ & 0 & $1,032,189$ \\
\hline Vehicle Fuel & 0 & 0 & 0 & 0 & 0 \\
\hline 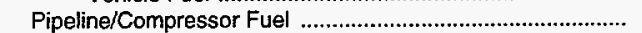 & $2,685,530$ & $2,590,860$ & 439,331 & 363 & 24 \\
\hline Other Disposition & 0 & 0 & 0 & 0 & 0 \\
\hline Unaccounted For & 798,974 & $2,908,864$ & 944,645 & 110,242 & 196,524 \\
\hline Total Disposition & $336,495,317$ & $660,381,104$ & $182,117,703$ & $72,931,684$ & $37,887,825$ \\
\hline
\end{tabular}

Source: Energy Information Administration (EIA), Form ElA-176, "Annual Report of Natural and Supplemental Gas Supply and Disposition."

Table 23. Koch Gateway Pipeline Co., Interstate Flows of Natural Gas, 1993 (Thousand Cubic Feet)

\begin{tabular}{|c|c|}
\hline Company and State & Volume \\
\hline $\begin{array}{l}\text { Koch Gateway Pipeline Co. } \\
\text { Texas To } \\
\text { Louisiana } \\
\text { Louisiana To } \\
\text { Mississippi } \\
\text { Alabama To } \\
\text { Florida } \\
\text { Mississippi }\end{array}$ & $\begin{array}{l}37,887,825 \\
13,790,249\end{array}$ \\
\hline
\end{tabular}

Source: Energy Information Administration (EIA), Form EIA-176, "Annual Report of Natural and Supplemental Gas Supply and Disposition." 


\section{Lone Star Gas Co.}

Lone Star Gas Company, a subsidiary of ENSERCH Corporation, serves over 1.25 million customers in Texas. ENSERCH Corporation is an integrated company with operations in natural gas exploration, production, processing, transportation, and marketing.

ENSERCH's pipeline system is made up of more than 32,000 miles of transportation, gathering, and transmission lines. It is an intrastate system that not only moves gas within Texas, but also links major producing areas on the Texas Gulf Coast, south Texas, and west Texas with markets throughout the country by connecting with interstate pipelines.
In the State of Texas, ENSERCH'S transmission and distribution activities are carried out primarily by Lone Star. Lone Star, with more than 9,000 miles of gathering and transmission pipelines in north Texas, is the 15th largest system in the country in terms of pipeline mileage. Lone Star also has 22,800 miles of distribution lines serving nearly 1.3 million customers in 550 cities and towns in north Texas. The majority of these customers are concentrated in the Dallas/Fort Worth area.

In 1993, Lone Star introduced a program enabling residential real estate developers to install their own natural gas mains while assuring quality standards. The program

Figure 18. Lone Star Gas Co., 1993

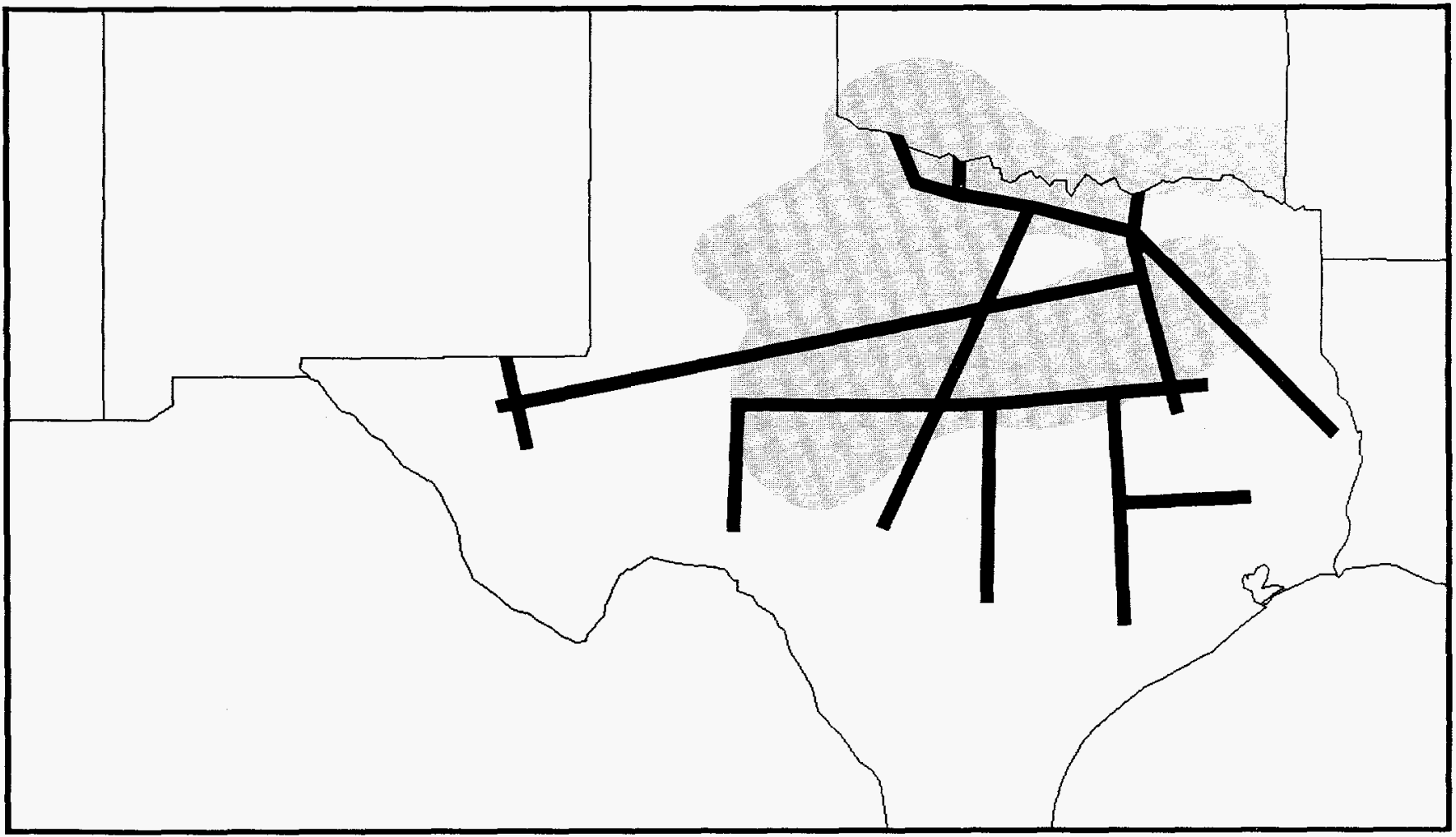

Source: Energy Information Administration (ElA), Form ElA-176, "Annual Report of Natural and Supplemental Gas Supply and Disposition."

Service Area Lone Star Gas Co. 
encourages the use of natural gas because it reduces the developers' costs. There were 24,000 new housing starts in Lone Star's service area in 1993, compared to 21,000 in 1992. Approximately 90 percent of the new homes constructed in 1993 used natural gas compared with 84 percent of new construction in 1992.

Nearly 7,000 tons of natural gas cooling equipment were installed on Lone Star's system in 1993, slightly less than in 1992 but more than double the tonnage sold in 1991. In the multifamily housing market, six new projects with a total of 1,900 units are being served by Lone Star. Each unit is equipped with combination space heating and water heating systems as well as gas logs.

To meet increased demand for west-to-east natural gas flow, Lone Star is adding compressor facilities to its pipeline system to increase capacity by at least 150,000 thousand cubic feet per day from Waha in west Texas to Katy in southwest Texas by May 1, 1995. This is Lone Star's second recent compression enhancement on the Waha-to-Katy pipeline segment. In 1993, the company increased capacity by 175,000 thousand cubic feet per day on that route. In addition to the Waha and Katy hubs, the Lone Star system is also connected to the Carthage hub in northeast Texas as well as to eight gas-producing basins in the State.

Increasingly, gas supplies from Canada and the Rocky Mountain area are being delivered to the California market, which had primarily been served by gas from west Texas and New Mexico. Lone Star's pipeline, running from Waha to Katy, enables producers of gas in west Texas to move their gas within the State to tie-in with interstate pipelines that transport their product farther eastward.

Lone Star's natural gas vehicle (NGV) business grew in 1993, primarily due to increasing national and local level support for alternative-energy vehicles. More than 300 vehicles were converted to natural gas for other firms by a joint-venture affiliate of the ENSERCH Corporation, with a backlog at the end of 1993 of 120 units under contract to be converted.

At the end of 1993, ENSERCH was a part owner in six public NGV refueling stations that are serving more than 1,000 vehicles in the greater Dallas/Fort Worth area, with four new stations under construction. Lone Star Gas Company also plans to build seven more stations in the Dallas/Fort Worth area during 1994. 
Table 24. Lone Star Gas Co., Natural Gas Data, 1993 (Thousand Cubic Feet)

\begin{tabular}{|c|c|c|}
\hline \multirow{2}{*}{ Supply/Disposition } & Lone Star Gas Co. & National Pipeline Co. \\
\hline & Texas & Oklahoma \\
\hline \multicolumn{3}{|l|}{ SUPPLY } \\
\hline \multicolumn{3}{|l|}{$\begin{array}{l}\text { Produced Onsystem } \\
\text { Receipls }\end{array}$} \\
\hline Purchases & $178,742,204$ & 0 \\
\hline 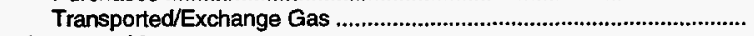 & $405,780,861$ & 812,479 \\
\hline \multicolumn{3}{|l|}{ Interstate Movements } \\
\hline 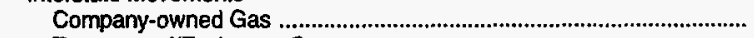 & 0 & 0 \\
\hline 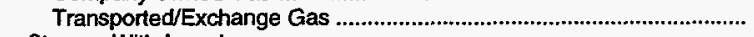 & 0 & 0 \\
\hline 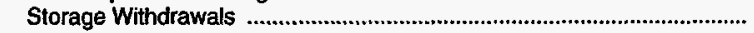 & $26,961,110$ & 0 \\
\hline Received from Storage Operators & 0 & 0 \\
\hline 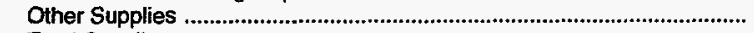 & 2,940 & 0 \\
\hline 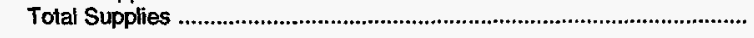 & $611,487,115$ & 812,479 \\
\hline \multicolumn{3}{|l|}{ DISPOSITION } \\
\hline Lease and Plant Use & $5,215,660$ & 0 \\
\hline Retumed to Formations & 0 & 0 \\
\hline \multicolumn{3}{|l|}{ Interstate Movements } \\
\hline 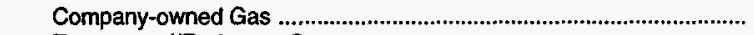 & 0 & 0 \\
\hline 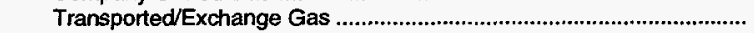 & $10,995,986$ & 0 \\
\hline 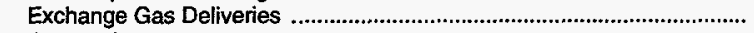 & $31,586,539$ & 0 \\
\hline Storage Injections & $26,476,573$ & 0 \\
\hline \multicolumn{3}{|l|}{ Deliveries of Company-owned Gas } \\
\hline To Storage Operators & 0 & 0 \\
\hline To Other Pipelines & 0 & 0 \\
\hline 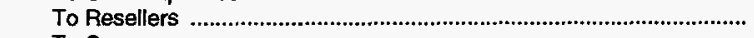 & 15,492 & 0 \\
\hline \multicolumn{3}{|l|}{ To Consumers } \\
\hline Residential & $100,491,297$ & 0 \\
\hline Commercial & $39,521,600$ & 0 \\
\hline Industriat & $13,833,316$ & 0 \\
\hline Electric Utility & $12,310,220$ & 0 \\
\hline 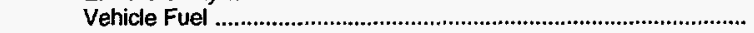 & 0 & 0 \\
\hline \multicolumn{3}{|l|}{ Transported for the Account of Others } \\
\hline 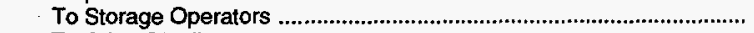 & 0 & 0 \\
\hline 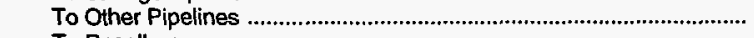 & 0 & 812,479 \\
\hline 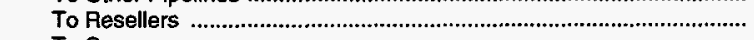 & 0 & 0 \\
\hline \multicolumn{3}{|l|}{ To Consumers } \\
\hline Residential & 0 & 0 \\
\hline 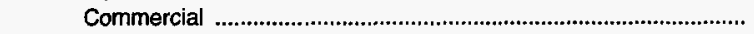 & $1,235,482$ & 0 \\
\hline 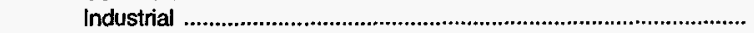 & $331,839,157$ & 0 \\
\hline 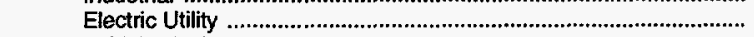 & $41,224,335$ & 0 \\
\hline 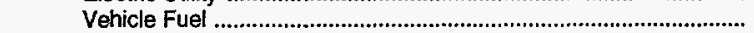 & 0 & 0 \\
\hline 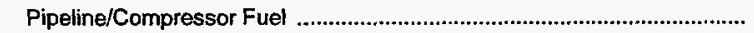 & 0 & 0 \\
\hline Other Disposition & 576,655 & 0 \\
\hline 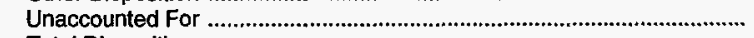 & $-3,835,197$ & 0 \\
\hline Total Disposition & $611,487,115$ & 812,479 \\
\hline
\end{tabular}

Source: Energy Information Administration (EIA), Form EIA-176, "Annual Report of Natural and Supplemental Gas Supply and Disposition." 



\section{Michigan Consolidated Gas Co.}

Michigan Consolidated Gas Company (MichCon), Citizens Gas Fuel Company, and MCN Investment Corporation are subsidiaries of MCN Corporation. MichCon, one of the largest natural gas distributors in the United States and the largest in Michigan, is a distribution, transmission, and storage company. MichCon's service area includes more than 500 communities and more than 1.1 million customers. Citizens is a natural gas utility company located in Michigan serving approximately 12,000 customers. Citizens is served by one intrastate pipeline company, Westside Pipeline, and two interstate pipeline companies, Panhandle Eastern Pipeline and ANR. MCN Investment Corporation owns subsidiaries involved in gas services, computer operations services, and natural gas technology.
MichCon's distribution system includes more than 14,000 miles of distribution mains, more than 1 million service lines, and more than 1 million active meters. Twenty-five hundred miles of pipeline are owned by ${ }^{3}$ MichCon for natural gas distribution and to connect its storage fields to supply sources and market areas. Properties associated with five storage fields owned by MichCon have a combined working storage capacity of approximately 130 billion cubic feet.

MichCon plans to build and operate a 59-mile pipeline north of Detroit, and has filed a request with the Michigan Public Service Commission for its approval of the pipeline project. The pipeline, to be called Milford-Belle River

Figure 19. Michigan Consolidated Gas Co., 1993

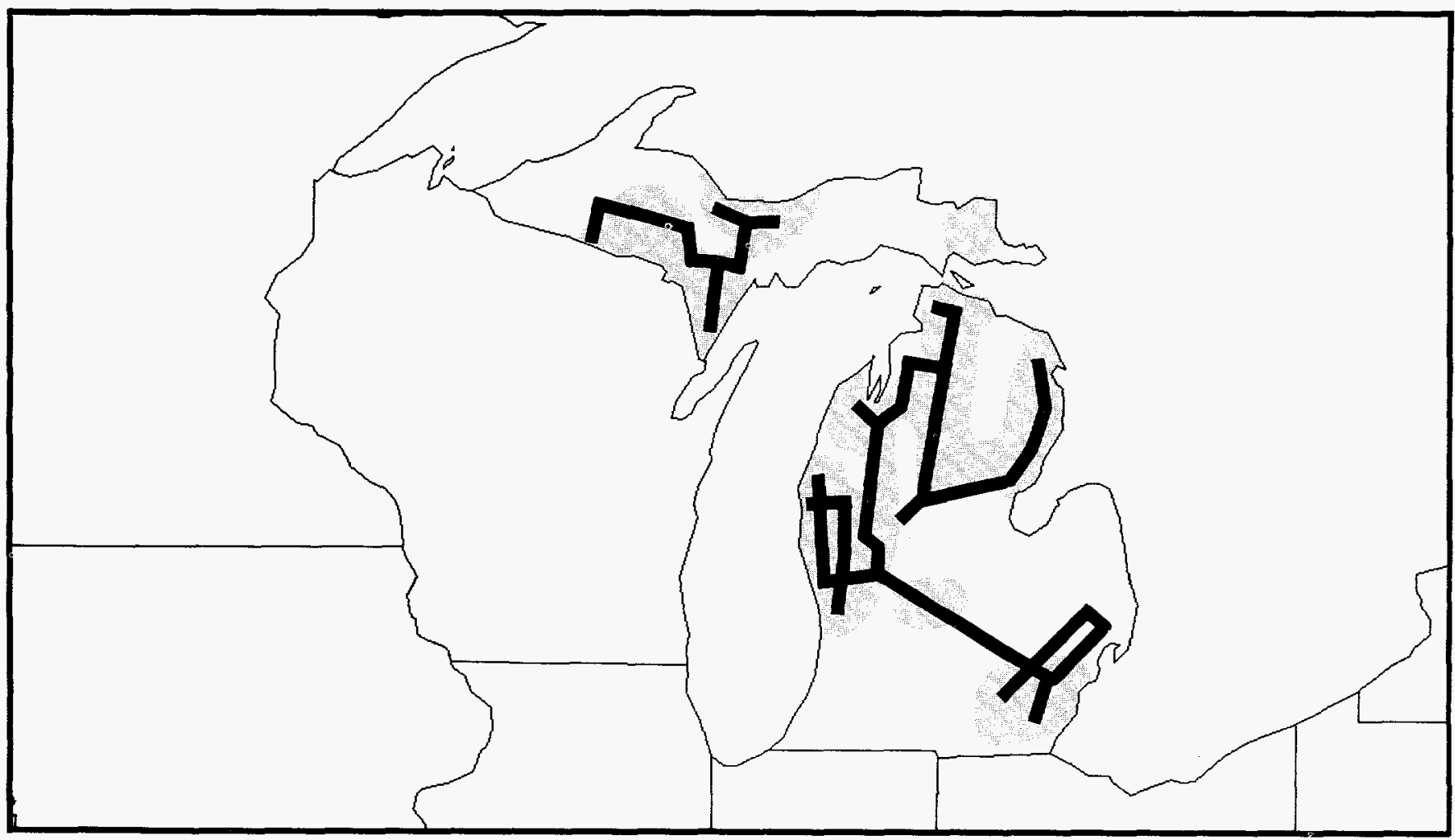

Source: Energy Information Administration (EIA), Form ElA-176, "Annual Report of Natural and Supplemental Gas Supply and Disposition." 
Table 25. Michigan Consolidated Gas Co., Natural Gas Data, 1993 (Thousand Cubic Feet)

\begin{tabular}{|c|c|c|}
\hline \multirow{2}{*}{ Supply/Disposition } & \multirow{2}{*}{$\begin{array}{c}\text { MichCon Gas Co. } \\
\text { Michigan } \\
\end{array}$} & \multirow{2}{*}{$\begin{array}{c}\text { Citizens Gas Fuel Co. } \\
\text { Michigan }\end{array}$} \\
\hline & & \\
\hline \multicolumn{3}{|l|}{ SUPPLY } \\
\hline $\begin{array}{l}\text { Produced Onsystem } \\
\text { Receipts }\end{array}$ & $1,355,205$ & 0 \\
\hline 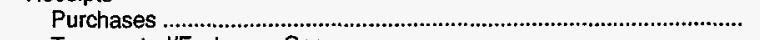 & $157,094,817$ & $3,626,319$ \\
\hline 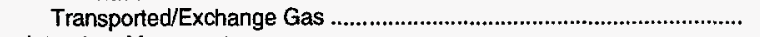 & $417,492,726$ & 230,385 \\
\hline \multicolumn{3}{|l|}{ Interstate Movements } \\
\hline Company-owned Gas & 0 & 0 \\
\hline 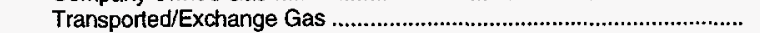 & $27,920,155$ & 0 \\
\hline 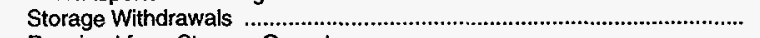 & $113,081,780$ & 0 \\
\hline 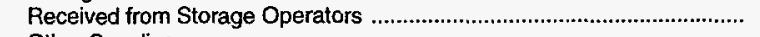 & $33,379,061$ & 0 \\
\hline 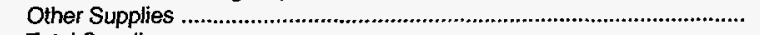 & 0 & 999 \\
\hline 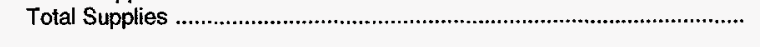 & $750,323,744$ & $3,857,703$ \\
\hline \multicolumn{3}{|l|}{ DISPOSITION } \\
\hline Lease and Plant Use & $5,664,311$ & 0 \\
\hline 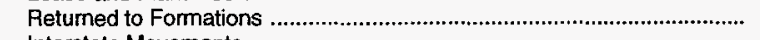 & 0 & 0 \\
\hline \multicolumn{3}{|l|}{ Interstate Movements } \\
\hline 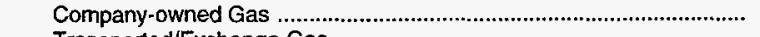 & 0 & 0 \\
\hline 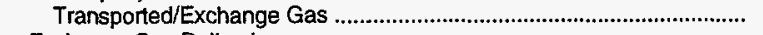 & 0 & 0 \\
\hline 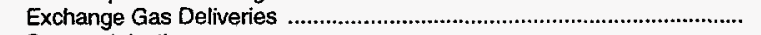 & 0 & 0 \\
\hline 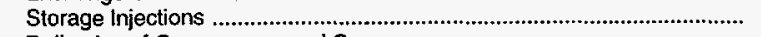 & $113,206,513$ & 0 \\
\hline \multicolumn{3}{|l|}{ Deliveries of Company-owned Gas } \\
\hline 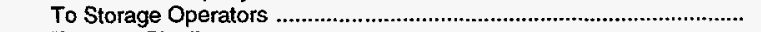 & $3,242,177$ & 0 \\
\hline 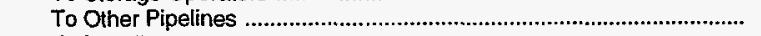 & 0 & 0 \\
\hline 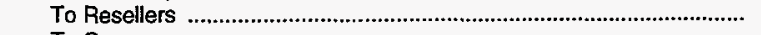 & 0 & 714,213 \\
\hline \multicolumn{3}{|l|}{ To Consumers } \\
\hline 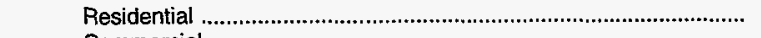 & $150,964,211$ & $1,396,348$ \\
\hline 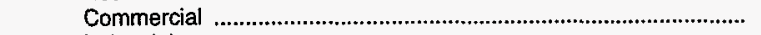 & $47,480,733$ & 603,059 \\
\hline 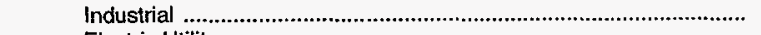 & $2,327,871$ & 895,995 \\
\hline 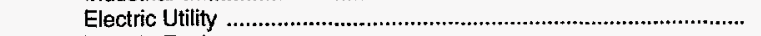 & 121,632 & 0 \\
\hline 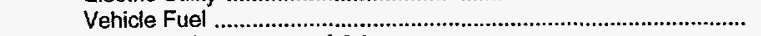 & 0 & 0 \\
\hline \multicolumn{3}{|l|}{ Transported for the Account of Others } \\
\hline 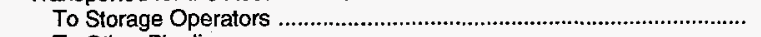 & $27,875,981$ & 0 \\
\hline 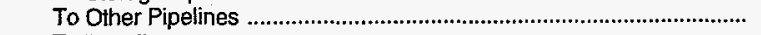 & $268,705,556$ & 0 \\
\hline 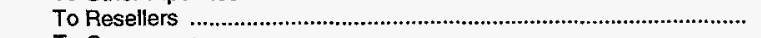 & 0 & 0 \\
\hline \multicolumn{3}{|l|}{ To Consumers } \\
\hline 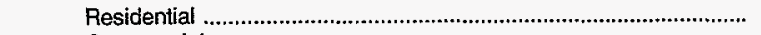 & $1,978,710$ & 0 \\
\hline 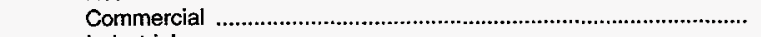 & $37,612,549$ & 0 \\
\hline Industrial & $87,003,615$ & 233,474 \\
\hline 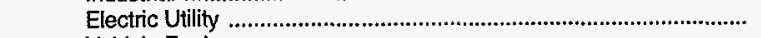 & $1,111,305$ & 0 \\
\hline Vehicle Fuel & 0 & 0 \\
\hline 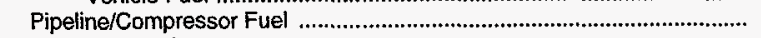 & $2,506,070$ & 0 \\
\hline Other Disposition & 0 & 0 \\
\hline 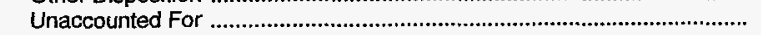 & 522,510 & 14,614 \\
\hline Total Disposition & $750,323,744$ & $3,857,703$ \\
\hline
\end{tabular}

Source: Energy Information Administration (EIA), Form EIA-176, "Annual Report of Natural and Supplemental Gas Supply and Disposition."

Loop, will be constructed between Oakland County and St. Clair County, Michigan. The new pipeline will be constructed parallel to an existing pipeline. The MilfordBelle River Loop will serve as a back-up for the existing line.

MichCon and CMS Gas Transmission plan to build a 25-mile pipeline and treating facilities to serve Michigan's Antrim gas production. This project, subject to approval by the Michigan Public Service Commission, will make Antrim gas accessible to Consumers Power, Great Lakes Transmission, ANR Pipeline, and MichCon's transmission system.
An application has also been filed to construct a pipeline to serve a natural gas cogeneration plant owned by Dow Chemical's Ludington, Michigan complex. The proposed pipeline will be capable of transporting approximately 33 million cubic feet per day.

Michcon has developed a new adsorbed natural gas technology that stores natural gas in containers at one-tenth the conventional pressure. This technology was successfully tested on a fleet of 80 golf cars at a local golf course. Michcon is now exploring the application of this technology in both on- and off-road vehicles. 


\section{MidCon Corp.}

MidCon Corporation is a subsidiary of Occidental Petroleum Corporation, an energy resource company with oil, gas, and coal operations. MidCon owns and operates nearly 1,700 miles of pipeline located in 12 States. MidCon is also the owner of two pipeline subsidiaries: Natural Gas Pipe Line Company of America (NGPL) and MidCon Texas Pipeline.

NGPL, one of the Nation's top storage operators, owns total storage capacity of approximately 600 billion cubic feet located in producing States and in the Midwest consuming States. MidCon Texas Pipeline constructed a salt dome storage cavern, and the construction of a second salt cavern facility is near completion.
MidCon's extensive network of transmission lines and huge storage capacity give the company the flexibility necessary to meet ever-changing customer needs. The company expects that increasing environmental concerns and legislation will boost natural gas consumption, particularly in the transportation and electric generation markets. Natural gas sales by the pipelines decreased in 1993 as restructuring of the industry neared completion. Increased throughput under transportation agreements more than offset the decline in company sales. MidCon Texas Pipeline and Houston Lighting and Power Company signed a sales and transportation agreement under which MidCon Texas will provide Houston Lighting with approximately 60 billion cubic feet of natural gas annually.

Figure 20. MidCon Corp., 1993

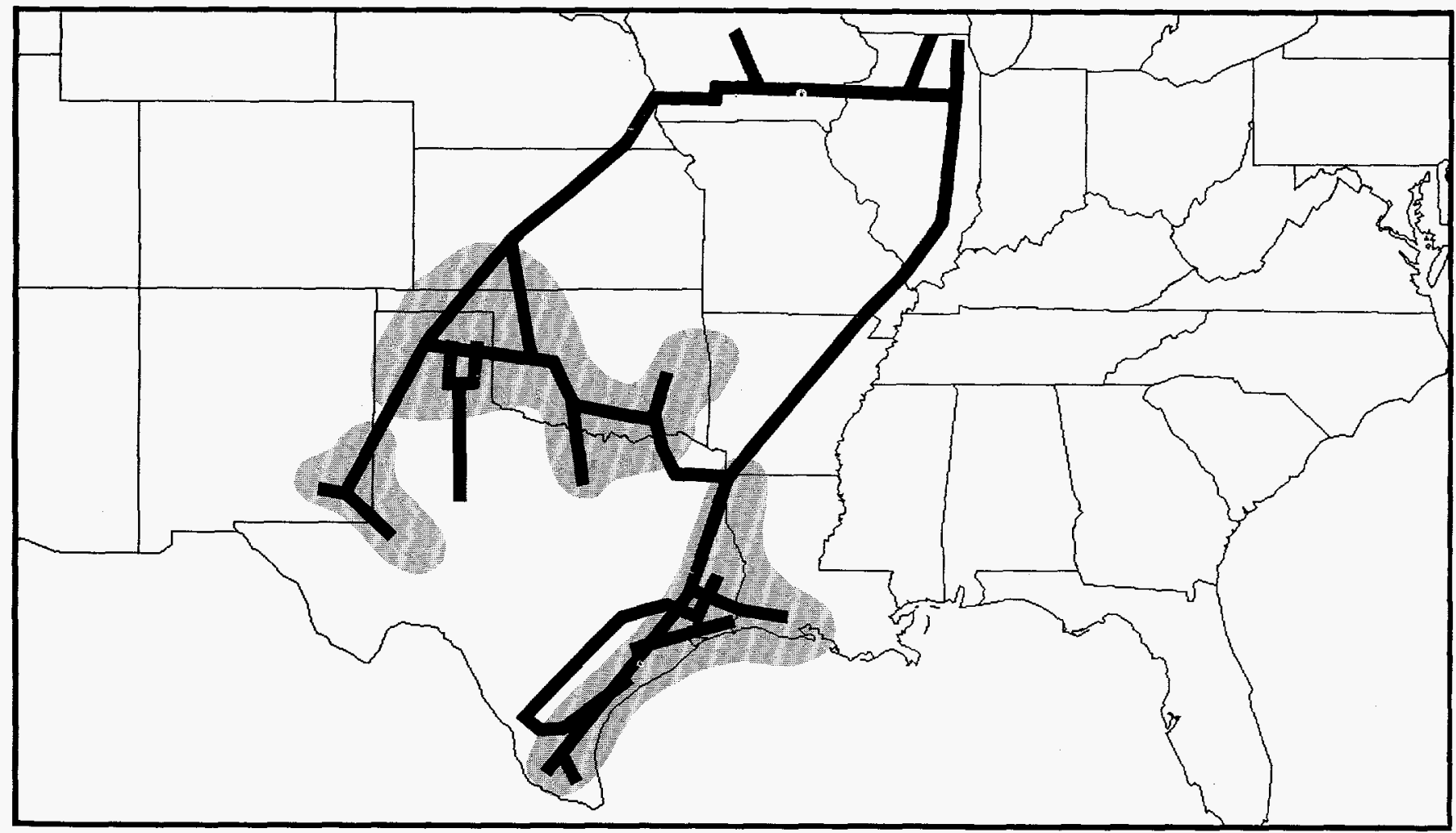

Source: Energy Information Administration (E|A), Form EIA-176, "Annual Report of Natural and Supplemental Gas Supply and Disposition." 
Table 26. MidCon Corp., Natural Gas Data, 1993

(Thousand Cubic Feet)

\begin{tabular}{|c|c|c|c|c|c|}
\hline \multirow{2}{*}{ Supply/Disposition } & \multicolumn{5}{|c|}{ Natural Gas Pipeline Co. of America } \\
\hline & Louisiana & Texas & Arkansas & Missouri & Illinois \\
\hline \multicolumn{6}{|l|}{ SUPPLY } \\
\hline $\begin{array}{l}\text { Produced Onsystem } \\
\text { Receipts }\end{array}$ & 0 & 0 & 0 & 0 & 0 \\
\hline 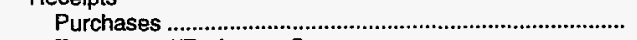 & $12,698,227$ & $50,487,326$ & 217,901 & 0 & $4,921,757$ \\
\hline 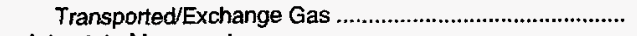 & $276,406,992$ & $563,898,650$ & $58,117,752$ & 64,462 & $177,130,913$ \\
\hline \multicolumn{6}{|l|}{ Interstate Movements } \\
\hline Company-owned Gas & 0 & 0 & 0 & 0 & 0 \\
\hline 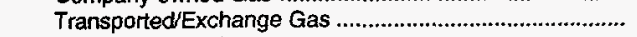 & $68,110,337$ & $228,758,314$ & $315,307,538$ & $358,988,591$ & $763,660,388$ \\
\hline 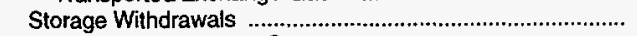 & 0 & $57,082,868$ & 0 & 0 & $45,937,302$ \\
\hline 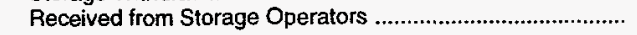 & 0 & $7,692,649$ & 0 & 0 & 0 \\
\hline 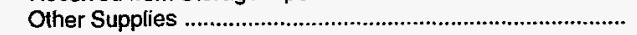 & 0 & 0 & 0 & 0 & 0 \\
\hline 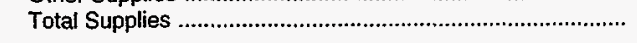 & $357,215,556$ & $907,919,807$ & $373,643,191$ & $359,053,053$ & $991,650,360$ \\
\hline \multicolumn{6}{|l|}{ DISPOSITION } \\
\hline Lease and Plant Use & 0 & $3,997,215$ & 88,409 & 0 & 0 \\
\hline 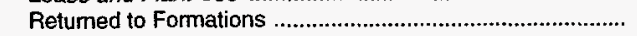 & 0 & 0 & 0 & 0 & 0 \\
\hline \multicolumn{6}{|l|}{ Interstate Movements } \\
\hline 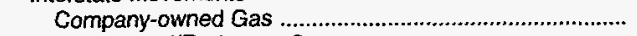 & 0 & 0 & 0 & 0 & $21,429,468$ \\
\hline 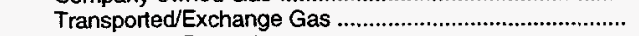 & 0 & $538,501,004$ & $358,988,591$ & $356,083,196$ & $195,381,676$ \\
\hline 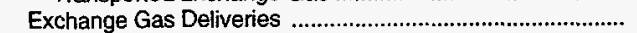 & $5,838,360$ & $33,168,423$ & $1,061,263$ & 129,735 & $100,636,494$ \\
\hline Storage Injections & 0 & $64,371,416$ & 0 & 0 & $49,706,340$ \\
\hline \multicolumn{6}{|l|}{ Deliveries of Company-owned Gas } \\
\hline 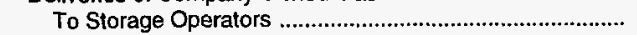 & 0 & 0 & 0 & 0 & 0 \\
\hline To Other Pipelines & $20,835,653$ & $26,788,308$ & 902,585 & 0 & $14,325,137$ \\
\hline To Resellers & 121,268 & 78,794 & 0 & 394,342 & $140,564,125$ \\
\hline \multicolumn{6}{|l|}{ To Consumers } \\
\hline 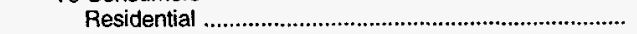 & 0 & 0 & 0 & 0 & 0 \\
\hline 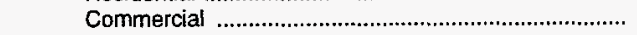 & 0 & 0 & 0 & 0 & 0 \\
\hline Industrial & 0 & 5,398 & 0 & 0 & 0 \\
\hline Electric Utility & 0 & 0 & 0 & 0 & 0 \\
\hline Vehicle Fuel & 0 & 0 & 0 & 0 & 0 \\
\hline \multicolumn{6}{|l|}{ Transported for the Account of Others } \\
\hline To Storage Operators & 0 & $11,457,356$ & 0 & 0 & 0 \\
\hline 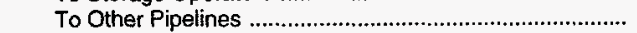 & $329,256,954$ & $213,377,690$ & $1,069,213$ & 0 & $54,061,611$ \\
\hline 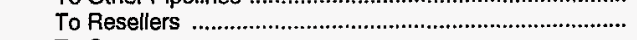 & 214,127 & $1,131,096$ & 0 & $1,265,398$ & $399,304,742$ \\
\hline \multicolumn{6}{|l|}{ To Consumers } \\
\hline 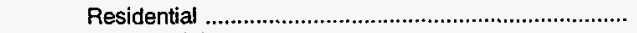 & 0 & 0 & 0 & 0 & 0 \\
\hline 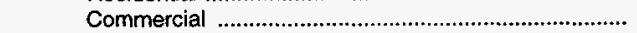 & 4,015 & 37,920 & 0 & 0 & $4,177,865$ \\
\hline 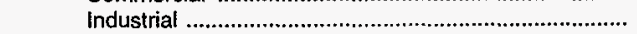 & 0 & $4,699,244$ & $7,339,880$ & 0 & $5,664,184$ \\
\hline 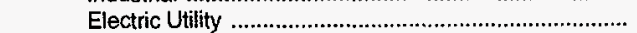 & 0 & 0 & 0 & 0 & 0 \\
\hline Vehicle Fuel & 0 & 0 & 0 & 0 & 0 \\
\hline Pipeline/Compressor Fuel & 515,020 & $5,844,368$ & $2,646,535$ & 659,813 & $2,942,438$ \\
\hline 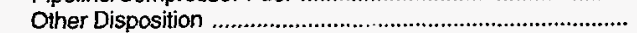 & 1,879 & 19,491 & 6,757 & 2,274 & 12,953 \\
\hline 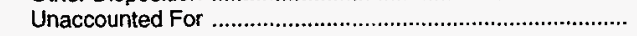 & 428,280 & $4,442,084$ & $1,539,958$ & 518,295 & $3,443,327$ \\
\hline 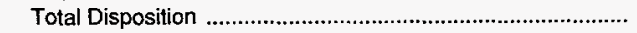 & $357,215,556$ & $907,919,807$ & $373,643,191$ & $359,053,053$ & $991,650,360$ \\
\hline
\end{tabular}

In October 1993, MidCon and a partner completed a 13.4-mile extension of the Stingray system, which extends more than 100 miles into the Gulf of Mexico. This extension provides additional capacity to transport gas from the Outer Continental Shelf to NGPL in Louisiana.

In 1994, NGPL received approval to complete the final phase of its Amarillo 1 Upgrade Program. The program, begun in 1982, will complete the replacement of NGPL's Amarillo 1 Line that extends from Texas and Oklahoma to the Chicago area. The final phase, which will be completed in 1995 or 1996, is not intended to increase delivery capacity to Chicago and its surrounding areas, but rather to replace transmission lines in the NGPL system, some of which were originally laid in the 1930's.
Under Federal Energy Regulatory Commission Order 636, MidCon reorganized into two business divisions. Under the new organization, the regulated business division manages all of MidCon's pipeline operations, including the marketing and sales of NGPL's regulated transportation and storage services, and managing volume control for the pipelines. The nonregulated business division includes MidCon Texas Pipeline, MidCon Marketing, as well as gas supply, marketing, and sales functions previously performed by NGPL.

In 1993, MidCon established two new subsidiaries. MidCon Development will explore new gas markets with an emphasis on natural gas vehicle and power generation, and MidCon Gas Products will serve markets in such areas as natural gas production, gathering, and liquids processing. 
Table 26. MidCon Corp., Natural Gas Data, 1993 (Continued)

(Thousand Cubic Feet)

\begin{tabular}{|c|c|c|c|c|c|}
\hline \multirow{2}{*}{ Supply/Disposition } & \multicolumn{5}{|c|}{ Natural Gas Pipeline Co. of America } \\
\hline & New Mexico & Oklahoma & Kansas & Nebraska & lowa \\
\hline \multicolumn{6}{|l|}{ SUPPLY } \\
\hline $\begin{array}{l}\text { Produced Onsystem } \\
\text { Receipts }\end{array}$ & & 0 & 0 & 0 & 0 \\
\hline 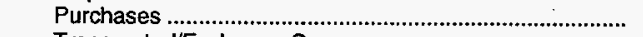 & $5,060,937$ & $18,712,876$ & 965,678 & $1,231,124$ & $4,008,658$ \\
\hline $\begin{array}{l}\text { Transported/Exchange Gas } \\
\text { Interstate Movements }\end{array}$ & $74,643,859$ & $225,613,667$ & $27,408,205$ & $92,826,956$ & $217,054,561$ \\
\hline 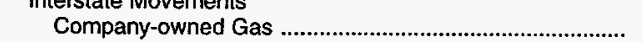 & \\
\hline 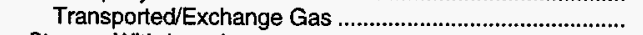 & 0 & $155,083,129$ & $179,800,974$ & $201,957,058$ & $263,623,661$ \\
\hline 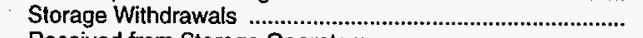 & 0 & $54,158,176$ & 0 & $\begin{array}{r}201,008,030 \\
0\end{array}$ & $\begin{array}{r}203,023,061 \\
37,976,922\end{array}$ \\
\hline 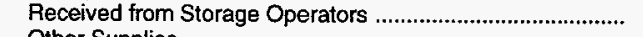 & $6,976,526$ & 0 & 0 & 0 & 0 \\
\hline 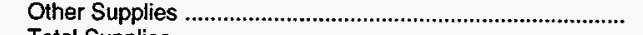 & 0 & 0 & 0 & 0 & 0 \\
\hline 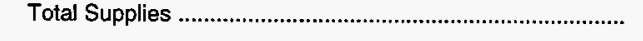 & $86,681,322$ & $453,567,848$ & $208,174,857$ & $296,015,138$ & $522,663,802$ \\
\hline \multicolumn{6}{|l|}{ DISPOSITION } \\
\hline Lease and Plant Use & 0 & 0 & 0 & 0 & 0 \\
\hline 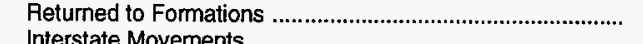 & 0 & 0 & 0 & 0 & 0 \\
\hline \\
\hline 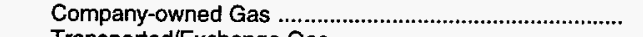 & 0 & 0 & 0 & 0 & 0 \\
\hline 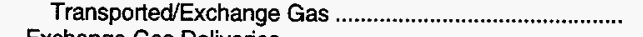 & $57,564,730$ & $350,994,558$ & $201,957,058$ & $263,623,661$ & $407,577,192$ \\
\hline 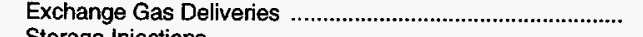 & $2,590,041$ & $5,799,203$ & 559,086 & 654,455 & $8,232,643$ \\
\hline \multicolumn{4}{|l|}{ Deliveries of Company-owned Gas } & 0 & $37,780,518$ \\
\hline 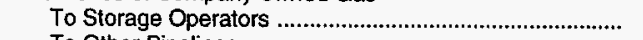 & 0 & 0 & 0 & 0 & 0 \\
\hline 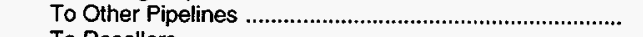 & $1,478,248$ & 329,597 & 395,303 & $9,714,871$ & $1,755,000$ \\
\hline 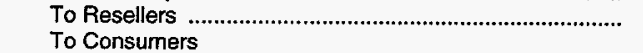 & 0 & 22,503 & 122,900 & 602,645 & $6,524,204$ \\
\hline 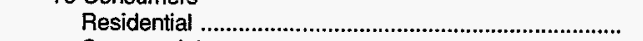 & 0 & 0 & 0 & & 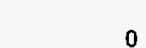 \\
\hline 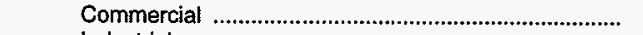 & 0 & 0 & 0 & 0 & 0 \\
\hline 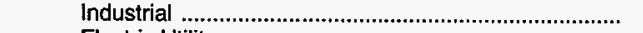 & 0 & 0 & 0 & 0 & 7,400 \\
\hline 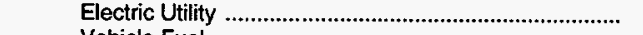 & 0 & 0 & 77,580 & 0 & 0 \\
\hline Vehicle Fuel & 0 & 0 & 0 & 0 & 0 \\
\hline \multicolumn{6}{|l|}{ Transported for the Account of Others } \\
\hline 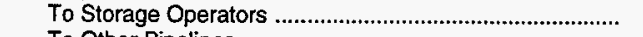 & $2,477,329$ & 0 & 0 & 0 & 0 \\
\hline 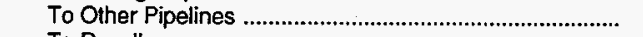 & $19,670,769$ & $38,042,161$ & $2,058,139$ & $6,442,610$ & $16,097,312$ \\
\hline 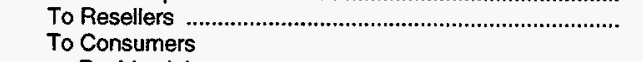 & $1,709,698$ & 4,050 & To Consumers & $8,193,034$ & $34,829,434$ \\
\hline Residential & 0 & 0 & 0 & 0 & 0 \\
\hline 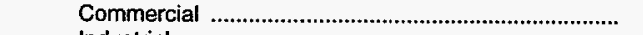 & 0 & 0 & 0 & 0 & 0 \\
\hline 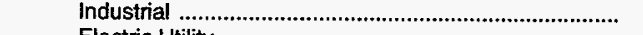 & 580,591 & 0 & 0 & $6,097,605$ & $5,783,961$ \\
\hline 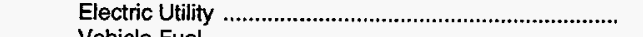 & 0 & 667,093 & 68,129 & 0 & 0 \\
\hline Vehicle Fuel & 0 & 0 & 0 & 0 & 0 \\
\hline 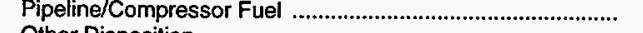 & 186,654 & $1,131,240$ & $1,269,184$ & 202,421 & $2,708,144$ \\
\hline 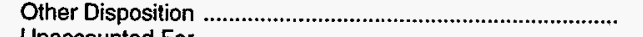 & 1,849 & 5,453 & 6,754 & 2,114 & 5,976 \\
\hline 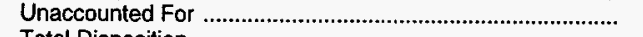 & 421,413 & 507,655 & $1,539,361$ & 481,722 & $1,362,018$ \\
\hline Total Disposition & $86,681,322$ & $453,567,848$ & $208,174,857$ & $296,015,138$ & $522,663,802$ \\
\hline
\end{tabular}


Table 26. MidCon Corp., Natural Gas Data, 1993 (Continued) (Thousand Cubic Feet)

\begin{tabular}{|c|c|c|c|c|c|}
\hline \multirow{2}{*}{ Supply/Disposition } & \multirow{2}{*}{$\begin{array}{c}\begin{array}{c}\text { Natural Gas } \\
\text { Pipeline Co. of } \\
\text { America }\end{array} \\
\text { Wisconsin }\end{array}$} & \multirow{2}{*}{$\begin{array}{c}\text { MidCon Texas } \\
\text { Pipeline Corp. } \\
\text { Texas }\end{array}$} & \multicolumn{3}{|c|}{ Trailblazer Pipeline System } \\
\hline & & & Colorado & Wyoming & Nebraska \\
\hline \multicolumn{6}{|l|}{ SUPPLY } \\
\hline $\begin{array}{l}\text { Produced Onsystem } \\
\text { Receipts }\end{array}$ & 0 & 0 & 0 & 0 & 0 \\
\hline 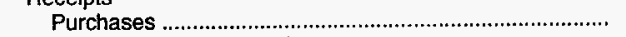 & 0 & $144,172,961$ & 0 & 0 & 0 \\
\hline 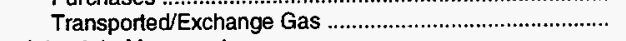 & 0 & $512,861,061$ & $107,244,681$ & 0 & $4,027,838$ \\
\hline & & & & & \\
\hline 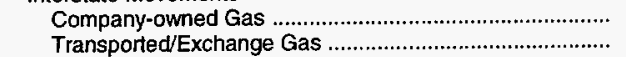 & 0 & 0 & 0 & $\begin{array}{r}0 \\
92432436\end{array}$ & $\begin{array}{r}0 \\
194858.157\end{array}$ \\
\hline & 0 & 0 & $92,396,511$ & $\begin{array}{r}92,432,436 \\
0\end{array}$ & $\begin{array}{r}194,858,157 \\
0\end{array}$ \\
\hline $\begin{array}{l}\text { Storage Withdrawals } \\
\text { Received from Storage Operators }\end{array}$ & $\stackrel{0}{0}$ & $\begin{array}{l}0 \\
0\end{array}$ & $\begin{array}{l}0 \\
0\end{array}$ & 0 & $\begin{array}{l}0 \\
0\end{array}$ \\
\hline $\begin{array}{l}\text { Received from Storage Operators } \\
\text { Other Supplies }\end{array}$ & $\begin{array}{l}0 \\
0\end{array}$ & $\begin{array}{l}0 \\
0\end{array}$ & 2,936 & 146 & 8,018 \\
\hline Other Supplies & $\begin{array}{l}0 \\
0\end{array}$ & $657,034,022$ & $199,644,128$ & $92,432,582$ & $198,894,013$ \\
\hline \multicolumn{6}{|l|}{ DISPOSITION } \\
\hline 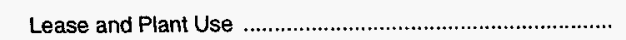 & 0 & 189,283 & 0 & 0 & 0 \\
\hline & 0 & 0 & 0 & 0 & 0 \\
\hline 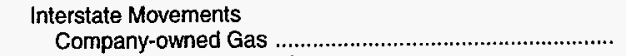 & & & 0 & 0 & \\
\hline $\begin{array}{l}\text { Company-owned Gas } \\
\text { Transported/Exchange Gas }\end{array}$ & $\begin{array}{l}0 \\
0\end{array}$ & $\begin{array}{l}0 \\
0\end{array}$ & $194,864,469$ & $92,426,124$ & $92,396,511$ \\
\hline $\begin{array}{l}\text { Transported/Exchange Gas } \\
\text { Exchange Gas Deliveries }\end{array}$ & $\begin{array}{l}0 \\
0\end{array}$ & $244,691,367$ & $2,484,888$ & 0 & $\begin{array}{r}92,396,511 \\
227,652\end{array}$ \\
\hline 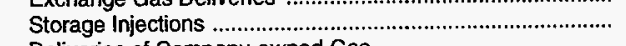 & 0 & 0 & 0 & 0 & 0 \\
\hline \multicolumn{6}{|l|}{$\begin{array}{l}\text { Storage Injections } \\
\text { Deliveries of Company-owned Gas }\end{array}$} \\
\hline To Storage Operators & 0 & 0 & 0 & 0 & 0 \\
\hline 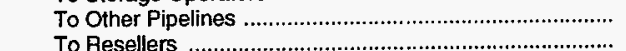 & 0 & $13,510,669$ & 0 & 0 & 0 \\
\hline 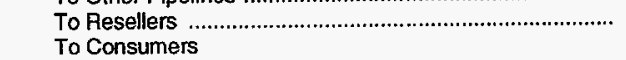 & 0 & $13,082,454$ & 0 & 0 & 0 \\
\hline 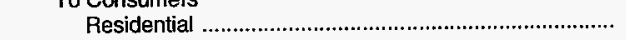 & 0 & 0 & 0 & 0 & 0 \\
\hline 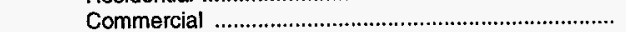 & 0 & 0 & 0 & 0 & 0 \\
\hline 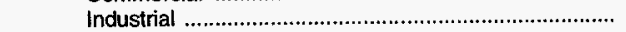 & 0 & $120,272,237$ & 0 & 0 & 0 \\
\hline 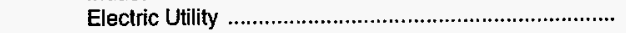 & 0 & 0 & 0 & 0 & 0 \\
\hline Vehicle Fuel & 0 & 0 & 0 & 0 & 0 \\
\hline \multicolumn{6}{|l|}{$\begin{array}{l}\text { Vehicle Fuel } \\
\text { Transported for the Account of Others }\end{array}$} \\
\hline 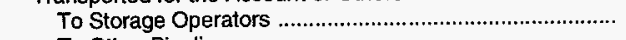 & 0 & 0 & 0 & 0 & 0 \\
\hline 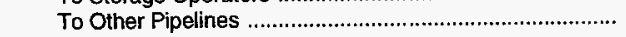 & 0 & $25,874,317$ & $2,164,804$ & 0 & $105,639,761$ \\
\hline 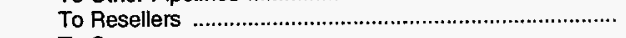 & 0 & $34,654,236$ & 0 & 0 & 275,254 \\
\hline \multicolumn{5}{|l|}{$\begin{array}{l}\text { To Resellers } \\
\text { To Consumers }\end{array}$} & 0 \\
\hline 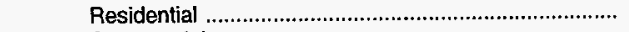 & 0 & $\begin{array}{l}0 \\
0\end{array}$ & 0 & 0 & 0 \\
\hline 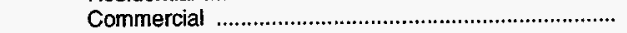 & 0 & & 0 & 0 & $\begin{array}{l}0 \\
0\end{array}$ \\
\hline 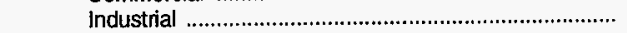 & 0 & $\begin{array}{r}162,361,567 \\
50,706,775\end{array}$ & $\begin{array}{l}0 \\
0\end{array}$ & $\begin{array}{l}0 \\
0\end{array}$ & $\begin{array}{l}0 \\
0\end{array}$ \\
\hline 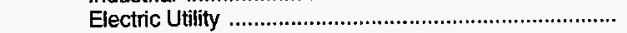 & 0 & $\begin{array}{r}50,706,775 \\
0\end{array}$ & $\begin{array}{l}0 \\
0\end{array}$ & $\begin{array}{l}0 \\
0\end{array}$ & $\begin{array}{l}0 \\
0\end{array}$ \\
\hline Vehicle Fuel & 0 & & & & $\begin{array}{r}0 \\
1526\end{array}$ \\
\hline 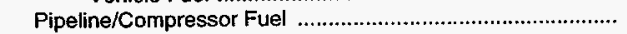 & 0 & $2,231,646$ & 559 & 28 & 1,526 \\
\hline Other Disposition & 0 & 0 & 0 & 0 & 0 \\
\hline 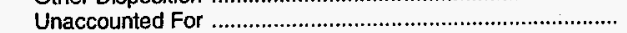 & 0 & $-10,540,529$ & 129,408 & 6,430 & 353,309 \\
\hline 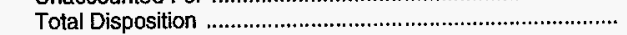 & 0 & $657,034,022$ & $199,644,128$ & $92,432,582$ & $198,894,013$ \\
\hline
\end{tabular}


Table 26. MidCon Corp., Natural Gas Data, 1993 (Continued) (Thousand Cubic Feet)

\begin{tabular}{|c|c|c|c|c|c|}
\hline \multirow{2}{*}{ Supply/Disposition } & \multicolumn{2}{|c|}{ Stingray Pipeline Co. } & \multirow{2}{*}{$\frac{\begin{array}{c}\text { Texas Industrial } \\
\text { Energy Co. }\end{array}}{\text { Texas }}$} & \multirow{2}{*}{$\begin{array}{c}\begin{array}{c}\text { Palo Duro Pipeline } \\
\text { Co. }\end{array} \\
\text { Texas }\end{array}$} & \multirow{2}{*}{$\begin{array}{c}\begin{array}{c}\text { Kansas Gas Supply } \\
\text { Corp. }\end{array} \\
\text { Kansas }\end{array}$} \\
\hline & Texas & Louisiana & & & \\
\hline \multicolumn{6}{|l|}{ SUPPLY } \\
\hline $\begin{array}{l}\text { Produced Onsystem } \\
\text { Receipts }\end{array}$ & 0 & 0 & 0 & 0 & 0 \\
\hline Purchases & 0 & 0 & 0 & $1,000,105$ & $14,161,906$ \\
\hline 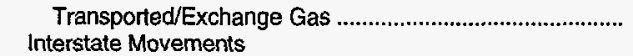 & $7,373,782$ & $221,785,261$ & $20,415,121$ & $5,296,069$ & $6,239,632$ \\
\hline Company-owned Gas & 0 & 0 & 0 & 0 & 0 \\
\hline 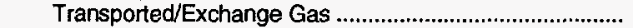 & 0 & $7,375,022$ & 0 & 0 & 0 \\
\hline 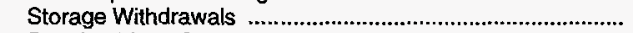 & 0 & 0 & 0 & 0 & 0 \\
\hline Received from Storage Operators & 0 & 0 & 0 & 0 & 0 \\
\hline 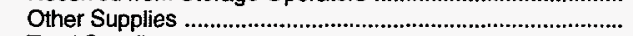 & 27 & 35,473 & 0 & 0 & 0 \\
\hline 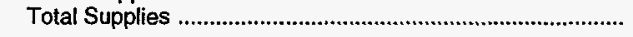 & $7,373,809$ & $229,195,756$ & $20,415,121$ & $6,296,174$ & $20,401,538$ \\
\hline \multicolumn{6}{|l|}{ DISPOSITION } \\
\hline Lease and Plant Use & 0 & $1,743,046$ & 0 & 0 & $2,056,085$ \\
\hline 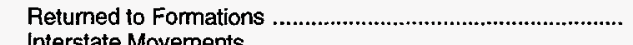 & 0 & 0 & 0 & 0 & 0 \\
\hline \multicolumn{6}{|l|}{ Interstate Movements } \\
\hline 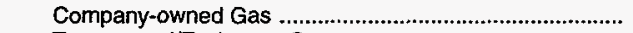 & 0 & 0 & 0 & 0 & 0 \\
\hline 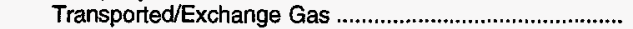 & $7,375,022$ & 0 & 0 & 0 & 0 \\
\hline 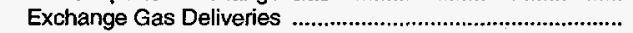 & 0 & 0 & 0 & 310,866 & 0 \\
\hline Storage Injections & 0 & 0 & 0 & 0 & 0 \\
\hline \multicolumn{6}{|l|}{ Deliveries of Company-owned Gas } \\
\hline To Storage Operators & 0 & 0 & 0 & 0 & 0 \\
\hline 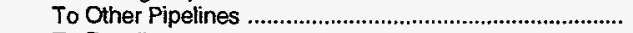 & 0 & 0 & 0 & 0 & 0 \\
\hline 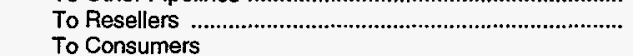 & 0 & 0 & 0 & 0 & $3,229,716$ \\
\hline 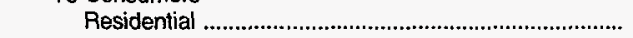 & 0 & 0 & 0 & 0 & 16,242 \\
\hline Commercial & 0 & 0 & 0 & 0 & 243,812 \\
\hline Industrial & 0 & 0 & 0 & 23,979 & $2,357,794$ \\
\hline 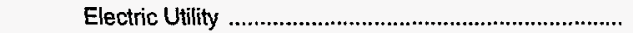 & 0 & 0 & 0 & 0 & $4,771,479$ \\
\hline Vehicle Fuel & 0 & 0 & 0 & 0 & 0 \\
\hline \multicolumn{6}{|l|}{ Transported for the Account of Others } \\
\hline 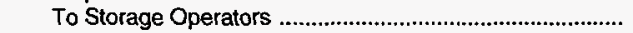 & 0 & 0 & 0 & 0 & 0 \\
\hline To Other Pipelines & 0 & $228,002,630$ & 0 & $5,825,282$ & $6,876,004$ \\
\hline $\begin{array}{l}\text { To Resellers } \\
\text { To Consumers }\end{array}$ & 0 & 0 & 0 & 0 & 0 \\
\hline 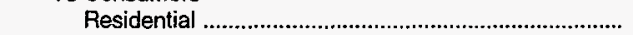 & 0 & 0 & 0 & 0 & 0 \\
\hline 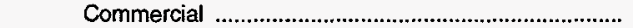 & 0 & 0 & 0 & 0 & 0 \\
\hline 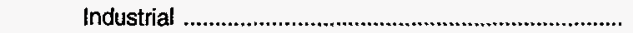 & 0 & 0 & $20,415,121$ & 0 & 0 \\
\hline 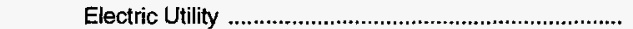 & 0 & 0 & 0 & 0 & 0 \\
\hline Vehicle Fuel & 0 & 0 & 0 & 0 & 0 \\
\hline 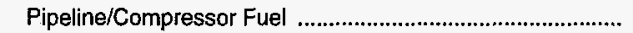 & 0 & $1,041,076$ & 0 & 30,043 & 496,824 \\
\hline 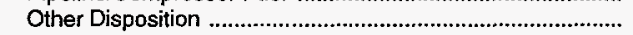 & 0 & 0 & 0 & 0 & 0 \\
\hline 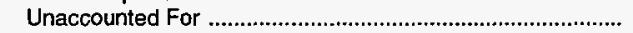 & $-1,213$ & $-1,590,996$ & 0 & 106,004 & 353,582 \\
\hline Total Disposition & $7,373,809$ & $229,195,756$ & $20,415,121$ & $6,296,174$ & $20,401,538$ \\
\hline
\end{tabular}

Source: Energy Information Administration (EIA), Form EIA-176, "Annual Report of Natural and Supplemental Gas Supply and Disposition." 
Table 27. MidCon Corp., Interstate Flows of Natural Gas, 1993

(Thousand Cubic Feet)

\begin{tabular}{|c|c|}
\hline Company and State & Volume \\
\hline \multicolumn{2}{|l|}{ Natural Gas Pipeline Co. of America } \\
\hline Arkansas & $315,307,538$ \\
\hline 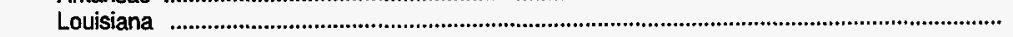 & $68,110,337$ \\
\hline Oklahoma & $155,083,129$ \\
\hline \multicolumn{2}{|l|}{ Arkansas To } \\
\hline 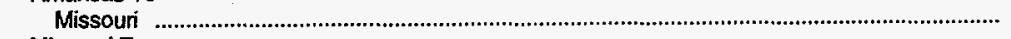 & $358,988,591$ \\
\hline \multicolumn{2}{|l|}{ Missouri To } \\
\hline 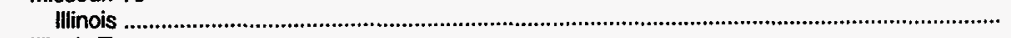 & $356,083,196$ \\
\hline \multicolumn{2}{|l|}{ Illinois To } \\
\hline 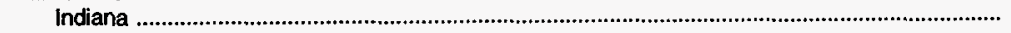 & $195,217,538$ \\
\hline 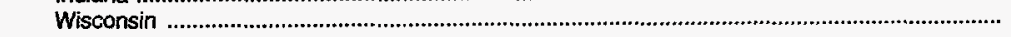 & $21,593,606$ \\
\hline \multicolumn{2}{|l|}{ New Mexico To } \\
\hline Texas & $57,564,730$ \\
\hline \multicolumn{2}{|l|}{ Oklahoma To } \\
\hline 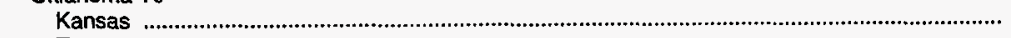 & $179,800,974$ \\
\hline 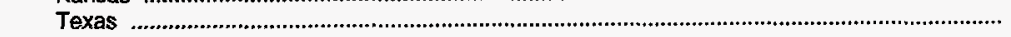 & $171,193,584$ \\
\hline \multicolumn{2}{|l|}{ Kansas To } \\
\hline Nebraska & $201,957,058$ \\
\hline \multicolumn{2}{|l|}{ Nebraska To } \\
\hline lowa & $263,623,661$ \\
\hline \multicolumn{2}{|l|}{ lowa To } \\
\hline \multicolumn{2}{|l|}{ 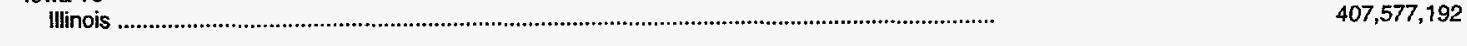 } \\
\hline \multicolumn{2}{|l|}{ Trailblazer Pipeline System } \\
\hline \multicolumn{2}{|l|}{ Colorado To } \\
\hline Nebraska & $102,432,033$ \\
\hline 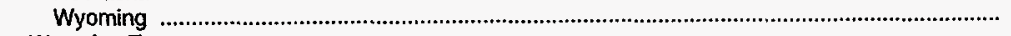 & $92,432,436$ \\
\hline \multicolumn{2}{|l|}{ Wyoming To } \\
\hline Nebraska & $92,426,124$ \\
\hline \multicolumn{2}{|l|}{ Nebraska To } \\
\hline 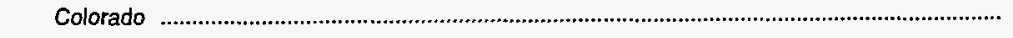 & $92,396,511$ \\
\hline \multicolumn{2}{|l|}{$\begin{array}{l}\text { Stingray Pipeline Co. } \\
\text { Texas To }\end{array}$} \\
\hline $\begin{array}{l}\text { Texas To } \\
\text { Louisiana }\end{array}$ & $7,375,022$ \\
\hline
\end{tabular}

Source: Energy Information Administration (EIA), Form EIA-176, "Annual Report of Natural and Supplemental Gas Supply and Disposition." 


\section{National Fuel Gas Co.}

National Fuel Gas Company (NFGC) is an integrated natural gas company with subsidiaries engaged in exploration, production, interstate pipeline operations, underground storage, and utility services. NFGC's exploration and production operations reached record levels in 1993, spurred primarily by the success of a subsidiary, Seneca Resources Corporation. Seneca drilled wells in the Gulf of Mexico as well as in central Texas where horizontal drilling through the Austin Chalk Trend was successful. Production increased to 19.9 billion cubic feet in 1993 compared to 12.1 billion cubic feet in 1992, an increase of 64 percent.

National Fuel Gas Supply Corporation (NFGSC), an NFGC subsidiary, owns and operates a 3,256-mile pipe- line network, and 27 underground natural gas storage fields. NFGSC is developing the Laurel Fields Storage Project in northwestern Pennsylvania and southwestern New York that will add 18 billion cubic feet of working storage capacity. Another NFGC subsidiary, Penn-York Energy Corporation, provides natural gas storage services to two interstate natural gas pipeline companies, and 17 nonaffiliated utilities, located in eight northwestern States.

On September 30, 1994, NFGC and Natural Gas Clearinghouse reached a final agreement for the establishment of a market area hub in north central Pennsylvania to be known as the Ellisburg-Leidy Northwestern Hub Company. The agreement establishes a general partnership

Figure 21. National Fuel Gas Co., 1993

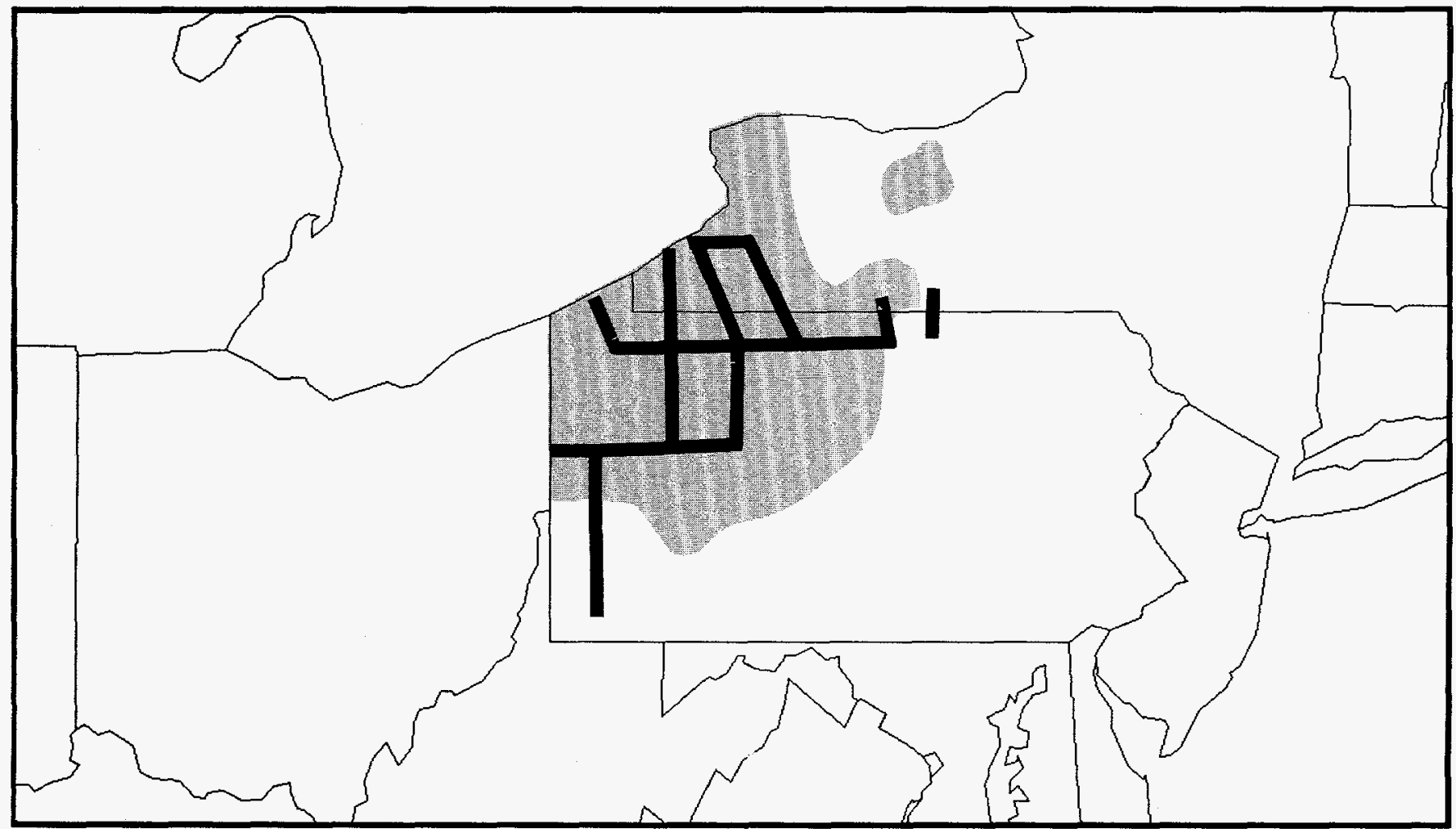

Source: Energy Information Administration (EIA), Form EIA-176, "Annual Report of Natural and Supplemental Gas Supply and Disposition." 
Table 28. National Fuel Gas Co., Natural Gas Data, 1993 (Thousand Cubic Feet)

\begin{tabular}{|c|c|c|c|c|c|c|}
\hline \multirow{2}{*}{ Supply/Disposition } & \multicolumn{2}{|c|}{ National Fuel Gas Supply Corp. } & \multicolumn{2}{|c|}{$\begin{array}{l}\text { National Fuel Gas Distribution } \\
\text { Corp. }\end{array}$} & \multicolumn{2}{|c|}{ Penn-York Energy Corp. } \\
\hline & Pennsylvania & New York & Pennsylvania & New York & New York & Pennsylvania \\
\hline \multicolumn{7}{|l|}{ SUPPLY } \\
\hline $\begin{array}{l}\text { Produced Onsystem } \\
\text { Receipts }\end{array}$ & $3,942,248$ & 233,313 & 0 & 0 & 0 & 0 \\
\hline 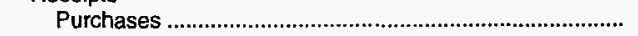 & $14,971,789$ & $6,665,796$ & $22,099,435$ & $55,826,093$ & 0 & 0 \\
\hline 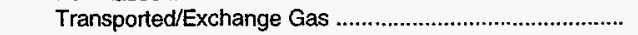 & $99,148,000$ & $119,029,055$ & $35,067,336$ & $65,936,190$ & 0 & $45,279,216$ \\
\hline \multicolumn{7}{|l|}{ Interstate Movements } \\
\hline 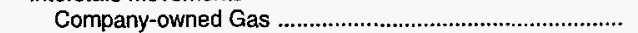 & 0 & 0 & 0 & 0 & 0 & 0 \\
\hline 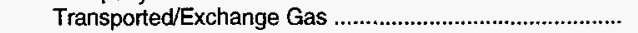 & $5,981,292$ & 0 & 0 & 0 & $13,167,320$ & $6,037,232$ \\
\hline Storage Withdrawals & $57,234,352$ & $25,105,940$ & 0 & 0 & $11,145,755$ & 0 \\
\hline 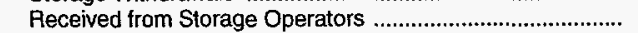 & 0 & 0 & 0 & 0 & 0 & $1,710,122$ \\
\hline 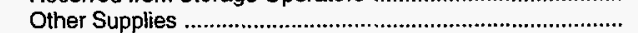 & 0 & 0 & 0 & 0 & 0 & 0 \\
\hline 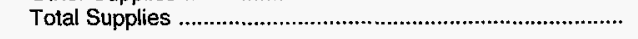 & $181,277,681$ & $151,034,104$ & $57,166,771$ & $121,762,283$ & $24,313,075$ & $53,026,570$ \\
\hline \multicolumn{7}{|l|}{ DISPOSITION } \\
\hline Lease and Plant Use & 0 & 0 & 0 & 0 & 0 & 0 \\
\hline Retumed to Formations & 0 & 0 & 0 & 0 & 0 & 0 \\
\hline \multicolumn{7}{|l|}{ Interstate Movements } \\
\hline 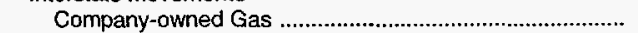 & 607,486 & 0 & 0 & 0 & 0 & 0 \\
\hline 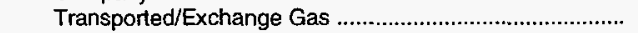 & 0 & $5,981,292$ & 0 & 0 & $6,037,232$ & $13,167,320$ \\
\hline 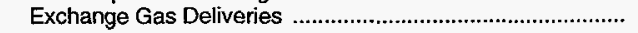 & $26,025,379$ & $3,353,427$ & 0 & 0 & 0 & 0 \\
\hline Storage Injections & $56,518,180$ & $26,297,900$ & 0 & 0 & $12,788,220$ & 0 \\
\hline \multicolumn{7}{|l|}{ Deliveries of Company-owned Gas } \\
\hline 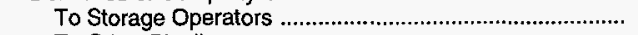 & 0 & 0 & 0 & 0 & 0 & 0 \\
\hline 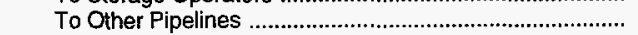 & 0 & 0 & 0 & 0 & 0 & 0 \\
\hline 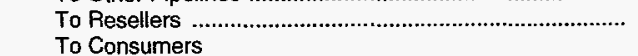 & $23,133,586$ & $52,710,988$ & 0 & 0 & 0 & 0 \\
\hline 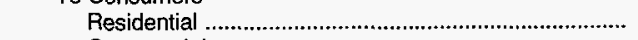 & 0 & 0 & $25,140,337$ & $60,402,474$ & 0 & 0 \\
\hline 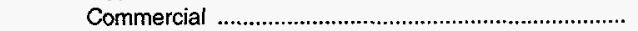 & 0 & 0 & $7,380,147$ & $17,727,982$ & 0 & 0 \\
\hline 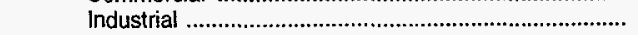 & 0 & 0 & $1,160,286$ & $4,885,401$ & 0 & 0 \\
\hline 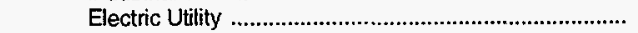 & 0 & 0 & 15,010 & 0 & 0 & 0 \\
\hline Vehicle Fuel & 0 & 0 & 0 & 0 & 0 & 0 \\
\hline \multicolumn{7}{|l|}{ Transported for the Account of Others } \\
\hline To Storage Operators & 0 & 0 & 0 & 0 & 0 & $1,710,122$ \\
\hline 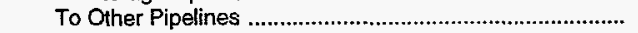 & $48,200,397$ & $4,429,994$ & 0 & 0 & $5,108,523$ & $38,149,128$ \\
\hline 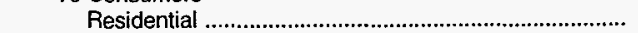 & 0 & 0 & 0 & 74,300 & 0 & 0 \\
\hline 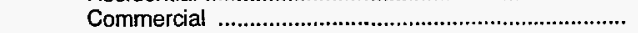 & 0 & 0 & $2,399,654$ & $7,062,193$ & 0 & 0 \\
\hline 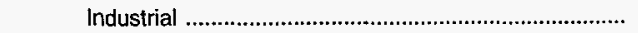 & 0 & 0 & $15,470,207$ & $24,965,198$ & 0 & 0 \\
\hline 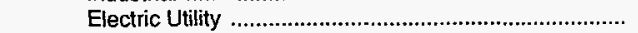 & 0 & 0 & 0 & 0 & 0 & 0 \\
\hline Vehicle Fuel & 0 & 0 & 0 & 0 & 0 & 0 \\
\hline Pipeline/Compressor Fuel & 594,711 & 98,922 & 836,828 & 908,314 & 379,100 & 0 \\
\hline Other Disposition & 0 & 0 & 0 & 0 & 0 & 0 \\
\hline 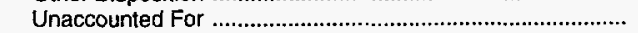 & $3,253,132$ & $2,699,665$ & $4,764,302$ & $5,736,421$ & 0 & \\
\hline 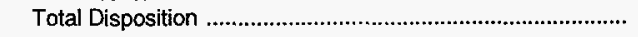 & $181,277,681$ & $151,034,104$ & $57,166,771$ & $121,762,283$ & $24,313,075$ & $53,026,570$ \\
\hline
\end{tabular}

Source: Energy Information Administration (EIA), Form EIA-176, "Annual Report of Natural and Supplemental Gas Supply and Disposition."

comprised of Hub Services, Inc., an affiliate of Natural Gas Clearinghouse, and Leidy Hub, Inc., a subsidiary of National Fuel.

National Fuel's utility operation is concentrating on some of the newer technologies such as natural gas vehicles (NGV), cogeneration, and fuel cells. The Niagara Frontier Transportation Authority in Buffalo, New York began using five dedicated natural gas buses in March
1993. National Fuel also operates more than 350 NGV's in its own fleet as well as 4 refueling stations.

In September 1993, the Oxbow Power Corporation came on line with a new 55-megawatt cogeneration facility, which sells electricity to a utility and steam to a greenhouse development. National Fuel also conducted a field demonstration of a phosphoric acid fuel cell that converts natural gas fuel into electricity and heat. 
Table 29. National Fuel Gas Co., Interstate Flows of Natural Gas, 1993 (Thousand Cubic Feet)

\begin{tabular}{|c|c|}
\hline Company and State & Volume \\
\hline \multicolumn{2}{|l|}{$\begin{array}{l}\text { National Fuel Gas Supply Corp. } \\
\text { Pennsylvania To }\end{array}$} \\
\hline 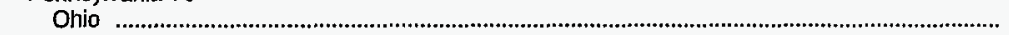 & 607,486 \\
\hline \multicolumn{2}{|l|}{ New York To } \\
\hline Pennsylvania & $5,981,292$ \\
\hline \multicolumn{2}{|l|}{$\begin{array}{l}\text { Penn-York Energy Corp. } \\
\text { New York To }\end{array}$} \\
\hline \multirow{2}{*}{\multicolumn{2}{|c|}{$\begin{array}{c}\text { Pennsylvania } \\
\text { Pennsylvania To }\end{array}$}} \\
\hline & \\
\hline New York & $13,167,320$ \\
\hline
\end{tabular}

Source: Energy Information Administration (EIA), Form ElA-176, "Annual Report of Natural and Supplemental Gas Supply and Disposition." 



\section{Niagara Mohawk Power Corp.}

Niagara Mohawk Power Corporation is an investor-owned combination gas and electric utility. The company operates in New York State and is principally engaged in the production, purchase, transmission, distribution, and sale of electricity and natural gas. Approximately 15 percent of Niagara Mohawk's corporate revenues are derived from the natural gas side of the business.

The company operates subsidiaries with facilities in both Canada and the United States. Opinac Energy Corporation, a Canadian-based subsidiary, owns Opinac Exploration Limited and Canadian Niagara Power Company, Limited. Opinac Exploration is engaged in the exploration and development of oil and gas properties in Canada. Canadian Niagara Power generates electricity at its Niagara Falls, Ontario hydroelectric plant. HYDRO-CO Enterprises, Inc, another of the company's subsidiaries, develops, owns, and operates cogeneration and small power plants.

The service area of Niagara Mohawk's natural gas distribution operations extends laterally across the middle part of New York State from Oswego to Albany. Some branches of the company's distribution area are not linked by company-owned transportation or distribution lines. For instance, nine separate distribution areas are con-

Figure 22. Niagara Mohawk Power Corp., 1993

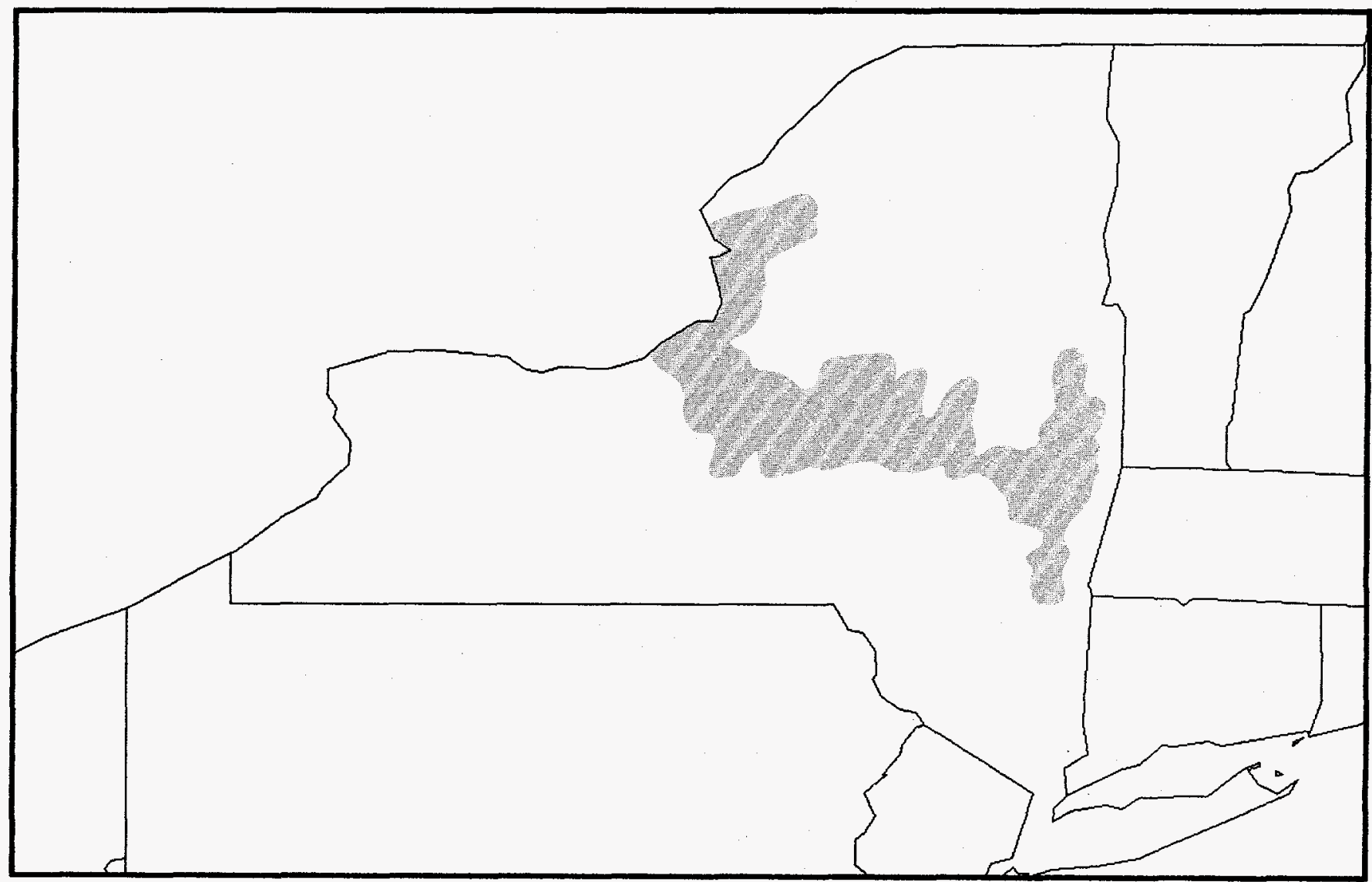

Source: Energy Information Administration (EIA), Form EIA-176, "Annual Report of Natural and Supplemental Gas Supply and Disposition."

\section{Service Area}


Table 30. Niagara Mohawk Power Corp., Natural Gas Data, 1993 (Thousand Cubic Feet)

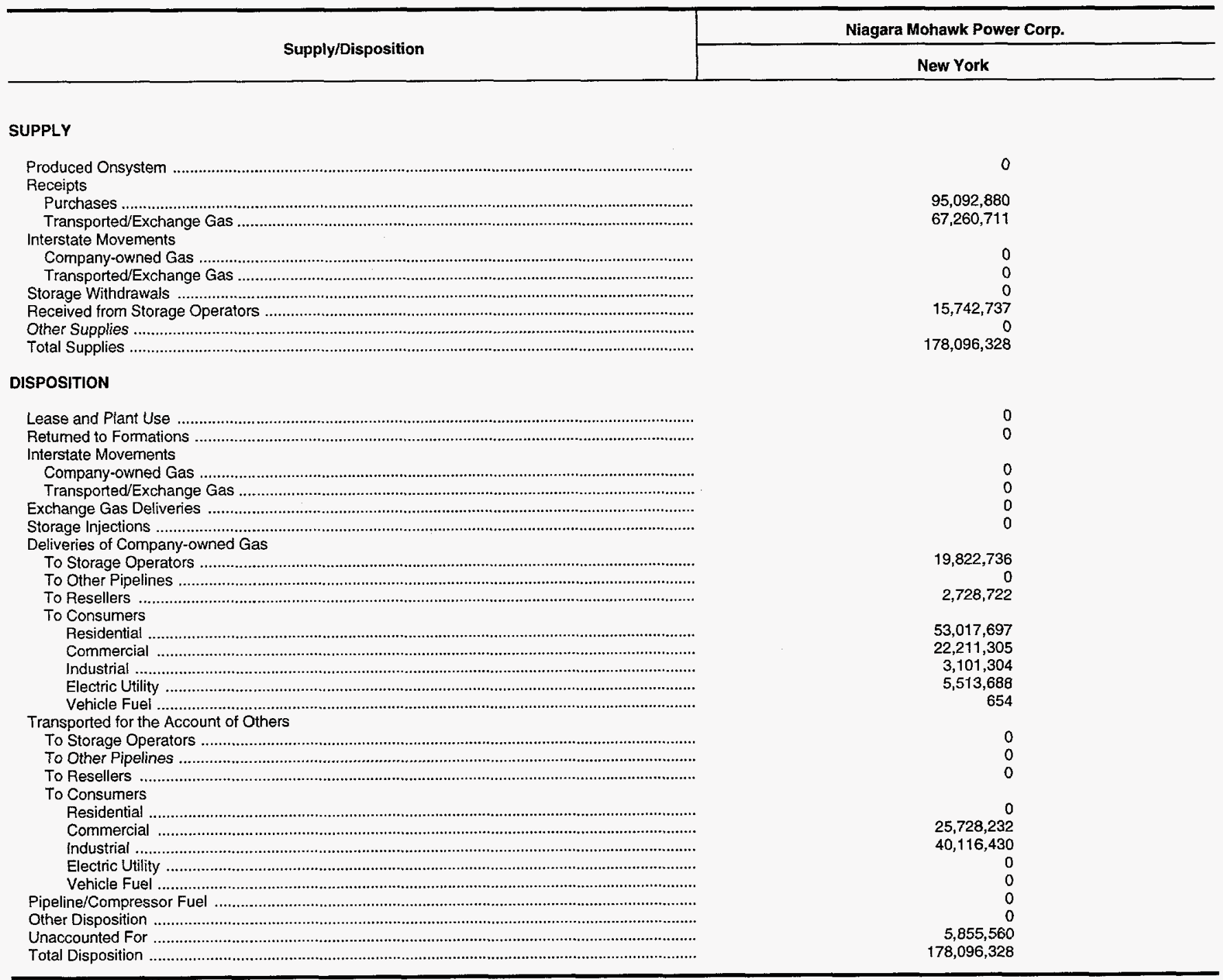

Source: Energy information Administration (EIA), Form EIA-176, "Annual Report of Natural and Supplemental Gas Supply and Disposition."

nected directly to CNG Transmission Corporation. Niagara Mohawk purchases gas brought into the State from Canada by Iroquois Gas Transmission System. The gas is then transported from the Iroquois pipeline to Niagara Mohawk's distribution area by CNG. The delivery to end-use consumers is made through Niagara Mohawk distribution facilities.
Niagara Mohawk's deliveries of natural gas to consumers decreased by 4 percent between 1992 and 1993 as a result of a large decline in deliveries to electric utilities. Increased deliveries to the residential, commercial, industrial, and vehicle fuel sectors were not sufficient to offset a net decline in total deliveries to consumers. 


\section{NorAm Energy Corp.}

NorAm Energy Corporation is one of the largest natural gas companies in the United States. Its primary businesses are natural gas distribution, transmission, marketing, and gathering. NorAm wholly owns two interstate pipelines: NorAm Gas Transmission Company and Mississippi River Transmission Corporation; along with three distribution companies: Entex, Arkla, and Minnegasco. NorAm Energy Services functions as the company's major natural gas supply and marketing group.

Prompted by the expansion of its operations territory, the company changed its name to NorAm Energy Corporation in May 1994. The former name, Arkla, Inc., reflected its roots in the Arkansas-Louisiana area. With Mississippi River Transmission, Entex, and Minnegasco, the company has outgrown the original Arkansas-Louisiana geographic boundaries suggested by the former name. The new name, short for North American, signifies the company's expansion to a much broader area of operations that reaches from the Canadian border to the border of Mexico.

Over the past 2 years, NorAm/Arkla undertook several streamlining efforts. The company sold two major assets, Louisiana Intrastate Gas and Arkla Exploration Company. To refocus its distribution operations in core areas,

Figure 23. NorAm Energy Corp., Inc., 1993

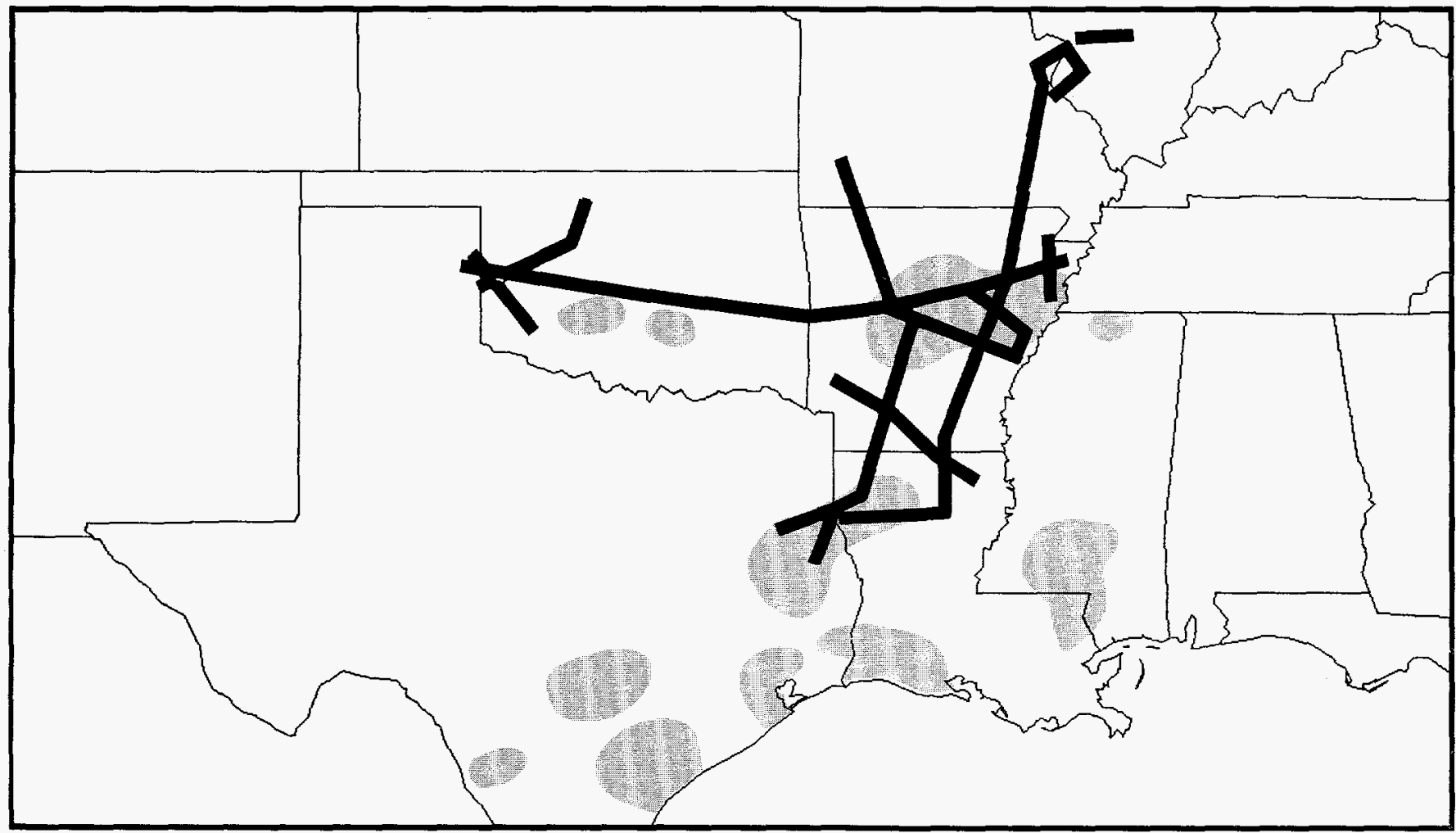

Source: Energy Information Administration (ElA), Form ElA-176, "Annual Report of Natural and Supplemental Gas Supply and Disposition." 
Arkla sold its gas distribution system in Nebraska to UtiliCorp United in February 1993, sold its Kansas distribution and transmission facility to Peoples Natural Gas in May 1993, and exchanged Minnegasco's South Dakota distribution properties for Midwest Gas Co.'s Minnesota properties. In addition, the spindown of Arkla Energy Resources (AER), a pipeline division, was completed in 1993, and moved AER to a wholly owned subsidiary.

The three natural gas distribution divisions of NorAm-Entex, Arkla, and Minnegasco--serve approximately 2.7 million customers in Arkansas, Kansas, Louisiana, Minnesota, Mississippi, Oklahoma, and Texas. Arkla, NorAm's original gas distribution company, serves 713,000 customers primarily in Arkansas, north Louisiana, Oklahoma, and east Texas. This service area includes the cities of Little Rock, Arkansas, and Shreveport, Louisiana.

Entex serves over 1.3 million customers in Louisiana, Mississippi and Texas, including the city of Houston. Its service area includes the major industrial markets in the south Gulf region. The largest natural gas distributor in Minnesota, Minnegasco serves 600,000 customers in the State, which has extremely cold winter weather.

Each of NorAm's three distribution divisions is involved in promoting new technological developments such as the futuristic home known as the "Smart House," natural gas cooling, and compressed natural gas as an alternative vehicle fuel. The use of natural gas vehicles by these divisions has continued for more than 10 years. Entex leads in the development of natural gas vehicles with 13 fueling stations serving 770 vehicles, followed by Minnegasco (117 vehicles) and Arkla (46 vehicles).

The NorAm pipeline network spans 12,000 miles and includes two interstate pipelines, NorAm Gas Transmission (NGT) and Mississippi River Transmission (MRT). The NGT pipeline accesses the prolific natural-gas-producing basins of the Midcontinent. It brings these supplies to Shreveport and Little Rock, and connects with the Nation's naturalgas pipeline grid to move gas to northern, midwestern, and eastern markets. MRT brings gas to

Figure 24. Minnegasco, Inc., 1993

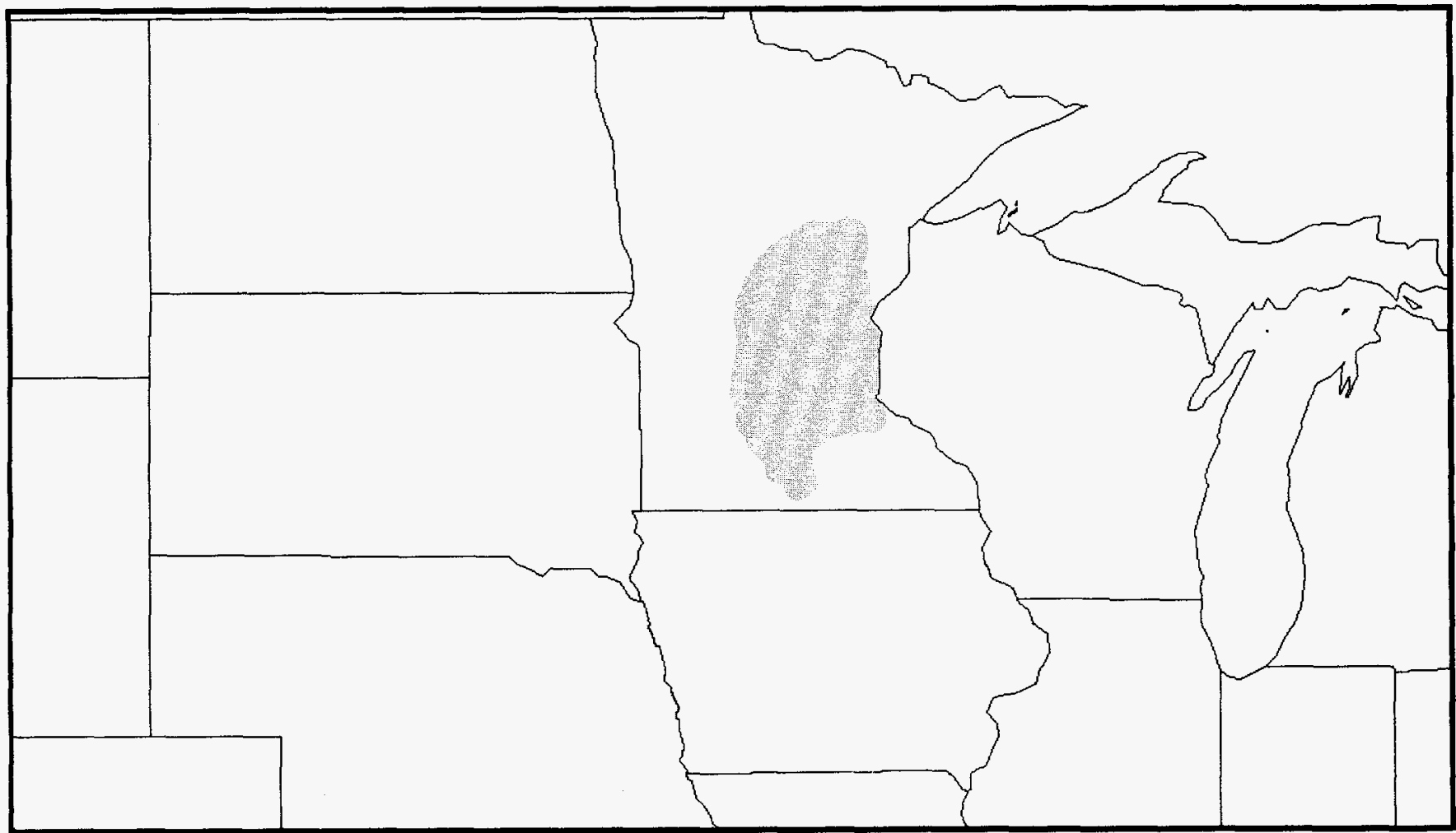

Source: Energy Information Administration (E|A), Form ElA- 176, "Annual Report of Natural and Supplemental Gas Supply and Disposition." 
Table 31. NorAm Energy Corp., Inc., Natural Gas Data, 1993 (Thousand Cubic Feet)

\begin{tabular}{|c|c|c|c|c|c|c|}
\hline \multirow{2}{*}{ Supply/Disposition } & \multicolumn{2}{|c|}{ Arkansas Louisiana Gas Co. } & \multicolumn{4}{|c|}{ NorAm Gas Transmission Co. } \\
\hline & Louisiana & Arkansas & Louisiana & Arkansas & Missouri & Texas \\
\hline \multicolumn{7}{|l|}{ SUPPLY } \\
\hline $\begin{array}{l}\text { Produced Onsystem } \\
\text { Receipts }\end{array}$ & 0 & 0 & 0 & 0 & 0 & 0 \\
\hline 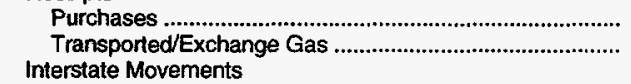 & $\begin{array}{r}13,722,056 \\
2,623,338\end{array}$ & $\begin{array}{l}54,237,576 \\
14,660,445\end{array}$ & $\begin{array}{r}2,830,076 \\
255,933,856\end{array}$ & $\begin{array}{r}6,334,281 \\
131,792,983\end{array}$ & $\begin{array}{r}0 \\
4,644,914\end{array}$ & $\begin{array}{r}5,132,032 \\
94,319,008\end{array}$ \\
\hline Company-owned Gas & 0 & 0 & 0 & 0 & 0 & 0 \\
\hline 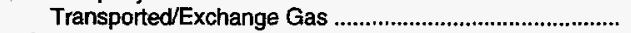 & 0 & 0 & $49,112,458$ & $358,878,048$ & $1,357,583$ & $6,219,689$ \\
\hline 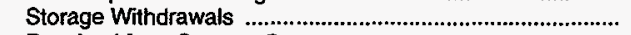 & 867,084 & $3,666,688$ & $1,464,163$ & 0 & 0 & 0 \\
\hline 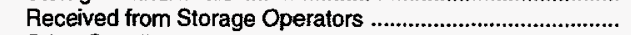 & 0 & 0 & $7,046,994$ & 0 & 0 & 0 \\
\hline Other Supplies & 0 & 0 & 0 & $\mathbf{a}$ & 0 & 0 \\
\hline 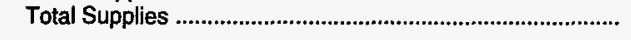 & $17,212,478$ & $72,564,709$ & $316,387,547$ & $497,005,312$ & $6,002,497$ & $105,670,729$ \\
\hline \multicolumn{7}{|l|}{ DISPOSITION } \\
\hline Lease and Plant Use & 0 & 0 & 72,115 & 2,783 & 0 & 332,628 \\
\hline $\begin{array}{l}\text { Retumed to Formations } \\
\text { Interstate Movements }\end{array}$ & 0 & 0 & 0 & 0 & 0 & 0 \\
\hline 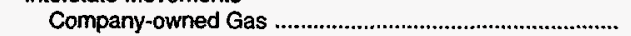 & 0 & 0 & 0 & 0 & 0 & 0 \\
\hline 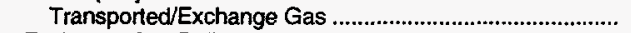 & 0 & 0 & $19,862,139$ & $6,883,989$ & $2,709,893$ & $58,811,772$ \\
\hline 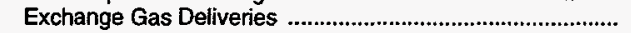 & 0 & 0 & $1,899,600$ & $2,502,547$ & 0 & $25,782,711$ \\
\hline 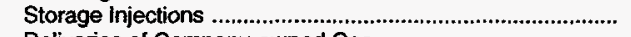 & 0 & 0 & $1,380,564$ & 0 & 0 & 0 \\
\hline \multicolumn{7}{|l|}{ Deliveries of Company-owned Gas } \\
\hline 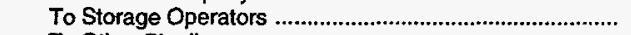 & 0 & 0 & $5,768,627$ & 0 & 0 & 0 \\
\hline 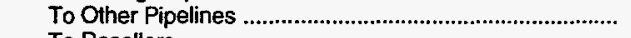 & 0 & 0 & 0 & 0 & 0 & 0 \\
\hline 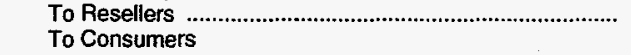 & 0 & 0 & $5,158,487$ & $20,625,085$ & 0 & $2,058,506$ \\
\hline 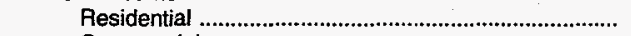 & $8,982,707$ & $31,899,343$ & 0 & 0 & 0 & 0 \\
\hline 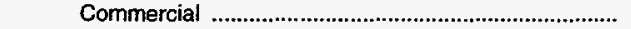 & $3,854,462$ & $17,275,684$ & 0 & 0 & 0 & 0 \\
\hline 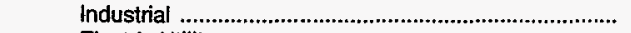 & $1,022,366$ & $6,009,191$ & 0 & 5,855 & 0 & 0 \\
\hline Electric Utility & 0 & 0 & 0 & 0 & 0 & 0 \\
\hline Vehicle Fuel & 0 & 0 & 0 & 0 & 0 & 0 \\
\hline \multicolumn{7}{|l|}{ Transported for the Account of Others } \\
\hline To Storage Operators & 0 & 0 & $2,567,539$ & 0 & 0 & 0 \\
\hline 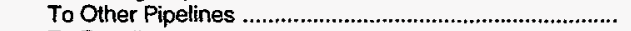 & 0 & 0 & $8,047,545$ & $210,984,223$ & 0 & 0 \\
\hline $\begin{array}{l}\text { To Resellers } \\
\text { To Consumers }\end{array}$ & 0 & 0 & $250,263,775$ & $163,953,621$ & $3,370,604$ & $8,917,559$ \\
\hline Residential & 0 & 0 & 0 & 0 & 0 & 0 \\
\hline Commercial & 0 & $2,390,204$ & 0 & 0 & 0 & 0 \\
\hline 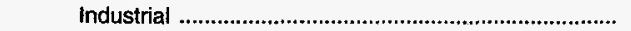 & $2,623,338$ & $12,270,241$ & $18,426,013$ & $75,674,444$ & 0 & $10,369,119$ \\
\hline Electric Utility & 0 & 0 & $2,019,661$ & $13,980,177$ & 0 & 0 \\
\hline 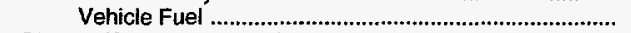 & 0 & 0 & 0 & 0 & 0 & 0 \\
\hline 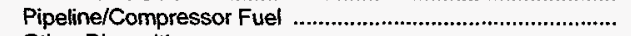 & 10,774 & 98,714 & 661,487 & $3,644,972$ & 0 & 393,720 \\
\hline Other Disposition & 0 & 0 & 0 & 7,931 & 0 & 0 \\
\hline Unaccounted For & 718,831 & $2,621,332$ & 259,995 & $-1,260,315$ & $-78,000$ & $-995,286$ \\
\hline Total Disposition & $17,212,478$ & $72,564,709$ & $316,387,547$ & $497,005,312$ & $6,002,497$ & $105,670,729$ \\
\hline
\end{tabular}

markets in the St. Louis, Missouri area and connects with other pipelines that supply the upper Midwest and the Northeast.

NorAm's Perryville Hub is located near Monroe, Louisiana and provides an array of natural gas services. Most major pipelines that bring natural gas from the Gulf Coast and Midcontinent converge in this location. The Hub has access to supplies from six major onshore producing basins and to two major storage reservoirs. Currently, there are five pipeline interconnections at Perryville: ANR, Koch Gateway, Texas Eastern, Texas Gas, and Trunkline. Four other pipelines are proposed for interconnections: Columbia Gas, Sonat, Tennessee Gas 100, and Tennessee Gas 800 . 
Table 31. NorAm Energy Corp., Inc., Natural Gas Data, 1993 (Continued) (Thousand Cubic Feet)

\begin{tabular}{|c|c|c|c|c|c|c|}
\hline \multirow{2}{*}{ Supply/Disposition } & \multicolumn{2}{|c|}{ NorAm Gas Transmission Co. } & \multicolumn{3}{|c|}{ Entex Division of NorAm Energy Corp. } & \multirow{2}{*}{$\begin{array}{c}\begin{array}{c}\text { Mississippi } \\
\text { River }\end{array} \\
\begin{array}{c}\text { Transmission } \\
\text { Corp. }\end{array} \\
\text { Oklahoma }\end{array}$} \\
\hline & Oklahoma & Kansas & Louisiana & Texas & Mississippi & \\
\hline \multicolumn{7}{|l|}{ SUPPLY } \\
\hline \\
\hline 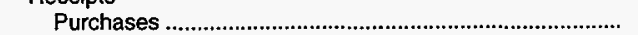 & $10,054,003$ & 44,781 & $26,681,732$ & $120,681,215$ & $19,950,489$ & 57,740 \\
\hline $\begin{array}{l}\text { Transported/Exchange Gas ............................................................ } \\
\text { Interstate Movements }\end{array}$ & & 223,718 \\
\hline 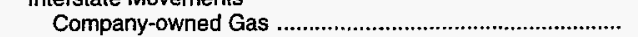 & 0 & 0 & 0 & 0 & 0 & 0 \\
\hline 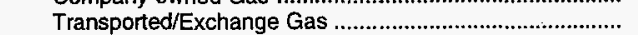 & $11,206,040$ & $8,196,075$ & 0 & 0 & 0 & 0 \\
\hline Storage Withdrawals & $18,968,696$ & 3,534 & 0 & 0 & 0 & 0 \\
\hline Received from Storage Operators & 0 & 0 & 0 & 0 & 0 & 0 \\
\hline 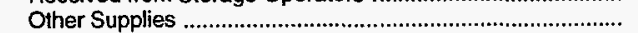 & 0 & 0 & 0 & 0 & 0 & 0 \\
\hline 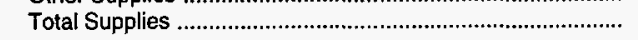 & $432,094,341$ & $11,320,474$ & $26,681,732$ & $128,817,812$ & $19,950,489$ & 281,458 \\
\hline \multicolumn{7}{|l|}{ DISPOSITION } \\
\hline 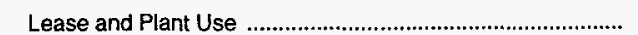 & 1,020 & 0 & 0 & 0 & 0 & 0 \\
\hline $\begin{array}{l}\text { Returned to Formations } \\
\text { Interstate Movements }\end{array}$ & 0 & 0 & 0 & 0 & 0 & 0 \\
\hline 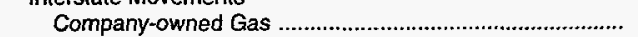 & 0 & 0 & 0 & 0 & 0 & 0 \\
\hline 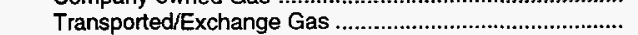 & $346,263,925$ & 438,175 & 0 & 0 & 0 & 0 \\
\hline Exchange Gas Deliveries & $8,326,232$ & 77,190 & 0 & 0 & 0 & 0 \\
\hline Storage Injections & $21,029,956$ & 0 & 0 & 0 & 0 & 0 \\
\hline \multicolumn{7}{|l|}{ Deliveries of Company-owned Gas } \\
\hline 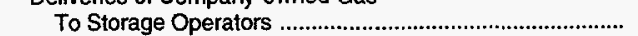 & 0 & 0 & 0 & 0 & 0 & 0 \\
\hline To Other Pipelines & 0 & 0 & 0 & 0 & 0 & 0 \\
\hline 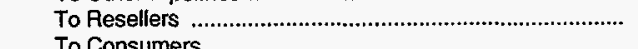 & \multirow{2}{*}{\multicolumn{4}{|c|}{ To Consumers }} & 0 & 57,740 \\
\hline $\begin{array}{l}\text { To Consumers } \\
\text { Residential }\end{array}$ & 0 & 0 & $6,992,660$ & $58,606,950$ & & \\
\hline 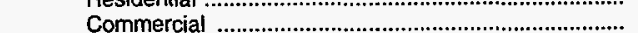 & 0 & 0 & $2,619,079$ & $38,975,860$ & $\begin{array}{l}0,3 / 0,199 \\
4,475,934\end{array}$ & $\begin{array}{l}0 \\
0\end{array}$ \\
\hline Industrial & 0 & 53,566 & $17,026,207$ & $18,582,205$ & $8,349,890$ & 0 \\
\hline Electric Utility & 21,907 & 0 & 0 & 0 & $\begin{array}{r}0,040,000 \\
0\end{array}$ & 0 \\
\hline Vehicle Fuel & 0 & 0 & 0 & 0 & 0 & 0 \\
\hline \multicolumn{7}{|l|}{ Transported for the Account of Others } \\
\hline 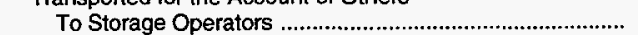 & 0 & 0 & 0 & 0 & 0 & 0 \\
\hline To Other Pipelines & 0 & 0 & 0 & 0 & 0 & 187,265 \\
\hline 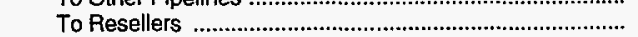 & $41,161,770$ & $4,414,439$ & 0 & 0 & 0 & 0 \\
\hline \multicolumn{7}{|l|}{ To Consumers } \\
\hline 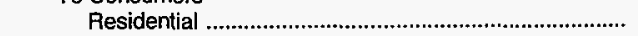 & 0 & 0 & 0 & 0 & 0 & 0 \\
\hline 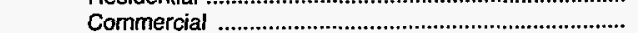 & 0 & 0 & 0 & $8,136,597$ & 56,684 & 0 \\
\hline Industrial & $2,832,846$ & $4,382,389$ & 0 & 0 & 0 & 0 \\
\hline Electric Utility & 371,318 & 0 & 0 & 0 & 0 & 0 \\
\hline Vehicle Fuel & 0 & 0 & 0 & 0 & 0 & 0 \\
\hline Pipeline/Compressor Fuel & $2,151,972$ & 2,485 & 0 & 0 & 0 & 31,741 \\
\hline Other Disposition & 0 & 0 & 0 & 0 & 0 & 0 \\
\hline Unaccounted For & $1,275,766$ & 124,934 & 43,786 & $4,516,200$ & 691,782 & 4,712 \\
\hline 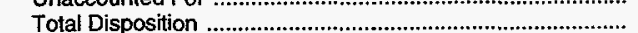 & $432,094,341$ & $11,320,474$ & $26,681,732$ & $128,817,812$ & $19,950,489$ & 281,458 \\
\hline
\end{tabular}


Table 31. NorAm Energy Corp., Inc., Natural Gas Data, 1993 (Continued)

(Thousand Cubic Feet)

\begin{tabular}{|c|c|c|c|c|c|}
\hline \multirow{2}{*}{ Supply/Disposition } & \multicolumn{5}{|c|}{ Mississippi River Transmission Corp. } \\
\hline & Texas & Louisiana & Arkansas & Missouri & Illinois \\
\hline \multicolumn{6}{|l|}{ SUPPLY } \\
\hline \multicolumn{6}{|l|}{$\begin{array}{l}\text { Produced Onsystem } \\
\text { Receipts }\end{array}$} \\
\hline Purchases & $1,198,892$ & $3,105,773$ & $56,405,274$ & 0 & $16,854,518$ \\
\hline \multicolumn{6}{|l|}{ Interstate Movements } \\
\hline 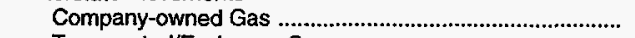 & 0 & 0 & 0 & 0 & 0 \\
\hline 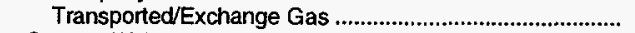 & 0 & $148,946,718$ & 0 & $112,956,255$ & $14,326,171$ \\
\hline Storage Withdrawals & 0 & $22,005,030$ & 0 & 0 & 683,658 \\
\hline 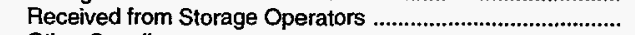 & 0 & 0 & 0 & 0 & 0 \\
\hline 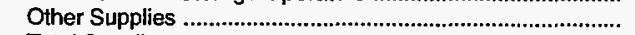 & 0 & 0 & 0 & 0 & 0 \\
\hline 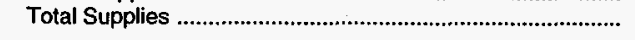 & $17,618,667$ & $195,519,987$ & $257,960,295$ & $112,956,255$ & $47,788,077$ \\
\hline \multicolumn{6}{|l|}{ DISPOSITION } \\
\hline Lease and Plant Use & 0 & 0 & 0 & 0 & 0 \\
\hline $\begin{array}{l}\text { Retumed to Formations } \\
\text { Interstate Movements }\end{array}$ & 0 & 0 & 0 & 0 & 0 \\
\hline 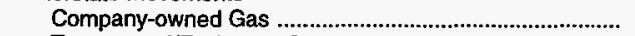 & 0 & 0 & 0 & 0 & 0 \\
\hline 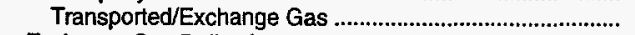 & $15,604,574$ & 0 & $246,298,399$ & $14,326,171$ & 0 \\
\hline 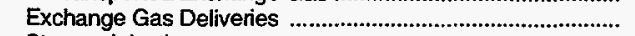 & 0 & 450,474 & $2,413,020$ & 0 & 583,102 \\
\hline Storage Injections & 0 & $31,460,239$ & 0 & 0 & 627,798 \\
\hline \multicolumn{6}{|l|}{ Deliveries of Company-owned Gas } \\
\hline 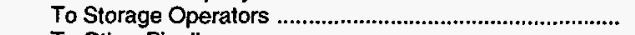 & 0 & 0 & 0 & 0 & 0 \\
\hline 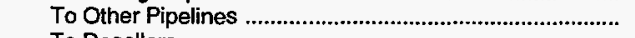 & 266,404 & 0 & 0 & 0 & 0 \\
\hline 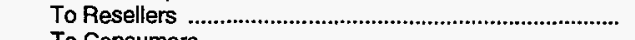 & 0 & 0 & $3,012,978$ & $44,425,470$ & $6,251,076$ \\
\hline \multicolumn{6}{|l|}{ To Consumers } \\
\hline 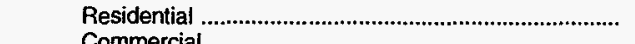 & 0 & 0 & 0 & 0 & 0 \\
\hline & 0 & 0 & 0 & 0 & 0 \\
\hline 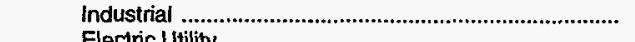 & 0 & 14,197 & 0 & 132,772 & $1,772,644$ \\
\hline 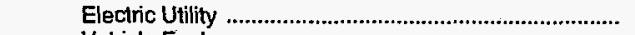 & 0 & 0 & 0 & $1,233,649$ & 419,505 \\
\hline 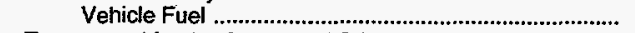 & 0 & 0 & 0 & 0 & 0 \\
\hline \multicolumn{6}{|l|}{ Transported for the Account of Others } \\
\hline 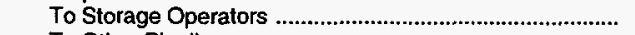 & 0 & 0 & 0 & 0 & 0 \\
\hline 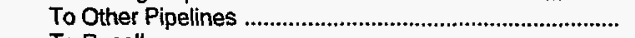 & 0 & $162,224,945$ & 0 & 0 & 0 \\
\hline 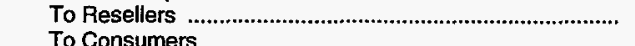 & $1,574,979$ & 0 & 999,841 & $48,840,139$ & $8,072,986$ \\
\hline 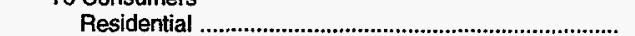 & 0 & 0 & 0 & 0 & To Consumers \\
\hline 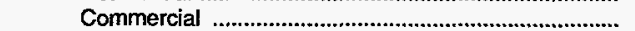 & 0 & 0 & 0 & 0 & $\begin{array}{l}0 \\
0\end{array}$ \\
\hline 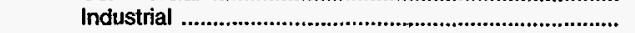 & 0 & 0 & $2,942,723$ & $2,691,488$ & $25,864,433$ \\
\hline 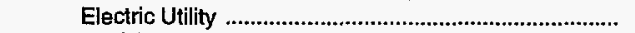 & 0 & 0 & 0 & 856,996 & $2,999,744$ \\
\hline 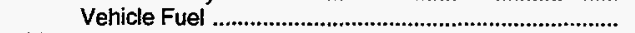 & 0 & 0 & 0 & 0 & 0 \\
\hline 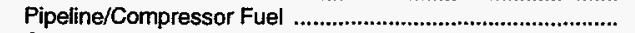 & 96,533 & 930,051 & $1,281,817$ & 253,913 & 490,683 \\
\hline 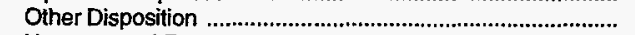 & 0 & 0 & 0 & 0 & 0 \\
\hline Unaccounted For & 76,177 & 440,081 & $1,011,517$ & 195,657 & 706,106 \\
\hline Total Disposition & $17,618,667$ & $195,519,987$ & $257,960,295$ & $112,956,255$ & $47,788,077$ \\
\hline
\end{tabular}


Table 31. NorAm Energy Corp., Inc., Natural Gas Data, 1993 (Continued) (Thousand Cubic Feet)

\begin{tabular}{|c|c|c|c|c|c|}
\hline \multirow{2}{*}{ Supply/Disposition } & \multirow{2}{*}{$\frac{\begin{array}{c}\text { Unit Gas } \\
\text { Transmission Co. }\end{array}}{\text { Texas }}$} & \multirow{2}{*}{$\frac{\begin{array}{c}\text { Industrial Gas } \\
\text { Supply Corp. }\end{array}}{\text { Texas }}$} & \multirow{2}{*}{$\begin{array}{l}\text { Tuscaloosa } \\
\text { Pipeline Co. } \\
\text { Louisiana }\end{array}$} & \multicolumn{2}{|c|}{ Minnegasco, Inc. } \\
\hline & & & & Minnesota & South Dakota \\
\hline \multicolumn{6}{|l|}{ SUPPLY } \\
\hline $\begin{array}{l}\text { Produced Onsystem } \\
\text { Receipts }\end{array}$ & 0 & 0 & 0 & 0 & 0 \\
\hline 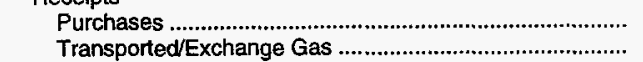 & $\begin{array}{r}16,003,892 \\
7,580,896\end{array}$ & $\begin{array}{l}10,409,876 \\
20,548,678\end{array}$ & $\begin{array}{r}12,978,081 \\
0\end{array}$ & $\begin{array}{r}122,874,056 \\
6,655,922\end{array}$ & $\begin{array}{r}5,543,915 \\
263,418\end{array}$ \\
\hline 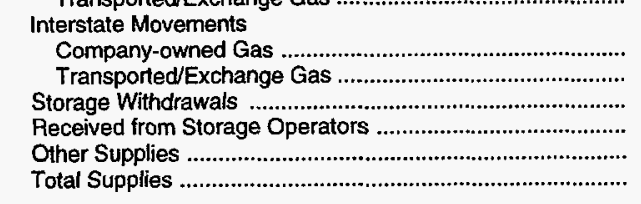 & $\begin{array}{r}0 \\
0 \\
0 \\
0 \\
0 \\
23,584,788\end{array}$ & $\begin{array}{r}0 \\
0 \\
0 \\
0 \\
0 \\
30,958,554\end{array}$ & $\begin{array}{r}0 \\
0 \\
0 \\
0 \\
0 \\
12,978,081\end{array}$ & $\begin{array}{r}0 \\
0 \\
2,022,929 \\
0 \\
55,050 \\
131,607,957\end{array}$ & $\begin{array}{r}0 \\
0 \\
0 \\
0 \\
0 \\
5,807,333\end{array}$ \\
\hline \multicolumn{6}{|l|}{ DISPOSITION } \\
\hline 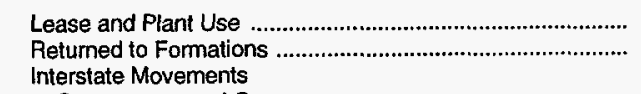 & $\begin{array}{l}0 \\
0\end{array}$ & $\begin{array}{l}0 \\
0\end{array}$ & $\begin{array}{l}0 \\
0\end{array}$ & $\begin{array}{l}0 \\
0\end{array}$ & $\begin{array}{l}0 \\
0\end{array}$ \\
\hline 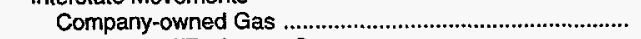 & 0 & 0 & 0 & 0 & 0 \\
\hline 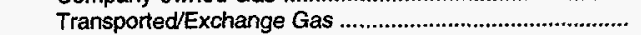 & 0 & 0 & 0 & 0 & 0 \\
\hline 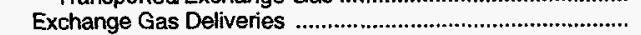 & 0 & 0 & 0 & 0 & 0 \\
\hline $\begin{array}{l}\text { Storage Injections } \\
\text { Deliveries of Company-owned Gas }\end{array}$ & 0 & 0 & 0 & $1,983,259$ & 0 \\
\hline 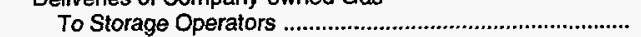 & 0 & 0 & 0 & 0 & 0 \\
\hline 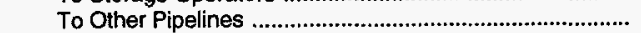 & 0 & 0 & 0 & 0 & 0 \\
\hline $\begin{array}{l}\text { To Gonsumers } \\
\text { Residential }\end{array}$ & 0 & 0 & 0 & $61,261,681$ & $2,754,160$ \\
\hline 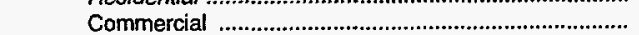 & 14,134 & 49,447 & 0 & $48,416,782$ & $2,505,186$ \\
\hline Industrial & $15,742,766$ & $10,360,429$ & $13,334,230$ & $12,839,061$ & 267,363 \\
\hline Electric Utility & 0 & 0 & 0 & 492,132 & 12,531 \\
\hline 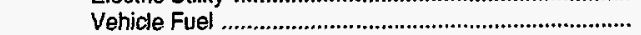 & 0 & 0 & 0 & 0 & 0 \\
\hline Transported for the Account of Others & & & & & \\
\hline 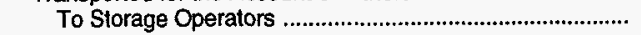 & 0 & 0 & 0 & 0 & 0 \\
\hline 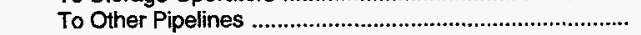 & 0 & 0 & 0 & 0 & 0 \\
\hline 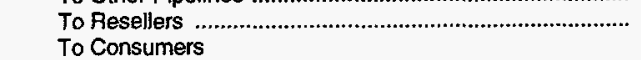 & 0 & 0 & 0 & 0 & 0 \\
\hline 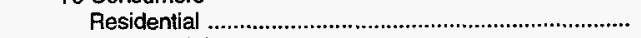 & 0 & 0 & 0 & 0 & 0 \\
\hline 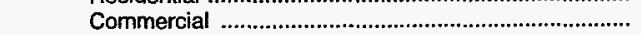 & $1,258,165$ & 0 & 0 & 225,979 & 0 \\
\hline Industrial & $6,322,711$ & $20,548,678$ & 0 & $6,308,888$ & 223,532 \\
\hline Electric Utility & 0 & 0 & 0 & 121,055 & 39,886 \\
\hline Vehicle Fuel & 0 & 0 & 0 & 0 & 0 \\
\hline 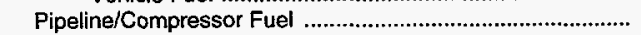 & 0 & 0 & 0 & 136,622 & 6,394 \\
\hline 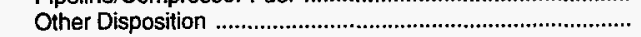 & 0 & 0 & 0 & 0 & 0 \\
\hline Unaccounted For & 52,155 & 0 & $-356,149$ & $-177,502$ & $-1,719$ \\
\hline 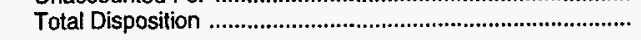 & $23,584,788$ & $30,958,554$ & $12,978,081$ & $131,607,957$ & $5,807,333$ \\
\hline
\end{tabular}

Source: Energy Information Administration (EIA), Form ElA-176, "Annual Report of Natural and Supplemental Gas Supply and Disposition." 
Table 32. NorAm Energy Corp., Inc., Interstate Flows of Natural Gas, 1993 (Thousand Cubic Feet)

\begin{tabular}{|c|c|}
\hline Company and State & Volume \\
\hline \multicolumn{2}{|l|}{ NorAm Gas Transmission Co. } \\
\hline Arkansas & $18,144,515$ \\
\hline Texas & $1,717,624$ \\
\hline \multicolumn{2}{|l|}{ Arkansas To } \\
\hline Louisiana & $1,068,551$ \\
\hline Missouri & $1,357,583$ \\
\hline Texas & $4,457,855$ \\
\hline \multicolumn{2}{|l|}{ Missouri To } \\
\hline Arkansas & $2,709,893$ \\
\hline \multicolumn{2}{|l|}{ Texas To } \\
\hline Louisiana & $48,043,907$ \\
\hline Oklahoma & $10,767,865$ \\
\hline \multicolumn{2}{|l|}{ Oklahoma To } \\
\hline 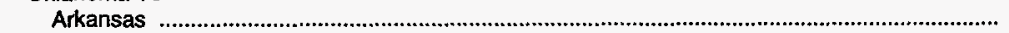 & $338,023,640$ \\
\hline Kansas & $8,196,075$ \\
\hline 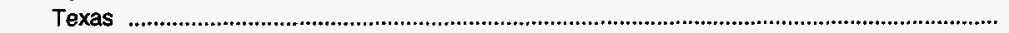 & 44,210 \\
\hline \multicolumn{2}{|l|}{ Kansas To } \\
\hline Oklahoma & 438,175 \\
\hline \multicolumn{2}{|l|}{$\begin{array}{l}\text { Mississippi River Transmission Corp. } \\
\text { Texas To }\end{array}$} \\
\hline \multirow{2}{*}{\multicolumn{2}{|c|}{$\begin{array}{c}\text { Louisiana } \\
\text { Arkansas To }\end{array}$}} \\
\hline & \\
\hline 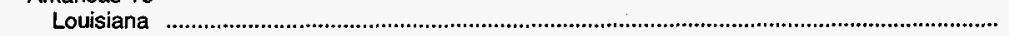 & $133,342,144$ \\
\hline Missouri & $112,956,255$ \\
\hline \multicolumn{2}{|l|}{ Missouri To } \\
\hline 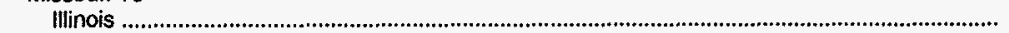 & $14,326,171$ \\
\hline
\end{tabular}

Source: Energy Information Administration (EIA), Form EIA-176, "Annual Report of Natural and Supplemental Gas Supply and Disposition." 


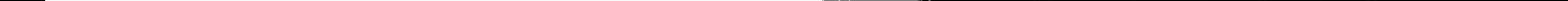




\section{Northern Illinois Gas Co.}

Northern Illinois Gas Company distributes natural gas to nearly 1.8 million customers in the northern third of Illinois, outside the city of Chicago. The company is a wholly owned subsidiary of NICOR, Inc., a holding company. During 1993, NICOR sold its oil and gas exploration and production subsidiary.

Northern Illinois delivers approximately one-half of its natural gas to residential customers; industrial and electric utility customers receive about one-third, and commercial customers receive the balance. For commercial and industrial consumers who make their own arrangements for purchasing natural gas supplies, Northern Illinois offers transportation, storage, and supply backup services.
Federal Energy Regulatory Commission (FERC) Order 636 , the restructuring order for the natural gas industry, went into effect beginning with the 1993-1994 heating season. Under Order 636, gas distributors purchase their supplies directly from producers, marketers, and pipeline affiliates, and storage often plays a more prominent role in supplementing supplies. During the severe winter of 1994, demand for gas reached a record level in a 24-hour period in January in the Northern Illinois service area. Withdrawals from underground storage helped the company meet this record demand.

Some of the emerging natural gas markets that Northern Illinois hopes to develop are: gas-fired electric power

Figure 25. Northern Illinois Gas Co., 1993

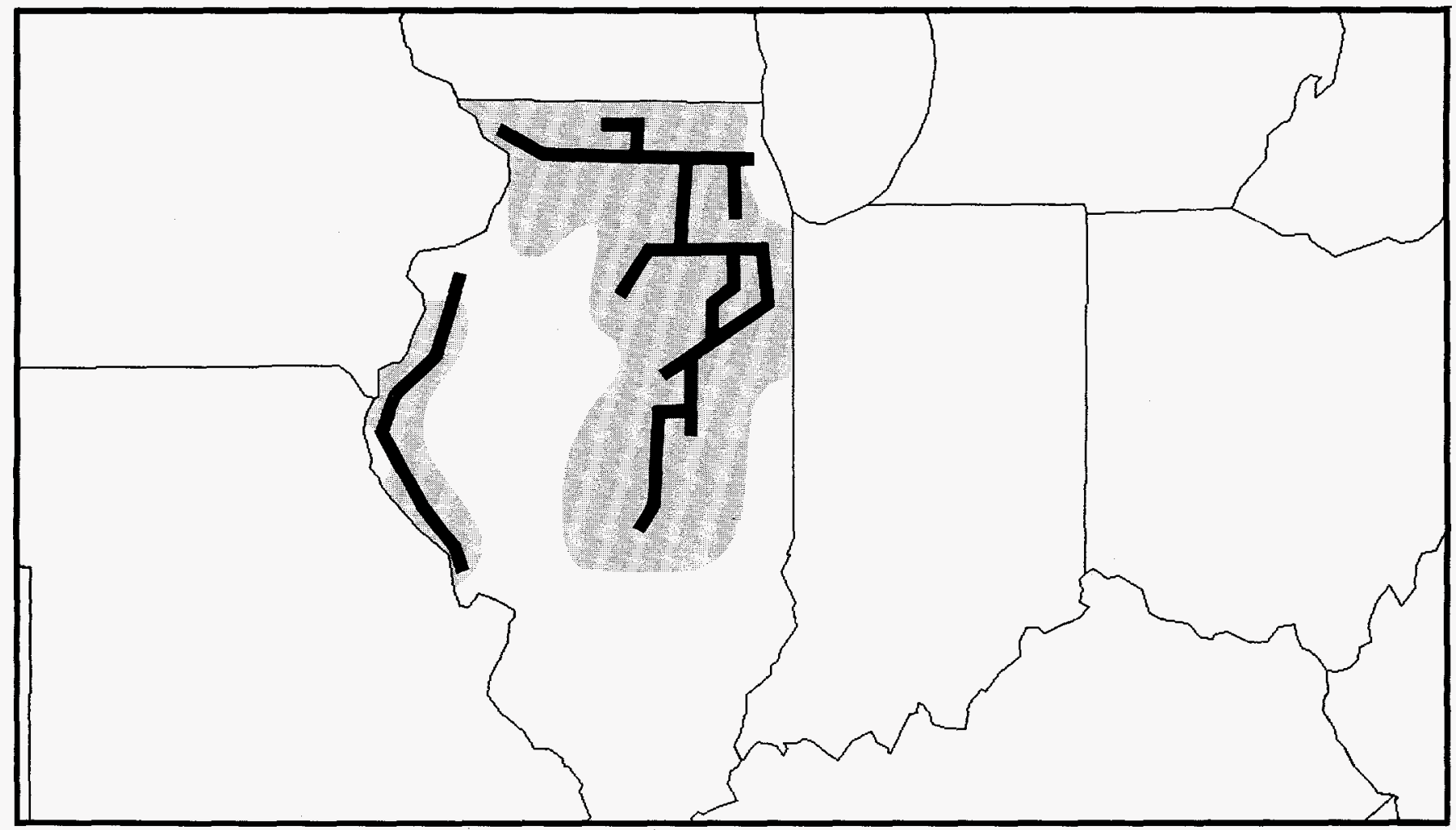

Source: Energy Information Administration (EIA), Form EIA-176, "Annual Report of Natural and Supplemental Gas Supply and Disposition." 
Table 33. Northern Illinois Gas Co., Natural Gas Data, 1993 (Thousand Cubic Feet)

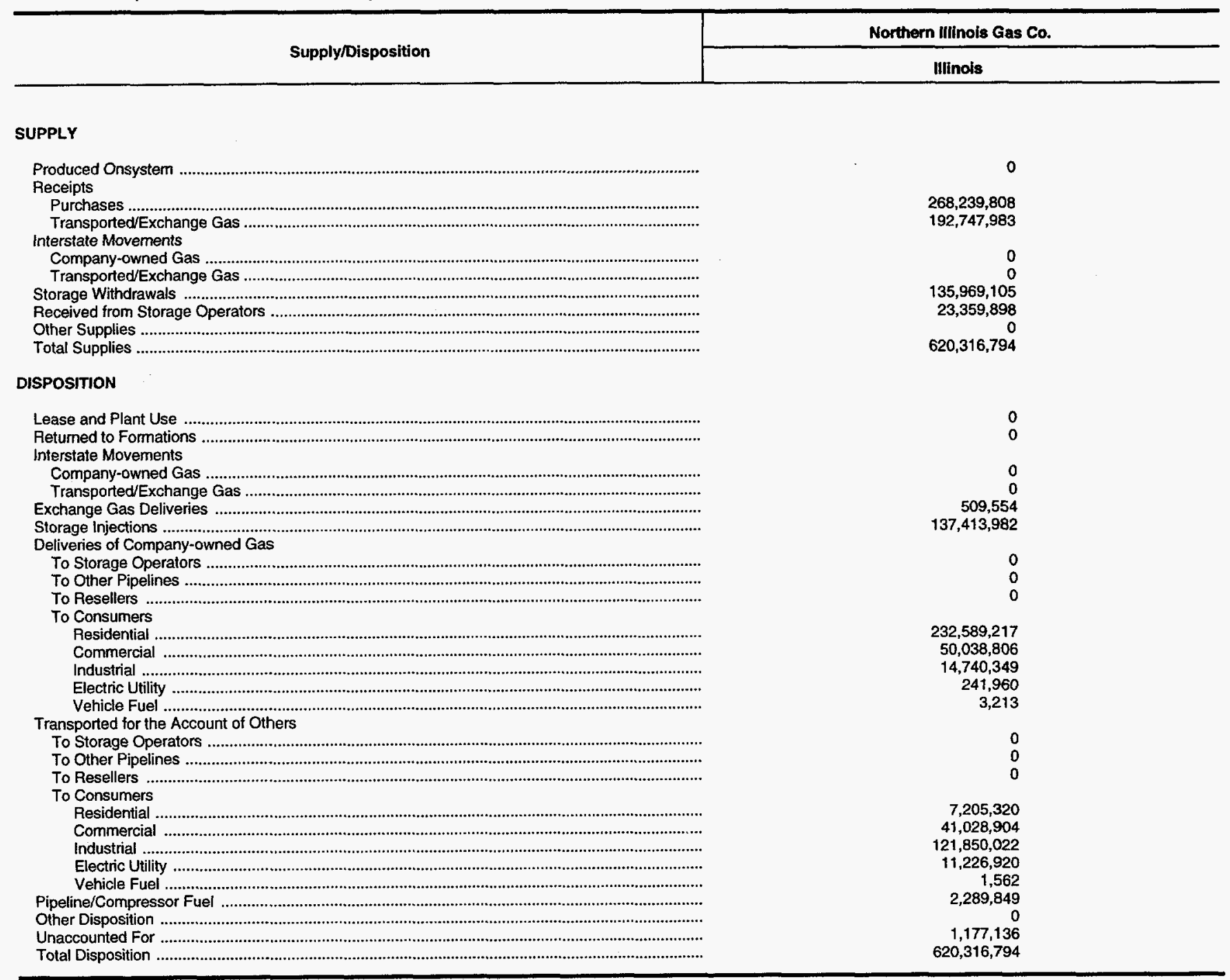

Source: Energy Information Administration (EIA), Form ElA-176, "Annual Report of Natural and Supplemental Gas Supply and Disposition."

generation, cogeneration, large-tonnage gas air conditioning, and natural gas vehicles. In May 1993, the company began to deliver gas to a large Commonwealth Edison Company electric-generating station. Also in 1993, 10 small-vehicle fleets in the Northern Illinois service area converted to natural gas.
The company opened the Chicago Hub in June 1993 to serve as a marketplace for gas buyers and sellers to conduct negotiations. Northern Illinois expects the volume of transactions at the Hub to increase as the deregulated natural gas market grows. 


\section{Northern Indiana Public Service Co.}

Northern Indiana Public Service Company (NIPSCO) delivers natural gas and electricity throughout a $12,000-$ square-mile area extending across the northern third of Indiana that has a population of approximately 2.1 million. It is the 13th largest gas distribution company in the United States, serving approximately 600,000 gas customers in Indiana. NIPSCO is the principal subsidiary of NIPSCO Industries, Incorporated, an energy-based holding company.

Other primary NIPSCO Industries subsidiaries include: Northern Indiana Fuel and Light Company, Incorporated, which became a wholly owned subsidiary in 1993, adding approximately 28,700 natural gas customers in northeast- ern Indiana; NIPSCO Development Company, Incorporated, which coordinates strategic investments in power projects and real estate; Kokomo Gas and Fuel Company, which provides natural gas to approximately 31,000 customers in the city of Kokomo and the surrounding rural territory; and NIPSCO Energy Services Incorporated, which is engaged in energy-related investments and owns gas storage facilities in addition to an interest in two intrastate pipelines.

Within NIPSCO Energy Services Incorporated, there are three subsidiaries: NIPSCO Fuel Company, which invests in gas and oil exploration and development ventures; NIPSCO Energy Trading Company, which brokers gas

Figure 26. Northern Indiana Public Service Co., 1993

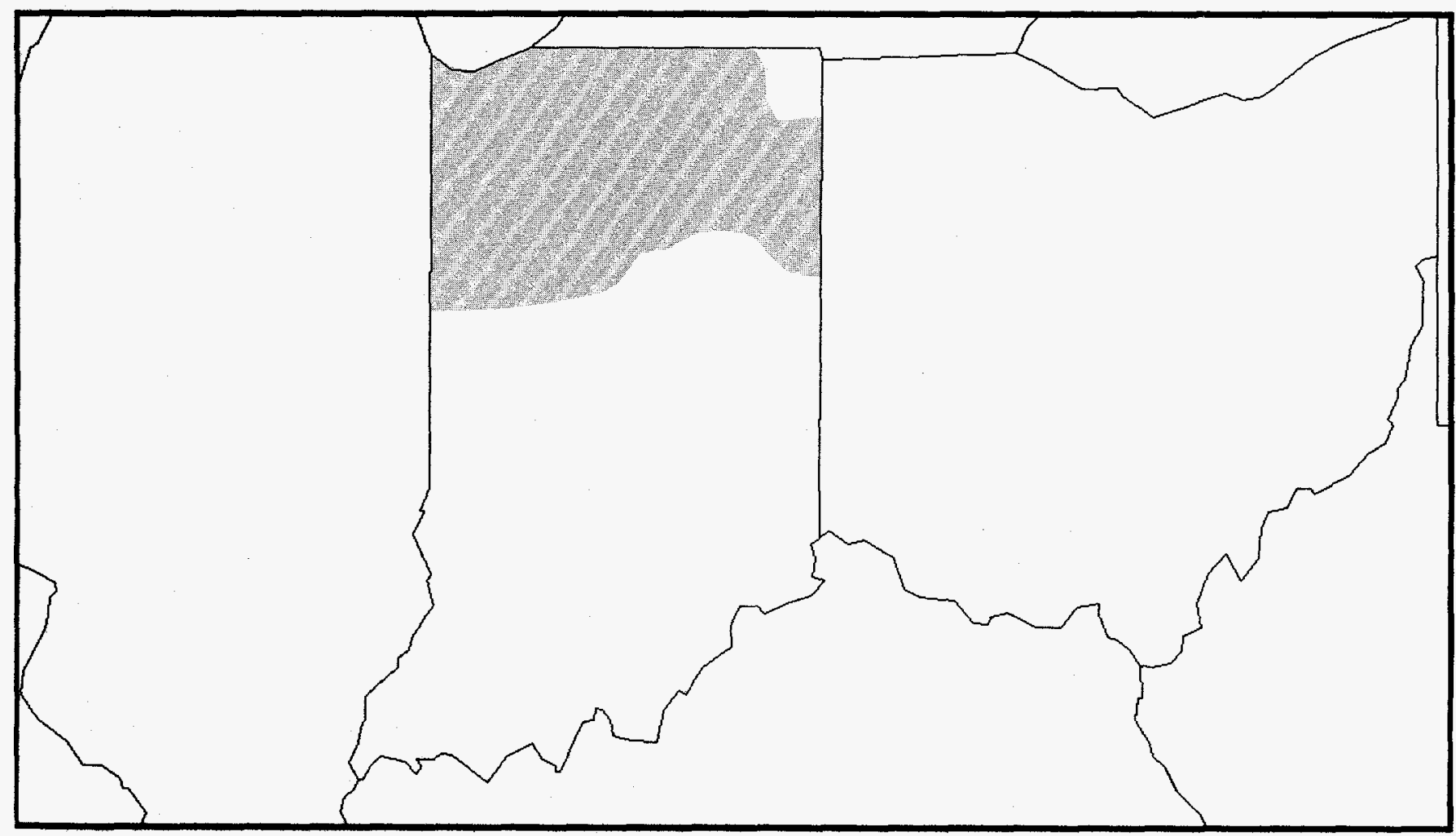

Source: Energy Information Administration (EIA), Form EIA-176, "Annual Report of Natural and Supplemental Gas Supply and Disposition."

Service Area 
Table 34. Northern Indiana Public Service Co., Natural Gas Data, 1993 (Thousand Cubic Feet)

\begin{tabular}{l|c|c|c|}
\hline \multirow{2}{*}{ Supply/Disposition } & Northern Indiana Public Service Co. & Kokomo Gas \& Fuel Co. & Indiana \\
\cline { 2 - 3 } & Indiana & \\
\hline
\end{tabular}

\section{SUPPLY}

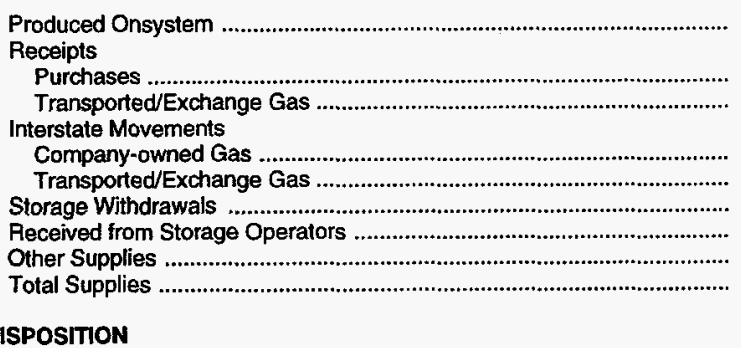

DISPOSITION $\begin{array}{lll}0 & 0 \\ 0 & 0\end{array}$<smiles>C1CCCCC1</smiles>

$6,443,694$

0
0
0

$68,915,503$

$27,208,527$

$13,044,227$

454,584

50,328

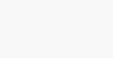

0
0
$161,204,247$

$3,885,231$

0
101,784
0

$2,973,021$

$284,281,146$
0

87,243

$8,593,018$
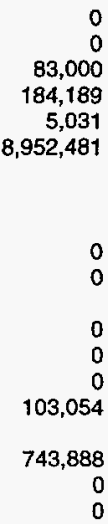

$3,312,632$

$3,312,632$
$1,397,560$

$1, \mathbf{5 5 7}, 820$

0

0

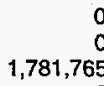

$1,781,765$

0
0

30,312

25,450

$8,952,481$

Source: Energy Information Administration (EIA), Form EIA-176, "Annual Report of Natural and Supplemental Gas Supply and Disposition."

and other energy products; and NI-TEX Incorporated, an intrastate natural gas transmission and gas supply company.

In 1994, NIPSCO separated its gas and electricity business into independent units to help the company compete on the basis of cost and service. NIPSCO serves 11 of America's industrial giants, in addition to five of the Nation's key steel producers. The company is currently working on a series of joint projects in cooperation with its industrial customers (such as U.S. Steel, Inland Steel, and Praxair). These projects, for example, will turn byproduct steam from the steel-making process into 200 to 300 megawatts of cost-efficient electric power.

NIPSCO subsidiaries have direct access to every major gas supply basin in the United States. In April 1993, NIPSCO purchased Crossroads Pipeline Company, a 20inch crude-oil pipeline that extends 202 miles east from the Illinois/Indiana State line to Cygnet, Ohio. The pipe- line was converted from oil to natural gas to provide access to major gas supplies in the United States and to increase reliability for customers in bad weather conditions.

NIPSCO has a continued interest in the natural gas vehicle market. It entered into a venture with Liquid Carbonic to remove propane and ethane from excess peakshaving fuel resulting in a liquid methane that is a cleaner burning and more efficient vehicle fuel. Liquid Carbonic installed and is testing an LNG purifier at NIPSCO's Laporte, Indiana plant near its Rolling Prairie storage site. This facility holds the liquid equivalent of 4 billion cubic feet of natural gas in two large tanks. NIPSCO and Liquid Carbonic jointly operate the purifier, supplying the natural gas through NIPSCO Energy Trading. This methane purification project is believed to be the only one of its kind in the United States. For the purification process to be successful, the purified fuel should be 99.5 percent pure methane. 


\section{Northwest Natural Gas Co.}

Northwest Natural Gas Company, headquartered in Portland, Oregon, is a diversified energy company whose primary business is providing sales and transportation service to natural gas customers in northwestern Oregon and southwestern Washington. Northwest Natural's subsidiaries are engaged in the exploration, development, and production of natural gas and oil, as well as nonenergy-related business.

Northwest Natural, the largest distributor on the Northwest Pipeline System, is served by Northwest Pipeline Corporation and Pacific Gas Transmission. Natural gas flows into the Northwest Natural distribution system from Canada and the Rocky Mountain States. Because of the demand for natural gas in the Pacific Northwest market area, Northwest Pipeline is responding to customers' needs for additional capacity by designing new facilities.

Northwest will be upgrading its least cost plan to incorporate several new industry developments; including changes due to gas-fired electric generation, the effect of industrial and transportation-only load on future demand, the possibility of acquiring and selling released pipeline capacity, storage alternatives as a supply-side option, and demand-side management for firm industrial users. Because of a strong regional economy, a flow of new residents from other States, construction of new homes and businesses, and conversions from other fuels, Northwest Natural has continued to grow in 1993 for the fifth consecutive year.

Figure 27. Northwest Natural Gas Co., 1993

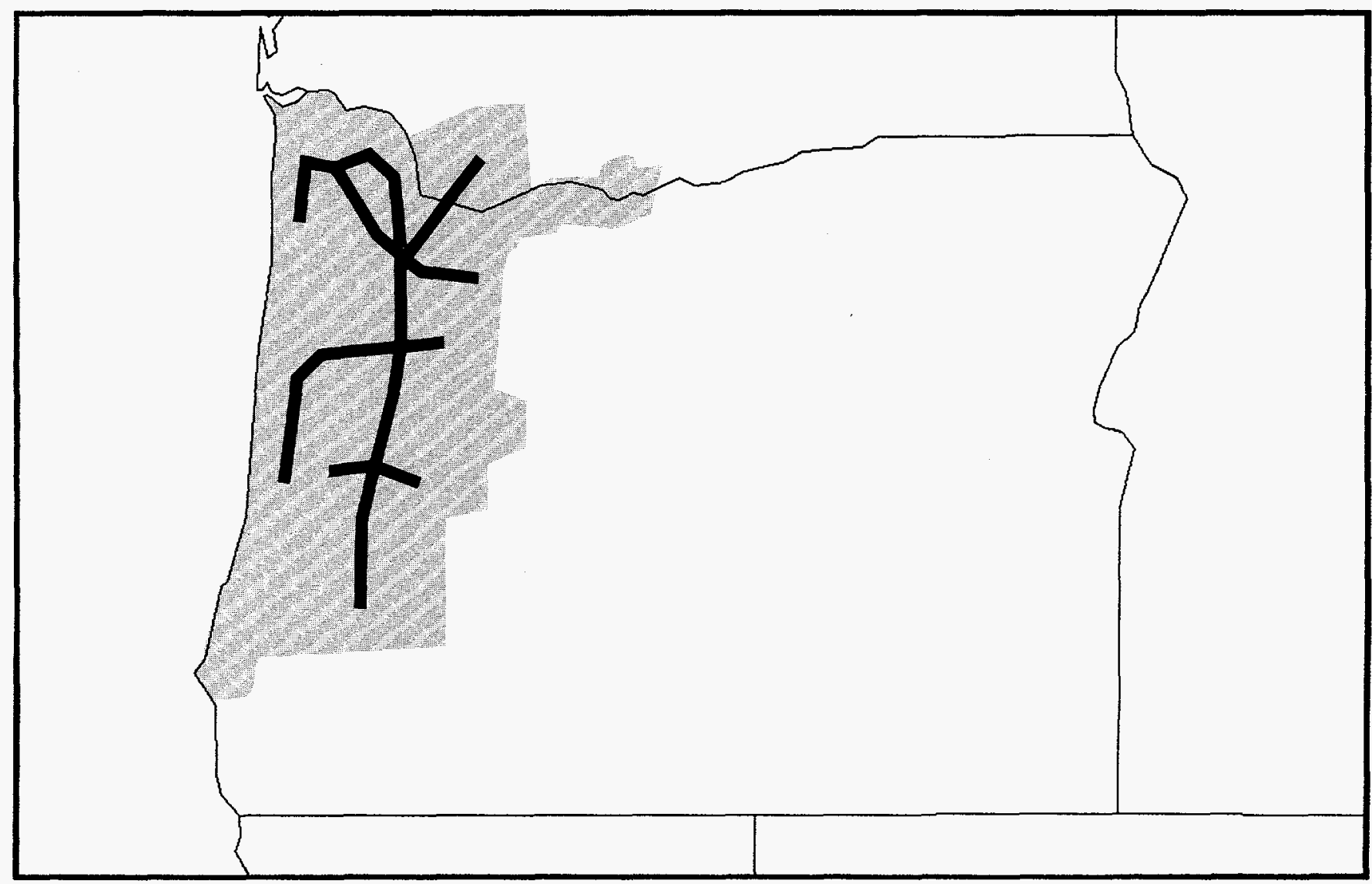

Source: Energy Information Administration (ElA), Form ElA-176, "Annual Report of Natural and Supplemental Gas Supply and Disposition." 
Table 35. Northwest Natural Gas Co., Natural Gas Data, 1993 (Thousand Cubic Feet)

\begin{tabular}{|c|c|c|}
\hline \multirow{2}{*}{ Supply/Disposition } & \multicolumn{2}{|c|}{ Northwest Natural Gas Co. } \\
\hline & Oregon & Washington \\
\hline \multicolumn{3}{|l|}{ SUPPLY } \\
\hline Produced Onsystem & 0 & 0 \\
\hline 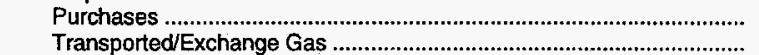 & $\begin{array}{l}56,740,691 \\
33,240,237\end{array}$ & $\begin{array}{l}3,190,401 \\
6,788,251\end{array}$ \\
\hline \multicolumn{3}{|l|}{ Interstate Movements } \\
\hline 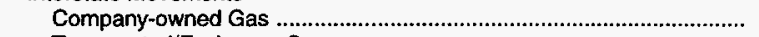 & 0 & 0 \\
\hline 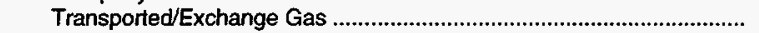 & 0 & 0 \\
\hline Storage Withdrawals & $7,456,528$ & 0 \\
\hline Received from Storage Operators & 0 & 0 \\
\hline Other Supplies & 2,495 & 0 \\
\hline 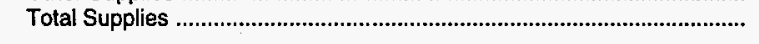 & $97,439,951$ & $9,978,652$ \\
\hline \multicolumn{3}{|l|}{ DISPOSITION } \\
\hline Lease and Plant Use & 0 & 0 \\
\hline Returned to Formations & 0 & 0 \\
\hline \multicolumn{3}{|l|}{ Interstate Movements } \\
\hline Company-owned Gas & 0 & 0 \\
\hline 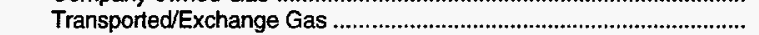 & 0 & 0 \\
\hline Exchange Gas Deliveries & 0 & 0 \\
\hline Storage Injections & $7,339,429$ & 0 \\
\hline \multicolumn{3}{|l|}{ Deliveries of Company-owned Gas } \\
\hline 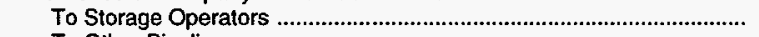 & 0 & 0 \\
\hline To Other Pipelines & 0 & 0 \\
\hline 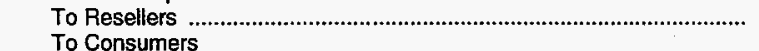 & 0 & 0 \\
\hline 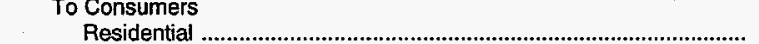 & & 1122130 \\
\hline 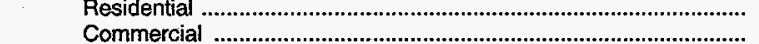 & $24,752,631$ & $1,122,130$ \\
\hline 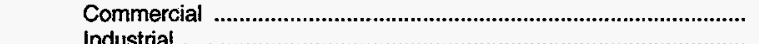 & $18,950,160$ & $1,352,162$ \\
\hline 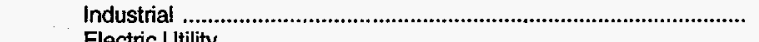 & $13,422,938$ & 729,928 \\
\hline 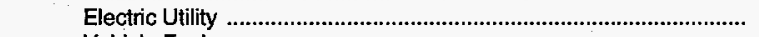 & 0 & 0 \\
\hline Vehicle Fuel & 7,648 & 0 \\
\hline \multicolumn{3}{|l|}{ Transported for the Account of Others } \\
\hline 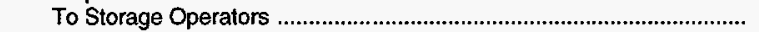 & 0 & 0 \\
\hline 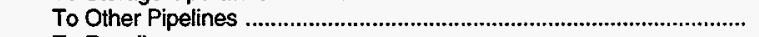 & 0 & 0 \\
\hline 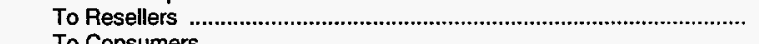 & 0 & 0 \\
\hline \multicolumn{3}{|l|}{ To Consumers } \\
\hline 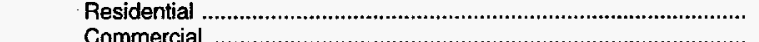 & 0 & 0 \\
\hline 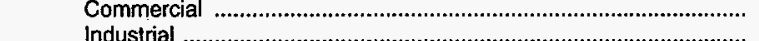 & 0 & 0 \\
\hline Industrial & $32,078,384$ & $6,788,251$ \\
\hline 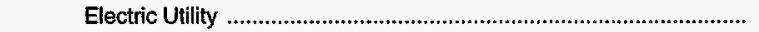 & $1,161,853$ & 0 \\
\hline 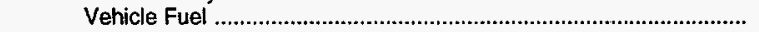 & 0 & 0 \\
\hline Pipeline/Compressor Fuel & 262,993 & 0 \\
\hline Other Disposition & 0 & 0 \\
\hline Unaccounted For & $-536,085$ & $-13,819$ \\
\hline Total Disposition & $97,439,951$ & $9,978,652$ \\
\hline
\end{tabular}

Source: Energy Information Administration (EIA), Form ElA-176, "Annual Report of Natural and Supplemental Gas Supply and Disposition." 


\section{ONEOK, Inc.}

ONEOK, Inc. (pronounced "won-oak") is made up of three major divisions and a number of wholly owned subsidiaries. Headquartered in Tulsa, Oklahoma, it operates the State's largest natural gas utility, consisting of two divisions. The Oklahoma Natural Gas Company (ONG) handles the purchasing, distribution, and selling of natural gas and the leasing of pipeline capacity to industrial and commercial customers for transporting gas. The ONG Transmission Company is responsible for gathering, compressing, transporting, and storing natural gas, and is also responsible for the company's links to interstate transmission systems.
In 1980, ONEOK developed a strategy to increase its nonutility activities and created the Energy Companies of ONEOK. Five subsidiaries now operate in the Company. ONEOK Exploration Company and ONEOK Resources Company are engaged in oil and gas exploration and production. ONEOK Gas Marketing Company, formed in 1992, represents ONEOK's partnership with Ward Gas Services to pursue gas marketing opportunities with local distribution companies and industrial customers throughout the United States. ONEOK Drilling Company, with its 12 rigs, is a contract drilling business. The fifth subsidiary, ONEOK Products Co., along with its joint-interest

Figure 28. ONEOK, Inc., 1993

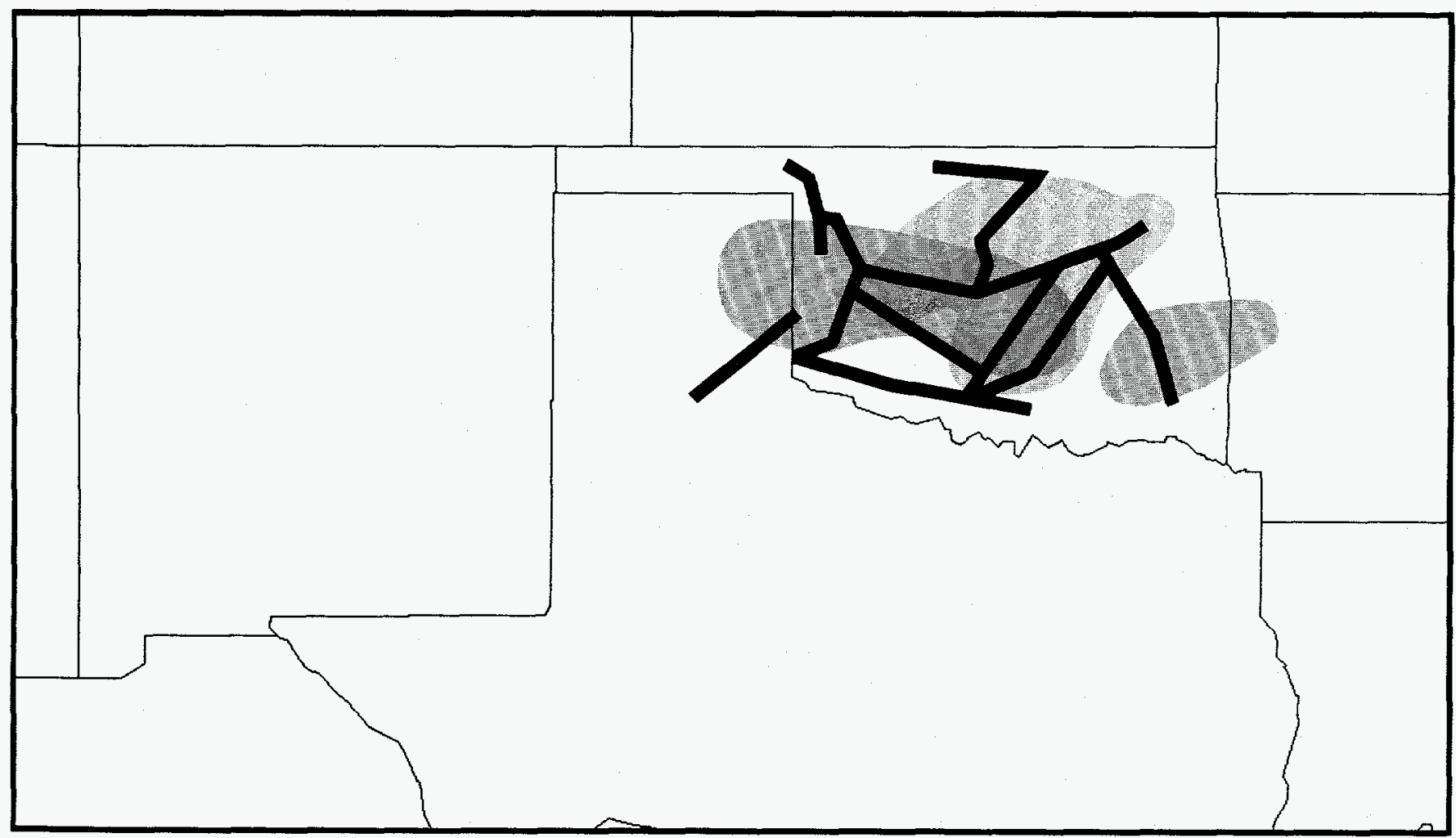

Source: Energy Information Administration (EIA), Form EIA-176, "Annual Report of Natural and Supplemental Gas Supply and Disposition." 
plant partner, Tonkawa Gas Processing Co., have relocated a 90 million cubic feet per day cryogenic processing plant to the construction site where Delhi Gas Pipeline is expanding their pipeline. Oklahoma Natural Gas, ANR Pipeline, and Arkla Energy Resources Co. will connect to Delhi at the plant tailgate.

Oklahoma Natural Gas Company's service territory covers 75 percent of the State-the Nation's third-largest gasproducing State. It owns more than 13,000 miles of distribution pipeline serving 700,000 customers in 331 communities in the State. ONG has direct access to a large number of gas supply sources.

ONG Transmission Company, established in 1985 as a separate company from Oklahoma Natural Gas, has almost 5,000 miles of gathering and transmission pipelines. The system connects supply sources and processing plants throughout the State and transports and sells natural gas to interstate connections. In 1993, ONG Transmission transported over 54 billion cubic feet of natural gas to markets throughout the United States through its links with 10 interstate pipelines. This volume represents 29 percent more than was transported the year before.

ONG includes two subsidiaries that own interests in partnerships operating natural gas transmission systems. One, the Caney River Transmission Company, owns a 25-percent interest in the Ozark Transmission system located in the Arkoma Basin. The other subsidiary, TransTex Pipeline Company, owns 25 percent interest in Red River Pipeline, a general partnership, which owns 361 miles of transmission pipeline and related facilities located in the panhandle of Texas.

ONG Western, Inc., operates a 525-mile transmission system connecting ONG Transmission's major pipeline system with the Anadarko Basin in western Oklahoma.
An additional 8-mile long pipeline that interconnects ONG Western with the Red River Pipeline was purchased in 1993 from United Gas Pipeline and Amoco Production Company. It is expected to improve ONG's ability to deliver gas to customers and to increase transportation services. ONG Sayre Storage Company purchases, stores, and delivers gas in western Oklahoma. ONG Red Oak Transmission Company purchases gas in southeastern Oklahoma and delivers it to other natural gas markets through a 90-mile pipeline ending near Tulsa.

Another small 4-1/2-mile pipeline was purchased from Associated Natural Gas, Inc., last year. It will interconnect ONEOK's pipeline system in western Oklahoma with Northern Natural Gas Company.

Oklahoma Natural Gas utilizes five underground natural gas storage facilities. On peak winter days, the storage facilities can supply up to 50 percent of their deliverability requirements to retail customers throughout Oklahoma. The largest markets are in the Oklahoma City and Tulsa metropolitan areas.

OK Tex Pipeline Company, another subsidiary of ONEOK, owns several transmission pipelines in Oklahoma and Texas that connect ONG's intrastate system to Lone Star Gas Company and to the Red River Pipeline. OK Tex has the capacity to move 200 million cubic feet of gas per day.

Oklahoma Natural Gas is one of the Nation's leading operators of natural gas vehicles (NGV), and in 1993, about 1,300 NGV's were operating in the State. Tinker Air Force Base has the largest NGV fleet operating in any Department of Defense facility. Also, the Tulsa Public School System operates the Nation's largest fleet of school buses fueled by natural gas. 
Table 36. ONEOK, Inc., Natural Gas Data, 1993

(Thousand Cubic Feet)

\begin{tabular}{|c|c|c|c|c|c|c|}
\hline \multirow[t]{2}{*}{ Supply/Disposition } & $\begin{array}{c}\text { Oklahoma } \\
\text { Natural Gas Co. }\end{array}$ & $\begin{array}{l}\text { ONG Sayre } \\
\text { Storage Co. }\end{array}$ & $\begin{array}{l}\text { ONG Red Oak } \\
\text { Transmission } \\
\text { Co. }\end{array}$ & $\begin{array}{l}\text { ONG Western, } \\
\text { Inc. }\end{array}$ & $\begin{array}{l}\text { Oktex Pipeline } \\
\text { Co. }\end{array}$ & $\begin{array}{l}\text { Red River } \\
\text { Pipeline Co. }\end{array}$ \\
\hline & Oklahoma & Oklahoma & Oklahoma & Oklahoma & Oklahoma & Texas \\
\hline \multicolumn{7}{|l|}{ SUPPLY } \\
\hline Produced Onsystem & 0 & 0 & 0 & 0 & 0 & 0 \\
\hline 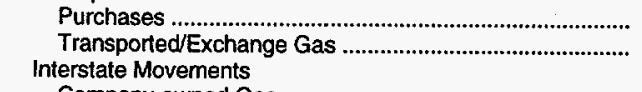 & $\begin{array}{l}148,003,247 \\
202,780,658\end{array}$ & $\begin{array}{r}723,995 \\
4,407,755\end{array}$ & $68,238,166$ & $\begin{array}{r}8,039,623 \\
89,701,839\end{array}$ & $\begin{array}{l}0 \\
0\end{array}$ & $\begin{array}{r}23,061 \\
23,728,643\end{array}$ \\
\hline 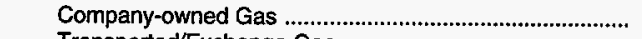 & 0 & 0 & 0 & 0 & 0 & 0 \\
\hline 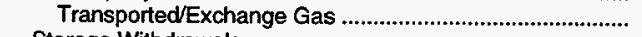 & 0 & 0 & 0 & 0 & $10,995,986$ & 0 \\
\hline Storage Withdrawals & $30,939,389$ & 0 & 0 & 0 & 0 & 0 \\
\hline Received from Storage Operators & $1,676,912$ & $1,676,912$ & 0 & 0 & 0 & 0 \\
\hline 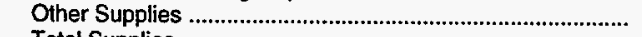 & 0 & 0 & 0 & 0 & 0 & 0 \\
\hline 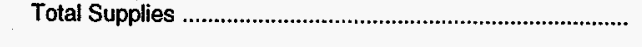 & $383,400,206$ & $6,808,662$ & $68,238,166$ & $97,741,462$ & $10,995,986$ & $23,751,704$ \\
\hline \multicolumn{7}{|l|}{ DISPOSITION } \\
\hline Lease and Plant Use & 240,732 & 0 & 0 & 0 & 0 & 0 \\
\hline $\begin{array}{l}\text { Retumed to Formations } \\
\text { Interstate Movements }\end{array}$ & 0 & 0 & 0 & 0 & 0 & 0 \\
\hline \multicolumn{6}{|l|}{ Interstate Movements } & \\
\hline & $\begin{array}{l}0 \\
0\end{array}$ & 0 & $\begin{array}{l}0 \\
0\end{array}$ & 0 & 0 & 0 \\
\hline 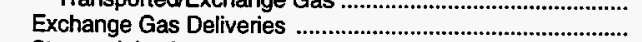 & & $4,494,206$ & $\begin{array}{r}0 \\
67,734,330\end{array}$ & 0 & 0 & 0 \\
\hline & $\begin{array}{l}79,351,080 \\
36,236,052\end{array}$ & 0 & & $88,118,805$ & 0 & 440,160 \\
\hline \multicolumn{6}{|l|}{ Deliveries of Company-owned Gas } & \\
\hline To Storage Operators & $2,518,595$ & 0 & 0 & 0 & 0 & 0 \\
\hline 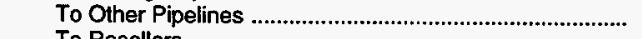 & & 0 & 0 & 0 & 0 & 0 \\
\hline 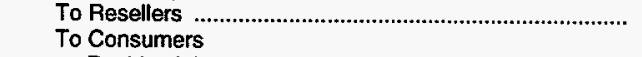 & $1,631,538$ & 723,995 & 0 & $8,039,623$ & 0 & 0 \\
\hline 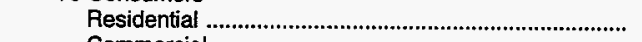 & $61,633,711$ & 0 & 0 & 0 & 0 & 0 \\
\hline 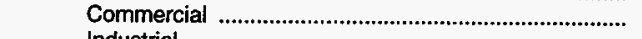 & $28,272,782$ & 0 & 0 & 0 & 0 & 0 \\
\hline 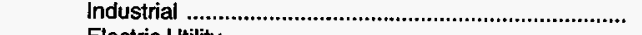 & $52,874,701$ & 0 & 0 & 0 & 0 & 0 \\
\hline 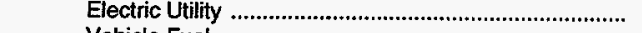 & $1,402,573$ & 0 & 0 & 0 & 0 & 0 \\
\hline 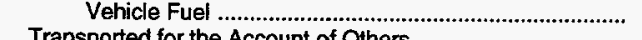 & 105,548 & 0 & 0 & 0 & 0 & 0 \\
\hline \multicolumn{7}{|l|}{ Transported for the Account of Others } \\
\hline $\begin{array}{l}\text { To Storage Operators } \\
\text { To Other Pipelines }\end{array}$ & 0 & $2,518,595$ & 0 & 0 & 0 & 0 \\
\hline 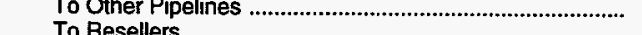 & 0 & 0 & 0 & 0 & 0 & $23,512,823$ \\
\hline $\begin{array}{l}\text { To Resellers } \\
\text { To Consumers }\end{array}$ & $1,848,850$ & 0 & 0 & 0 & $10,995,986$ & 0 \\
\hline 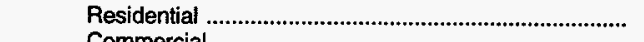 & 0 & 0 & 0 & 0 & 0 & 0 \\
\hline 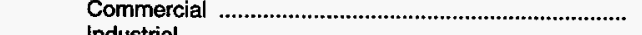 & $2,119,905$ & 0 & 0 & 0 & 0 & 0 \\
\hline 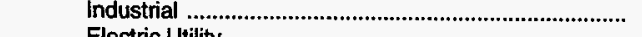 & $104,680,617$ & 0 & 0 & 0 & 0 & 0 \\
\hline 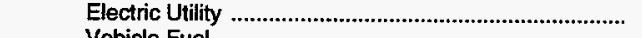 & $5,343,831$ & 0 & 0 & 0 & 0 & 0 \\
\hline 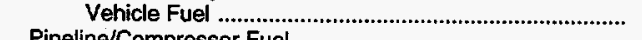 & $\begin{array}{r}0 \\
0\end{array}$ & 0 & 0 & 0 & 0 & 0 \\
\hline 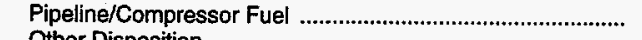 & $1,141,761$ & 0 & 147 & 392 & 0 & 0 \\
\hline 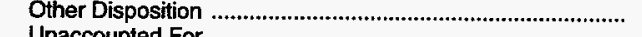 & 0 & 0 & 0 & 0 & 0 & 0 \\
\hline 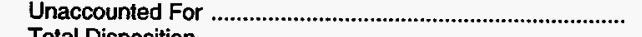 & $3,997,930$ & $-928,134$ & 503,689 & $1,582,642$ & 0 & $-201,279$ \\
\hline 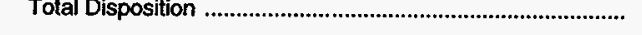 & $383,400,206$ & $6,808,662$ & $68,238,166$ & $97,741,462$ & $10,995,986$ & $23,751,704$ \\
\hline
\end{tabular}

Source: Energy Information Administration (EIA), Form EIA-176, "Annual Report of Natural and Supplemental Gas Supply and Disposition." 



\section{Pacific Gas and Electric Co.}

Pacific Gas and Electric Company (PG\&E), the Nation's largest investor-owned gas and electric company, has operations throughout northern and central California. PG\&E's service territory encompasses 94,000 squaremiles and extends from Eureka to Bakersfield, California. The company provides natural gas service to 3.6 million customers in northern and central California. Its gas supplies come primarily from Canada, with lesser amounts from the Southwestern United States and California.

Pacific Gas Transmission (PGT), a wholly owned subsidiary of PG\&E, transports gas from Canada through the Pacific Northwest into California. Expansion of the pipeline from Alberta, Canada to California was completed and placed into service in November 1993. This expansion will bring an additional 903 million cubic feet per day of firm capacity to California and the Northwestern States. Because many more customers are buying their own gas supplies, PG\&E is becoming more of an intrastate transporter of gas rather than a market. It will no longer purchase as much gas and plans to sell its interest in pipelines and other natural gas facilities outside of California. For the past several years, PG\&E has been considering the sale of PGT to TransCanada Pipelines. However, during the summer of 1994, PG\&E decided not to sell because both companies could not mutually agree on a sale price.

Figure 29. Pacific Gas and Electric Co., 1993

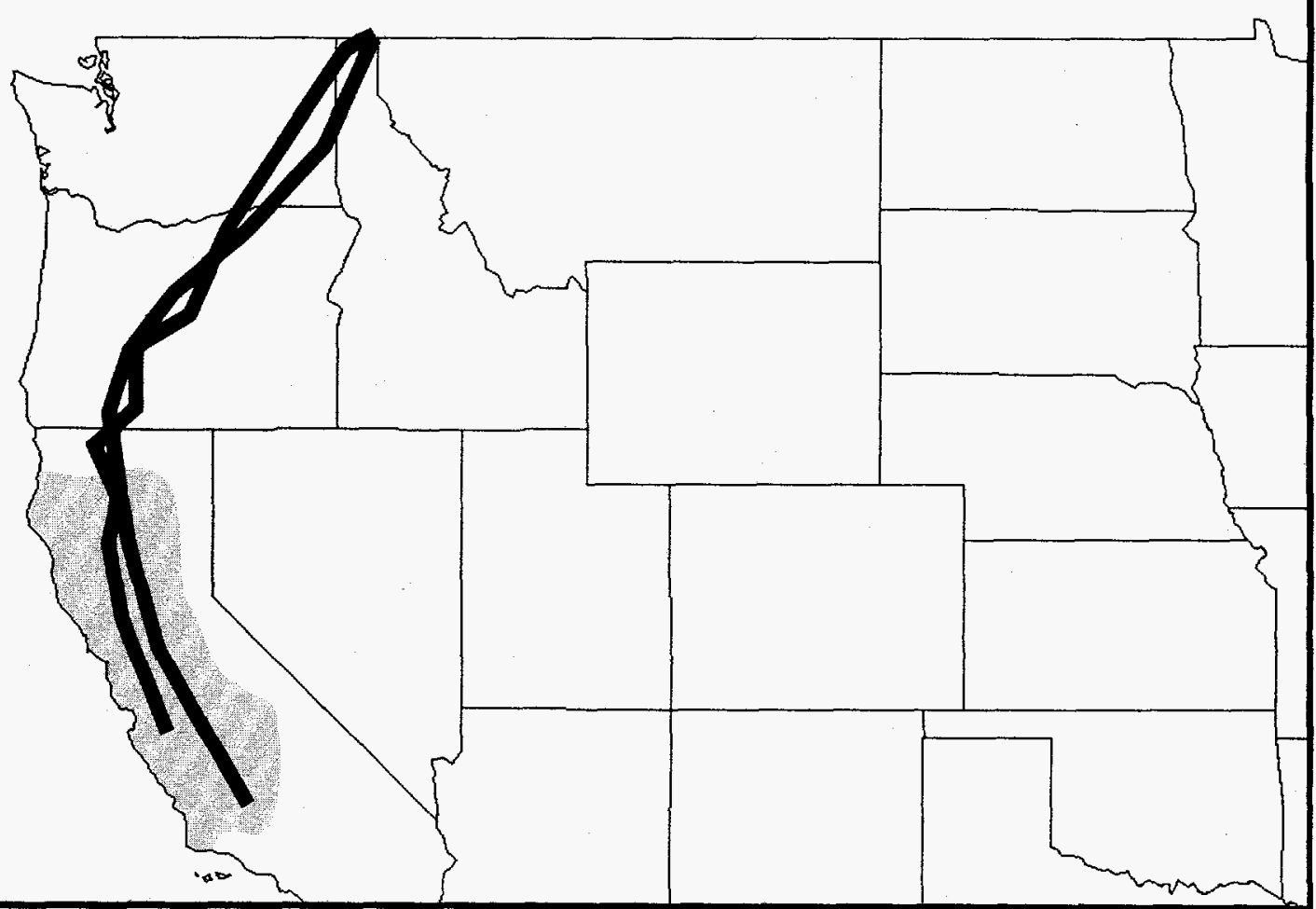

Source: Energy Information Administration (EIA), Form EIA-176, "Annual Report of Natural and Supplemental Gas Supply and Disposition." 
Table 37. Pacific Gas and Electric Co., Natural Gas Data, 1993 (Thousand Cubic Feet)

\begin{tabular}{|c|c|c|c|c|}
\hline \multirow{2}{*}{ Supply/Disposition } & \multirow{2}{*}{$\frac{\text { Pacific Gas \& Electric Co. }}{\text { California }}$} & \multicolumn{3}{|c|}{ Pacific Gas Transmission Co. } \\
\hline & & Idaho & Washington & Oregon \\
\hline \multicolumn{5}{|l|}{ SUPPLY } \\
\hline \multicolumn{4}{|l|}{ Receipts } & 0 \\
\hline 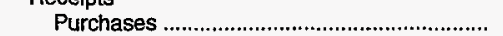 & $33,609,000$ & 0 & 0 & 0 \\
\hline $\begin{array}{l}\text { Transported/Exchange Gas ................................ } \\
\text { Interstate Movements }\end{array}$ & \multicolumn{3}{|c|}{ Interstate Movements } & $14,453,103$ \\
\hline 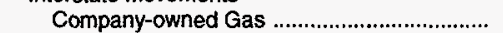 & $296,784,839$ & $267,644,194$ & 0 & 0 \\
\hline 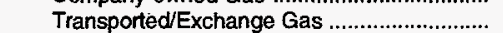 & $310,519,683$ & $244,091,494$ & $515,637,473$ & $473,576,300$ \\
\hline 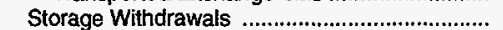 & $32,229,000$ & 0 & 0 & 0 \\
\hline 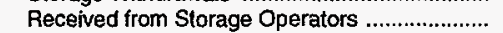 & 0 & 0 & 0 & 0 \\
\hline Other Supplies & 319,200 & 0 & 0 & 0 \\
\hline 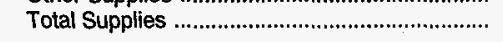 & $782,663,722$ & $511,735,688$ & $515,646,528$ & $488,029,403$ \\
\hline \multicolumn{5}{|l|}{ DISPOSITION } \\
\hline Lease and Plant Use & 0 & 0 & 0 & 0 \\
\hline Returned to Formations .......................................... & 0 & 0 & 0 & 0 \\
\hline \multicolumn{5}{|l|}{ Interstate Movements } \\
\hline 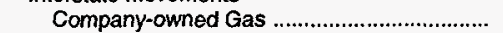 & 0 & $266,406,012$ & $265,240,866$ & $263,818,062$ \\
\hline 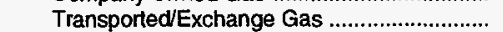 & 0 & $249,231,461$ & $208,344,489$ & $115,445,777$ \\
\hline 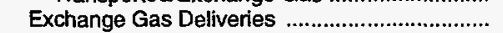 & 0 & 0 & 0 & 0 \\
\hline Storage Injections & $42,465,000$ & 0 & 0 & 0 \\
\hline \multicolumn{5}{|l|}{ Deliveries of Company-owned Gas } \\
\hline 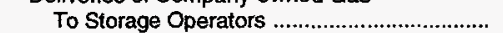 & 0 & 0 & 0 & 0 \\
\hline To Other Pipelines & 0 & 0 & 0 & 0 \\
\hline $\begin{array}{l}\text { To Resellers } \\
\text { To Consumers }\end{array}$ & $8,499,759$ & 0 & 0 & 0 \\
\hline 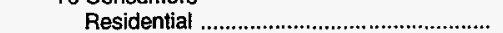 & $207,109,689$ & 0 & 0 & 0 \\
\hline 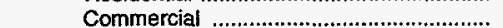 & $115,784,393$ & 0 & 0 & 0 \\
\hline 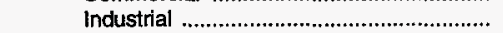 & $98,507,979$ & 0 & 0 & 0 \\
\hline Electric Utility & $75,115,179$ & 0 & 0 & 0 \\
\hline Vehicle Fuel & 111,395 & 0 & 0 & 0 \\
\hline \multicolumn{5}{|l|}{ Transported for the Account of Others } \\
\hline 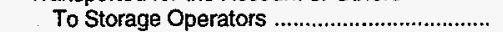 & 0 & 0 & 0 & 0 \\
\hline To Other Pipelines & $19,729,693$ & 850,194 & $39,983,831$ & $103,999,135$ \\
\hline To Resellers & 939,168 & 0 & 0 & 0 \\
\hline \multicolumn{5}{|l|}{ To Consumers } \\
\hline 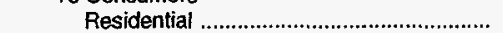 & 0 & 0 & 0 & 0 \\
\hline 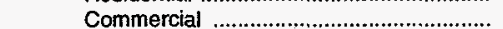 & $12,649,375$ & 0 & 0 & 0 \\
\hline Industrial & $74,875,637$ & 0 & 0 & 0 \\
\hline Electric Utility & $105,910,301$ & 0 & 0 & 0 \\
\hline Vehicle Fuel & 0 & 0 & 0 & 0 \\
\hline 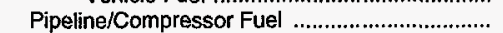 & $5,714,119$ & $1,999,653$ & $1,563,676$ & $4,096,375$ \\
\hline Other Disposition & 733,170 & 99,833 & 173,333 & 218,405 \\
\hline Unaccounted For & $14,518,865$ & $-6,851,465$ & 340,333 & 451,649 \\
\hline Total Disposition & $782,663,722$ & $511,735,688$ & $515,646,528$ & $488,029,403$ \\
\hline
\end{tabular}

Source: Energy Information Administration (EIA), Form EIA-176, "Annual Report of Natural and Supplemental Gas Supply and Disposition."

Nearly 20 years ago, PG\&E began experimenting with natural gas vehicles (NGV). Currently, the Company and its customers operate nearly 2,000 NGV's. By the end of the decade, PG\&E hopes to have 125,000 fleet vehicles operating on natural gas in northern and central California. The Company continues to fund and install refueling facilities for natural gas vehicles. 
Table 38. Pacific Gas and Electric Co., Interstate Flows of Natural Gas, 1993 (Thousand Cubic Feet)

\begin{tabular}{|c|c|}
\hline Company and State & Volume \\
\hline $\begin{array}{l}\text { Pacific Gas \& Electric Co. } \\
\text { California From } \\
\text { Arizona } \\
\text { Oregon }\end{array}$ & $\begin{array}{l}228,040,683 \\
379,263,839\end{array}$ \\
\hline $\begin{array}{l}\text { Pacific Gas Transmission Co. } \\
\text { Canada To } \\
\text { Idaho } \\
\text { Idaho To } \\
\text { Washington } \\
\text { Washington To } \\
\text { Idaho } \\
\text { Oregon } \\
\text { Oregon To } \\
\text { Califomia }\end{array}$ & $\begin{array}{r}511,726,633 \\
515,637,473 \\
9,055 \\
473,576,300 \\
379,263,839\end{array}$ \\
\hline
\end{tabular}

Source: Energy Information Administration (EIA), Form ElA-176, "Annual Report of Natural and Supplemental Gas Supply and Disposition." 


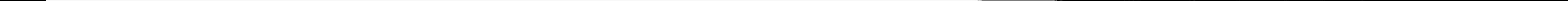




\section{Panhandle Eastern Corp.}

Panhandle Eastern Corporation provides natural gas transportation and related services through four interstate pipeline subsidiaries: Texas Eastern Transmission Corporation (TETCO), Algonquin Gas Transmission Company, Panhandle Eastern Pipeline Company (PEPL), and Trunkline Gas Company. Panhandle operates and owns one of the largest natural gas transmission networks in the Nation. Trunkline LNG Company, Centana Energy Corporation, and Panhandle Trading Company are also subsidiaries of Panhandle Eastern Corporation.

Trunkline LNG operates a liquefied natural gas terminal in Lake Charles, Louisiana that receives and processes shipments from Algeria. Centana is a natural gas gatherer and processor involved in marketing natural gas liquids and producing helium. Panhandle Trading is a nonjurisdictional buyer and seller of natural gas.

On September 1, 1993, Panhandle Eastern Pipeline became the first multi-pipeline operator to fully implement the service restructuring called for in FERC Order 636 by providing unbundled services including storage, gathering, and separate transportation. In May, PEPL started providing restructured services, TETCO and Algonquin began in June, and Trunkline began in September. An approval from the FERC was requested by PEPL to transfer west-end gathering facilities to a subsidiary, Panhandle Field Services Company. The subsidiary was created to operate the gathering system on a nonjurisdictional, openaccess basis.

Figure 30. Panhandle Eastern Corp., 1993

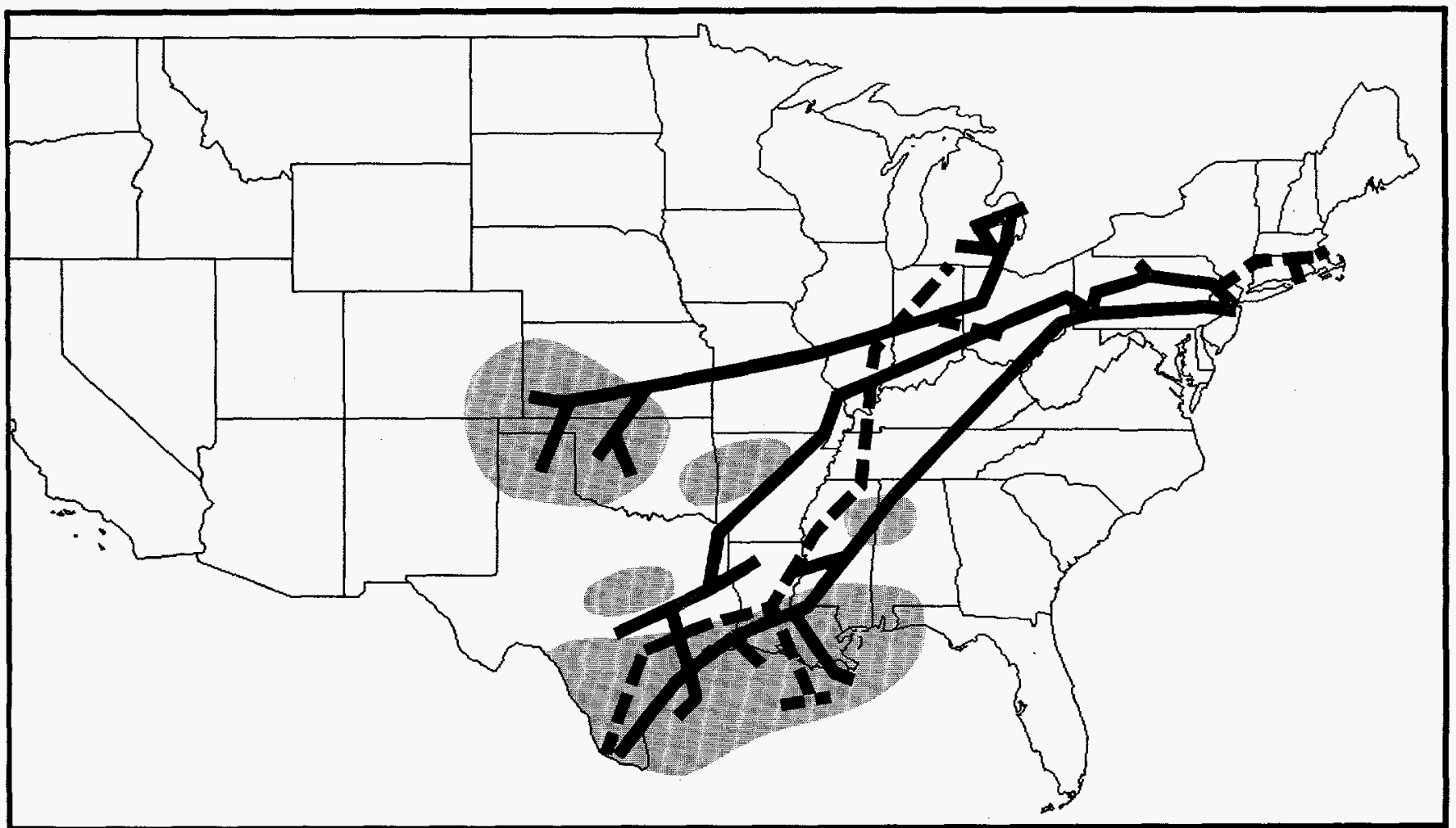

Source: Energy Information Administration (EIA), Form ElA-176, "Annual Report of Natural and Supplemental Gas Supply and Disposition." 
Table 39. Panhandle Eastern Corp., Natural Gas Data, 1993 (Thousand Cubic Feet)

\begin{tabular}{|c|c|c|c|c|c|c|}
\hline \multirow{2}{*}{ Supply/Disposition } & \multicolumn{6}{|c|}{ Panhandle Eastern Pipeline Co. } \\
\hline & Texas & Oklahoma & Wyoming & Colorado & Kansas & Missouri \\
\hline \multicolumn{7}{|l|}{ SUPPLY } \\
\hline $\begin{array}{l}\text { Produced Onsystem } \\
\text { Receipts }\end{array}$ & 0 & 0 & 0 & 0 & 0 & 0 \\
\hline 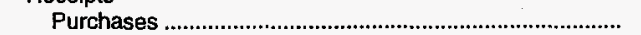 & 818,280 & $4,883,500$ & $1,633,456$ & $3,774,646$ & $5,959,576$ & 0 \\
\hline 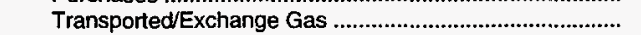 & $59,102,372$ & $182,199,791$ & $2,183,522$ & $17,810,670$ & $209,860,617$ & 577,359 \\
\hline Interstate Movements & & & & & & \\
\hline 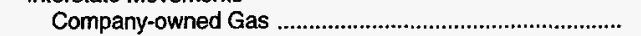 & 0 & 0 & 0 & 0 & 0 & 0 \\
\hline 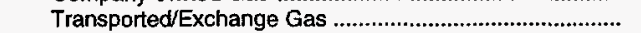 & $15,981,821$ & $75,222,656$ & 0 & 0 & $246,135,939$ & $414,991,246$ \\
\hline Storage Withdrawals & 0 & $5,937,587$ & 0 & 0 & 0 & 0 \\
\hline 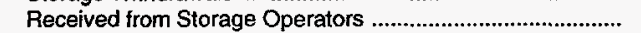 & 0 & 0 & 0 & 0 & $10,552,594$ & 0 \\
\hline Other Supplies & 0 & 0 & 0 & 0 & 0 & 0 \\
\hline Total Supplies & $75,902,473$ & $268,243,534$ & $3,816,978$ & $21,585,316$ & $472,508,726$ & $415,568,605$ \\
\hline \multicolumn{7}{|l|}{ DISPOSITION } \\
\hline Lease and Plant Use & 0 & 41,201 & 1,362 & 3,117 & 0 & 0 \\
\hline Returned to Formations & 0 & 0 & 0 & 0 & 0 & 0 \\
\hline \multicolumn{7}{|l|}{ Interstate Movements } \\
\hline 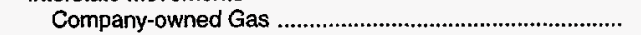 & 0 & 0 & 0 & 0 & 0 & 0 \\
\hline 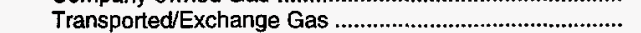 & $75,222,656$ & $262,117,760$ & 0 & 0 & $414,991,246$ & $370,577,791$ \\
\hline Exchange Gas Deliveries & 440,562 & 516,663 & 213,548 & 0 & $12,343,080$ & 0 \\
\hline Storage Injections & 0 & $6,499,650$ & 0 & 0 & 0 & 0 \\
\hline \multicolumn{7}{|l|}{ Deliveries of Company-owned Gas } \\
\hline 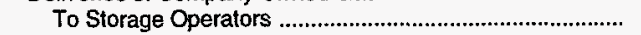 & 0 & 0 & 0 & 0 & $3,937,197$ & 0 \\
\hline 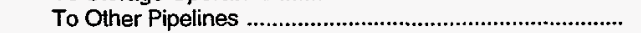 & 0 & 0 & 0 & 0 & 0 & 0 \\
\hline 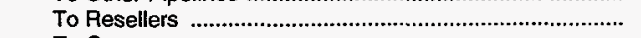 & 512 & 0 & 0 & 0 & $6,686,023$ & $2,850,549$ \\
\hline \multicolumn{7}{|l|}{ To Consumers } \\
\hline 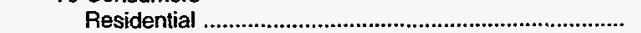 & 7,212 & 13,290 & 0 & 0 & 38,399 & 72,489 \\
\hline 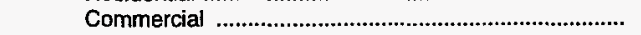 & 25,124 & 13,072 & 0 & 1,208 & 16,682 & 3,744 \\
\hline Indestrial & 0 & 0 & 0 & 0 & 16,045 & 38,309 \\
\hline 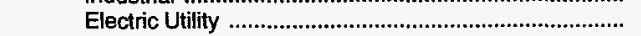 & 0 & 0 & 0 & 0 & 0 & 0 \\
\hline Vehicle Fuel & 0 & 0 & 0 & 0 & 0 & 0 \\
\hline \multicolumn{7}{|l|}{ Transported for the Account of Others } \\
\hline 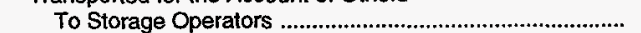 & 0 & 0 & 0 & 0 & 0 & 0 \\
\hline 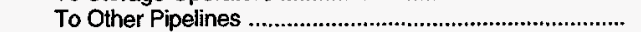 & 0 & 0 & $3,161,956$ & $8,470,823$ & 0 & 0 \\
\hline 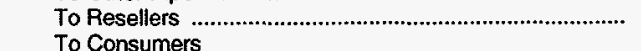 & 201,126 & $1,201,248$ & 0 & $12,431,804$ & $17,410,086$ & $38,906,532$ \\
\hline 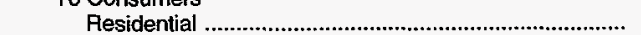 & 0 & 0 & 0 & 0 & 0 & 0 \\
\hline 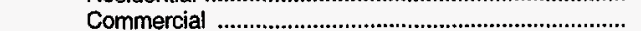 & 0 & 0 & 0 & 0 & 0 & 0 \\
\hline Industrial & 0 & 240,890 & 0 & 0 & $16,713,330$ & $2,879,396$ \\
\hline Electric Utility & 0 & 0 & 0 & 0 & 0 & $\begin{array}{r}0,0700 \\
0\end{array}$ \\
\hline Vehicle Fuel & 0 & 0 & 0 & 0 & 0 & 0 \\
\hline Pipeline/Compressor Fuel & 20,511 & 69,375 & 0 & 0 & 4,447 & 7,669 \\
\hline Other Disposition & 0 & 0 & 0 & 0 & 0 & 0 \\
\hline Unaccounted For & $-15,230$ & $-2,469,615$ & 440,112 & 678,364 & 352,191 & 232,126 \\
\hline Total Disposition & $75,902,473$ & $268,243,534$ & $3,816,978$ & $21,585,316$ & $472,508,726$ & $415,568,605$ \\
\hline
\end{tabular}

In early 1993, 1 Source Corporation was created to pursue new services to increase efficiency and flexibility for natural gas transportation customers. Flex-X and Minute man are original expansion programs coordinated by 1 Source. The Flex-X program has the ability to add capacity in increments tailored to customers' needs rather than constructing a single major expansion project. In Flex$X$ 's first open-season, 145 million cubic feet per day of new capacity was requested. Flex-X's 10-year commitment to new expansion projects would utilize four Panhandle pipelines to support its plan to add 700 million cubic feet per day of firm natural gas transportation service.

The Minuteman project is designed with 100 -plus miles of high pressure pipeline to meet service needs of natural gas utilities, electric power generators, and other customers in the company's northeast markets. Minuteman, operated by Algonquin, will extend from the natural gas pipeline transportation hub at Mendon, Massachusetts to direct customer connections.

PEPL succeeded in Midwest market expansions, signing a 10-year agreement to provide firm transportation and storage service of 75 million cubic feet per day to the expanding Dayton, Ohio market. Partial service began in April 1994.

PEPL is a 50-percent owner of Riverside Storage Company, which is constructing a 3.1 billion cubic feet storage facility in Pennsylvania that is scheduled to begin service in 1995. Also, PEPL is a 10-percent partner in the 42 billion cubic feet Washington 10 storage project, which will convert a depleted natural gas field in the Midwest. Service is slated to begin in 1996. 
Table 39. Panhandle Eastern Corp., Natural Gas Data, 1993 (Continued) (Thousand Cubic Feet)

\begin{tabular}{|c|c|c|c|c|c|c|}
\hline \multirow{2}{*}{ Supply/Disposition } & \multicolumn{4}{|c|}{ Panhandle Eastern Pipeline Co. } & \multicolumn{2}{|c|}{$\begin{array}{l}\text { Texas Eastern Transmission } \\
\text { Corp. }\end{array}$} \\
\hline & Illinois & Indiana & Ohio & Michigan & Texas & Louisiana \\
\hline \multicolumn{7}{|l|}{ SUPPLY } \\
\hline $\begin{array}{l}\text { Produced Onsystem } \\
\text { Receipts }\end{array}$ & 0 & 0 & 0 & 0 & 0 & 0 \\
\hline 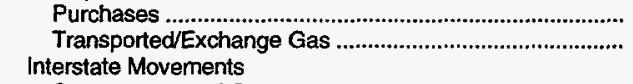 & $81,011,482$ & $\begin{array}{r}0 \\
22,028,578\end{array}$ & $\begin{array}{r}0 \\
7,793,589\end{array}$ & $9,218,321$ & $\begin{array}{r}4,716,494 \\
352,825,958\end{array}$ & $\begin{array}{r}16,570,895 \\
550,201,227\end{array}$ \\
\hline 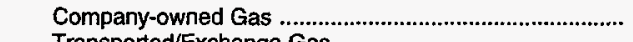 & $\begin{array}{r}0 \\
0709\end{array}$ & 0 & 0 & 0 & 0 & 0 \\
\hline 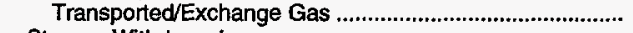 & $370,577,791$ & $364,657,337$ & $213,820,695$ & $107,791,511$ & $69,063,005$ & $255,655,709$ \\
\hline 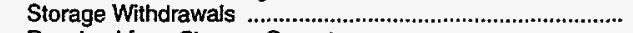 & $1,999,025$ & 0 & 0 & $11,466,658$ & 0 & 0 \\
\hline Received from Storage Operators & 0 & 0 & 0 & $1,911,325$ & 0 & 0 \\
\hline Other Supplies & 0 & 0 & 0 & 0 & 0 & 0 \\
\hline 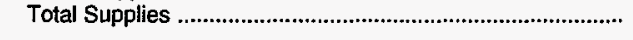 & $453,588,298$ & $386,685,915$ & $221,614,284$ & $130,387,815$ & $426,605,457$ & $822,427,831$ \\
\hline \multicolumn{7}{|l|}{ DISPOSITION } \\
\hline 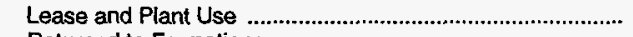 & 0 & 0 & 0 & 0 & $2,901,112$ & $4,759,876$ \\
\hline $\begin{array}{l}\text { Returned to Formations } \\
\text { interstate Movements }\end{array}$ & 0 & 0 & 0 & 0 & 0 & 0 \\
\hline Company-owned Gas & 0 & 0 & 0 & 0 & 0 & 0 \\
\hline 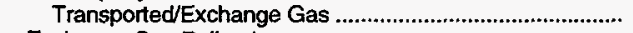 & $364,657,337$ & $213,820,695$ & $100,838,043$ & $13,170,656$ & $358,739,148$ & $710,106,514$ \\
\hline 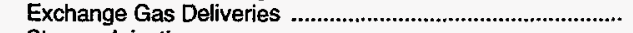 & 0 & 173,086 & 0 & 28,969 & 644,006 & $11,454,890$ \\
\hline 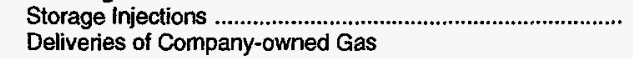 & $3,761,502$ & 0 & 0 & $15,118,056$ & 0 & 0 \\
\hline To Storage Operators & 0 & 0 & 0 & $3,597,754$ & 0 & 0 \\
\hline 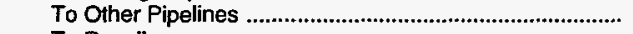 & 0 & 0 & 7,434 & 0 & 0 & 0 \\
\hline 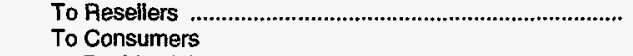 & $1,630,069$ & $1,201,561$ & $2,803,767$ & $6,612,269$ & $2,119,571$ & $9,865,392$ \\
\hline 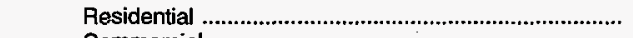 & 0 & 0 & 0 & 0 & 0 & 0 \\
\hline 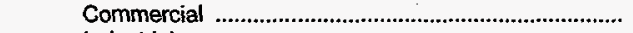 & 0 & 0 & 13,082 & 0 & 0 & 0 \\
\hline Industrial & 58,518 & 4,631 & 7,080 & 930 & 0 & 0 \\
\hline 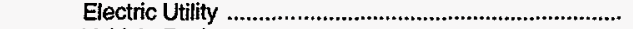 & 38,629 & 0 & 1,628 & 0 & 0 & 0 \\
\hline 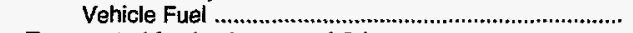 & 0 & 0 & 0 & 0 & 0 & 0 \\
\hline \multicolumn{7}{|l|}{ Transported for the Account of Others } \\
\hline To Storage Operators & 0 & 0 & 0 & 0 & 0 & 0 \\
\hline To Other Pipelines & 0 & $37,025,649$ & 0 & $12,784,940$ & 0 & 6,534 \\
\hline 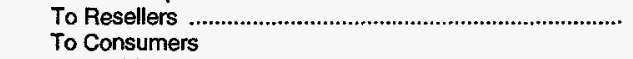 & $60,202,811$ & $126,111,617$ & $103,496,744$ & $41,237,653$ & $69,155,025$ & $81,259,385$ \\
\hline 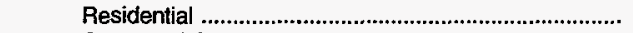 & 0 & 0 & 0 & 0 & 0 & 0 \\
\hline 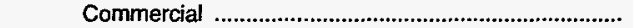 & 0 & 0 & 0 & 0 & 0 & 0 \\
\hline 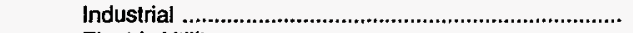 & $24,045,636$ & $8,241,421$ & $14,292,830$ & $43,064,905$ & 0 & 0 \\
\hline 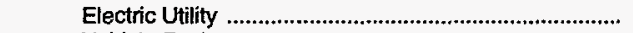 & 0 & 0 & 0 & 0 & 0 & 0 \\
\hline Vehicle Fuel & 0 & 0 & 0 & 0 & 0 & 0 \\
\hline 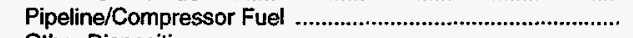 & 198,208 & 73,288 & 102,092 & 436,245 & $1,944,549$ & $4,295,794$ \\
\hline Other Disposition & 0 & 0 & 0 & 0 & 0 & 0 \\
\hline Unaccounted For & $-1,004,412$ & 33,967 & 51,584 & $-5,664,562$ & $-8,897,954$ & 679,446 \\
\hline Total Disposition & $453,588,298$ & $386,685,915$ & $221,614,284$ & $130,387,815$ & $426,605,457$ & $822,427,831$ \\
\hline
\end{tabular}

During 1993, Panhandle concentrated on market expansion. TETCO added 63 million cubic feet per day of transportation service to customers under long-term firm contracts. Algonquin added 90 million cubic feet per day of incremental firm transportation service to New England customers. Trunkline Gas Company started providing 30 million cubic feet per day of firm upstream transportation from the Crossroads Pipeline.

TETCO will provide 80 million cubic feet per day of service to a Philadelphia power facility, and 75 million cubic feet per day of interruptible service will be provided by Algonquin to a Cape Cod generation facility, pending approval from the FERC and minor facilities construction.

A March 12-13, 1994, winter storm caused damage to Texas Eastern's compressor stations in Alabama and Pennsylvania. The damage resulted in a 450 million cubic feet per day reduction in TETCO's throughput; as a result, Algonquin, which receives its supply from Texas Eastern, suffered a 25-percent reduction in deliveries to its customers. By March 17, 1994, repairs were completed and both pipelines resumed deliveries. 
Table 39. Panhandle Eastern Corp., Natural Gas Data, 1993 (Continued) (Thousand Cubic Feet)

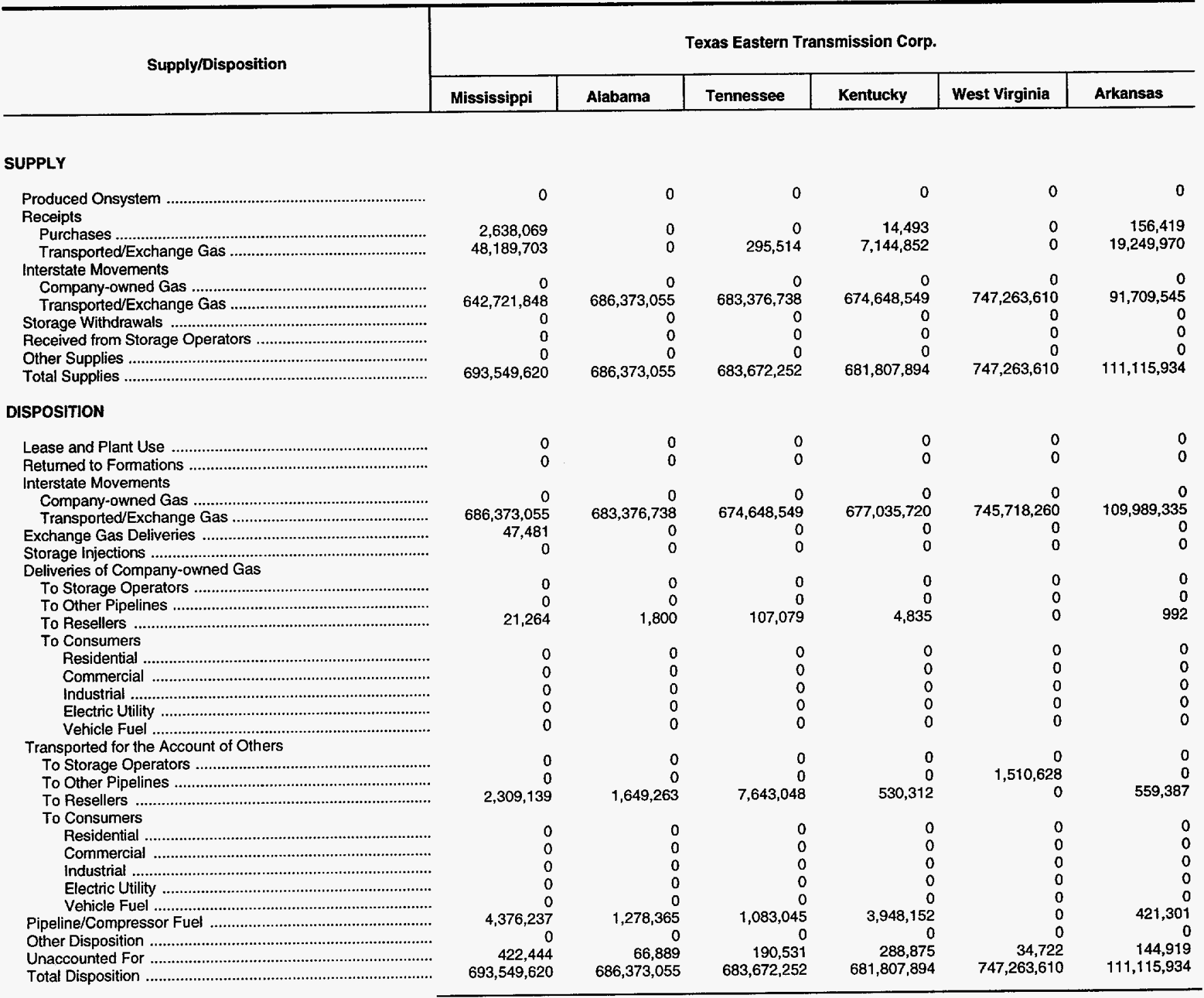


Table 39. Panhandle Eastern Corp., Natural Gas Data, 1993 (Continued) (Thousand Cubic Feet)

\begin{tabular}{|c|c|c|c|c|c|c|}
\hline \multirow{2}{*}{ Supply/Disposition } & \multicolumn{6}{|c|}{ Texas Eastem Transmission Corp. } \\
\hline & Missouri & Illinois & Indiana & Ohio & Pennsylvania & New York \\
\hline \multicolumn{7}{|l|}{ SUPPLY } \\
\hline \multicolumn{7}{|l|}{ Receipts } \\
\hline 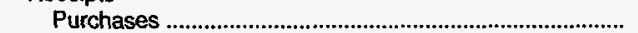 & 0 & 0 & 0 & 24 & 0 & 0 \\
\hline 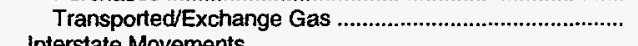 & 93,960 & $42,187,750$ & $19,342,328$ & $74,575,195$ & $95,199,576$ & $19,944,142$ \\
\hline \multicolumn{7}{|l|}{ Interstate Movements } \\
\hline 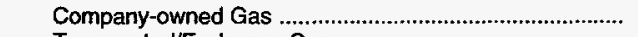 & 0 & 0 & 0 & 0 & 0 & 0 \\
\hline 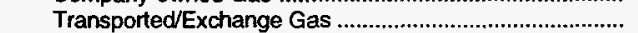 & $109,989,335$ & $104,094,303$ & $141,571,322$ & $833,863,932$ & $745,718,260$ & $61,245,769$ \\
\hline Storage Withdrawals & 0 & 0 & 0 & 0 & 0 & 0 \\
\hline Received from Storage Operators & 0 & 0 & 0 & 0 & $10,950,848$ & 0 \\
\hline Other Supplies & 0 & 0 & 0 & 0 & 0 & 0 \\
\hline 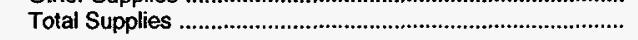 & $110,083,295$ & $146,282,053$ & $160,913,650$ & $908,439,151$ & $851,900,879$ & $81,189,911$ \\
\hline \multicolumn{7}{|l|}{ DISPOSITION } \\
\hline Lease and Plant Use & 0 & 0 & 0 & 0 & 0 & 0 \\
\hline 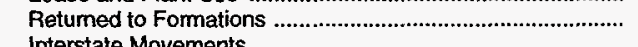 & 0 & 0 & 0 & 0 & 0 & 0 \\
\hline \multicolumn{7}{|l|}{ Interstate Movements } \\
\hline 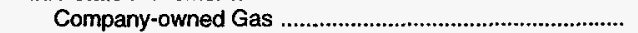 & 0 & 0 & 0 & 0 & 0 & 0 \\
\hline 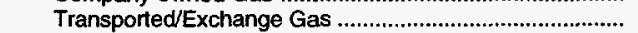 & $104,094,303$ & $141,571,322$ & $156,828,212$ & $747,263,610$ & $345,555,243$ & 0 \\
\hline 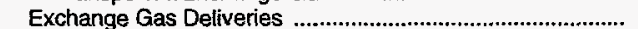 & 0 & 0 & 0 & 0 & $12,397,723$ & 0 \\
\hline Storage Injections & 0 & 0 & 0 & 0 & 0 & 0 \\
\hline \multicolumn{7}{|l|}{ Deliveries of Company-owned Gas } \\
\hline 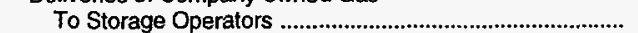 & 0 & 0 & 0 & 0 & $5,372,700$ & 0 \\
\hline To Other Pipelines & 0 & 0 & 2,485 & $2,526,434$ & $1,196,510$ & 0 \\
\hline 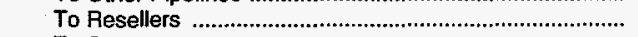 & 356,289 & 115,992 & 194,665 & 160,124 & $14,352,597$ & 930,038 \\
\hline \multicolumn{7}{|l|}{ To Consumers } \\
\hline 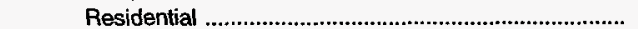 & 0 & 0 & 0 & 0 & 0 & 0 \\
\hline Commercial & 0 & 0 & 0 & 0 & 0 & 0 \\
\hline 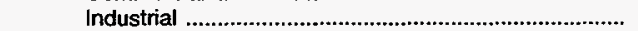 & 0 & 0 & 0 & 0 & 0 & 0 \\
\hline 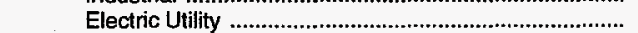 & 0 & 0 & 0 & 0 & 0 & 0 \\
\hline 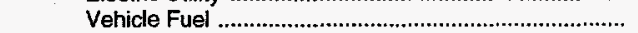 & 0 & 0 & 0 & 0 & 0 & 0 \\
\hline \multicolumn{7}{|l|}{ Transported for the Account of Others } \\
\hline 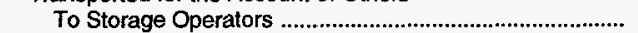 & 0 & 0 & 0 & 0 & 0 & 0 \\
\hline To Other Pipelines & 0 & 0 & 0 & $18,636,032$ & $101,028,471$ & $1,724,343$ \\
\hline To Resellers & $5,195,414$ & $4,289,160$ & $3,450,168$ & $137,057,991$ & $359,928,947$ & $78,533,394$ \\
\hline \multicolumn{7}{|l|}{ To Consumers } \\
\hline 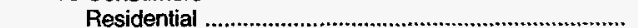 & 0 & 0 & 0 & 0 & 0 & 0 \\
\hline 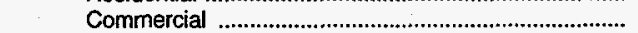 & 0 & 0 & 0 & 0 & 0 & 0 \\
\hline Industrial & 0 & 0 & 0 & 0 & 0 & 0 \\
\hline 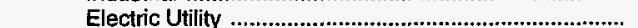 & 0 & 0 & 0 & 0 & 0 & 0 \\
\hline Vehicle Fuel & 0 & 0 & 0 & 0 & 0 & 0 \\
\hline 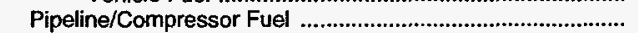 & 406,881 & 259,464 & 334,247 & $2,440,284$ & $11,285,537$ & 0 \\
\hline 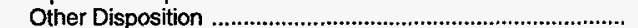 & 0 & 0 & 0 & 0 & 0 & 0 \\
\hline Unaccounted For & 30,408 & 46,115 & 103,873 & 354,676 & 783,151 & 2,136 \\
\hline Total Disposition & $110,083,295$ & $146,282,053$ & $160,913,650$ & $908,439,151$ & $851,900,879$ & $81,189,911$ \\
\hline
\end{tabular}


Table 39. Panhandle Eastern Corp., Natural Gas Data, 1993 (Continued) (Thousand Cubic Feet)

\begin{tabular}{|c|c|c|c|c|c|c|}
\hline \multirow{2}{*}{ Supply/Disposition } & \multicolumn{2}{|c|}{$\begin{array}{l}\text { Texas Eastern Transmission } \\
\text { Corp. }\end{array}$} & \multicolumn{4}{|c|}{ Algonquin Gas Transmission Co. } \\
\hline & New Jersey & Maryland & New Jersey & New York & Connecticut & Rhode Island \\
\hline \multicolumn{7}{|l|}{ SUPPLY } \\
\hline $\begin{array}{l}\text { Produced Onsystem } \\
\text { Receipts }\end{array}$ & 0 & 0 & 0 & 0 & 0 & 0 \\
\hline 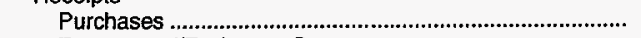 & 0 & 0 & $1,388,470$ & 0 & 0 & 0 \\
\hline 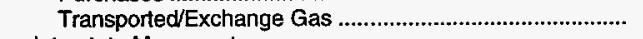 & $33,350,304$ & 0 & $176,387,936$ & 0 & $17,061,579$ & 0 \\
\hline \multicolumn{7}{|l|}{ Interstate Movements } \\
\hline 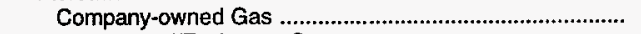 & 0 & 0 & 0 & 0 & 0 & 0 \\
\hline 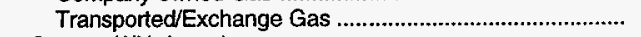 & $341,828,046$ & 333,292 & 0 & $172,721,964$ & $148,585,243$ & $116,522,205$ \\
\hline 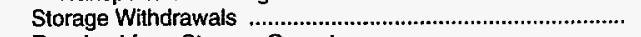 & 0 & $16,179,390$ & 0 & 0 & 0 & 0 \\
\hline 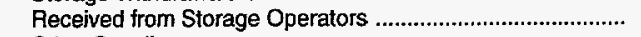 & 0 & 0 & 0 & 0 & 0 & 0 \\
\hline 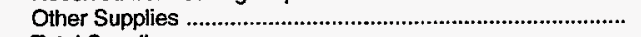 & 0 & 0 & 0 & 0 & 0 & 0 \\
\hline 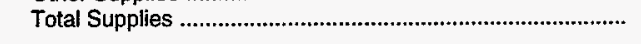 & $375,178,350$ & $16,512,682$ & $177,776,406$ & $172,721,964$ & $165,646,822$ & $116,522,205$ \\
\hline \multicolumn{7}{|l|}{ DISPOSITION } \\
\hline Lease and Plant Use & 0 & 0 & 0 & 0 & 0 & 0 \\
\hline 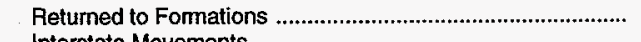 & 0 & 0 & 0 & 0 & 0 & 0 \\
\hline Interstate Movements & & & & & & \\
\hline 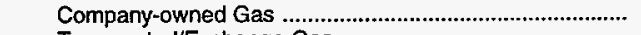 & $\begin{array}{r}0 \\
61,245,769\end{array}$ & $\begin{array}{l}0 \\
0\end{array}$ & $1,734,107$ & $1,665,511$ & $1,230,082$ & 902,984 \\
\hline 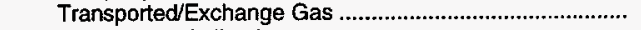 & $61,245,769$ & 0 & $170,987,857$ & $146,919,732$ & $115,292,123$ & $95,131,407$ \\
\hline 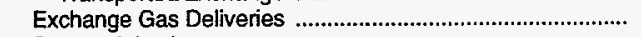 & 0 & 0 & $4,745,259$ & 0 & 0 & 0 \\
\hline Storage Injections & 0 & $15,251,750$ & 0 & 0 & 0 & 0 \\
\hline \multicolumn{7}{|l|}{ Deliveries of Company-owned Gas } \\
\hline 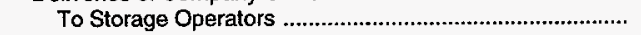 & 0 & 0 & 0 & 0 & 0 & 0 \\
\hline 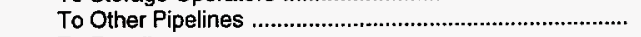 & 23,975 & 0 & 0 & 0 & 0 & 0 \\
\hline $\begin{array}{l}\text { To Resellers } \\
\text { To Consumers }\end{array}$ & 730,338 & $11,918,464$ & 191,550 & 68,596 & 435,429 & 327,097 \\
\hline 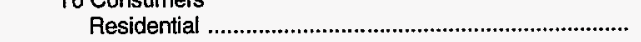 & 0 & 0 & 0 & 0 & 0 & 0 \\
\hline 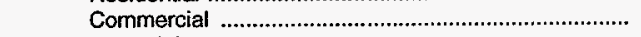 & 0 & 0 & 0 & 0 & 0 & 0 \\
\hline 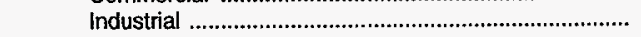 & 0 & 0 & 0 & 0 & 0 & 0 \\
\hline 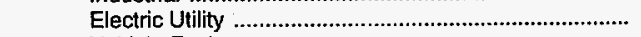 & 0 & 0 & 0 & 0 & 0 & 0 \\
\hline 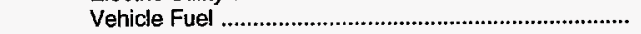 & 0 & 0 & 0 & 0 & 0 & 0 \\
\hline \multicolumn{7}{|l|}{ Transported for the Account of Others } \\
\hline To Storage Operators & 0 & 0 & 0 & 0 & 0 & 0 \\
\hline 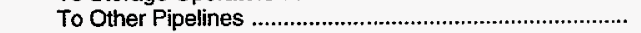 & $7,133,711$ & 0 & 0 & 0 & 0 & 0 \\
\hline 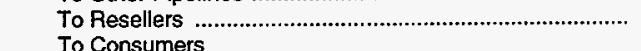 & $305,456,789$ & 0 & 110,053 & $23,642,134$ & $48,482,592$ & $19,805,725$ \\
\hline 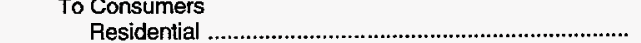 & 0 & 0 & 0 & 0 & 0 & 0 \\
\hline & 0 & 0 & 0 & 0 & 0 & 0 \\
\hline Industrial & 0 & 0 & 0 & 0 & 0 & 247,705 \\
\hline 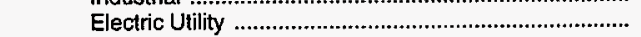 & 0 & 0 & 0 & 0 & 0 & 0 \\
\hline 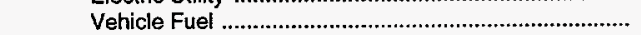 & 0 & 0 & 0 & 0 & 0 & 0 \\
\hline 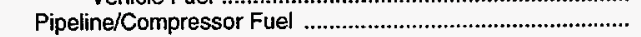 & 483,476 & 613,360 & 24,477 & 506,682 & 370,824 & 175,779 \\
\hline Other Disposition & 0 & 0 & 0 & 0 & 0 & 0 \\
\hline Unaccounted For & 104,292 & $-11,270,892$ & $-16,897$ & $-80,691$ & $-164,228$ & $-68,492$ \\
\hline Total Disposition & $375,178,350$ & $16,512,682$ & $177,776,406$ & $172,721,964$ & $165,646,822$ & $116,522,205$ \\
\hline
\end{tabular}


Table 39. Panhandle Eastern Corp., Natural Gas Data, 1993 (Continued) (Thousand Cubic Feet)

\begin{tabular}{|c|c|c|c|c|c|c|}
\hline \multirow{2}{*}{ Supply/Disposition } & \multirow{2}{*}{$\begin{array}{c}\begin{array}{c}\text { Algonquin Gas } \\
\text { Transmission } \\
\text { Co. }\end{array} \\
\text { Massachusetts }\end{array}$} & \multicolumn{5}{|c|}{ Trunkline Gas Co. } \\
\hline & & Texas & Louisiana & Arkansas & Mississippi & Tennessee \\
\hline \multicolumn{7}{|l|}{ SUPPLY } \\
\hline \multicolumn{7}{|l|}{ Receipts } \\
\hline Purchases & 0 & $1,888,703$ & $14,723,930$ & 0 & 0 & 0 \\
\hline & 0 \\
\hline Company-owned Gas & 0 & 0 & 0 & 0 & 0 & 0 \\
\hline 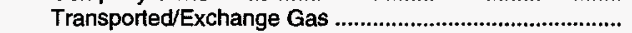 & $96,034,391$ & 0 & $96,205,934$ & $436,765,158$ & $436,766,836$ & $433,957,165$ \\
\hline Storage Withdrawals & 0 & 0 & $10,763,451$ & 0 & 0 & 0 \\
\hline 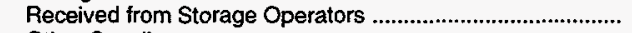 & 0 & 0 & 0 & 0 & 0 & 0 \\
\hline 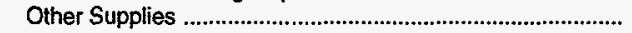 & 0 & 0 & 0 & $\mathbf{0}$ & 0 & 0 \\
\hline 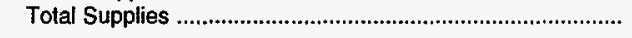 & $133,001,472$ & $104,924,964$ & $813,330,112$ & $436,765,158$ & $436,766,836$ & $433,957,165$ \\
\hline \multicolumn{7}{|l|}{ DISPOSITION } \\
\hline 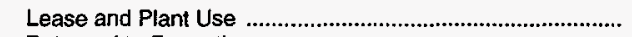 & 0 & 0 & $10,991,675$ & 0 & 0 & 0 \\
\hline $\begin{array}{l}\text { Returned to Formations } \\
\text { Interstate Movements }\end{array}$ & \multicolumn{5}{|c|}{ Interstate Movements } & 0 \\
\hline 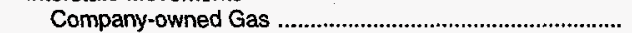 & 0 & 0 & 0 & 0 & 0 & 0 \\
\hline 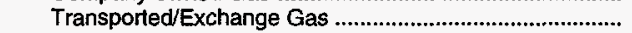 & 0 & $96,205,934$ & $436,765,158$ & $436,766,836$ & $433,957,165$ & $400,730,493$ \\
\hline 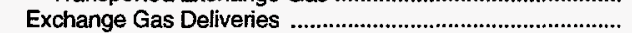 & 0 & 0 & 299,357 & 0 & 0 & 0 \\
\hline Storage Injections & 0 & 0 & $8,873,741$ & 0 & 0 & 0 \\
\hline \multicolumn{7}{|l|}{ Deliveries of Company-owned Gas } \\
\hline To Storage Operators & 0 & 0 & 0 & 0 & 0 & 0 \\
\hline 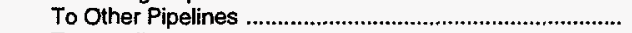 & 6,544 & 0 & 0 & 0 & 0 & 0 \\
\hline $\begin{array}{l}\text { To Resellers } \\
\text { To Consumers }\end{array}$ & 896,441 & 5,995 & 62,474 & 0 & 442,937 & 433,999 \\
\hline 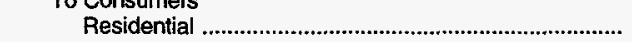 & 0 & 0 & 0 & 0 & 0 & 0 \\
\hline 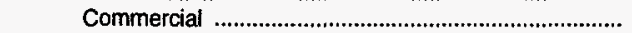 & 0 & 50,508 & 8,608 & 0 & 0 & 0 \\
\hline 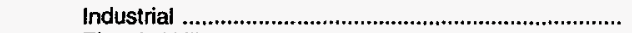 & 0 & 1,551 & 12,441 & 0 & 0 & 17,247 \\
\hline Electric Utility & 0 & 0 & 0 & 0 & 0 & 0 \\
\hline Vehicle Fuel & 0 & 0 & 0 & 0 & 0 & 0 \\
\hline \multicolumn{7}{|l|}{ Transported for the Account of Others } \\
\hline 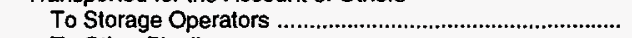 & 0 & 0 & 0 & 0 & 0 & 0 \\
\hline To Other Pipelines & $3,183,637$ & $7,574,467$ & $351,246,532$ & 0 & 539,930 & $32,187,637$ \\
\hline 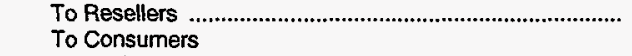 & $105,393,865$ & 0 & 0 & 0 & 0 & \\
\hline 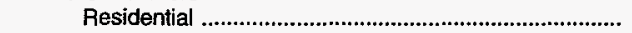 & 0 & 0 & 0 & 0 & 0 & 0 \\
\hline Commercial & 0 & 0 & 0 & 0 & 0 & 0 \\
\hline 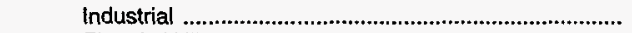 & $23,571,334$ & 0 & 0 & 0 & 0 & 0 \\
\hline 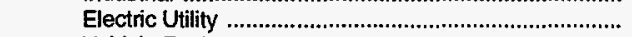 & 337,554 & 0 & 0 & 0 & 0 & 0 \\
\hline Vehicle Fuel & 0 & 0 & 0 & 0 & 0 & 0 \\
\hline 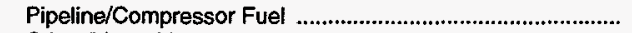 & 56,733 & $1,105,939$ & $5,123,242$ & 0 & $1,839,692$ & 597,155 \\
\hline Other Disposition & 0 & 0 & 0 & 0 & 0 & 0 \\
\hline Unaccounted For & $-444,636$ & $-19,430$ & $-53,116$ & $-1,678$ & $-12,888$ & $-9,366$ \\
\hline Total Disposition & $133,001,472$ & $104,924,964$ & $813,330,112$ & $436,765,158$ & $436,766,836$ & $433,957,165$ \\
\hline
\end{tabular}


Table 39. Panhandle Eastern Corp., Natural Gas Data, 1993 (Continued) (Thousand Cubic Feet)

\begin{tabular}{|c|c|c|c|c|c|c|}
\hline \multirow{2}{*}{ Supply/Disposition } & \multicolumn{4}{|c|}{ Trunkline Gas Co. } & \multirow{2}{*}{$\begin{array}{c}\begin{array}{c}\text { Trunkline LNG } \\
\text { Co. }\end{array} \\
\text { Louisiana } \\
\end{array}$} & \multirow{2}{*}{$\begin{array}{c}\begin{array}{c}\text { Southwest Gas } \\
\text { Storage Co. }\end{array} \\
\text { Kansas }\end{array}$} \\
\hline & Kentucky & Illinois & Indiana & Ohio & & \\
\hline \multicolumn{7}{|l|}{ SUPPLY } \\
\hline $\begin{array}{l}\text { Produced Onsystem } \\
\text { Receipts }\end{array}$ & 0 & 0 & 0 & 0 & 0 & 0 \\
\hline 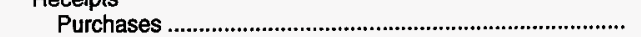 & 0 & 0 & 0 & 0 & 0 & 0 \\
\hline Transported/Exchange Gas & 0 & $1,291,227$ & $38,366,347$ & $39,735,037$ & $1,803,491$ & $18,546,542$ \\
\hline \multicolumn{7}{|l|}{ Interstate Movements } \\
\hline 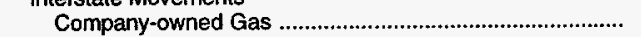 & 0 & 0 & 0 & 0 & 0 & 0 \\
\hline 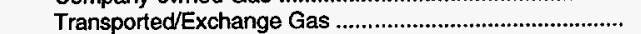 & $400,730,493$ & $400,226,736$ & $217,613,443$ & $38,382,643$ & $30,789,801$ & 0 \\
\hline Storage Withdrawals & 0 & 0 & 0 & 0 & $32,032,666$ & $16,095,316$ \\
\hline 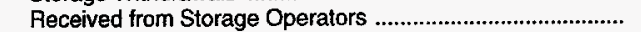 & 0 & 0 & 0 & 0 & 0 & 0 \\
\hline Other Supplies & 0 & 0 & 0 & 0 & 0 & 0 \\
\hline Total Supplies & $400,730,493$ & $401,517,963$ & $255,979,790$ & $78,117,680$ & $64,625,958$ & $34,641,858$ \\
\hline \multicolumn{7}{|l|}{ DISPOSITION } \\
\hline 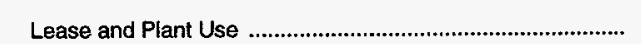 & 0 & 0 & 0 & 0 & 871,003 & 0 \\
\hline 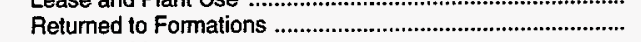 & 0 & 0 & 0 & 0 & 0 & 0 \\
\hline Interstate Movements & & & & & & \\
\hline 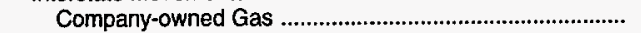 & 0 & 0 & $61,201,288$ & 0 & 0 & 0 \\
\hline 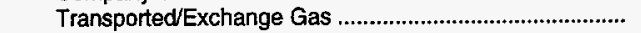 & $400,226,736$ & $217,182,274$ & $151,612,460$ & 0 & 0 & 0 \\
\hline 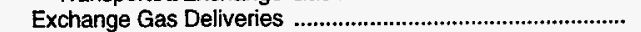 & 0 & 0 & 0 & 0 & $1,674,850$ & 0 \\
\hline Storage Injections & 0 & 0 & 0 & 0 & $30,676,538$ & $18,546,542$ \\
\hline \multicolumn{7}{|l|}{ Deliveries of Company-owned Gas } \\
\hline To Storage Operators & 0 & 0 & 0 & 0 & 0 & 0 \\
\hline To Other Pipelines & 0 & 0 & 0 & 0 & 0 & 0 \\
\hline 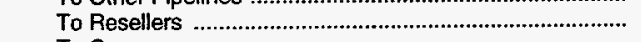 & 261,095 & $2,580,771$ & 751,853 & 0 & $31,070,882$ & 0 \\
\hline To Consumers & & & & & & \\
\hline 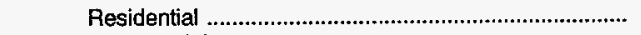 & 0 & 0 & 0 & 0 & 0 & 0 \\
\hline 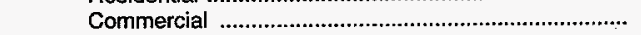 & 0 & 0 & 0 & 0 & 0 & 0 \\
\hline 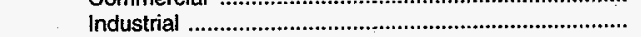 & 0 & 0 & 0 & 0 & 0 & 0 \\
\hline 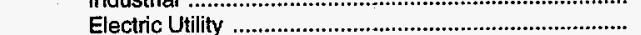 & 0 & 0 & 0 & 0 & 0 & 0 \\
\hline 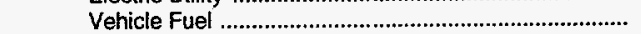 & 0 & 0 & 0 & 0 & 0 & 0 \\
\hline \multicolumn{7}{|l|}{ Transported for the Account of Others } \\
\hline 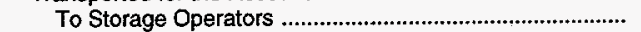 & 0 & 0 & 0 & 0 & 0 & 0 \\
\hline 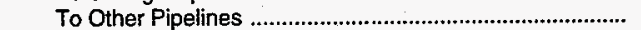 & 246,954 & $179,896,552$ & $42,019,502$ & $78,174,829$ & 0 & $16,027,298$ \\
\hline 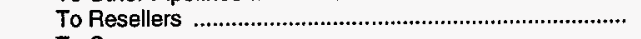 & 0 & 0 & 0 & 0 & 0 & 0 \\
\hline \multicolumn{7}{|l|}{ To Consumers } \\
\hline 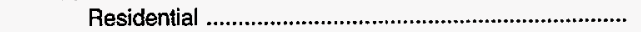 & 0 & 0 & 0 & 0 & 0 & 0 \\
\hline Commercial & 0 & 0 & 0 & 0 & 0 & 0 \\
\hline Industrial & 0 & 0 & 0 & 0 & 0 & 0 \\
\hline Electric Utility & 0 & 0 & 0 & 0 & 0 & 0 \\
\hline Vehicle Fuel & 0 & 0 & 0 & 0 & 0 & 0 \\
\hline 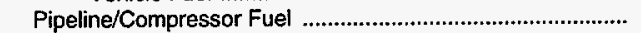 & 0 & $1,878,615$ & 430,067 & 0 & 219,422 & 0 \\
\hline Other Disposition & 0 & 0 & 0 & 0 & 113,263 & 0 \\
\hline Unaccounted For & $-4,292$ & $-20,249$ & $-35,380$ & $-57,149$ & & 68,018 \\
\hline Total Disposition & $400,730,493$ & $401,517,963$ & $255,979,790$ & $78,117,680$ & $64,625,958$ & $34,641,858$ \\
\hline
\end{tabular}

Source: Energy Information Administration (ElA), Form ElA-176, "Annual Report of Natural and Supplemental Gas Supply and Disposition." 
Table 40. Panhandle Eastern Corp., Interstate Flows of Natural Gas, 1993 (Thousand Cubic Feet)

\begin{tabular}{|c|c|}
\hline Company and State & Volume \\
\hline \multicolumn{2}{|l|}{$\begin{array}{l}\text { Panhandle Eastem Pipeline Co. } \\
\text { Texas To }\end{array}$} \\
\hline \multicolumn{2}{|l|}{ Oklahoma To } \\
\hline Kansas . & $246,135,939$ \\
\hline Texas & $15,981,821$ \\
\hline \multicolumn{2}{|l|}{ Kansas To } \\
\hline Missouni & $414,991,246$ \\
\hline \multicolumn{2}{|l|}{ Missouri To } \\
\hline 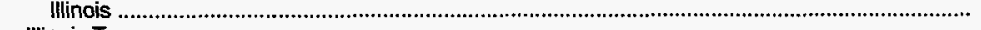 & $370,577,791$ \\
\hline \multicolumn{2}{|l|}{ Illinois To } \\
\hline \multirow{2}{*}{\multicolumn{2}{|c|}{ Indiana To }} \\
\hline & \\
\hline \multirow{2}{*}{\multicolumn{2}{|c|}{ Ohio To }} \\
\hline $\begin{array}{l}\text { Ohio To } \\
\text { Michigan }\end{array}$ & \\
\hline \multicolumn{2}{|l|}{ Michigan To } \\
\hline 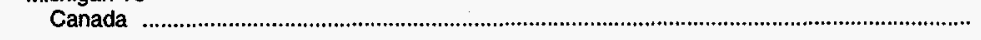 & $13,170,656$ \\
\hline \multicolumn{2}{|l|}{$\begin{array}{l}\text { Texas Eastern Transmission Corp. } \\
\text { Texas To }\end{array}$} \\
\hline \multirow{2}{*}{\multicolumn{2}{|c|}{$\begin{array}{l}\text { Texas To } \\
\text { Arkansas }\end{array}$}} \\
\hline & \\
\hline Louisiana & $255,655,709$ \\
\hline Mexico & $11,373,894$ \\
\hline Louisiana To & \\
\hline Mississippi & $642,721,848$ \\
\hline 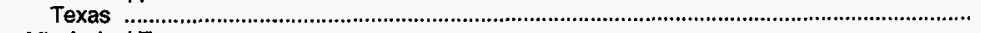 & $67,384,666$ \\
\hline Mississippi To & \\
\hline Alabama & $686,373,055$ \\
\hline Alabama To & \\
\hline 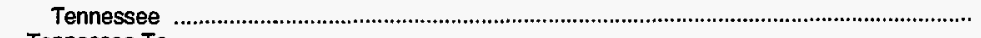 & $683,376,738$ \\
\hline Tennessee To & \\
\hline 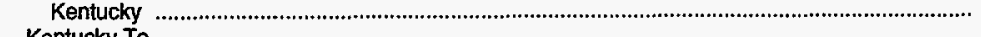 & $674,648,549$ \\
\hline Kentucky To & \\
\hline West Virginia To & $677,035,720$ \\
\hline Pennsylvania & $745,718,260$ \\
\hline Arkansas To & \\
\hline 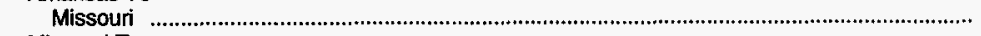 & $109,989,335$ \\
\hline Missouri To & \\
\hline Illinois & $104,094,303$ \\
\hline Illinois To & \\
\hline Indiana & $141,571,322$ \\
\hline Indiana To & \\
\hline & $156,828,212$ \\
\hline $\begin{array}{l}\text { Ohio To } \\
\text { West Virginia }\end{array}$ & \\
\hline $\begin{array}{l}\text { West Virginia } \\
\text { Pennsylvania To }\end{array}$ & $747,263,610$ \\
\hline Delaware & $3,393,905$ \\
\hline 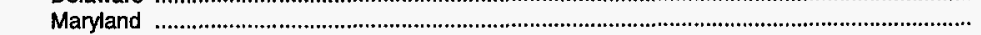 & $\begin{array}{r}3,393,905 \\
333,292\end{array}$ \\
\hline 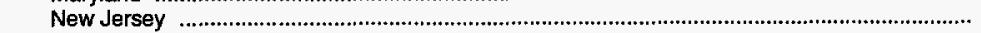 & $341,828,046$ \\
\hline New Jersey To & \\
\hline 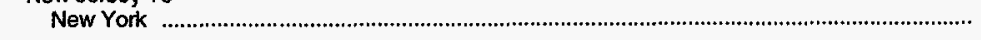 & $61,245,769$ \\
\hline Algonquin Gas Transmission Co. & \\
\hline New Jersey To & \\
\hline New York & $172,721,964$ \\
\hline New York To & \\
\hline 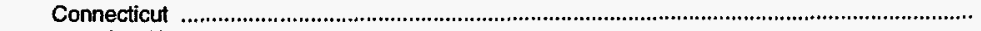 & $148,585,243$ \\
\hline Connecticut To & \\
\hline Rhode Isiand & $\uparrow 16,522,205$ \\
\hline Rhode Island To & \\
\hline 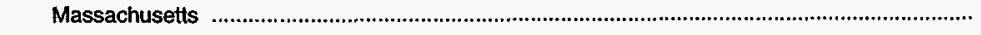 & $96,034,391$ \\
\hline Trunkline Gas Co. & \\
\hline Texas To & \\
\hline 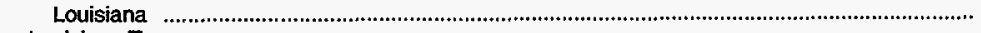 & $96,205,934$ \\
\hline Louisiana To & \\
\hline Arkansas & $436,765,158$ \\
\hline Arkansas To & \\
\hline 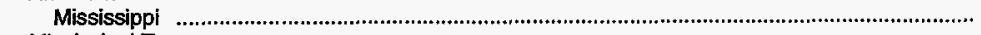 & $436,766,836$ \\
\hline Mississippi To & \\
\hline 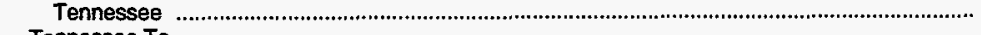 & $433,957,165$ \\
\hline Tennessee To & \\
\hline 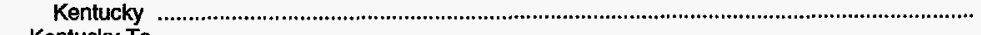 & $400,730,493$ \\
\hline Kentucky To & \\
\hline Illinois & $400,226,736$ \\
\hline Illinois To & \\
\hline 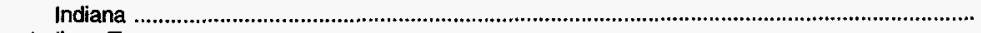 & $217,182,274$ \\
\hline Indiana To & \\
\hline Ohio & $251,196,391$ \\
\hline Trunkline LNG Co. & \\
\hline $\begin{array}{l}\text { Algena To } \\
\text { Louisiana }\end{array}$ & \\
\hline Loulsiana ......, 1, & $30,789,801$ \\
\hline
\end{tabular}

Source: Energy Information Administration (EIA), Form EIA-176, "Annual Report of Natural and Supplemental Gas Supply and Disposition." 



\section{Peoples Gas Light and Coke Co.}

Peoples Energy Corporation is the holding company for Peoples Gas Light and Coke Company, North Shore Gas Company, and Peoples District Energy Corporation. Peoples Gas and North Shore Gas are public utilities primarily engaged in the purchase, production, storage, distribution, sale, and transportation of natural gas in two of the Nation's largest gas-consuming markets. Peoples Gas has approximately 843,000 residential, commercial, and industrial retail sales and transportation customers within the city of Chicago. North Shore Gas, based in Waukegan, Illinois, has more than 126,000 residential, commercial, and industrial retail sales and transportation customers within approximately 275 square miles in Northeastern Illinois.
Peoples Gas and North Shore Gas supply sources are derived from connections to four interstate pipelines as well as from such storage facilities as the Manlove Field underground storage facility, a liquefied natural gas (LNG) plant near Champaign, Illinois, and a synthetic natural gas plant near Joliet, Illinois. Peoples Gas recently signed 5-year storage contracts with both ANR Pipeline and Grands Lacs Market Center. Both projects are in Michigan. The ANR agreement covers 50 days of annual storage with withdrawal rights of 200 million cubic feet per day (10 billion cubic feet total). Grands Lacs will provide up to 150 million cubic feet per day.

Figure 31. Peoples Gas Light and Coke Co., 1993

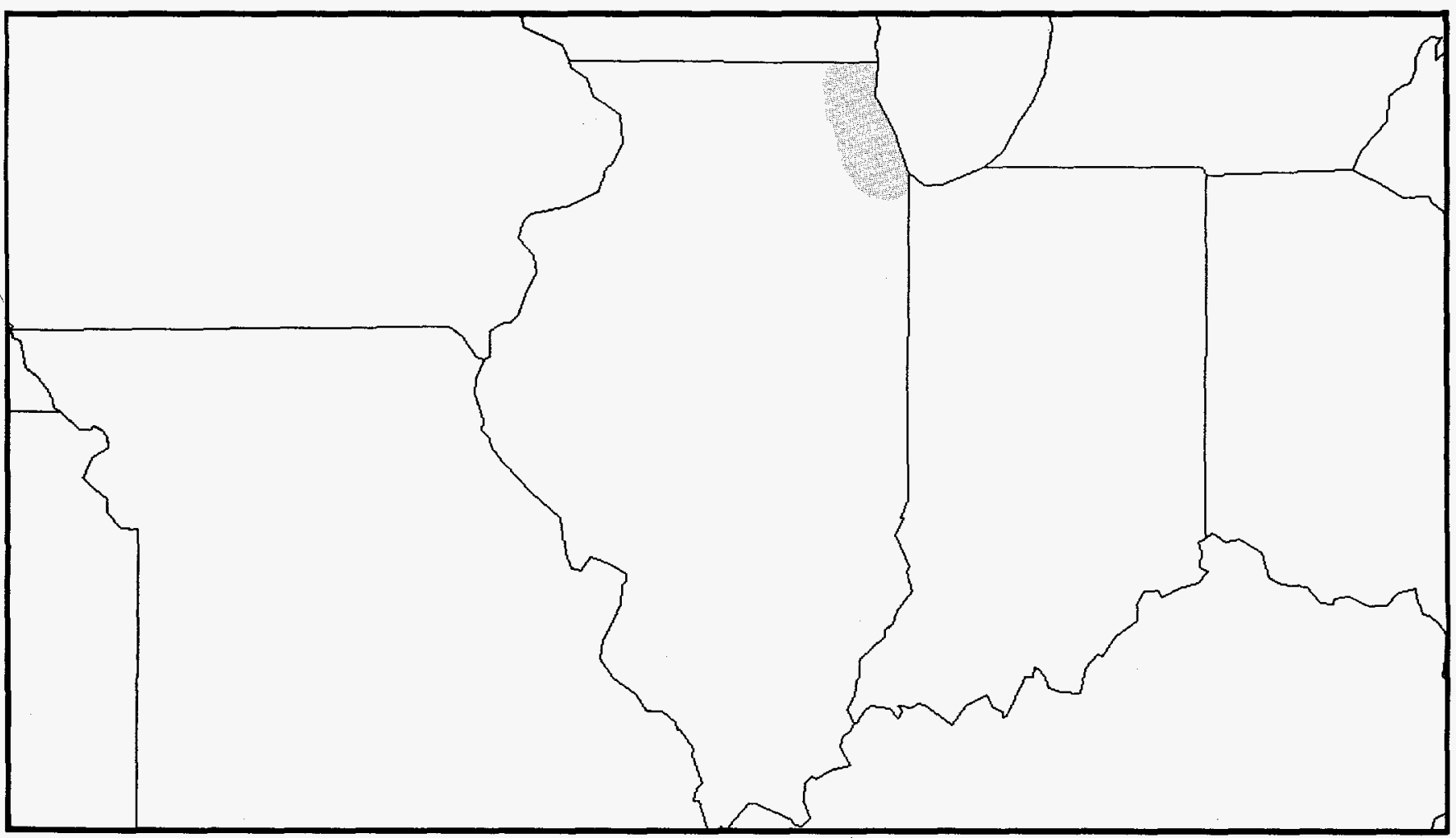

Source: Energy Information Administration (EIA), Form ElA-176, "Annual Report of Natural and Supplemental Gas Supply and Disposition."

\section{Service Area}


Table 41. Peoples Gas Light and Coke Co., Natural Gas Data, 1993 (Thousand Cubic Feet)

\begin{tabular}{l|c|c|c|}
\hline \multirow{2}{*}{ Supply/Disposition } & Peoples Gas Light \& Coke Co. & North Shore Gas Co. \\
\cline { 2 - 4 } & Illinois & Illinois \\
\hline
\end{tabular}

SUPPLY

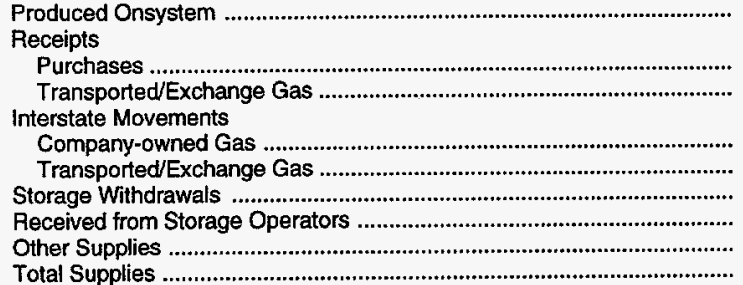

Total Supplies

DISPOSITION
$145,422,786$ $91,797,933$

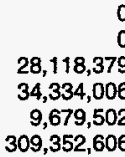

$1,606,042$

(1)

0
0
$2,292,073$

$31,035,549$

$36,943,702$

0

$119,307,895$

$21,923,347$

$5,999,043$

257,641

0

0

0

$23,858,706$

$34,327,624$

$28,257,199$

0
0

$1,014,358$

$2,529,427$

$309,352,606$
0

$25,551,380$

$11,862,211$

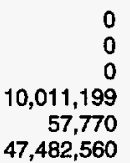

57,770
$47,482,560$

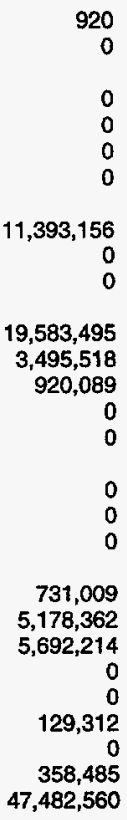

Source: Energy Information Administration (EIA), Form EIA-176, "Annual Report of Natural and Supplemental Gas Supply and Disposition."

During 1993, North Shore installed approximately 14.6 miles of transmission pipeline. Peoples Gas added 8 wells at its Manlove underground storage reservoir, bringing the total to 14 wells drilled through the second year of this 4-year project. Six wells remain to be added. Peoples Gas also replaced several LNG vaporizers at its Manlove Field.

The company continues to expand its nontraditional markets. In 1993, several large local fleets volunteered to test natural gas-fueled vehicles. Recent conversions have included several fleet vans, a double-deck sightseeing bus, and a commercial limousine. Gas absorption chillers, totaling 6,000 tons of cooling capacity, were installed in Peoples Gas and North Shore service areas in 1993.

In the cogeneration market, Peoples added a second hospital to its customer base and expanded another cogeneration customer's existing system by adding a gas-fired engine. As of September 1993, the company had 10 cogeneration customers, who used a total of more than 1 billion cubic feet of gas annually.

Peoples District Energy, a recently formed subsidiary of Peoples Gas, became a 50-percent participant in a partnership formed to provide heating and cooling services to Chicago's McCormick Place exposition and convention center as well as to other large buildings in the vicinity. The partnership, known as Trigen-Peoples District Energy Corporation, will provide service from a central plant known as District Energy. Construction began in the spring of 1993 and is expected to be completed within 4 years. The plant will use a highly efficient technology to supply chilled water and steam, and will be fueled primarily by natural gas. By tying into District Energy systems, businesses can obtain efficient heating and cooling services without investing capital. 


\section{Public Service Co. of Colorado}

Public Service Company of Colorado is an investorowned electric, natural gas, and thermal energy utility serving approximately 2.7 million people throughout Colorado and, through its wholly owned subsidiary, Cheyenne Light, Fuel and Power Company, in the area around Cheyenne, Wyoming. The company's natural gas system consists of more than 13,800 miles of distribution mains.

On January 1, 1993, Western Gas Supply Co. (WestGas) was merged into the company; the three existing WestGas subsidiaries--WestGas Interstate, Inc., WestGas TransColorado, Inc., and WestGas Gathering, Inc.--became wholly owned subsidiaries of Public Service. In August 1994, Public Service sold WestGas Gathering, Inc. Most of the WestGas assets involved in the sale are in Southwest Colorado on the Southern Ute Indian reservation. The new owner and the Southern Ute Tribe plan to form a joint venture, Red Cedar Gathering Company, to operate the facilities.

Decommissioning continued at the company's idle (since 1989) Fort St. Vrain nuclear power plant. The facility will be converted to a natural-gas-burning power plant when the company completes construction of two pipelines that will supply the plant with natural gas to generate electricity. The new pipelines, which will also supply gas to outlying homes, are to be completed by December 1995 .

Figure 32. Public Service Co. of Colorado, 1993

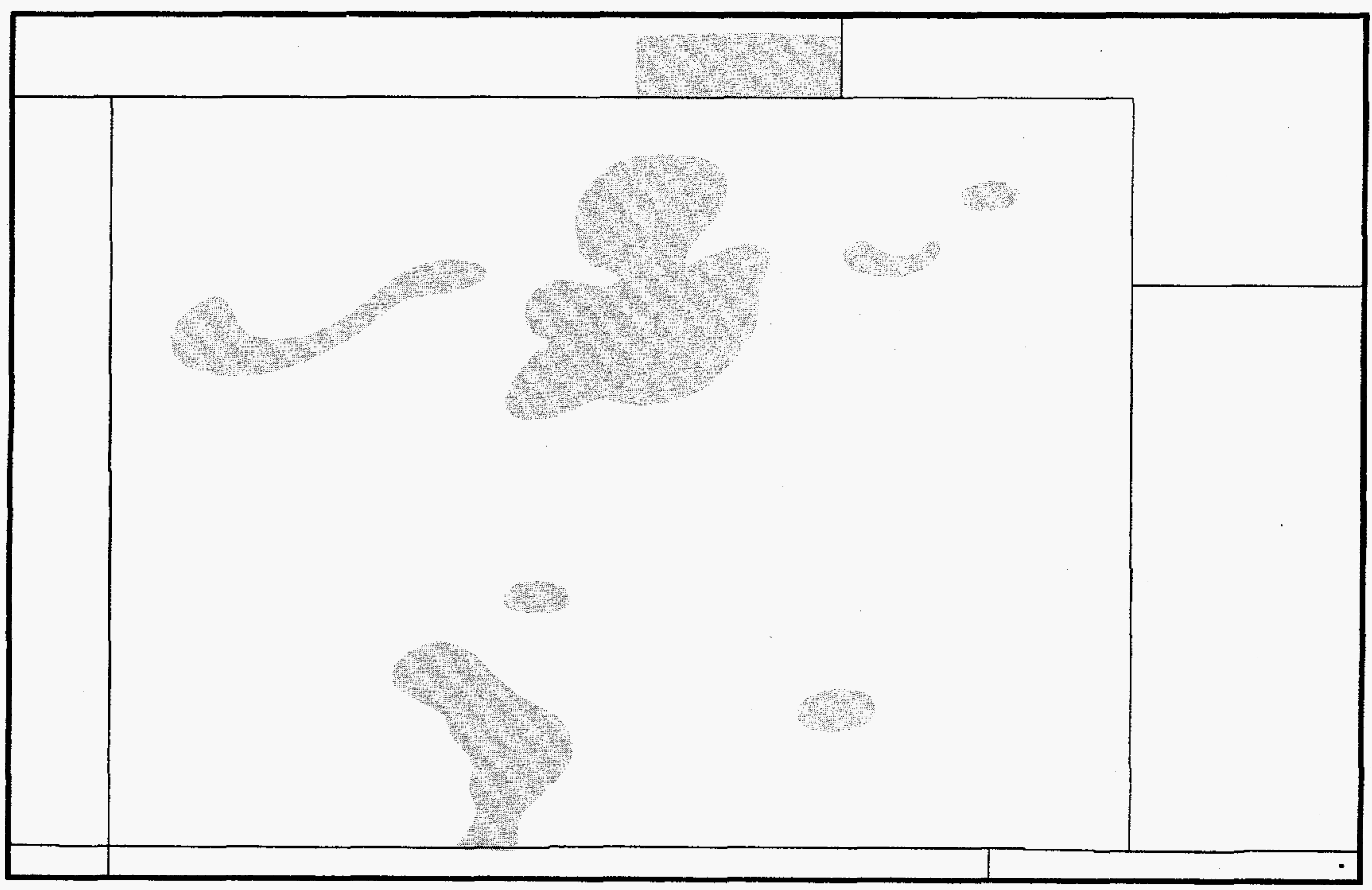

Source: Energy Information Administration (EIA), Form EIA-176, "Annual Report of Natural and Supplemental Gas Supply and Disposition." 
Table 42. Public Service Co. of Colorado Natural Gas Data, 1993 (Thousand Cubic Feet)

\begin{tabular}{|c|c|c|}
\hline \multirow{2}{*}{ Supply/Disposition } & \multirow{2}{*}{$\frac{\text { Public Service Co. of Colorado }}{\text { Colorado }}$} & \multirow{2}{*}{$\frac{\text { Cheyenne Light, Fuel and Power Co. }}{\text { Wyoming }}$} \\
\hline & & \\
\hline \multicolumn{3}{|l|}{ SUPPLY } \\
\hline $\begin{array}{l}\text { Produced Onsystem } \\
\text { Receipts }\end{array}$ & 0 & 0 \\
\hline Pransported/Exchange Gas & $\begin{array}{r}132,384,366 \\
51,834,502\end{array}$ & $\begin{array}{r}4,466,502 \\
11,240,190\end{array}$ \\
\hline 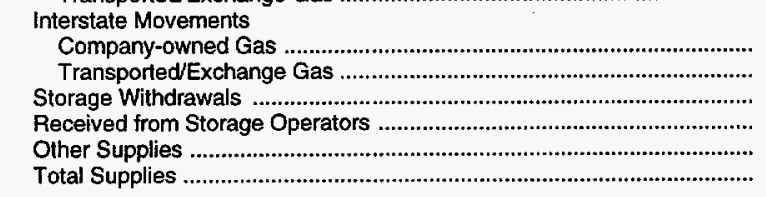 & $\begin{array}{r}0 \\
0 \\
11,719,567 \\
0 \\
470,138 \\
196,408,573\end{array}$ & $\begin{array}{r}0 \\
0 \\
0 \\
0 \\
0 \\
15,706,692\end{array}$ \\
\hline \multicolumn{3}{|l|}{ DISPOSITION } \\
\hline $\begin{array}{l}\text { Lease and Plant Use } \\
\text { Retumed to Formations } \\
\text { Interstate Movements }\end{array}$ & $\begin{array}{r}419,962 \\
0\end{array}$ & $\begin{array}{l}0 \\
0\end{array}$ \\
\hline 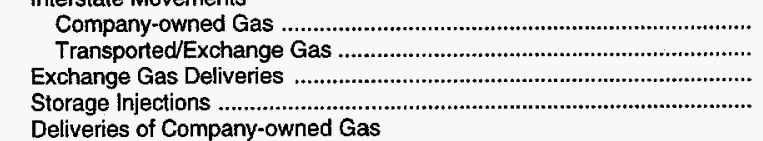 & $\begin{array}{r}0 \\
0 \\
0 \\
8,586,907\end{array}$ & $\begin{array}{l}0 \\
0 \\
0 \\
0\end{array}$ \\
\hline $\begin{array}{l}\text { To Storage Operators } \\
\text { To Other Pipelines } \\
\text { To Resellers } \\
\text { To Consumers }\end{array}$ & $\begin{array}{r}0 \\
0 \\
658,330\end{array}$ & $\begin{array}{l}0 \\
0 \\
0\end{array}$ \\
\hline 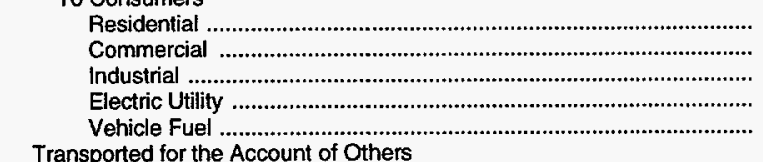 & $\begin{array}{r}80,652,932 \\
51,385,739 \\
0 \\
204,114 \\
0\end{array}$ & $\begin{array}{r}2,392,117 \\
2,118,571 \\
0 \\
0 \\
0\end{array}$ \\
\hline $\begin{array}{l}\text { Transported for the Account of Others } \\
\text { To Storage Operators } \\
\text { To Other Pipelines } \\
\text { To Resellers } \\
\text { To Consumers }\end{array}$ & $\begin{array}{r}0 \\
9,566,590 \\
3,286,754\end{array}$ & $\begin{array}{l}0 \\
0 \\
0\end{array}$ \\
\hline 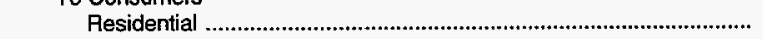 & 0 & 0 \\
\hline 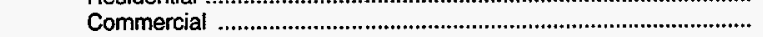 & 0 & 0 \\
\hline Industrial & $36,713,184$ & $11,015,386$ \\
\hline Electric Utility & 133,185 & 0 \\
\hline Vehicle Fuel & 0 & 0 \\
\hline 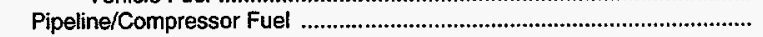 & 522,676 & 0 \\
\hline Other Disposition & 0 & 0 \\
\hline $\begin{array}{l}\text { Unaccounted For } \\
\text { Total Disposition }\end{array}$ & $\begin{array}{r}4,278,200 \\
196,408,573\end{array}$ & $\begin{array}{r}180,618 \\
15,706,692\end{array}$ \\
\hline
\end{tabular}

Source: Energy Information Administration (E|A), Form ElA-176, "Annual Report of Natural and Supplemental Gas Supply and Disposition."

Public Service actively promotes the use of natural gas as an alternative fuel for vehicles and has converted a number of company fleet vehicles to natural gas. During 1994 , the company plans to obtain a total of 40 additional vehicles capable of being fueled with natural gas. To further encourage the use of natural gas as a vehicle fuel, the company is a participant in building natural gas refueling stations. 


\section{Public Service Electric and Gas Co.}

Public Service Electric and Gas Company (PSE\&G), New Jersey's largest utility, is the third largest regulated combined electric and gas operating utility in the United States. PSE\&G provides both electric and gas service to approximately 2 million customers. Certain areas of New Jersey are served with both electric and gas services, while other areas are served by electric only or gas only. The corridor serviced by PSE\&G's electric and gas service is approximately 2,600 square miles, which runs diagonally across New Jersey from the northeastern part of Bergen County to an area below the southwestern part of the City of Camden. PSE\&G's natural gas distribution system is composed of approximately 15,000 miles of distribution line. The territory is composed mostly of New Jersey's six largest municipalities: Camden, Elizabeth, Jersey City, Newark, Paterson, and Trenton.

Current expansion projects include repowering projects at the Burlington Station and the Bergen Generation Station. The repowering will help reduce emissions and increase generating capacity. An oil-fired unit was replaced with a gas-fired unit at the Burlington Station, the Bergen Generation Station project is a combined-cycle conversion that should be completed in the summer of 1995 .

Shell Oil Company and PSE\&G signed an agreement to install the first natural gas vehicle fueling station at a site in Jersey City, by the end of 1994. The public facility

Figure 33. Public Service Electric and Gas Co., 1993

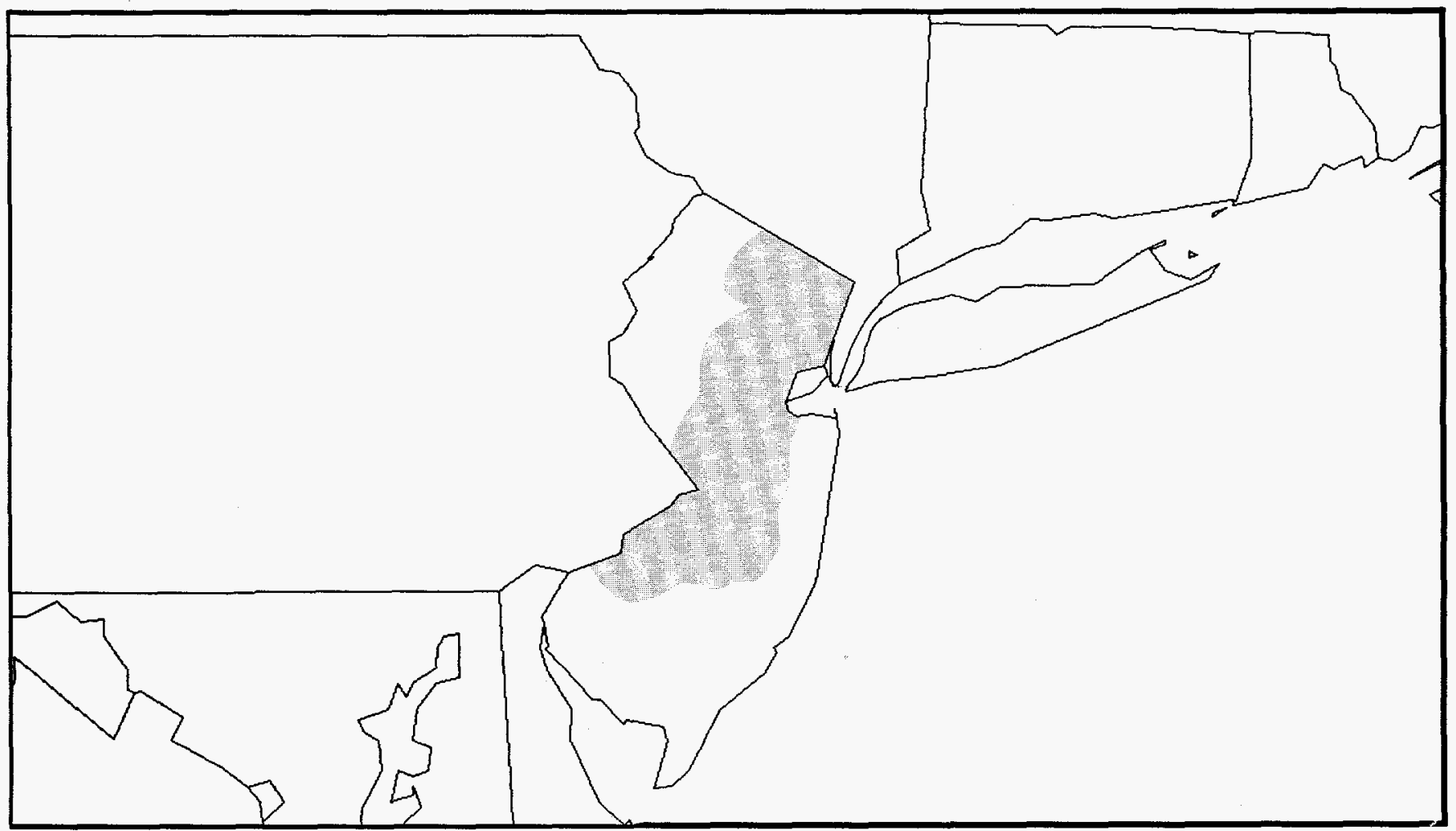

Source: Energy Information Administration (EIA), Form EIA-176, "Annual Report of Natural and Supplemental Gas Supply and Disposition."

Service Area 
Table 43. Public Service Electric and Gas Co., Natural Gas Data, 1993

(Thousand Cubic Feet)

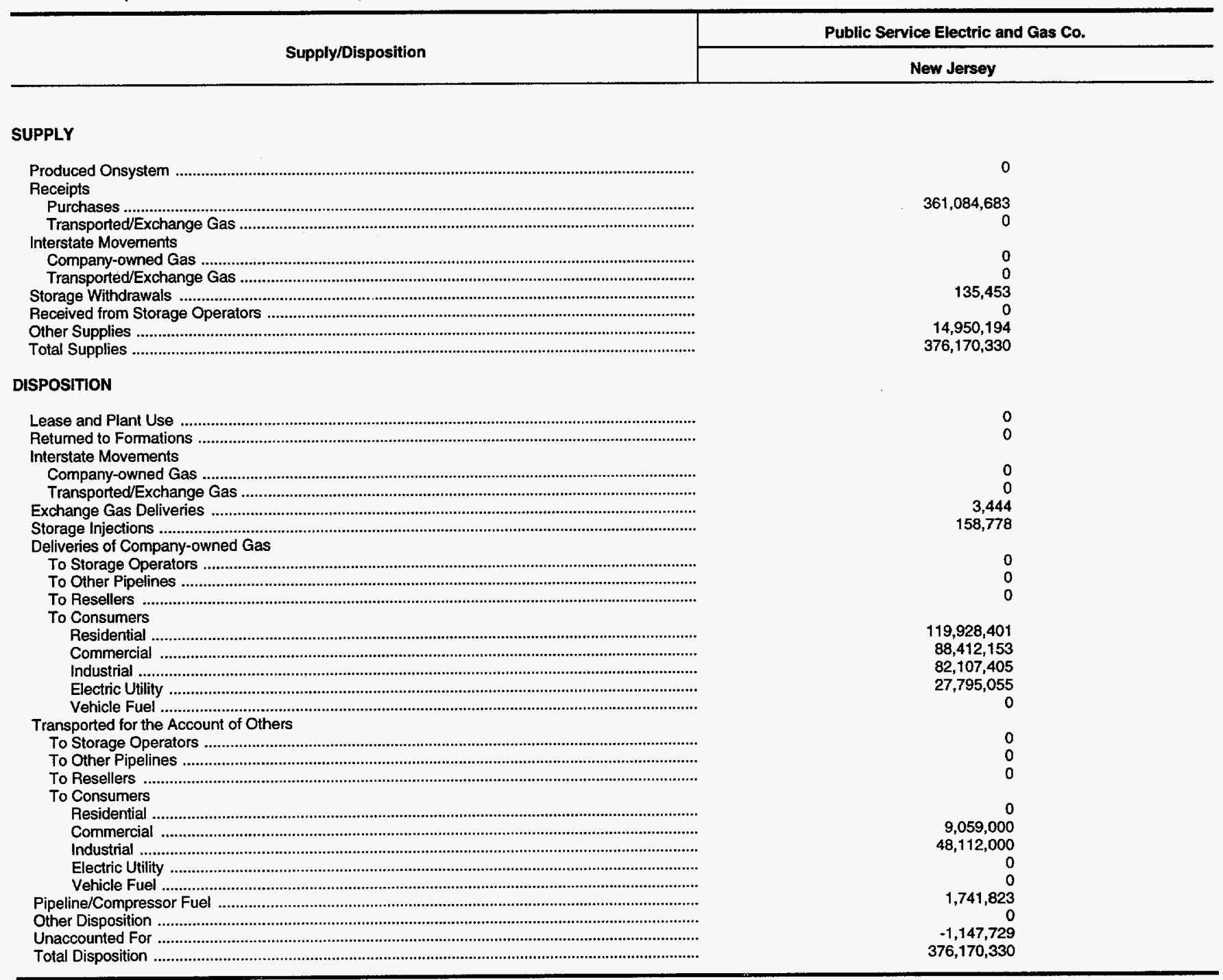

Source: Energy Information Administration (EIA), Form ElA-176, "Annual Report of Natural and Supplemental Gas Supply and Disposition."

will be used by fleet operators and others who are interested in converting to natural gas vehicles (NGV). PSE\&G's NGV program includes projects with Bell Atlantic, the State of New Jersey, and New Jersey Transit. PSE\&G refueling services support more than 350 privately owned natural gas vehicles.

PSE\&G has approximately 4,000 vehicles in its fleet, 150 of which are natural gas vehicles. By the year 2000, PSE\&G plans to have more than half of its service vehicle fleet powered by natural gas and to have 60 percent of its automotive fleet consist of alternative fuel vehicles. PSE\&G's goal is 75 percent by the year 2002 .
In early 1994, Public Service Gas Marketing Company, a subsidiary of PSE\&G, joined the Cincinnati Gas and Electric Company and formed a retail natural gas marketing partnership to sell natural gas to consumers.

In October 1994, PSE\&G of New Jersey proposed guidelines that offered gas transportation and other services to approximately 170,000 industrial and commercial gas customers. This service enables customers to participate in the competitive marketplace by giving them the option of purchasing gas directly from producers and marketers. The customers joined the 342 large-business customers that pay PSE\&G to transport and deliver the gas. 


\section{Questar Corp.}

Questar Corporation's eventual founding originated in 1929 with the construction of a natural gas pipeline from southwestern Wyoming to Utah markets. In 1935, the holding companies were consolidated resulting in Mountain Fuel Supply Company being designated the parent company. When Questar, an investor-owned, integrated energy company, was created in 1984, Mountain Fuel became a wholly owned natural gas distribution subsidiary. Headquartered in Salt Lake City, Utah, Questar's three main businesses are gas and oil exploration, production, and marketing; interstate gas gathering, transportation, and storage; and retail gas distribution.
Questar Pipeline Company, an interstate natural gas gathering and transmission system, manages 2,500 miles of gathering and distribution pipeline in Colorado, Wyoming, and Utah, as well as a large underground storage facility in northeastern Utah. Recently, the Clay Basin underground storage capacity was expanded from 100 billion cubic feet to 110 billion cubic feet. In September 1994, Questar Pipeline and Coastal Gas Gathering and Processing announced plans to operate the 84 million cubic feet per day Black Fork gas processing plant in Wyoming.

Figure 34. Questar Corp., 1993

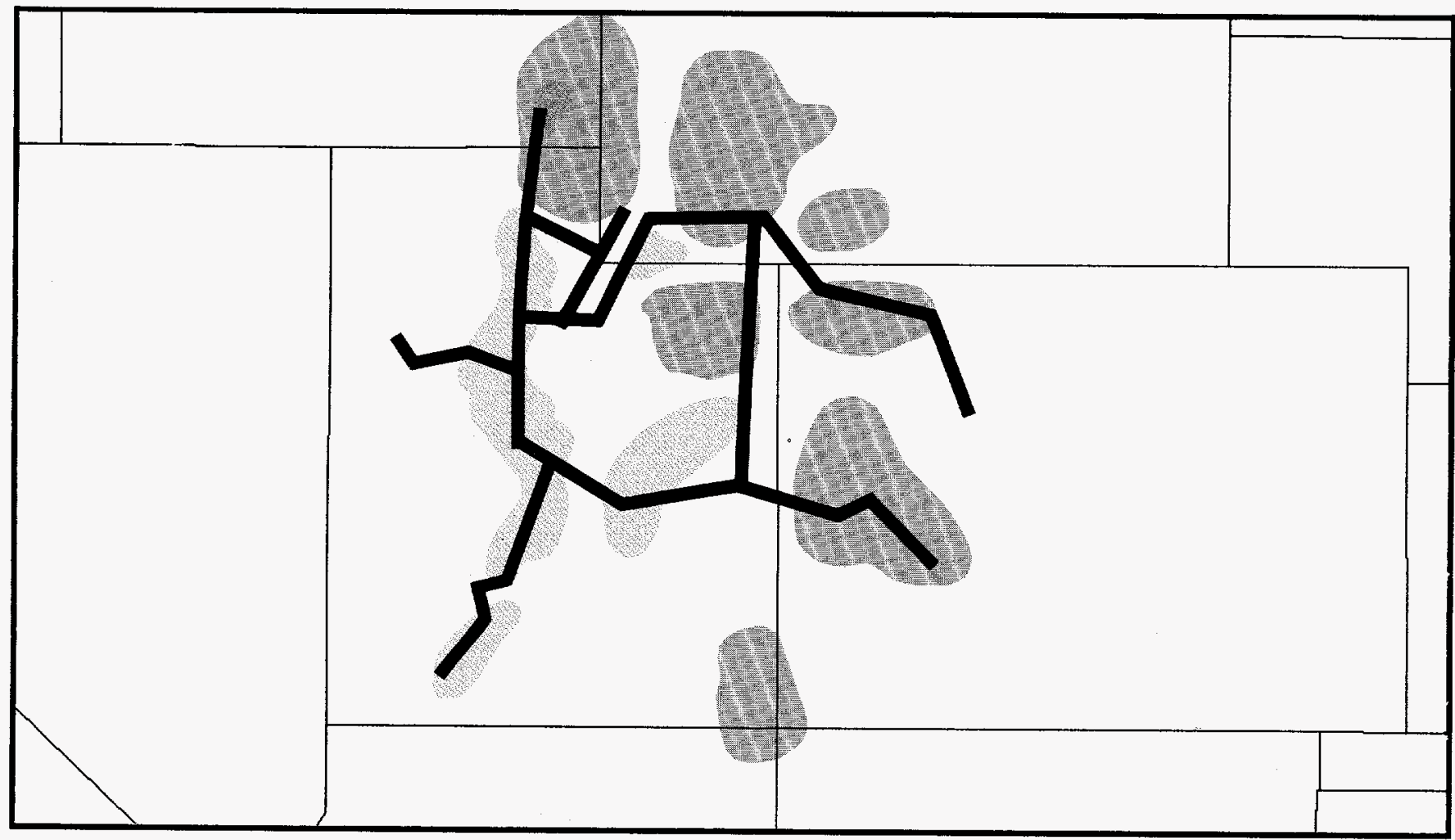

Source: Energy Information Administration (EIA), Form EIA-176, "Annual Report of Natural and Supplemental Gas Supply and Disposition."

Supply Areas Service Areas
Questar Pipeline Co.

Mountain Fuel Supply Co. 
Table 44. Questar Corp., Natural Gas Data, 1993

(Thousand Cubic Feet)

\begin{tabular}{l|c|c|c|c|}
\hline \multirow{2}{*}{ Supply/Disposition } & \multicolumn{3}{|c|}{ Questar Pipeline Co. } & $\begin{array}{c}\text { Mountain Fuel Supply } \\
\text { Co. }\end{array}$ \\
\cline { 2 - 6 } & Colorado & Utah & Wyoming & Utah \\
\hline
\end{tabular}

SUPPLY

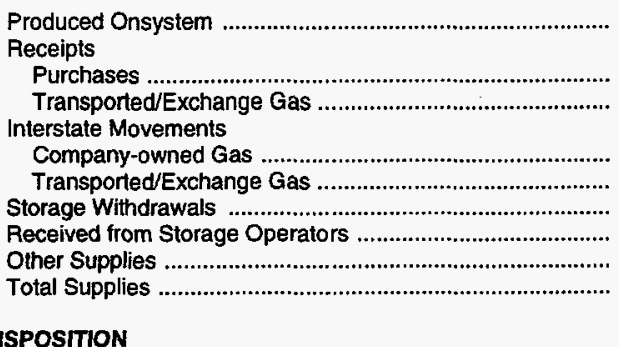

DISPOSITION

Lease and Plant Use ........................................................

Returned to Formations

Interstate Movements

Company-owned Gas

.

Transported/Exchange Gas ...............................................

Exchange Gas Deliveries ......................................................

Storage Injections .......

Deliveries of Company-owned Gas

To Storage Operators

To Other Pipelines

To Resellers

To Consumers

Residential

Industrial .

Electric Utility

To Storage Operators ..................................................

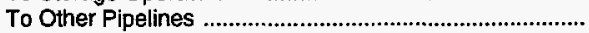

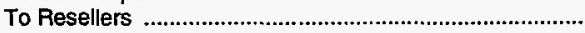

To Consumers

Residential

Commercial

Industrial.

Electric Utility

Vehicle Fuel

Pipeline/Compressor Fuel

Other Disposition

Unaccounted For

Total Disposition
0

$3,241,573$
$22,433,850$
0
0
283,294
0
0
0
$25,958,717$

42,229

0
$22,474,034$
0
0

0
0

2,365

0

0

0

0

0
$3,021,827$
0

a

0
0
0
0
158,285
0
259,977

$25,958,717$
0

$11,754,322$

$51,902,210$

0
$105,728,263$
$27,123,047$
0
0
$196,507,842$

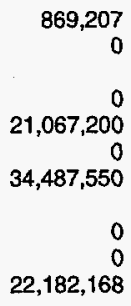

0

0

0

0
$115,246,173$

0

0
0

0
238,498

$2,417,046$

$2,417,046$
$196,507,842$
0

$11,530,110$

0
$33,778,265$
852,047
0
0
$190,325,299$

499,826

0
$96,248,588$
0

901,159

0
0

$1,095,411$

0
0
0

0
0
0
0

$88,566,237$

0

0
0
0
0
0
$1,135,567$
0
$1,878,511$
$190,325,299$
0
0
0
0
0

$123,860,298$

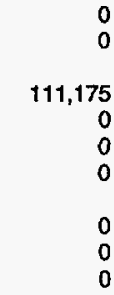

$51,382,805$

$22,196,527$

$2,672,889$

4,775

33,159
Questar Pipeline also serves five major gas-producing basins that interconnect five major interstate pipelines. On September 1, 1993, Questar Pipeline became one of the first companies to start operating under FERC Order 636. As a result, Mountain Fuel obtained full control of gas supplies and assumed all available working gas storage capacity.

Colder than normal temperatures in early 1993, coupled with expanded industrial usage, helped increase total deliveries. Mountain Fuel provided natural gas distribution service to more than 550,000 residential, commercial, and industrial customers in Idaho, Utah, and Wyoming, 18,000 more customers than in 1992.
Questar is a one-third owner in the proposed 292-mile Trans-Colorado pipeline project. On a north-south route, this pipeline will connect production fields in the Piceance Basin in northwestern Colorado with pipelines in the San Juan Basin in northwestern New Mexico. Pending FERC approval, construction is scheduled to begin in 1995 .

Questar is close to completing the initial phase of its environmental marketing program--a natural gas refueling network in Utah and southwestern Wyoming, consisting of 30 natural gas refueling facilities, 21 of which will have public access. Questar's ongoing marketing of natural gas vehicles (NGV) is best illustrated by the current use of 300 NGV's by 37 private firms and government agen- 
Table 44. Questar Corp., Natural Gas Data, 1993 (Continued) (Thousand Cubic Feet)

\begin{tabular}{|c|c|c|c|}
\hline \multirow{2}{*}{ Supply/Disposition } & \multicolumn{2}{|c|}{ Mountain Fuel Supply Co. } & \multirow{2}{*}{$\begin{array}{c}\text { Overthrust Pipeline Co. } \\
\text { Wyoming }\end{array}$} \\
\hline & Wyoming & Idaho & \\
\hline \multicolumn{4}{|l|}{ SUPPLY } \\
\hline \multicolumn{4}{|l|}{ 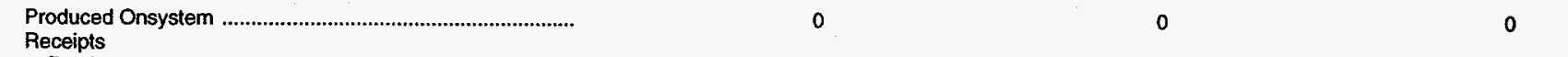 } \\
\hline Purchases & $1,097,777$ & 0 & 0 \\
\hline 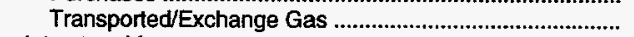 & $6,839,494$ & 0 & $15,665,356$ \\
\hline \multicolumn{4}{|l|}{ Interstate Movements } \\
\hline Company-owned Gas & 0 & .0 & 0 \\
\hline 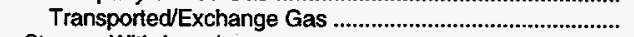 & 0 & 111,175 & 0 \\
\hline 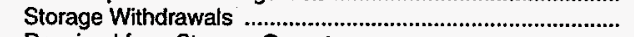 & 0 & 0 & 0 \\
\hline Received from Storage Operators & 0 & 0 & 0 \\
\hline 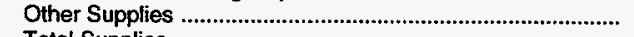 & 0 & 0 & 0 \\
\hline Total Supplies & $7,937,271$ & 111,175 & $15,665,356$ \\
\hline \multicolumn{4}{|l|}{ DISPOSITION } \\
\hline 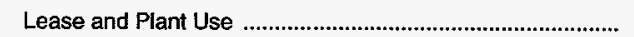 & 0 & 0 & 0 \\
\hline Retumed to Fomations & 0 & 0 & 0 \\
\hline \multicolumn{4}{|l|}{ Interstate Movements } \\
\hline 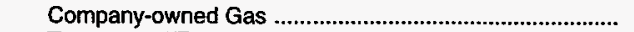 & 0 & 0 & 0 \\
\hline 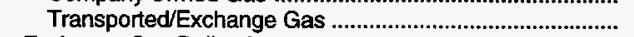 & 0 & 0 & 0 \\
\hline Exchange Gas Deliveries & 0 & 0 & $15,852,612$ \\
\hline Storage Injections & 0 & 0 & 0 \\
\hline \multicolumn{4}{|l|}{ Deliveries of Company-owned Gas } \\
\hline To Storage Operators & 0 & 0 & 0 \\
\hline To Other Pipelines & 0 & 0 & 0 \\
\hline 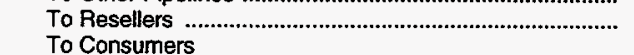 & \multicolumn{3}{|c|}{ To Consumers } \\
\hline 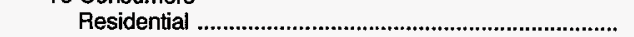 & $2,057,389$ & 64,227 & 0 \\
\hline 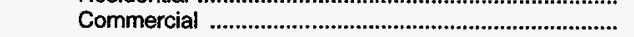 & $1,630,102$ & 46,948 & 0 \\
\hline 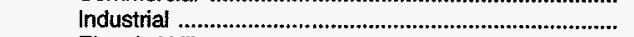 & 38,715 & 0 & 0 \\
\hline 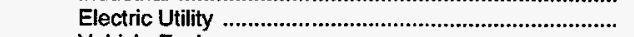 & 0 & 0 & 0 \\
\hline Vehicle Fuel & 11,316 & 0 & 0 \\
\hline \multicolumn{4}{|l|}{ Transported for the Account of Others } \\
\hline 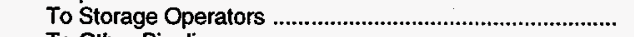 & 0 & 0 & 0 \\
\hline 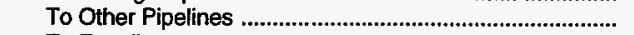 & 0 & 0 & 0 \\
\hline 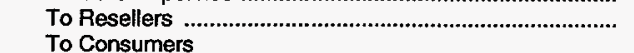 & 0 & 0 & 0 \\
\hline \multicolumn{4}{|l|}{ To Consumers } \\
\hline & $\begin{array}{r}0 \\
44,011\end{array}$ & 0 & 0 \\
\hline $\begin{array}{l}\text { Commercial } \\
\text { Industrial }\end{array}$ & $\begin{array}{r}44,011 \\
3,703,208\end{array}$ & 0 & 0 \\
\hline & $\begin{array}{r}3,703,208 \\
0\end{array}$ & $\begin{array}{l}0 \\
0\end{array}$ & $\begin{array}{l}0 \\
0\end{array}$ \\
\hline Vehicle Fuel & 0 & 0 & $\begin{array}{l}0 \\
0\end{array}$ \\
\hline 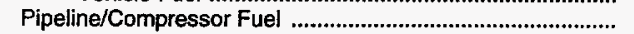 & 7,936 & 0 & 0 \\
\hline 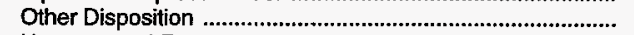 & 0 & 0 & 0 \\
\hline 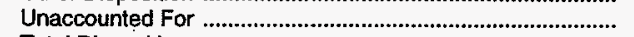 & 444,594 & 0 & $-187,256$ \\
\hline Total Disposition & $7,937,271$ & 111,175 & $15,665,356$ \\
\hline
\end{tabular}

Source: Energy Information Administration (EIA), Form EIA-176, "Annual Report of Natural and Supplemental Gas Supply and Disposition."

cies, including the Salt Lake City International Airport. NGV's include automobiles, forklifts, transit buses, and school buses. The public market is now being targeted.

Questar is a 35-percent owner of the Western Market Center, a full-service marketing hub scheduled to open in late 1994 at Muddy Creek, near Opal, Wyoming. A vari- ety of services, such as title transfers, wheeling, peaking, parking, and balancing gas will be offered through independent operation. Storage will be available through Questar's Clay Basin field. An electronic bulletin board will provide financial, regulatory, weather, and industry news and allow cash trading to match bids and offers of physical gas. 
Table 45. Questar Corp., Interstate Flows of Natural Gas, 1993 (Thousand Cubic Feet)

\begin{tabular}{|c|c|}
\hline Company and State & Volume \\
\hline $\begin{array}{l}\text { Questar Pipeline Co. } \\
\text { Colorado To } \\
\text { Utah } \\
\text { Wyoming } \\
\text { Utah To } \\
\text { Colorado } \\
\text { Wyoming } \\
\text { Wyoming To } \\
\text { Utah }\end{array}$ & $\begin{array}{r}9,479,675 \\
12,994,359 \\
283,294 \\
20,783,906 \\
96,248,588\end{array}$ \\
\hline $\begin{array}{l}\text { Mountain Fuel Supply Co. } \\
\text { Utah To } \\
\text { Idaho }\end{array}$ & 111,175 \\
\hline
\end{tabular}

Source: Energy Information Administration (EIA), Form EIA-176, "Annual Report of Natural and Supplemental Gas Supply and Disposition." 


\section{Sabine Pipe Line Company}

Sabine Pipe Line Company, a wholly owned subsidiary of Texaco Inc., was founded in 1963. Sabine's mainline extends 131 miles from the Henry Hub near Erath, Louisiana to Port Arthur, Texas. It is an open-access, interstate pipeline that transports natural gas in Louisiana, Texas, and the Outer Continental Shelf off Louisiana. From 1964 to the mid-1980's, Sabine transported primarily for Texaco and affiliated companies. In addition, Sabine operates three offshore laterals.

In 1987, the Henry Hub, a major gas interchange center jointly owned by Sabine and Texaco, was created in Southern Louisiana and began operations in May 1988. In 1989, the New York Mercantile Exchange designated the Henry Hub as the delivery location for their natural gas futures contract which began trading in April 1990.
Sabine's Henry Hub and mainline pipeline system interconnect with more than 20 interstate and intrastate pipeline systems. Solely a short-haul transporter, Sabine does not buy or sell natural gas and currently does not have storage facilities on its mainline system.

On July 1, 1994, CNG Transmission Corporation, a subsidiary of Consolidated Natural Gas Company, and Sabine Pipeline Company opened a "Super Hub" natural gas center in the Northeast. The hub, called the CNG/SABINE Center, offers services at points along CNG's 7,400mile pipeline across Maryland, New York, Ohio, Pennsylvania, Virginia, and West Virginia.

Figure 35. Sabine Pipe Line Co., 1993

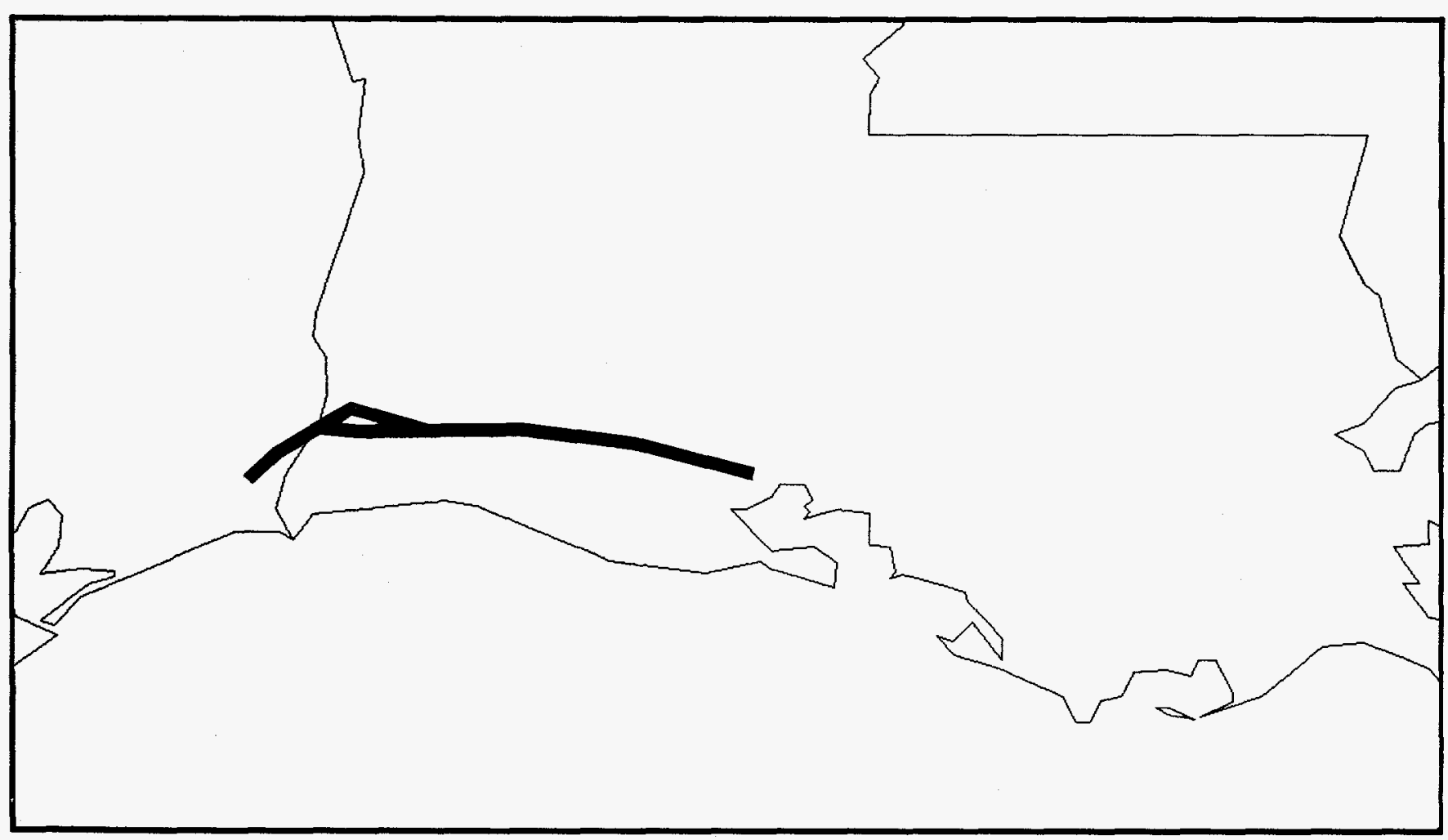

Source: Energy Information Administration (EIA), Form EIA-176, "Annual Report of Natural and Supplemental Gas Supply and Disposition." 
Table 46. Sabine Pipe Line Co., Natural Gas Data, 1993 (Thousand Cubic Feet)

\begin{tabular}{|c|c|c|c|c|}
\hline \multirow{2}{*}{ Supply/Disposition } & \multicolumn{2}{|c|}{ Sabine Pipe Line Co. } & \multirow{2}{*}{$\frac{\text { Riverway Gas Pipeline Co. }}{\text { Louisiana }}$} & \multirow{2}{*}{\begin{tabular}{|c|}
$\begin{array}{c}\text { Bridgeline Gas Distribution } \\
\text { Co. }\end{array}$ \\
Louisiana \\
\end{tabular}} \\
\hline & Louisiana & Texas & & \\
\hline \multicolumn{5}{|l|}{ SUPPLY } \\
\hline $\begin{array}{l}\text { Produced Onsystem } \\
\text { Receipts }\end{array}$ & 0 & 0 & 0 & 0 \\
\hline 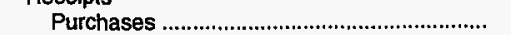 & 0 & 0 & 0 & $93,017,393$ \\
\hline 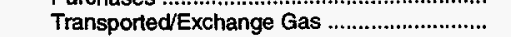 & $149,184,843$ & $25,712,841$ & $14,365,939$ & $145,811,982$ \\
\hline \multicolumn{5}{|l|}{ Interstate Movements } \\
\hline 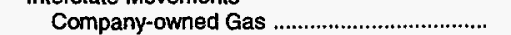 & 0 & 0 & 0 & 0 \\
\hline 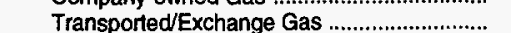 & $15,459,430$ & $3,765,708$ & 0 & 0 \\
\hline 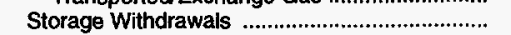 & 0 & 0 & 0 & $6,180,392$ \\
\hline 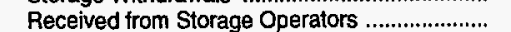 & 0 & 0 & 0 & 0 \\
\hline 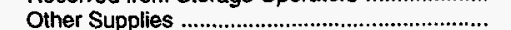 & 0 & 0 & 0 & 0 \\
\hline Total Supplies & $164,644,273$ & $29,478,549$ & $14,365,939$ & $245,009,767$ \\
\hline \multicolumn{5}{|l|}{ DISPOSITION } \\
\hline 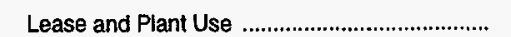 & 0 & 0 & 0 & 0 \\
\hline 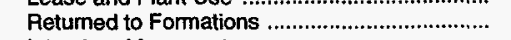 & 0 & 0 & 0 & 0 \\
\hline \multicolumn{5}{|l|}{ Interstate Movements } \\
\hline 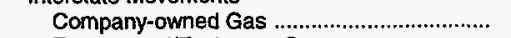 & 0 & 0 & 0 & 0 \\
\hline 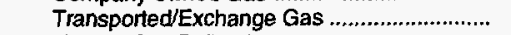 & $3,765,708$ & $15,459,430$ & 0 & 0 \\
\hline 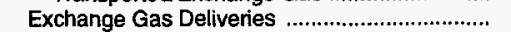 & 0 & 0 & 0 & $58,154,623$ \\
\hline Storage Injections & 0 & 0 & 0 & $5,963,226$ \\
\hline \multicolumn{5}{|l|}{ Deliveries of Company-owned Gas } \\
\hline 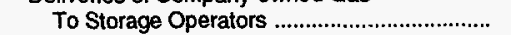 & 0 & 0 & 0 & 0 \\
\hline To Other Pipelines & 0 & 0 & 0 & 0 \\
\hline To Resellers & 0 & 0 & 0 & 0 \\
\hline \multicolumn{5}{|l|}{ To Consumers } \\
\hline 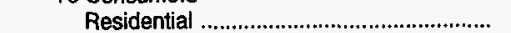 & 0 & 0 & 0 & 0 \\
\hline 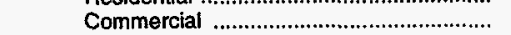 & 0 & 0 & 0 & 0 \\
\hline Industrial & 0 & 0 & 0 & $113,638,304$ \\
\hline 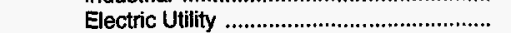 & 0 & 0 & $6,784,478$ & $10,221,502$ \\
\hline Vehicle Fuel & 0 & 0 & 0 & 0 \\
\hline \multicolumn{5}{|l|}{ Transported for the Account of Others } \\
\hline 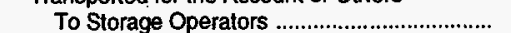 & 0 & 0 & 0 & 0 \\
\hline To Other Pipelines & $160,249,100$ & $14,134,314$ & 0 & 0 \\
\hline 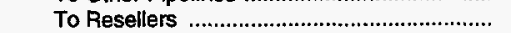 & 0 & 0 & 0 & 0 \\
\hline \multicolumn{5}{|l|}{ To Consumers } \\
\hline 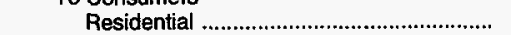 & 0 & 0 & 0 & 0 \\
\hline 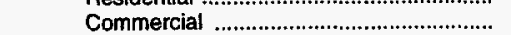 & 0 & 0 & 0 & 0 \\
\hline 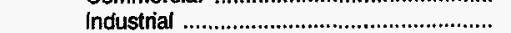 & 0 & 0 & 0 & $22,796,753$ \\
\hline Electric Utility & 0 & 0 & $7,581,461$ & $33,853,635$ \\
\hline Vehicle Fuel & 0 & 0 & 0 & 0 \\
\hline Pipeline/Compressor Fuel & 0 & 0 & 0 & 81,155 \\
\hline Other Disposition & 0 & 0 & 0 & 0 \\
\hline 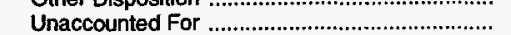 & 629,465 & $-115,195$ & 0 & 300,569 \\
\hline Total Disposition & $164,644,273$ & $29,478,549$ & $14,365,939$ & $245,009,767$ \\
\hline
\end{tabular}

Source: Energy Information Administration (ElA), Form ElA-176, "Annual Report of Natural and Supplemental Gas Supply and Disposition." 


\section{San Diego Gas and Electric Co.}

San Diego Gas and Electric (SDG\&E), an operating public utility, is engaged in the gas and electric business. The company purchases and distributes natural gas to 690,000 customers in San Diego County; and generates, purchases, and distributes electric energy to $1,129,000$ customers in San Diego County and parts of southern Orange County. SDG\&E also transports gas and electricity for other companies.

The Federal Energy Regulatory Commission's Order 636 requires interstate pipeline companies to make pipeline capacity available directly to retail and wholesale customers. The California Public Utilities Commission's (CPUC) gas capacity brokering decision requires the Southern California Gas Company (SoCal) to make available to retail and wholesale customers its long-term rights to interstate capacity. As a result of these decisions, SDG\&E reduced its commitment to SoCal for firm interstate pipeline capacity to reflect its core customer demand. SDG\&E is allowed to bid for capacity to meet its noncore customer demand on a short-term, as-needed basis.

Figure 36. San Diego Gas and Electric Co., 1993

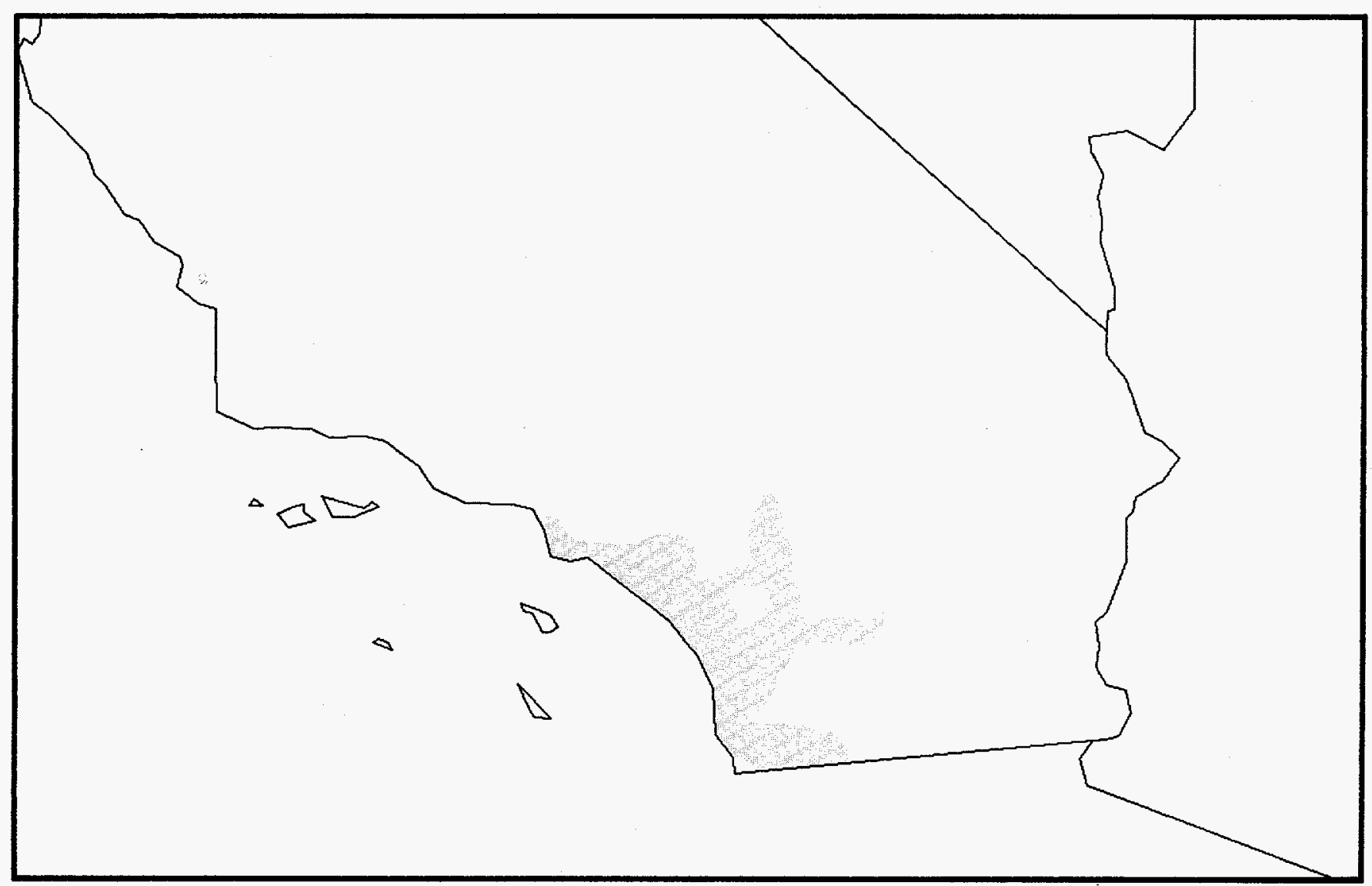

Source: Energy Information Administration (EIA), Form ElA-176, "Annual Report of Natural and Supplemental Gas Supply and Disposition."

\section{Service Area}


Table 47. San Diego Gas and Electric Co., Natural Gas Data, 1993 (Thousand Cubic Feet)

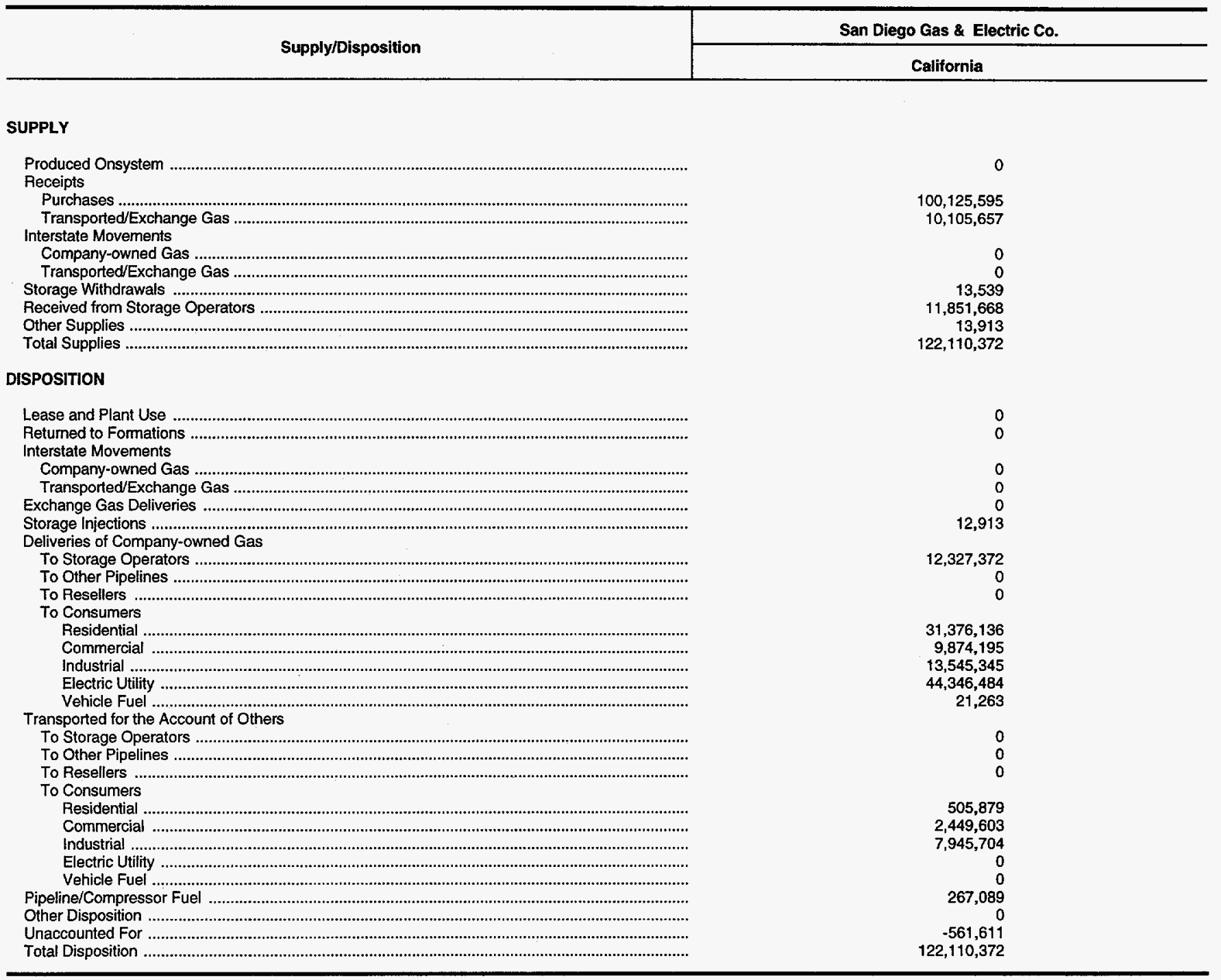

Source: Energy Information Administration (E|A), Form ElA-176, "Annual Report of Natural and Supplemental Gas Supply and Disposition."

San Diego Gas \& Electric benefitted from the Gas Bypass Rulemaking by the CPUC. The CPUC allowed SDG\&E to discount their prices in order to avoid losing customers who would have their gas transported by others. The CPUC also decided to allocate a larger portion of gas transportation costs to residential customers, resulting in lower rates for large industrial customers. The decision was reached to make it possible for industries in California to become more competitive and reduce the risk of losing large industrial customers from the utilities' systems.
The Federal Energy Regulatory Commission granted SDG\&E approval to construct an 80-mile pipeline from SoCal's service territory to the Mexican border for transporting natural gas to Mexico. The Mexican government is to give the required final approval. The FERC issued a permit in August 1993 to SDG\&E and SoCal, making natural gas available to Mexico at the Tijuana border. The project will provide gas to the nearby Rosarito Power Plant, which if expanded and converted from an oil-fired to a gas-fired plant, will reduce air pollution in California and Mexico. 


\section{Southern California Gas Co.}

Southern California Gas Company (SOCAL), a public utility, owns and operates the largest natural gas distribution system in the United States. SOCAL, the principal subsidiary of Pacific Enterprises, provides service to parts of Central California and most of Southern California. Pacific Enterprises also owns Pacific Interstate Pipeline Company that transports natural gas for resale to SOCAL. Pacific Interstate, in turn, owns Pacific Offshore Pipeline Company. Pacific Enterprises' interstate transportation system primarily moves natural gas originating in Western Canada, while Pacific Offshore brings natural gas produced in the Santa Barbara offshore area to the SOCAL distribution area.
SOCAL's service area includes 535 communities within an area of 23,000 square miles. In addition, SOCAL delivers natural gas for resale to the distribution systems of the City of Long Beach, California and the San Diego Gas and Electric Company. In January 1994, SOCAL's service area was struck by a major earthquake that disrupted natural gas service to approximately 150,000 customers and caused damage to some of the company's facilities. Nearly all customers were back on line within 12 days of the quake.

On September 1, 1994, SOCAL received authority from the Federal Energy Regulatory Commission (FERC) for

Figure 37. Southern California Gas Co., 1993

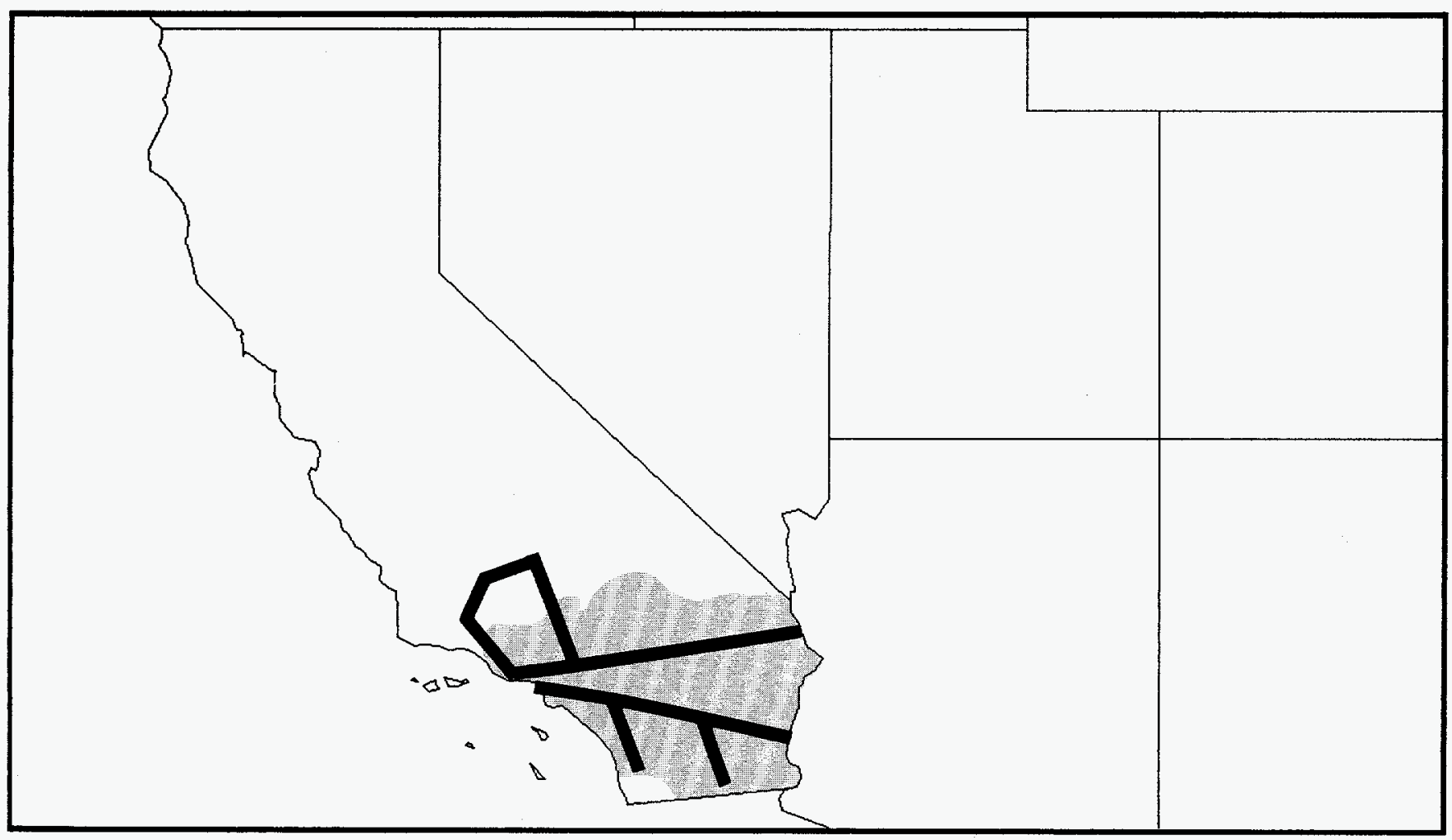

Source: Energy Information Administration (E|A), Form ElA-176, "Annual Report of Natural and Supplemental Gas Supply and Disposition."

Service Area Southern California Gas Co. 
Table 48. Southern California Gas Co., Natural Gas Data, 1993 (Thousand Cubic Feet)

\begin{tabular}{|c|c|c|}
\hline \multirow{2}{*}{ Supply/Disposition } & Southern California Gas Co. & \multirow{2}{*}{$\frac{\text { Pacific Offshore Pipeline Co. }}{\text { California }}$} \\
\hline & California & \\
\hline \multicolumn{3}{|l|}{ SUPPLY } \\
\hline 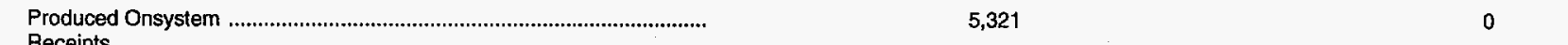 & 5,321 & 0 \\
\hline 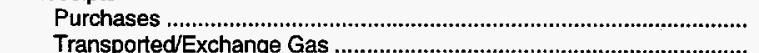 & $144,300,824$ & $10,581,809$ \\
\hline \multicolumn{3}{|l|}{ Interstate Movements } \\
\hline Company-owned Gas & $224,252,326$ & 0 \\
\hline 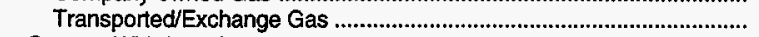 & $445,276,090$ & 0 \\
\hline 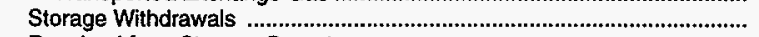 & $102,608,776$ & 0 \\
\hline 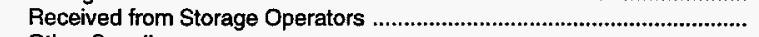 & 0 & 0 \\
\hline Other Supplies & 0 & 0 \\
\hline 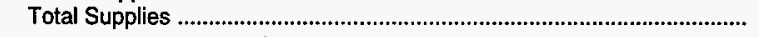 & $1,261,341,837$ & $10,581,809$ \\
\hline \multicolumn{3}{|l|}{ DISPOSITION } \\
\hline Lease and Plant Use & 0 & $1,233,726$ \\
\hline Retumed to Formations & 0 & 0 \\
\hline \multicolumn{3}{|l|}{ Interstate Movements } \\
\hline Company-owned Gas & 0 & 0 \\
\hline 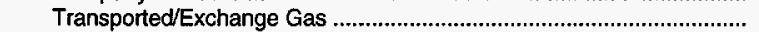 & 0 & 0 \\
\hline 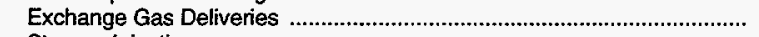 & $16,969,433$ & 0 \\
\hline Storage Injections & $112,106,037$ & 0 \\
\hline \multicolumn{3}{|l|}{ Deliveries of Company-owned Gas } \\
\hline To Storage Operators & 0 & 0 \\
\hline To Other Pipelines & 0 & 0 \\
\hline 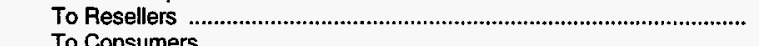 & 0 & $9,371,352$ \\
\hline \multicolumn{3}{|l|}{ To Consumers } \\
\hline 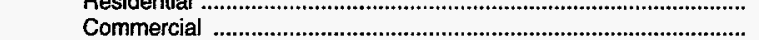 & $\begin{array}{r}244,312,438 \\
60,726,092\end{array}$ & $\begin{array}{l}0 \\
0\end{array}$ \\
\hline Industrial & $35,866,556$ & 0 \\
\hline Electric Utility & 54,142 & 0 \\
\hline Vehicle Fuel & 82,714 & 0 \\
\hline \multicolumn{3}{|l|}{ Transported for the Account of Others } \\
\hline To Storage Operators & 0 & 0 \\
\hline 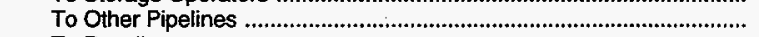 & 0 & 0 \\
\hline 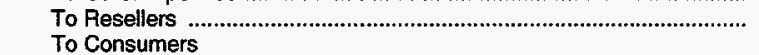 & $147,977,671$ & 0 \\
\hline 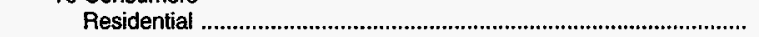 & $3,194,430$ & 0 \\
\hline Commercial & $42,572,179$ & 0 \\
\hline Industrial & $200,429,468$ & 0 \\
\hline 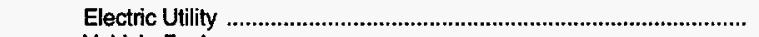 & $212,665,945$ & 0 \\
\hline Vehicle Fuel & 28,274 & 0 \\
\hline Pipeline/Compressor Fuel & $5,778,970$ & 0 \\
\hline 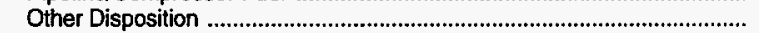 & 0 & 0 \\
\hline Unaccounted For & $178,577,488$ & $-23,269$ \\
\hline Total Disposition & $1,261,341,837$ & $10,581,809$ \\
\hline
\end{tabular}

Source: Energy Information Administration (EIA), Form ElA-176, "Annual Report of Natural and Supplemental Gas Supply and Disposition."

siting, construction, and operation of border crossing facilities on the U.S.-Mexican border near Calexico, California. These facilities will enable exports of up to 40 million cubic feet of gas per day. The border system will connect SOCAL's natural gas system with transportation facilities to be built and operated by Petroleos Mexicanos (PEMEX) for service to new and existing markets in Mexicali, Mexico. The facilities would be built in conjunction with "Project Vecinos," a joint venture by SOCAL and San Diego Gas and Electric Company that was authorized by FERC in 1993 to deliver natural gas to PEMEX for redelivery to electric generation and other markets in Baja, California and Norte, Mexico.
SOCAL has 290 vehicles capable of running on natural gas and is continuing to encourage development of a natural gas vehicle (NGV) market. The utility joined with other companies to form the NGV Ecotrans Technology Center. Owned and managed jointly by SOCAL and NGV Systems Inc., the center converts new and existing fleet vehicles to run on natural gas. There are 57 natural gas refueling stations operating in SOCAL's service territory, about 70 stations in all of Southern California and 106 Statewide. In July 1994, SOCAL completed and began operating California's first liquefied natural gas (LNG) refueling station at the Los Angeles International Airport to service airport shuttle buses powered by LNG. 


\section{Southern Natural Gas Co.}

Southern Natural Gas Company owns and operates a major natural gas pipeline system located in the Southeastern United States. With the implementation in late 1993 of the Federal Energy Regulatory Commission Order 636, the restructuring order for the natural gas industry, Southern Natural no longer sells gas. Now the company provides transportation services for customers who have contracted with producers or marketers for natural gas supplies.

Sonat, Inc., the parent company of Southern Natural Gas, has an interest in a 14,000-mile natural gas pipeline network. In addition to the Southern Natural system, this network includes pipelines operated by Sea Robin Pipeline Company and South Georgia Natural Gas Company.
Florida Gas Transmission Company, the principal natural gas pipeline in Florida, is a subsidiary of Citrus Corp., a 50-percent owned Sonat company. A Florida Gas pipeline expansion project that will increase capacity by 530 million cubic feet per day is expected to be completed in early 1995. In another pipeline expansion project, Southern Natural will add 40 million cubic feet per day of capacity in northern Florida and southwestern Georgia.

Sonat Exploration Company, also a Sonat subsidiary, engages in natural gas and oil exploration and production. It has lease interests in Alabama, Arkansas, Louisiana, Oklahoma, Texas, and the Gulf of Mexico. In 1993, Sonat Exploration drilled 301 development wells.

Figure 38. Southern Natural Gas Co., 1993

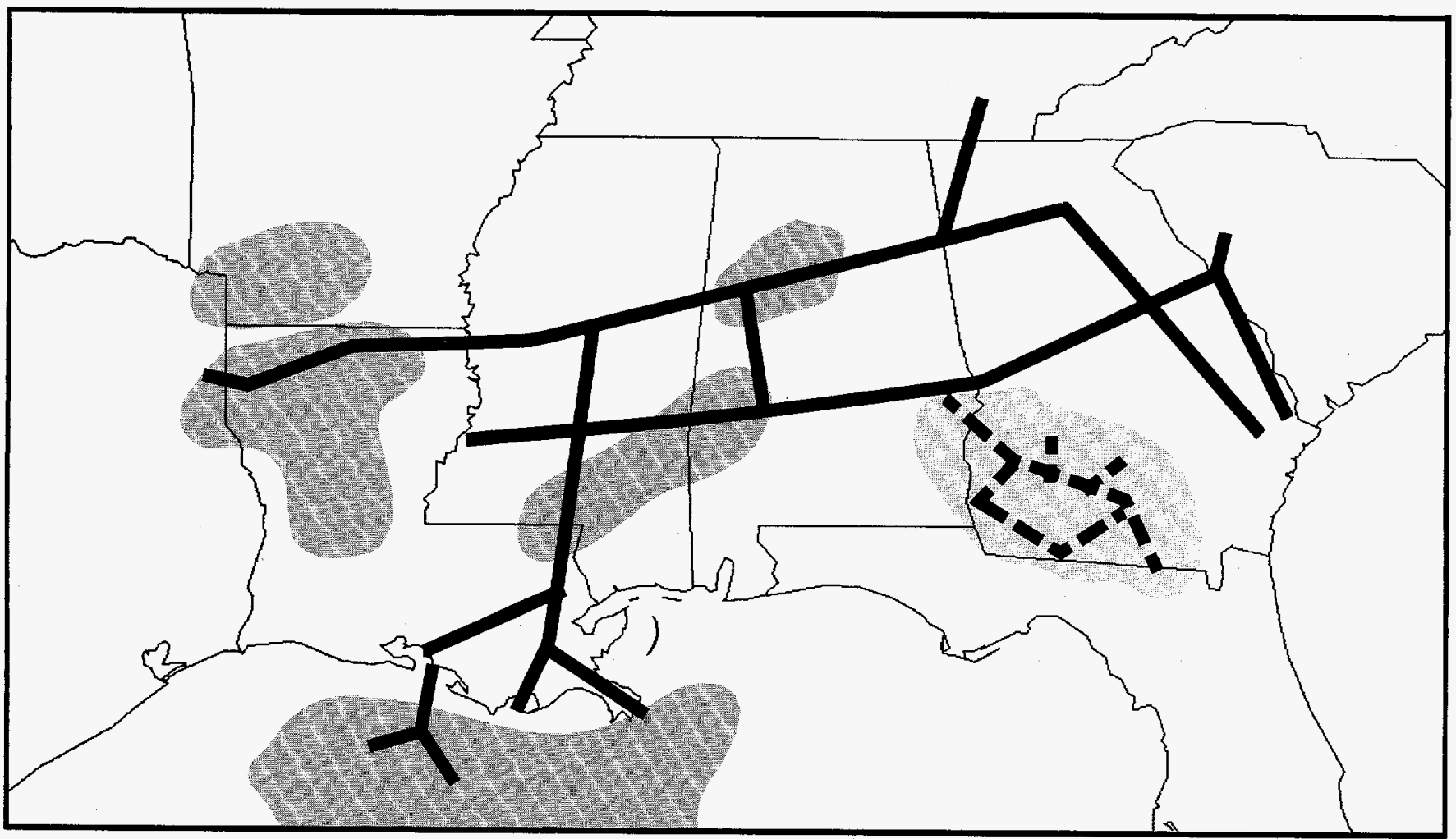

Source: Energy Information Administration (EIA), Form EIA-176, "Annual Report of Natural and Supplemental Gas Supply and Disposition."

Supply Areas Service Area
Southern Natural Gas Co.

- South Georgia Natural Gas Co. 
Table 49. Southern Natural Gas Co., Natural Gas Data, 1993 (Thousand Cubic Feet)

\begin{tabular}{|c|c|c|c|c|c|c|}
\hline \multirow{2}{*}{ Supply/Disposition } & \multicolumn{6}{|c|}{ Southern Natural Gas Co. } \\
\hline & Texas & Louisiana & Mississippi & Alabama & Georgia & South Carolina \\
\hline \multicolumn{7}{|l|}{ SUPPLY } \\
\hline $\begin{array}{l}\text { Produced Onsystem } \\
\text { Receipts }\end{array}$ & 0 & 0 & 0 & 0 & 0 & 0 \\
\hline Purchases & 0 & $37,823,477$ & $5,419,902$ & $23,153,202$ & 0 & 0 \\
\hline $\begin{array}{l}\text { Transported/Exchange Gas ............................................................. } \\
\text { Interstate Movements }\end{array}$ & $38,174,296$ & $406,506,661$ & $34,956,976$ & $72,233,148$ & 100,668 & 0 \\
\hline 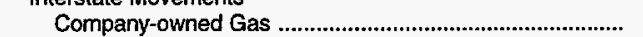 & 0 & 0 & 0 & 0 & 0 & 0 \\
\hline 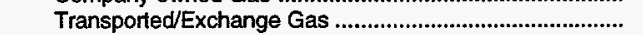 & 0 & $38,174,296$ & $385,753,240$ & $394,340,242$ & $333,142,338$ & $78,179,055$ \\
\hline Storage Withdrawals & 0 & 0 & $33,474,671$ & 0 & 0 & 0 \\
\hline Received from Storage Operators & 0 & $21,617,098$ & 0 & 0 & 0 & 0 \\
\hline 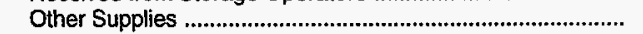 & 0 & 0 & 0 & 0 & 0 & 0 \\
\hline 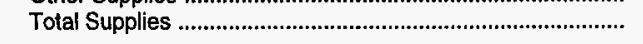 & $38,174,296$ & $504,121,532$ & $459,604,789$ & $489,726,592$ & $333,243,006$ & $78,179,055$ \\
\hline \multicolumn{7}{|l|}{ DISPOSITION } \\
\hline Lease and Plant Use & 0 & 0 & 0 & 0 & 0 & 0 \\
\hline 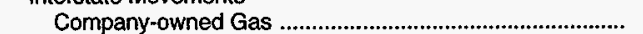 & 0 & 0 & 0 & 0 & 0 & 0 \\
\hline 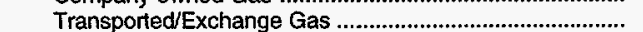 & $38,174,296$ & $385,753,240$ & $394,340,242$ & $333,142,338$ & $83,534,079$ & 0 \\
\hline 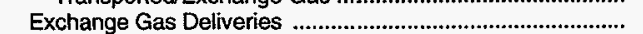 & 0 & $56,954,455$ & $18,033,278$ & $97,600,822$ & $181,290,758$ & $67,760,135$ \\
\hline Storage Injections & 0 & 0 & $32,771,564$ & 0 & 0 & 0 \\
\hline \multicolumn{7}{|l|}{ Deliveries of Company-owned Gas } \\
\hline 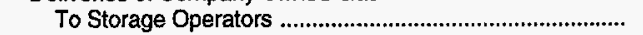 & 0 & $31,209,580$ & 0 & 0 & 0 & 0 \\
\hline 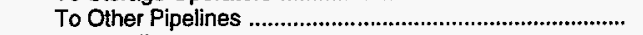 & 0 & 0 & 0 & 0 & 0 & 0 \\
\hline 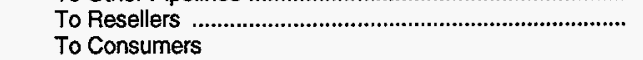 & 0 & 13,824 & $1,134,959$ & $22,441,727$ & $38,657,300$ & $7,954,196$ \\
\hline 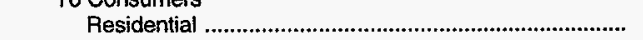 & 0 & 0 & 0 & 0 & 0 & 0 \\
\hline 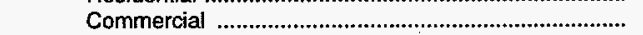 & 0 & 8,184 & 12,866 & 288 & 404 & 0 \\
\hline 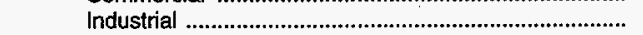 & 0 & 0 & 1,031 & 56,808 & 558 & 154 \\
\hline 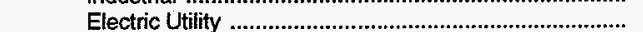 & 0 & 0 & 0 & 0 & 0 & 0 \\
\hline 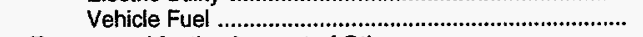 & 0 & 0 & 0 & 0 & 0 & 0 \\
\hline \multicolumn{7}{|l|}{ Transported for the Account of Others } \\
\hline To Storage Operators & 0 & 0 & 0 & 0 & 0 & 0 \\
\hline To Other Pipelines & 0 & 0 & 0 & 0 & 0 & 0 \\
\hline 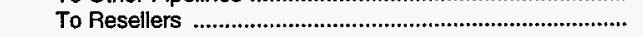 & 0 & 0 & 0 & 0 & 0 & 0 \\
\hline \multicolumn{7}{|l|}{ To Consumers } \\
\hline 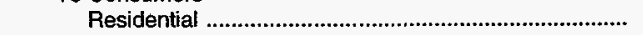 & 0 & 0 & 0 & 0 & 0 & 0 \\
\hline 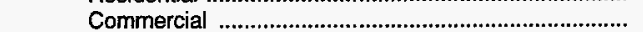 & 0 & 0 & 0 & 0 & 0 & 0 \\
\hline Industrial & 0 & $25,812,022$ & $9,315,683$ & $32,172,219$ & $27,817,852$ & $2,238,565$ \\
\hline 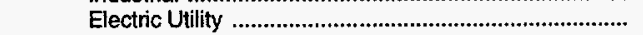 & 0 & 0 & 0 & 33,132 & 0 & 0 \\
\hline 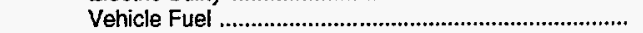 & 0 & 0 & 0 & 0 & 0 & 0 \\
\hline Pipeline/Compressor Fuel & 0 & $3,951,235$ & $3,780,762$ & $3,802,070$ & $1,179,250$ & 0 \\
\hline Other Disposition & 0 & 76,806 & 25,733 & 24,525 & 40,931 & 0 \\
\hline 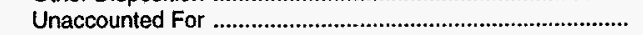 & 0 & 342,186 & 188,671 & 452,663 & 721,874 & 226,005 \\
\hline 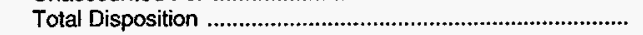 & $38,174,296$ & $504,121,532$ & $459,604,789$ & $489,726,592$ & $333,243,006$ & $78,179,055$ \\
\hline
\end{tabular}

Sonat Energy Services Company provides natural gas marketing, gathering, and intrastate natural gas pipeline services for Sonat, Inc. Sonat Marketing, one of the Energy Services companies, markets Sonat Exploration Company's production. Another Energy Services company, Sonat Ventures, promotes the development of facilities in the Southeast for natural gas vehicles. Currently, Sonat Ventures has entered into two joint ventures with local distribution companies in Alabama and Florida to provide natural gas vehicle services.
In December 1993, AES/Sonat Power L.L.C. (a 50-percent owned company) won a bid for the Hunter's Point 221-megawatt cogeneration plant to be located near San Francisco. The California Public Utilities Commission ordered the solicitations. Sonat will be responsible for the gas supply requirements for the new facility, and AES Corp., will manage its construction and operation. The plant is scheduled to be completed by mid- 1997 . 
Table 49. Southern Natural Gas Co., Natural Gas Data, 1993 (Continued) (Thousand Cubic Feet)

\begin{tabular}{|c|c|c|c|c|c|c|}
\hline \multirow{2}{*}{ Supply/Disposition } & \multirow{2}{*}{$\begin{array}{c}\begin{array}{c}\text { Southern } \\
\text { Natural Gas Co. }\end{array} \\
\text { Tennessee }\end{array}$} & \multirow{2}{*}{$\begin{array}{l}\begin{array}{c}\text { Bear Creek } \\
\text { Storage Co. }\end{array} \\
\text { Louisiana }\end{array}$} & \multirow{2}{*}{$\begin{array}{c}\begin{array}{c}\text { Sea Robin } \\
\text { Pipeline Co. }\end{array} \\
\text { Louisiana }\end{array}$} & \multicolumn{3}{|c|}{ South Georgia Natural Gas Co. } \\
\hline & & & & Alabama & Georgia & Florida \\
\hline \multicolumn{7}{|l|}{ SUPPLY } \\
\hline $\begin{array}{l}\text { Produced Onsystem } \\
\text { Receipts }\end{array}$ & 0 & 0 & 0 & 0 & 0 & 0 \\
\hline 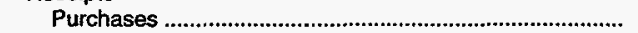 & 0 & 0 & 0 & 0 & 0 & 0 \\
\hline 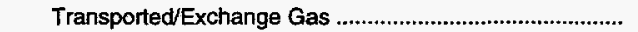 & 0 & $56,546,115$ & $288,309,199$ & $32,321,831$ & 0 & 0 \\
\hline \multicolumn{7}{|l|}{ Interstate Movements } \\
\hline Company-owned Gas & 0 & 0 & 0 & 0 & 0 & 0 \\
\hline 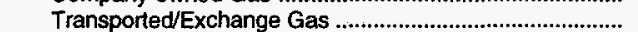 & $5,355,024$ & 0 & 0 & 0 & $32,321,831$ & $12,370,954$ \\
\hline Storage Withdrawals & 0 & $55,781,960$ & 0 & 0 & 0 & 0 \\
\hline 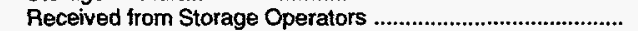 & 0 & 0 & 0 & 0 & 0 & 0 \\
\hline 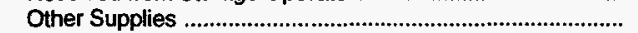 & 0 & 0 & 0 & 0 & 0 & 0 \\
\hline 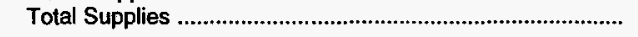 & $5,355,024$ & $112,328,075$ & $288,309,199$ & $32,321,831$ & $32,321,831$ & $12,370,954$ \\
\hline \multicolumn{7}{|l|}{ DISPOSITION } \\
\hline Lease and Plant Use & 0 & 0 & 0 & 0 & 0 & 0 \\
\hline $\begin{array}{l}\text { Returned to Fomations } \\
\text { Interstate Movements }\end{array}$ & 0 & 0 & 0 & 0 & 0 & 0 \\
\hline 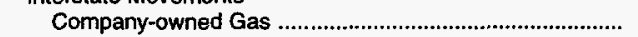 & 0 & 0 & 0 & 0 & 0 & 0 \\
\hline 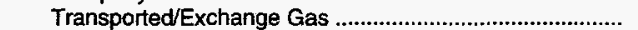 & 0 & 0 & 0 & $32,321,831$ & $12,370,954$ & 0 \\
\hline 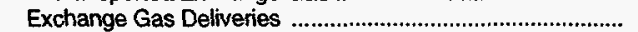 & $4,575,054$ & $55,781,960$ & $283,468,292$ & 0 & 0 & 0 \\
\hline 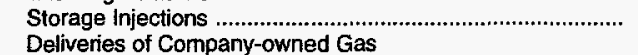 & \multicolumn{5}{|c|}{ Deliveries of Company-owned Gas } & 0 \\
\hline 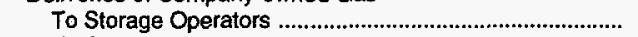 & 0 & 0 & 0 & 0 & 0 & 0 \\
\hline To Other Pipelines & 0 & 0 & 0 & 0 & 0 & 0 \\
\hline 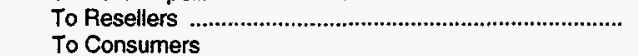 & 764,490 & 0 & 0 & 0 & 0 & 0 \\
\hline 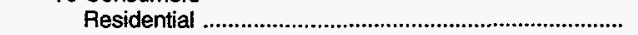 & 0 & 0 & 0 & 0 & 0 & 0 \\
\hline 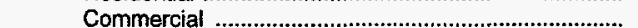 & 0 & 0 & 0 & 0 & 0 & 0 \\
\hline 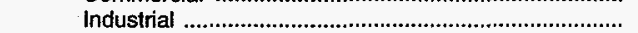 & 0 & 0 & 0 & 0 & 0 & 0 \\
\hline 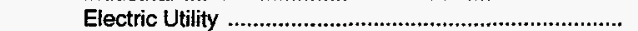 & 0 & 0 & 0 & 0 & 0 & 0 \\
\hline Vehicle Fuel & 0 & 0 & 0 & 0 & 0 & 0 \\
\hline \multicolumn{7}{|l|}{ Transported for the Account of Others } \\
\hline 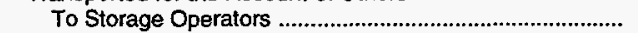 & 0 & 0 & 0 & 0 & 0 & 0 \\
\hline 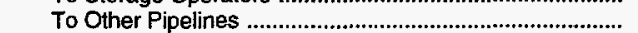 & 0 & 0 & $4,841,199$ & 0 & 0 & 0 \\
\hline 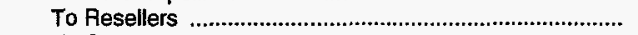 & 0 & 0 & 0 & 0 & $14,129,278$ & $9,296,112$ \\
\hline \multicolumn{7}{|l|}{ To Consumers } \\
\hline 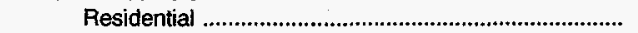 & 0 & 0 & 0 & 0 & 0 & 0 \\
\hline 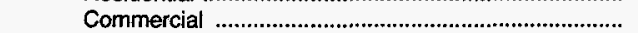 & 0 & 0 & 0 & 0 & 0 & 0 \\
\hline Industrial & 0 & 0 & 0 & 0 & $5,739,512$ & $3,023,942$ \\
\hline 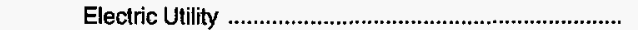 & 0 & 0 & 0 & 0 & 0 & 0 \\
\hline Vehicle Fuel & 0 & 0 & 0 & 0 & 0 & 0 \\
\hline 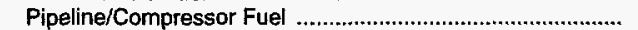 & 0 & 0 . & 2,332 & 0 & 0 & 0 \\
\hline Other Disposition & 0 & 0 & 0 & 0 & 0 & 0 \\
\hline Unaccounted For & 15,480 & 0 & $-2,624$ & 0 & 82,087 & 50,900 \\
\hline 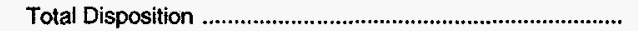 & $5,355,024$ & $112,328,075$ & $288,309,199$ & $32,321,831$ & $32,321,831$ & $12,370,954$ \\
\hline
\end{tabular}

Source: Energy Information Administration (EIA), Form EIA-176, "Annual Report of Natural and Supplemental Gas Supply and Disposition." 
Table 50. Southern Natural Gas Co., Interstate Flows of Natural Gas, 1993

(Thousand Cubic Feet)

\begin{tabular}{|c|c|}
\hline Company and State & Volume \\
\hline $\begin{array}{l}\text { Southem Natural Gas Co. } \\
\text { Texas To } \\
\text { Louisiana } \\
\text { Louisiana To } \\
\text { Mississippi } \\
\text { Mississippi To } \\
\text { Alabama } \\
\text { Alabama To } \\
\text { Georgia } \\
\text { Georgia To } \\
\text { South Carolina } \\
\text { Tennessee }\end{array}$ & $\begin{array}{r}38,174,296 \\
385,753,240 \\
394,340,242 \\
333,142,338 \\
\\
78,179,055 \\
5,355,024\end{array}$ \\
\hline $\begin{array}{l}\text { South Georgia Natural Gas Co. } \\
\text { Alabama To } \\
\text { Georgia } \\
\text { Georgia To } \\
\text { Florida }\end{array}$ & $\begin{array}{r}32,321,831 \\
12,370,954\end{array}$ \\
\hline
\end{tabular}

Source: Energy information Administration (EIA), Form EIA-176, "Annual Report of Natural and Supplemental Gas Supply and Disposition." 


\section{Southwest Gas Corp.}

Southwest Gas Corporation was incorporated in 1931 under California laws. Based in Las Vegas, Nevada, this diversified natural gas distribution company is responsible for natural gas transmission and distribution services and operates in Arizona, California, and Nevada.

Southwest, one of the fastest growing natural gas distribution companies in the country, operates over 16,000 miles of pipeline. In 1993, it added 35,000 new customers (a4-percent annual growth rate) and now serves approximately 932,000 customers. The company, with 563,000 total customers, is the largest distributor in Arizona. Southwest is also the largest distributor and transporter of gas in Nevada, where it added 20,000 new customers for a total of 266,000 customers. In California, an additional
2,000 customers brought its total customers to 103,000 . By the end of 1994, Southwest expects to add another 36,000 customers.

Ninety-four percent of Southwest's total customers are residential. In addition, the company has secured another 57,000 commitments from new home units for natural gas service for four-use gas homes (spaceheating, water heating, cooking, and clothes drying). Some homes may even include gas fireplaces, barbecue grills, pool and spa heaters, or outdoor lighting.

The commercial sector accounted for only 6 percent of Southwest's customers but this sector accounted for 25 percent of Southwest's gas deliveries. Southwest ranks

Figure 39. Southwest Gas Corp., 1993

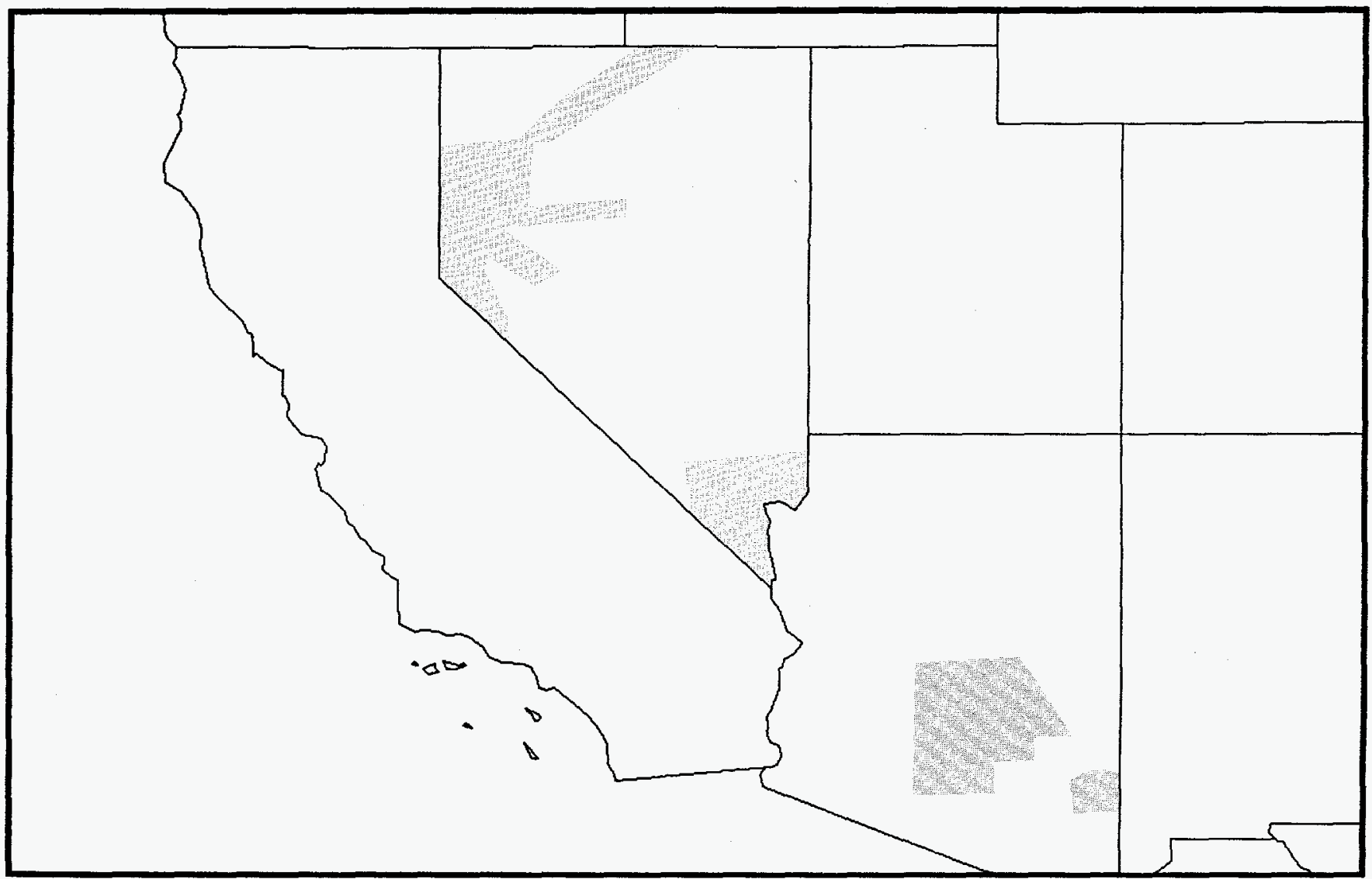

Source: Energy Information Administration (EIA), Form EIA-176, "Annual Report of Natural and Supplemental Gas Supply and Disposition." 
Table 51. Southwest Gas Corp., Natural Gas Data, 1993

(Thousand Cubic Feet)

\begin{tabular}{l|l|l|l|}
\hline \multirow{2}{*}{ Supply/Disposition } & \multicolumn{2}{|c}{ Southwest Gas Corp. } \\
\cline { 2 - 4 } & & Arizona & Nevada \\
\hline
\end{tabular}

SUPPLY

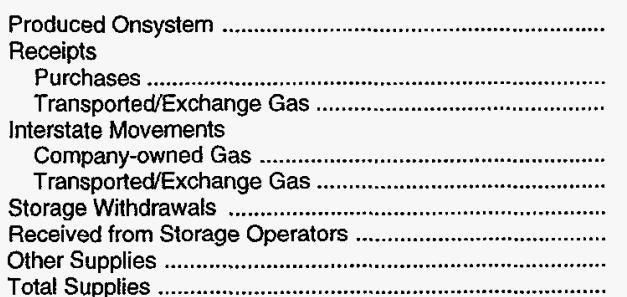

0

$18,810,986$

$38,138,491$

0
0
0
0
0
$56,949,477$

DISPOSITION

Lease and Plant Use

Returned to Formations

Interstate Movements

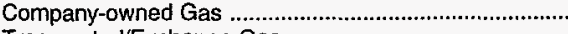

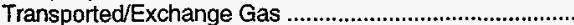

Exchange Gas Deliveries

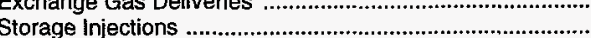

Deliveries of Company-owned Gas

To Storage Operators

.

To Resellers

To Consumers

Commercial

Industrial

Electric Utility

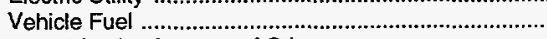

Transported for the Account of Others

To Storage Operators .

To Other Pipelines

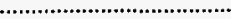

Restlers .......................................................................

To Consumers

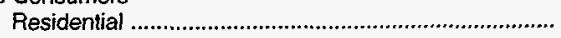

Commercial

Industrial

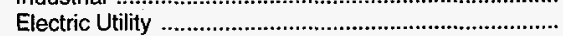

Vehicle Fuel ..................................................................

Pipeline/Compressor Fuel

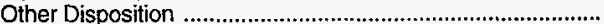

Unaccounted For

Total Disposition

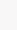

Source: Energy Information Administration (EIA), Form ElA-176, "Annual Report of Natural and Supplemental Gas Supply and Disposition."

with the top five companies who nationally operate utility and nonutility natural gas vehicles (NGV), and continues to promote NGV's in public and private fleet operations. More than 1300 NGV's currently operate within Southwest's service areas. In October 1993, the first public NGV refueling station opened in the city of Las Vegas. Concurrently, the city of Las Vegas was designated a "Clean City" by the Department of Energy because of the city's efforts to reduce auto emissions through NGV's.

Industrial and transportation customers comprised less than 1 percent of Southwest's total customer base. Natural gas delivered for cogeneration also continued to grow, mostly for large industrial customers who wanted to produce their own electricity. The company is also exploring other technologies, such as natural gas cooling with gas powered absorption chillers. A residential gas heat pump is still being tested.

Paiute Pipeline Company, a wholly owned subsidiary, transports gas for Southwest. One of the two major pipeline transmission systems Paiute operates extends from the Idaho-Nevada border to the Reno Sparks and Carson City areas and the Lake Tahoe area of California and Nevada, and northern and western Nevada. The second major pipeline system stretches from the Colorado River at the Southern tip of Nevada to the Las Vegas distribution area. Transportation of gas supplies goes through the pipeline systems of El Paso, Kern River, Northwest Pipeline Corporation, and Southern California Gas Company. 
Table 52. Southwest Gas Corp., Interstate Flows of Natural Gas, 1993 (Thousand Cubic Feet)

\begin{tabular}{|c|c|}
\hline Company and State & Volume \\
\hline $\begin{array}{l}\text { Southwest Gas Corp. } \\
\text { Nevada To } \\
\text { Califomia } \\
\text { Nevada From } \\
\text { Arizona } \\
\text { Jdaho } \\
\text { Califomia From } \\
\text { Arizona }\end{array}$ & $\begin{array}{r}930,728 \\
35,893,045 \\
39,196,714 \\
837,395,703\end{array}$ \\
\hline
\end{tabular}

Source: Energy Information Administration (EIA), Form EIA-176, "Annual Report of Natural and Supplemental Gas Supply and Disp-osition." 


\section{Tenneco, Inc.}

Tenneco is ranked as one of the 30 largest industrial companies in the United States. It also ranks among the top 100 natural gas marketers and provides approximately 5 percent of the supply for total domestic consumption. The Company is involved in various areas of the natural gas industry, including power generation, international gas projects, and providing funds for oil and gas exploration and production. It markets and transports approximately 16 percent of the natural gas consumed in the United States and operates more than 19,000 miles of pipeline in 26 States. The Company owns and operates the largest offshore natural gas pipeline system in the Gulf of Mexico.
In 1993, the Tenneco Power/International group completed construction of the 236-megawatt Mass Power facility near Springfield, Massachusetts, its first cogeneration project, and has rights to negotiate a 125-megawatt power facility elsewhere in New England. Also, the Tenneco Power/International group was selected to build, operate, and develop a 72-megawatt cogeneration and utility service plant near Texas A\&M University. The Tenneco Power/International group has also joined other utilities to convert three power plants to seasonal use of natural gas, and to supply natural gas as the primary fuel for two new power plants. In the near future, Tennessee Gas Pipeline Co., a subsidiary of Tenneco, plans to

Figure 40. Tenneco, Inc., 1993

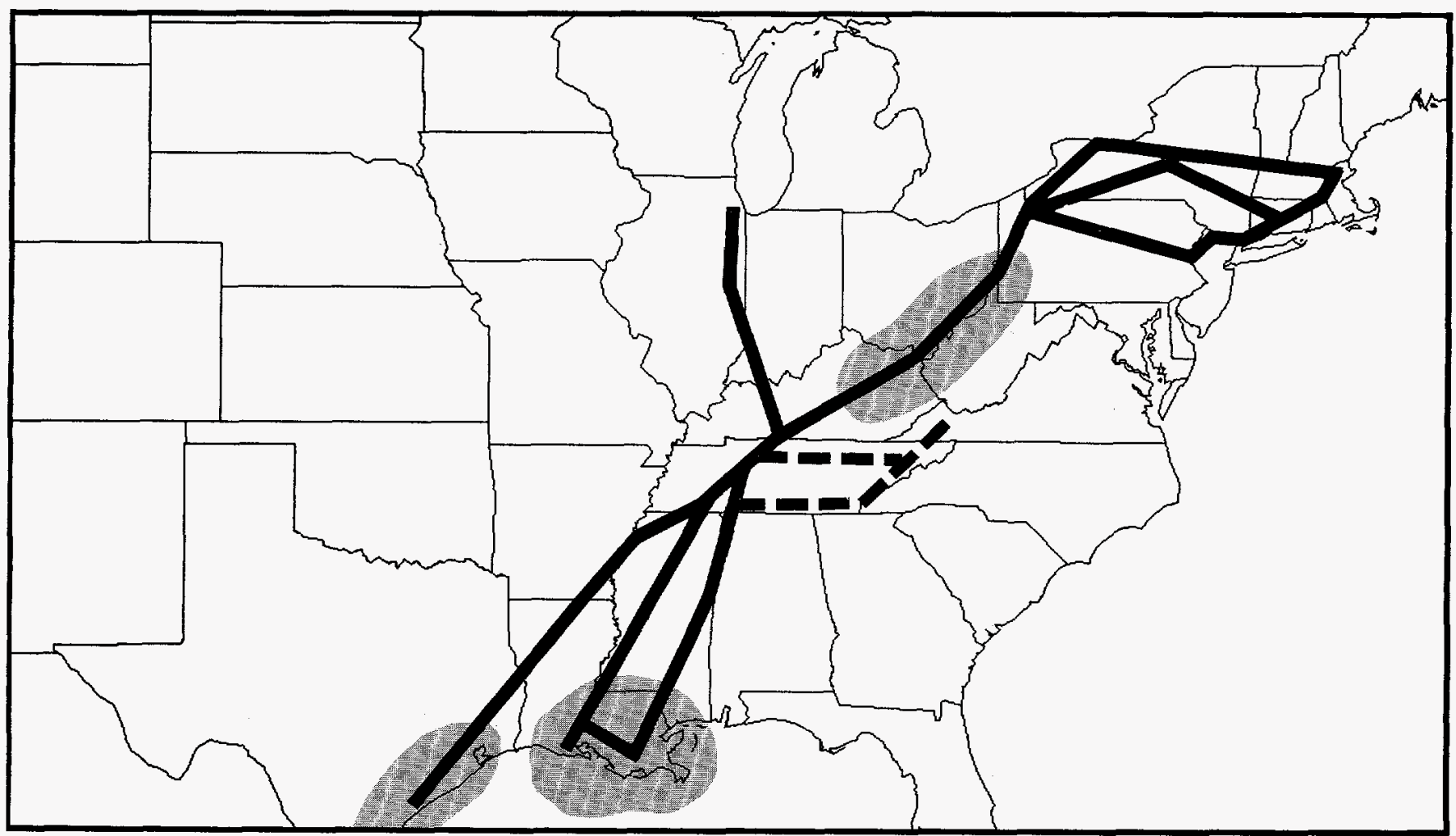

Source: Energy Information Administration (EIA), Form EIA-176, "Annual Report of Natural and Supplemental Gas Supply and Disposition."

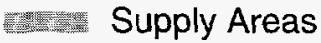
Tennessee Gas Pipeline Co. Midwestern Gas Transmission Co. ש East Tennessee Natural Gas Co. 
Table 53. Tenneco, Inc., Natural Gas Data, 1993 (Thousand Cubic Feet)

\begin{tabular}{|c|c|c|c|c|c|c|}
\hline \multirow{2}{*}{ Supply/Disposition } & \multicolumn{6}{|c|}{ Tennessee Gas Pipeline Co. } \\
\hline & Texas & Louisiana & Arkansas & Mississippi & Alabama & Tennessee \\
\hline \multicolumn{7}{|l|}{ SUPPLY } \\
\hline $\begin{array}{l}\text { Produced Onsystem } \\
\text { Receipts }\end{array}$ & 0 & $1,261,469$ & 0 & 0 & 0 & 0 \\
\hline 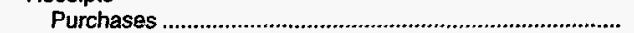 & $23,617,289$ & $53,390,599$ & 0 & 126,006 & 1,526 & 0 \\
\hline 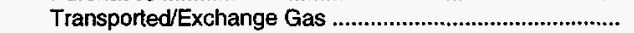 & $335,464,811$ & $1,094,854,522$ & 0 & $13,315,707$ & $8,576,577$ & 0 \\
\hline \multicolumn{7}{|l|}{ Interstate Movements } \\
\hline 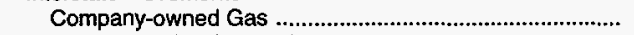 & 0 & 0 & 0 & 0 & 0 & 0 \\
\hline 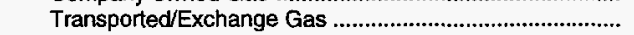 & 0 & $316,962,544$ & $323,995,352$ & $1,101,656,175$ & $543,409,611$ & $964,645,256$ \\
\hline 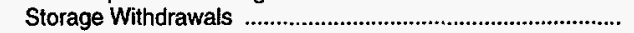 & 0 & 0 & 0 & 0 & 0 & 0 \\
\hline 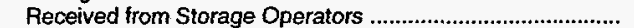 & 0 & $47,512,173$ & 0 & 224,998 & 0 & 0 \\
\hline 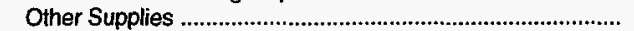 & 0 & 0 & 0 & 0 & 0 & 0 \\
\hline 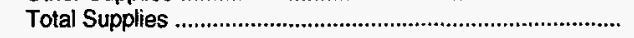 & $359,082,100$ & $1,513,981,307$ & $323,995,352$ & $1,115,322,886$ & $551,987,714$ & $964,645,256$ \\
\hline \multicolumn{7}{|l|}{ DISPOSITION } \\
\hline Lease and Plant Use & 0 & 65,592 & 0 & 0 & 0 & 0 \\
\hline Retumed to Formations & 0 & 0 & 0 & 0 & 0 & 0 \\
\hline \multicolumn{7}{|l|}{ Interstate Movements } \\
\hline 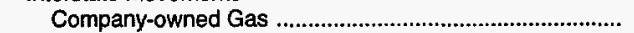 & $7,377,970$ & $70,779,685$ & $20,755,999$ & $67,709,988$ & $33,370,868$ & $31,305,238$ \\
\hline 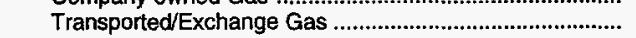 & $309,584,574$ & $1,031,245,324$ & $302,870,519$ & $928,640,601$ & $478,333,410$ & $770,596,304$ \\
\hline 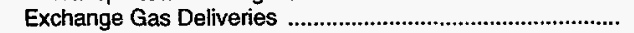 & $2,491,074$ & $66,702,941$ & 0 & $22,838,510$ & 2,814 & 977,231 \\
\hline 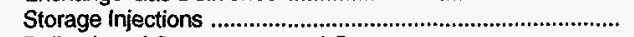 & 0 & 0 & 0 & 0 & 0 & 0 \\
\hline \multicolumn{7}{|l|}{ Deliveries of Company-owned Gas } \\
\hline To Storage Operators & 0 & $13,457,238$ & 0 & 224,998 & 0 & 0 \\
\hline 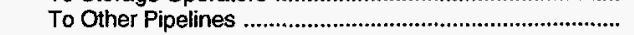 & 0 & 0 & 53,228 & 0 & $3,084,839$ & $20,862,067$ \\
\hline 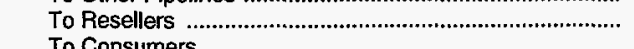 & $16,143,948$ & $25,213,250$ & 0 & $3,142,475$ & 474,846 & $11,984,524$ \\
\hline $\begin{array}{l}\text { To Consumers } \\
\text { Residential }\end{array}$ & 0 & 0 & 0 & 0 & 0 & 0 \\
\hline 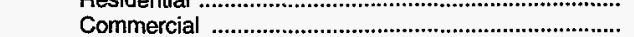 & 0 & 0 & 0 & 0 & 0 & 0 \\
\hline Industrial & 95,371 & 26,446 & 0 & 0 & 0 & 0 \\
\hline 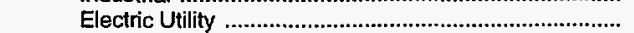 & 0 & 0 & 0 & 0 & 0 & 0 \\
\hline Vehicle Fuel & 0 & 0 & 0 & 0 & 0 & 0 \\
\hline \multicolumn{7}{|l|}{ Transported for the Account of Others } \\
\hline 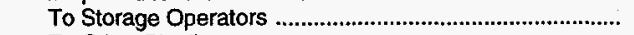 & 0 & $12,255,376$ & 0 & 0 & 0 & 0 \\
\hline To Other Pipelines & $14,496,405$ & $276,926,508$ & 7,366 & $78,964,300$ & $34,998,155$ & $119,673,896$ \\
\hline 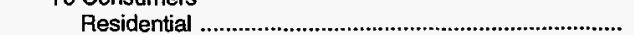 & 0 & 0 & 0 & 0 & 0 & 0 \\
\hline 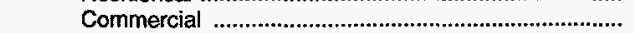 & 0 & 0 & 0 & 0 & 0 & 0 \\
\hline 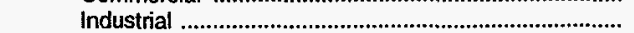 & 0 & 0 & 0 & 0 & 0 & 0 \\
\hline 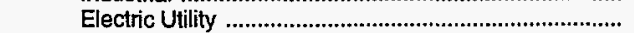 & 0 & 0 & 0 & 0 & 0 & 0 \\
\hline 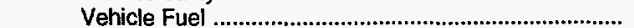 & 0 & 0 & 0 & 0 & 0 & $\mathbf{0}$ \\
\hline 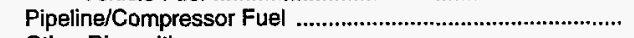 & $3,960,249$ & $10,613,958$ & 0 & $10,211,777$ & $1,226,234$ & $6,587,340$ \\
\hline Other Disposition & 0 & 0 & 0 & 0 & 0 & 0 \\
\hline Unaccounted For & $4,932,509$ & $6,694,989$ & 308,240 & $3,590,237$ & 496,548 & $2,658,656$ \\
\hline 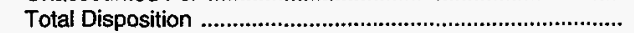 & $359,082,100$ & $1,513,981,307$ & $323,995,352$ & $1,115,322,886$ & $551,987,714$ & $964,645,256$ \\
\hline
\end{tabular}

construct a 24-inch pipeline from Rhode Island to supply Boston Edison's electric generation plant in south Boston.

In 1994, Tenneco Mobile Bay Gathering Company, in a joint venture, acquired a 50-percent interest in the Dauphin Island Gathering Systems (DIGS). The venture involves the construction of a 30 -mile offshore natural gas gathering system to connect wells in Mobile Bay, Alabama to the Viosca Knoll Gathering System in the Gulf of Mexico. This project is part of a long-range strategy to pursue nonregulated business opportunities in the natural gas marketplace. When completed, DIGS will have the capacity to transport more than 200 million cubic feet of gas per day.
Also in 1994, Tenneco Gas Gathering and the Leviathan Gas Pipeline Partners agreed jointly to construct a pipeline system to gather natural gas from producing properties located offshore of Louisiana. The properties under construction are located at the Viosca Knoll Gathering System and Main Pass areas. The joint venture includes the construction of approximately 95 miles of 20-inch pipeline from a Shell Offshore platform in Main Pass Block 252 to an interconnect with Tennessee Gas Pipeline in South Pass Block 55. In addition, the partnership plans to build a 16-inch, 6-mile pipeline from Viosca Knoll to an interconnect at a platform with Southern Natural Gas Company in Main Pass Block 289. The system will transport approximately 400 million cubic feet of gas per day. 
Table 53. Tenneco, Inc., Natural Gas Data, 1993 (Continued) (Thousand Cubic Feet)

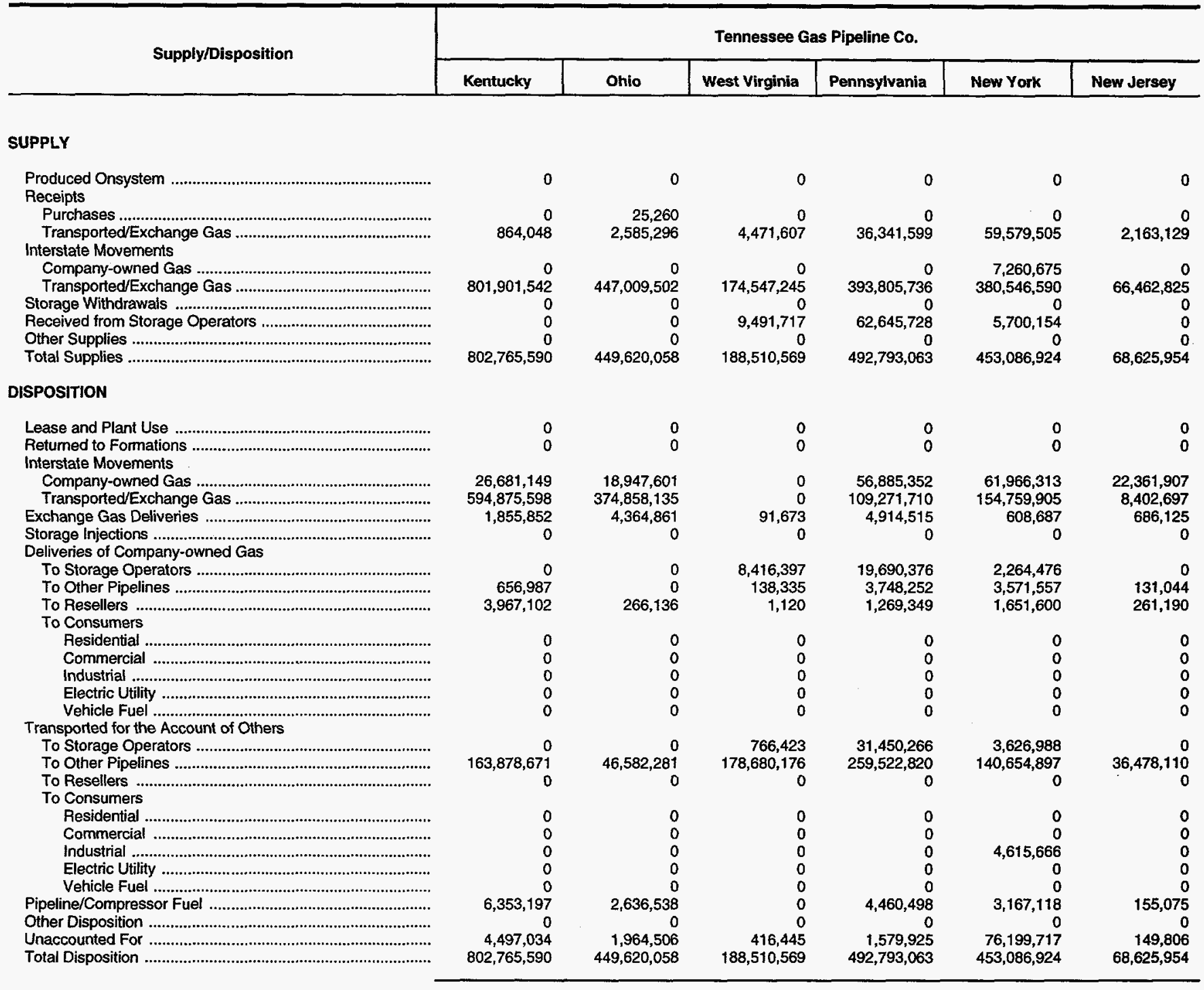

By 1995, Tenneco, Questar Corporation, and Entech Incorporated (a division of Montana Power) are expected to build the first full service natural gas hub near Opal, Wyoming. The hub will be located at a pipeline interconnect called Muddy Creek and will link Wyoming's gasproducing area with five major pipelines that serve markets in the West and Midwest. The hub will enable Muddy Creek pipelines to flow gas in many directions. Also, the hub will be set up to provide gas sellers and buyers with such varied services as: wheeling, peaking, parking, balancing, title tracking and electronic gas trading that will give customers the choice of redirecting their gas flows to take advantage of new market and supply opportunities. Customers will have access to approximately 1.8 billion cubic feet of gas per day.

Tennessee Gas Pipeline's expansion of its northeastern natural gas pipeline is expected to be completed in 1997. The expanded pipeline will add approximately 300 million cubic feet of additional capacity and provide new access between the Gulf Coast and Mid-Continent. 
Table 53. Tenneco, Inc., Natural Gas Data, 1993 (Continued) (Thousand Cubic Feet)

\begin{tabular}{|c|c|c|c|c|c|}
\hline \multirow{2}{*}{ Supply/Disposition } & \multicolumn{4}{|c|}{ Tennessee Gas Pipeline Co. } & \multirow{2}{*}{$\begin{array}{c}\text { Midwestern Gas } \\
\text { Transmission Co. } \\
\text { Tennessee }\end{array}$} \\
\hline & Connecticut & Rhode Island & Massachuselts & New Hampshire & \\
\hline \multicolumn{6}{|l|}{ SUPPLY } \\
\hline $\begin{array}{l}\text { Produced Onsystem } \\
\text { Receipts }\end{array}$ & 0 & 0 & 0 & 0 & 0 \\
\hline 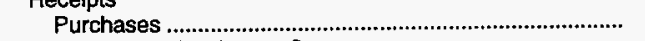 & 0 & 0 & 0 & 0 & $23,142,338$ \\
\hline 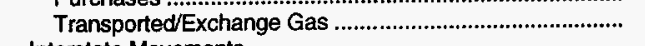 & $17,029,376$ & $41,217,159$ & $17,734,090$ & 163,466 & $56,910,663$ \\
\hline 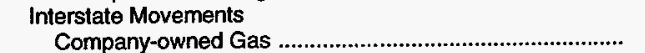 & 0 & & & & \\
\hline $\begin{array}{l}\text { Company-owned Gas } \\
\text { Transported/Exchange Gas }\end{array}$ & $\begin{array}{r}0 \\
35,995,218\end{array}$ & $\begin{array}{r}0 \\
42,686,673\end{array}$ & $\begin{array}{r}0 \\
182,890,713\end{array}$ & $\begin{array}{r}0 \\
9,531,666\end{array}$ & $\begin{array}{l}0 \\
0\end{array}$ \\
\hline Storage Withdrawals & 0 & $\begin{array}{r}42,686,6 / 3 \\
0\end{array}$ & 0 & 0 & 0 \\
\hline 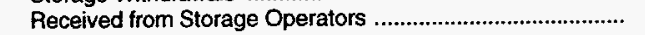 & 0 & 0 & 0 & 0 & 0 \\
\hline 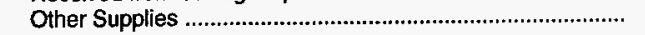 & 0 & 0 & 0 & 0 & 0 \\
\hline 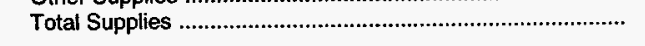 & $53,024,594$ & $83,903,832$ & $200,624,803$ & $9,695,132$ & $80,053,001$ \\
\hline \multicolumn{6}{|l|}{ DISPOSITION } \\
\hline 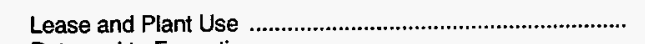 & 0 & 0 & 0 & 0 & 0 \\
\hline 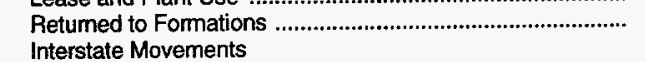 & 0 & 0 & 0 & 0 & 0 \\
\hline 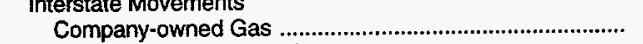 & 0 & 0 & $35,509,368$ & 0 & 0 \\
\hline 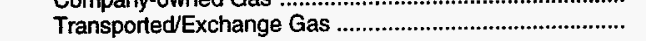 & 0 & 0 & $23,631,906$ & 0 & $79,777,102$ \\
\hline Exchange Gas Deliveries & 892,651 & $1,354,077$ & $8,107,623$ & 40,064 & 0 \\
\hline 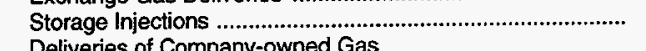 & 0 & 0 & 0 & 0 & 0 \\
\hline & 0 & 0 & $\begin{array}{r}0 \\
4,720,310\end{array}$ & $\begin{array}{l}0 \\
0\end{array}$ & 0 \\
\hline To Other Pipelines & 0 & 0 & $\begin{array}{r}4,720,310 \\
14050,490\end{array}$ & $\begin{array}{r}0 \\
871,533\end{array}$ & 0 \\
\hline & 14 & $2,085,711$ & & 871,533 & 0 \\
\hline 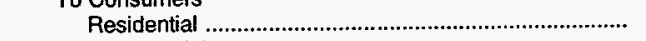 & 0 & 0 & 0 & 0 & 0 \\
\hline 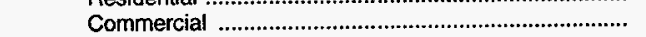 & 0 & 0 & 0 & 0 & 0 \\
\hline Industrial & 0 & 0 & 0 & 0 & 0 \\
\hline 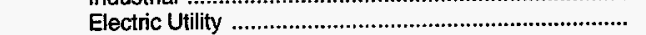 & 0 & 0 & 0 & 0 & 0 \\
\hline Vehicle Fuel & 0 & 0 & 0 & 0 & 0 \\
\hline \multicolumn{6}{|l|}{ Transported for the Account of Others } \\
\hline 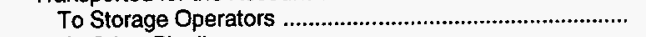 & 0 & 0 & 0 & $\begin{array}{r}0 \\
8.596 .997\end{array}$ & 0 \\
\hline To Other Pipelines & $51,852,473$ & $44,106,314$ & $117,390,609$ & $8,596,997$ & 0 \\
\hline 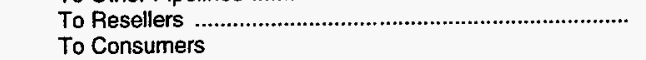 & 0 & 0 & 0 & 0 & 0 \\
\hline 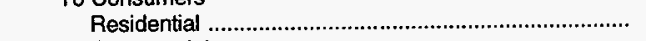 & 0 & 0 & 0 & 0 & 0 \\
\hline 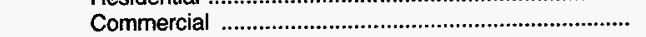 & 0 & 0 & 0 & 0 & 0 \\
\hline 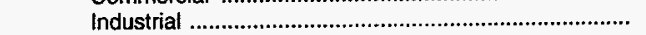 & 0 & $36,344,492$ & 0 & 0 & 0 \\
\hline 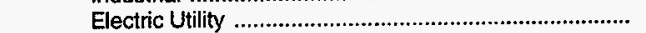 & 0 & 0 & 0 & 0 & 0 \\
\hline Vehicle Fuel & 0 & 0 & 0 & 0 & 0 \\
\hline 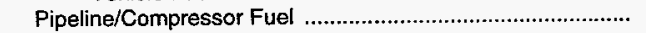 & 22,771 & 0 & 792,112 & 0 & 272,803 \\
\hline 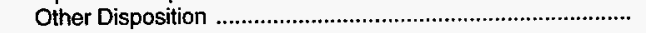 & 0 & 0 & 0 & 0 & 0 \\
\hline 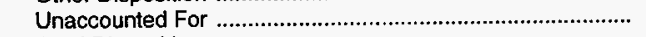 & 256,685 & 13,238 & $-3,577,615$ & 186,538 & 3,096 \\
\hline 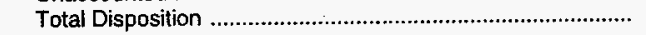 & $53,024,594$ & $83,903,832$ & $200,624,803$ & $9,695,132$ & $80,053,001$ \\
\hline
\end{tabular}


Table 53. Tenneco, Inc., Natural Gas Data, 1993 (Continued) (Thousand Cubic Feet)

\begin{tabular}{|c|c|c|c|c|c|}
\hline \multirow{2}{*}{ Supply/Disposition } & \multicolumn{3}{|c|}{ Midwestern Gas Transmission Co. } & \multicolumn{2}{|c|}{ Viking Gas Transmission Co. } \\
\hline & Kentucky & Indiana & Illinois & North Dakota & Minnesota \\
\hline \multicolumn{6}{|l|}{ SUPPLY } \\
\hline $\begin{array}{l}\text { Produced Onsystem } \\
\text { Receipts }\end{array}$ & 0 & 0 & 0 & 0 & 0 \\
\hline Purchases & 0 & 0 & 92,864 & 0 & 0 \\
\hline 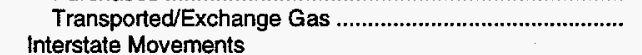 & $60,407,691$ & 69 & $15,489,134$ & 0 & $1,819,263$ \\
\hline Company-owned Gas & 0 & 0 & 0 & 0 & 0 \\
\hline 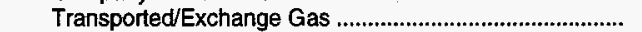 & $79,777,102$ & $139,157,523$ & $125,315,876$ & $5,536,921$ & $135,710,678$ \\
\hline 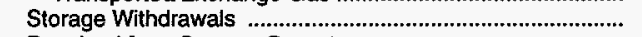 & 0 & 0 & 0 & 0 & 0 \\
\hline 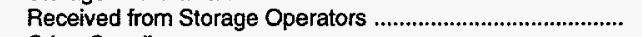 & 0 & 0 & 0 & 0 & 0 \\
\hline Other Supplies & 0 & 0 & 0 & 0 & 0 \\
\hline 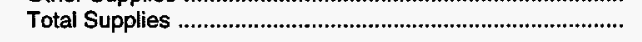 & $140,184,793$ & $139,157,592$ & $140,897,874$ & $5,536,921$ & $137,529,941$ \\
\hline \multicolumn{6}{|l|}{ DISPOSITION } \\
\hline Lease and Plant Use & 0 & 0 & 0 & 0 & 0 \\
\hline $\begin{array}{l}\text { Returned to Formations } \\
\text { Interstate Movements }\end{array}$ & 0 & 0 & 0 & 0 & 0 \\
\hline 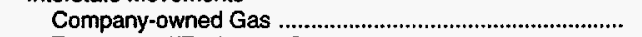 & 0 & 0 & 0 & 0 & 0 \\
\hline 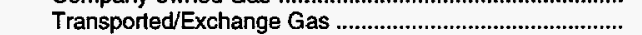 & $139,157,523$ & $125,315,876$ & 0 & 0 & $110,115,886$ \\
\hline Exchange Gas Deliveries & 0 & 0 & 0 & 0 & 0 \\
\hline Storage Injections & 0 & 0 & 0 & 0 & 0 \\
\hline \multicolumn{6}{|l|}{ Deliveries of Company-owned Gas } \\
\hline 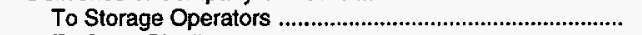 & 0 & 0 & 0 & 0 & 0 \\
\hline 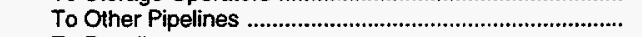 & 0 & 0 & 0 & 0 & 0 \\
\hline $\begin{array}{l}\text { To Resellers } \\
\text { To Consumers }\end{array}$ & 376,280 & 99,926 & $29,966,643$ & 0 & 0 \\
\hline 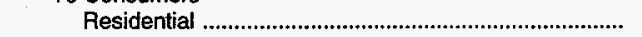 & 0 & 0 & 0 & 0 & 0 \\
\hline 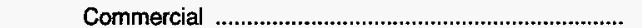 & 0 & 0 & 0 & 0 & 0 \\
\hline 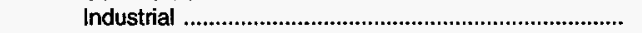 & 0 & 0 & 0 & 0 & 0 \\
\hline 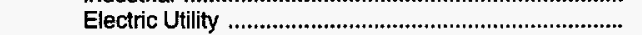 & 0 & 0 & 0 & 0 & 0 \\
\hline 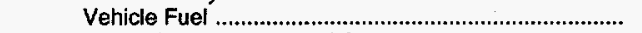 & 0 & 0 & 0 & 0 & 0 \\
\hline \multicolumn{6}{|l|}{ Transported for the Account of Others } \\
\hline To Storage Operators & 0 & 0 & 0 & 0 & 0 \\
\hline 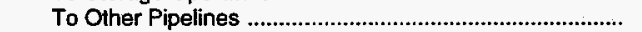 & 419,165 & $13,067,660$ & $110,434,001$ & $5,537,064$ & $26,320,827$ \\
\hline $\begin{array}{l}\text { To Resellers } \\
\text { To Consumers }\end{array}$ & \multicolumn{4}{|c|}{ To Consumers } & 0 \\
\hline 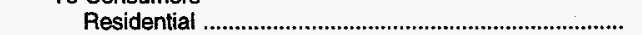 & 0 & 0 & 0 & 0 & 0 \\
\hline 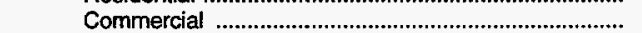 & 0 & 0 & 0 & 0 & 0 \\
\hline 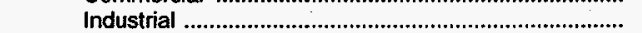 & 0 & 0 & 0 & 0 & 0 \\
\hline Electric Utility & 0 & 0 & 0 & 0 & 0 \\
\hline 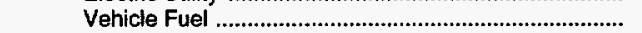 & 0 & 0 & 0 & 0 & 0 \\
\hline 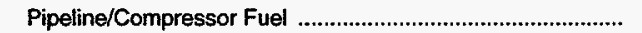 & 119,192 & 544,232 & 321,851 & 0 & $2,113,218$ \\
\hline 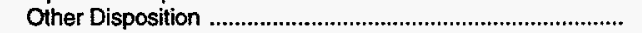 & 0 & 0 & 0 & 0 & 0 \\
\hline Unaccounted For & 112,633 & 129,898 & 175,379 & -143 & $-1,019,990$ \\
\hline Total Disposition & $140,184,793$ & $139,157,592$ & $140,897,874$ & $5,536,921$ & $137,529,941$ \\
\hline
\end{tabular}


Table 53. Tenneco, Inc., Natural Gas Data, 1993 (Continued) (Thousand Cubic Feet)

\begin{tabular}{|c|c|c|c|c|c|}
\hline \multirow{2}{*}{ Supply/Disposition } & \multirow{2}{*}{$\begin{array}{c}\text { Viking Gas } \\
\text { Transmission Co. } \\
\text { Wisconsin }\end{array}$} & \multicolumn{2}{|c|}{ East Tennessee Natural Gas Co. } & \multirow{2}{*}{$\begin{array}{c}\begin{array}{c}\text { Channel Industries } \\
\text { Gas Co. }\end{array} \\
\text { Texas }\end{array}$} & \multirow{2}{*}{$\begin{array}{c}\begin{array}{c}\text { Creole Gas Pipeline } \\
\text { Corp. }\end{array} \\
\text { Louisiana }\end{array}$} \\
\hline & & Tennessee & Virginia & & \\
\hline \multicolumn{6}{|l|}{ SUPPLY } \\
\hline $\begin{array}{l}\text { Produced Onsystem } \\
\text { Receipts }\end{array}$ & .0 & 0 & 0 & 0 & 0 \\
\hline 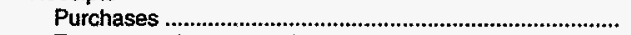 & 0 & $26,153,991$ & $9,129,371$ & 0 & $26,498,410$ \\
\hline 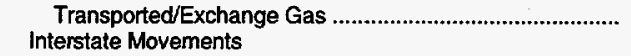 & $1,474,549$ & $74,207,981$ & 245,092 & $471,960,943$ & 847,937 \\
\hline Company-owned Gas & 0 & 0 & 0 & 0 & 0 \\
\hline 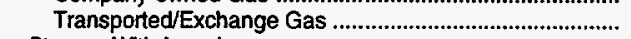 & $104,578,965$ & $2,335,045$ & 0 & 0 & 0 \\
\hline Storage Withdrawals & 0 & $1,279,594$ & 0 & 0 & 0 \\
\hline 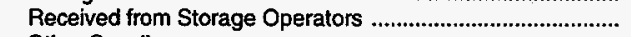 & 0 & $15,272,991$ & 0 & $8,184,178$ & 0 \\
\hline 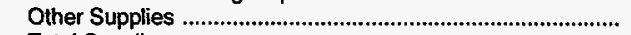 & 0 & 0 & 0 & 0 & 0 \\
\hline Total Supplies & $106,053,514$ & $119,249,602$ & $9,374,463$ & $480,145,121$ & $27,346,347$ \\
\hline \multicolumn{6}{|l|}{ DISPOSITION } \\
\hline Lease and Plant Use & 0 & 0 & 0 & 0 & 0 \\
\hline $\begin{array}{l}\text { Retumed to Formations } \\
\text { Interstate Movements }\end{array}$ & 0 & 0 & 0 & 0 & 0 \\
\hline 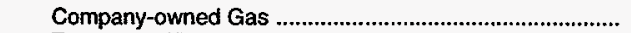 & 0 & 0 & 0 & 0 & 0 \\
\hline 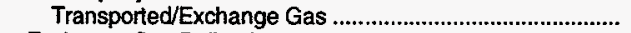 & 0 & 0 & $2,335,045$ & 0 & 0 \\
\hline 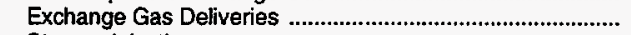 & 0 & 0 & 0 & 0 & 0 \\
\hline $\begin{array}{l}\text { Storage Injections .................................................................... } \\
\text { Deliveries of Company-owned Gas }\end{array}$ & 0 & $1,249,980$ & 0 & 0 & 0 \\
\hline To Storage Operators & 0 & $5,829,965$ & 0 & 0 & 0 \\
\hline 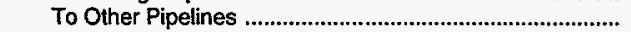 & 0 & 0 & 0 & 0 & 0 \\
\hline 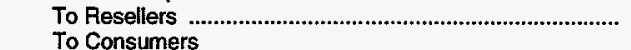 & 0 & $36,146,168$ & $2,692,996$ & 0 & 0 \\
\hline 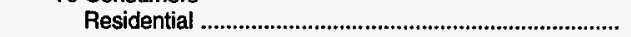 & 0 & 0 & 0 & 0 & 0 \\
\hline 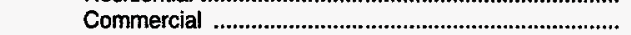 & 0 & 20,524 & 0 & 0 & 0 \\
\hline 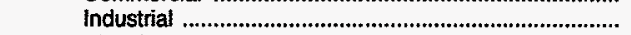 & 0 & $5,214,912$ & 0 & 0 & $18,734,575$ \\
\hline 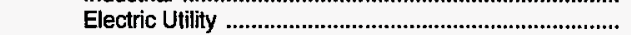 & 0 & 0 & 0 & 0 & $7,721,462$ \\
\hline Vehicle Fuel & 0 & 0 & 0 & 0 & 0 \\
\hline \multicolumn{6}{|l|}{ Transported for the Account of Others } \\
\hline To Storage Operators & 0 & 0 & 0 & $8,329,331$ & 0 \\
\hline 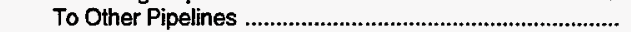 & $105,819,852$ & 0 & 0 & $258,196,485$ & 0 \\
\hline 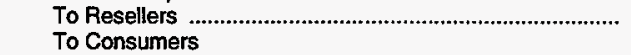 & 0 & $57,076,260$ & $4,860,989$ & 41,836 & 0 \\
\hline 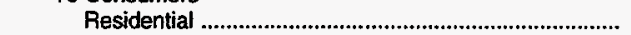 & 0 & 0 & 0 & 0 & 0 \\
\hline 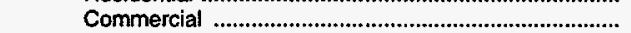 & 0 & 0 & 0 & 14,867 & 0 \\
\hline Industrial & 0 & $11,937,971$ & 0 & $139,770,655$ & $1,153,099$ \\
\hline 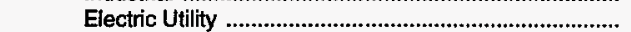 & 0 & 0 & 0 & $70,812,468$ & 0 \\
\hline 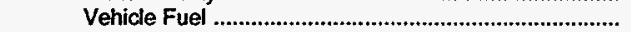 & 0 & 0 & 0 & 0 & 0 \\
\hline 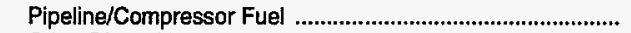 & 299,452 & $1,472,812$ & 272,037 & 789,747 & 0 \\
\hline 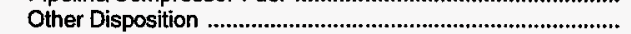 & 0 & 0 & 0 & 14,287 & 0 \\
\hline Unaccounted For & $-65,790$ & 301,010 & $-786,604$ & $2,175,445$ & $-262,789$ \\
\hline Total Disposition & $106,053,514$ & $119,249,602$ & $9,374,463$ & $480,145,121$ & $27,346,347$ \\
\hline
\end{tabular}

Source: Energy Information Administration (ElA), Form ElA-176, "Annual Report of Natural and Supplemental Gas Supply and Disposition." 
Table 54. Tenneco, Inc., Interstate Flows of Natural Gas, 1993

(Thousand Cubic Feet)

\begin{tabular}{|c|c|}
\hline Company and State & Volume \\
\hline \multicolumn{2}{|l|}{$\begin{array}{l}\text { Tennessee Gas Pipeline Co. } \\
\text { Texas To }\end{array}$} \\
\hline \multicolumn{2}{|l|}{ Louisiana To } \\
\hline 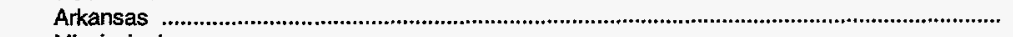 & $323,995,352$ \\
\hline Mississippi & $778,029,657$ \\
\hline Arkansas To & \\
\hline Mississippi & $323,626,518$ \\
\hline \multicolumn{2}{|l|}{ Mississippi To } \\
\hline $\begin{array}{l}\text { Alabama } \\
\text { Tennessee }\end{array}$ & $\begin{array}{l}543,409,611 \\
452,940,978\end{array}$ \\
\hline \multicolumn{2}{|l|}{ Alabama To } \\
\hline \multirow{2}{*}{\multicolumn{2}{|c|}{$\begin{array}{l}\text { Tennessee } \\
\text { Tennessee To }\end{array}$}} \\
\hline & \\
\hline \multicolumn{2}{|l|}{ (1) } \\
\hline Ohio & $447,009,502$ \\
\hline West Virginia & $174,547,245$ \\
\hline \multicolumn{2}{|l|}{ Ohio To } \\
\hline \multirow{2}{*}{\multicolumn{2}{|c|}{ Pennsylvania To }} \\
\hline & \\
\hline New Jersey & $66,462,825$ \\
\hline 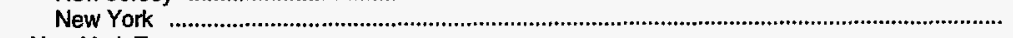 & $99,694,237$ \\
\hline \multicolumn{2}{|l|}{ New York To } \\
\hline Connecticut . . & $33,835,505$ \\
\hline 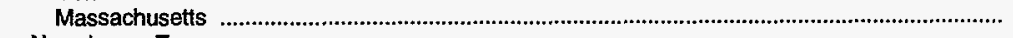 & $182,890,713$ \\
\hline \multicolumn{2}{|l|}{ New Jersey To } \\
\hline 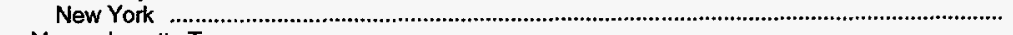 & $30,764,604$ \\
\hline \multicolumn{2}{|l|}{ Massachusetts To } \\
\hline Connecticut & $2,159,713$ \\
\hline New Hampshire & $23,826,554$ \\
\hline Rhode Island & $42,686,673$ \\
\hline \multicolumn{2}{|l|}{$\begin{array}{l}\text { Midwestern Gas Transmission Co. } \\
\text { Tennessee To }\end{array}$} \\
\hline 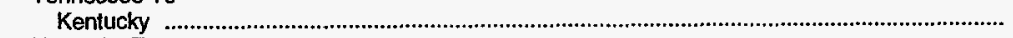 & $79,777,102$ \\
\hline \multicolumn{2}{|l|}{ Kentucky To } \\
\hline Indiana & $139,157,523$ \\
\hline \multicolumn{2}{|l|}{ Indiana To } \\
\hline \multicolumn{2}{|l|}{ Illinois } \\
\hline \multicolumn{2}{|l|}{$\begin{array}{l}\text { East Tennessee Natural Gas Co. } \\
\text { Virginia To }\end{array}$} \\
\hline Tennessee & $2,335,045$ \\
\hline \multicolumn{2}{|l|}{$\begin{array}{l}\text { Viking Gas Transmission Co. } \\
\text { Canada To }\end{array}$} \\
\hline Minnnesota & $135,710,678$ \\
\hline \multicolumn{2}{|l|}{ Minnesota To } \\
\hline North Dakota & $5,536,921$ \\
\hline 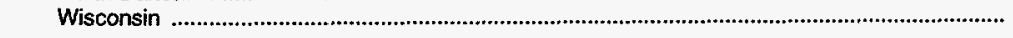 & $104,578,965$ \\
\hline
\end{tabular}

Source: Energy Information Administration (EIA), Form EIA-176, "Annual Report of Natural and Supplemental Gas Supply and Disposition." 



\section{Transco Gas Co.}

Transco Gas Company is engaged primarily in the interstate natural gas pipeline and natural gas marketing businesses. Transco also has investments in natural gas liquids processing, natural gas gathering, coal mining, and marketing operations. Transco also had a nonoperating interest in a coalbed methane project in Alabama. During 1993, the company completed the transfer of its interest in coalbed methane properties in Alabama's Black Warrior Basin to TECO Energy, Inc. Transco also regained control of gathering pipelines that it had held in partnership with Corpus Christi Gas Gathering.

The company's pipeline business is conducted through Transcontinental Gas Pipe Line Corporation (TGPL) and
Texas Gas Transmission Corporation (Texas Gas), wholly owned subsidiaries of Transco, which in turn, is wholly owned by Transco Energy Company. TGPL and Texas Gas are principally engaged in the transport of natural gas from the Gulf of Mexico and the Gulf Coast regions to markets in the eastern half of the United States.

TGPL's 10,500-mile pipeline system extends from the Gulf Coast supply areas to New York City. Its principal markets encompass 11 Southeastern and Atlantic seaboard States, including the New York City and Philadelphia metropolitan areas. The company has total peak mainline delivery capacity of approximately 3.3 billion cubic feet per day, and additional peak-day delivery

Figure 41. Transco Gas Co., 1993

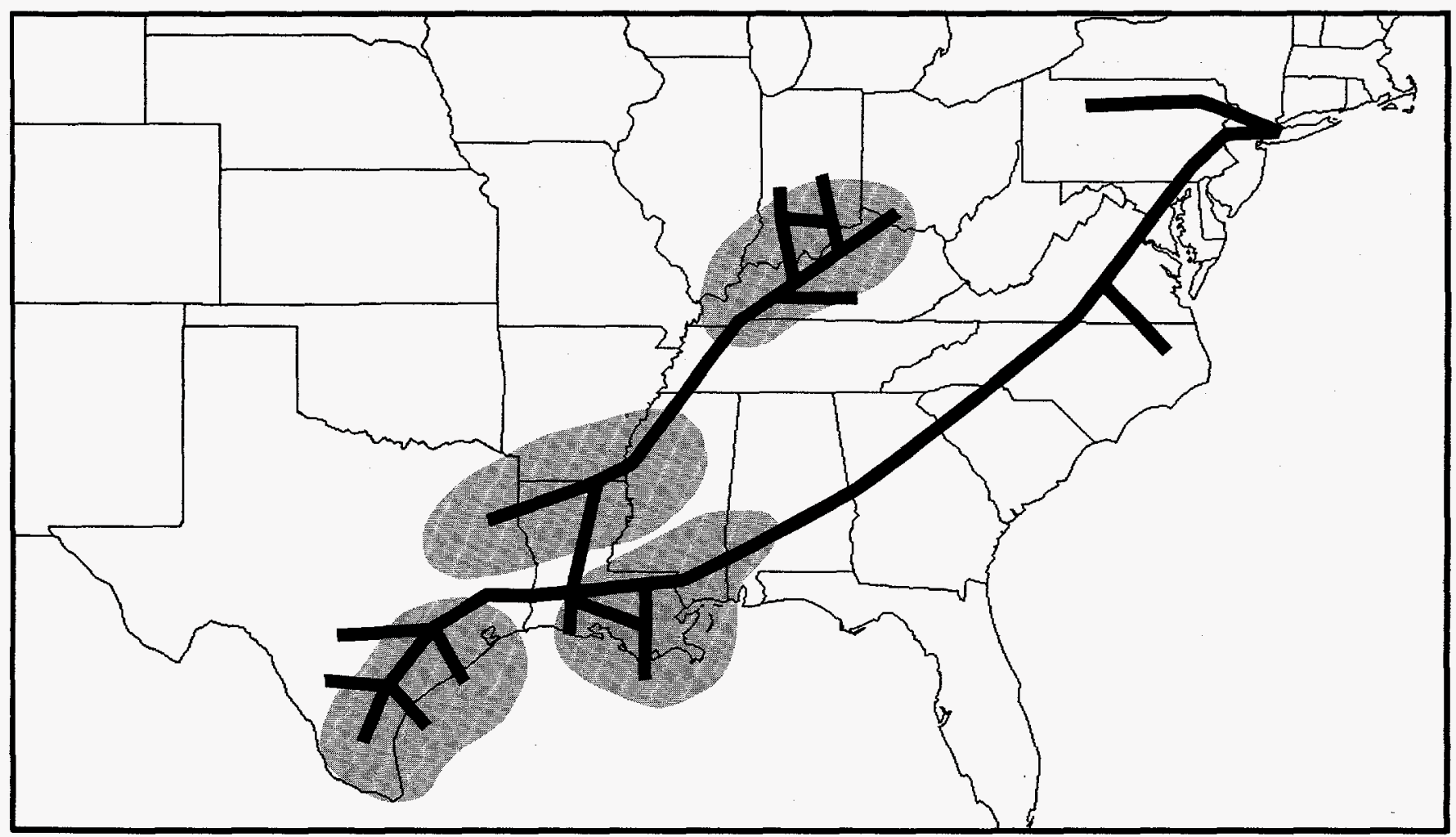

Source: Energy Information Administration (EIA), Form EIA-176, "Annual Report of Natural and Supplemental Gas Supply and Disposition." 
Table 55. Transco Gas Co., Natural Gas Data, 1993

(Thousand Cubic Feet)

\begin{tabular}{|c|c|c|c|c|c|c|}
\hline \multirow{2}{*}{ Supply/Disposition } & \multicolumn{6}{|c|}{ Transcontinental Gas Pipe Line Corp. } \\
\hline & Texas & Louisiana & Mississippi & Alabama & Georgia & South Carolina \\
\hline \multicolumn{7}{|l|}{ SUPPLY } \\
\hline \multicolumn{7}{|l|}{ Receipts } \\
\hline 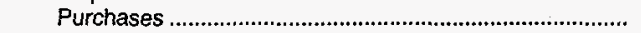 & $54,190,444$ & $60,282,168$ & $58,262,429$ & $13,119,862$ & 0 & 0 \\
\hline 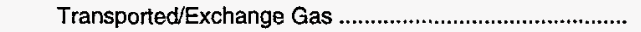 & $374,719,313$ & $692,181,735$ & $30,997,149$ & $83,517,593$ & 344,547 & 0 \\
\hline \multicolumn{7}{|l|}{ Interstate Movements } \\
\hline 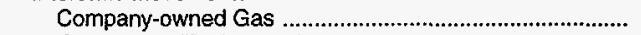 & 0 & 0 & 0 & 0 & 0 & 0 \\
\hline 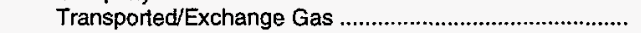 & 0 & $228,108,399$ & $855,456,809$ & $936,880,295$ & $1,005,947,097$ & $945,946,167$ \\
\hline 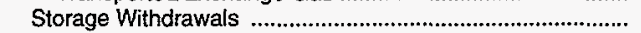 & 0 & $66,277,100$ & $6,851,229$ & 0 & 0 & 0 \\
\hline 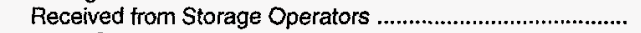 & 0 & 0 & 0 & 0 & 0 & 0 \\
\hline 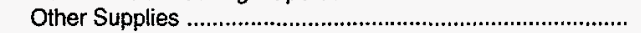 & 0 & 0 & 0 & 0 & 0 & 0 \\
\hline 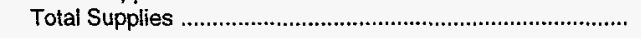 & $428,909,757$ & $1,046,849,402$ & $951,567,616$ & $1,033,517,750$ & $1,006,291,644$ & $945,946,167$ \\
\hline \multicolumn{7}{|l|}{ DISPOSITION } \\
\hline 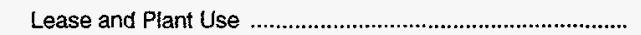 & $1,009,753$ & $21,125,118$ & 0 & 0 & 0 & 0 \\
\hline Returned to Formations & 0 & 0 & 0 & 0 & 0 & 0 \\
\hline \multicolumn{7}{|l|}{ Interstate Movements } \\
\hline 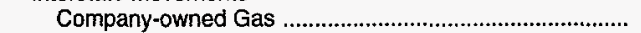 & 0 & 0 & 0 & 0 & 0 & 0 \\
\hline 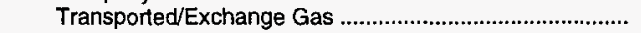 & $228,108,399$ & $855,456,809$ & $936,880,295$ & $1,005,947,097$ & $945,946,167$ & $893,469,368$ \\
\hline 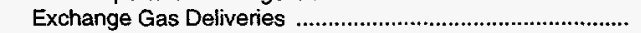 & $51,059,252$ & $7,400,034$ & 0 & 0 & 0 & 0 \\
\hline 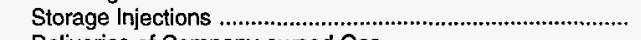 & 0 & $55,523,440$ & $9,691,906$ & 0 & 0 & 0 \\
\hline \multicolumn{7}{|l|}{ Deliveries of Company-owned Gas } \\
\hline To Storage Operators & 0 & 0 & 0 & 0 & 0 & 0 \\
\hline 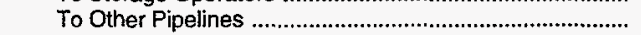 & 0 & 0 & 0 & 0 & 0 & 0 \\
\hline 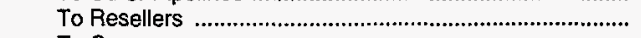 & 0 & 0 & 0 & 9,319 & 7,065 & 5,568 \\
\hline \multicolumn{7}{|l|}{ To Consumers } \\
\hline Residential & 0 & 0 & 0 & 0 & 0 & 0 \\
\hline 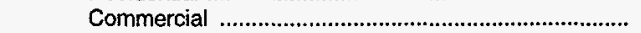 & 0 & 0 & 0 & 0 & 0 & 0 \\
\hline 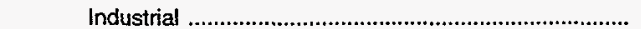 & 0 & 0 & 0 & 0 & 0 & 0 \\
\hline Electric Utility & 0 & 0 & 0 & 0 & 0 & 0 \\
\hline Vehicle Fuel & 0 & 0 & 0 & 0 & 0 & 0 \\
\hline \multicolumn{7}{|l|}{ Transported for the Account of Others } \\
\hline 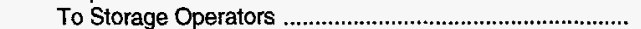 & 0 & 0 & 0 & 0 & 0 & 0 \\
\hline 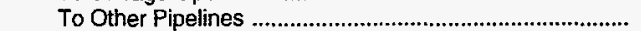 & $146,619,422$ & $99,422,227$ & 191,087 & 8,200 & $55,325,436$ & 0 \\
\hline $\begin{array}{l}\text { To Resellers } \\
\text { To Consumers }\end{array}$ & 307 & $1,911,115$ & 0 & $19,030,975$ & 0 & $48,153,620$ \\
\hline To Consumers & 0 & 0 & 0 & 0 & 0 & 0 \\
\hline 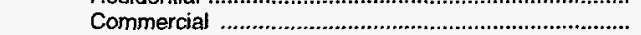 & 0 & 0 & 0 & 0 & 0 & 0 \\
\hline Industrial & 0 & 123,879 & 0 & 0 & 0 & $1,916,272$ \\
\hline 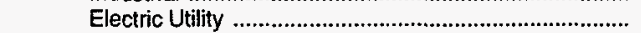 & 0 & 0 & 0 & 0 & 0 & 0 \\
\hline Vehicle Fuel & 0 & 0 & 0 & $\mathbf{0}$ & 0 & 0 \\
\hline 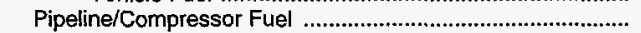 & $2,112,624$ & $5,886,780$ & $4,804,328$ & $8,522,159$ & $5,012,976$ & $2,401,339$ \\
\hline Other Disposition & 0 & 0 & 0 & 0 & 0 & 0 \\
\hline Unaccounted For & 0 & 0 & 0 & 0 & 0 & 0 \\
\hline 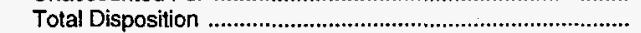 & $428,909,757$ & $1,046,849,402$ & $951,567,616$ & $1,033,517,750$ & $1,006,291,644$ & $945,946,167$ \\
\hline
\end{tabular}

capacity of 2.6 billion cubic feet per day through the Leidy line and market area facilities. TGPL operates an extensive gas-gathering system both onshore and offshore in the Gulf Coast area. TGPL also has 213 billion cubic feet of working gas storage capacity in five underground storage fields located on or near its pipeline system or market areas.

Texas Gas's 6,050-mile pipeline system extends from Louisiana and Texas to Illinois. Direct market areas encompass Louisiana, Arkansas, Mississippi, Tennessee, Kentucky, Indiana, Ohio and Illinois, including the Memphis, Tennessee; Louisville, Kentucky; Cincinnati and Dayton, Ohio; and Indianapolis, Indiana metropolitan areas. Texas Gas has indirect market access to northeast markets through interconnections with Columbia Gas Transmission Corporation, CNG Transmission Corporation, and Texas Eastern Transmission Corporation. The company also owns and operates 10 underground storage reservoirs with 86.5 billion cubic feet of working gas storage capacity.

In September 1993, the Federal Energy Regulatory Commission authorized joint ownership and expansion of TGPL's Mobile Bay lateral with Florida Gas Transmission Company. The lateral transports gas from the Mobile Bay gas supply basin to the TGPL mainline, near Butler, Alabama. This expansion will involve adding compression facilities and a new interconnect on the lateral with the Florida Gas mainline. TGPL's portion of pipeline capacity will be increased by roughly 58.6 million to 520.6 million cubic feet per day. The Mobile Bay lateral is expected to be in service by December 1994. In addition, TGPL and Exxon are constructing a 2-mile pipeline to connect the Mobile Bay lateral with Exxon's recently constructed treatment plant. 
Table 55. Transco Gas Co., Natural Gas Data, 1993 (Continued) (Thousand Cubic Feet)

\begin{tabular}{|c|c|c|c|c|c|}
\hline \multirow{2}{*}{ Supply/Disposition } & \multicolumn{5}{|c|}{ Transcontinental Gas Pipe Line Corp. } \\
\hline & North Carolina & Virginia & Maryland & Pennsylvania & New Jersey \\
\hline \multicolumn{6}{|l|}{ SUPPLY } \\
\hline $\begin{array}{l}\text { Produced Onsystem } \\
\text { Receipts }\end{array}$ & 0 & 0 & 0 & 0 & 0 \\
\hline 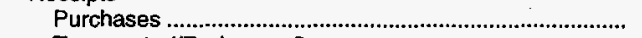 & 0 & 0 & 0 & 0 & 0 \\
\hline $\begin{array}{l}\text { Transported/Exchange Gas } \\
\text { Interstate Movements }\end{array}$ & 0 & $8,776,165$ & 0 & $198,503,370$ & $23,714,037$ \\
\hline 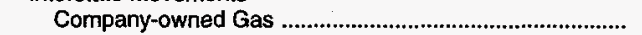 & 0 & 0 & 0 & 0 & 0 \\
\hline 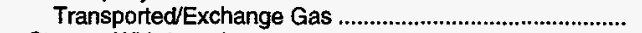 & $893,469,368$ & $700,015,243$ & $648,038,004$ & $633,484,844$ & $573,915,014$ \\
\hline 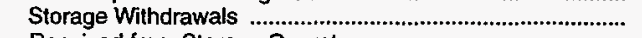 & 0 & 0 & 0 & 0 & $1,362,420$ \\
\hline 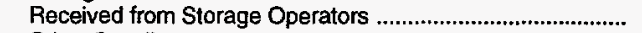 & 0 & 0 & 0 & 0 & 0 \\
\hline 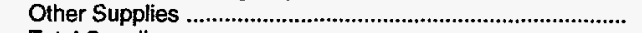 & 0 & 0 & 0 & 0 & 0 \\
\hline 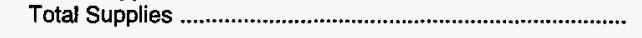 & $893,469,368$ & $708,791,408$ & $648,038,004$ & $831,988,214$ & $598,991,471$ \\
\hline \multicolumn{6}{|l|}{ DISPOSITION } \\
\hline Lease and Plant Use & 0 & 0 & 0 & 0 & 0 \\
\hline $\begin{array}{l}\text { Returned to Formations } \\
\text { Interstate Movements }\end{array}$ & 0 & 0 & 0 & 0 & 0 \\
\hline 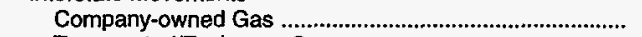 & 0 & 0 & 0 & 0 & 0 \\
\hline 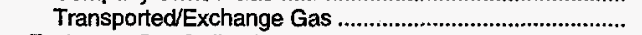 & $700,015,243$ & $648,038,004$ & $633,484,844$ & $591,485,647$ & $288,171,087$ \\
\hline 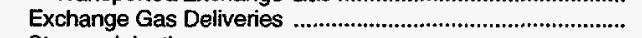 & 0 & 0 & 0 & $11,316,259$ & 0 \\
\hline Storage Injections & 0 & 0 & 0 & 0 & $1,981,426$ \\
\hline \multicolumn{6}{|l|}{ Deliveries of Company-owned Gas } \\
\hline 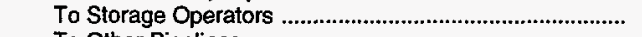 & 0 & 0 & 0 & 0 & 0 \\
\hline 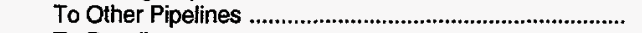 & 0 & 0 & 0 & 0 & 0 \\
\hline 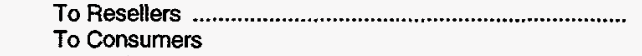 & 3,094 & 0 & 0 & 0 & 0 \\
\hline Residential & 0 & 0 & 0 & 0 & 0 \\
\hline 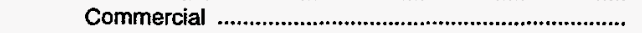 & 0 & 0 & 0 & 0 & 0 \\
\hline Industrial & 0 & 0 & 0 & 0 & 0 \\
\hline Electric Utility & 0 & 0 & 0 & 0 & 0 \\
\hline Vehicle Fuel & 0 & 0 & 0 & 0 & 0 \\
\hline \multicolumn{6}{|l|}{ Transported for the Account of Others } \\
\hline To Storage Operators & 0 & 0 & 0 & 0 & 0 \\
\hline 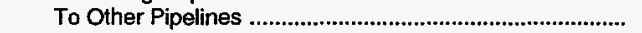 & 0 & $2,093,009$ & 7,337 & $14,368,225$ & $12,437,999$ \\
\hline $\begin{array}{l}\text { To Resellers ....................................................................... } \\
\text { To Consumers }\end{array}$ & $187,854,425$ & $54,175,919$ & $12,829,638$ & $+97,396,873$ & $292,217,056$ \\
\hline 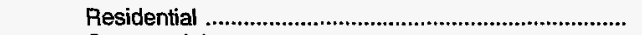 & 0 & 0 & 0 & 0 & 0 \\
\hline 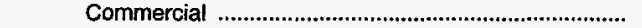 & 0 & 0 & 0 & 0 & 0 \\
\hline Industrial & 0 & 0 & 0 & $13,406,541$ & $3,923,680$ \\
\hline 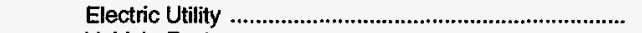 & 0 & 0 & 0 & 0 & 0 \\
\hline Vehicle Fuel & 0 & 0 & 0 & 0 & 0 \\
\hline 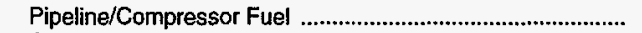 & $5,596,606$ & $4,484,476$ & $1,716,185$ & $4,014,669$ & 260,223 \\
\hline Other Disposition & 0 & 0 & 0 & 0 & 0 \\
\hline 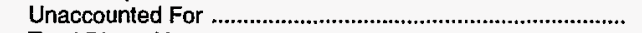 & 0 & 0 & 0 & 0 & 0 \\
\hline 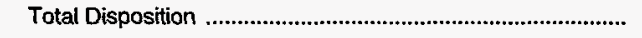 & $893,469,368$ & $708,791,408$ & $648,038,004$ & $831,988,214$ & $598,991,471$ \\
\hline
\end{tabular}

During 1993, TGPL completed the first phase of its Eminence storage field expansion project, expanding the working capacity from 6 billion to 9 billion cubic feet and increasing the withdrawal rate from 750 million cubic feet per day to 1.3 billion cubic feet per day. Further expansions are planned in 1994 and 1995, allowing for withdrawals of up to 1.5 billion cubic feet per day and ultimately increasing the working capacity of the storage field to 15 billion cubic feet.

In May 1994, the FERC granted a final certificate to TGPL for the company's 1994 Southeast Expansion Project. This project will add 35 million cubic feet per day of new capacity to markets in Alabama, Georgia, North Carolina, South Carolina, and Virginia for the 1994-1995 heating season. The 1994 project represents the first of three incremental mainline capacity expansions announced last year. In late June 1994, FERC provided preliminary approval for the 1995-1996 Southeast Expansion Project. Final approval is awaiting the resolution of environmental issues. The 1995-1996 project will add increased capacity of 115 million cubic feet per day for the 1995-1996 heating season, and 50 million cubic feet per day for the 19961997 heating season. Combined expansion facilities will include approximately 25 miles of pipeline replacement and looping and the installation of additional compression totaling approximately 70,000 horsepower.

Sponsors of the Liberty Pipeline in the New York City area have asked FERC to indefinitely postpone review of the 
Table 55. Transco Gas Co., Natural Gas Data, 1993 (Continued) (Thousand Cubic Feet)

\begin{tabular}{|c|c|c|c|c|c|}
\hline \multirow{2}{*}{ Supply/Disposition } & \multirow{2}{*}{$\begin{array}{c}\begin{array}{c}\text { Transcontinental } \\
\text { Gas Pipe Line } \\
\text { Corp. }\end{array} \\
\text { New York }\end{array}$} & \multicolumn{4}{|c|}{ Texas Gas Transmission Corp. } \\
\hline & & Texas & Louisiana & Arkansas & Mississippi \\
\hline \multicolumn{6}{|l|}{ SUPPLY } \\
\hline $\begin{array}{l}\text { Produced Onsystem } \\
\text { Receipts }\end{array}$ & 0 & 0 & 0 & 0 & 0 \\
\hline 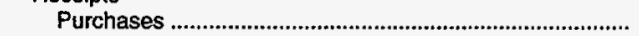 & 0 & $1,834,593$ & $15,553,068$ & 0 & 657,067 \\
\hline 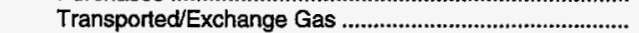 & 0 & $46,141,031$ & $719,802,090$ & $27,862,778$ & 0 \\
\hline \multicolumn{6}{|l|}{ Interstate Movements } \\
\hline 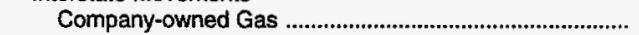 & 0 & 0 & 0 & 0 & 0 \\
\hline 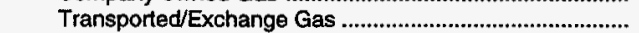 & $288,171,087$ & 0 & $46,439,943$ & $494,490,114$ & $517,548,178$ \\
\hline 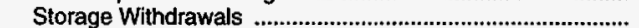 & 0 & 0 & 0 & 0 & 0 \\
\hline 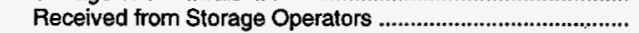 & 0 & 0 & 0 & 0 & 0 \\
\hline Other Supplies & 204,873 & 0 & 0 & 0 & 0 \\
\hline 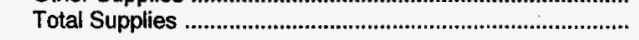 & $288,375,960$ & $47,975,624$ & $781,795,101$ & $522,352,892$ & $518,205,245$ \\
\hline \multicolumn{6}{|l|}{ DISPOSITION } \\
\hline Lease and Plant Use & 0 & 0 & 0 & 0 & 0 \\
\hline 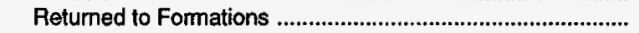 & 0 & 0 & 0 & 0 & 0 \\
\hline \multicolumn{6}{|l|}{ Interstate Movements } \\
\hline Company-owned Gas & 0 & 0 & 0 & 0 & 0 \\
\hline 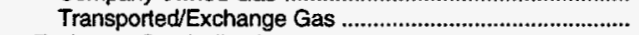 & 0 & $46,439,943$ & $494,490,114$ & $517,548,178$ & $497,004,043$ \\
\hline Exchange Gas Deliveries & 0 & 0 & 0 & 0 & 0 \\
\hline Storage Injections & 0 & 0 & 0 & 0 & 0 \\
\hline \multicolumn{6}{|l|}{ Deliveries of Company-owned Gas } \\
\hline To Storage Operators & 0 & 0 & 0 & 0 & 0 \\
\hline To Other Pipelines & 0 & 0 & 0 & 395,233 & $1,896,901$ \\
\hline 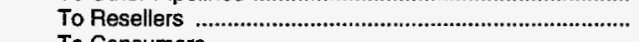 & 0 & 0 & 284,335 & 75,238 & $2,849,560$ \\
\hline \multicolumn{6}{|l|}{ To Consumers } \\
\hline 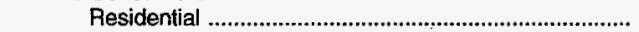 & 0 & 0 & 0 & 0 & 0 \\
\hline 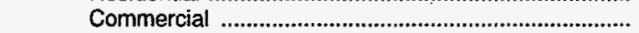 & 0 & 0 & 0 & 0 & 0 \\
\hline Industrial & 0 & 0 & 0 & 0 & 0 \\
\hline 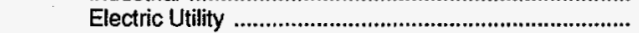 & 0 & 0 & 0 & 0 & 0 \\
\hline 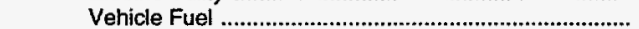 & 0 & 0 & 0 & 0 & 0 \\
\hline \multicolumn{6}{|l|}{ Transported for the Account of Others } \\
\hline To Storage Operators & 0 & 0 & 0 & 0 & 0 \\
\hline To Other Pipelines & 0 & $1,426,374$ & $125,145,822$ & 0 & 0 \\
\hline 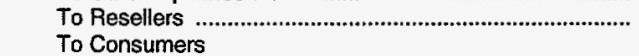 & $286,085,909$ & 111,310 & $149,505,499$ & $1,792,660$ & $11,083,920$ \\
\hline 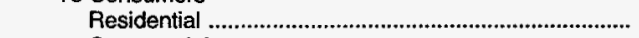 & 0 & 0 & 0 & 0 & 0 \\
\hline 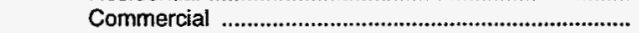 & 0 & 0 & 0 & 0 & 0 \\
\hline 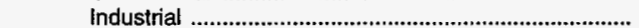 & 0 & 0 & $10,440,818$ & 0 & 0 \\
\hline 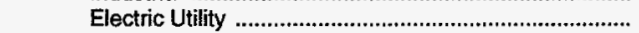 & 0 & 0 & 0 & $2,547,946$ & $2,033,551$ \\
\hline 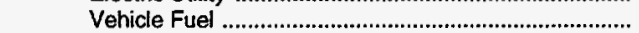 & 0 & 0 & 0 & 0 & 0 \\
\hline Pipeline/Compressor Fuel & 0 & 0 & $2,309,333$ & 0 & $3,365,313$ \\
\hline Other Disposition & 0 & 0 & 0 & 0 & 0 \\
\hline Unaccounted For & $2,290,051$ & $-2,003$ & $-380,820$ & $-6,363$ & $-28,043$ \\
\hline 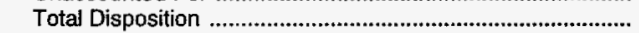 & $288,375,960$ & $47,975,624$ & $781,795,101$ & $522,352,892$ & $518,205,245$ \\
\hline
\end{tabular}

project, following withdrawal in June 1994 of one key shipper and project partner, and the more recent withdrawal of another shipper. The partners are continuing to pursue plans for an additional delivery point in New York as proposed by the Liberty project. As originally planned, the 38-mile pipeline would have provided a new transportation route from New Jersey to Long Island, N.Y., providing up to 500 million cubic feet per day of firm service to the greater New York area. Liberty's sponsors include subsidiaries of Transco Energy Company, Panhandle Eastern Corporation, The Coastal Corporation as well as two New York local distribution companies, The Brooklyn Union Gas Company and Consolidated Edison Company of New York. Pipeline companies affiliated with the Liberty project are: TGPL, Texas Gas, Panhandle Eastern
Pipe Line Company, Texas Eastern Transmission Corporation, Trunkline Gas Company, and ANR Pipeline Company. The pipeline subsidiaries transport gas to midwest and northeast markets.

In the fall of 1994, Transco acquired Tren-Fuels, Inc., a compressed natural gas (CNG) fueling company. TrenFuels, through its FleetStar subsidiary, provides CNG as a vehicle fuel through its network of public access fueling sites and its patented mobile fueling system. Tren-Fuels operates 11 CNG stations in Arizona, California, and Nevada; and in partnership with other companies, owns 17 fueling stations and 2 vehicle conversion facilities in Texas. 
Table 55. Transco Gas Co., Natural Gas Data, 1993 (Continued) (Thousand Cubic Feet)

\begin{tabular}{|c|c|c|c|c|c|}
\hline \multirow{2}{*}{ Supply/Disposition } & \multicolumn{5}{|c|}{ Texas Gas Transmission Corp. } \\
\hline & Tennessee & Kentucky & Illinois & Indiana & Ohio \\
\hline \multicolumn{6}{|l|}{ SUPPLY } \\
\hline $\begin{array}{l}\text { Produced Onsystem } \\
\text { Receipts }\end{array}$ & 0 & 3,544 & 0 & 0 & 0 \\
\hline 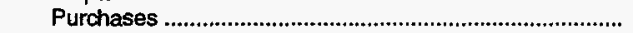 & 19,470 & 40,935 & 0 & 0 & 0 \\
\hline 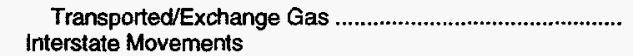 & $5,363,007$ & $15,938,284$ & 0 & 102,844 & 0 \\
\hline 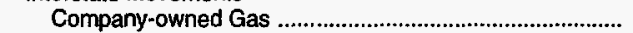 & 0 & 0 & 0 & 0 & 0 \\
\hline 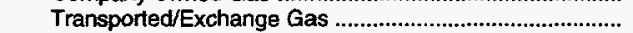 & $497,004,043$ & $447,353,517$ & $12,811,944$ & $345,003,151$ & $240,963,037$ \\
\hline 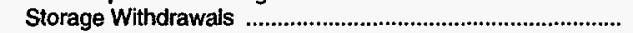 & 0 & $37,724,977$ & 0 & $5,121,465$ & 0 \\
\hline 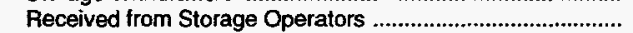 & 0 & 0 & 0 & 0 & 0 \\
\hline 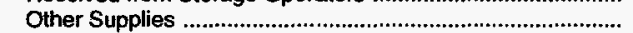 & 0 & 0 & 0 & 0 & 0 \\
\hline Total Supplies & $502,386,520$ & $501,061,257$ & $12,811,944$ & $350,227,460$ & $240,963,037$ \\
\hline \multicolumn{6}{|l|}{ DISPOSITION } \\
\hline Lease and Plant Use & 0 & 0 & 0 & 0 & 0 \\
\hline Retumed to Formations & 0 & 0 & 0 & 0 & 0 \\
\hline Interstate Movements & $\omega$ & & & & \\
\hline 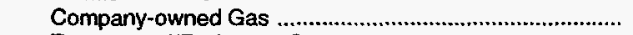 & 0 & 0 & 0 & 0 & 0 \\
\hline 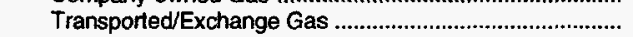 & $447,353,517$ & $345,003,151$ & 0 & $253,774,981$ & 0 \\
\hline 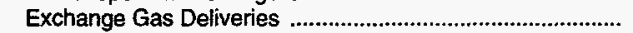 & 0 & $2,130,024$ & 0 & 0 & 0 \\
\hline 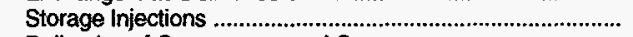 & 0 & $29,230,593$ & 0 & $4,743,656$ & 0 \\
\hline \multicolumn{6}{|l|}{ Deliveries of Company-owned Gas } \\
\hline To Storage Operators & 0 & 0 & 0 & 0 & 0 \\
\hline To Other Pipelines & 0 & 0 & 0 & 0 & 0 \\
\hline 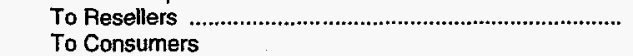 & $11,601,578$ & $19,632,745$ & $1,835,190$ & $12,719,357$ & $2,498,405$ \\
\hline 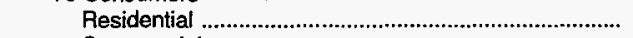 & 0 & 0 & 0 & 0 & 0 \\
\hline 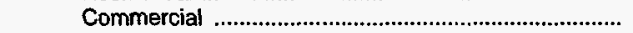 & 0 & 0 & 0 & 0 & 0 \\
\hline 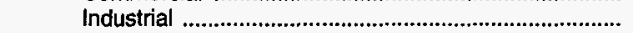 & 0 & 0 & 0 & 0 & 0 \\
\hline 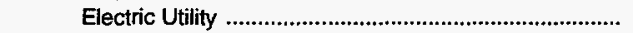 & 0 & 0 & 0 & 0 & 0 \\
\hline Vehicle Fuel & 0 & 0 & 0 & 0 & 0 \\
\hline \multicolumn{6}{|l|}{ Transported for the Account of Others } \\
\hline 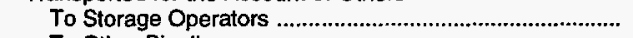 & 0 & 0 & 0 & 0 & 0 \\
\hline 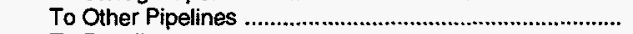 & 0 & 0 & 0 & 0 & $102,467,903$ \\
\hline 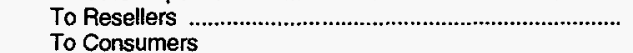 & $31,630,122$ & $94,281,182$ & $10,959,787$ & $77,892,202$ & $134,545,776$ \\
\hline 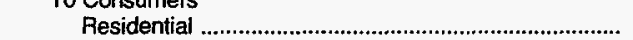 & 0 & 0 & 0 & 0 & 0 \\
\hline 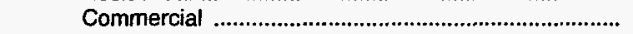 & 0 & 465,524 & 0 & 3,696 & 0 \\
\hline 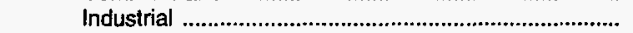 & $9,549,149$ & $6,312,291$ & 0 & 470,254 & $1,787,466$ \\
\hline 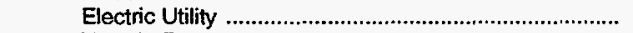 & 0 & 0 & 0 & 0 & 0 \\
\hline Vehicle Fuel & 0 & 0 & 0 & 0 & 0 \\
\hline 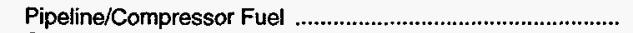 & $2,325,090$ & $4,210,535$ & 0 & 785,095 & 0 \\
\hline 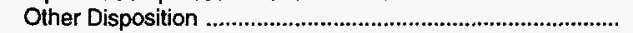 & 0 & 0 & 0 & 0 & 0 \\
\hline Unaccounted For & $-72,936$ & $-204,788$ & 16,967 & $-161,781$ & $-336,513$ \\
\hline Total Disposition & $502,386,520$ & $501,061,257$ & $12,811,944$ & $350,227,460$ & $240,963,037$ \\
\hline
\end{tabular}

Source: Energy Information Administration (EIA), Form ElA-176, "Annual Report of Natural and Supplemental Gas Supply and Disposition." 
Table 56. Transco Gas Co., Interstate Flows of Natural Gas, 1993

(Thousand Cubic Feet)

\begin{tabular}{|c|c|}
\hline Company and State & Volume \\
\hline \multicolumn{2}{|l|}{$\begin{array}{l}\text { Transcontinental Gas Pipe Line Corp. } \\
\text { Texas To }\end{array}$} \\
\hline 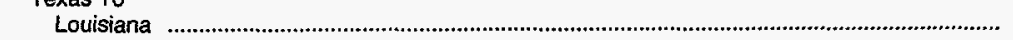 & $228,108,399$ \\
\hline \multicolumn{2}{|l|}{ Louisiana To } \\
\hline Mississippi & $855,456,809$ \\
\hline \multicolumn{2}{|l|}{ Mississippi To } \\
\hline Alabama & $936,880,295$ \\
\hline \multicolumn{2}{|l|}{ Alabama To } \\
\hline \multirow{2}{*}{\multicolumn{2}{|c|}{$\begin{array}{l}\text { Georgia } \\
\text { Georgia To }\end{array}$}} \\
\hline & \\
\hline South Carolina & $945,946,167$ \\
\hline \multicolumn{2}{|l|}{ South Carolina To } \\
\hline North Carolina & $893,469,368$ \\
\hline \multicolumn{2}{|l|}{ North Carolina To } \\
\hline Virginia & $700,015,243$ \\
\hline \multicolumn{2}{|l|}{$\begin{array}{l}\text { Virginia } \\
\text { Virginia To }\end{array}$} \\
\hline (1) & $648,038,004$ \\
\hline \multicolumn{2}{|l|}{ Maryland To } \\
\hline Pennsylvania & $633,484,844$ \\
\hline \multicolumn{2}{|l|}{ Pennsylvania To } \\
\hline Delaware & $17,570,633$ \\
\hline New Jersey & $573,915,014$ \\
\hline \multicolumn{2}{|l|}{ New Jersey To } \\
\hline \multicolumn{2}{|l|}{ New York } \\
\hline \multicolumn{2}{|l|}{$\begin{array}{l}\text { Texas Gas Transmission Corp. } \\
\text { Texas To }\end{array}$} \\
\hline $\begin{array}{l}\text { Texas To } \\
\text { Louisiana }\end{array}$ & $46,439,943$ \\
\hline \multicolumn{2}{|l|}{ Louisiana To } \\
\hline \multirow{2}{*}{\multicolumn{2}{|c|}{$\begin{array}{l}\text { Arkansas . } \\
\text { Arkansas To }\end{array}$}} \\
\hline & \\
\hline $\begin{array}{l}\text { Arkansas To } \\
\text { Mississippi }\end{array}$ & $517,548,178$ \\
\hline \multicolumn{2}{|l|}{ Mississippi To } \\
\hline \multirow{2}{*}{\multicolumn{2}{|c|}{ Tennessee ... }} \\
\hline & \\
\hline 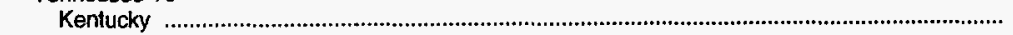 & $447,353,517$ \\
\hline \multicolumn{2}{|l|}{ Kentucky To } \\
\hline 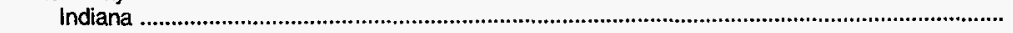 & $345,003,151$ \\
\hline \multicolumn{2}{|l|}{ Indiana To } \\
\hline 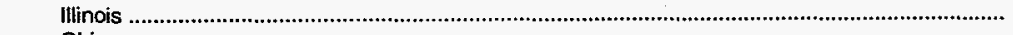 & $12,811,944$ \\
\hline Ohio & $240,963,037$ \\
\hline
\end{tabular}

Source: Energy Information Administration (EIA), Form EIA-176, "Annual Report of Natural and Supplemental Gas Supply and Disposition." 


\section{Valero Energy Corp.}

Valero Energy Corporation is a diversified energy company that is involved in natural gas marketing and transportation services. It has agreed to merge with Valero Gas Partners Limited Partnership, to regain complete control of the natural gas marketing, pipeline, and liquids operation that it sold in 1987. The partnership operates 7,200 miles of natural gas gathering and transmission lines in Texas. Valero Energy Corporation sold its natural gas retail distribution subsidiary, Rio Grande Valley Company, in 1993 for \$31 million.

Valero has increased the amount of its available storage capacity by using third-party storage. It has added six billion cubic feet of storage capacity to the approximate seven billion cubic feet of company-owned storage capacity used for the 1993-94 winter heating season.

Valero Transmission LP, a group of subsidiaries of the corporation, petitioned the Federal Energy Regulatory Commission to amend its existing permit to allow gas to be imported from Mexico at McAllen, Texas through its existing facilities. These facilities were previously being used exclusively to export gas. The company does not intend to buy or sell gas but to offer open access to shippers or suppliers. Imports from Mexico started flowing in December 1993 for the first time since 1984.

Figure 42. Valero Energy Corp., 1993

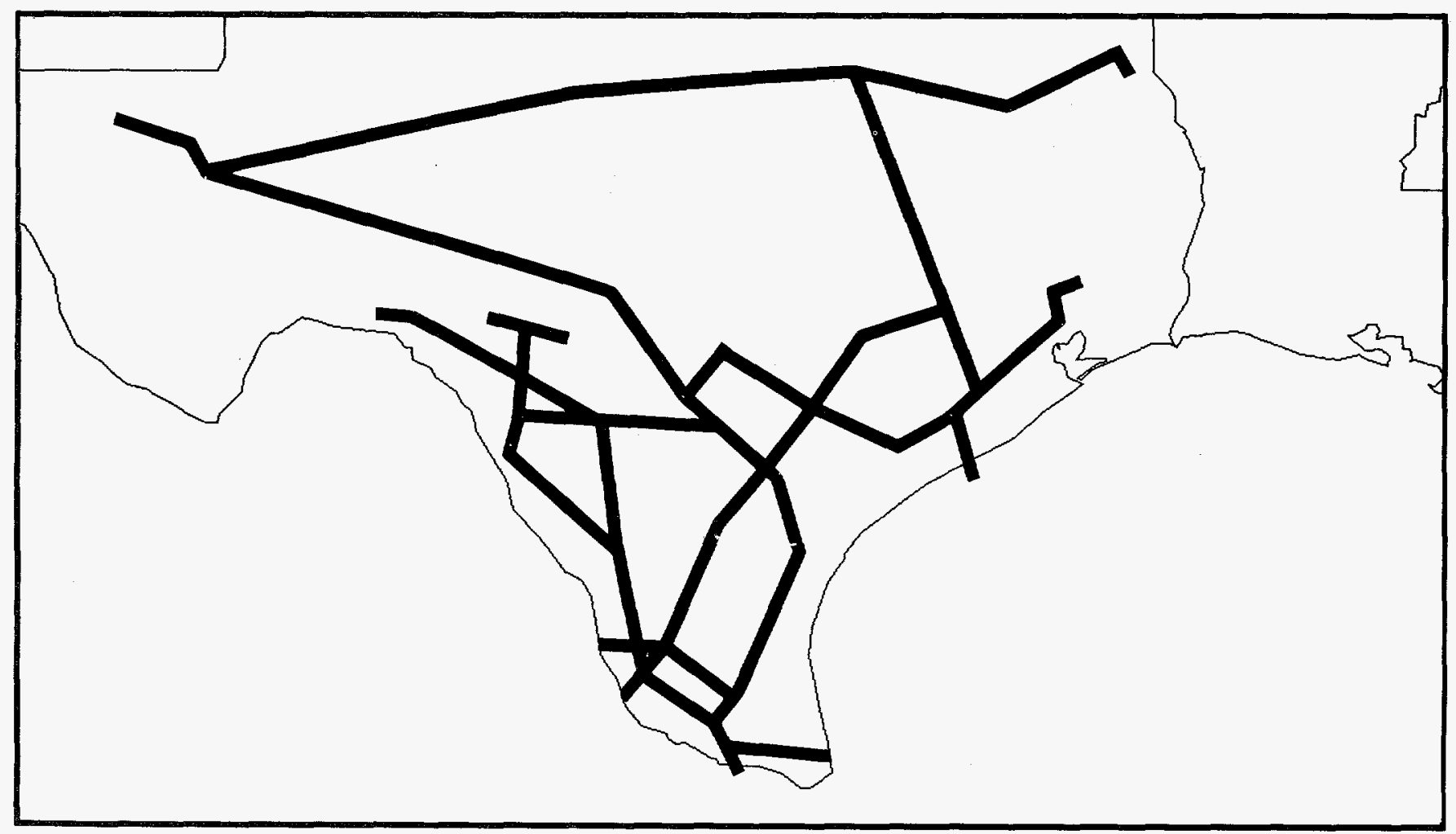

Source: Energy Information Administration (E|A), Form ElA-176, "Annual Report of Natural and Supplemental Gas Supply and Disposition." 
Table 57. Valero Energy Corp., Natural Gas Data, 1993 (Thousand Cubic Feet)

\begin{tabular}{|c|c|c|c|c|}
\hline \multirow{2}{*}{ Supply/Disposition } & $\begin{array}{l}\text { Valero Interstate } \\
\text { Transmission Co. }\end{array}$ & $\begin{array}{l}\text { Valero Transmission } \\
\text { Limited Partnership }\end{array}$ & Valero Gas Storage & Val Gas Limited Partnership \\
\hline & Texas & Texas & Texas & Texas \\
\hline \multicolumn{5}{|l|}{ SUPPLY } \\
\hline $\begin{array}{l}\text { Produced Onsystem } \\
\text { Receipts }\end{array}$ & 0 & 0 & 0 & 0 \\
\hline 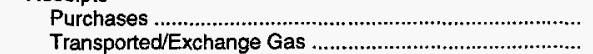 & $\begin{array}{r}7,823,003 \\
51,381,464\end{array}$ & $\begin{array}{r}21,822,358 \\
859,781,486\end{array}$ & $\begin{array}{r}0 \\
17,127,345\end{array}$ & $\begin{array}{r}5,406,619 \\
14,493,655\end{array}$ \\
\hline \multicolumn{5}{|l|}{ Interstate Movements } \\
\hline Company-awned Gas & 0 & 0 & 0 & 0 \\
\hline 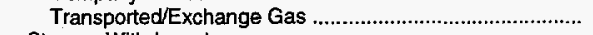 & 0 & 0 & 0 & 0 \\
\hline Storage Withdrawals & 0 & 0 & $18,338,670$ & 0 \\
\hline Received from Storage Operators & 0 & 0 & 0 & 0 \\
\hline 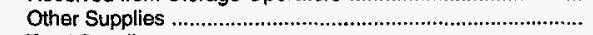 & 0 & 0 & 0 & 0 \\
\hline 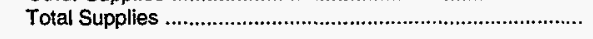 & $59,204,467$ & $881,603,844$ & $35,466,015$ & $19,900,274$ \\
\hline \multicolumn{5}{|l|}{ DISPOSITION } \\
\hline 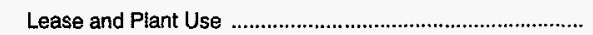 & 0 & $1,030,288$ & 0 & 0 \\
\hline 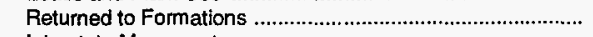 & 0 & 0 & 0 & 0 \\
\hline \multicolumn{5}{|l|}{ Interstate Movements } \\
\hline 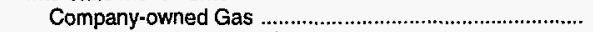 & 0 & 0 & 0 & 0 \\
\hline 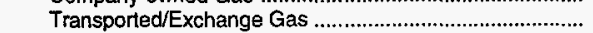 & 0 & $16,272,666$ & 0 & 0 \\
\hline 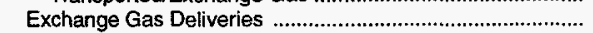 & 152,614 & $17,128,765$ & $18,338,670$ & 0 \\
\hline 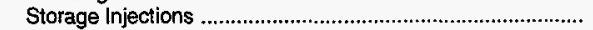 & 0 & 0 & $17,127,345$ & 0 \\
\hline \multicolumn{5}{|l|}{ Deliveries of Company-owned Gas } \\
\hline To Storage Operators & 0 & 0 & 0 & 0 \\
\hline 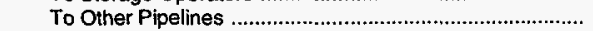 & $7,823,003$ & 0 & 0 & 0 \\
\hline To Resellers & 0 & $20,424,660$ & 0 & 214,200 \\
\hline \multicolumn{5}{|l|}{ To Consumers } \\
\hline 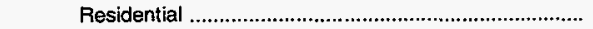 & 0 & 3,133 & 0 & 0 \\
\hline 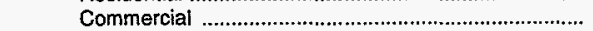 & 0 & 37,491 & 0 & 0 \\
\hline Industria! & 0 & 53,508 & 0 & $2,108,907$ \\
\hline Electric Utility & 0 & 0 & 0 & $2,940,232$ \\
\hline Vehicle Fuel & 0 & 0 & 0 & 0 \\
\hline \multicolumn{5}{|l|}{ Transported for the Account of Others } \\
\hline 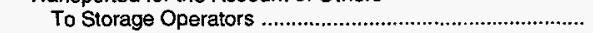 & 0 & 0 & 0 & 0 \\
\hline To Other Pipelines & $51,057,704$ & $655,038,666$ & $\ldots$ & $13,124,810$ \\
\hline 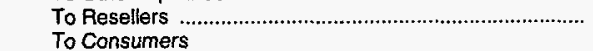 & 0 & 0 & 0 & 0 \\
\hline 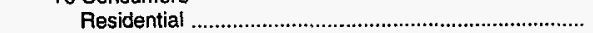 & 0 & 0 & 0 & 0 \\
\hline 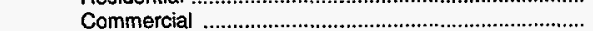 & 0 & $3,181,035$ & 0 & 0 \\
\hline Industrial & 0 & $20,960,306$ & 0 & $1,366,583$ \\
\hline Electric Utility & 0 & $158,388,981$ & 0 & 0 \\
\hline Vehicle Fuel & 0 & 0 & 0 & 0 \\
\hline Pipeline/Compressor Fuel & 111,644 & $6,371,808$ & 0 & 3,066 \\
\hline Other Disposition & 0 & 0 & 0 & 0 \\
\hline 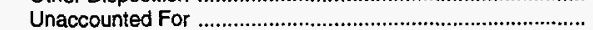 & 59,502 & $-17,287,463$ & 0 & 142,476 \\
\hline Total Disposition & $59,204,467$ & $881,603,844$ & $35,466,015$ & $19,900,274$ \\
\hline
\end{tabular}

In 1993, Valero signed a 10-year agreement with Shell Oil to process, transport, and purchase natural gas produced by Shell in southern Texas. Also, the company purchased a 48-mile pipeline from Shell that extends from the Mc-
Allen Ranch Field in Hidalgo County to Falfurrias in Brooks County, and will carry Shell-produced natural gas to the Valero pipeline. 
Table 57. Valero Energy Corp., Natural Gas Data, 1993 (Continued) (Thousand Cubic Feet)

\begin{tabular}{|c|c|c|c|}
\hline \multirow{2}{*}{ Supply/Disposition } & $\begin{array}{l}\text { V.H.C. Pipeline Limited } \\
\text { Partnership }\end{array}$ & Val Gas Co. & $\begin{array}{l}\text { Val Gathering Limited } \\
\text { Partnership }\end{array}$ \\
\hline & Texas & Texas & Texas \\
\hline \multicolumn{4}{|l|}{ SUPPLY } \\
\hline \multicolumn{4}{|l|}{ Receipts } \\
\hline Purchases & 0 & 74,906 & 0 \\
\hline 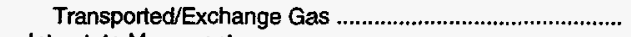 & $16,932,881$ & $4,914,200$ & $18,584,274$ \\
\hline \multicolumn{4}{|l|}{ Interstate Movements } \\
\hline 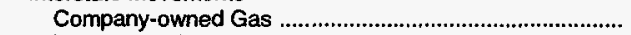 & 0 & 0 & 0 \\
\hline Transported/Exchange Gas & 0 & 0 & 0 \\
\hline Storage Withdrawals & 0 & 0 & 0 \\
\hline 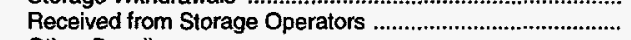 & 0 & 0 & 0 \\
\hline 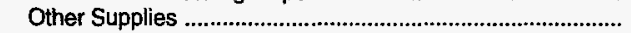 & 0 & 0 & 0 \\
\hline Total Supplies & $16,932,881$ & $4,989,106$ & $18,584,274$ \\
\hline \multicolumn{4}{|l|}{ DISPOSITION } \\
\hline Lease and Plant Use & 0 & 0 & 0 \\
\hline 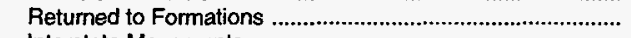 & 0 & 0 & 0 \\
\hline \multicolumn{4}{|l|}{ Interstate Movements } \\
\hline 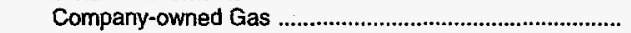 & 0 & 0 & 0 \\
\hline 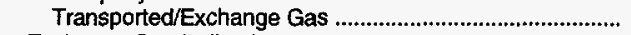 & 0 & 0 & 0 \\
\hline 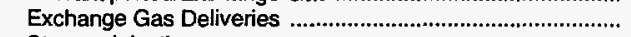 & 0 & 0 & 0 \\
\hline Storage Injections & 0 & 0 & 0 \\
\hline \multicolumn{4}{|l|}{ Deliveries of Company-owned Gas } \\
\hline To Storage Operators & 0 & 0 & 0 \\
\hline To Other Pipelines & 0 & 0 & 0 \\
\hline 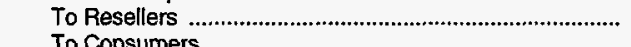 & 0 & 75,189 & 0 \\
\hline $\begin{array}{l}\text { To Consumers } \\
\text { Residential }\end{array}$ & 0 & 0 & 0 \\
\hline 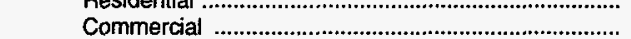 & 0 & 0 & 0 \\
\hline 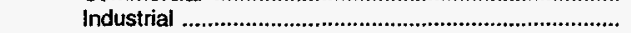 & 0 & 0 & 0 \\
\hline 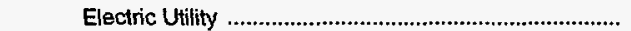 & 0 & 0 & 0 \\
\hline Vehicle Fuel & 0 & 0 & 0 \\
\hline \multicolumn{4}{|l|}{ Transported for the Account of Others } \\
\hline 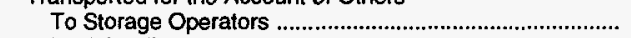 & 0 & 0 & 0 \\
\hline 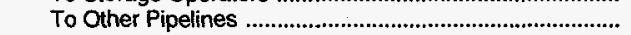 & $16,932,881$ & 0 & $18,584,274$ \\
\hline 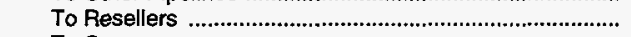 & 0 & $4,914,200$ & 0 \\
\hline \multicolumn{4}{|l|}{ To Consumers } \\
\hline 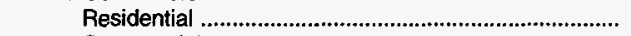 & 0 & 0 & 0 \\
\hline 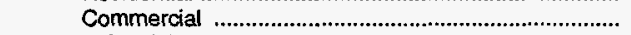 & 0 & 0 & 0 \\
\hline Industrial & 0 & 0 & 0 \\
\hline 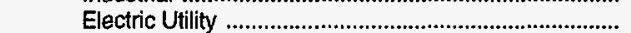 & 0 & 0 & 0 \\
\hline Vehicle Fuel & 0 & 0 & 0 \\
\hline Pipeline/Compressor Fuel ............................................... & 0 & 0 & 0 \\
\hline 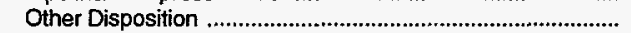 & 0 & 0 & 0 \\
\hline Unaccounted For & 0 & -283 & 0 \\
\hline Total Disposition & $16,932,881$ & $4,989,106$ & $18,584,274$ \\
\hline
\end{tabular}

Source: Energy Information Administration (EIA), Form EIA-176, "Annual Report of Natural and Supplemental Gas Supply and Disposition." 


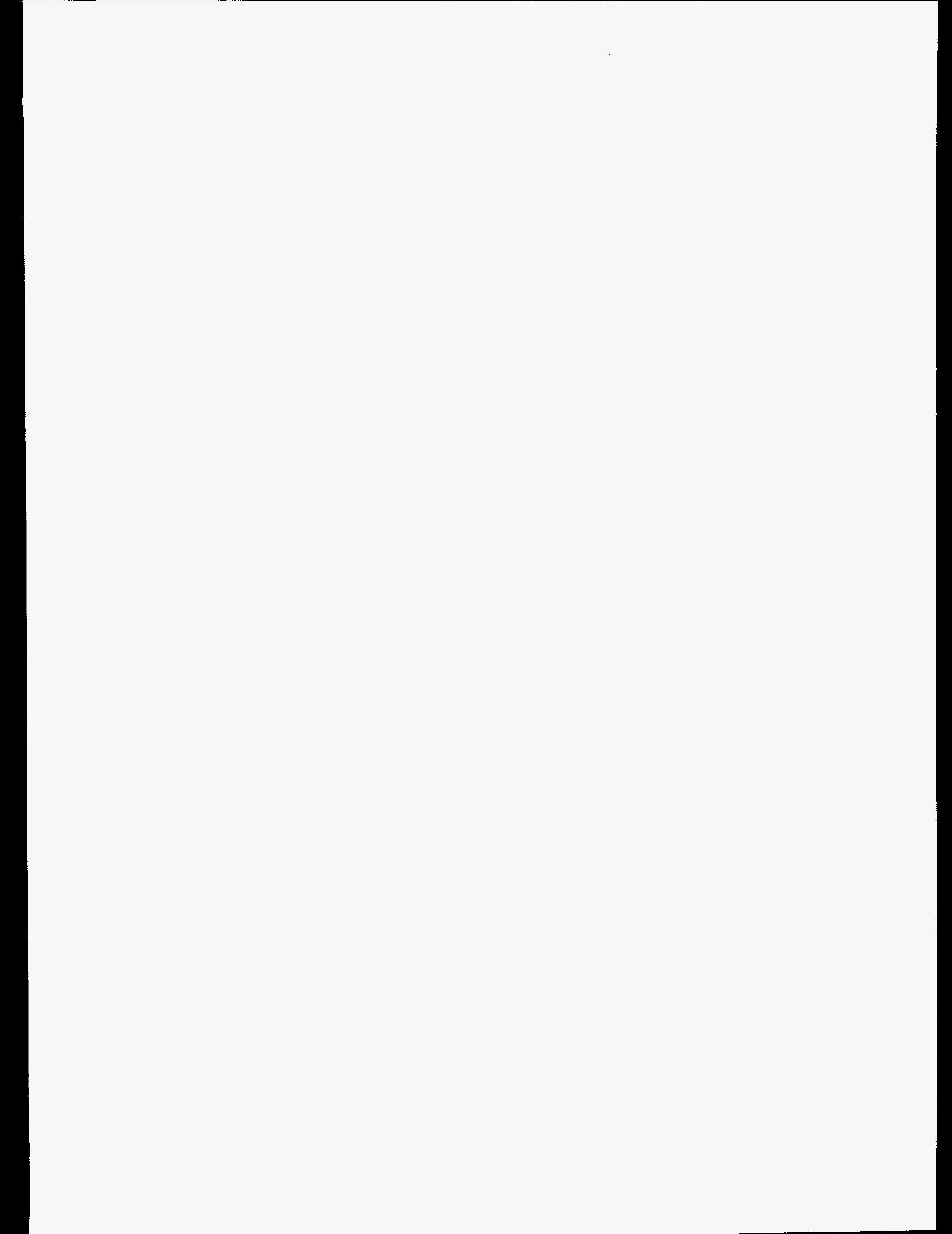




\section{Washington Gas Light Co.}

Washington Gas Light Company and its distribution subsidiaries, Shenandoah Gas and Frederick Gas, provide natural gas service to over 700,000 customers in the Washington, D. C. metropolitan area and surrounding region. The company meets almost two-thirds of its peakday requirements from company-owned facilities, two propane-air plants and an underground storage facility in Hampshire, West Virginia.

Three natural gas pipelines serve Washington Gas. Columbia Gas Transmission's pipeline goes through the Virginia, Maryland and West Virginia portion of its service area. CNG Transmission's pipeline goes through the Frederick, Maryland and Northern Virginia portion of the company service area. Transco goes through the Maryland and Virginia portions of the service area.

A 9-mile pipeline in Prince George's County, Maryland was completed in March 1994. This new pipeline will support future growth and improve reliability of service. This area experienced curtailment as a result of low pressure during the extreme cold of the winter in 1993-1994. The severe cold caused record send-outs of gas day after day, the likes of which had not been seen in over one hundred years.

Federal Energy Regulatory Commission Order 636 has changed the way pipelines operate in that they no longer sell gas, but provide storage and transportation services.

Figure 43. Washington Gas Light Co., 1993

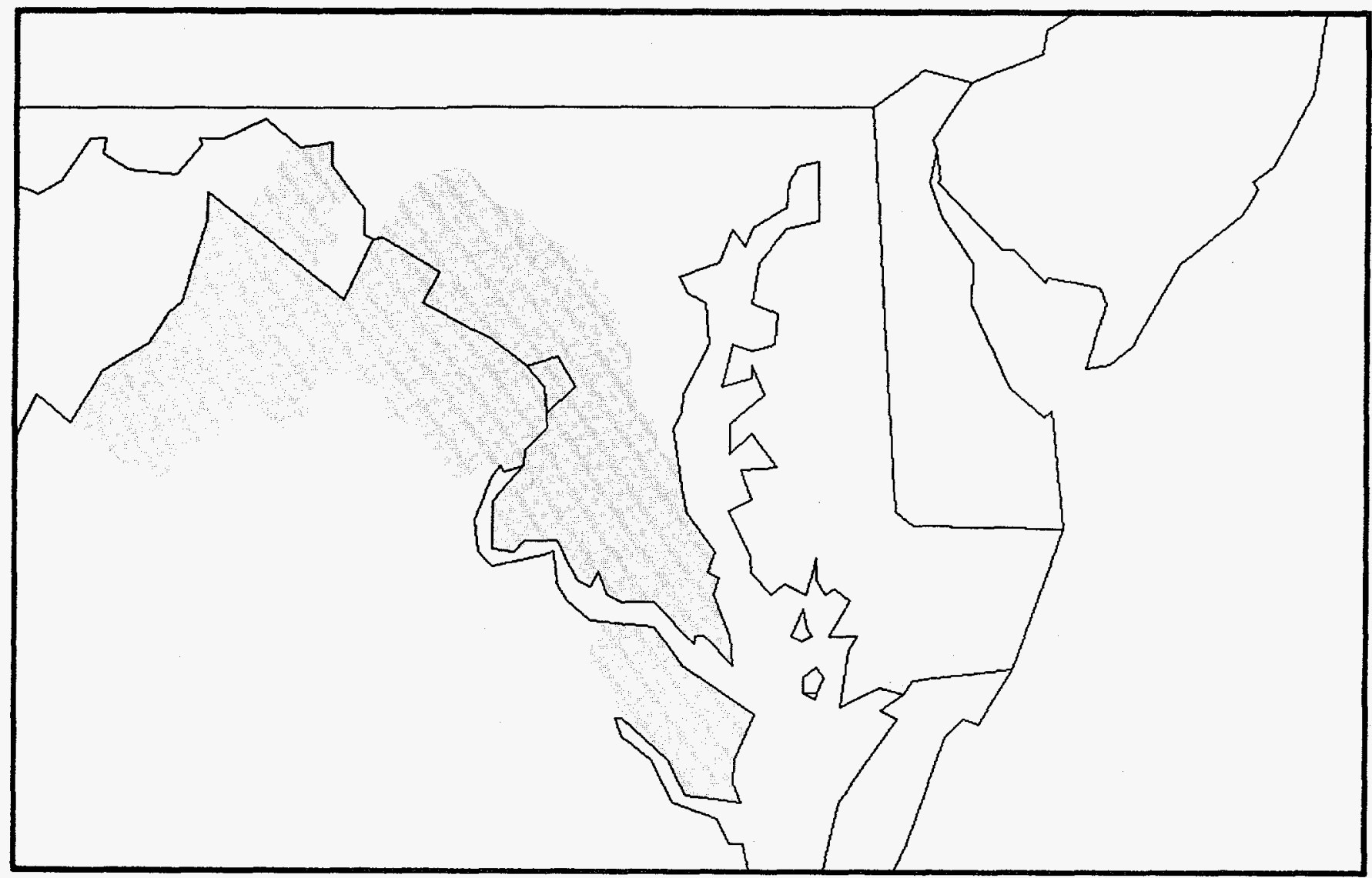

Source: Energy Information Administration (EIA), Form EIA-176, “Annual Report of Natural and Supplemental Gas Supply and Disposition.” 
Table 58. Washington Gas Light Co., Natural Gas Data, 1993 (Thousand Cubic Feet)

\begin{tabular}{|c|c|c|c|c|}
\hline \multirow{2}{*}{ Supply/Disposition } & \multicolumn{3}{|c|}{ Washington Gas Light Co. } & \multirow{2}{*}{$\frac{\text { Shenandoah Gas Co. }}{\text { Virginla }}$} \\
\hline & Virginia & D.C. & Maryland & \\
\hline \multicolumn{5}{|l|}{ SUPPLY } \\
\hline Produced Onsystem & 0 & 0 & 0 & 0 \\
\hline \multicolumn{5}{|l|}{ Receipts } \\
\hline 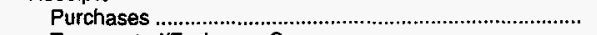 & $17,023,818$ & 0 & $24,030,241$ & $1,488,679$ \\
\hline 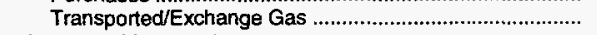 & $51,305,453$ & 330,561 & $38,767,973$ & $3,760,130$ \\
\hline \multicolumn{5}{|l|}{ Interstate Movements } \\
\hline Company-owned Gas & 0 & 0 & 0 & 0 \\
\hline 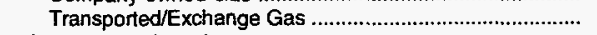 & 0 & $33,372,864$ & $3,892,338$ & 0 \\
\hline Storage Withdrawals & 0 & 0 & 0 & 0 \\
\hline 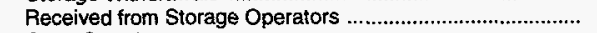 & 0 & 0 & 0 & 0 \\
\hline 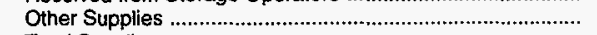 & 290,578 & 0 & 166,690 & 0 \\
\hline 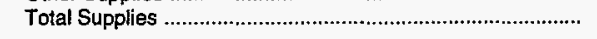 & $68,619,849$ & $33,703,425$ & $66,857,242$ & $5,248,809$ \\
\hline \multicolumn{5}{|l|}{ DISPOSTION } \\
\hline Lease and Plant Use & 0 & 0 & 0 & 0 \\
\hline 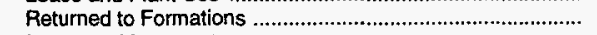 & 0 & 0 & 0 & 0 \\
\hline \multicolumn{5}{|l|}{ Interstate Movements } \\
\hline 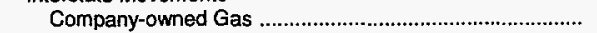 & 0 & 0 & 0 & $2,228,037$ \\
\hline 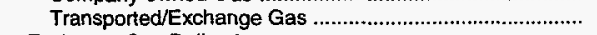 & $26,897,718$ & 0 & $10,367,484$ & 22,091 \\
\hline 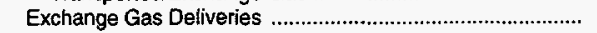 & 0 & 0 & 0 & 0 \\
\hline Storage Injections & 0 & 0 & 15,599 & 0 \\
\hline \multicolumn{5}{|l|}{ Deliveries of Company-owned Gas } \\
\hline 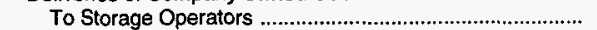 & 0 & 0 & 0 & 0 \\
\hline 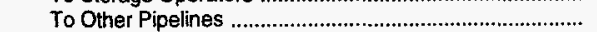 & 0 & 0 & 0 & 0 \\
\hline 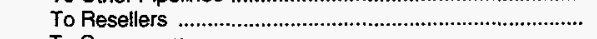 & 0 & 0 & 0 & 0 \\
\hline \multicolumn{5}{|l|}{ To Consumers } \\
\hline 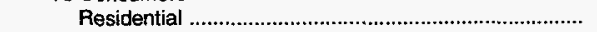 & $26,225,558$ & $16,589,064$ & $33,971,712$ & 493,412 \\
\hline 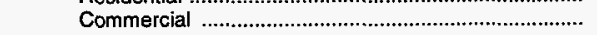 & $13,481,159$ & $15,897,117$ & $17,305,278$ & $2,289,600$ \\
\hline 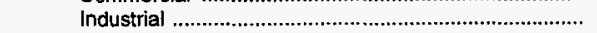 & 0 & 0 & 0 & 0 \\
\hline 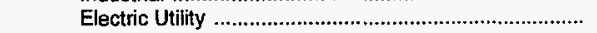 & 0 & 0 & $3,892,338$ & 0 \\
\hline 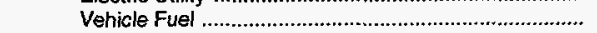 & 0 & 0 & 0 & 0 \\
\hline \multicolumn{5}{|l|}{ Transported for the Account of Others } \\
\hline To Storage Operators & 0 & 0 & 0 & 0 \\
\hline 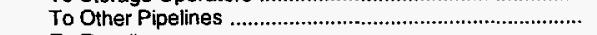 & 0 & 0 & 0 & 0 \\
\hline 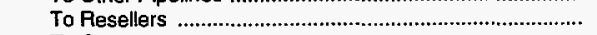 & 0 & 0 & 0 & 0 \\
\hline \multicolumn{5}{|l|}{ To Consumers } \\
\hline 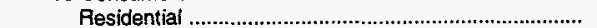 & 0 & 0 & 0 & 0 \\
\hline 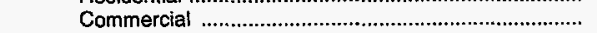 & $1,045,664$ & 332,124 & 277,912 & 91,257 \\
\hline Industrial & 0 & 0 & 0 & 0 \\
\hline 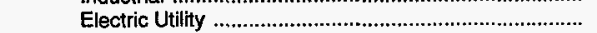 & 0 & 0 & 0 & 0 \\
\hline Vehicle Fuel & 0 & 0 & 0 & 0 \\
\hline Pipeline/Compressor Fuel & 224,213 & 275,156 & 64,138 & 7,368 \\
\hline Other Disposition & 0 & 0 & 0 & 0 \\
\hline 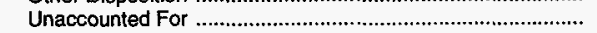 & 745,537 & 609,964 & 962,781 & 117,044 \\
\hline 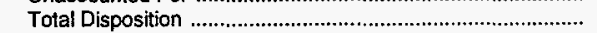 & $68,619,849$ & $33,703,425$ & $66,857,242$ & $5,248,809$ \\
\hline
\end{tabular}

Washington Gas has contracted for 80 percent of its gas through long-term contracts with marketers and producers at market-sensitive prices. The remaining 20 percent will come from short-term contracts and the spot market.

A plan is underway to reactivate Columbia LNG Corporation's Cove Point, Maryland facility in order to provide peaking service to area utilities. Washington Gas is also working with PANDA-Brandywine L.P. to build a pipeline to provide transportation service.
Washington Gas expects to have continued strong growth, as the Washington area remains highly attractive for new businesses, creating the need for more residential and commercial development. Washington Gas projects that 1,500 natural gas vehicles will be operating in the Washington area in 1994. There are now 19 natural gas fueling stations in the area. Washington Gas plans to add another 20 stations by the end of 1994 . 
Table 58. Washington Gas Light Co., Natural Gas Data, 1993 (Continued) (Thousand Cubic Feet)

\begin{tabular}{l|c|c|c|}
\hline \multirow{2}{*}{ Supply/Disposition } & Shenandoah Gas Co. & Frederick Gas Co. & Hampshire Gas Co. \\
\cline { 2 - 4 } & West Virginia & Maryland & West Virginia \\
\hline
\end{tabular}

SUPPLY

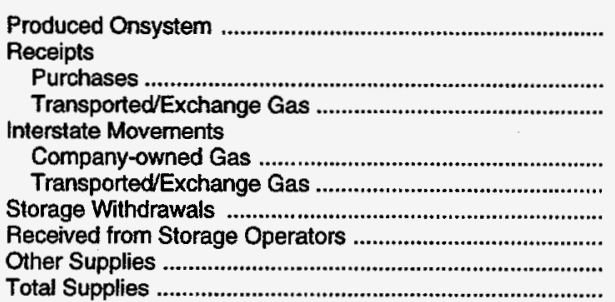

Total Supplies

DISPOSITION

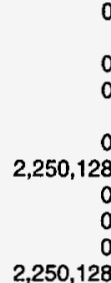

0

0
0
0
0
0
250,128

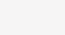

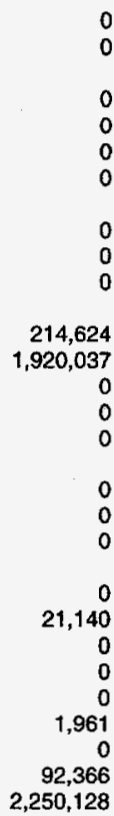

$3,774,914$

37,648

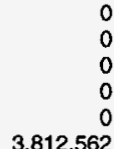

$3,812,562$

(1)

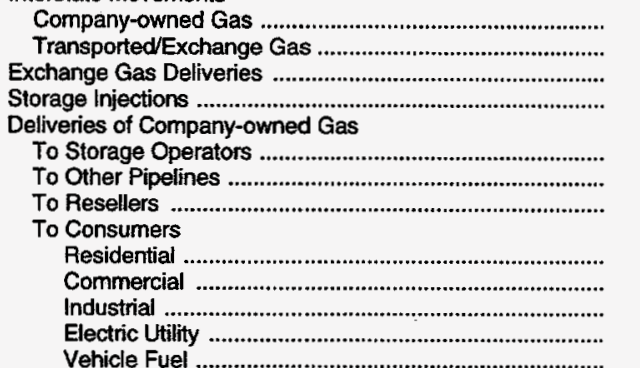

Transported for the Account of Others

To Storage Operators

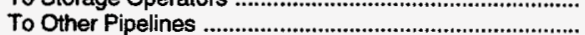

To Resellers

To Consumers

Residential ...

Commerci

Industrial

Electric Utility

Pipeline/Compressor Fuel

Other Disposition

Unaccounted For

Source: Energy Information Administration (EIA), Form EIA-176, "Annual Report of Natural and Supplemental Gas Supply and Disposition."

Table 59. Washington Gas Light Co., Interstate Flows of Natural Gas, 1993 (Thousand Cubic Feet)

\begin{tabular}{|c|c|}
\hline Company and State & Volume \\
\hline \multicolumn{2}{|l|}{$\begin{array}{l}\text { Washington Gas Light Co. } \\
\text { Virginia To }\end{array}$} \\
\hline 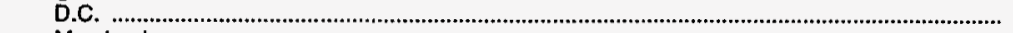 & $23,005,380$ \\
\hline Maryland & $3,892,338$ \\
\hline \multicolumn{2}{|l|}{ Maryland To } \\
\hline D.C. & $10,367,484$ \\
\hline \multicolumn{2}{|l|}{$\begin{array}{l}\text { Shenandoah Gas Co. } \\
\text { Virginia To }\end{array}$} \\
\hline West Virginia & $2,250,128$ \\
\hline
\end{tabular}

Source: Energy Information Administration (E1A), Form ElA-176, "Annual Report of Natural and Supplemental Gas Supply and Disposition." 


\section{Western Resources, Inc.}

Western Resources, Inc., was formed in 1992 as a result of a merger of Kansas Power and Light Company and Kansas Gas and Electric Company. During 1993, the company provided both gas and electricity to Kansas, Missouri and Oklahoma. In January 1994, the company completed the sale to Southern Union of most of its Missouri natural gas properties. This sale removed approximately 40 percent of the company's natural gas customers.

The company owns a 1,750-mile intrastate natural gas pipeline as well as a natural gas underground storage facility, both in Kansas. The company is also adding to their fleet vehicles that burn either natural gas or bio-diesel fuel to gain expansion into this market. One of Western Resources' employees has built and has raced competitively a natural gas drag car.

The temperatures in 1993 were colder than the above-normal temperatures in 1992, resulting in increased sales to both residential and commercial customers. The flood of 1993 caused disruptions to many company customers. No major facilities were damaged, however, and the company was able to deliver gas to all customers who could receive it. Some customers had to have their meters turned off until they could clean up the flood damage.

Figure 44. Western Resources, Inc., 1993

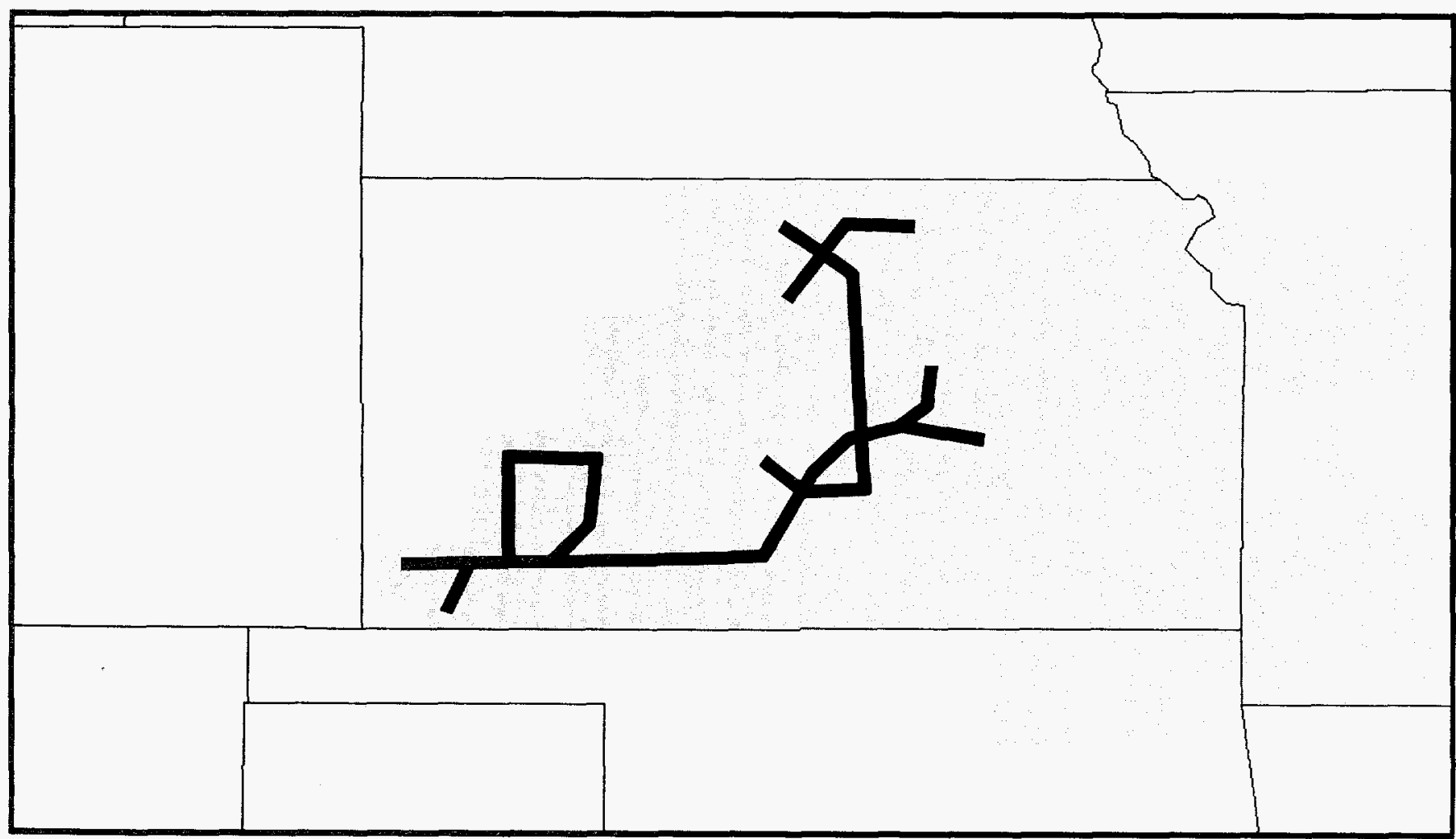

Source: Energy Information Administration (EIA), Form EIA-176, "Annual Report of Natural and Supplemental Gas Supply and Disposition."

Service Area

Western Resources, Inc. 
Table 60. Western Resources, Inc., Natural Gas Data, 1993 (Thousand Cubic Feet)

\begin{tabular}{|c|c|c|c|}
\hline \multirow{2}{*}{ Supply/Disposition } & \multicolumn{3}{|c|}{ Western Resources, Inc. } \\
\hline & Kansas & Missouri & Oklahoma \\
\hline \multicolumn{4}{|l|}{ SUPPLY } \\
\hline \multicolumn{4}{|l|}{$\begin{array}{l}\text { Produced Onsystem } \\
\text { Receipts }\end{array}$} \\
\hline 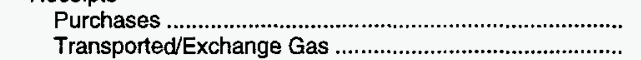 & $\begin{array}{l}88,247,288 \\
42,279,000\end{array}$ & $\begin{array}{l}71,407,689 \\
29,499,818\end{array}$ & $\begin{array}{r}4,728,902 \\
975,563\end{array}$ \\
\hline \multicolumn{4}{|l|}{ Interstate Movements } \\
\hline 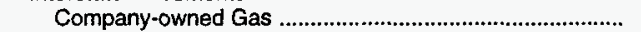 & 0 & 0 & 0 \\
\hline Transported/Exchange Gas ............................................... & 0 & 0 & 0 \\
\hline Storage Withdrawals & $1,709,883$ & 0 & 0 \\
\hline Received from Storage Operators & $38,075,474$ & $16,300,360$ & 0 \\
\hline Other Supplies & 0 & 0 & 0 \\
\hline 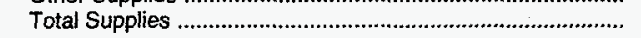 & $170,311,645$ & $117,207,867$ & $5,704,465$ \\
\hline \multicolumn{4}{|l|}{ DISPOSITION } \\
\hline Lease and Plant Use & 109,203 & 0 & 0 \\
\hline Returned to Formations & 0 & 0 & 0 \\
\hline \multicolumn{4}{|l|}{ Interstate Movements } \\
\hline 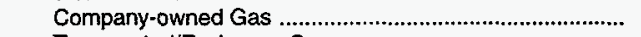 & 0 & 0 & 0 \\
\hline 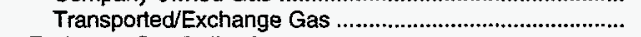 & 0 & 0 & 0 \\
\hline 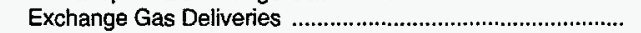 & 0 & 0 & 0 \\
\hline Storage Injections & $1,967,187$ & 0 & 0 \\
\hline \multicolumn{4}{|l|}{ Deliveries of Company-awned Gas } \\
\hline 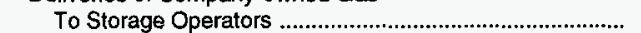 & $37,818,169$ & $16,300,360$ & 0 \\
\hline To Other Pipelines & 0 & 0 & 0 \\
\hline $\begin{array}{l}\text { To Resellers . } \\
\text { To Consumers }\end{array}$ & \multicolumn{2}{|c|}{ To Consumers } & 0 \\
\hline 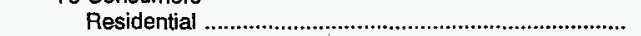 & $59,125,152$ & $47,136,383$ & $3,188,819$ \\
\hline 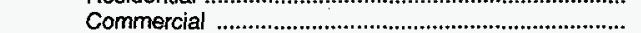 & $23,442,811$ & $22,586,125$ & $1,252,309$ \\
\hline 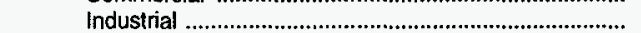 & $1,055,589$ & 299,799 & 264 \\
\hline 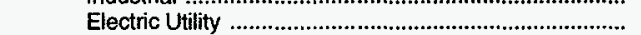 & 82,939 & 49,349 & 0 \\
\hline 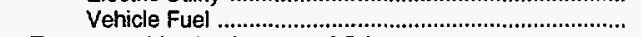 & 0 & 0 & 0 \\
\hline \multicolumn{4}{|l|}{ Transported for the Account of Others } \\
\hline 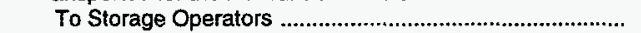 & 0 & 0 & 0 \\
\hline 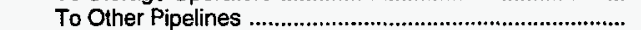 & 0 & 0 & 0 \\
\hline 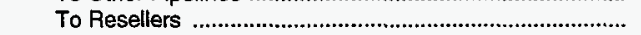 & $1,415,279$ & 0 & 0 \\
\hline \multicolumn{4}{|l|}{ To Consumers } \\
\hline 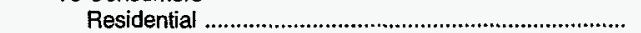 & 0 & 0 & 0 \\
\hline 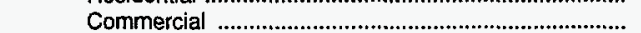 & $8,079,208$ & $6,128,607$ & 205,023 \\
\hline 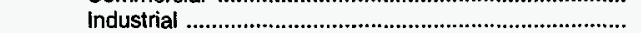 & $29,182,880$ & $22,150,377$ & 770,540 \\
\hline 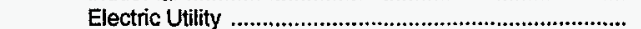 & $4,023,804$ & $1,220,834$ & 0 \\
\hline Vehicle Fuel & 0 & 0 & 0 \\
\hline Pipeline/Compressor Fuel & $1,537,563$ & 0 & 0 \\
\hline 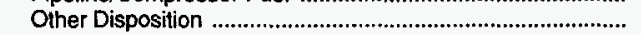 & 0 & 0 & 0 \\
\hline Unaccounted For & $2,438,049$ & $1,336,033$ & 287,510 \\
\hline Total Disposition & $170,311,645$ & $117,207,867$ & $5,704,465$ \\
\hline
\end{tabular}

Source: Energy Information Administration (EIA), Form EIA-176, "Annual Report of Natural and Supplemental Gas Supply and Disposition." 


\section{The Williams Companies, Inc.}

The Williams Companies, Inc., provides regional transmission, gathering, and processing services. Its natural gas subsidiaries include Northwest Pipeline Corporation and Williams Natural Gas Company. It also owns 50 percent of Kern River Pipeline Company. In addition, it owns two gas-gathering and processing systems located in the San Juan Basin of New Mexico and Colorado and the Rocky Mountain producing area of Wyoming, a petroleum products pipeline operating in the middle section of the country, and a major telecommunications company.

Northwest Pipeline is a 3,500-mile pipeline system that connects Canada and the Pacific Northwest with the
Rocky Mountain area. Northwest completed a mainline expansion in 1993 that enables it to carry an additional 2.5 million cubic feet per day, and is planning a second expansion in 1995. Kern River, which provides gas supplies to the California market, operated at its full 700 million cubic feet per day design capacity during 1993. Planned expansion of Kern River, expected to be completed in 1998, will increase the capacity by an additional 300 million cubic feet per day.

Williams Natural Gas Company serves seven Midcontinent States. The company operates a 9,300-mile pipeline system, receiving supplies from Colorado, Kansas, Okla

Figure 45. The Williams Companies, Inc., 1993

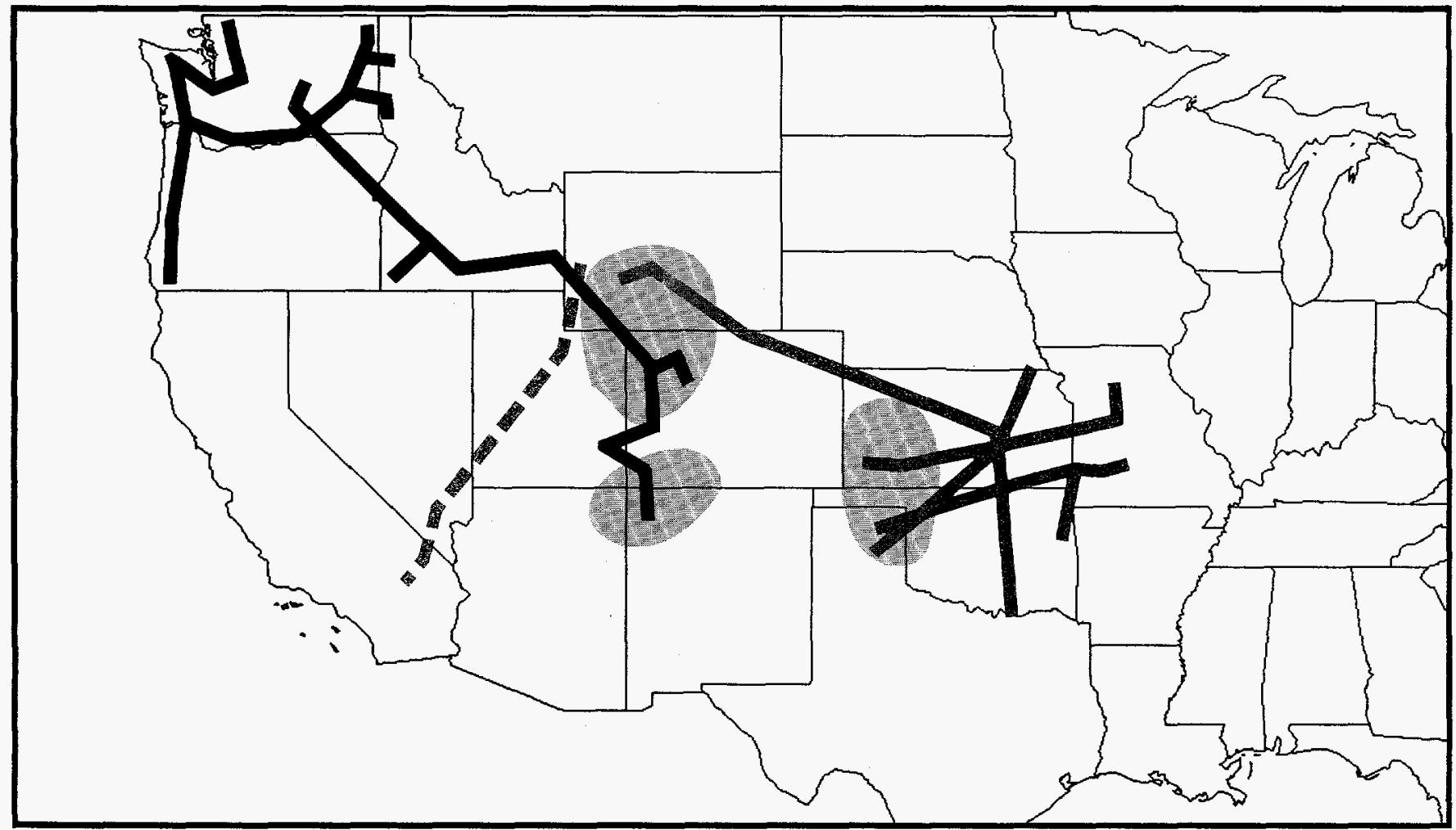

Source: Energy Information Administration (EIA), Form EIA-176, "Annual Report of Natural and Supplemental Gas Supply and Disposition." 
Table 61. The Williams Companies, Inc., Natural Gas Data, 1993 (Thousand Cubic Feet)

\begin{tabular}{|c|c|c|c|c|c|}
\hline \multirow{2}{*}{ Supply/Disposition } & \multicolumn{5}{|c|}{ Williams Natural Gas Co. } \\
\hline & Texas & Oklahoma & Wyoming & Colorado & Kansas \\
\hline \multicolumn{6}{|l|}{ SUPPLY } \\
\hline \multicolumn{5}{|l|}{ Receipts } & 0 \\
\hline 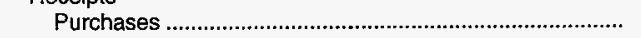 & $9,552,510$ & $27,606,164$ & $4,543,690$ & 607,460 & $13,043,089$ \\
\hline 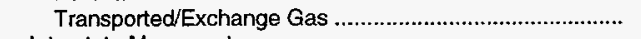 & $19,378,244$ & $84,773,102$ & $41,415,642$ & $3,244,863$ & $197,033,136$ \\
\hline \multicolumn{6}{|l|}{ Interstate Movements } \\
\hline 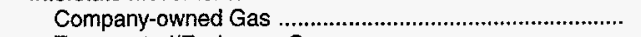 & 0 & 0 & 0 & 0 & 0 \\
\hline 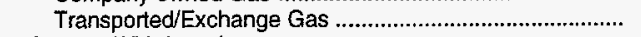 & 722,405 & $46,305,022$ & 0 & $43,469,898$ & $162,276,455$ \\
\hline Storage Withdrawals & 0 & $13,176,531$ & 0 & 0 & $41,858,699$ \\
\hline 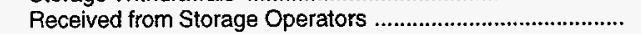 & 0 & 0 & 0 & 0 & 0 \\
\hline 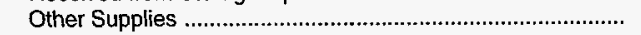 & 0 & 0 & 0 & 0 & 0 \\
\hline Total Supplies & $29,653,159$ & $171,860,819$ & $45,959,332$ & $47,322,221$ & $414,211,379$ \\
\hline \multicolumn{6}{|l|}{ DISPOSITION } \\
\hline 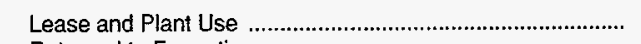 & 229,798 & 119,139 & 446,313 & 0 & 0 \\
\hline 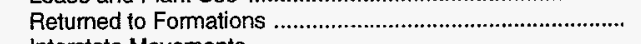 & 0 & 0 & 0 & 0 & 0 \\
\hline \multicolumn{6}{|l|}{ Interstate Movements } \\
\hline 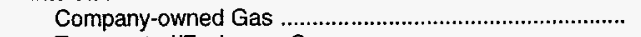 & 0 & 0 & 0 & 0 & 0 \\
\hline 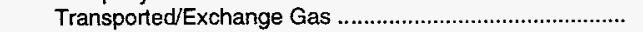 & $27,105,950$ & $130,397,288$ & $43,469,898$ & $46,816,062$ & $120,123,911$ \\
\hline 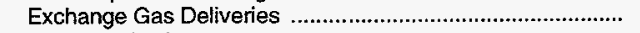 & 21,394 & 404,887 & $1,077,797$ & 0 & $4,331,824$ \\
\hline 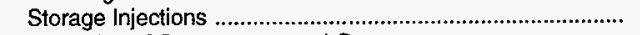 & 0 & $14,563,360$ & 0 & 0 & $45,487,580$ \\
\hline \multicolumn{6}{|l|}{ Deliveries of Company-owned Gas } \\
\hline 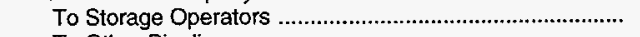 & 0 & 0 & 0 & 0 & 0 \\
\hline 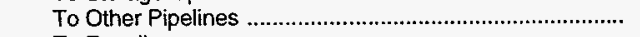 & 0 & 0 & 0 & 0 & 0 \\
\hline 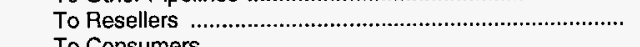 & 3,733 & $1,670,440$ & 0 & 12,120 & $23,394,754$ \\
\hline \multicolumn{6}{|l|}{ To Consumers } \\
\hline 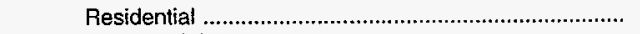 & 5,071 & 19,176 & 0 & 0 & 67,241 \\
\hline 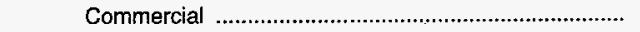 & 375 & 23,676 & 0 & 0 & 54,681 \\
\hline 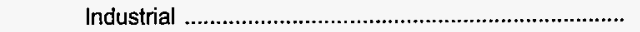 & 5,002 & 14,699 & 0 & 0 & 435,463 \\
\hline 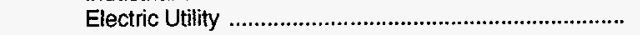 & 0 & 0 & 0 & 0 & 81,646 \\
\hline 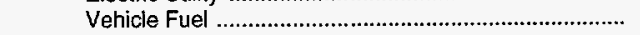 & 0 & 0 & 0 & 0 & 0 \\
\hline \multicolumn{6}{|l|}{ Transported for the Account of Others } \\
\hline 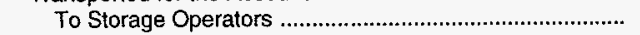 & 0 & 0 & 0 & 0 & 0 \\
\hline 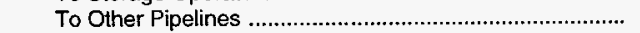 & $1,545,739$ & 296,971 & 0 & 0 & $62,313,506$ \\
\hline 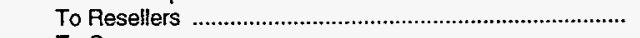 & 2,886 & $13,494,466$ & 90,897 & 7,507 & $88,143,152$ \\
\hline \multicolumn{6}{|l|}{ To Consumers } \\
\hline 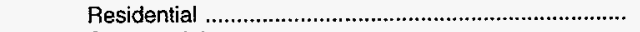 & 0 & 0 & 0 & 0 & 0 \\
\hline Commercial & 69 & 193,374 & 0 & 0 & 189,958 \\
\hline 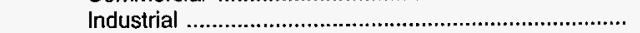 & 14,215 & $5,212,036$ & 0 & 0 & $51,171,574$ \\
\hline 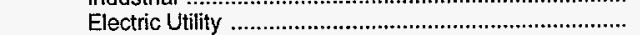 & 0 & 0 & 0 & 0 & $3,921,041$ \\
\hline Vehicle Fuel & 0 & 0 & 0 & 0 & 0 \\
\hline 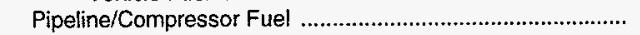 & 356,816 & $2,780,378$ & 405,671 & 224,123 & $8,875,159$ \\
\hline Other Disposition & 0 & 0 & 0 & 0 & 0 \\
\hline 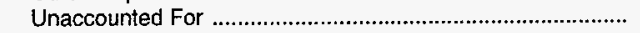 & 362,111 & $2,670,929$ & 468,756 & 262,409 & $5,619,889$ \\
\hline 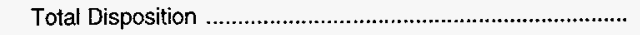 & $29,653,159$ & $171,860,819$ & $45,959,332$ & $47,322,221$ & $414,211,379$ \\
\hline
\end{tabular}

homa, Texas, and Wyoming. The company's main lines of business are the gathering, storing, and transporting of natural gas, and it operates natural gas storage fields in Oklahoma and Kansas with a total working gas capacity of 40 billion cubic feet. Since, under FERC Order 636, the company no longer purchases and stores natural gas for its own account, the gas in storage is for various of its customers. During 1993, the company's customers fully utilized those storage facilities. 
Table 61. The Williams Companies, Inc., Natural Gas Data, 1993 (Continued) (Thousand Cubic Feet)

\begin{tabular}{|c|c|c|c|c|c|}
\hline \multirow{2}{*}{ Supply/Disposition } & \multicolumn{2}{|c|}{ Williams Natural Gas Co. } & \multicolumn{3}{|c|}{ Northwest Pipeline Corp. } \\
\hline & Missouri & Nebraska & Washington & Oregon & Idaho \\
\hline \multicolumn{6}{|l|}{ SUPPLY } \\
\hline $\begin{array}{l}\text { Produced Onsystem } \\
\text { Receipts }\end{array}$ & 0 & 0 & 0 & 0 & 0 \\
\hline 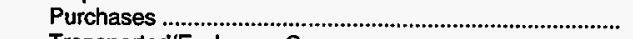 & 701,997 & 0 & 0 & 0 & 0 \\
\hline 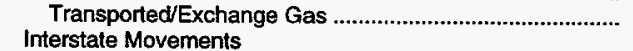 & 658,267 & 0 & $32,478,896$ & $85,166,989$ & 0 \\
\hline 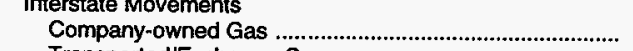 & 0 & 0 & 0 & 0 & 0 \\
\hline 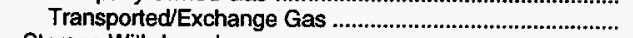 & $114,515,193$ & 902,200 & $306,030,160$ & $67,125,255$ & $91,846,907$ \\
\hline 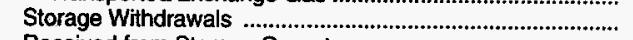 & 0 & 0 & $7,095,338$ & 0 & $\begin{array}{r}91,0+0,908 \\
0\end{array}$ \\
\hline Received from Storage Operators & 0 & 0 & $17,881,849$ & 0 & 0 \\
\hline Other Supplies & 0 & 0 & 0 & 0 & 0 \\
\hline 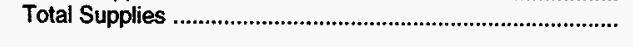 & $115,875,457$ & 902,200 & $363,486,243$ & $152,292,244$ & $91,846,907$ \\
\hline \multicolumn{6}{|l|}{ DISPOSITION } \\
\hline 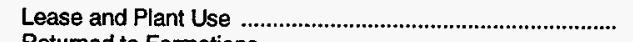 & 0 & 0 & 0 & 0 & 0 \\
\hline $\begin{array}{l}\text { Returned to Formations } \\
\text { Interstate Movements }\end{array}$ & 0 & 0 & 0 & 0 & 0 \\
\hline 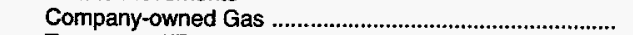 & 0 & 0 & 0 & 0 & 0 \\
\hline 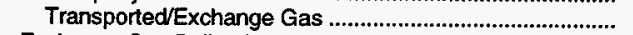 & 278,064 & 0 & $67,125,255$ & $55,738,240$ & $39,196,714$ \\
\hline 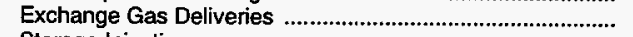 & 77,642 & 0 & 0 & 0 & 0 \\
\hline 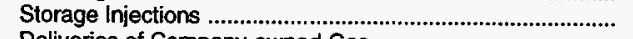 & 0 & 0 & $3,167,421$ & 0 & 0 \\
\hline \multicolumn{6}{|l|}{ Deliveries of Company-owned Gas } \\
\hline 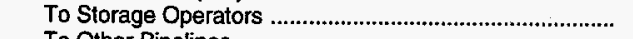 & 0 & 0 & $3,851,016$ & 0 & 0 \\
\hline To Other Pipelines & 0 & 0 & 0 & 0 & 0 \\
\hline $\begin{array}{l}\text { To Resellers } \\
\text { To Consumers }\end{array}$ & $24,576,772$ & 128,828 & $12,839,085$ & 689,649 & $1,458,629$ \\
\hline 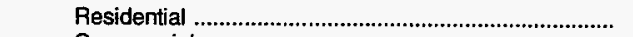 & 5,583 & 0 & 0 & 0 & 0 \\
\hline 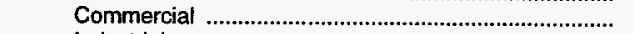 & 15,372 & 0 & 0 & 0 & 0 \\
\hline Industrial & 8,297 & 2,421 & 0 & 0 & 0 \\
\hline 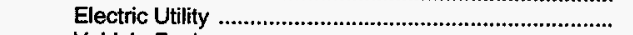 & 493 & 0 & 0 & 0 & 0 \\
\hline 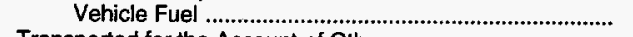 & 0 & 0 & 0 & 0 & 0 \\
\hline \multicolumn{6}{|l|}{ Transported for the Account of Others } \\
\hline To Storage Operators & 0 & 0 & $15,508,136$ & 0 & 0 \\
\hline 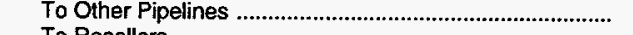 & $2,631,890$ & 0 & 0 & 0 & 0 \\
\hline 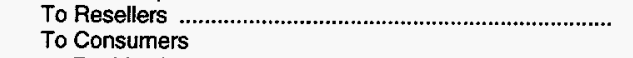 & $85,980,300$ & 765,904 & $201,194,279$ & $73,590,418$ & $49,113,684$ \\
\hline 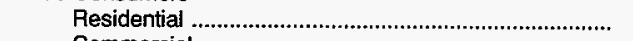 & 0 & 0 & 0 & 0 & 0 \\
\hline 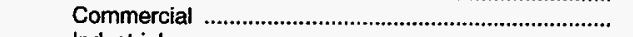 & 36,815 & 0 & 0 & 0 & 0 \\
\hline 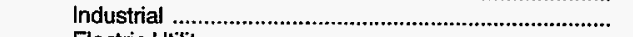 & $1,179,398$ & 1,198 & $7,611,995$ & $6,408,483$ & 0 \\
\hline 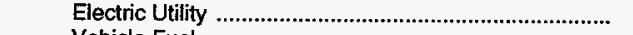 & 367,013 & 0 & 0 & $14,879,179$ & 0 \\
\hline Vehicle Fuel & 0 & 0 & 0 & 0 & 0 \\
\hline 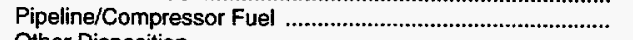 & 106,877 & 0 & $2,648,533$ & 515,257 & $1,629,810$ \\
\hline 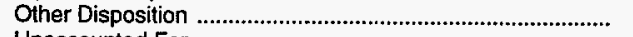 & 0 & 0 & 0 & 0 & 0 \\
\hline 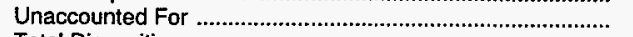 & 610,941 & 3,849 & $49,540,523$ & 471,018 & 448,070 \\
\hline 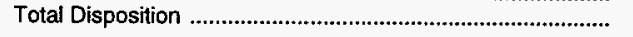 & $115,875,457$ & 902,200 & $363,486,243$ & $152,292,244$ & $91,846,907$ \\
\hline
\end{tabular}


Table 61. The Williams Companies, Inc., Natural Gas Data, 1993 (Continued) (Thousand Cubic Feet)

\begin{tabular}{|c|c|c|c|c|c|}
\hline \multirow{2}{*}{ Supply/Disposition } & \multicolumn{4}{|c|}{ Northwest Pipeline Corp. } & \multirow{2}{*}{$\begin{array}{c}\begin{array}{c}\text { Williams Gas } \\
\text { Marketing Co. }\end{array} \\
\text { Oklahoma }\end{array}$} \\
\hline & Wyoming & Utah & Colorado & New Mexico & \\
\hline \multicolumn{6}{|l|}{ SUPPLY } \\
\hline $\begin{array}{l}\text { Produced Onsystem } \\
\text { Receipts }\end{array}$ & 0 & 0 & 0 & 0 & 0 \\
\hline 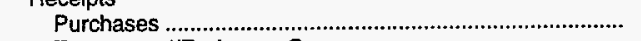 & 0 & 0 & 0 & 0 & 0 \\
\hline 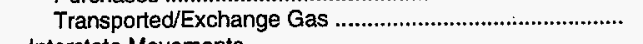 & $82,230,325$ & $15,025,715$ & $139,145,463$ & 0 & 936,411 \\
\hline \multicolumn{6}{|l|}{ Interstate Movements } \\
\hline Company-owned Gas & 0 & 0 & 0 & 0 & 0 \\
\hline $\begin{array}{l}\text { Transported/Exchange Gas } \\
\text { Storage Withdrawals }\end{array}$ & 0 & $26,961,951$ & $40,002,622$ & $41,589,001$ & 0 \\
\hline 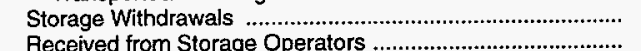 & 0 & 0 & 0 & 0 & 0 \\
\hline 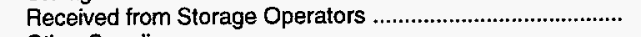 & 0 & $13,214,455$ & 0 & 0 & 0 \\
\hline 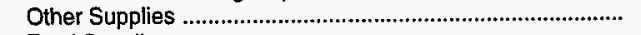 & 0 & 0 & 0 & 0 & 0 \\
\hline Total Supplies & $82,230,325$ & $55,202,121$ & $179,148,085$ & $41,589,001$ & 936,411 \\
\hline \multicolumn{6}{|l|}{ DISPOSITION } \\
\hline Lease and Plant Use & 0 & 0 & 0 & 0 & 0 \\
\hline 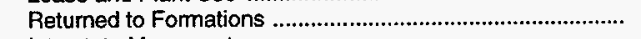 & 0 & 0 & 0 & 0 & 0 \\
\hline \multicolumn{6}{|l|}{ Interstate Movements } \\
\hline 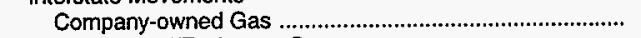 & 0 & 0 & 0 & 0 & 0 \\
\hline 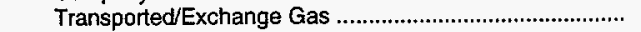 & $63,070,618$ & $40,002,622$ & $41,589,001$ & 0 & 0 \\
\hline 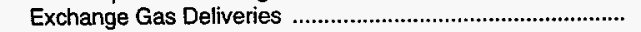 & 0 & 0 & 0 & 0 & 0 \\
\hline Storage Injections & 0 & 0 & 0 & 0 & 0 \\
\hline \multicolumn{6}{|l|}{ Deliveries of Company-owned Gas } \\
\hline To Storage Operators & 0 & $2,991,587$ & 0 & 0 & 0 \\
\hline 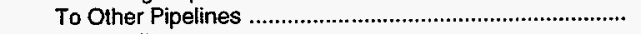 & 0 & 0 & 0 & 0 & 0 \\
\hline 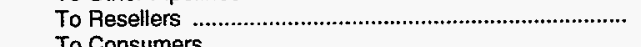 & 0 & 45,256 & $2,027,170$ & 0 & 0 \\
\hline $\begin{array}{l}\text { To Consumers } \\
\text { Residential }\end{array}$ & & & & 0 & 0 \\
\hline 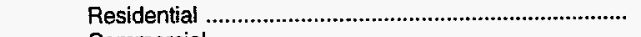 & 0 & 0 & & $\begin{array}{l}0 \\
0\end{array}$ & 0 \\
\hline 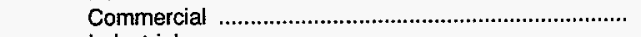 & 0 & $\begin{array}{l}0 \\
0\end{array}$ & $\begin{array}{l}0 \\
0\end{array}$ & $\begin{array}{l}0 \\
0\end{array}$ & 0 \\
\hline 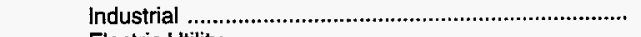 & $\begin{array}{l}0 \\
0\end{array}$ & $\begin{array}{l}0 \\
0\end{array}$ & 0 & 0 & 0 \\
\hline $\begin{array}{l}\text { Electric Utility } \\
\text { Vehicle Fuel }\end{array}$ & $\begin{array}{l}0 \\
0\end{array}$ & 0 & 0 & 0 & 0 \\
\hline \multicolumn{6}{|l|}{ Transported for the Account of Others } \\
\hline To Storage Operators & 0 & $10,740,961$ & 0 & 0 & 0 \\
\hline 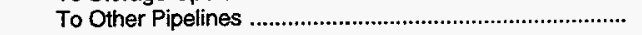 & $16,929,003$ & 0 & $86,536,861$ & $41,386,111$ & 0 \\
\hline 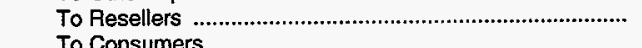 & $1,619,456$ & $1,104,921$ & $47,444,615$ & 0 & 0 \\
\hline \multicolumn{5}{|l|}{ To Consumers } & 0 \\
\hline 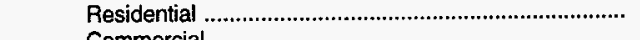 & $\begin{array}{l}0 \\
0\end{array}$ & $\begin{array}{l}0 \\
0\end{array}$ & $\begin{array}{l}0 \\
0\end{array}$ & 0 & $\begin{array}{l}0 \\
0\end{array}$ \\
\hline 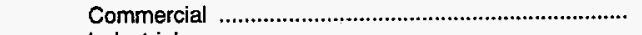 & $\begin{array}{l}0 \\
0\end{array}$ & 0 & $\begin{array}{l}0 \\
0\end{array}$ & 0 & 936,411 \\
\hline Industrial & 0 & 0 & & 0 & $\begin{array}{r}930,411 \\
0\end{array}$ \\
\hline 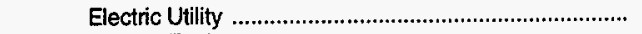 & 0 & 0 & $\begin{array}{l}0 \\
0\end{array}$ & 0 & 0 \\
\hline 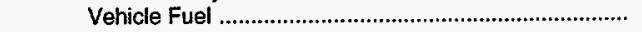 & 0 & 0 & $\begin{array}{r}0 \\
879,364\end{array}$ & 0 & 0 \\
\hline 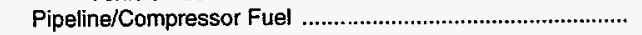 & 517,778 & 242,624 & $\begin{array}{r}879,364 \\
0\end{array}$ & 0 & 0 \\
\hline 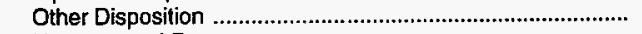 & $\begin{aligned} 0 \\
0\end{aligned}$ & $\begin{array}{r}0 \\
-150\end{array}$ & $\begin{array}{r}0 \\
671,074\end{array}$ & 202,890 & 0 \\
\hline $\begin{array}{l}\text { Unaccounted For } \\
\text { Total Disposition }\end{array}$ & $\begin{array}{r}93,470 \\
82,230,325\end{array}$ & $\begin{array}{r}74,150 \\
55,202,121\end{array}$ & $\begin{array}{r}671,074 \\
179,148,085\end{array}$ & $\begin{array}{r}202,890 \\
41,589,001\end{array}$ & 936,411 \\
\hline
\end{tabular}


Table 61. The Williams Companies, Inc., Natural Gas Data, 1993 (Continued) (Thousand Cubic Feet)

\begin{tabular}{|c|c|c|c|c|c|}
\hline \multirow{2}{*}{ Supply/Disposition } & \multicolumn{4}{|c|}{ Kern River Gas Transmission Co. } & \multirow{2}{*}{$\begin{array}{c}\begin{array}{c}\text { Louisiana } \\
\text { Resources Co }\end{array} \\
\text { Louisiana }\end{array}$} \\
\hline & Wyoming & Utah & Nevada & California & \\
\hline \multicolumn{6}{|l|}{ SUPPLY } \\
\hline $\begin{array}{l}\text { Produced Onsystem } \\
\text { Receipts }\end{array}$ & 0 & 0 & 0 & 0 & 0 \\
\hline 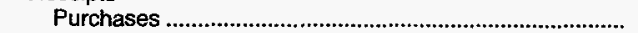 & 0 & 0 & 0 & 0 & 0 \\
\hline 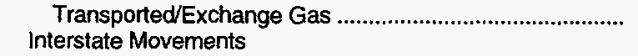 & $258,468,246$ & 0 & 0 & 0 & $269,472,944$ \\
\hline 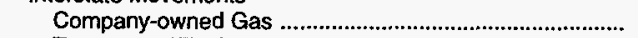 & 0 & 0 & 0 & 0 & 0 \\
\hline 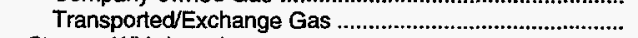 & 0 & $256,475,285$ & $254,756,612$ & $240,539,903$ & 0 \\
\hline Storage Withdrawals & 0 & 0 & 0 & 0 & 0 \\
\hline Received from Storage Operators & 0 & 0 & 0 & 0 & 0 \\
\hline 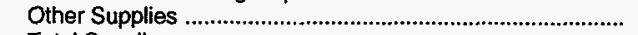 & 0 & $\because \quad 0$ & 7,261 & $2,550,901$ & 0 \\
\hline 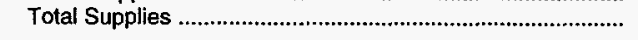 & $258,468,246$ & $256,475,285$ & $254,763,873$ & $243,090,804$ & $269,472,944$ \\
\hline \multicolumn{6}{|l|}{ DISPOSITION } \\
\hline Lease and Plant Use & 0 & 0 & 0 & 0 & 0 \\
\hline $\begin{array}{l}\text { Returned to Formations } \\
\text { Interstate Movements }\end{array}$ & 0 & 0 & 0 & 0 & 0 \\
\hline 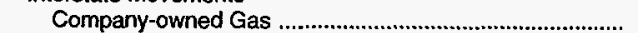 & 0 & 0 & 0 & 0 & 0 \\
\hline 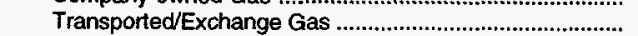 & $256,475,285$ & $254,756,612$ & $240,539,903$ & 0 & 0 \\
\hline Exchange Gas Deliveries & 0 & 0 & $\begin{array}{r}240,000,000 \\
0\end{array}$ & 0 & 0 \\
\hline \multicolumn{6}{|l|}{ Deliveries of Company-owned Gas } \\
\hline 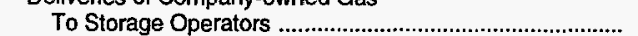 & 0 & 0 & 0 & 0 & 0 \\
\hline To Other Pipelines & 0 & 0 & 0 & 0 & 0 \\
\hline 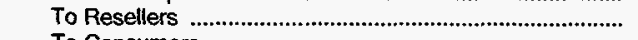 & 0 & 0 & 0 & 0 & 0 \\
\hline \multicolumn{6}{|l|}{ To Consumers } \\
\hline 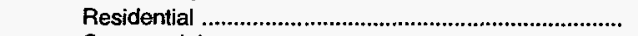 & 0 & 0 & 0 & 0 & 0 \\
\hline 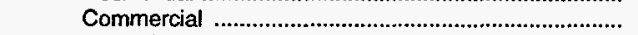 & 0 & 0 & 0 & 0 & 0 \\
\hline 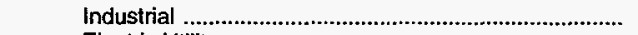 & 0 & 0 & 0 & 0 & 0 \\
\hline 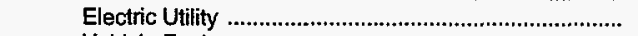 & 0 & 0 & 0 & 0 & 0 \\
\hline Vehicle Fuel' & 0 & 0 & 0 & 0 & 0 \\
\hline \multicolumn{6}{|l|}{ Transported for the Account of Others } \\
\hline 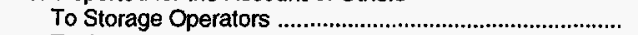 & 0 & 0 & 0 & 0 & 0 \\
\hline 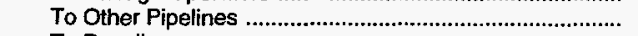 & 487,492 & $1,027,423$ & $13,666,966$ & $131,477,166$ & $273,739,270$ \\
\hline 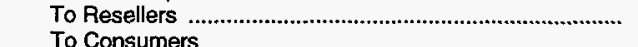 & 0 & 0 & 0 & 0 & 0 \\
\hline \multicolumn{6}{|l|}{ To Consumers } \\
\hline Residential & 0 & 0 & 0 & 0 & 0 \\
\hline 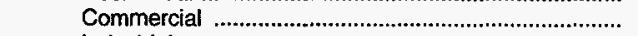 & 0 & 0 & 0 & 0 & 0 \\
\hline 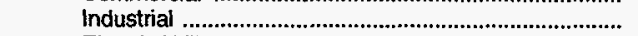 & 0 & 0 & 0 & $105,499,266$ & 0 \\
\hline 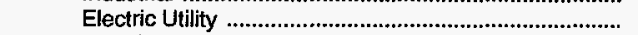 & 0 & 0 & 0 & $6,079,622$ & 0 \\
\hline 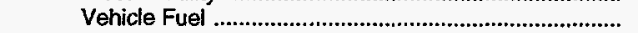 & 0 & 0 & 0 & 0 & 0 \\
\hline 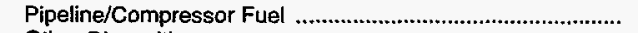 & $1,487,845$ & 685,444 & 557,004 & 0 & 705,524 \\
\hline 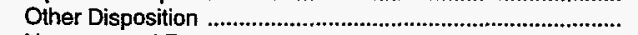 & 17,624 & 5,806 & 0 & 34,750 & 18,235 \\
\hline 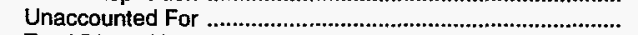 & 0 & 0 & 0 & 0 & $-4,990,085$ \\
\hline Total Disposition & $258,468,246$ & $256,475,285$ & $254,763,873$ & $243,090,804$ & $269,472,944$ \\
\hline
\end{tabular}

Source: Energy Information Administration (EIA), Form EIA-176, "Annual Report of Natural and Supplemental Gas Supply and Disposition." 
Table 62. The Williams Companies, Inc., Interstate Flows of Natural Gas, 1993 (Thousand Cubic Feet)

\begin{tabular}{|c|c|}
\hline Company and State & Volume \\
\hline \multicolumn{2}{|l|}{$\begin{array}{l}\text { Williams Natural Gas Co. } \\
\text { Texas To }\end{array}$} \\
\hline Oklahoma To & $27,105,950$ \\
\hline 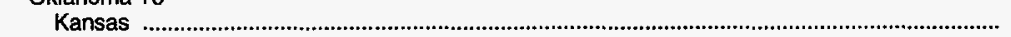 & $115,460,393$ \\
\hline 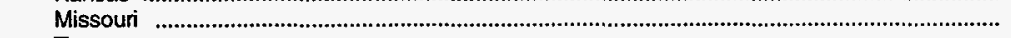 & $14,214,490$ \\
\hline 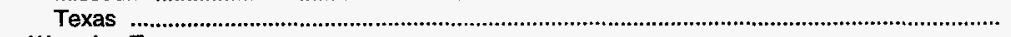 & 722,405 \\
\hline \multicolumn{2}{|l|}{ Wyoming To } \\
\hline Colorado .................. & $43,469,898$ \\
\hline \multicolumn{2}{|l|}{ Colorado To } \\
\hline 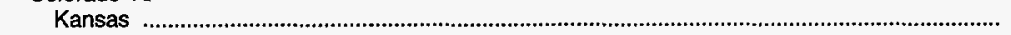 & $46,816,062$ \\
\hline \multicolumn{2}{|l|}{ Kansas To } \\
\hline Missouri & $100,300,703$ \\
\hline Nebraska & 902,200 \\
\hline Oklahoma & $18,921,008$ \\
\hline \multicolumn{2}{|l|}{ Missouri To } \\
\hline Oklahoma & 278,064 \\
\hline \multicolumn{2}{|l|}{ Northwest Pipeline Corp. } \\
\hline Washington & $306,030,160$ \\
\hline \multicolumn{2}{|l|}{ Washington To } \\
\hline \multirow{2}{*}{\multicolumn{2}{|c|}{$\begin{array}{l}\text { Oregon } \\
\text { Oregon To }\end{array}$}} \\
\hline & \\
\hline Idaho & $55,738,240$ \\
\hline \multicolumn{2}{|l|}{$\begin{array}{l}\text { Idaho } \\
\text { Idaho To }\end{array}$} \\
\hline Nevada & $39,196,714$ \\
\hline \multicolumn{2}{|l|}{ Wyoming To } \\
\hline Idaho & $36,108,667$ \\
\hline Utah & $26,961,951$ \\
\hline \multicolumn{2}{|l|}{ Utah To } \\
\hline Colorado & $40,002,622$ \\
\hline \multicolumn{2}{|l|}{ Colorado To } \\
\hline New Mexico & $41,589,001$ \\
\hline \multicolumn{2}{|l|}{ Kern River Gas Transmission Co. } \\
\hline \multirow{2}{*}{\multicolumn{2}{|c|}{ Utah }} \\
\hline & \\
\hline Nevada & $254,756,612$ \\
\hline \multicolumn{2}{|l|}{ Nevada To } \\
\hline California & $240,539,903$ \\
\hline
\end{tabular}

Source: Energy Information Administration (EIA), Form ElA-176, "Annual Report of Natural and Supplemental Gas Supply and Disposition." 


\section{Williston Basin Interstate Pipeline Co.}

Williston Basin Interstate Pipeline Company is a wholly owned subsidiary of Montana-Dakota Utility Resource Group, a diversified natural resource company comprised of an electric and gas utility; a natural gas transmission company; mining and construction material operations; an oil and natural gas production unit; and an energy marketing firm with bulk propane distribution services. Montana-Dakota Utilities (MDU) is the public utility division serving communities in Montana, North Dakota, and Wyoming. MDU has five subsidiaries: Williston Basin Interstate Pipeline Company, Knife River Coal Mining Company, Fidelity Oil Group, Prairielands Energy Marketing, Inc., and Williston Basin Interstate Canadian Pipeline.
These subsidiaries are engaged in natural gas transmission, underground storage, and exploration and production ventures throughout the Western United States, Canada, and the Gulf of Mexico. In addition, they provide energy marketing and management services throughout the Midwest.

In 1993, Williston Basin extended natural gas distribution service to north-central South Dakota communities. The company also acquired two underground propane distribution systems and expanded utility services to new markets. Williston Basin promoted its natural gas vehicles (NGV's) program that provides approximately 500 vehicles with natural gas supplied by 13 refueling stations

Figure 46. Williston Basin Interstate Pipeline Co., 1993

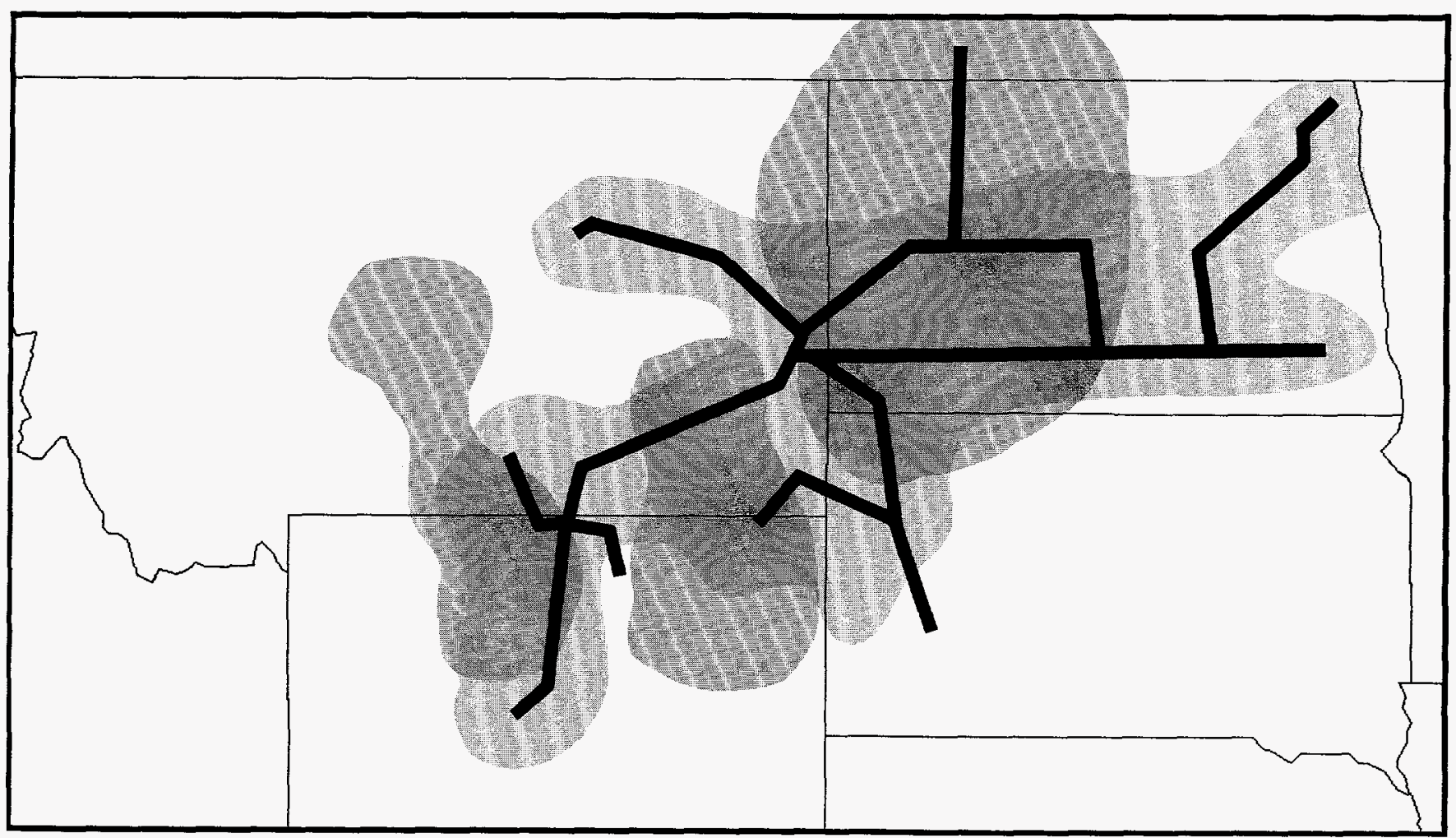

Source: Energy Information Administration (EIA), Form EIA-176, "Annual Report of Natural and Supplemental Gas Supply and Disposition." 
Table 63. Williston Basin Interstate Pipeline Co., Natural Gas Data, 1993 (Thousand Cubic Feet)

\begin{tabular}{|c|c|c|c|c|}
\hline \multirow{2}{*}{ Supply/Disposition } & \multicolumn{4}{|c|}{ Williston Basin Interstate Pipeline Co. } \\
\hline & Montana & North Dakota & South Dakota & Wyoming \\
\hline \multicolumn{5}{|l|}{ SUPPLY } \\
\hline $\begin{array}{l}\text { Produced Onsystem } \\
\text { Receipts }\end{array}$ & 0 & 0 & 0 & 0 \\
\hline 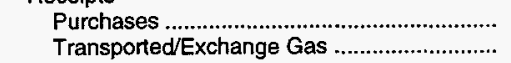 & $\begin{array}{l}4,383,170 \\
9,006,834\end{array}$ & $\begin{array}{r}1,077,201 \\
17,565,537\end{array}$ & $\begin{array}{l}198,236 \\
659,505\end{array}$ & $\begin{array}{r}2,823,014 \\
15,170,391\end{array}$ \\
\hline \multicolumn{5}{|l|}{ Interstate Movements } \\
\hline 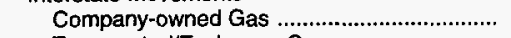 & 0 & 0 & 0 & 0 \\
\hline Transported/Exchange Gas ............................. & $19,183,159$ & $20,311,663$ & $7,186,817$ & $11,298,341$ \\
\hline Storage Withdrawals & $25,796,698$ & 0 & 0 & $10,296,738$ \\
\hline Received from Storage Operators ..................... & 0 & 0 & 0 & 0 \\
\hline 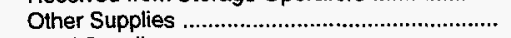 & 0 & 0 & 0 & 0 \\
\hline 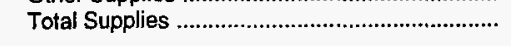 & $58,369,861$ & $38,954,401$ & $8,044,558$ & $39,588,484$ \\
\hline \multicolumn{5}{|l|}{ DISPOSITION } \\
\hline Lease and Plant Use & 0 & 43,072 & 0 & 25,681 \\
\hline 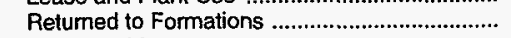 & 0 & 0 & 0 & 0 \\
\hline \multicolumn{5}{|l|}{ Interstate Movements } \\
\hline 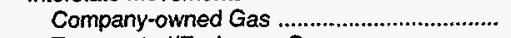 & 0 & 0 & 0 & 0 \\
\hline 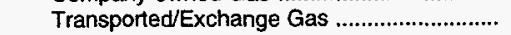 & $38,386,973$ & $9,594,509$ & 409,848 & $9,588,650$ \\
\hline Exchange Gas Deliveries ..................................... & 0 & 0 & 0 & 0 \\
\hline 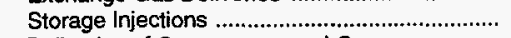 & $2,427,263$ & 0 & 0 & $11,571,869$ \\
\hline \multicolumn{5}{|l|}{ Deliveries of Company-owned Gas } \\
\hline 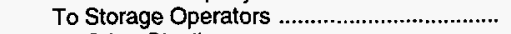 & 0 & 0 & 0 & 0 \\
\hline 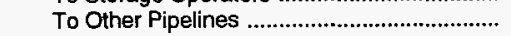 & 0 & 0 & 0 & 0 \\
\hline To Resellers & $3,909,533$ & $4,884,200$ & $2,088,082$ & $1,616,636$ \\
\hline \multicolumn{5}{|l|}{ To Consumers } \\
\hline 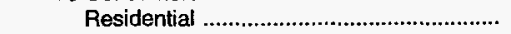 & 0 & 0 & 0 & 0 \\
\hline 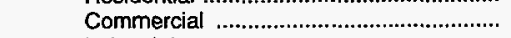 & 0 & 0 & 0 & 0 \\
\hline 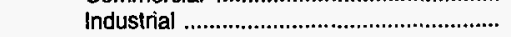 & 0 & 0 & 0 & 0 \\
\hline 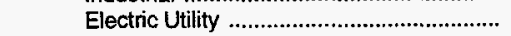 & 0 & 0 & 0 & 0 \\
\hline Vehicle Fuel & 0 & 0 & 0 & 0 \\
\hline \multicolumn{5}{|l|}{ Transported for the Account of Others } \\
\hline To Storage Operators & 0 & 0 & 0 & 0 \\
\hline 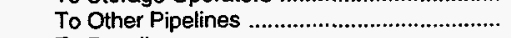 & $1,376,332$ & $8,400,634$ & 0 & $12,806,341$ \\
\hline 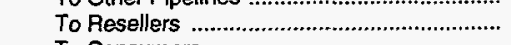 & $9,472,759$ & $14,733,343$ & $4,925,548$ & $3,518,365$ \\
\hline \multicolumn{5}{|l|}{ To Consumers } \\
\hline 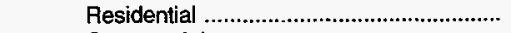 & 0 & 0 & 0 & 0 \\
\hline 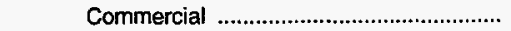 & 0 & 0 & 0 & 0 \\
\hline Industrial & 165,343 & $1,001,184$ & 502,525 & 10,808 \\
\hline Electric Utility & 0 & 0 & 0 & 0 \\
\hline 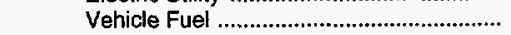 & 0 & 0 & 0 & 0 \\
\hline 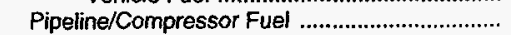 & $1,826,489$ & 375,057 & 66,303 & 654,937 \\
\hline 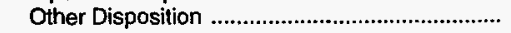 & 0 & 0 & 0 & 0 \\
\hline 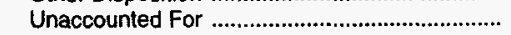 & 805,169 & $-77,598$ & 52,252 & $-204,803$ \\
\hline 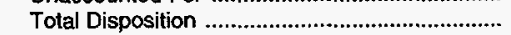 & $58,369,861$ & $38,954,401$ & $8,044,558$ & $39,588,484$ \\
\hline
\end{tabular}

throughout Montana, North Dakota, South Dakota, and Wyoming.

Williston Basin interconnects with six other interstate pipelines located in Montana, North Dakota, South Dakota, and Wyoming. In 1993, Canada's National Energy Board approved an application by Williston Basin to construct a natural gas pipeline from North Portal, Saskatchewan to the U.S.-Canadian border where it will connect with a new pipeline in the United States. On the Canadian side, this new pipeline will connect with the newly constructed 35.6 kilometer TransGas line extending to Steelman, Saskatchewan from North Portal--the "Steelman/North Portal Extension."

In October 1993, Williston Basin Interstate Canadian Pipeline started construction of a 0.72 -mile gas pipeline that would link with a 10.6 -mile section of pipe owned by Williston Basin in Saskatchewan to bring natural gas to North Dakota. This line, operated by a unit of Williston Basin in Bismarck, North Dakota, also delivers gas from TransGas Ltd.

Williston Basin expanded deliverability of two storage fields to provide an additional 95 thousand cubic feet per day of firm withdrawal capacity to Montana-Dakota Utilities. Williston Basin drilled six new wells to increase its deliverability by 54,685 thousand cubic feet per day at its Elk Basin storage field in Carbon County, Montana, and Park County, Wyoming. The company also added 40,315 thousand cubic feet per day deliverability at Baker storage field in Fallon County, Montana, the largest jurisdictional storage facility in the United States. 


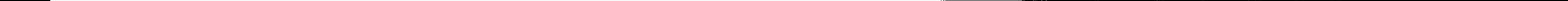




\section{Wisconsin Gas Co.}

Wisconsin Gas Company, the largest subsidiary of WICOR, Inc., is both the oldest, dating back to 1852 , and also the largest gas distributor in Wisconsin. Wisconsin Gas is becoming more aggressive in its marketing strategies to obtain new customers. In 1993, it added more than 14,000 new customers and more than 1,000 miles of new gas mains throughout the State.
In response to the deregulation of interstate pipelines pursuant to FERC Order 636, Wisconsin Gas Company began buying and managing gas supplies for its customers. In 1993, the Company introduced and implemented a new turnkey gas supply management service for large-volume customers. Wisconsin Gas is also marketing equipment leasing in such areas as natural gas vehicles, food service equipment, and air conditioning.

Figure 47. Wisconsin Gas Co., 1993

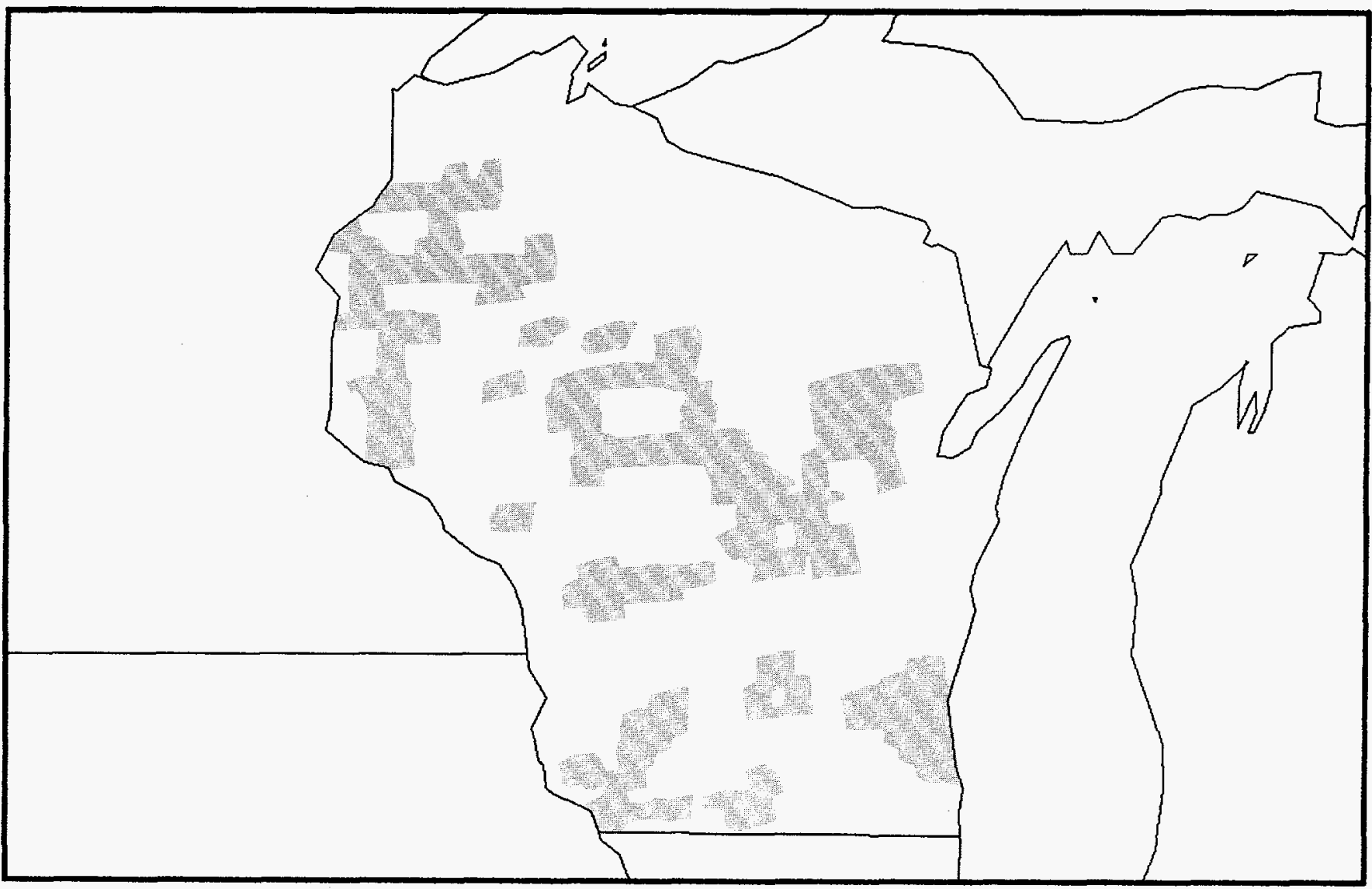

Source: Energy Information Administration (EIA), Form EIA-176, "Annual Report of Natural and Supplemental Gas Supply and Disposition."

\section{Service Areas}


Table 65. Wisconsin Gas Co., Natural Gas Data, 1993 (Thousand Cubic Feet)

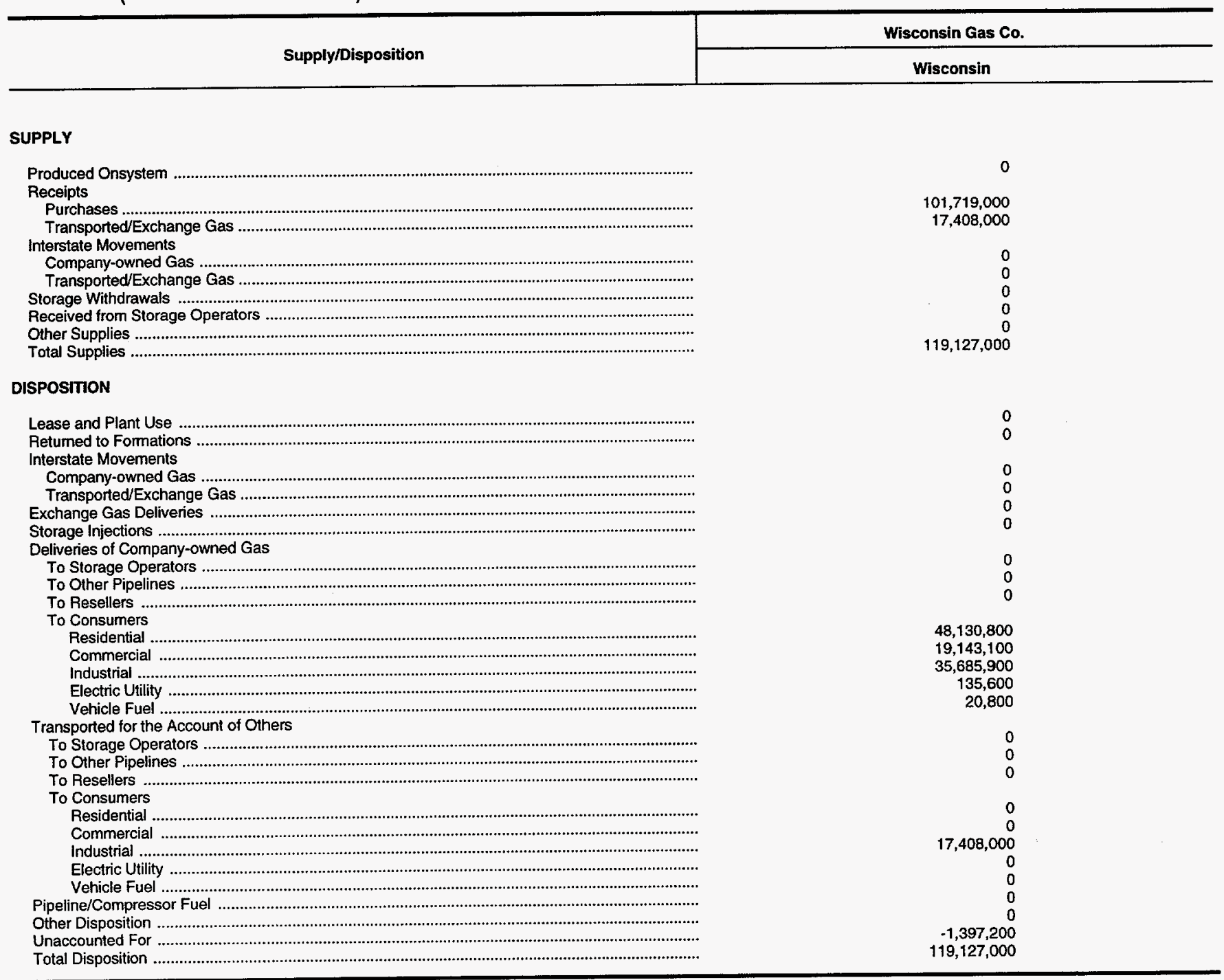

Source: Energy Information Administration (EIA), Form EIA-176, "Annual Report of Natural and Supplemental Gas Supply and Disposition." 
Appendix A

Summary of Data Collection and Report Methodology 


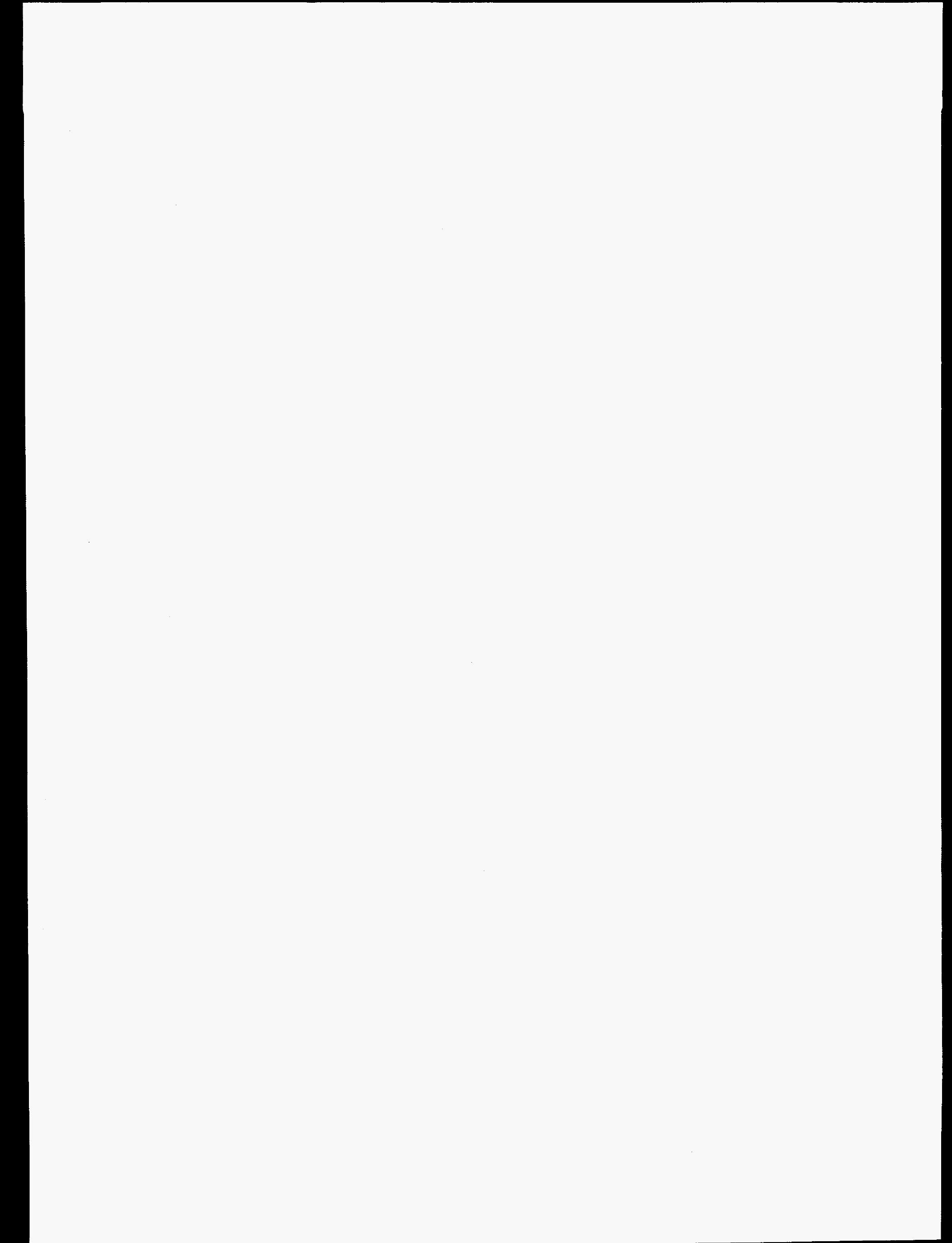




\section{Summary of Data Collection and Report Methodology}

The data for the Natural Gas Annual 1993 Supplement: Company Profiles are taken from Form EIA-176, "Annual Report of Natural and Supplemental Gas Supply and Disposition." A copy of the form is shown as Figure A1. Other sources of information include industry literature and corporate annual reports to shareholders.

The companies appearing in this report are major interstate natural gas pipeline companies, large distribution companies, or combination companies with both pipeline and distribution operations. The report contains case profiles of 41 corporate families. They were selected in a multistep process using knowledge of the industry and specific information included on Form EIA-176 submissions. First, the interstate pipeline companies (representing distinct corporate families) with the greatest reported volumes of gas transported were selected. Other companies not in the same corporate family as any of the first group were selected from the top companies in volumes delivered to end users. Finally, additional companies belonging to the same corporate family as companies in either of the first two groups were included in the profiles.

\section{Data Collection Operations}

\section{Survey Design}

The original version of Form EIA-176 was approved in 1980 with a mandatory response requirement. Prior to 1980 , published data were based on voluntary responses to Bureau of Mines, U.S. Department of the Interior, predecessor Forms BOM-6-1340-A and BOM-6-1341-A of the same title.

In 1982, the scope of the revised EIA-176 survey was expanded to collect the number of electric utility consumers in each State, volumes of gas transported to industrial and electric utility consumers, detailed information on volumes transported across State borders by the respondent for others and for the responding company, and detailed information on other disposition. These changes were incorporated to provide more complete survey information with a minimal change in respondent burden.
The 1982 revision of the Form EIA-176 continues to be the basis for the current version of this form.

In 1988, the Form EIA-176 was revised to include data collection for deliveries of natural gas to commercial consumers for the account of others. The revised form was approved for use during report years 1987 through 1989 . A short version of Form EIA-176 was also approved in 1988. Companies engaged in purchase and delivery activities but not in transportation and storage activities may file the short form. Usually, these companies are municipals handling small volumes of gas.

In 1990, the Form EIA-176 was revised to include more detailed information for gas withdrawn from storage facilities, gas added to storage facilities, deliveries of company-owned natural gas and natural gas transported for the account of others. The revised form was approved for use beginning with report year 1990 .

Upon the Office of Management and Budget's approval in 1993, the Form EIA-176 was revised. All deliveries to consumers are now categorized as firm or interruptible. Commercial and industrial consumers are further categorized as nonutility power producers or as those excluding nonutility power producers. Data reported on this form are no longer collected under a confidentiality agreement with respondents. Response to the form continues to be mandatory.

In February 1994, forms for report year 1993 were mailed to all identified interstate natural gas pipeline companies; intrastate natural gas pipeline companies; investor and municipally owned natural gas distributors; underground natural gas storage operators; synthetic natural gas plant operators; field, well, or processing-plant operators that deliver natural gas directly to consumers (including their own industrial facilities) other than for lease or plant use or processing; and field, well, or processing-plant operators that transport gas to, across, or from a State border through field or gathering facilities. Detailed instructions for completing the form were included in each survey package. Completed forms were returned to the Data Operations Branch of the Reserves and Natural Gas Division, where each was checked for errors, corrected as necessary, and processed into computer-generated State and national data summaries. 


\section{Response Statistics}

Each company and its parent company or subsidiaries were required to file if they met the survey specifications. The original mailing totaled 2,065 questionnaire packages. To this original mailing, 7 names were added and 72 were deleted as a result of the survey processing. Additions were the result of comparisons of the mailing list to other survey mailing lists. Deletions result from post office returns and determinations that companies were out of business, sold, or not within the scope of the survey. After all updates, the survey universe was 2,039 responses from approximately 1,800 companies.

\section{Report Methodology}

\section{Natural Gas Consumed as a Vehicle Fuel}

Data on deliveries of natural gas for use as a vehicle fuel were collected for the first time in 1990.

\section{Natural Gas Unaccounted For}

Natural gas unaccounted for represents the difference between the sum of the components of natural gas supply and the sum of the components of natural gas disposition. When this calculation results in a negative quantity, the natural gas unaccounted for represents an excess of reported supply in relation to reported disposition, and positive quantities indicate the opposite situation.

The differences between supply and demand represent quantities lost, the net result of gas company conversions of flow data metered at varying temperature and pressure conditions to a standard temperature and pressure base, metering inaccuracies, the effect of variations in company accounting and billing practices, and differences between billing cycle and calendar-period time frames.

A listing of parent companies along with their affiliates is shown on the following page. A copy of Form EIA-176 follows the listing. Table A1 shows the company profile data elements with their corresponding line numbers from the Form EIA-176. Table A2 provides an index of affiliate companies with their associated parent company. 
The following list presents the parent companies along with their affiliates featured in the Natural Gas Annual 1993 Supplement: Company Profiles

Atlanta Gas Light Co.

Chatanooga Gas Co.

Baltimore Gas and Electric Co.

Boston Gas Co.

Brooklyn Union Gas Co.

Coastal Corp.

ANR Pipeline Co.

ANR Production Co.

ANR Storage Co.

Coastal Gas Gathering Co.

Colorado Interstate Gas Co.

Eaton Rapids Gas Storage System

Great Lakes Gas Transmission Ltd.

High Island Offshore System

Steuben Gas Storage Co.

U-T Offshore System

Wyoming Interstate Co.

Columbia Gas System, Inc.

Columbia Gas of Kentucky

Columbia Gas of Maryland

Columbia Gas of Ohio

Columbia Gas of Pennsylvania

Columbia Gas Transmission Corp.

Columbia Gulf Transmission Corp.

Columbia LNG Corp.

Commonwealth Gas Service Inc.

Consolidated Edison Co. of New York, Inc.

Consolidated Natural Gas Co.

CNG Transmission Corp.

East Ohio Gas Co.

Hope Gas, Inc.

Peoples Natural Gas Co.

River Gas Co.

Virginia Natural Gas, Inc.

West Ohio Gas Co.

Consumers Power Co.

Michigan Gas Storage Co.

El Paso Natural Gas Co.

Mojave Pipeline Co.
Enron Corp.

Black Marlin Pipeline Co.

Enron Industrial Natural Gas Co.

Florida Gas Transmission Co.

Houston Pipe Line Co.

Intratex Gas Co.

Northern Border Pipeline Co.

Northern Natural Gas Co.

Transwestern Pipeline Co.

Valley Pipeline Inc.

Equitable Resources, Inc.

Equitable Gas Co.

Equitrans, Inc.

Kentucky West Virginia Gas Co.

Louisiana Intrastate Gas Co.

Gas Company of New Mexico

Iroquois Gas Transmission System

K N Energy, Inc.

Northern Gas Co.

Northern Gas of Wyoming

RMNG Gathering Co.

Rocky Mountain Natural Gas Co.

Rocky Mountain Natural Gas Div.

Koch Gateway Pipeline Co.

Lone Star Gas Co.

National Pipeline Co.

Michigan Consolidated Gas Co.

Citizens Gas Fuel Co.

MichCon Gas Co.

MidCon Corp.

Kansas Gas Supply Corp.

MidCon Texas Pipeline Co.

Moraine Pipeline Co.

Natural Gas Pipeline Co. of America

Palo Duro Pipeline Co.

Stingray Pipeline Co.

Texas Industrial Energy Co.

Trailblazer Pipeline System

National Fuel Gas Co.

National Fuel Gas Distribution Corp. 
National Fuel Gas Supply Corp.

Penn-York Energy Corp

Niagara Mohawk Power Corp.

NorAm Energy Corp., Inc.

Arkansas Louisiana Gas Co.

Entex Div. of NorAm Energy Corp.

Industrial Gas Supply Corp.

Minnegasco, Inc.

Mississippi River Transmission Corp.

NorAm Gas Transmission Co.

Tuscaloosa Pipeline Co.

Unit Gas Transmission Co.

Northern Illinois Gas Co.

Northern Indiana Public Service Co. Kokomo Gas and Fuel Co.

Northwest Natural Gas Co.

ONEOK, Inc.

Oklahoma Natural Gas Co.

Oktex Pipeline Co.

ONG Red Oak Transmission Co.

ONG Sayre Storage Co.

ONG Western, Inc.

Red River Pipeline Co.

Pacific Gas \& Electric Co.

Pacific Gas Transmission Co.

Panhandle Eastern Corp.

Algonquin Gas Transmission Co.

Panhandle Eastern Pipe Line Co.

Southwest Gas Storage Co.

Texas Eastern Transmission Corp.

Trunkline Gas Co.

Trunkline LNG Co.

Peoples Gas Light and Coke Co.

North Shore Gas Co.

Public Service Co. of Colorado

Cheyenne Light Fuel and Power Co.

Public Service Electric and Gas Co.

Questar Corp.

Mountain Fuel Supply Co.

Overthrust Pipeline Co.

Questar Pipeline Co.
Sabine Pipe Line Co.

Bridgeline Gas Distribution Co.

Riverway Gas Pipeline Co.

San Diego Gas and Electric Co.

Southern California Gas Co. Pacific Offshore Pipeline Co.

Southern Natural Gas Co.

Bear Creek Storage Co.

Sea Robin Pipeline Co.

South Georgia Natural Gas Co

Southwest Gas Corp.

Tenneco, Inc.

Channel Industries Gas Co.

Creole Gas Pipeline Corp.

East Tennessee Natural Gas Co.

Midwestern Gas Transmission Co.

Tennessee Gas Pipeline Co.

Viking Gas Transmission Co.

Transco Gas Co.

Texas Gas Transmission Corp.

Transcontinental Gas Pipe Line Corp.

Valero Energy Corp.

Val Gas Co.

Val Gas Limited Partnership

Val Gathering Limited Partnership

Valero Gas Storage

Valero Interstate Transmission Co.

Valero Transmission Limited Partnership

V.H.C. Pipeline Limited Partnership

Washington Gas Light Co.

Frederick Gas Co.

Hampshire Gas Co.

Shenandoah Gas Co.

Western Resources, Inc.

Williams Companies, Inc., The

Kern River Gas Transmission Co.

Louisiana Resources Co.

Northwest Pipeline Corp.

Williams Gas Marketing Co.

Williams Natural Gas Co.

Williston Basin Interstate Pipeline Co. Montana-Dakota Utilities Co.

Wisconsin Gas Co. 
Figure A1. Form EIA-176

ElA-176 (Revised 1993)

U.S. DEPARTMENT OF ENERGY

Form Approved

Energy Information Administration

OMB No. 1905017 Washington DC 20585

\section{ANNUAL REPORT OF NATURAL AND SUPPLEMENTAL GAS SUPPLY AND DISPOSITION, 19}

This report is mandatory under the Federal Energy Administration Act of 1974 (Public Law 93-275). For the provisions conceming the confidentiality of intormation and sanctions statement, see Sections VI and VII of the instructions. Public reporting burden for this collection of information is estimated to average 20.8 hours per response, including the time of reviewing instructions, searching existing data sources, gathering and maintaining the data needed, and completing and reviewing the collection of information. Send comments regarding this burden esitmate or any other aspect of this collection of intormation including, suggestions for reducing this burden, to the Energy Information Administration, Office of Statistical Standards, 1000 Independence Ave. SW, Washington, DC 20585; and to the Office of Information and Regulatory Affairs, Office of Management and Budget, Washington, DC 20503 EIA USE

RESPONDENT COPY

Retain for your files.
Control (ID) No

Affix mailing label or enter mail address

Name: . . . . . . .

Operations in (State): . . . . .

Street or Post Office Box: . . .

City, State, Zip Code: . . . . .

Attention: .........

\begin{tabular}{|r|}
\hline PART I: ID \\
\hline 1.0 Control No \\
\hline 5.0 Company \\
\end{tabular}

5.1 Change company name and/or address to:
a. Company Name:
b. Operations in (State):
c. Street or Post Office Box:
d. City, State, Zip Code:
e. Attention:

\subsection{Contact person:}

Name:

Name and address on mailing label are correct.

Change name, attention line, and/or mall address as indicated below.

Company went out of business. Customer accounts taken over by company entered below.

Other changes, corrections, or comments:

\section{PART II: CERTIFICATION AND DISCLOSUAE STATEMENT}

1.0 I certify that (Check appropriate box):

a. $\square$

The information provided herein and appended hereto is true and accurate or, where indicated on the form, are reasonable estimates to the best of my knowledge.

b. $\square$

My company does not meet any of the criteria set forth in Section II, "Who must report," of the instructions and is therefore not required to complete and submit a Form ElA-176 for the report State.

\begin{tabular}{|l|l|}
\hline 2.0 Name & 3.0 Title \\
\hline 4.0 Signature & 5.0 Date \\
$\begin{array}{l}\text { Title 18, USC 1001, makes it a crime for any person knowingly and willingly to make to any agency or department of the United States any false, fictitious } \\
\text { or fraudulent statements as to any matter within its jurisdiction. }\end{array}$ \\
\hline
\end{tabular}


EIA-176, ANNUAL REPORT OF NATURAL AND SUPPLEMENTAL GAS SUPPLY AND DISPOSITION, 19

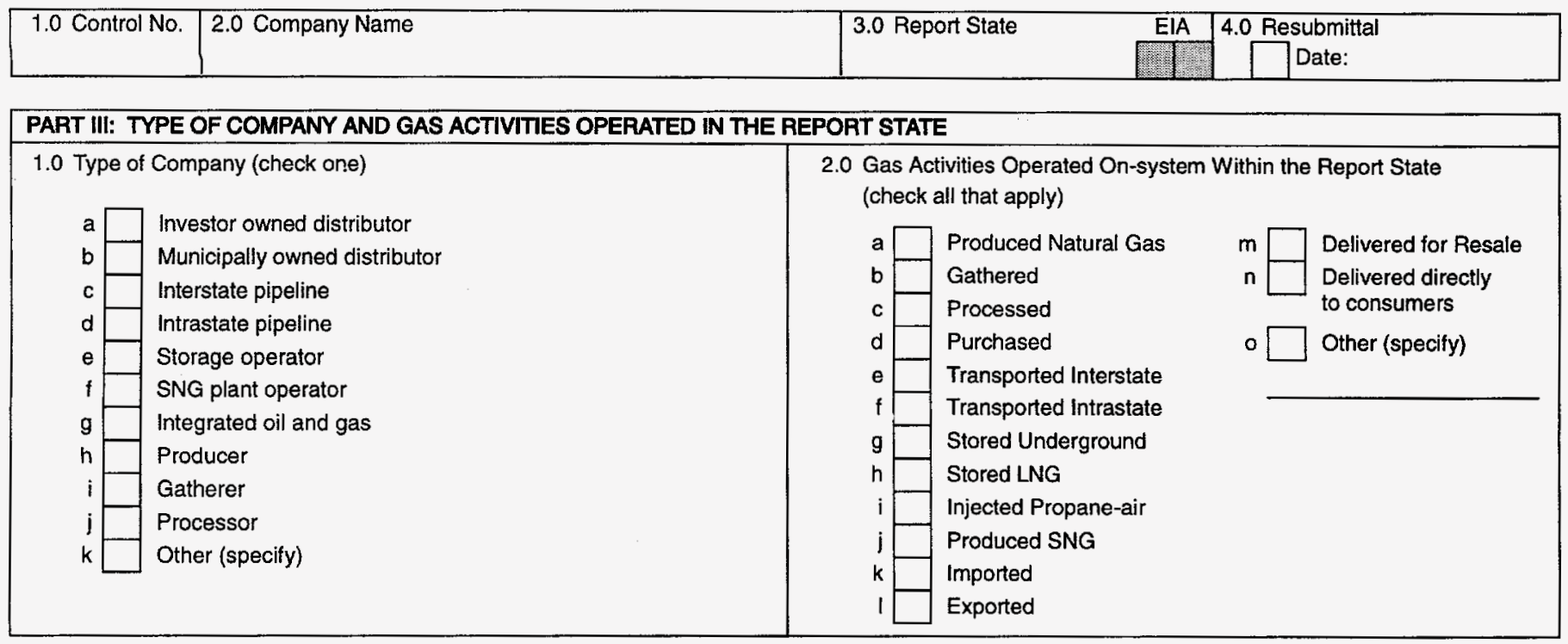

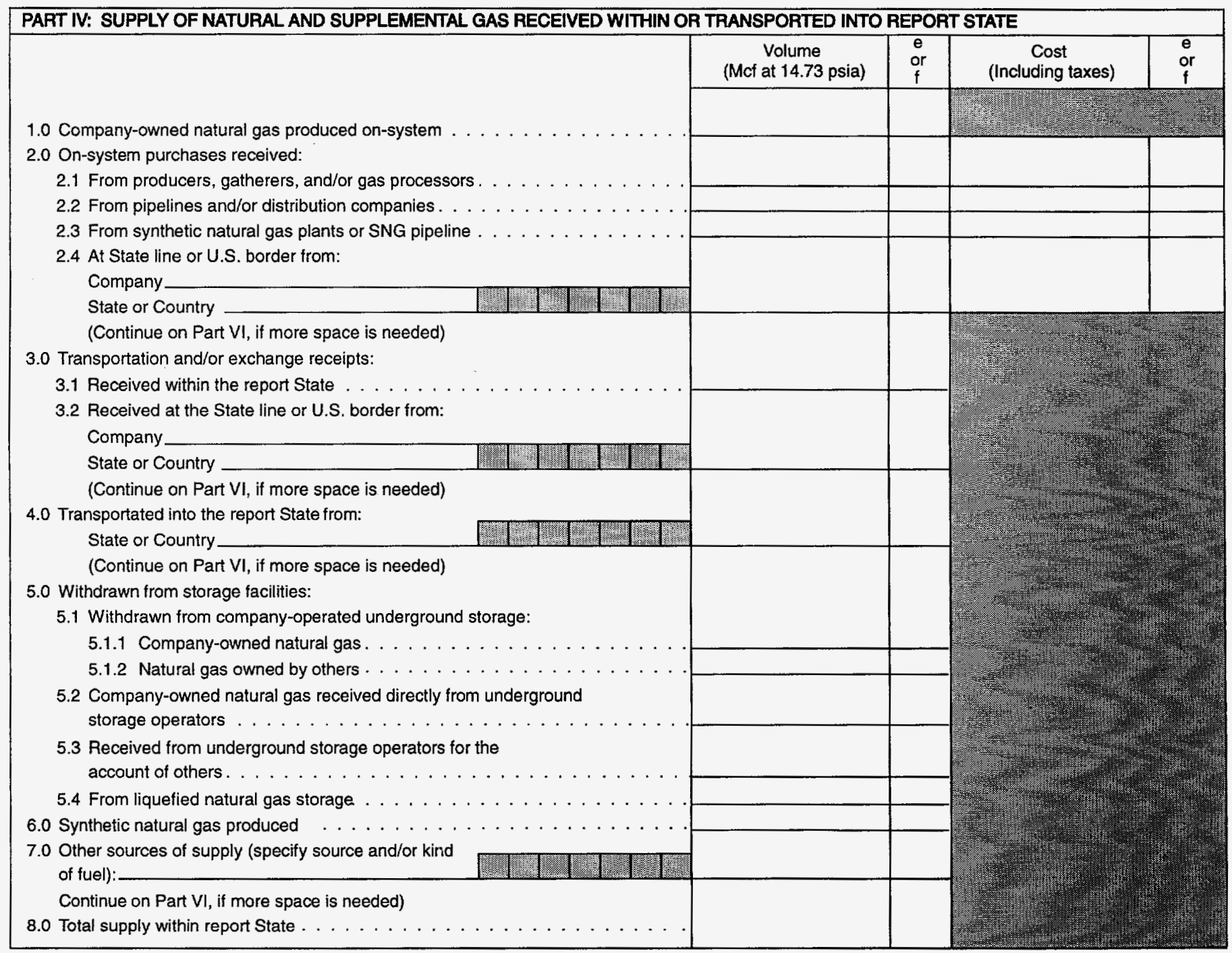


EIA-176, ANNUAL REPORT OF NATURAL AND SUPPLEMENTAL GAS SUPPLY AND DISPOSITION, 19

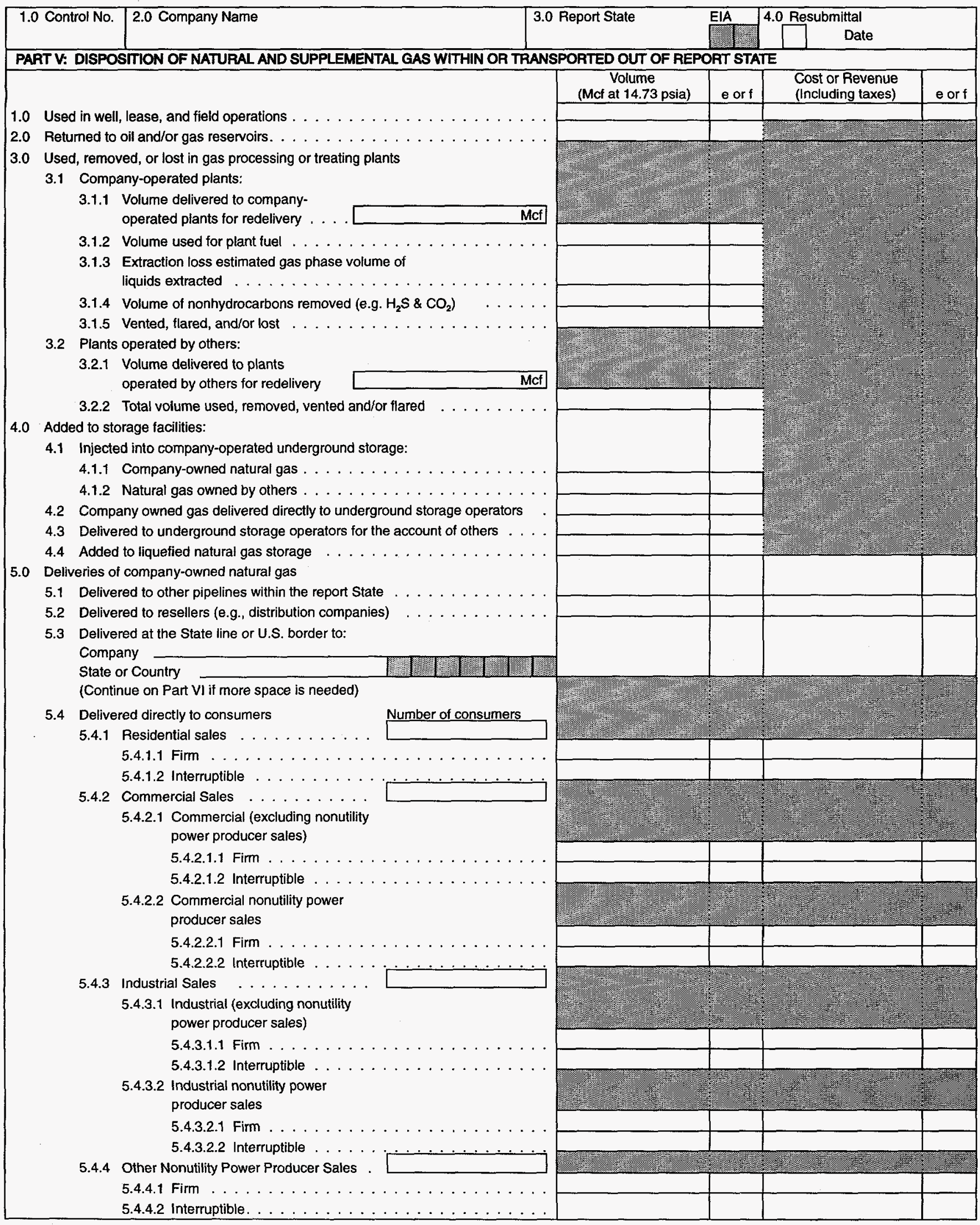


EIA-176, ANNUAL REPORT OF NATURAL AND SUPPLEMENTAL GAS SUPPLY AND DISPOSITION, $19 \square$

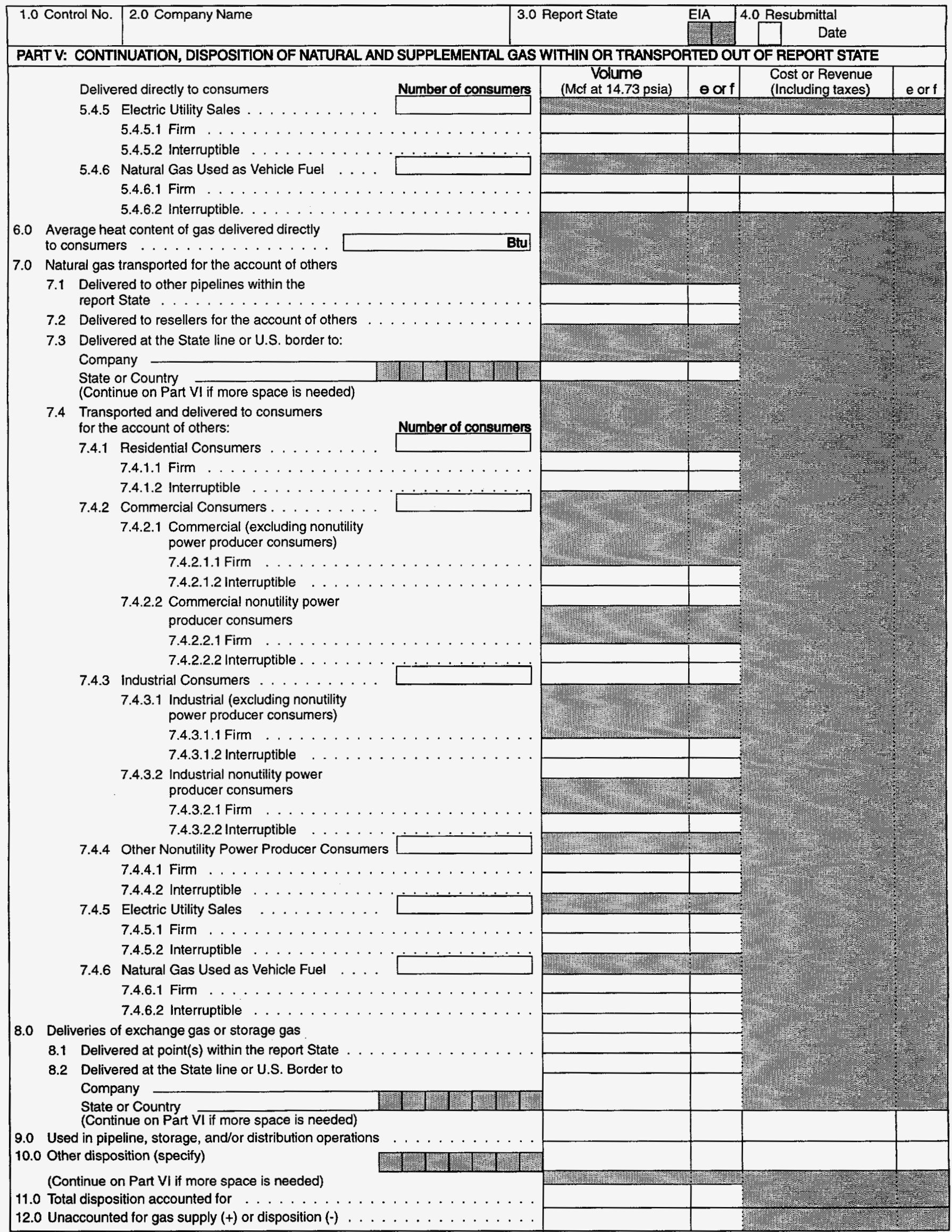


EIA-176, ANNUAL REPORT OF NATURAL AND SUPPLEMENTAL GAS SUPPLY AND DISPOSITION, 19

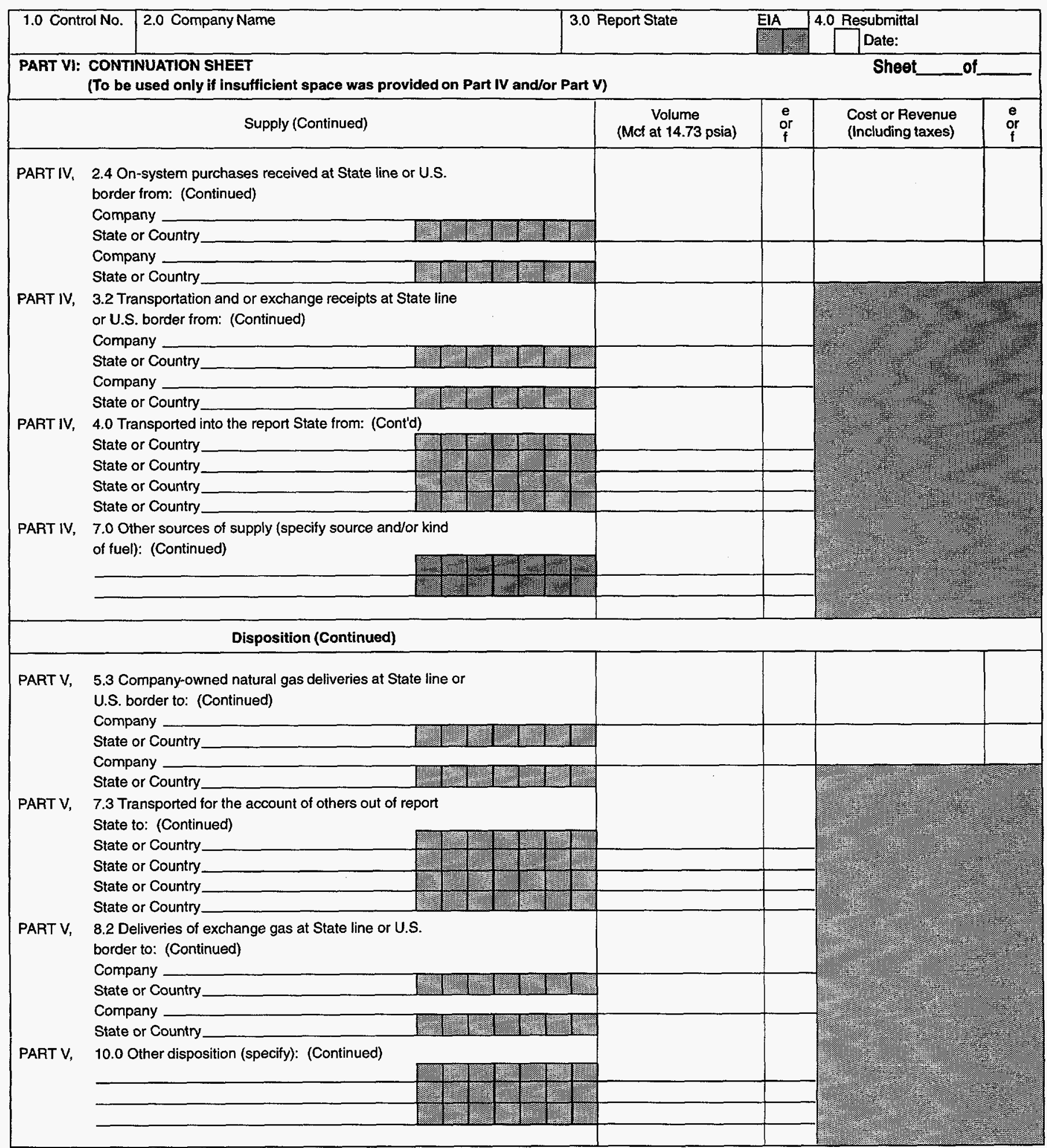


EIA-176, ANNUAL REPORT OF NATURAL AND SUPPLEMENTAL GAS SUPPLY AND DISPOSITION, 19

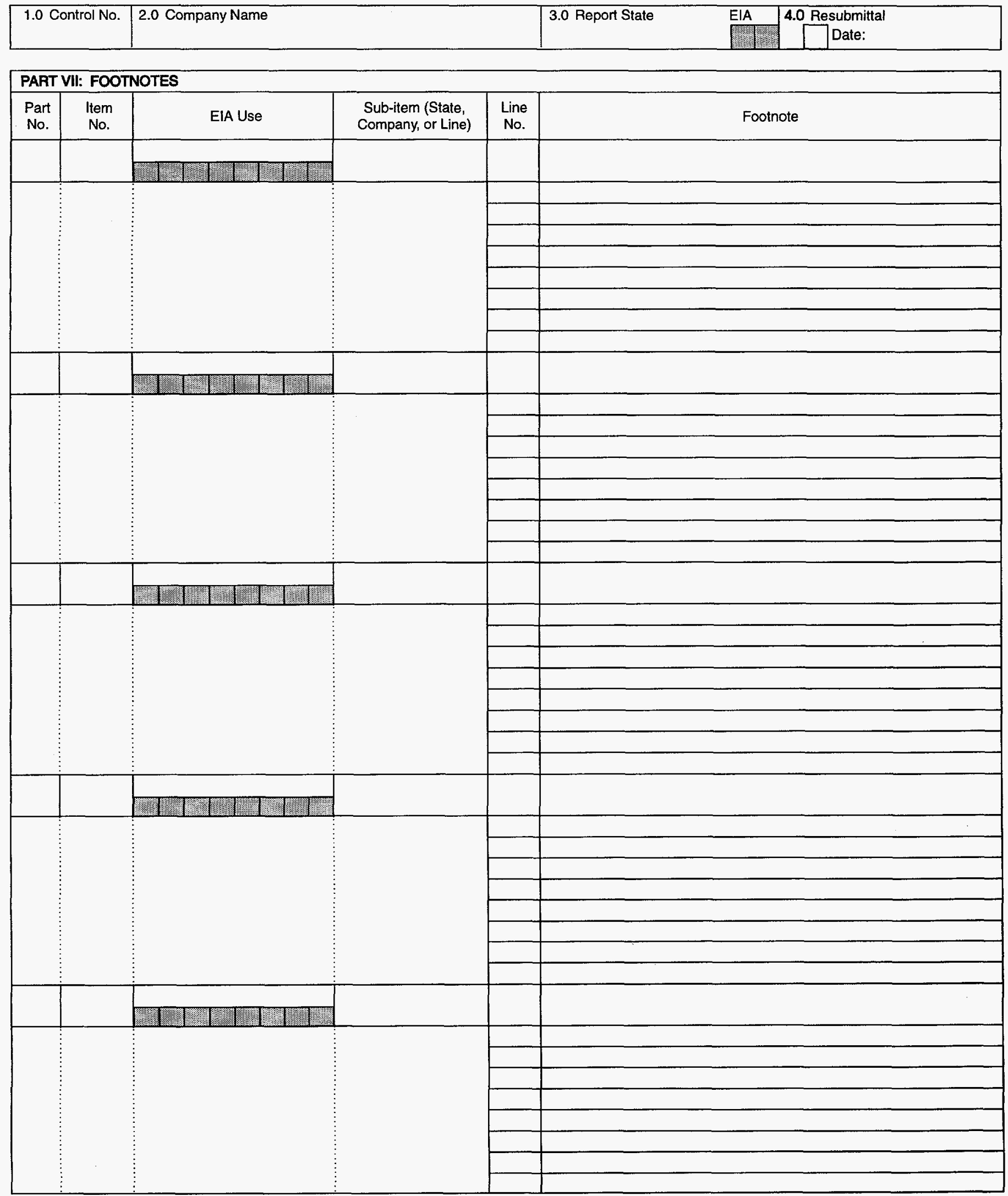


Supply (Part IV)

Produced Onsystem

Receipts

Purchases

Transported/Exchange Gas

Interstate Movements

Company-Owned Gas . . . . . . . . . . . . . . 2.4

Transportation/Exchange Gas . . . . . . . . . . . . $3.2+4.0$

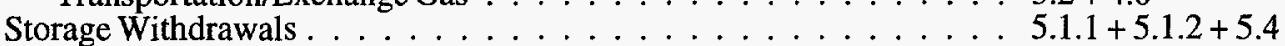

Received from Storage Operators . . . . . . . . . . . . . . . 5.2+5.3

Other Supplies . . . . . . . . . . . . . . 6.0 + 7.0

Total Supplies

8.0

Disposition (Part V)

Line Number

Lease and Plant Use

$1.0+3.1 .2+3.1 .3+$

$3.1 .4+3.1 .5+3.2 .2$

Returned to Formations

2.0

Interstate Movements

Company-owned Gas . . . . . . . . . . . . . 5.3

Transported $/$ Exchange Gas . . . . . . . . . . . . . $7.3+8.2$

Exchange Gas Deliveries . . . . . . . . . . . . . . . 8.1

Storage Injections . . . . . . . . . . . . . . . . . . 4.1.1+4.1.2+4.4

Deliveries of Company-owned Gas

To Storage Operators . . . . . . . . . . . . . 4.2

To Other Pipelines . . . . . . . . . . . . . . 5.1

To Resellers . . . . . . . . . . . . . . . . . . . 5.2

Residential . . . . . . . . . . . . . . . 5.4.1.1 +5.4.1.2

Commercial . . . . . . . . . . . . . 5.4.2.1.1+5.4.2.1.2+

$5.4 .2 .2 .1+5.4 .2 .2 .2$

Industrial $\ldots \ldots \ldots \ldots \ldots . \ldots \ldots . \ldots \ldots . . \ldots \ldots .1 .1+5.4 .3 .1$.

Electric Utilites

5.4.5.1+ 5.4.5.2+

$5.4 .4 .1+5.4 .4 .2$

$5.4 .6 .1+5.4 .6 .2$

Vehicle Fuel

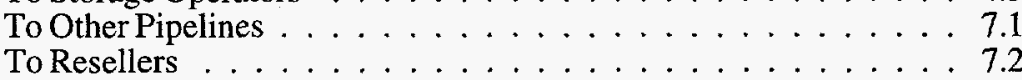

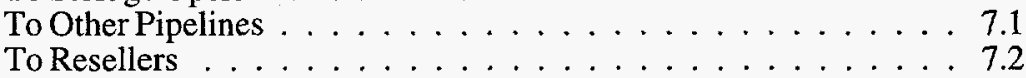

To Consumers

Residential . . . . . . . . . . . . . . . . . 7.4.1.1+7.4.1.2

Commercial . . . . . . . . . . . . 7.4.2.1.1+7.4.2.1.2+

7.4.2.2.1 + 7.4.2.2.2

Industrial .

7.4.3.1.1 +7.4.3.1.2+

7.4.3.2.1 +7.4.3.2.2

Electric Utilities

7.4.4.1+7.4.4.2+

$7.4 .5 .1+7.4 .5 .2$

Vehicle Fuel . . . . . . . . . . . . . . . 7.4.6.1 + 7.4.6.2

Pipeline/Compressor Fuel . . . . . . . . . . . . . . 9.0

Other Disposition . . . . . . . . . . . . . . . . 10.0

Unaccounted For . . . . . . . . . . . . . . . . 12.0

Total Disposition . . . . . . . . . . . . . . . 11.0

Source: Energy Information Administration (EIA), Form EIA-176, "Annual Report of Natural and Supplemental Gas Supply and Disposition." 


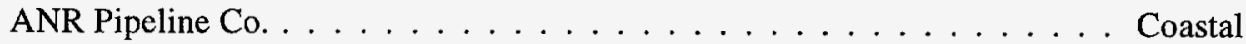

ANR Production Co. . . . . . . . . . . . . . . . . . Coastal

ANR Storage Co. . . . . . . . . . . . . . . . . . Coastal

Algonquin Gas Transmission Co. . . . . . . . . . . . . . . . . Panhandle Eastern

Arkansas Louisiana Gas Co. . . . . . . . . . . . . . . . . . . NorAm

Atlanta Gas Light Co

Baltimore Gas and Electric Co.

Bear Creek Storage Co. . . . . . . . . . . . . . . . . . Southern Natural

Black Marlin Pipeline Co. . . . . . . . . . . . . . . . Enron

Boston Gas Co.

Bridgeline Gas Distribution Co. . . . . . . . . . . . . . Sabine

Brooklyn Union Gas Co.

Channel Industries Gas Co. . . . . . . . . . . . . . . . . . . . . Tenneco

Chatanooga Gas Co. . . . . . . . . . . . . . . . . Atlanta

Cheyenne Light Fuel and Power Co. . . . . . . . . . . . . . Public Service Co. of Colorado

Citizens Gas Fuel Co. . . . . . . . . . . . . . . . . Michigan Consolidated

Coastal Corp.

Coastal Gas Gathering Co. . . . . . . . . . . . . . . . . . Coastal

Colorado Interstate Gas Co. . . . . . . . . . . . . . . . . . Coastal

Columbia Gas of Kentucky . . . . . . . . . . . . . . . . Columbia

Columbia Gas of Maryland . . . . . . . . . . . . . . . . . . Columbia

Columbia Gas of Ohio . . . . . . . . . . . . . . . . . Columbia

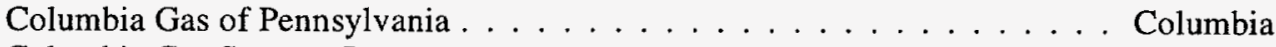

Columbia Gas System, Inc.

Columbia Gas Transmission Corp. . . . . . . . . . . . . . . . . . . . . . . . . . . . . . . . . . . . . . . . . . . .

Columbia Gulf Transmission Corp. . . . . . . . . . . . . . . . Columbia

Columbia LNG Corp. . . . . . . . . . . . . . . . . . . . Columbia

Commonwealth Gas Service Co. . . . . . . . . . . . . . . . . Columbia

Consolidated Edison Co. of New York, Inc.

Consolidated Natural Gas Co.

Consumers Power Co.

CNG Transmission Corp. . . . . . . . . . . . . . . . . . . . . Consolidated

Creole Gas Pipeline Corp. . . . . . . . . . . . . . . . . . Tenneco

East Ohio Gas Co. . . . . . . . . . . . . . . . . . Consolidated

East Tennessee Natural Gas Co. . . . . . . . . . . . . . . . . . . Tenneco

Eaton Rapids Gas Storage System . . . . . . . . . . . . . . . . Coastal

El Paso Natural Gas Co.

Enron Corp.

Enron Industrial Gas Co. . . . . . . . . . . . . . Enron

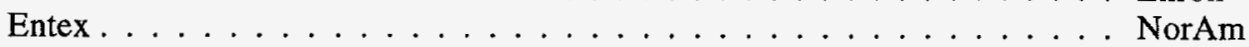

Equitable Gas Co. . . . . . . . . . . . . . . . . . . Equitable

Equitable Resources, Inc.

Equitrans, Inc. $\quad$ Equitable

Florida Gas Transmission Co. . . . . . . . . . . . . . . . Enron

Frederick Gas Co. . . . . . . . . . . . . . . . . . . . Washington Gas Light Co.

Gas Company of New Mexico

Great Lakes Gas Transmission Ltd. . . . . . . . . . . . . . . . . . . . . . Coastal 
Hampshire Gas Co.

Washington Gas Light Co.

High Island Offshore System $\ldots \ldots \ldots \ldots \ldots \ldots \ldots \ldots$ Coastal

Hope Gas, Inc. . . . . . . . . . . . . . . . . . . . Consolidated

Houston Pipe Line Co. . . . . . . . . . . . . . . . . . . . . Enron

Industrial Gas Supply Corp. . . . . . . . . . . . . . . . NorAm

Intratex Gas Co. . . . . . . . . . . . . . . . . . Enron

Iroquois Gas Transmission System

K N Energy, Inc.

Kansas Gas Supply Corp. . . . . . . . . . . . . . . . MidCon

Kentucky West Virginia Gas Co. . . . . . . . . . . . . . Equitable

Kern River Gas Transmission Co . . . . . . . . . . . . Williams

Koch Gateway Pipeline Co.

Kokomo Gas and Fuel Co. . . . . . . . . . . . . . . . . . Northern Indiana

Lone Star Gas Co. . . . . . . . . . . . . . . . . . . . .

Louisiana Intrastate Gas Co. . . . . . . . . . . . . . . . . . . Equitable

Louisiana Resources Co. . . . . . . . . . . . . . . . . Williams

MichCon Gas Co. . . . . . . . . . . . . . . . . Michigan Consolidated

Michigan Consolidated Gas Co.

Michigan Gas Storage Co. . . . . . . . . . . . . . . . Consumers Power

MidCon Corp.

MidCon Texas Pipeline Co. . . . . . . . . . . . . . . . . MidCon

Midwestern Gas Transmission Co. . . . . . . . . . . . . . . . . . Tenneco

Minnegasco, Inc. . . . . . . . . . . . . . . NorAm

Mississippi River Transmission Corp. . . . . . . . . . . . . . NorAm

Mojave Pipeline Co. . . . . . . . . . . . . . . . . El Paso

Montana-Dakota Utilities Co. . . . . . . . . . . . . . . . Williston Basin

Moraine Pipeline Co . . . . . . . . . . . . . . . . . . MidCon

Mountain Fuel Supply Co. . . . . . . . . . . . . . . Questar

National Fuel Gas Co.

National Fuel Gas Distribution Corp. . . . . . . . . . . . National Fuel

National Fuel Gas Supply Corp. . . . . . . . . . . . . . . . National Fuel

National Pipeline Co. . . . . . . . . . . . . . . . . . Lone Star

Natural Gas Pipeline Co. of America . . . . . . . . . . . MidCon

Niagara Mohawk Power Corp.

NorAm Energy Corp., Inc.

NorAm Gas Transmission Co. . . . . . . . . . . . . . NorAm

North Shore Gas Co. $\ldots \ldots \ldots \ldots \ldots \ldots \ldots \ldots$ Peoples Gas Light

Northern Border Pipeline Co. . . . . . . . . . . . . . . Enron

Northern Gas Co. . . . . . . . . . . . . . . . . . . . K N Energy

Northern Gas of Wyoming . . . . . . . . . . . . K N Energy

Northern Illinois Gas Co.

Northern Indiana Public Service Co.

Northern Natural Gas Co. . . . . . . . . . . . . . Enron

Northwest Natural Gas Co.

Northwest Pipeline Corp. . . . . . . . . . . . . . . . . . W Williams

Oklahoma Natural Gas Co. . . . . . . . . . . . . . . . . . ONEOK

Oktex Pipeline $\mathrm{Co} \ldots \ldots \ldots \ldots \ldots \ldots \ldots \ldots$ ONEO 
ONEOK, Inc.

ONG Red Oak Transmission Co. . . . . . . . . . . . . . . ONEOK

ONG Sayre Storage Co. $\ldots \ldots \ldots \ldots \ldots \ldots \ldots \ldots$ ONEOK

ONG Western Inc. . . . . . . . . . . . . . . . . . . ONEOK

Overthrust Pipeline Co. . . . . . . . . . . . . . . . . Questar

Pacific Gas \& Electric Co.

Pacific Gas Tranmission Co. . . . . . . . . . . . . . . . . . PG\&E

Pacific Offshore Pipeline Co. . . . . . . . . . . . . . . Southern California

Palo Duro Pipeline Co. . . . . . . . . . . . . . . . . MidCon

Panhandle Eastern Corp.

Panhandle Eastern Pipe Line Co.

Panhandle Eastern

Penn-York Energy Corp.

National Fuel Gas

Peoples Gas Light and Coke Co.

Peoples Natural Gas Co.

Consolidated

Public Service Co. of Colorado

Public Service Electric and Gas Co.

Questar Corp.

Questar Pipeline Co.

Red River Pipeline Co.

Questar

River Gas Co.

ONEOK

Riverway Gas Pipeline Co.

Consolidated

Co.................... Sabine

RMNG Gathering Co. . . . . . . . . . . . . . . . . . . . K N Energy

Rocky Mountain Natural Gas Co. . . . . . . . . . . . . . . K N Energy

Rocky Mountain Natural Gas Div. . . . . . . . . . . . . . . . K N Energy

Sabine Pipe Line Co.

San Diego Gas and Electric Co.

Sea Robin Pipeline Co. . . . . . . . . . . . . . . . . . Southern Natural

Shenandoah Gas Co. . . . . . . . . . . . . . . Washington Gas Light Co.

South Georgia Natural Gas Co. . . . . . . . . . . . . . . . Southern Natural

Southern California Cas Co.

Southern Natural Gas Co.

Southwest Gas Corp.

Southwest Gas Storage Co.

Panhandle Eastern

Steuben Gas Storage Co. . . . . . . . . . . . . . . . . Coastal

Stingray Pipeline Co. . . . . . . . . . . . . . . . . . MidCon

Tenneco, Inc.

Tennessee Gas Pipeline Co. . . . . . . . . . . . . . . . . . Tenneco

Texas Eastern Transmission Corp. . . . . . . . . . . . . . . . . . . Panhandle Eastern

Texas Gas Transmission Corp. . . . . . . . . . . . . . . . . . . Transco

Texas Industrial Energy Co. . . . . . . . . . . . . . MidCon

Trailblazer Pipeline System . . . . . . . . . . . . . MidCon

Transco Gas Co.

Transcontinental Gas Pipe Line Corp. . . . . . . . . . . . . . Transco

Transwestern Pipeline Co. . . . . . . . . . . . . . . . . . Enron

Trunkline Gas Co. . . . . . . . . . . . . . . . . . . Panhandle Eastern

Trunkline LNG Co. . . . . . . . . . . . . . . . . . . . . Panhandle Eastern

Tuscaloosa Pipeline Co. . . . . . . . . . . . . . . . NorAm 
U-T Offshore System . . . . . . . . . . . . . . . . Coastal

Unit Gas Transmission Co. . . . . . . . . . . . . . . . . . . . NorAm

Val Gas Co. . . . . . . . . . . . . . . . . . . . . Valero

Val Gas Limited Partnership . . . . . . . . . . . . . . . . . . Valero

Val Gathering Limited Partnership . . . . . . . . . . . . . . . . Valero

Valero Energy Corp. . . . . . . . . . . . . . . . . . . .

Valero Gas Storage . . . . . . . . . . . . . . . . . Valero

Valero Interstate Transmission Co. . . . . . . . . . . . . . Valero

Valero Transmission Limited Partnership . . . . . . . . . . . . Valero

Valley Pipeline, Inc. . . . . . . . . . . . . . . . . . E Enron

Viking Gas Transmission Co. . . . . . . . . . . . . Tenneco

Virginia Natural Gas, Inc. . . . . . . . . . . . . . . . . . . Consolidated

V.H.C. Pipeline Limited Partnership . . . . . . . . . . . . . . . Valero

Washington Gas Light Co.

West Ohio Gas Co. . . . . . . . . . . . . . . . . Consolidated

Western Resources, Inc.

Williams Companies, Inc., The

Williams Gas Marketing Co. . . . . . . . . . . . . . Williams

Williams Natural Gas Co. . . . . . . . . . . . . . . Williams

Williston Basin Interstate Pipeline Co.

Wisconsin Gas Co.

Wyoming Interstate Co.

Coastal

Source: Energy Information Administration (EIA), Form EIA-176, "Annual Report of Natural and Supplemental Gas Supply and Disposition." 

Appendix B

Selected

Natural Gas and Related Reports 



\section{Appendix B}

\section{Selected Natural Gas and Related Reports}

\section{Recurring Natural Gas Reports}

Natural Gas Monthly, DOE/EIA-0130.

Published monthly.

\section{Other Reports Covering Natural Gas, Natural Gas Liquids, and Other Energy Sources}

- U.S. Crude Oil, Natural Gas, and Natural Gas Liquids Reserves - 1993 Annual Report, DOE/EIA-0216(93), September 1994.

- Monthly Energy Review, DOE/EIA-0035. Published monthly. Provides national aggregate data for natural gas, natural gas liquids, and other energy sources.

- Annual Report to Congress 1993, DOE/ EIA0173(93), March 1994. Published annually.

- Annual Energy Outlook 1995, DOE/ EIA-0383(95), January 1995. Published annually.

- Annual Energy Review 1993, DOE/ EIA-0384(93), July 1994. Published annually.

- Short-Term Energy Outlook, DOE/EIA-0202. Published quarterly. Provides forecasts for next six quarters for natural gas and other energy sources.

- State Energy Data Report, Consumption Estimates, 1960-1992, DOE/EIA-0214(92), May 1994.

- State Energy Price and Expenditure Report 1990, DOE/EIA-0376(90), September 1992.

\section{One-Time Reports}

- U.S. Production of Natural Gas from Tight Reservoirs DOE/EIA-TR-0574, October 1993.

- Natural Gas 1994: Issues and Trends DOE/EIA0560(20), July 1994.

- Natural Gas Productive Capacity for the Lower 48 States 1980 through 1995, DOE/EIA-0542(95), July 1994.

- Largest U.S. Oil and Gas Fields, DOE/EIA-TR-0567, August 1993.

- Geologic Distributions of U.S. Oil and Gas, DOE/EIA-0557, July 1992.

- Capacity and Service on the Interstate Natural Gas Pipeline System 1990, DOE/EIA-0556, June 1992.

\section{Reference Reports}

- Directory of Energy Data Collection Forms, DOE/EIA-0249(93), December 1993.

- Oil and Gas Field Code Master List, 1992,EIA0370(92), December 1993. 



\section{Glossary}

Cofiring: The process of burning natural gas in conjunction with another fuel to reduce air pollutants.

Cogeneration: The simultaneous generation of steam and electricity from one fuel.

Commercial Consumption: Natural gas used by nonmanufacturing establishments or agencies primarily engaged in the sale of goods or services. Included are such establishments as hotels, restaurants, wholesale and retail stores, and other service enterprises; gas used by establishments engaged in agriculture, forestry, and fisheries; and gas used by local, State, and Federal agencies engaged in nonmanufacturing activities.

Compressed Natural Gas (CNG): Natural gas which is comprised primarily of methane, compressed to a pressure at or above 2,400 pounds per square inch and stored in special high pressure containers. It is used as a fuel for natural gas powered vehicles.

Compressor Station: Any combination of facilities which supplies the energy to move gas in transmission lines or into storage by increasing the pressure.

Delivered: The physical transfer of natural, synthetic, and/or supplemental gas from facilities operated by the responding company to facilities operated by others or to consumers.

Distribution: Use Natural gas used as fuel in the respondent's operations.

Electric Utilities: Establishments primarily engaged in the generation, transmission, and/or distribution of electricity for sale or resale.

Electric Utility Consumption: Natural gas used for gross generation, providing standby service, start-up and/or flame stabilization.

Exchange Agreement: A contractual agreement whereby one company agrees to deliver gas either directly or through intermediates to another company at one location, or in one time period, in exchange for the delivery by the second company to the first company of an equivalent volume or heat content at a different location or time period.

Exports: Natural gas deliveries out of the continental United States and Alaska to foreign countries.
Imports: Gas receipts into the United States from a foreign country.

Industrial Consumers: Establishments engaged in a process which creates or changes raw or unfinished materials into another form or product. Generation of electricity, other than by electric utilities, is included.

Industrial Consumption : Natural gas used by manufacturing and mining establishments for heat, power, and chemical feedstock.

Lease and Plant Fuel : Natural gas used in well, field, and lease operations (such as gas used in drilling operations, heaters, dehydrators, and field compressors), and as fuel in natural gas-processing plants.

Natural Gas: A mixture of hydrocarbon compounds and small quantities of various nonhydrocarbons existing in the gaseous phase or in solution with crude oil in natural underground reservoirs at reservoir conditions.

Nonutility Power Producer: A company which owns electric generating capacity and is not an electric utility. Nonutility power producers include qualifying cogenerators, qualifying small power producers, and other nonutility generators (including independent power producers).

Onsystem: Any point on or directly interconnected with, a transportation, storage, or distribution system operated by a natural gas company.

Pipeline: A continuous pipe conduit, complete with such equipment as valves, compressor stations, communications systems, and meters, for transporting natural and/or supplemental gas from one point to another, usually from a point in or beyond the producing field or processing plant to another pipeline or to points of use. Also refers to a company operating such facilities.

Pipeline Fuel: Gas consumed in the operation of pipelines, primarily in compressors.

Reburn : An advanced cofiring technique using natural gas to reduce pollution from electric power plants.

Receipts: Gas physically transferred into the responding company's transportation, storage, and/or distribution facilities. 
Repressuring : The injection of gas into oil or gas reservoir formations to effect greater ultimate recovery.

Residential Consumption: Natural gas used in private dwellings, including apartments, for heating, air conditioning, cooking, water heating, and other household uses.

Storage Additions:Volumes of gas injected or otherwise added to underground natural gas reservoirs or liquefied natural gas storage.

Storage Withdrawals: Volumes of gas withdrawn from underground storage or liquefied natural gas storage.

Supplemental Gaseous Fuels Supplies: Synthetic natural gas, propane-air, coke oven gas, refinery gas, biomass gas, air injected for Btu stabilization, and manufactured gas commingled and distributed with natural gas.
Unaccounted For: Represents differences between the sum of the components of natural gas supply and the sum of the components of natural gas disposition. These differences may be due to quantities lost or to the effects of data-reporting problems. Reporting problems include differences due to the net result of conversions of flow data metered at varying temperature and pressure bases and converted to a standard temperature and pressure base; the effect of variations in company accounting and billing practices; differences between billing cycle and calendar period time frames; and imbalances resulting from the merger of data reporting systems that vary in scope, format, definitions, and type of respondents.

Underground Storage: The storage of natural gas in underground reservoirs at a different location from which it was produced. 